\title{
Third-generation femtosecond technology
}

Hanieh Fattahi

München 2015 



\title{
Third-generation femtosecond technology
}

Hanieh Fattahi

\author{
Dissertation \\ an der Physik \\ der Ludwig-Maximilians-Universität \\ München \\ vorgelegt von \\ Hanieh Fattahi \\ aus Teheran, Iran
}

München, den 8.12.2014 
Erstgutachter: Prof. Ferenc Krausz

Zweitgutachter: Prof. Jens Biegert

Tag der mündlichen Prüfung: 15.01.2015 
to

- Asghar 



\footnotetext{
Every man is the creature of the age in which he lives: very fere are able to raise themselves above the ideas of the times.

The first principle is that you must not fool yourself. and you are the easiest person to fool. Richard Foymman 

This table is dedicated to Prof. Ferenc Krausz for his ambitions, intelligent insight and sense of humor!

\begin{tabular}{c|ccc} 
& power (hp) & awesomeness & where? \\
\hline enhancement cavity* & 536.4 & 1600 & LMU \\
\hline BMW i8 & 333 & 32.515 & Alte Heide \\
\hline BMW 6er Coupé & 320 & 13.69 & MPQ parking lot \\
\hline 2J thin-disk laser & 26.8 & 10 & TSL \\
\hline 2J-pumped OPCPA & 4.62 & 690 & --- \\
\hline thin-disk oscillator & 3.566 & 1.28 & LMU \\
\hline XCPA thin-disk laser & 1.34 & 0.5 & TSL/LEX \\
\hline PFS pump & 0.536 & 0.5 & MPQ \\
\hline LWSpro & 0.327 & 244 & LEX \\
\hline PFSpro & 0.27 & 40 & LEX \\
\hline CPA thin-disk laser & 0.08 & 0.04 & MPQ \\
\hline PFS OPA & 0.067 & 10 & MPQ \\
\hline LWS20 OPA & 0.067 & 11 & MPQ
\end{tabular}

awesomeness $_{\mathrm{car}}=$ power $\times$ price/mass

awesomeness $_{\text {laser }}=$ energy $\times$ repetition rate/pulse duration $(\mathrm{fs})$

* loachim is a bit cheating here!

Carstens et. al, OL, 2014

Brons et. al, OL, 2014

Fattahi et. al, Optica, 2014

Klingebiel, OE, 20 I I

Metzger et. al, OL, 2009

Skrobol et. al, OE, 2012

Herrmann et. al, OE, 2010 

I had the advantage of incredibly enjoyable scientific discussions about the material of this thesis with my best friend and office mate, Dr. Nicholas Karpowicz, that it scares me to not name him in advance. 



\section{Zusammenfassung}

Zur Erzeugung höchstintensiver, ultrakurzer Laserimpulse ist nach anfänglichen Erfolgen mit Farbstofflasern seit etwa zwanzig Jahren die Verwendung von breitbandig verstärkenden Festkörperlasern die vorherschende Methode. Dieser Technik verhalf die Erfindung der "chirped pulse amplification" (CPA) zum Durchbruch, bei der ein zeitlich gestreckter Lichtimpuls im Lasermedium verstärkt und erst nach der Verstärkung, einhergehend mit einer signifikanten Zunahme der Spitzenleistung, zu seiner kürzest möglichen Dauer komprimiert wird.

Um auch die Beschränkungen dieser Technologie zu überwinden, haben Forschungslaboratorien in jüngerer Zeit die "optical parametric chirped pulse amplification" (OPCPA) als Ausweg erkannt. Dank der zunehmenden Zerstörschwelle nichtlinearer Medien bei abnehmender Pumpimpulsdauer, verspricht dieses Laserprinzip einzigartige Betriebsparameter. Mit Kurzimplusscheibenlasern als Pumpquelle $\left(\tau_{\text {pump }} \leq 1 \mathrm{ps}\right.$ ) wird die Erzeugung von Laserimpulsen von nur wenigen Feldzyklen Dauer möglich, bei gleichzeitig bisher unerreichbar scheinenden Werten der Spitzenleistung im Terawattund der durchschnittlichen Leistung im Kilowattbereich.

Die vorliegende Arbeit betrachtet die wesentlichen theoretischen Gesichtspunkte, die der Planung eines OPCPA-Lasersystems zur Erzeugung von höchstintensiven, ultrakurzen Laserimpulsen zugrunde liegen und präsentiert ihre experimentelle Überprüfung.

Im Einzelnen werden die Erzeugung eines breitbandigen Seed Impulses mit fester Phasendifferenz zwischen der Trägerwelle und der Intensitätseinhüllenden diskutiert, praktische Erwägungen zum Betrieb eines Hochleistungs Scheibenlasers als Pumpquelle dargestellt und die experimentelle Realisierung eines mehrstufigen OPCPA-Systems mit anschließender Kompression der Laserimpulse beschrieben. Die Ergebnisse einer theoretischen Untersuchung zeigen die Möglichkeiten der Optimierung als wellenlängenabstimmbare oder besonders breitbandige Laserquelle auf.

Die Arbeit wird von der Konzeptstudie eines optischen Wellenformsynthetisators abgeschlossen, der mehrere Oktaven spektraler Bandbreite unterstützen und gleichzeitig Multi-Terawatt Spitzenimpulsleistungen erzielen 
kann. Basierend auf diesen Untersuchungen erscheint eine solche Laserquelle möglich und es werden die notwendigen experimentellen Voraussetzungen dargestellt. Ausgehend von den erwarteten Impulsparametern zeigt eine numerische Modellierung, dass mit der Verfügbarkeit solcher Laserimpulse Attosekundenspektroskopie bei Photonenenergien im Bereich harter Röntgenstrahlung möglich wird. 


\section{Abstract}

Chirped pulse amplification in solid-state lasers is currently the method of choice for producing high-energy ultrashort pulses, having surpassed the performance of dye lasers over 20 years ago. The third generation of femtosecond technology based on short-pulse-pumped optical parametric chirped pulse amplification (OPCPA) holds promise for providing few-cycle pulses with terawatt-scale peak powers and kilowatt-scale-average powers simultaneously, heralding the next wave of attosecond and femtosecond science.

OPCPA laser systems pumped by near-1-ps pulses support broadband and efficient amplification of few-cycle pulses due to their unrivaled gain per unit length. This is rooted in the high threshold for dielectric breakdown of the nonlinear crystals for even shorter pump pulse durations. Concomitantly, short pump pulses simplify dispersion management and improve the temporal contrast of the amplified signal.

This thesis covers the main experimental and theoretical steps required to design and operate a high-power, high-energy, few-cycle OPCPA. This includes the generation of a broadband, high-contrast, carrier envelope phase (CEP)-stable seed, the practical use of a high-power thin-disk regenerative amplifier, its efficient use for pumping a multi-stage OPCPA chain and compression of the resulting pulses. A theoretical exploration of the concept and its extension to different modes of operation, including widely-tunable, high-power multi-cycle pulse trains, and ultrabroadband waveform synthesis is presented.

Finally, a conceptual design of a field synthesizer with multi-terawatt, multi-octave light transients is discussed, which holds promise for extending the photon energy attainable via high harmonic generation to several kiloelectronvolts, nourishing the hope for attosecond spectroscopy at hard-x-ray wavelengths. 


\section{Contents}

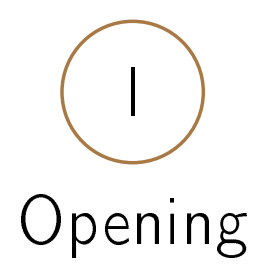

1 Introduction

7 Outline of the thesis

\section{(II) \\ Theories and experiments}

21 How to amplify photons

1.1 Optical parametric amplification (OPA) 23

Intensity and gain 29

Amplification and saturation 35

Optimum pump-pulse duration 37

Optimum pump-to-signal energy ratio 43

OPA phase 47

1.2 Yb:YAG based OPCPA systems 50

1.3 Tunable OPCPA 58

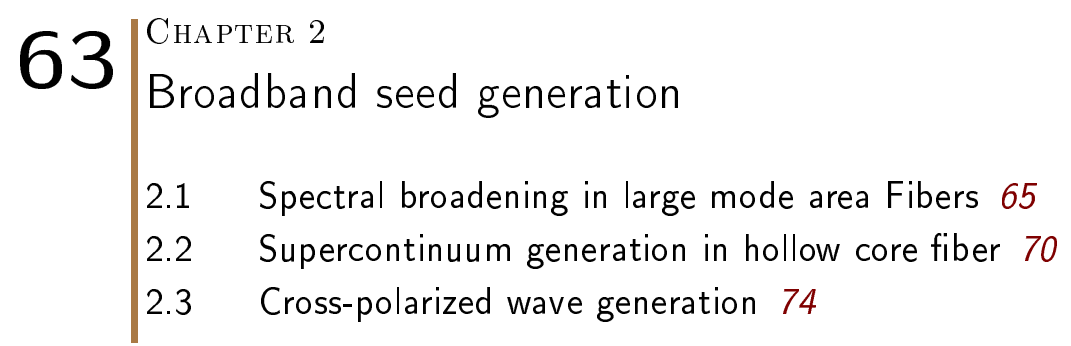


|2.4 Difference frequency generation 84
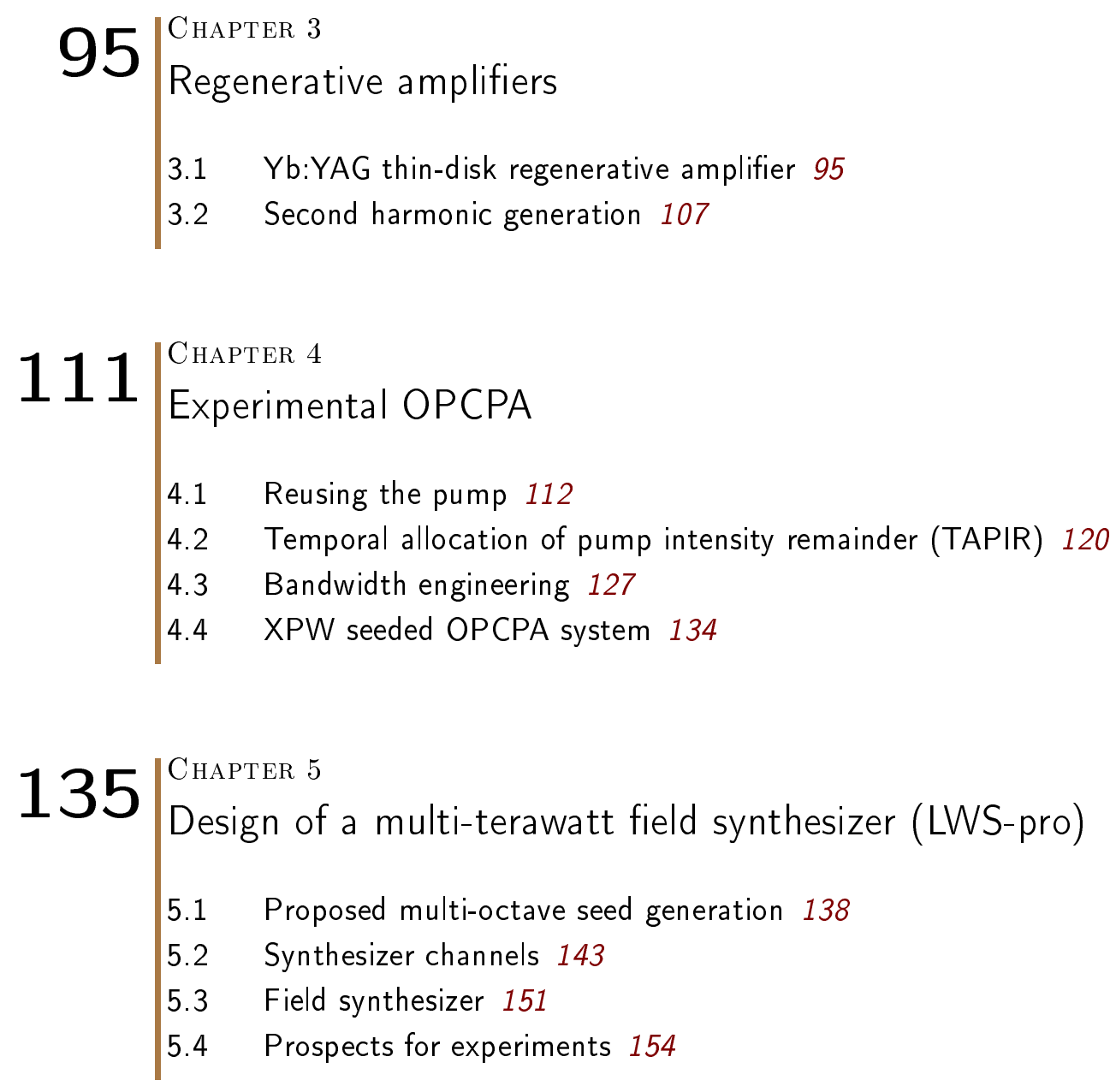

$\left.159\right|_{\text {Conclusion and outlook }} ^{\text {СhAрте } 6}$

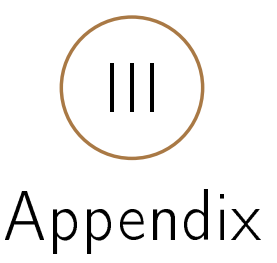


$165 \mid \begin{aligned} & \text { ChAртеR A } \\ & \text { In situ spectral interferometry as a pulse compression tool }\end{aligned}$

$\left.169\right|_{\text {Toolbox and ideas }} ^{\text {CHAPTER }}$

$\left.171\right|_{\text {Simulation details of Fig. } 0.2} ^{\text {CHAPTER } \mathrm{C}}$

$\left.173\right|_{1 \text {-ps-pumped supercontinuum generation }} ^{\text {СнартеR D }}$

$175 \mid \begin{aligned} & \text { ChAPTER E } \\ & \text { Publications }\end{aligned}$

E.1 Decoupling chaotic amplification and nonlinear phase in high-energy thin-disk amplifiers for stable OPCPA pumping 175

E.2 Third-generation femtosecond technology 184

E.3 Efficient, octave-spanning difference-frequency generation using fewcycle pulses in simple collinear geometry 204

E.4 High efficiency, multi-mJ, sub $10 \mathrm{fs}$, optical parametric amplifier at 3 $\mathrm{kHz} 209$

E.5 Pump-seed synchronization for $\mathrm{MHz}$ repetition rate, high-power optical parametric chirped pulse amplification 212

$221 \mid \begin{aligned} & \text { CHAPTER F } \\ & \text { Data archiving }\end{aligned}$ 
$225 \mid$ References 


\section{List of Figures}

0.1 Conceptual architecture of next generation laser systems . . . . 4

0.2 Comparison between different generations of femtosecond technology .......................... 5

1.1 Wave-picture and photon-picture of OPA . . . . . . . . . 23

1.2 Noncollinear OPA . . . . . . . . . . . . . . . . . 26

1.3 Intensity-dependent gain in $\mathrm{BBO} \ldots \ldots \ldots \ldots \ldots \ldots$

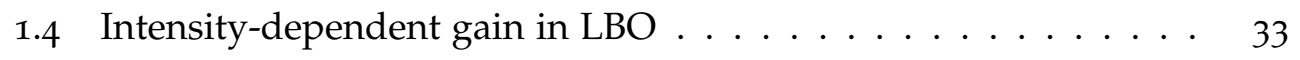

1.5 Phase-mismatch in OPCPA . . . . . . . . . . . . . . . . 34

1.6 OPCPA gain ........................ 35

1.7 Temporal profile of pump and signal pulses in OPCPA . . . . . 38

1.8 Spatial profile of pump beam after amplification in an OPCPA . 39

1.9 Spatial profile of seed beam after amplification in an OPCPA . . 40

1.10 Saturated OPCPA versus over-saturated OPCPA . . . . . . . . $44^{41}$

1.11 OPCPA with different pump-pulse duration $\ldots \ldots \ldots . . . . .42$

1.12 OPCPA gain and efficiency for different signal-pump energy ratio 44

1.13 OPCPA spectra for different signal-pump energy ratio . . . . . 45

1.14 Spectral behavior of a broadband OPCPA in saturation regime . 46

1.15 OPA phase . . . . . . . . . . . . . . . . . . . . . . 49

1.16 MIR OPA, crystals comparison . . . . . . . . . 51

1.17 Phase-matching, DOPA . . . . . . . . . . . . . 52

1.18 Phase-matching, LBO . . . . . . . . . . . . . . . . . . . . 53

1.19 Phase-matching, BBO and DKDP . . . . . . . . . . . . . . . 55

1.20 Broadband SHG in BBO and LBO . . . . . . . . . . . 57

1.21 Tunable OPA, general scheme . . . . . . . . . . . . . 59

2.1 LMA and HCF cross section . . . . . . . . . . . . . . . 65

2.2 Experimental setup of spectral broadening in LMA fiber . . . . 68

2.3 Parameters of a Yb:YAG oscillator . . . . . . . . . . . . 69

$2.4 n_{2}$ of different materials and the GDD curve of a set of chirped mirrors . . . . . . . . . . . . . . . . 69

2.5 SH-FROG measurement before HCF $\ldots \ldots \ldots \ldots . \ldots 72$ 
2.6 Experimental layout of spectral broadening in a HCF . . . . . 73

2.7 Cross-polarized wave generation . . . . . . . . . . 75

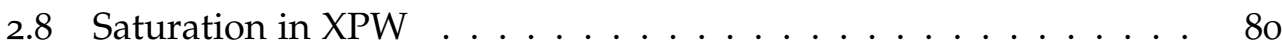

2.9 Experimental XPW-1 . . . . . . . . . . . . . . . . . 81

2.10 TG-FROG measurement of the pump of XPW . . . . . . . 82

2.11 Experimental XPW-2 . . . . . . . . . . . . . . . . . 83

2.12 Different DFG types in a BBO crystal . . . . . . . . . . 85

2.13 DFG spectra for different pump-signal energy ratio . . . . . . . 86

2.14 Efficiency of DFG . . . . . . . . . . . . . . . . . 88

2.15 Measured and simulated DFG spectra . . . . . . . . . . . 92

2.16 Redshift of the pump spectrum in DFG . . . . . . . . . . . . . . . 92

2.17 DFG, experimental layout . . . . . . . . . . . . . . 93

3.1 Effect of SPM in time and frequency domains . . . . . . . . . 98

3.2 SPM vs. round trip number in regenerative amplifier . . . . . . 99

3.3 Chaos in regenerative amplifiers ............ 100

3.4 Temporal evolution of regenerative amplifier pulses affected by

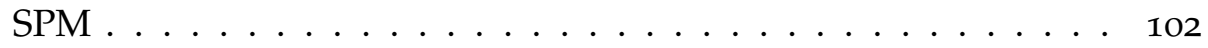

3.5 Effect of suboptimal pump pulses on OPA and SHG . . . . . 104

3.6 Simulated SHG in LBO and BBO . . . . . . . . . . . . 107

3.7 SHG in LBO and $\mathrm{BBO}$ experimental results . . . . . . . . . . . 109

$4.1 \quad$ OPCPA design-recycling pump energy $\ldots \ldots \ldots \ldots \ldots$

4.2 SHG of the OPCPA pump and XFROG measurement of the OPCPA

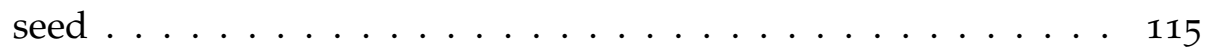

4.3 OPCPA, beam profiles . . . . . . . . . . . 116

4.4 OPCPA spectra, recycling the pump energy . . . . . . . . . 117

4.5 OPCPA spectrum and SH-FROG measurement of the compressed

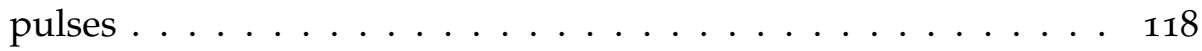

4.6 OPCPA design, temporal stacking . . . . . . . . . 121

4.7 Spatio-temporal profile of pump in the temporal stacking design of OPCPA . . . . . . . . . . . . . . . . . 122

4.8 Experimental OPCPA, temporal stacking design . . . . . . . . . 126

4.9 Bandwidth engineering in an OPA, theory . . . . . . . . 128

4.10 Bandwidth engineering in an OPA, experiment . . . . . . . . 129

4.11 OPCPA spectrum . . . . . . . . . . . . . . 131

4.12 OPCPA FROG measurement, seed . . . . . . . . . . . 132

4.13 OPCPA FROG measurement, second and third stage $\ldots \ldots . .133$

4.14 XPW-seeded OPCPA . . . . . . . . . . . . . . . . . . 134

5.1 Prototypical multi-octave high energy field synthesizer . . . . . 137

5.2 Multi-octave seed generation scheme $\ldots \ldots \ldots . . \ldots 139$ 
5.3 Alternative seed generation schemes . . . . . . . . . . . 140

5.4 Low-harmonic generation . . . . . . . . . . . . . . . . 144

5.5 Spectra of different channels of the designed synthesizer . . . . . 147

5.6 Simulated beam profile of different channels of the synthesizer . 148

5.7 Calculated synthesized waveforms . . . . . . . . . . 152

5.8 HHG, pulse duration dependence . . . . . . . . . . . . . . 154

5.9 HHG, CEP dependence . . . . . . . . . . . . . . . . . . . . 154

5.10 HHG, amplitude dependence . . . . . . . . . . . . . . . 156

5.11 HHG, chirp dependence . . . . . . . . . . . . . . . 157

A.1 Spectral interferometry . . . . . . . . . . . . . . . 167

D.1 1 -ps pumped supercontinuum generation in bulk . . . . . . 174

\section{List of Tables}

1.1 General parameters of the tunable OPCPA system. . . . . . . . 60

1.2 Detailed parameters of the tunable OPCPA system. . . . . . . . 61

2.1 Parameters of the supercontinuum generation in YAG and $\mathrm{YVO}_{4}$ crystals ......................... 66

2.2 Comparison between different techniques to enhance the temporal contrast of ultrashort pulses. . . . . . . . . 78

3.1 Possible approaches for the reduction of SPM in a thin-disk regenerative amplifier. . . . . . . . . . . 105

5.1 Detailed parameters of the simulated channels of the synthesizer 146

5.2 Summary of possible challenges for the designed synthesizer . . 150

C.1 Summary of the simulated OPCPA systems depicted in Fig. 0.2 . 172 


\section{Acronyms}

BOC Balanced Optical Cross Correlator

CEP Carrier Envelope Phase

COM Center Of Mass

CPA Chirped Pulse Amplification

CW Continues Wave

DFG Difference Frequency Generation

DOPA Degenerate Optical Parametric Amplification

DSLM Dual Spatial Light Modulator

FWHM Full Width at Half Maximum

GDD Group Delay Dispersion

GVD Group Velocity Dispersion

HCF Hollow Core Fiber

HHG High Harmonic Generation

KLM Kerr Lens Mode-locked

LMA Large Mode Area

MIR Mid Infrared

NCPM Non Critical Phase-Matching

NIR Near Infrared

NOPA Noncollinear Optical Parametric Amplification 
OPA Optical Parametric Amplification

OPCPA Optical Parametric Chirped Pulse Amplification

PC Pockels Cell

PCF Photonic Crystal Fiber

PPLN Periodically Poled $\mathrm{LiNbO}_{3}$

PPSLT Periodically Poled Stoichiometric $\mathrm{LiTaO}_{3}$

SFG Sum Frequency Generation

SHG Second Harmonic Generation

SH-FROG Second Harmonic - Frequency Resolved Optical Gating

SPM Self Phase Modulation

SVEA Slowly Varying Envelope Approximation

TAPIR Temporal Allocation of Pump Intensity Remainder

TG-FROG Transient Gating - Frequency Resolved Optical Gating

THG Third Harmonic Generation

TPA Two Photon Absorption

UV Ultra Violet

VIS Visible

XFROG Cross-Correlation Frequency Resolved Optical Gating

XPM Cross Phase Modulation

XPW Crossed Polarized Wave Generation

XUV Extreme Ultra Violet 


\section{Part I}

\section{Opening}





\section{Introduction}

It is a dream (one of mine, at least) to understand the hide and seek of photons, incognito messengers of information that let us explore the universe with quantum transitions. I am fascinated by the Greek and Persian philosophies of light, by the magic story of photons and what they make us perceive as reality. Physics and photonics are the grand attracting magnets as they make our further acquaintance with these tiny packets of energy possible. The story of the interconnection between life on earth and light spans a history of four billion years, when the first interaction between a bacterium and a photon took place. But the real success story of the human fascination with photons began a century ago.

Past The discovery of the principle of spontaneous and stimulated emission by Einstein (1917) is the root of almost all recent innovations in photonics. The suggested method for producing population inversion in the $\mathrm{PhD}$ thesis of Fabrikant in 1939 and optical pumping by Kastler (1950), paved the route for demonstration of the first maser by Gordon et al. (1955). In 1958, Schawlow and Townes introduced the concept of the optical maser (laser) (Schawlow and Townes, 1958) and two years later the first laser in a ruby crystal was demonstrated by Maiman (1960). The big question was on the table: What is this army of coherent photons oscillating at $\mathrm{PHz}$ frequencies with intensities around $10^{10} \mathrm{~W} / \mathrm{cm}^{2}$ good for?

One year later, lasers gave birth to the field of nonlinear optics by the generation of low-order optical harmonics in crystalline quartz (Franken et al., 1961). For electric field strengths less than the characteristic field strength of atoms, perturbative phenomena dominate. Here, the light perturbs the atoms, inducing a nonlinear (anharmonic) polarization and low-order harmonics may be generated.

In the 1970s, passively mode-locked dye lasers produced the first pulses shorter than 1 ps and gave birth to femtosecond technology (Ippen et al., 1972). Advances in laser oscillators led to pulse durations of a few tens of femtoseconds (Fork et al., 1981), while the poor energy storage capability of dye lasers limited the pulse energies to microjoules and the peak powers to 
megawatts (Knox et al., 1984). This first-generation femtosecond technology opened the door for direct time-domain investigations of processes that were once immeasurably fast, such as molecular dynamics, chemical reactions, and phase transitions in condensed matter (Rowlinson, 1969; Zewail, 2000).

By the end of the 1980s, broadband solid-state lasers with large energy storage capabilities offered the potential for additional pulse shortening as well as boosting of the pulse energy and peak power by many orders of magnitude (Moulton, 1986; Petričević et al., 1988).

Present Although the idea of optical stimulated emission was triggered by the same phenomena in the microwaves and masers, it took physicists fifteen years to exploit chirped-pulse amplification (Strickland and Mourou, 1985), a well-known technique in microwave technology (Cook, 1960), to solid-state lasers in particular Ti:sapphire-based systems (Nabekawa et al., 1998). At peak intensities on the order of $10^{14} \mathrm{~W} / \mathrm{cm}^{2}$ (Yanovsky et al., 2008; Sung et al., 2010), the laser field strength became comparable to the electric field experienced by a bound electron and nonlinear optics stepped into a new area, the so called non-perturbative regime.

In this regime electrons absorb photons in excess of the minimum required for ionization to occur (Agostini et al., 1979) and may lead to the generation of high-order harmonics, a coherent spectrum that could reach several kilo-electronvolts (Buth et al., 2013; Seres et al., 2005; Popmintchev et al., 2012). Ti:sapphire-based CPA systems combined with chirped multilayer mirrors form the second-generation of femtosecond technology, providing pulses with ultrahigh (petawatt) peak powers at relatively-low repetition rates (Chu et al., 2013) and moderate-peak-power (gigawatt) pulses at kilohertz repetition rates (Hädrich et al., 2013). Second-generation femtosecond technology paved the way for the emergence of entirely new research fields and technologies such as attosecond science (Krausz and Ivanov, 2009) and has been the workhorse for the generation of isolated attosecond pulses in many laboratories around the world for more than a decade (Cavalieri et al., 2007).

Future Electron dynamics in atoms and ions take place on the time scale of attoseconds. The capabilities of the current high-harmonic generation (HHG) sources are limited to energies around a few hundred $\mathrm{eV}$ and to pulse durations of several tens of attoseconds. Attosecond pulses at high repetition rates, with photon energies as high as several kiloelectronvolts in the regime of hard x-rays, would enable the probing of electron dynamics in inner shells and ${ }_{4} \mathrm{D}$ imaging of the electronic structure of matter with atomic temporal and spatial resolution, and open the door to time resolved intra- 
nuclear dynamics in addition to the ability to track the electron dynamics inside atoms.

Comprising optical parametric chirped-pulse amplification (OPCPA) (Dubietis et al., 1992) driven by terawatt-scale pulses from ytterbium-based lasers at kilowatt-scale average power, third-generation femtosecond technology will combine high (terawatt-scale) peak powers with high (kilowattscale) average powers in ultrashort optical pulse generation for the first time. This unprecedented parameter combination will allow the exploration of extreme nonlinearities in matter and extend ultrashort pulse generation from subnanometer to multimicrometer wavelengths at unforeseen flux levels, holding promise for yet another revolution in ultrafast science.

In this work it will be shown that diode-pumped ytterbium-doped thindisk lasers offer a promising route to fulfill requirements to implement thirdgeneration femtosecond technology in the conceptual architecture outlined in Fig. 0.1 and will outperform the first- and second- generations of femtosecond technology by reaching unprecedented peak and average powers, simultaneously (Fig. 0.2).

Besides a variety of operational modes, this next generation technology of waveform-controlled light offers an agile electromagnetic force whose strength can be increased from zero to maximum within hundred attoseconds and also kept nearly constant over several femtoseconds. The generation of synchronized $\mathrm{UV}$ and $\mathrm{X}$-ray light at $\mathrm{kHz}$ repetition rates open the door for novel attosecond techniques such as UV transient absorption spectroscopy, XUV pump-probe spectroscopy, and attosecond x-ray diffraction. This high-energy, high-power field synthesizer will make it possible to understand multi-electron phenomena in solids and let us explore the ultimate limits of solid-state electronics. The new insights it leads to could bring about the next breakthroughs in optoelectronics needed to speed up information technology to light frequencies, reaching the world of light-wave electronics (Krausz and Stockman, 2014).

This thesis combines the first experimental and theoretical steps towards the realization of a light source to fully exploit the potential of the third generation of femtosecond laser technology. It includes feasibility tests performed at the Laboratory for Attosecond Physics at the Max Planck Institute of Quantum Optics and the Ludwig-Maximillians-University, and simple theoretical models to understand them. It also contains my personal interpretation of the phenomena which I dealt with in the laboratory, over the course of the last six years. 


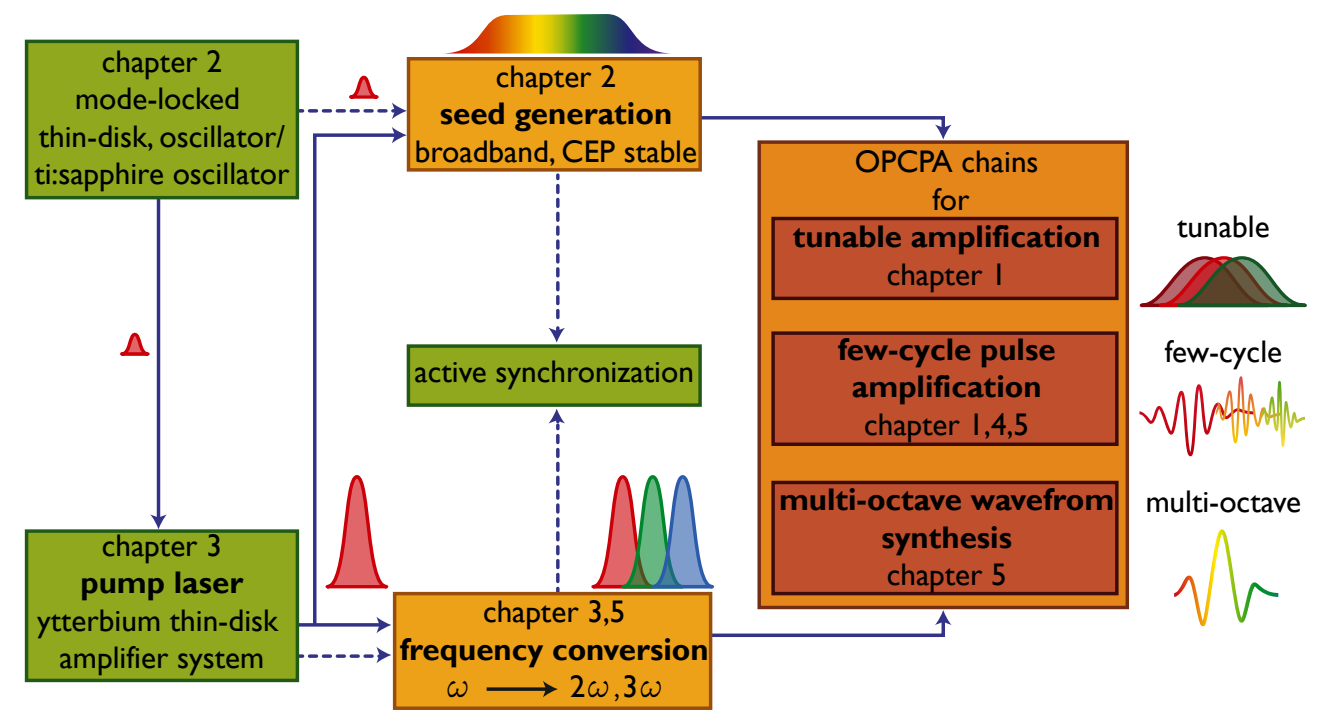

Figure 0.1: Basic conceptual architecture of an OPCPA system. A sub-ps ytterbium laser oscillator or a Ti:sapphire oscillator, seeds the pump source. The broadband seed can either be generated from the output of the picosecond pump laser (solid arrows) or directly from the oscillator (dashed arrows). In the latter case an active temporal synchronization is needed between the pump and seed pulses of the OPCPA chain. This system can be operated to generate i) widely tunable pulses of few-tens of femtoseconds duration, ii) few-cycle pulses in different spectral ranges, or iii) multi-octave controlled waveforms with sub-optical-cycle structure. These blocks are discussed in detail in the respective chapters as indicated in the figure (Fattahi et al., 2014a). 


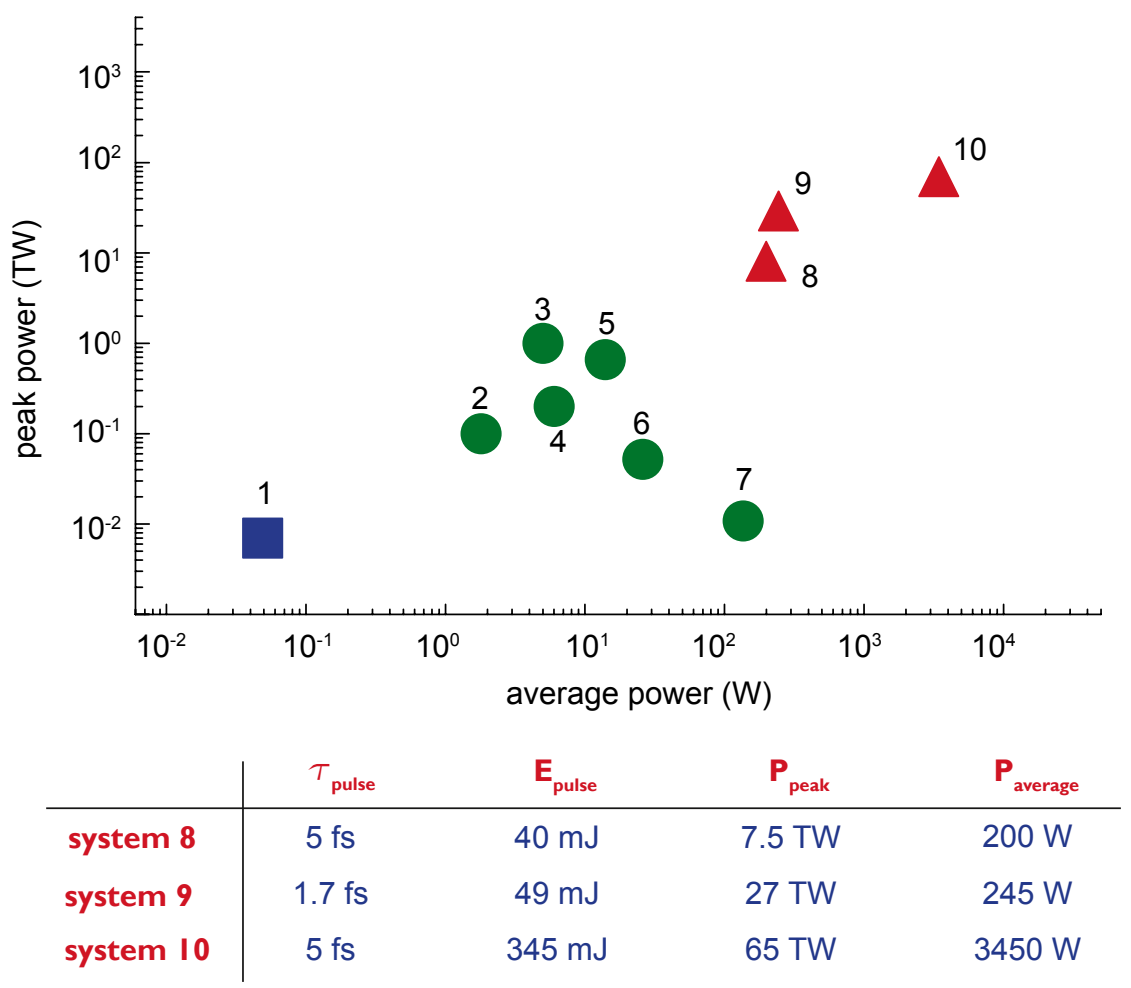

Figure 0.2: Comparison between different generations of laser systems: The blue dot represent the performance of the first-generation of femtosecond technology in terms of the average and peak power ( 1 , corresponds to (Rolland and Corkum, 1986)), the green dots contain the second-generation of femtosecond technology (2-7, corresponds to (Hentschel et al., 2000; Bohman et al., 2010; S. Chen et al., 2009; Nabekawa et al., 1998; Matsushima et al., 2006; Hädrich et al., 2013)) and the red dots show the simulated results for OPCPA based on pump sources under development and envisioned. The table summarizes the predicted output parameters of these systems. The details of the simulation are presented in Appendix C (Fattahi et al., 2014a). 



\section{Outline of the thesis and the main results}

This thesis presents experimental and theoretical evidences for short-pulse pumped OPCPA systems as the most promising route to achieve high-contrast, few-cycle pulses scalable in peak and average power simultaneously.

It will be shown that the optimum duration of pump pulses in an OPCPA system to achieve a broad amplification gain and high conversion efficiency concurrently is of the order of 1 ps. This constitutes a trade-off between a high resistance to optical damage (decreasing for longer pulses) and a small temporal walk-off between pump and signal pulses relative to their duration (increasing for shorter pulses).

The realizability of this approach is subject to the availability of pump lasers delivering scalable high-energy near-1ps-pulses with near diffraction limited beam quality at repetition rates in the $\mathrm{kHz}$ to $\mathrm{MHz}$ range. $\mathrm{Yb}$ :YAG think disk lasers, containing all the above mentioned parameters are the most developed approach to achieve this goal and therefore formed the heart of all the experimentally demonstrated and designed OPCPA systems presented in this thesis.

Having the concept of short-pulse pumped OPCPA in place along with reliable pump sources, all the necessary ingredients of an OPCPA system will be discussed. This includes the full study of: alternative schemes for generation of multi-octave coherent seed sources for OPCPA, optimization of the performance of $\mathrm{Yb}$ :YAG thin-disk lasers for daily use, and presenting alternative schemes for increasing conversion efficiency and amplification bandwidth.

The gain bandwidth and therefore the attainable pulse duration in an OPCPA system is limited to the phase-matching range of the nonlinear crystal. To generate confined, sub-cycle pulses with controlled variability of the electric field, few-cycle pulses at different carrier frequencies can be synthesized temporally. The outcome of combining this concept with short-pulsepumped OPCPA concept are sub-cycle pulses, scalable in peak and average power. It will be shown that few-cycle OPCPA pulses with $\mathrm{mJ}$ levels of en- 
ergy in the visible, near-infrared, and mid-infrared ranges pumped by different harmonics of an Yb:YAG think-disk laser can be synthesized to form multi-TW sub-cycle waveforms. This extends the horizon of high-energy attosecond pulse generation to $\mathrm{keV}$ range.

Chapter $\mathbb{1}$

SUMMARY POINTS

Chapter 1 gives an overview of optical parametric amplification and is dedicated to the theoretical description of the parametric amplification process. Numerical simulations for optimization of different parameters in OPCPA systems such as pump pulse duration, signal-topump energy ratio, and OPCPA phase are performed and discussed in detail. At the end of the chapter a general discussion about Yb:YAG thin-disk based OPCPA systems is presented. This section discusses the possibility of using fundamental, second- and third-harmonics of Yb:YAG lasers to pump few-cycle OPCPA systems in the visible, near-infrared, and mid-infrared. Here, different nonlinear crystals are analytically compared and the best candidates are suggested. The chapter is finalized by proposing a new scheme for a widely tunable OPCPA system (from ultraviolet to mid-infrared) based on Yb:YAG thin-disk lasers.

\section{MAIN RESULTS}

In this chapter the behavior of OPCPA efficiency and amplification bandwidth versus the pump pulse duration (from fs to ns range) are theoretically investigated. It is shown the optimum pump pulse duration is in the range of $1-10 \mathrm{ps}$ at FWHM. This study reveals that in this range, 1 ps pump pulses are favorable over $10 \mathrm{ps}$ pulse duration, as they provide higher damage threshold on materials. It is theoretically demonstrated that increase in the OPCPA's pump intensity results in the raise of the small signal gain and its amplification bandwidth. This indicates another advantage of the short-pulse-pumped OPCPA concept.

Another important parameter in designing OPCPA system, is the input signal-to-pump energy ratio. The performed simulations in this chapter indicate, for low input seed energy, saturation of different frequency components happens at different length of the nonlinear medium. This results in backconversion of energy for some frequency components while the others have not reached saturation yet. The optimum signal-to-pump energy ratio of $10^{-3}$ is suggested for relatively 
uniform amplification while high conversion efficiency and amplification gain is achieved.

This chapter also includes simulations demonstrating that the phasemismatch during the amplification process in an OPCPA system results in high-order phase. This additional phase is imprinted on the spectral phase of the signal. It is shown that increase in the amplification gain leads to increase in the OPA phase. This additional phase should be considered for Fourier limit pulse compression in OPCPA systems.

Finally a new scheme for an OPCPA-based tunable source is suggested. Here, a multi-octave seed pulse is temporally stretched to a pulse duration of several times the pump pulse duration. Using different harmonics of an Yb:YAG thin-disk laser, and by adjusting the temporal delay between seed and pump pulses, sub $30 \mathrm{fs}$ pulses with $\mathrm{mJ}$ level of energy are generated. This table-top tunnable source is scalable in peak- and average-power with tunnablity in central frequency and temporal duration of pulses.

\section{Chapter 2}

\section{SUMMARY POINTS}

Chapter 2 is dedicated to study alternative techniques for the generation of multi-octave broadband spectra suitable for seeding OPCPA systems. OPCPA seed pulses are required to have high temporal contrast, smooth spectral phase and preferably passively stable carrier envelope phase. This can be provided by broadband Ti:sapphire oscillators with $\mathrm{nJ}$ of energy or kerr-lens mode-locked Yb:YAG oscillators with $\mu \mathrm{J}$ level of energy. Both require an additional active temporal synchronization to overcome temporal jitter between seed and pump pulses and they are limited in energy. As an alternative approach a broadband seed with several hundreds of $\mu \mathrm{J}$ energy can be generated by employing a Ti:sapphire amplifier. However, this scheme also demands for an active temporal synchronization system. Generating seed pulses from a near-1 ps pulses of a Yb:YAG amplifier will eliminate the demand for any external synchronization system and provides the possibility of scaling the seed energy level to $\mathrm{mJ}$.

Therefore in this chapter a scheme to generate an octave seed pulses from a 1-ps Yb:YAG oscillator by combining spectral broadening in large mode area (LMA) fiber and supercontinuum generation in bulk 
is studied. Later, supercontinuum generation of $30 \mathrm{fs}$ pulses in a hollow-core-fibers (HCF) is discussed. The chapter concludes with a full experimental and theoretical study of cross-polarized-wavegeneration (XPW) and difference frequency generation (DFG) serving as suitable techniques for OPCPA seed generation.

\section{MAIN RESULTS}

In a cascaded broadening setup, 1 ps pulses centered at $1030 \mathrm{~nm}$ were spectrally broadened in an LMA fiber and temporally compressed to $30 \mathrm{fs}$ and used for supercontinuum generation in YAG and $\mathrm{YVO}_{4}$ crystal. In this approach the spectrum of the generated supercontinuum reached $500 \mathrm{~nm}$. However the bottle neck of this approach is the LMA fiber which is limited to pump pulse energies of the order of several $\mu \mathrm{J}$.

After that a full theoretical and experimental study on XPW generation as a technique for enhancing the temporal contrast of seed pulses is presented. It is shown that by employing a two-stage XPW, the efficiency of the process increase from $5 \%$ to $9 \%$. The demonstrated theoretical study and experimental evidence shows in an XPW signal which had reached energy saturation, the generated spectrum becomes modulated due to impact of the other third-order nonlinear processes along with the backconversion of energy from the XPW signal to the pump. These third-order nonlinear processes also result in spectral broadening and generation of new frequencies. It is experimentally shown, the input pump spectrum which spans from $600 \mathrm{~nm}$ to $1000 \mathrm{~nm}$ can be extended to $1400 \mathrm{~nm}$ in a single XPW stage using $1 \mathrm{~mm}$-thick $\mathrm{BaF}_{2}$. This makes XPW a useful technique for OPCPA seed generation.

A multi-octave spectrum spanning from visible to mid-infrared can be generated by spectral broadening of mid-infrared CEP-stable pulses in a gas or a gas-filled hollow core fiber. This method benefits from efficient generation of CEP-stable pulses in mid-infrared in a DFG process, as DFG pulses have high temporal contrast, smooth spectral phase and they are passively CEP-stable.

To increase the efficiency of a single-pulse DFG, a simple idea is implemented experimentally. Here the efficiency of a broadband DFG signal, pumped by a few-cycle near-infrared-to-visible pulses, is increased to $12 \%$. This increase in efficiency is achieved by employing 
a $42 \mu \mathrm{m}$-thick quartz wave plate before the $500 \mu \mathrm{m}$-thick BBO crystal used as the nonlinear medium. The wave plate optimizes the photon distribution on ordinary and extra-ordinary axes of the type I crystal. It will be shown that the type I DFG, has a higher efficiency due to its reduced sensitivity to the material dispersion, whereas in type II DFG, the generated signal is more stable.

This results will be used in Chapter 5 to suggest alternative seedgeneration schemes for a multi-TW, super-octave field synthesizer.

\section{Chapter 3}

\section{SUMMARY POINTS}

Chapter 3 presents the dynamics of chirped pulse amplification in $\mathrm{Yb}$ :YAG thin-disk regenerative amplifiers as the most promising pump source for OPCPA systems. Here numerous simulations are performed to investigate the accumulated nonlinear phase during the amplification process in a regenerative amplifier. This chapter also includes a full experimental and theoretical study about second harmonic generation of Yb:YAG thin-disk lasers.

\section{MAIN RESULTS}

It will be shown experimentally and theoretically that by increasing the input seed energy of a thin-disk regenerative amplifier from $0.1 \mathrm{~nJ}$ to $17 \mathrm{~nJ}$ a stable mode of operation without doubling and chaos can be achieved. Increasing the input seed energy also makes it possible to use less number of round trips in the amplifier and consequently reduce the accumulated nonlinear phase on the beam in the expense of using higher pump energy. Experimental and theoretical evidence indicate, that reproducible pumping of OPCPA systems requires a wellcontrolled balance between the demands of avoiding chaotic pulse train dynamics and providing a reproducible spectral phase.

The frequency doubling of an Yb:YAG thin-disk laser in type I BBO and LBO crystals is the second subject of this chapter. Up to $70 \%$ of energy conversion is achieved in a $4 \mathrm{~mm} \mathrm{LBO}$ and a $2 \mathrm{~mm} \mathrm{BBO}$ at the pump peak intensity of $70 \mathrm{GW} / \mathrm{cm}^{2}$. Although $\mathrm{LBO}$ has a lower nonlinear coefficient compared to $\mathrm{BBO}$, it results in a similar conversion efficiency due to its smaller temporal walk-off. The achieved high conversion efficiency indicates the good quality of thin-disk amplifier in time and space. 


\section{Chapter 4}

\section{SUMMARY POINTS}

Chapter 4 is devoted to second harmonic-pumped, near-infrared (NIR) OPCPA systems. The chapter begins with a theoretical design study optimized for increasing OPCPA pump-to-signal conversion efficiency by recycling the residual pump energy in a cascaded OPCPA stage. Further optimization of efficiency is studied in a scheme consisting of controlled deposition of pump energy in different part of the spectrum. In the following, different methods for spectral bandwidth engineering by using different kinds of nonlinear crystal are experimentally and theoretically investigated.

\section{MAIN RESULTS}

The conversion efficiency of a 3-stage few-cycle OPCPA system, centered at $800 \mathrm{~nm}$ is increased to an unprecedented value of $30 \%$. This is achieved by reusing the residual pump energy after the second OPCPA stage to pump the third OPCPA stage. The $2.5 \mathrm{~mJ}$ pulses with $7.5 \mathrm{~W}$ of average power are temporally compressed to $10 \mathrm{fs}$ employing a chirped-mirror compressor providing $0.23 \mathrm{TW}$ of peak power.

In the second approach the possibility of increasing the OPCPA conversion efficiency by controlling the allocation of pump energy on different spectral components is theoretically discussed. In this technique seed pulses are temporally stretched to longer than pump pulse duration. The pump is reused after each OPCPA stage and the pump energy in each OPCPA stage is deposited on different part of the seed spectrum by adjusting the relative temporal delay between pump and signal. This technique also leads to an smooth amplified spectrum, which is demonstrated experimentally.

And finally, near-1-cycle pulses with $1.8 \mathrm{~mJ}$ of energy are generated in a 3-stage noncollinear OPCPA system centered at $1 \mu \mathrm{m}$. Here by using a $2 \mathrm{~mm} \mathrm{LBO}, 2 \mathrm{~mm} \mathrm{BBO}$, and $3 \mathrm{~mm}$ LBO crystal, the amplification bandwidth is extended to support near-1-cycle pulses. Using a chirped-mirror compressor resulted in $9 \mathrm{fs}$ pulses.

And finally in an all LBO 3-stage OPCPA system, generated spectrum from the XPW setup shown in chapter 2 is amplified to $1.2 \mathrm{~mJ}$ of energy. Here the amplified spectrum is smooth without any sharp features, indicating the enhanced contrast of the amplified pulses. 


\section{Chapter 5}

\section{SUMMARY POINTS}

Chapter 5 consists of a design study of a multi-channel OPCPA system based on Yb:YAG thin-disk lasers. Here, different harmonics of the laser are used to pump different few-cycle OPCPA channels in visible, near-infrared, and mid-infrared. Next, the generation of a variety of ultra-short wave transients from the three-channel OPCPAbased synthesizer and their optimization as a driving field for HHG in order to reach $\mathrm{keV}$ photon energies is discussed theoretically.

\section{MAIN RESULTS}

The full design study of a multi-TW, multi-octave OPCPA based field synthesizer is shown in this chapter. An Yb:YAG thin-disk laser with $200 \mathrm{~mJ}$ of energy operating at $5 \mathrm{kHz}$ is considered as a pump source for this system. In a cascaded second-order nonlinear stage, $40 \mathrm{~mJ}$ pulses at $343 \mathrm{~nm}, 74 \mathrm{~mJ}$ pulses at $515 \mathrm{~nm}$ are generated, leaving $86 \mathrm{~mJ}$ energy at $1030 \mathrm{~nm}$.

These pulses are used to pump three OPCPA chains employing BBO, $\mathrm{LBO}$, and $\mathrm{LiNbO}_{3}$, alternatively. The amplified spectrum in $343 \mathrm{~nm}$ pumped OPCPA chain spans from $450 \mathrm{~nm}$ to $650 \mathrm{~nm}$ and contains $7.1 \mathrm{~mJ}$ of energy. In the near-infrared channel the $680-1300 \mathrm{~nm}$ amplified spectrum contains $22.7 \mathrm{~mJ}$ of energy and the spectrum in the mid-infrared channel from $1700 \mathrm{~nm}$ to $2800 \mathrm{~nm}$ has $19.2 \mathrm{~mJ}$ of energy.

By synthesizing these amplified spectra after their temporal compression, and varying temporal delays between the three different channels, a variety of confined sub-cycle multi-TW waveforms could be generated.

In a series of simulations, the advantages of controlled waveforms over one-cycle and multi-cycle pulses for high harmonic generation in terms of generated photon cut-off energy is shown. It is shown by optimizing the energy ratio between the visible, near-infrared, and mid-infrared channels in favor of mid-infrared channel, the photon cut off energy can be extended dramatically in the high harmonic generation process. Simulation results indicate, chirping the waveform by shifting the blue channel to the front of the pulse also leads to an increase of the photon cut off energy. This could be understood by the role of blue photons in the leading edge of the pulse in ionization and the longer wavelength photons in the trailing edge of the 
waveform in electron recombination and high harmonic generation.

Comparison between the ionization probability of a $4 \mathrm{fs}$ pulse at $800 \mathrm{~nm}$ and a synthesized waveform at $1 \mu \mathrm{m}$, shows lower ionization rate for the waveform for the case of similar cut-off energy. This demonstrate another advantage of a waveform over few-cycle pulses for high harmonic generation process.

\section{Chapter 6}

\section{SUMMARY POINTS}

Finally, Chapter 5 is followed by a summary of the findings and perspectives for the future.

\section{Appendix A and D}

SUMMARY POINTS

Appendix A and D, cover two important subjects. Spectral interferometry as an important tool for characterizing chirped-mirror compressor and supercontinuum generation in bulk material pumped by 1 ps pulses. 


\section{List of publications}

- H. Fattahi, A. Schwarz, X. Geng, S. Keiber, D. E. Kim, F. Krausz, N. Karpowicz, "Decoupling chaotic amplification and nonlinear phase in high-energy thin-disk amplifiers for stable OPCPA pumping", Optics Express, 22, 31440 (2014).

I took part in the measurement campaign, contributed in data analysis and wrote the paper with Dr. Nicholas Karpowicz.

- H. Fattahi, H. Barros, M. Gorjan, T. Nubbemeyer, B. Alsaif, C. Teisset, M. Schultze, S. Prinz, M Haefner, M. Ueffing, A. Alismail, L. Vamos, A. Schwarz, O. Pronin, J. Brons, X. Geng, G. Arisholm, M. Ciappina, V. Yakovlev, D.E. Kim, A. M. Azzer, N. Karpowicz, D. Sutter, Z. Major, T. Metzger, and F. Krausz, "Third-generation femtosecond technology", Optica 1, 45 (2014).

The design study, simulations and the majority of the experimental results shown in section 3 of this paper were performed by me. Also, this section was written by me and Prof. Ferenc Krausz.

- H. Fattahi, A. Schwarz, S. Keiber, and N. Karpowicz, "Efficient, octavespanning difference-frequency generation using few-cycle pulses in simple collinear geometry", Optics Letters 38, 4216 (2013).

The experiments were performed by me and Dr. Nicholas Karpowicz. I also helped to prepare the manuscript.

- Y. Deng, A. Schwarz, H. Fattahi, M. Ueffing, X. Gu, M. Ossiander, T. Metzger, V. Pervak, H. Ishizuki, T. Taira, T. Kobayashi, G. Marcus, F. Krausz, R. Kienberger, and N. Karpowicz, "Carrier-envelope-phasestable, 1.2 mJ, 1.5 cycle laser pulses at $2.1 \mu \mathrm{m}$ ", Optics Letters 37, 4973 (2012).

I had a direct contribution in building the MIR OPCPA with Dr. Yunpei Deng and Dr. Alexander Schwarz.

- H. Fattahi, C. Teisset, O. Pronin, A. Sugita, R. Graf, V. Pervak, X. Gu, T. Metzger, Z. Major, F. Krausz, A. Apolonski, "Pump-seed synchroniza- 
tion for $\mathrm{MHz}$ repetition rate, high-power optical parametric chirped pulse amplification", Optics Express 20, 9833 (2012).

I contributed in building the OPCPA system and the pulse compression. The heart of the work was done by Dr. Catherine Teisset and I helped her as the second PhD student on this project. I also prepared the manuscript.

- A. Schwarz, M. Ueffing, Y. Deng, X. Gu, H. Fattahi, T. Metzger, M. Ossiander, F. Krausz, and R. Kienberger, "Active stabilization for optically synchronized optical parametric chirped pulse amplification", Optics Express 20, 5557 (2012).

For this paper I took part in the discussions. The majority of the work was done by Dr. Alexander Schwarz, Moritz Ueffing and Dr. Yunpei Deng. 


\section{List of conference contribution}

- H. Fattahi, N. Karpowicz, T. Metzger, Zs. Major, and F. Krausz, “ Third-generation femtosecond technology" Conference on Lasers and Electro-Optics, invited talk, 2015.

The presented work in this publication is based on my simulation and experimental results.

- D. Franz, H. Fattahi, V. Pervak, M. Trubetskov, E. Fedulova, N. Karpowicz, Zs. Major, and F. Krausz, "Investigation of temporal compression of few-cycle pulses from an ultrabroadband, multi-mJ optical parametric amplifier" The European Conference on Lasers and Electro-Optics (CLEO-Europe), CFIEP3, 2013.

The experiment was performed by me and Dominik Franz in the fram of his Master thesis.

- H. Fattahi, C. Skrobol, M. Ueffing, Y. Deng, A. Schwarz, Y. Kida, V. Pervak, T. Metzger, Z. Major, and F. Krausz, "High efficiency, multi-mJ, sub 10 fs, optical parametric amplifier at $3 \mathrm{kHz}$ " Conference on Lasers and Electro-Optics, (Washington, D.C.), p. CTh1N.6, OSA, 2012.

The experiment was performed by me, with the great help of Dr. Alexander Schwarz and Dr. Yunpei Deng.

- H. Fattahi, M. Ueffing, Y. Deng, A. Schwarz, V. Pervak, Z. Major, T. Metzger, and F. Krausz, "Octave-spanning, multi-mJ, optical parametric chirped pulse amplifier at $3 \mathrm{kHz}$ repetition rate" NLO50, Barcelona, Spain, 2012.

The experiment was mainly performed by me. 



\section{Part II}

\section{Theories and experiments}





\section{Chapter 1}

\section{How to amplify photons}

Stimulated emission and optical parametric amplification (OPA) lead to efficient amplification of photons. In stimulated emission, the amplification is limited by amplified spontaneous emission (ASE) in the gain medium, and the amplification bandwidth to the energy level structure of a laser active medium and gain narrowing. In this process the energy is stored in the medium.

OPA however is an instantaneous process with a high single pass gain. Here, the energy of a typically narrowband, high-energy pulse is transferred to a possibly broadband, low-energy pulse in a nonlinear crystal. In the absence of low-energy photons and at high intensities, high-energy photons decay spontaneously to two photons with lower energy via the emission of two photons. The process is called parametric fluorescence and its amplification in subsequent OPA media limits the achievable gain.

OPA provides broad amplification bandwidth which can be tuned by crystal type, crystal thickness, temperature, and the geometry of the interacting beams. To increase the conversion efficiency, the low-energy seed pulses should be temporally stretched to the time window of high-energy pump pulses. This is therefore called optical parametric chirped pulse amplification (OPCPA), combining OPA with chirped pulse amplification (CPA), but with different objective in the stretching of the seed pulses than in standard laser CPAs.

As here, since no energy is stored in the material, the thermal load on the crystal is significantly smaller. However, at several hundreds of $\mathrm{kHz}$ repetition rate, the small absorption of idler or pump can become significant and active cooling of the nonlinear medium is needed.

One of the most important properties of OPCPA is its simultaneous scalability in peak- and average-power. This unique property demands for powerful, high-energy, high-power pump sources with optimum pulse duration. For long-pulse-pumped OPCPAs, the intensity damage threshold of ma- 
terials is on the order of $30 \mathrm{GW} / \mathrm{cm}^{2}$. Therefore for sufficient gain, thick crystals have to be used, limiting the amplification bandwidth. Decreasing the pump pulse duration results in the increase of the intensity damage threshold. However for pump pulses below $\sim 500 \mathrm{fs}$ the OPCPA conversion efficiency drops due to the increase in group velocity mismatch between the interacting pulses.

This chapter is devoted to the fundamentals of the OPCPA process. In the first section numerical simulations are performed to find the optimum pump pulse duration, optimum signal-to-pump energy ratio, and to study the induced spectral phase in an OPCPA system. In section 2 a general concept for an OPCPA system pumped by Yb:YAG thin-disk amplifiers is reviewed and at the end a scheme for a widely tunable source based on OPCPA is presented. 


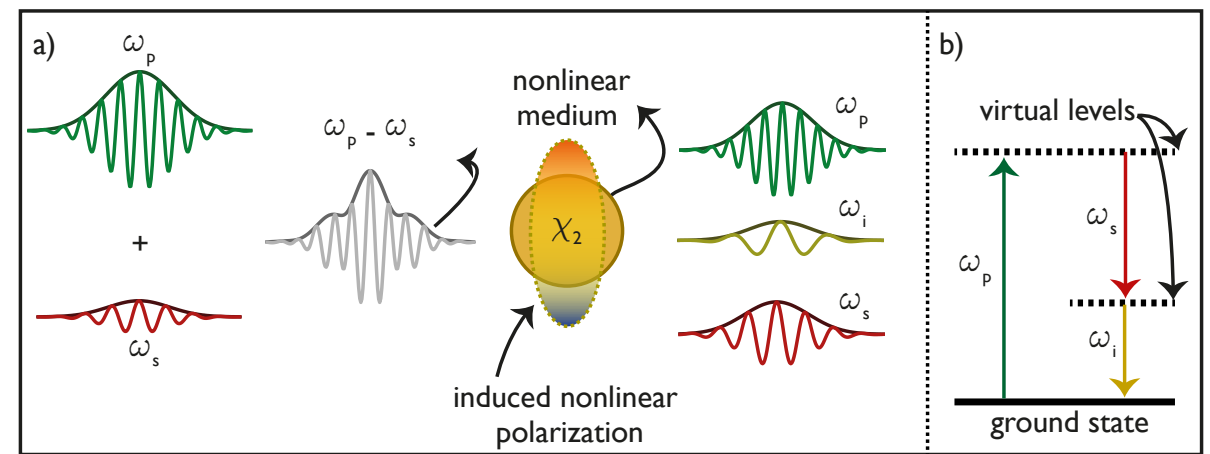

Figure 1.1: a) The wave-picture and b) the photon-picture of OPA process.

\subsection{Optical parametric amplification (OPA)}

When an electric field interacts with a material, a nonlinear polarization is induced. This polarization depends on the strength of the electric field (E) and the polarization response of the material, called the electric susceptibility, $\chi$.

Using a Taylor series expansion of the polarization in terms of the electric field and assuming an instantaneous response, the induced polarization is typically defined as:

$$
P=\epsilon_{0}\left(E+\chi^{(1)} E+\chi^{(2)} E^{2}+\chi^{(3)} E^{3}+\ldots\right) .
$$

OPA is a nonlinear process in the perturbative regime which is described by the second-order susceptibility $\left(\chi^{(2)}\right)$. This non-resonant process can be simply explained by considering the photon-picture of light. In this process, the medium is excited to a virtual level with a higher energy by a photon with the energy of $\hbar \omega_{p}$, where $\omega_{p}$ is the angular frequency of the photon. Coupling to a virtual level represents a transient, polarized state of the system. After a non-resonant field interacts with the medium, the population returns to the ground state nearly instantaneously, with a slight delay determined by how far the virtual level is from a real level. In a medium with a nonzero $\chi^{(2)}$, a photon with lower energy of $\hbar \omega_{s}\left(\omega_{s}\right.$ represents the angular frequency of the low-energy photon) may stimulate the decay, and consequently two photons, with energies of $\hbar \omega_{s}$ and $\hbar \omega_{p}-\hbar \omega_{s}$ will be generated (Fig. 1.1).

Considering the wave picture of light, when two electric fields with the carrier frequencies of $\omega_{p}$ and $\omega_{s}$ interact, the envelope of the intensity resulting from their superposition has a modulation with the frequency of $\omega_{p}-\omega_{s}$. In the interaction of this field with the medium, a new electric field at the carrier frequency of $\omega_{i}=\omega_{p}-\omega_{s}$ is generated. The emission of these photons is caused by the presence of a small perturbation on the 
envelope of the input field, and results in the growth of this feature via energy transfer from the higher frequency field. In a gedanken experiment, the spectrum of the spontaneous emission in a very thin medium could span from $\omega_{p}$ to zero. However, in reality the bandwidth of the spectrum is limited by absorption and the phase-matching conditions of the material. The concept of phase-matching is based on two unbreakable laws of physics: the conservation of energy and momentum. The conservation of energy, for three interacting beams with the central frequency of $\omega_{p}, \omega_{s}$, and $\omega_{i}$ (pump, signal, and idler respectively), is given by:

$$
\omega_{p}-\omega_{s}-\omega_{i}=0
$$

In reality, a pulse with a single frequency does not exist. Allowing a certain spectral bandwidth for each of these frequencies, the condition becomes:

$$
\omega_{p} \pm \Delta \omega_{p}=\omega_{s} \pm \Delta \omega_{s}+\omega_{i} \pm \Delta \omega_{i} .
$$

From the conservation of momentum:

$$
\overrightarrow{k_{p}}-\overrightarrow{k_{s}}-\overrightarrow{k_{i}}=0
$$

and including the spectral bandwidth:

$$
\overrightarrow{k_{p}} \pm \Delta \vec{k}_{p}-\left(\vec{k}_{s} \pm \Delta \vec{k}_{s}\right)-\left(\vec{k}_{i} \pm \Delta \vec{k}_{i}\right)=\overrightarrow{\Delta k},
$$

from Eq. (1.4) and Eq. (1.5):

$$
\Delta \vec{k}_{p}-\Delta \vec{k}_{s}-\Delta \vec{k}_{i}=\overrightarrow{\Delta k}
$$

By substituting Eq. (1.3) in Eq. (1.6) and the Taylor expansion to first order:

$$
\begin{gathered}
\frac{\partial k_{p}}{\partial \omega_{p}} \Delta \omega_{p}-\frac{\partial k_{s}}{\partial \omega_{s}} \Delta \omega_{s}-\frac{\partial k_{i}}{\partial \omega_{i}} \Delta \omega_{i}=\Delta k, \\
\frac{\partial k_{p}}{\partial \omega_{p}} \Delta \omega_{p}-\frac{\partial k_{s}}{\partial \omega_{s}} \Delta \omega_{s}-\frac{\partial k_{i}}{\partial \omega_{i}} \Delta \omega_{p}+\frac{\partial k_{i}}{\partial \omega_{i}} \Delta \omega_{s}=\Delta k, \\
\Delta \omega_{p}\left(\frac{\partial k_{p}}{\partial \omega_{p}}-\frac{\partial k_{i}}{\partial \omega_{i}}\right)+\Delta \omega_{s}\left(\frac{\partial k_{i}}{\partial \omega_{i}}-\frac{\partial k_{s}}{\partial \omega_{s}}\right)=\Delta k, \\
\Delta \omega_{p}\left(\frac{1}{v_{g p}}-\frac{1}{v_{g i}}\right)+\Delta \omega_{s}\left(\frac{1}{v_{g i}}-\frac{1}{v_{g s}}\right)=\Delta k .
\end{gathered}
$$

where $v_{g j}$ for $j=p, s, i$ is the group velocity of the interacting waves and is defined by $\frac{\mathrm{d} \omega_{j}}{\mathrm{~d} k_{j}}$. Eq. (1.10) suggests that the phase-mismatch in the OPA process originates from the different group velocities of the interacting waves. 
It also suggests for the case of a very narrow-band pump beam, where $\Delta \omega_{p} \ll \Delta \omega_{s}$, the phase-mismatch is dominated by the group velocity difference between the signal and idler waves.

For the case of a broadband pump, Eq. (1.10) is zero when $v_{g i}=v_{g s}=v_{g p}$ or when:

$$
\Delta \omega_{p}=\Delta \omega_{s}\left[\frac{\frac{1}{v_{g s}}-\frac{1}{v_{g i}}}{\frac{1}{v_{g p}}-\frac{1}{v_{g i}}}\right] .
$$

Eq. (1.11) indicates that the phase-matching bandwidth can be increased by using pump pulses with broader spectral bandwidth. This can be understood intuitively since in a broadband pump more pairs of frequencies meet the condition for zero phase-mismatch in comparison to a narrowband or a CW pump.

Under the assumption of an undepleted pump, the parametric gain bandwidth at full width at half maximum (FWHM) is:

$$
\Delta v=\frac{2(\ln 2)^{\frac{1}{2}}}{\pi}\left(\frac{\Gamma}{L}\right)^{\frac{1}{2}} \frac{1}{\left|\delta_{s i}\right|}
$$

where $\delta_{s i}$ is the group velocity mismatch between signal and idler, and $\Gamma$ is:

$$
\Gamma^{2}=\frac{\omega_{i} \omega_{s} d_{e f f}^{2}\left|A_{p}\right|^{2}}{n_{i} n_{s} c^{2}}=\frac{2 d_{e f f}^{2} \omega_{i} \omega_{s}}{c^{3} \epsilon_{0} n_{i} n_{s} n_{p}} I_{p}=\frac{8 \pi^{2} d_{e f f}^{2} I_{p}}{n_{i} n_{s} n_{p} \lambda_{i} \lambda_{s} \epsilon_{0} c^{2}},
$$

where, $L$ is the length of the nonlinear medium, $A_{p}$ is the envelope of the pump pulse, $I_{p}$ is the pump intensity, $d_{e f f}$ is the effective nonlinear optical coefficient, and $n_{j}$ and $\lambda_{j}, j=i, p, s$ indicate the refractive index of the nonlinear medium and the wavelength of the idler, signal, and pump, respectively.

Eq. (1.12) shows that the gain bandwidth is inversely proportional to the group velocity mismatch between the signal and idler. In the special case where the central frequency of the signal is exactly half that of the pump, the generated idler covers the same spectral range as the seed. In this case, $v_{g i}=v_{g s}$ and Eq. (1.12) loses validity and Eq. (1.6) must be extended to second order (Cerullo and De Silvestri, 2003):

$$
\Delta v=\frac{2(\ln 2)^{\frac{1}{4}}}{\pi}\left(\frac{\Gamma}{L}\right)^{\frac{1}{4}} \frac{1}{\left|\frac{\partial^{2} k_{s}}{\partial \omega_{s}^{2}}+\frac{\partial^{2} k_{i}}{\partial \omega_{i}^{2}}\right|} .
$$

In this case the gain bandwidth is proportional to the group velocity dispersion of the idler and signal. The Kramers-Kronig relation indicates that the frequency-dependent refractive index of materials changes dramatically between the electronic absorption band and phonon absorption bands of 


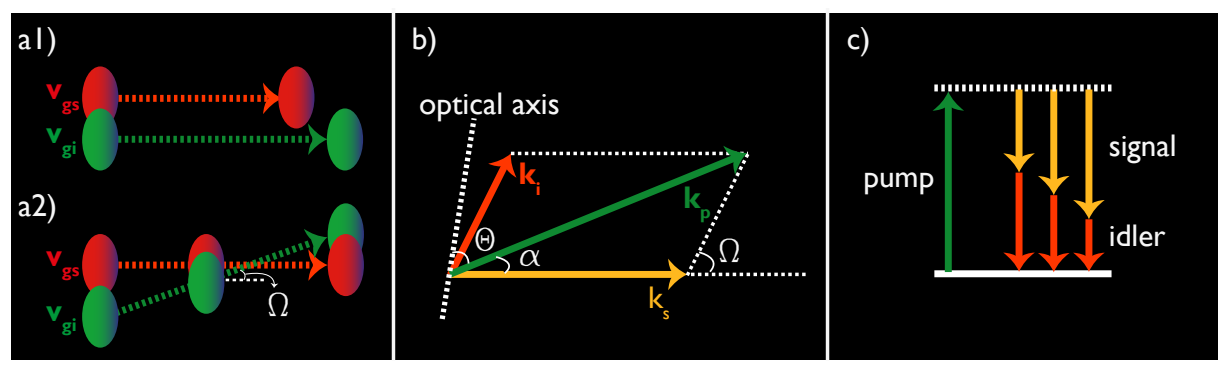

Figure 1.2: a1) The group velocity of the signal and idler pulses in a collinear OPA. Due to dispersion, pulses with longer wavelengths propagate faster than pulses with shorter wavelengths. a2) This group velocity mismatch is compensated in a noncollinear geometry. b) The phase-matching geometry for a noncollinear OPA. c) The energy diagram for a broadband optical parametric amplifier.

materials but at a saddle point between these two absorption regions, the group velocity dispersion (GVD) of materials becomes zero. For the frequencies in the vicinity of the saddle point, the deviation in dispersion, and therefore their temporal walk-off, is at its minimum.

This property of materials is utilized to phase-match and amplify a broad region of frequencies (Eq. (1.14)), in the so-called degenerate OPA (DOPA). In addition to parametric amplification in DOPA, second harmonic generation of the signal is also phase-matched, which causes an unwanted backward flow of energy from the signal to the pump. Ultimately, this modifies the shape of the amplified spectrum, limits the conversion efficiency, and can distort the spectral phase.

For frequencies away from the degeneracy point, the temporal walkoff depends on the group velocity (Eq. (1.12)) and can be compensated by exploiting a noncollinear geometry (Gale et al., 1995; Gale et al., 1998). Here, phase-matching is achieved by adding a noncollinear angle $(\alpha)$ between pump and signal, providing a longer temporal overlap between the pulses. In this case the group velocity of the signal is equal to the projection of the group velocity of the idler along the signal direction. In comparison with collinear geometry where signal and idler become quickly separated, in noncollinear geometry all three beams stay effectively overlapped. The idler generated in this process becomes angularly dispersed to fulfill the conservation of momentum (Fig. 1.2)(Riedle et al., 2000). Then the phase-matching condition becomes:

$$
v_{g s}=v_{g i} \cos \Omega,
$$

where $\Omega$ is the angle between the signal and idler wave vectors. Due to the angle between the interacting beams, this technique is called noncollinear 
optical parametric amplification (NOPA).

In DOPA and NOPA, ordinary and extraordinary axes of birefringence crystals are used. Here, the phase matching angle $(\theta)$ is defined as the angle between the optical axis of the crystal and the wave vector of the propagating beam. Two types of phase-matching can be realized in birefringent crystals. In type I phase-matching the two input pulses with lower frequencies have the same polarization, whereas in type II, they have different polarizations. A full study about this subject can be found in (Dmitriev et al., 1999).

Besides the mentioned phase-matching techniques, quasi phase-matching can be used to engineer and increase the coherence length in a nonlinear process. Here the phase-mismatch is compensated by inverting the orientation of the crystal axis, with a period equal to twice the coherence length. This reverses the sign of $d_{e f f}$ as soon as the field amplitude of the newly generated wave starts to decrease. The broadest spectral bandwidth can be achieved for frequencies around the degeneracy point of the material by poling a crystal with the period which compensates the wave vector mismatch of a frequency $\omega$ detuned from the zero group-delay dispersion (GDD) frequency.

Unlike DOPA and NOPA where ordinary and extra-ordinary axis of a birefringent crystal is exploited to compensate for phase-mismatch, quasi phase-matching permits the use of the same polarization for all three interacting beams. This often corresponds to the strongest element of the nonlinear tensor of the material which increases the conversion efficiency of the process. In the fabrication of periodically poled crystals, a permanent electrical polarization is induced into a ferroelectric material by applying an electric field. Therefore the quasi phase-matched crystals are limited in aperture size, as it is not yet possible to apply a uniform field on a crystal bigger than a certain size, generally a few millimeters. Consequently, this type of phase-matching is not applicable for high-energy OPCPA systems.

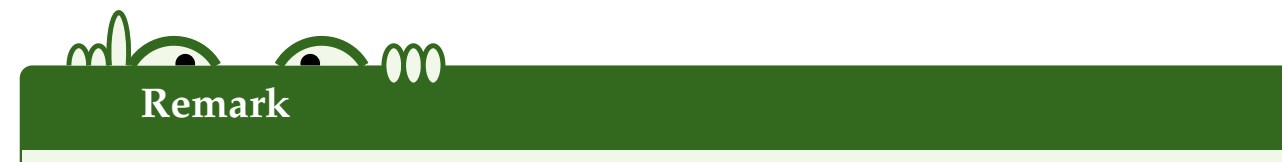

Eq. (1.10) indicates that the phase mismatch in an optical parametric amplifier is due to the group velocity difference between the interacting beams. It is also shown theoretically that using pulses with broad spectral bandwidth to pump OPCPAs result in a broader gain bandwidth.

To overcome the group velocity mismatch during the amplification, and to achieve broadband amplification, several different methods 
such as: noncollinear OPA, degenerate OPA, quasi-phase-matched OPA, and OPA in the Fourier domain (Schmidt et al., 2014), can be employed.

000 000 


\section{Intensity and gain}

Beginning with Maxwell's curl equation, the nonlinear wave equation assuming the instantaneous response for polarization can be obtained (Boyd, 2003):

$$
\nabla^{2} E-\frac{\epsilon}{c^{2}} \frac{\partial^{2} E}{\partial t^{2}}=\frac{1}{\epsilon_{0} c^{2}} \frac{\partial^{2} P^{n l}}{\partial t^{2}}
$$

For the phenomena studied in this thesis, it is assumed that the propagation length of interacting electromagnetic waves is much longer than their wavelength. The waves can be treated as light rays or plane waves, constant frequency waves with infinite parallel planes of constant peak-to-peak amplitude normal to the phase velocity vector. Sine and cosine functions meet the above criteria. For a linearly polarized wave propagating in the $z$ direction, one can write:

$$
E(z, t)=\Re[A(z) \exp (i \omega t-i k z)],
$$

and the nonlinear polarization:

$$
P^{n l}(z, t)=\Re\left[P^{n l}(z) \exp \left(i \omega t-i k_{p} z\right)\right] .
$$

By substituting Eq. (1.17) and Eq. (1.18) in Eq. (1.16):

$$
\begin{aligned}
& {\left[\frac{\mathrm{d}^{2} A(z)}{\mathrm{d} z^{2}}-2 i k \frac{\mathrm{d} A(z)}{\mathrm{d} z}-A(z) k^{2}+\frac{\epsilon \omega^{2}}{c^{2}} A(z)\right] \exp (i \omega t-i k z)=} \\
& -\frac{\omega^{2}}{\epsilon_{0} c^{2}} P^{n l}(z) \exp \left(i \omega t-i k_{p} z\right) .
\end{aligned}
$$

As the envelope of the wave varies slowly in time and space in comparison to its wavelength, the slowly varying envelope approximation (SVEA) is often employed:

$$
\frac{\mathrm{d}^{2} A}{\mathrm{~d} z^{2}} \ll 2 k \frac{\mathrm{d} A}{\mathrm{~d} z}
$$

Taking into account that:

$$
k=\frac{n \omega}{c}
$$

Eq. (1.19) becomes:

$$
\frac{\mathrm{d} A}{\mathrm{~d} z}=-i \frac{\mu_{0} c \omega}{2 n} P^{n l} \exp \left(i k z-i k_{p} z\right)
$$

where $n$ is the refractive index at frequency $\omega$. This general equation shows that the amplitude variation of the propagating wave is caused by the nonlinear source term on the right hand side of Eq. (1.22). As OPA is a secondorder nonlinear process, Eq. (1.1) can be written as:

$$
P_{i}^{n l}=\epsilon_{0} \chi_{i j k}^{(2)} E_{j} E_{k}
$$


where $i, j$, and $k$ refers to three interacting waves, at frequencies $\omega_{p}, \omega_{s}$, and $\omega_{i}$ and $\chi^{(2)}$ is the second-order susceptibility tensor. By assuming the simple case of collinear geometry the following coupled wave equations can be derived from Eq. (1.23) and Eq. (1.22):

$$
\begin{aligned}
\frac{\mathrm{d} A_{i}}{\mathrm{~d} z} & =-i \frac{\omega_{i} d_{e f f}}{n_{i} c} A_{s}^{*} A_{p} \exp (-i \Delta k z), \\
\frac{\mathrm{d} A_{s}}{\mathrm{~d} z} & =-i \frac{\omega_{s} d_{e f f}}{n_{s} c} A_{i}^{*} A_{p} \exp (-i \Delta k z), \\
\frac{\mathrm{d} A_{p}}{\mathrm{~d} z} & =-i \frac{\omega_{p} d_{e f f}}{n_{p} c} A_{i}^{*} A_{s} \exp (i \Delta k z),
\end{aligned}
$$

where $d_{e f f}$ is the effective nonlinear optical coefficient, which depends on the propagation direction and polarization of the three beams, and $\Delta k=$ $k_{p}-k_{s}-k_{i}$ is the phase-mismatch.

Assuming an initial signal intensity $A_{s 0}$, zero initial idler intensity $A_{i 0}=$ 0 and neglecting pump depletion $A_{p} \cong$ const., the coupled wave equation for a nonlinear crystal of length $L$ can be solved for the intensity gain $G$ :

$$
G=\frac{I_{s}}{I_{s 0}}=1+\left[\frac{\Gamma}{g} \sinh (g L)\right]^{2},
$$

where $\mathrm{g}$ is the small signal gain:

$$
g=\sqrt{\left(\Gamma^{2}-\left(\frac{\Delta k}{2}\right)^{2}\right)} .
$$

It can be seen from Eq. (1.28) that the small signal gain depends on the phase-mismatch between the interacting beams and $\Gamma$, where $\Gamma$ is proportional to the pump intensity $I_{p}$. This suggests that there are two parameters which have the main role in the shape of the amplified spectrum: pump intensity and phase-mismatch of the interacting beams. These two parameters can be used to optimise the small signal gain of an OPA. For the case of ideal phase-matching $(\Delta k=0)$, the gain becomes maximal $g=\Gamma$. But when $\Delta k \neq 0$, the shape of the small signal gain is defined by the interplay between the pump intensity and phase-mismatch. Eq. (1.28) suggests that by increasing the pump intensity one could tailor the spectral shape of the amplification gain and minimize the effect of $\Delta k$ and the resulting accumulated phase-mismatching, $\Delta k L$.

To study this effect, numerical simulations (using SISYFOS code, for details see Appendix B) for OPCPA at two different pump intensities $\left(50 \mathrm{GW} / \mathrm{cm}^{2}\right.$ and $100 \mathrm{GW} / \mathrm{cm}^{2}$ ) and two different crystals with different phase-mismatch 
(BBO and $\mathrm{LBO}$ ) are performed. In these simulations it is assumed that the $1 \mathrm{~mJ}$ pump pulses, centered at $515 \mathrm{~nm}$, have a Gaussian distribution in time and space with a pulse duration of 1 ps at FWHM. The signal pulse energy is set to $3 \mu \mathrm{J}$. In each simulation, the crystal thickness is optimized to reach energy saturation at each intensity.

Fig. 1.3 shows the temporal and spectral evolution of the pump pulses and the spectral evolution of the signal pulses over the length of a BBO crystal. The amplification for pump intensities of $100 \mathrm{GW} / \mathrm{cm}^{2}$ and $50 \mathrm{GW} / \mathrm{cm}^{2}$ reaches saturation in $0.9 \mathrm{~mm}$-thick and $1.3 \mathrm{~mm}$-thick $\mathrm{BBO}$ crystals, respectively. For both intensities the pump energy is depleted, which results in spectral broadening of the pump pulses (Fig. 1.3-b). The gain curves and therefore the amplified spectra for both cases are nearly identical. Fig. 1.5-b shows the accumulated phase-mismatch $(\Delta k L)$ for amplification in these two cases.

Due to the lower nonlinearity of LBO, a longer crystal length is required to reach saturation: $5.4 \mathrm{~mm}$ for the pump intensity of $50 \mathrm{GW} / \mathrm{cm}^{2}$ and $2.5 \mathrm{~mm}$ for $100 \mathrm{GW} / \mathrm{cm}^{2}$. Fig. 1.4 shows that the saturation dynamics are different for the case of an LBO crystal. Here, for the pump intensity of $50 \mathrm{GW} / \mathrm{cm}^{2}$ the pump energy is not depleted, although the amplification reaches saturation. As the signal is linearly chirped, and therefore the frequencies which satisfy phase-matching have temporal overlap with the wings of the pump pulses, mostly the energy of the temporal wings of the pump is extracted. Little spectral broadening in the pump spectrum is observed (Fig. 1.4-b) and the amplified spectrum has a hole in the middle, which matches very well with the phase-mismatch curve shown in Fig. 1.5a. At $100 \mathrm{GW} / \mathrm{cm}^{2}$ pump intensity the situation becomes similar to the BBO crystal. Despite the large phase-mismatch in the central frequencies of the spectrum, due to the effect of the higher pump intensity on the gain bandwidth, no hole is present.

Therefore it can be concluded that high peak intensity of pump pulses, achievable in short-pulse-pumped OPCPA systems, ensures high amplification gain which results in saturation in a shorter crystal length. Additionally, as was shown above, it is a parameter for tailoring the amplification gain.

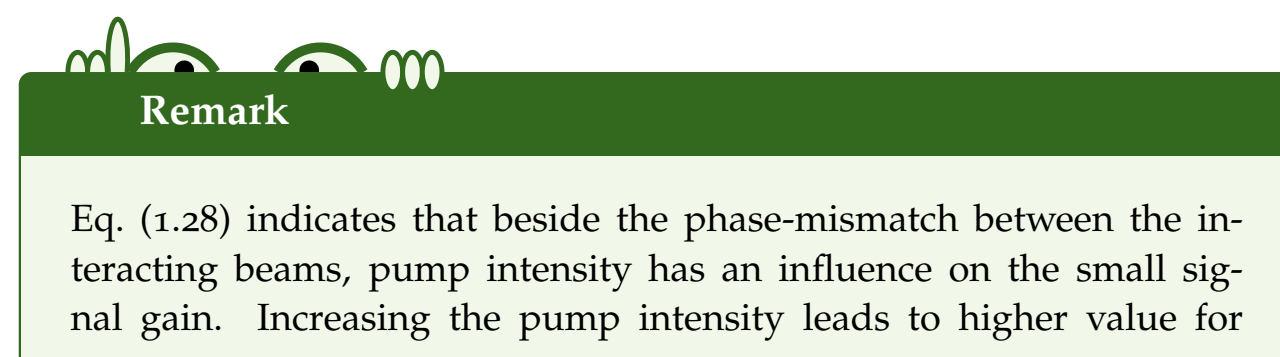


small signal gain and compensate for a degree of phase-mismatch, allowing gain at wavelengths that are not amplified at lower intensity. This shows one of the advantages of short-pule-pumped OPCPAs, as higher pump intensity can be used without damaging the crystal.

ou vov 


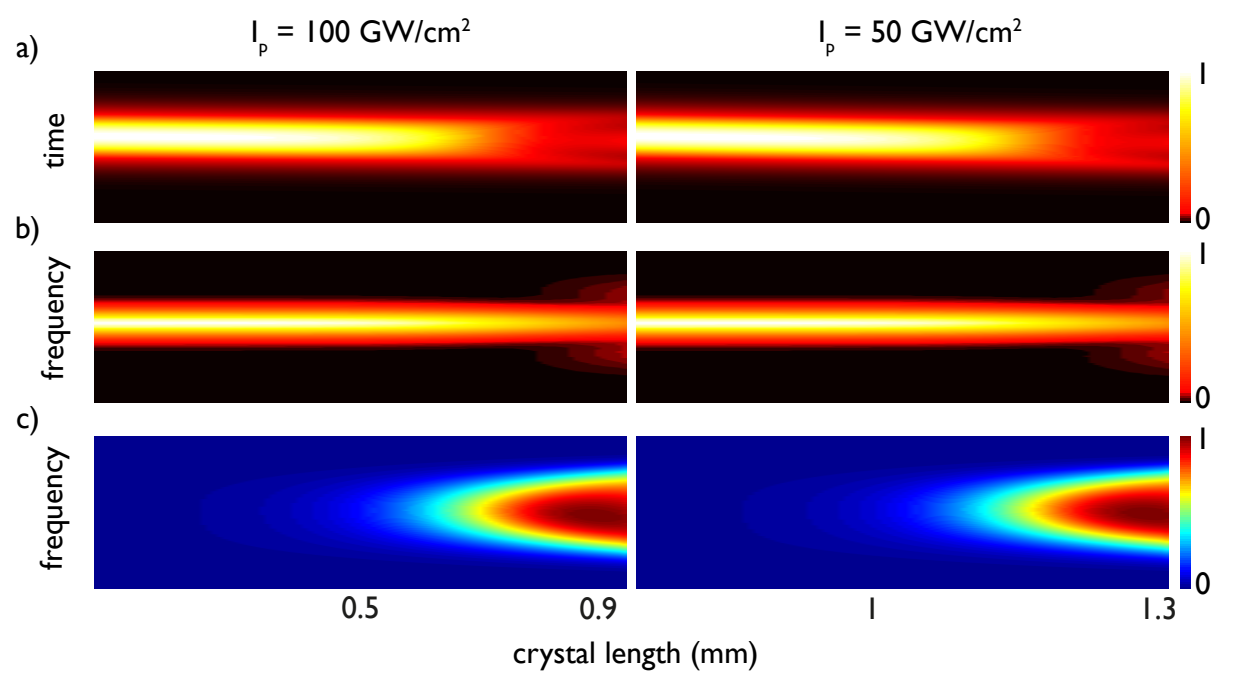

Figure 1.3: Intensity-dependent gain in BBO. a) The temporal profile of pump pulses over crystal length at two different pump intensities, and b) their corresponding spectra. c) Amplified signal spectrum over crystal length for BBO crystal used as a nonlinear medium for a saturated OPCPA.

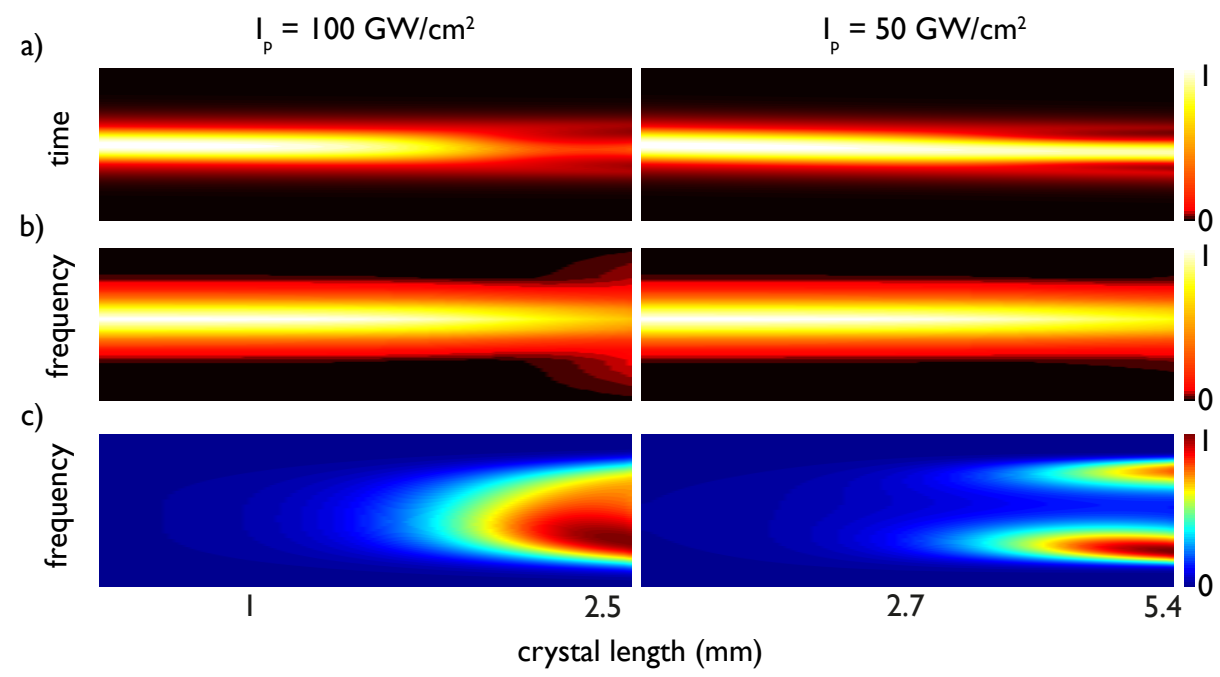

Figure 1.4: Intensity dependent gain in LBO. a) The temporal profile of pump pulses over crystal length at two different pump intensities, and b) their corresponding spectra. c) Amplified signal spectrum over crystal length for LBO crystal used as a nonlinear medium for a saturated OPCPA. 

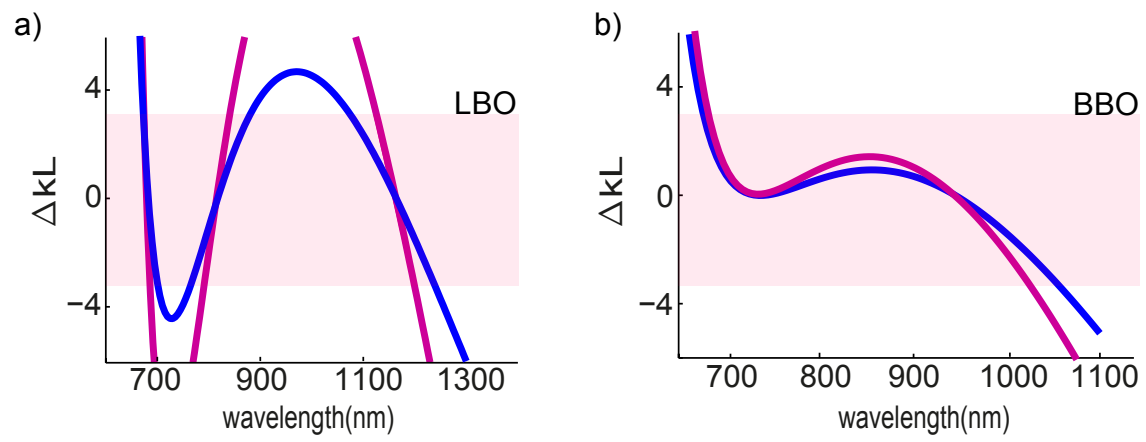

Figure 1.5: a) Residual phase-mismatch of a saturated OPCPA with a pump intensity of $100 \mathrm{GW} / \mathrm{cm}^{2}$ (blue) and $50 \mathrm{GW} / \mathrm{cm}^{2}$ (red) utilizing a $2.5 \mathrm{~mm}$ and $5.4 \mathrm{~mm}$ LBO crystal for the nonlinear medium and, b) using a $0.9 \mathrm{~mm}$ and $1.5 \mathrm{~mm} \mathrm{BBO}$ crystal as the nonlinear medium. The pink area shows the phase-mismatch between $-\pi$ and $\pi$. 


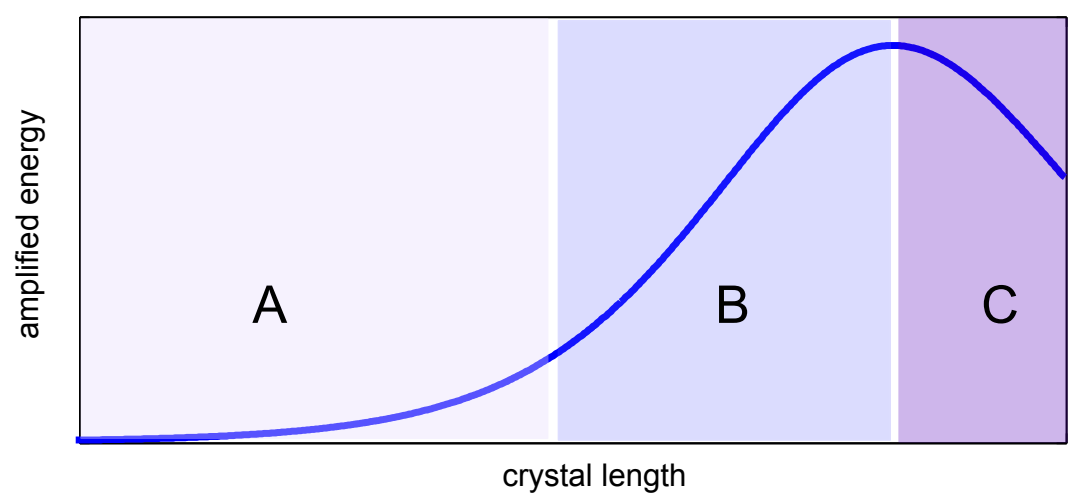

Figure 1.6: Typical OPA gain curve as a function of crystal thickness. For the description of the regions A, B and C, see Section 1.1.

\section{Amplification and saturation}

In the large gain approximation $(\Gamma L \gg 1)$, Eq. (1.27) simplifies to:

$$
\begin{gathered}
I_{S}(L) \cong \frac{1}{4} I_{S 0} \exp (2 \Gamma L) . \\
G=\frac{I_{S}(L)}{I_{S 0}}=\frac{1}{4} \exp (2 \Gamma L) .
\end{gathered}
$$

The exponential growth in OPA shown in (Eq. (1.30)), is qualitatively different from the quadratic growth of other second order nonlinear phenomena. In an OPA, an initial signal photon stimulates the generation of an additional signal and an idler photon. Likewise, due to the symmetry of the signal and idler, the generated idler photon stimulates the generation of a signal photon. Therefore the generation of a signal field reinforces the generation of an idler field and viceversa. This generates the positive feedback responsible for the exponential growth of the signal.

The OPA amplification gain can be divided to three different regions (Fig. 1.6). Region $A$, with exponential behavior, for $E_{i} \ll E_{p}$, where $E_{i}$ and $E_{p}$ are idler and pump electric fields, respectively. Region $B$, linear amplification until $E_{i} \approx E_{p}$ and region $C$, where $E_{i} \gg E_{p}$ and the direction of energy flow is reversed. Here the backconversion of energy from the idler and signal to the pump (sum frequency generation of idler and signal) overtakes the amplification process. Due to the symmetry in conservation of energy and momentum, energy flow from pump photons to signal photons and from signal photons to the pump photons happens simultaneously. But the backconversion of energy becomes noticeable when the total energy of idler and signal pulses becomes comparable to or more than the pump en- 
ergy. In an adequately long crystal operating beyond region $C$, the energy between pump and signal is shuffled repeatedly back and forth.

\section{Remark}

The generation of the idler is the initial fuel for the exponential raise in the gain of parametric amplification, as is shown in Fig. 1.6- region A. In region $B$, the behavior of the gain becomes linear. Therefore the elimination and control of the idler would lead to better energy extraction by the signal pulses and consequently higher efficiency. This is the basics for the efficient OPCPA systems, which will be discussed in Chapter 4.

ouv vov 


\section{Optimum pump-pulse duration}

In an OPCPA, the pulse duration of the pump laser has an important role in the amplification process. On one hand, the damage threshold intensity for transparent materials increases roughly proportional to the inverse of the square root of the pulse duration $\left(I_{t h} \propto \tau^{-\frac{1}{2}}\right)$ for pulsed lasers, following the dynamics of impact ionization. For lasers with pulses longer than $\mu$, thermal damage mechanisms may dominate, but such laser sources are not considered here (Stuart et al., 1996).

On the other hand, the impact of temporal walk-off between pump and seed pulses increases with decreasing pulse duration. These two effects place opposing demands on the pulse duration, suggesting that an optimal compromise exists. To study this, a series of simulations were performed to find the optimum pump-pulse duration for broadband OPCPAs by varying the pump-pulse duration from $100 \mathrm{fs}$ to $100 \mathrm{ps}$. In the first series, the peak intensity on the crystal is set to $100 \mathrm{GW} / \mathrm{cm}^{2}$ for all pump-pulse durations in order to decouple the effect of the following parameters: intensitydependent gain, temporal walk-off between signal and pump pulses and the damage threshold intensity. Here, the shape of the amplified spectrum is affected by the temporal walk-off between pump and seed pulses and the influence of the intensity-dependent gain is decoupled by using a similar intensity for all pump-pulse durations.

In the following simulations, a linearly chirped signal with a fourthorder super-Gaussian spectrum, centered at $820 \mathrm{~nm}$, is assumed. A Gaussian temporal and spatial profile is considered for the pump pulse centered at $515 \mathrm{~nm}$. A BBO crystal with $d_{e f f}=2.3168 \mathrm{pm} / \mathrm{V}$ is used and the simulation is performed for pump-pulse durations of $100 \mathrm{fs}, 1 \mathrm{ps}, 10 \mathrm{ps}, 100 \mathrm{ps}$. In each simulation the pump-beam size is set to provide $100 \mathrm{GW} / \mathrm{cm}^{2}$ on-axis peak intensity and the signal-to-pump energy ratio in all cases is set to $3 \times 10^{-3}$. The pulse fronts of the pump and signal beams are both perpendicular to the the propagation direction of signal beam.

Fig. 1.7-a shows the temporal profile of the signal and pump pulses in a saturated OPCPA with different pump-pulse durations. For long pump pulses on the order of tens and hundreds of ps, the temporal walk-off between pump and seed pulses in the OPCPA nonlinear crystal is negligible. In the saturated regime the backconversion of energy is visible in the center of the pulse, due to the Gaussian temporal profile of the pump. For $1 \mathrm{ps}$ pump pulses, the temporal walk-off between the interacting beams becomes visible and finally, for $100 \mathrm{fs}$ pump pulses, the temporal walk-off limits the efficient energy extraction from the pump, as the signal and pump pulses quickly become temporally separated before backconversion of the energy to the pump pulse starts. This phenomenon causes local saturation points 


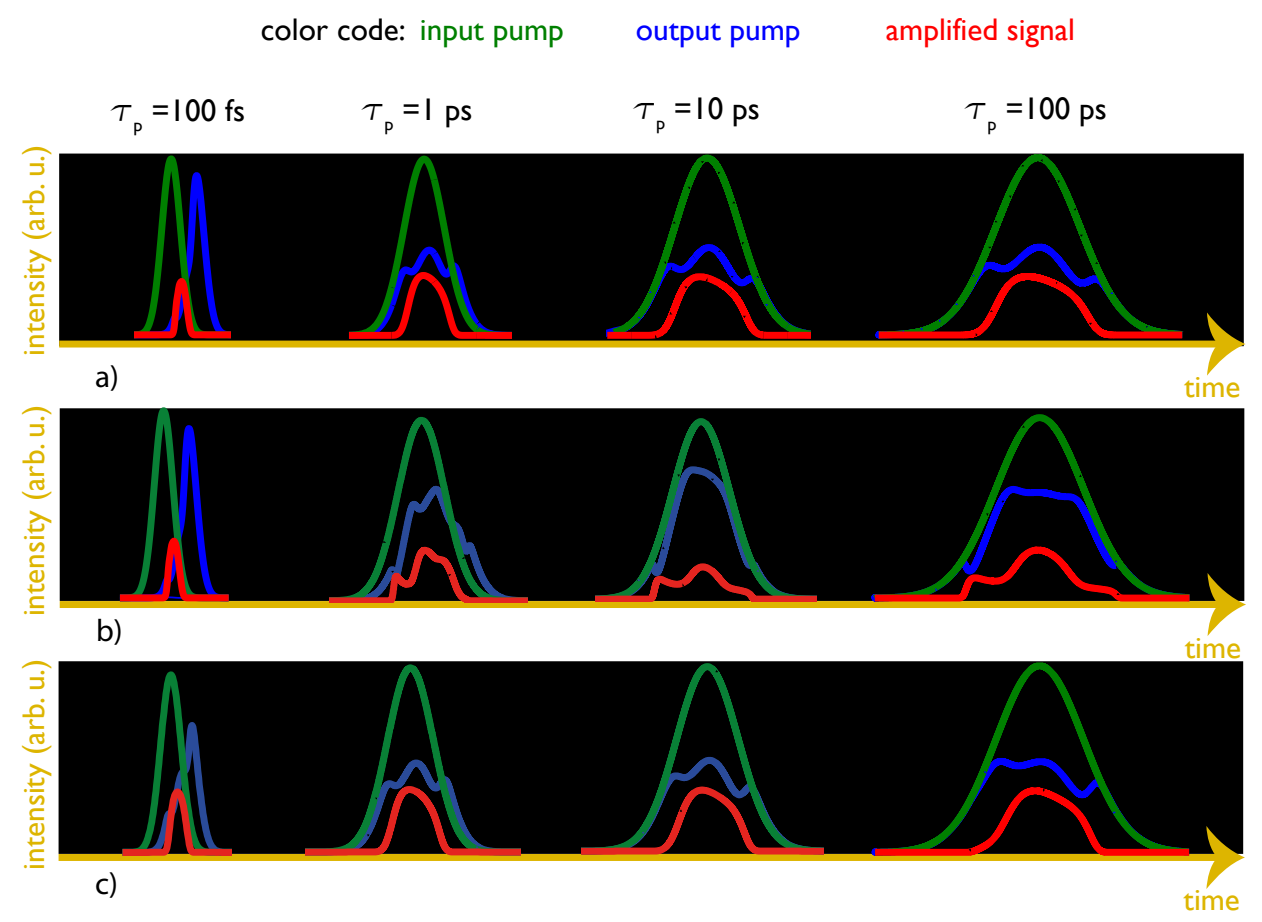

Figure 1.7: Temporal profile of pump and signal pulses in a a) saturated and $b$ ) over-saturated OPCPA for different input pump-pulse durations. The temporal walk-off for long pulses is negligible. c) Saturated OPCPA in which the pump intensity is scaled relative to the pump-pulse duration. Green: pump temporal profile before amplification, blue: residual pump temporal profile after amplification, red: amplified signal temporal profile.

in the gain of the OPCPA.

Fig. 1.7-b shows the behavior of these cases in an over-saturated OPCPA, where a noticeable amount of amplified signal energy goes back to the pump pulse, causing modulations on the temporal profile of the amplified signal.

This line of reasoning also applies to the spatial profile of the pump pulses. Fig. 1.8-a shows the effect of saturation in space. Poor energy extraction in $100 \mathrm{fs}$ pump pulses leaves the spatial shape of the pump almost unchanged, whereas for longer pump pulse durations the amplification reaches saturation and the energy backconversion is visible in the center of the beam, which carries the highest intensity. In the case of an over-saturated OPCPA (Fig. 1.8-b) the residual pump beam profile undergoes severe changes with its inverse imprinted on the amplified signal beam profile(Fig. 1.9-b), except for $100 \mathrm{fs}$ pump pulses, due to localized saturation.

As can be seen in Fig. 1.10-b pump pulse durations longer than $1 \mathrm{ps}$ at the same peak intensity result in approximately the same amplified signal 


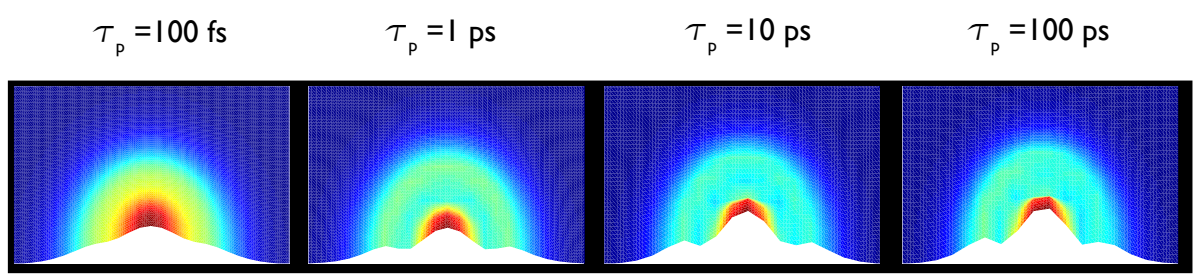

a)

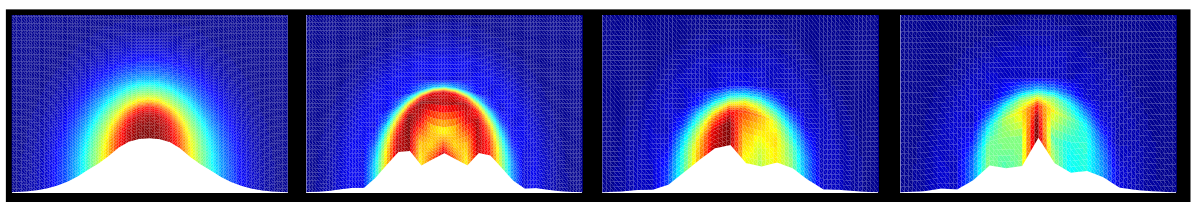

b)

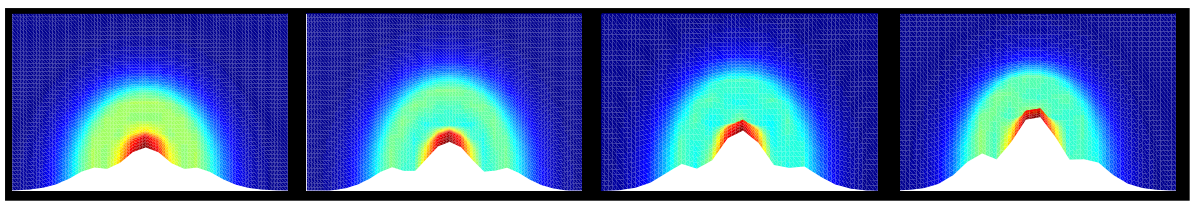

c)

Figure 1.8: Spatial profile of pump beam after amplification in a a) saturated and $b$ ) over-saturated OPCPA for different pump-pulse durations. The effect of spatial walk-off for 10 ps pump pulses which leads to asymmetric energy extraction of the pump is clear. c) Saturated OPCPA in which the pump intensity is scaled relative to the pump-pulse duration. The white areas shows the on-axis beam profile.

peak power while the amplified peak power drops for $100 \mathrm{fs}$ pump pulses. This indicates the negligible effect of temporal walk-off for pump pulses longer than 1 ps and the advantage of near-1-ps-pumped OPCPAs.

As the signal has a linear positive chirp, the center of mass of the amplified spectrum moves to longer wavelengths for shorter pump-pulse durations, because the leading edge of the signal pulse always overlaps the undepleted pump pulse regions due to the temporal walk-off between pump and signal (Fig. 1.10-a,-b). In an over-saturated OPCPA, the amplified signal spectrum becomes modulated and broader. Here, imperfectly phasematched spectral components of the signal spectrum have a chance to be amplified, because of energy backconversion of the phase-matched spectral components and result in a broader amplified spectrum. The longer nonlinear crystal used for the over-saturated OPCPA causes a larger temporal walk-off and shifts the spectral center of mass to even longer wavelengths compared to the saturated OPCPA (Fig. 1.10-c,-d).

In the second series of simulations, all of the input parameters are the same as the previous simulations except for the on-axis pump intensity 


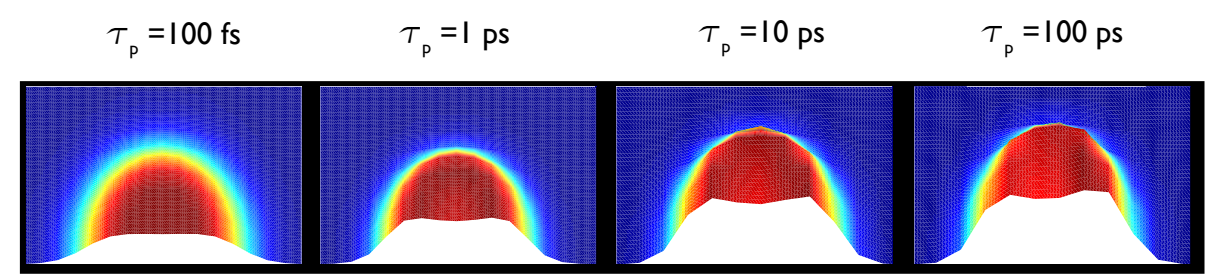

a)

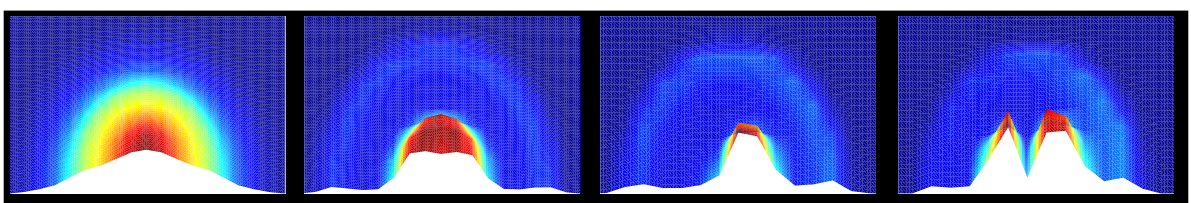

b)

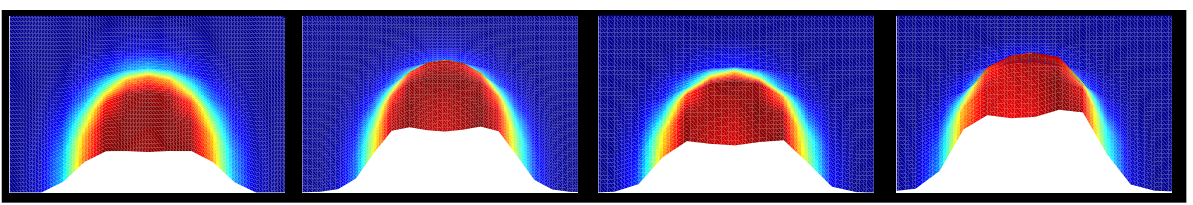

c)

Figure 1.9: Spatial profile of seed beam after amplification in a a) saturated and b) over-saturated OPCPA for different pump-pulse duration. c) Saturated OPCPA in which the pump intensity is scaled relative to the pump pulse-duration. The white areas show the on-axis beam profile.

in the nonlinear crystal. Here the pump intensity is scaled relative to the pump-pulse duration to stay below the damage threshold intensity. The damage threshold intensity for 1 ps pulses is assumed to be $100 \mathrm{GW} / \mathrm{cm}^{2}$ based on experimental measurements and the relative intensity is scaled with the square root of the pump-pulse duration. As the damage threshold intensity is higher for short pump pulses, the energy extraction of pump pulses becomes more efficient (Fig. 1.7-c,Fig. 1.8-c), and for different pumppulse durations, saturation occurs at different crystal thicknesses (Fig. 1.11). Therefore, for a longer pump pulse duration a thicker nonlinear crystal is needed to reach saturation. This limits the bandwidth of the amplified spectrum. The combination of all these parameters indicates that near-1-ps pump-pulse duration is the optimum for achieving the highest peak power in an OPCPA (Fig. 1.11). 
a)

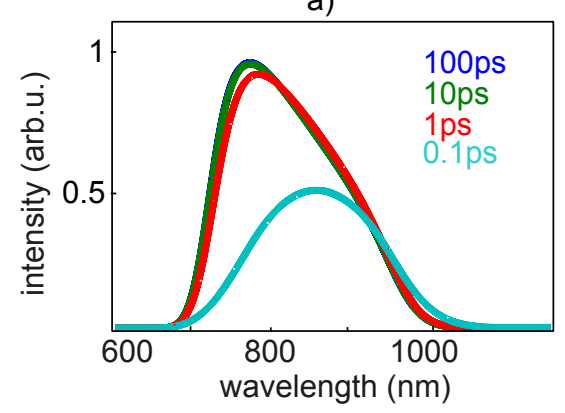

c)

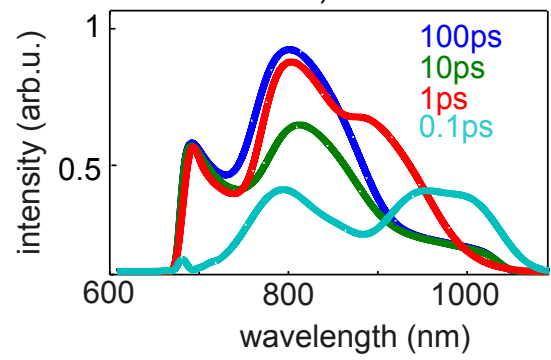

b)

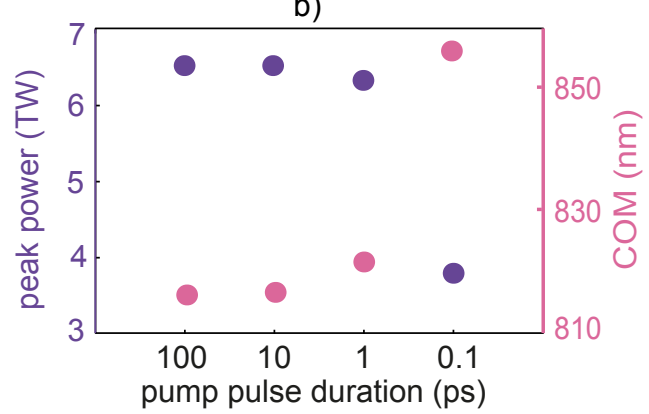

d)

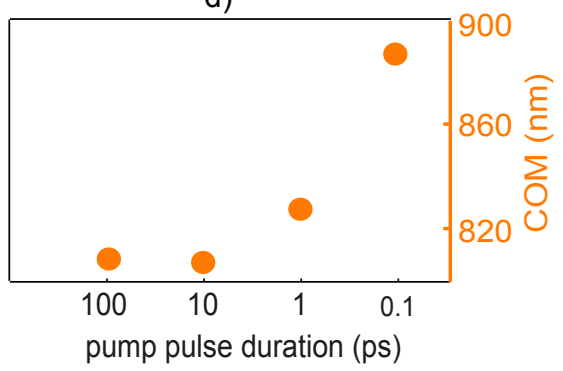

Figure 1.10: a) Amplified spectra for different pump-pulse durations in a saturated OPCPA. The amplified spectra of $10 \mathrm{ps}$ and $100 \mathrm{ps}$ pump pulses are overlapped. b) Peak power of the amplified signal energy and the corresponding spectral center of mass $(\mathrm{COM})$ in a saturated OPCPA versus pump-pulse duration. c) Amplified spectra for different pump-pulse durations and, d) peak powers of the amplified signal energy and the corresponding spectral center of mass (COM) in an over-saturated OPCPA.

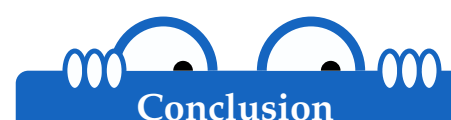

Pump pulse durations in the range of $1-10$ ps are optimum for OPCPA systems in terms of spectral bandwidth and conversion efficiency. In this range the temporal walk-off between the interacting beam is negligible, the intensity damage threshold of materials are relatively high and thin crystals with high single-pass gain can be employed allowing large amplification bandwidth.

Yb-doped amplifiers, in thin-disk, slab, and bulk geometry provide such high energy pulses, making them suitable for pumping highenergy, high-power OPCPA systems. 
a)

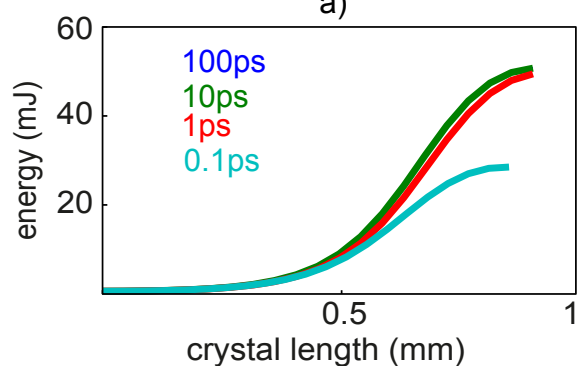

c)

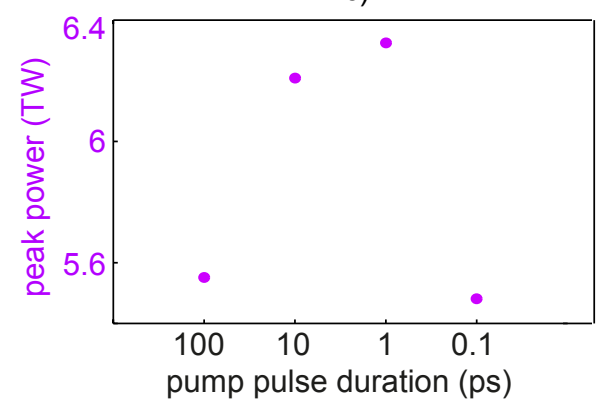

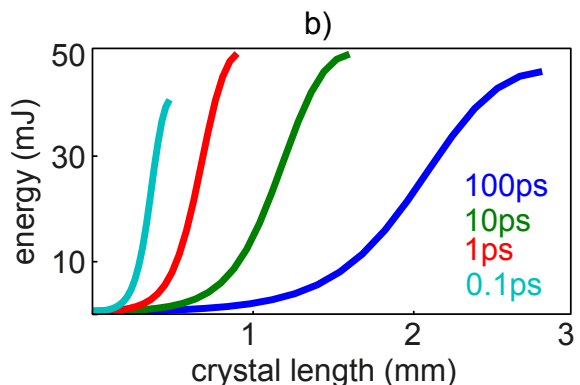

d)

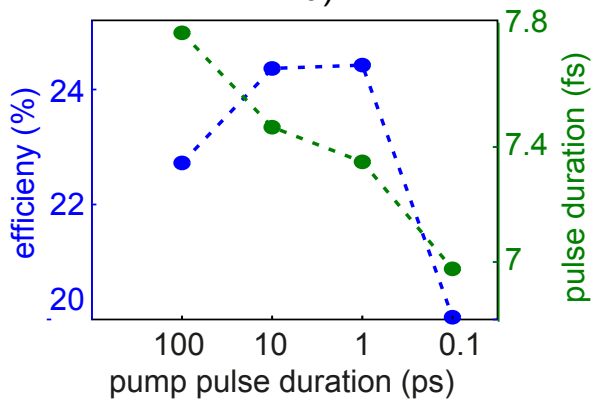

Figure 1.11: a) Amplified signal energy over crystal length for different pump-pulse durations with a fixed peak intensity of $100 \mathrm{GW} / \mathrm{cm}^{2}$. The energy curves for $100 \mathrm{ps}$ and $10 \mathrm{ps}$ overlaps. b) Amplified signal-energy scaling with nonlinear crystal length for different pulse durations and with the pump intensity scaled relative to the pump-pulse duration. c) Peak power of the amplified signal. d) Pump-to-signal conversion efficiency and the amplified signal-pulse duration of OPCPAs with different pump pulse durations. 


\section{Optimum pump-to-signal energy ratio}

To understand efficiency and gain, let us assume an oak tree with 100 acorns. For a small number of hungry squirrels, there are more than enough acorns to go around and each squirrel has a chance to gather as many as he or she likes, or scientifically speaking, each squirrel has a high gain. But, because there are far fewer squirrels than acorns, their efficiency in picking up the acorns is low. Increasing the number of squirrels will increase the thoroughness of the consumption of acorns but at the same time decrease the share of acorns for each squirrel. A similar phenomenon happens in an OPA, by considering signal photons as squirrels and pump photons as acorns. By increasing the signal-to-pump energy ratio, the energy extraction and OPA efficiency increases.

The process can be explained by looking at the role of the idler. For a high input signal energy, saturation occurs in a shorter crystal length. As a result, the newly generated idler has a broader spectral bandwidth. These new photons generated at the edge of the idler spectrum correspond to amplification of seed photons that would not get amplified in a longer crystal, and result in a higher net efficiency. Essentially, the increased gain bandwidth allows for a seed pulse with the same stretched duration, normalized to the pump duration, to make use of a wider temporal window of pump pulse energy. For a low input-signal energy, energy saturation happens in a longer crystal length, which limits the bandwidth of the generated idler and therefore the energy-extraction efficiency.

For signal-pulse energies higher than the pump-pulse energy, although unfamiliar to our common sense, amplification still happens due to the conservation of momentum and energy in the generation of the idler. Fig. 1.12 shows the calculated gain and efficiency for different signal-to-pump energy ratios. A signal-pump energy ratio of the order of $10^{-3}$ is the optimum for having a high amplification gain and efficiency simultaneously.

The signal-to-pump energy ratio also has an effect on the saturation behavior of an OPCPA. Fig. 1.13 (right column) shows the amplification gain for different input signal energy. When the input signal is equal to the input pump energy, the amplified signal reaches saturation very quickly, $46 \%$ of the amplified energy converts back to the pump and the process reaches a steady state for longer crystal lengths. By decreasing the input signal energy, the ratio of the back-converted energy decreases. For lower input signal energy, the steady-state is not observed and in addition to the first saturation point, a second one with higher energy appears (for energy ratio=0.0001). The local saturation points are due to the fact that different spectral components reach saturation at different crystal lengths Fig. 1.13 (left column). For high signal energies, all of the frequencies reach saturation in a very short 


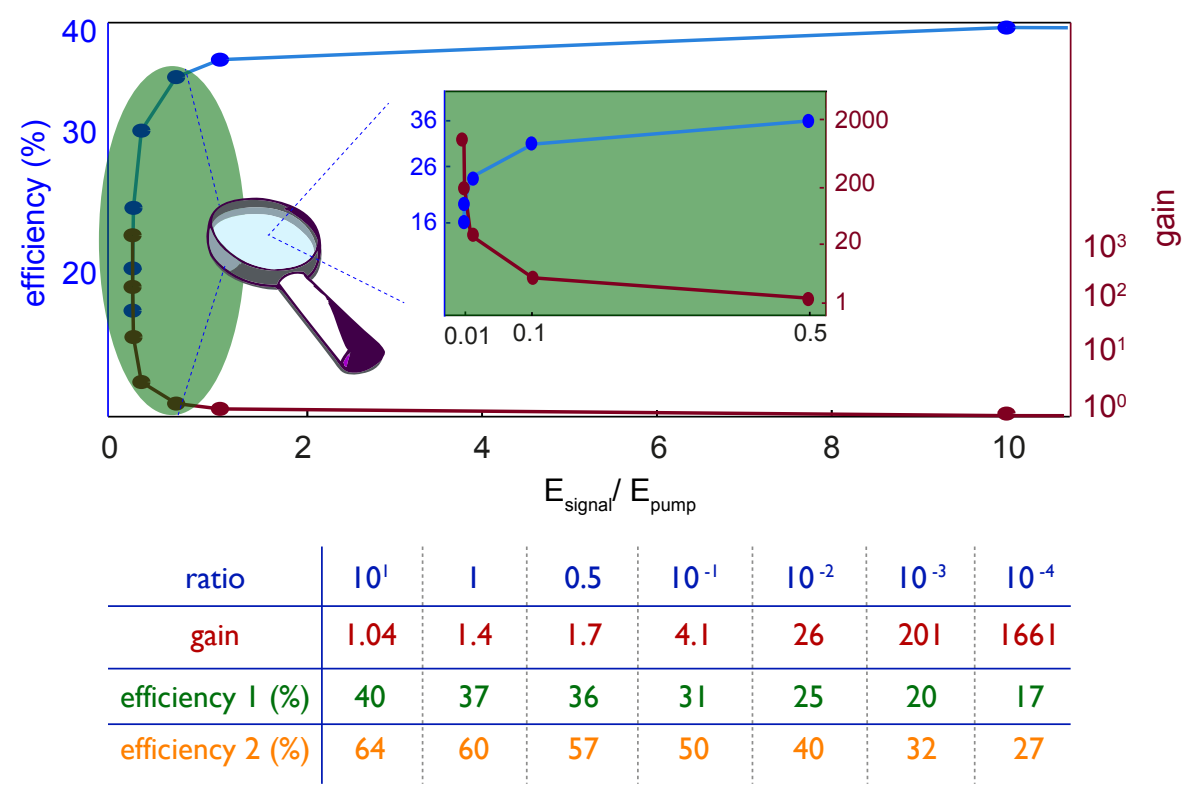

Figure 1.12: Calculated gain and efficiency for different signal-pump energy ratios in an OPCPA pumped with $1 \mathrm{~mJ}$ pulses at $515 \mathrm{~nm}$. The amplified spectrum in a BBO crystal covers $680-1050 \mathrm{~nm}$. The amplification gain is calculated by dividing the amplified signal energy over the input signal energy. Efficiency $I$ is the net increase in signal energy divided by the input pump energy and efficiency 2 is the sum of the net increase in signal energy plus the idler energy divided by the input pump energy.

length of the crystal and by decreasing the signal energy, the saturation points spread over a larger range of crystal lengths.

Spectral components at $720 \mathrm{~nm}, 790 \mathrm{~nm}, 860 \mathrm{~nm}, 930 \mathrm{~nm}, 1000 \mathrm{~nm}$, and $1070 \mathrm{~nm}$ are considered for detailed study. Fig. 1.14-a shows the difference between the crystal length where the first wavelength and the last wavelength reach saturation $\left(\Delta L_{s a t}\right)$, divided by the saturation length $\left(L_{s a t}\right)$ of the whole spectrum in the crystal. Fig. 1.14-b and -c shows the relative saturation length for different wavelengths and input signal energies and their corresponding amplification gain, alternatively. Saturation is reached quickest at $870 \mathrm{~nm}$ due to the small phase-mismatch. For lower signal energies and longer crystal lengths, the saturation lengths for different wavelengths become more dispersed. 

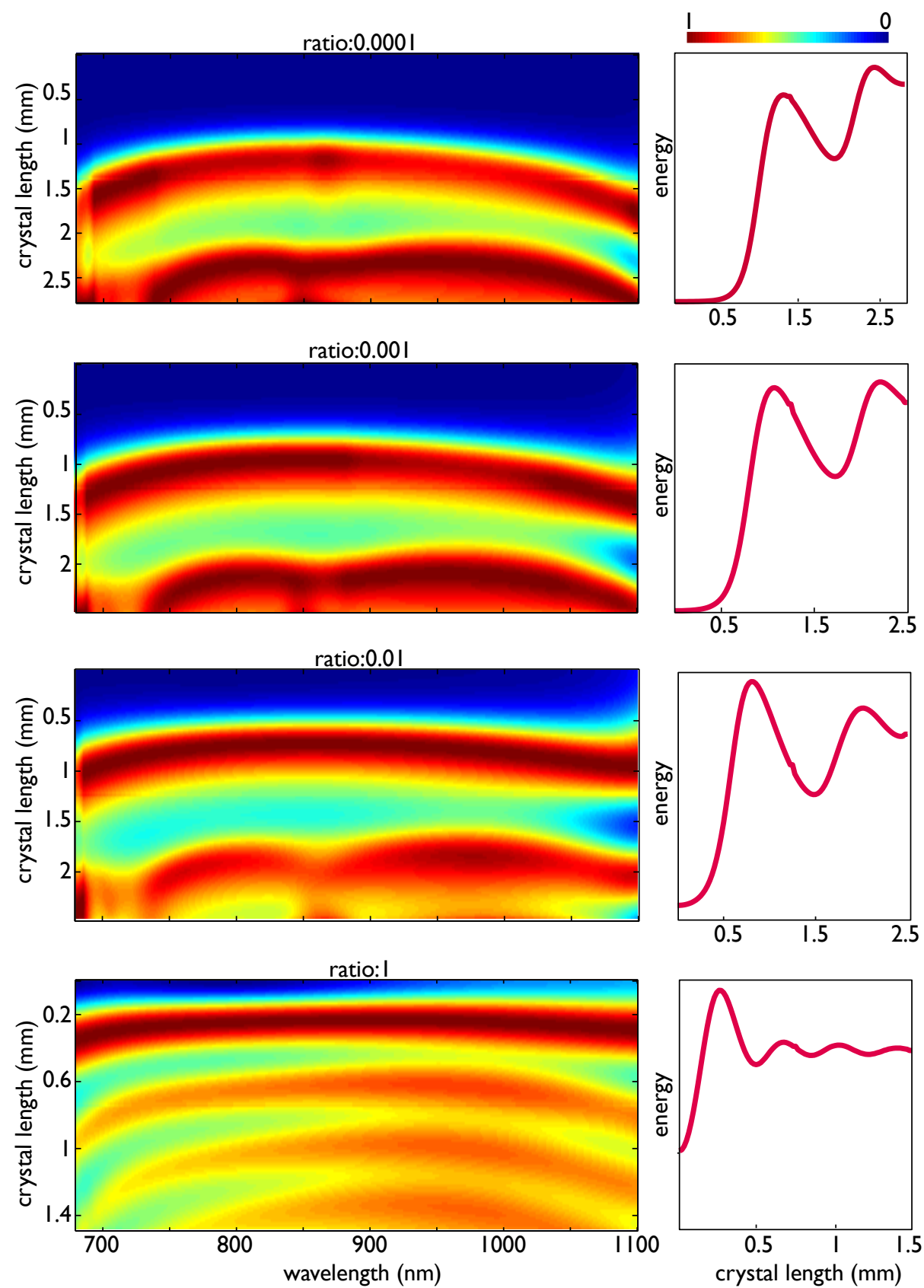

Figure 1.13: Spectral behavior of an amplified signal for different seed-topump energy ratios. Left: spectral distribution of the amplified signal at each position in the crystal. The amplitude of each spectral component over the crystal length is normalized to one. Right: The integrated spectrum at each crystal position. 
a)

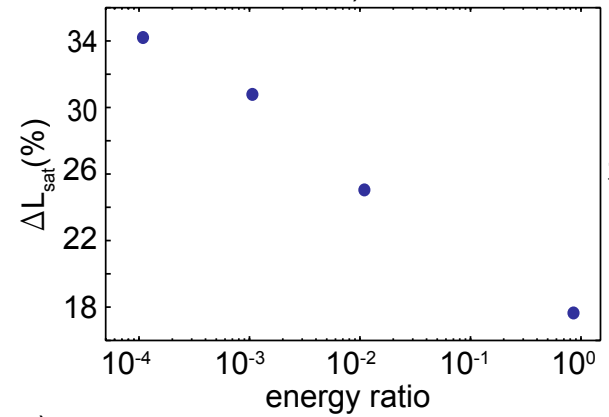

b)

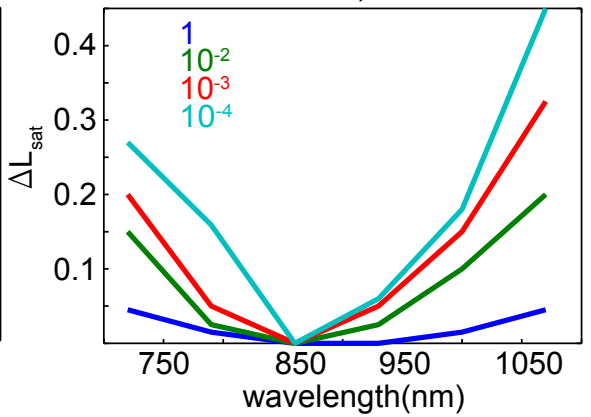

c)

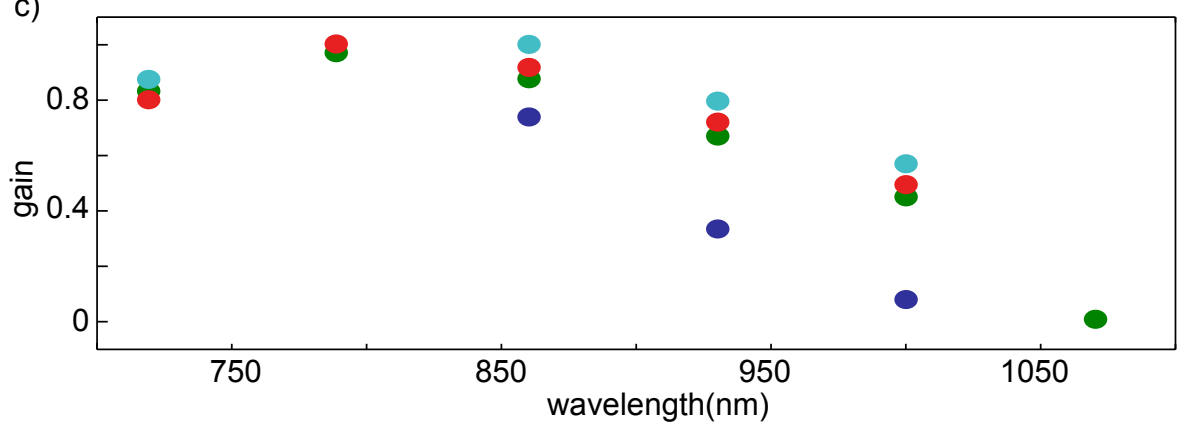

Figure 1.14: a) $\Delta L_{s a t} \times 100 / L_{s a t}$ for different input seed energy. b) Relative saturation length and c) relative amplification gain for different spectral components of a signal with different input energies.

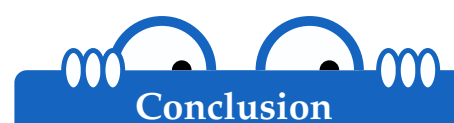

Conclusion

The signal-to-pump energy ratio is a crucial parameter in an OPCPA, as it directly affects the amplification gain, conversion efficiency and the level of superfluorescence. Based on the analytical study in this section, it is shown that this parameter influences the gain behavior of broadband OPCPA dramatically. In high-seeded OPCPA, saturation of all the signal frequencies occur simultaneously whereas for for the case of low-seeded OPCPA the saturation of different frequencies is spread over a larger length of the crystal. This modifies the shape of the amplified spectrum and limits the efficient energy extraction from the pump pulses.

It is shown analytically that the signal-to-pump energy ratio of $10^{-3}$ results in a simultaneous high amplification gain and high conversion efficiency.

\section{0 vor}




\section{OPA phase}

In the OPA process, due to the accumulated phase slip $|\Delta k L|$, caused by the phase-mismatch between the interacting waves, an additional phase is imprinted on the signal wave during amplification (Herrmann et al., 2010c). In the case of undepleted pump, the so called OPA phase is defined as:

$$
\Phi_{s}(z=L)=\Phi_{s(0)}-\frac{\Delta k L}{2}+\arctan \frac{\Delta k \times \tanh \left[L\left(g^{2}-(\Delta k / 2)^{2}\right)^{1 / 2}\right]}{2\left(g^{2}-(\Delta k / 2)^{2}\right)^{1 / 2}},
$$

Where $\Phi_{s(0)}$ is the initial signal spectral phase, $\Delta k$ is the wavevector mismatch between the interacting waves, $g$ is the small signal gain, and $L$ is the length of the OPA crystal. The dependence of the signal phase on the pump intensity, shown in Eq. (1.31), makes the compensation of the spectral phase in OPCPA very tricky and often demands an adaptive compensation element.

To study the OPCPA phase for the case of a depleted pump, the following simulations are performed (Appendix B). A Gaussian structure for the pump in both space and time and a Gaussian structure for the seed in space is assumed. The super-Gaussian seed spectrum (fourth order), is linearly chirped to $1.6 \mathrm{ps}$ pulse duration. The signal is amplified in a type I LBO crystal in a noncollinear geometry with an internal pump-signal angle of $1.1^{\circ}$ and a phase-matching angle of $14.4^{\circ}$, assuming the $d_{e f f}$ value of $0.82 \mathrm{pm} / \mathrm{V}$. The initial pump and signal energies are set to $3 \mathrm{~mJ}$ and $3 \mu \mathrm{J}$, respectively. The beam size of the pump is adjusted to provide $100 \mathrm{GW} / \mathrm{cm}^{2}$ peak intensity on the crystal. The simulation is performed for different amounts of gain by changing the crystal thickness.

The OPCPA reaches saturation in a $2.5 \mathrm{~mm}$ thick crystal. To extract the OPCPA phase, the beam after amplification is focused analytically using a convex Bk7-lens with a radius of curvature of $0.6 \mathrm{~m}$. The spectral phase of the central point of the focused beam is then calculated and subtracted from the spectral phase of the input signal. Therefore the calculated phase just includes the OPCPA phase and the introduced chirp on the pulse due to the dispersion of LBO. Later this dispersion is compensated numerically.

Fig. 1.15 shows the residual higher order OPCPA phase for different gain factors and the corresponding pulses in the time domain. For low gain, the shown third order OPCPA phase follows the phase-mismatch of the interacting waves in the LBO crystal (as shown in Fig. 1.5). In a saturated and an over-saturated OPCPA (green and blue curves in Fig. 1.15-a, respectively) the residual phase, mainly third order, increases and results in pronounced satellite pulses in the time domain. Therefore, to compensate the chirp of amplified pulses in an OPCPA system, the OPCPA phase should be carefully measured and included in the design of the compressor. 


\section{0}

Conclusion

The analytical study on the behavior of the imposed spectral phase on a signal pulse during amplification is presented in this section.

The results indicate that in addition to the spectral phase caused by dispersion in the OPCPA nonlinear crystal, the accumulated phasemismatch between the interacting beams results in a higher order dispersion (OPA phase). This higher order dispersion increases when the amplification is saturated due to the additional effect of the temporally modified pump pulses in this regime.

The intensity dependence of the OPCPA phase suggests the correlation of the wavefront of the amplified beam to intensity. Therefore in the amplified beam, each frequency may have a different divergence.

000 000 

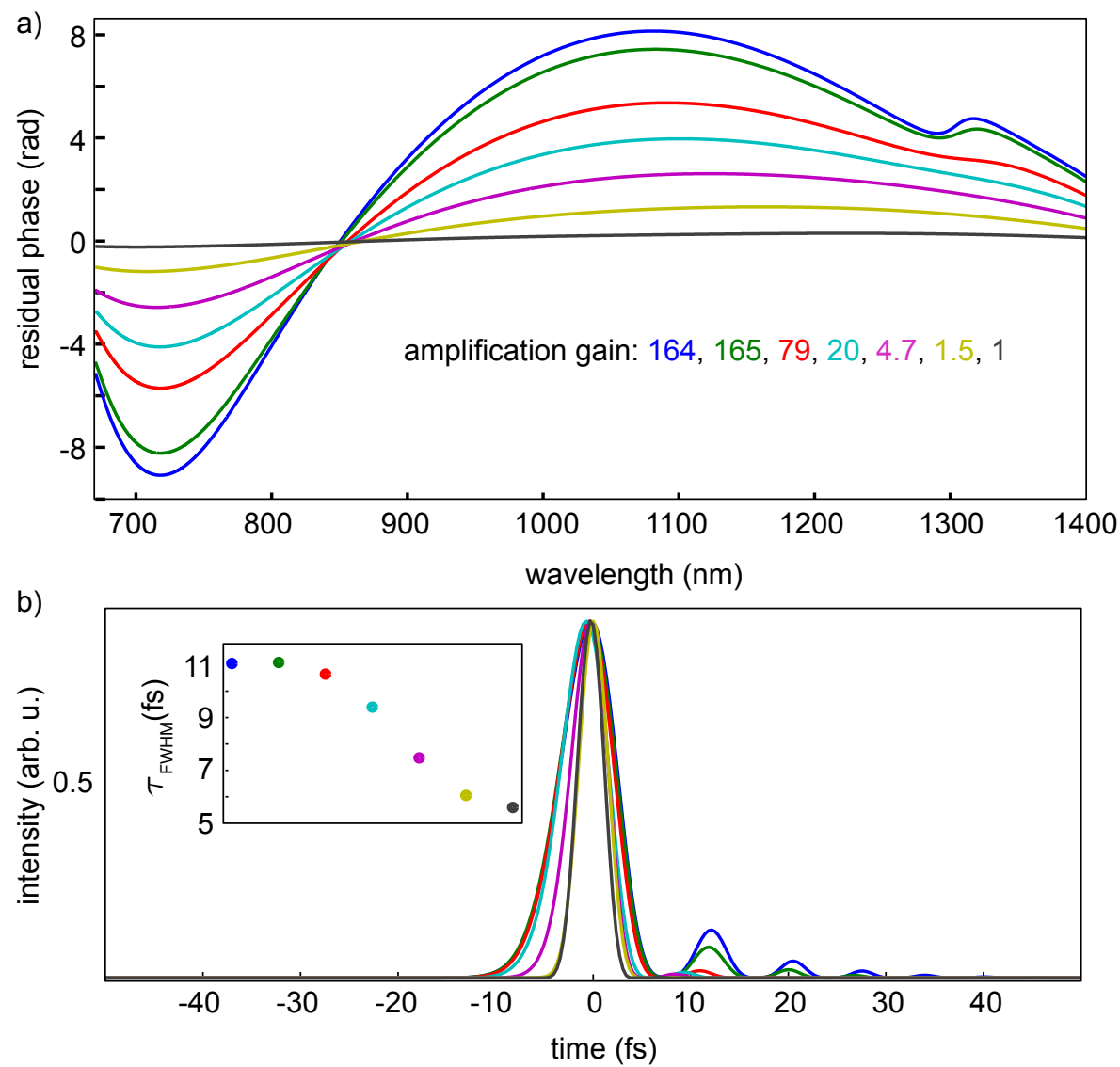

Figure 1.15: a) The residual spectral phase in OPCPAs with different gain after compensation of the linear dispersion of the amplifier and b) their corresponding pulses in the time domain. Inset: the pulse duration at FWHM. 


\subsection{Yb:YAG based OPCPA systems}

Considering the concept of OPCPA explained in the previous sections, different low-order harmonics of high-energy narrowband lasers could be utilized to amplify a broad range of frequencies extending from ultraviolet to infrared. This allows a variety of operational modes, offering multicycle pulses tunable over several octaves, few-cycle pulses at different carrier wavelengths, and multi-octave synthesis of light waveforms.

Diode-pumped ytterbium-doped (Yb-doped) thin-disk lasers are reliable, cost-effective, and power-scalable sources of high-energy 1-ps-scale laser pulses. These characteristics make them suitable sources for pumping OPCPA systems. In what follows, the feasibility of a few-cycle OPCPA and a tunable OPCPA based on diode-pumped $\mathrm{Yb}$-doped thin-disk lasers are discussed, while Chapter 4 and Chapter 5 cover the detailed study of few-cycle OPCPA and subcycle light waveforms.

Fundamental-pumped OPCPA Three types of phase-matching schemes can be used to amplify a broadband spectrum supporting ultra-short intense pulses, utilizing near-1-ps pulses centered at the fundamental wavelength of $1030 \mathrm{~nm}$ : collinear, noncollinear and quasi phase-matched.

Periodically poled $\mathrm{LiNbO}_{3}$ (PPLN) and periodically poled stoichiometric $\mathrm{LiTaO}_{3}$ (PPSLT) are suitable crystals for few-cycle OPCPA systems in the MIR range, as they have a very high nonlinearity, e.g. $d_{33}=25 \mathrm{pm} / \mathrm{V}$ for $\mathrm{LiNbO}_{3}$, resulting in a high amplification gain and therefore efficiency. However the limited aperture size of the periodically poled crystals limits their usability to low-energy-pumped OPCPA systems or pre-amplification stages to boost the signal energy-level before the high-energy OPCPA stages. Alternatively, bulk crystals like $\mathrm{LiNbO}_{3}, \mathrm{LiIO}_{3}$ or $\mathrm{BBO}$ can be used for amplification.

Phase-matching in a BBO crystal can be achieved in a noncollinear type-I geometry with an internal pump-signal angle of $2.2^{\circ}$ and a phase-matching angle of $20^{\circ}$. Although amplification in a BBO crystal with a length of $2 \mathrm{~mm}$ supports pulses as short as $19 \mathrm{fs}$ with a carrier wavelength of $2 \mu \mathrm{m}$, the linear absorption coefficient of the crystal at $2.55 \mu \mathrm{m}$ reaches $0.5 \mathrm{~cm}^{-1}$ and the accumulated heat in this process may impede the OPCPA conversion efficiency or cause crystal damage. For an internal pump-signal angle of $3^{\circ}$, the center of the amplified spectrum moves to higher frequencies, but the amplified spectral bandwidth only supports $43 \mathrm{fs}$ pulses (Fig. 1.16).

On the other hand, using a type $\mathrm{IiNbO}_{3}$ crystal with a phase-matching angle of $47^{\circ}$ at the degeneracy point in a collinear geometry or a type $\mathrm{I} \mathrm{LiO}_{3}$ supports few-cycle pulses (Fig. 1.16), but $\mathrm{LiIO}_{3}$ suffers from a low damage threshold intensity. 


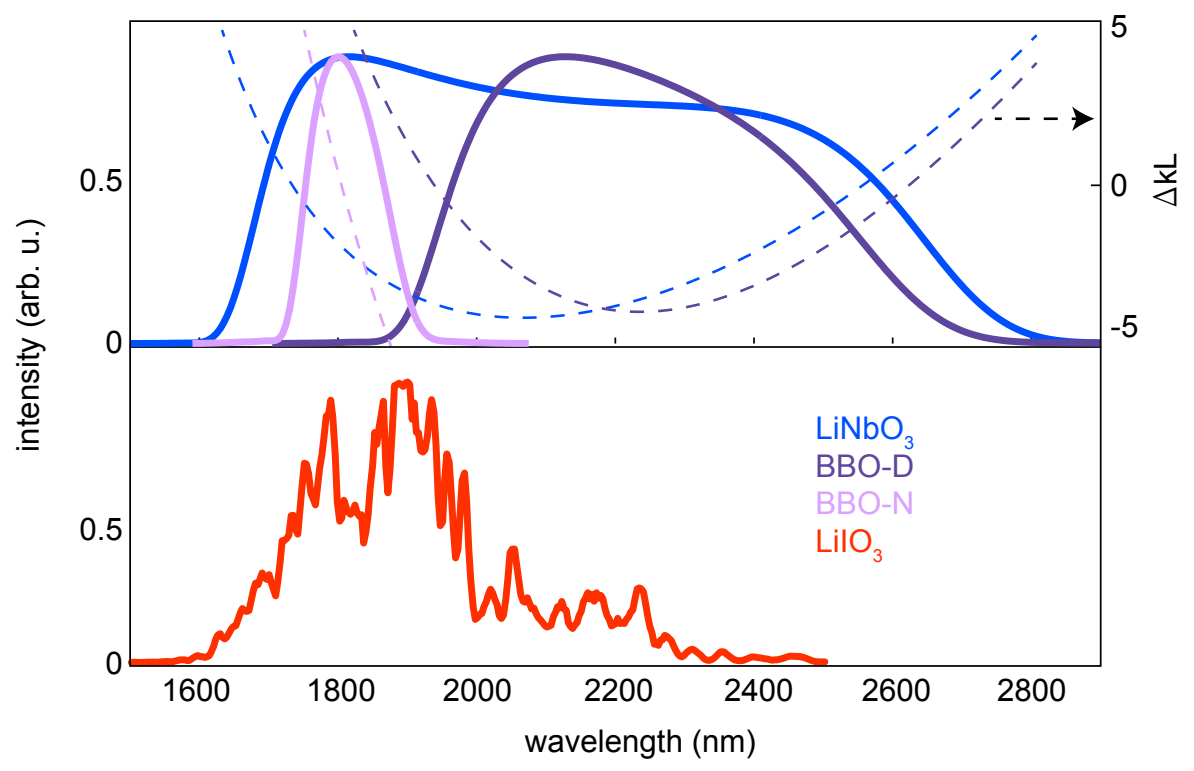

Figure 1.16: Top: Amplified spectrum in a $4 \mathrm{~mm} \mathrm{LiNbO}_{3}$, a $4 \mathrm{~mm} \mathrm{BBO}$ crystal in a collinear geometry (BBO-D), and a $2.1 \mathrm{~mm}$ BBO crystal in a noncollinear geometry (BBO-N). In the mentioned simulations, the pump energy of a $1.6 \mathrm{ps}$ pump pulses, centered at $1030 \mathrm{~nm}$, with the Gaussian profile in time and space is set to $1 \mathrm{~mJ}$. The stretched broadband seed pulses have a duration of $1.6 \mathrm{ps}$, a Gaussian profile in time, and contain $0.5 \mu \mathrm{J}$ of energy. The dashed lines show the accumulated OPA phase over $2 \mathrm{~mm}$-thick crystals. Bottom: the amplified spectrum in a $2 \mathrm{~mm}$ thick $\mathrm{LiIO}_{3}$ crystal in the first stage of the OPCPA system described in (Deng et al., 2012).

In reality, even in a "collinear" degenerate OPCPA a small angle between the pump and seed beams is required to spatially separate the signal and the idler in order to avoid interference between them. This separation is necessary when the carrier envelope phase (CEP) offset of signal pulses are stable, as the generated idler pulses are not CEP stable. Even for an OPCPA with a CEP stabilized pump and signal, which results in a CEP stabilized idler, a small angle is required for pulse compression as the idler and signal possess opposite chirp. Fig. 1.17 shows the effect of the noncollinear angle on the shape of the amplified spectrum and the OPCPA gain in a $4 \mathrm{~mm}$-thick $\mathrm{LiNbO}_{3}$ crystal. The simulation is performed for a pump energy of $1 \mathrm{~mJ}$ at $1030 \mathrm{~nm}$ and a signal input energy of $0.5 \mu \mathrm{J}$.

Among the mentioned crystals, $\mathrm{LiNbO}_{3}$ is the most suitable one for highenergy OPCPA systems as it supports a broad amplification bandwidth, is available with large apertures, and has a high $\mathrm{d}_{\text {eff }}$ value of $3.3 \mathrm{pm} / \mathrm{V}$ where $\mathrm{d}_{31}=4.35 \mathrm{pm} / \mathrm{V}$; although its $n_{2}$ value is three times higher than $\mathrm{BBO}$ 


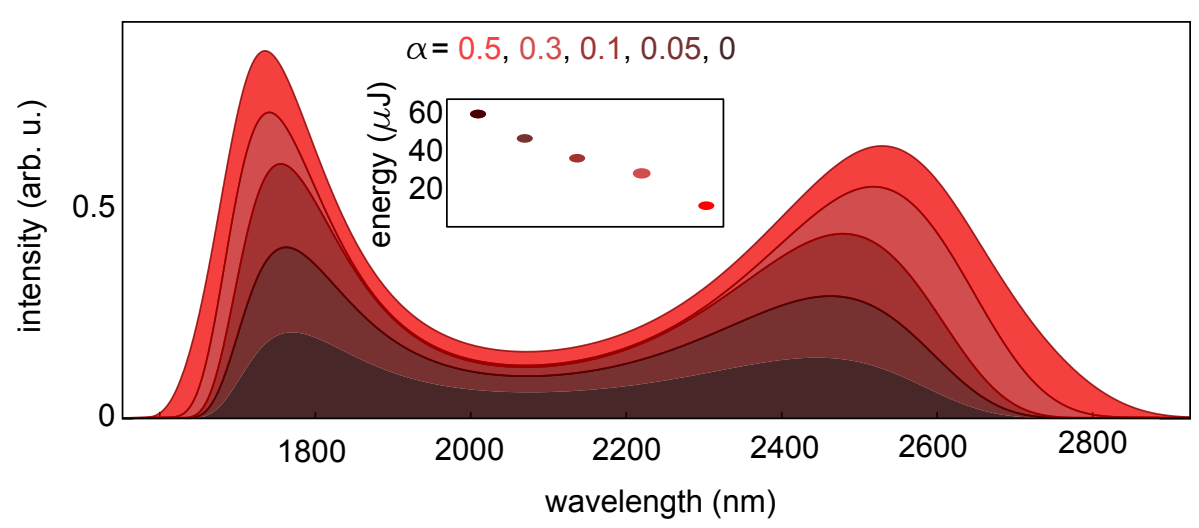

Figure 1.17: The effect of the noncollinear angle $(\alpha)$ on the amplified spectrum in a $4 \mathrm{~mm} \mathrm{LiNbO}_{3}$ crystal. Inset: the corresponding effect of the noncollinear angle on the amplification gain. In this graph, the relative amplitude of the spectra does not show their relative energy.

and the applicable pump intensity in the crystal is limited to a value below $70 \mathrm{GW} / \mathrm{cm}^{2}$ due to photorefraction (Furukawa et al., 2000; Augstov and Shvarts, 1980).

Second-harmonic pumped OPCPA By using the frequency doubled output of $\mathrm{Yb}$-doped lasers to pump an OPCPA, the amplification gain can be shifted to the NIR range of the spectrum, extending from about $650 \mathrm{~nm}$ to $1400 \mathrm{~nm}$. As the zero-dispersion point of the majority of nonlinear crystals is above $1300 \mathrm{~nm}$, broadband-collinear-degenerate OPCPA is not an option. Therefore, to achieve a broadband amplification, noncollinear geometry should be used.

Crystals like YCOB (Pires et al., 2014), BBO (Bromage et al., 2011; Rothhardt et al., 2012; Schultze et al., 2010b), LBO (Thai et al., 2010) and DKDP (Skrobol et al., 2012), pumped at $515 \mathrm{~nm}$, support broadband phase-matching in a noncollinear geometry. Among these crystals, LBO and BBO are distinguished by their high nonlinearities and broadband amplification gain spectra.

$\mathrm{BBO}$ is a robust, negative uniaxial crystal but limited in available aperture size to $25 \times 25 \mathrm{~mm}^{2}$. LBO is a biaxial crystal supporting broad phasematching in the $X Y$ plane, where $\theta=90^{\circ}$ (Zhao et al., 2008). The crystal in this plane can be treated similarly to a negative uniaxial crystal with $n_{0}=n_{z}$ and

$$
\begin{gathered}
\frac{1}{\lambda_{p}}=\frac{1}{\lambda_{s}}+\frac{1}{\lambda_{i}} \\
\left(\frac{n_{i}}{\lambda_{i}}\right)^{2}=\left(\frac{n_{s}}{\lambda_{s}}\right)^{2}+\left(\frac{n_{p}}{\lambda_{p}}\right)^{2}-2 \cos (\alpha) \frac{n_{s} n_{p}}{\lambda_{s} \lambda_{p}},
\end{gathered}
$$




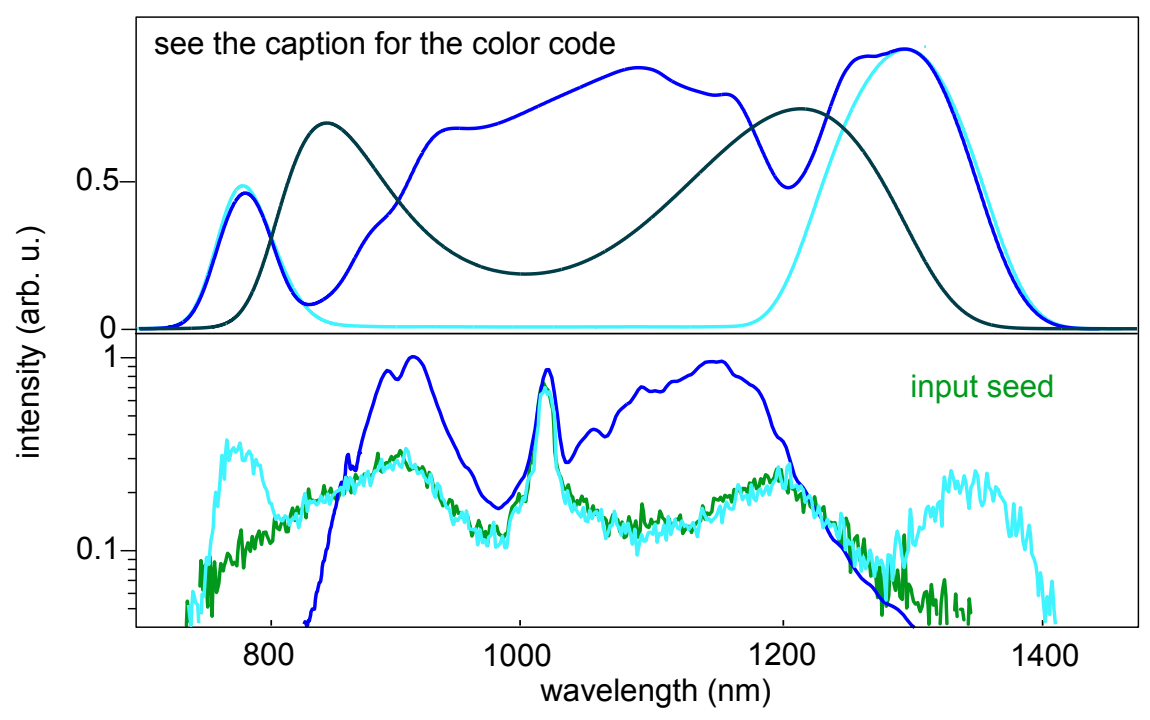

Figure 1.18: Top: simulated amplified spectra in a 2-stage OPCPA employing LBO crystals with different phase-matching angles. In the first OPCPA stage, the phase-matching angle of $\theta: 13.8^{\circ}$ is used to amplify the input spectrum in its wings (light blue line). In the second stage the phase matching is tuned to $\theta: 14.9^{\circ}$, to amplify the middle of the input spectrum (dark blue line). The black line shows the amplified spectrum in a 2-stage OPCPA system using LBO crystals with a phase-matching angle of $14.4^{\circ}$, for comparison. It can be seen in the first case broader spectrum is amplified. Bottom: Experimental proof of the mentioned simulation. Here by using a $4 \mathrm{~mm}$ LBO crystal and for different phase-matching angles, the wings or the middle of the input spectrum is amplified. The OPCPA seed in this measurement was generated in a $4 \mathrm{~mm}$ TGG crystal. The super-continuum is passed through a $95 \%$ output coupler to suppress the intense spectral components in the vicinity of $1030 \mathrm{~nm}$ (Appendix D).

$$
\frac{1}{n_{p}^{2}}=\frac{\cos ^{2}(\theta)}{n_{p y}^{2}}+\frac{\sin ^{2}(\theta)}{n_{p x}^{2}} .
$$

One of the advantages of the noncollinear geometry is the possibility of adjusting the amplified spectrum by changing the phase-matching angle and the noncollinear angle. Here, by changing the phase-matching angle $\theta$, the amplification bandwidth can be tuned around a fixed central frequency. Fig. 1.18 shows the simulated amplified spectrum in a 2-stage OPCPA system. The simulation is performed for a Gaussian pump pulse in time and space with an energy of $1 \mathrm{~mJ}$ and a pulse duration at FWHM of $1.6 \mathrm{ps}$ while the beam size is set to $740 \mu \mathrm{m}$ at FWHM. The input seed energy is $1 \mu \mathrm{J}$. 
In the first amplification stage, with a phase-matching angle of $13.8^{\circ}$ and a noncollinear angle of $1.1^{\circ}$, the amplification gain is moved to the wings of the spectrum. Changing the phase-matching angle of the second stage to $14.9^{\circ}$ shifts the gain to the center of the spectrum. This spectrum is compared with a 2-stage OPCPA system with LBO crystals at a phase-matching angle of $14.4^{\circ}$. As shown in Fig. 1.18, the first scheme results in a somewhat broader spectrum. Fig. 1.18-bottom shows the experimental demonstration of this process in an OPCPA stage seeded with a supercontinuum generated in a TGG crystal (Appendix D).

This flexibility for the gain bandwidth engineering in NOPA systems is theoretically and experimentally investigated in Section 4.3 by utilizing BBO and LBO crystals. 
Third-harmonic pumped OPCPA The third-harmonic of an Yb-doped laser can be obtained via sum frequency generation using the fundamental frequency and the second harmonic of the laser. The OPCPA crystal in this range should have a high band-gap energy to avoid resonant absorption in the OPCPA. Among different groups of nonlinear crystals, the borate group is the most suitable to be used in the ultraviolet region due to their high nonlinear coefficients, transparency and high laser induced damage thresholds. A broadband amplification gain in a noncollinear OPCPA, pumped at $343 \mathrm{~nm}$, can be achieved in DKDP and BBO crystals (Fig. 1.19), but BBO is more favorable due to its higher $d_{\text {eff }}$ value.

\begin{tabular}{|c|c|c|c|c|c|}
\hline material & LBO & BBO & KBBF & KDA & DKDP \\
\hline $\mathrm{d}_{\text {eff }}(\mathrm{Pm} / \mathrm{V})$ & 0.8 & 2 & 0.45 & 0.5 & 0.3 \\
\hline
\end{tabular}

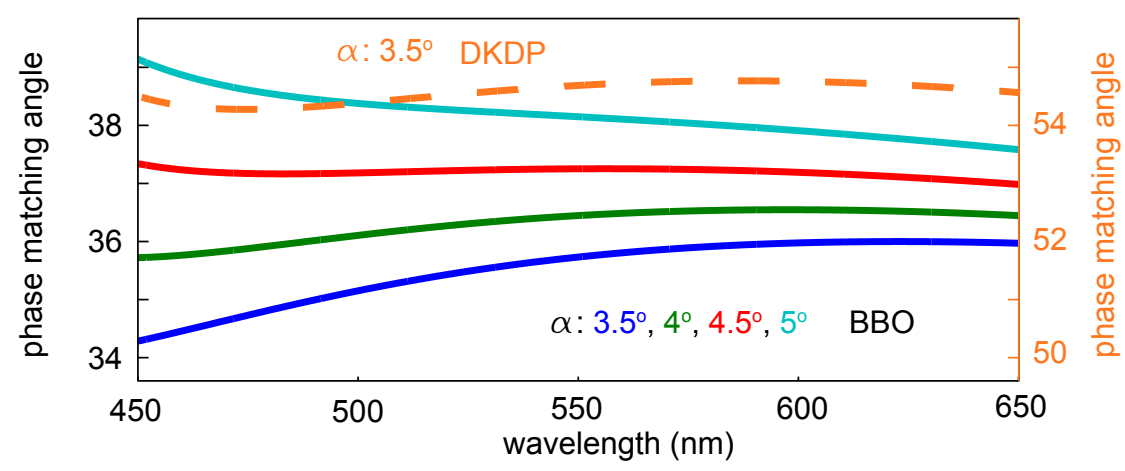

Figure 1.19: Top: comparison between the nonlinearity of different crystals suitable for third harmonic-pumped OPCPA. Bottom: Phase-matching angles for broadband amplification in BBO and DKDP crystals pumped at $343 \mathrm{~nm}$.

A serious challenge in short-pulse-pumped and short-wavelength-pumped OPCPAs are higher-order nonlinear effects in the wavelength range of the pump beam. As the pump wavelength gets closer to the resonance frequency of the crystal, $\chi^{(3)}$ grows rapidly and as a result, the efficiency of third order nonlinear effects like self-phase modulation (SPM) and thirdharmonic generation (THG) in the crystal increases drastically. The third harmonic of the pump at $343 \mathrm{~nm}$ is at $114 \mathrm{~nm}$. The linear absorption coefficient of BBO for $266 \mathrm{~nm}$ is $0.15 \mathrm{~cm}^{-1}$ and the crystal is not transparent for wavelengths below $190 \mathrm{~nm}$. This absorption could lead to damage in the crystal.

The other issue in this range is two photon absorption (TPA). The re- 
ported TPA coefficient at $211 \mathrm{~nm}$ is $2.43 \mathrm{~cm} / \mathrm{GW}$ for $\mathrm{BBO}$ and $1.03 \mathrm{~cm} / \mathrm{GW}$ for LBO. These numbers decrease at $264 \mathrm{~nm}$ to $0.93 \mathrm{~cm} / \mathrm{GW}$ for $\mathrm{BBO}$ and $0.15 \mathrm{~cm} / \mathrm{GW}$ for LBO. Due to the UV cutoff edge at $189 \mathrm{~nm}$ for BBO and $160 \mathrm{~nm}$ for LBO, TPA is a severe energy loss mechanism for intense ultrashort UV pulses, which also contributes to pulse broadening (Dubietis et al., 2000).

A numerical simulation software SISYFOS (Appendix B), is used to estimate TPA in a $343 \mathrm{~nm}$-pumped OPCPA. The matrix used to describe the third order effects consists of $[i \times n b+j]$ elements, which describes how beam $j$ induces absorption of beam $i$, where $n b$ is the number of beams. As there is hardly any measured data on non-degenerate TPA coefficients and the beam with the highest frequency has the maximum TPA, the TPA is only calculated for the pump beam. The TPA coefficient for a BBO crystal at $354.7 \mathrm{~nm}$ and a $17 \mathrm{ps}$ pulse is $1 \times 10^{-11} \mathrm{~cm} / \mathrm{W}$. Therefore, for the pump pulses with an intensity of $100 \mathrm{GW} / \mathrm{cm}^{2}$, the absorption coefficient becomes $1 \mathrm{~cm}^{-1}$.

The measured damage threshold intensity for a $\mathrm{BBO}$ crystal irradiated with several ns-pump pulses is $48 \mathrm{GW} / \mathrm{cm}^{2}$ at $532 \mathrm{~nm}$, and $25 \mathrm{GW} / \mathrm{cm}^{2}$ at $355 \mathrm{~nm}$ (Nakatani et al., 1988), which is expected to increase for ps-pumplasers. The estimated TPA, using the mentioned parameters, is negligible for $40 \mathrm{~mJ}, 1.6 \mathrm{ps}$ pulses at $343 \mathrm{~nm}$.

In an alternative approach, to generate a few-cycle pulse in the VIS, a thin BBO crystal can be used to generate the second harmonic of a broadband OPCPA signal, pumped by the second harmonic of the laser. The gain bandwidth of the second-harmonic signal is inversely proportional to the length of the crystal and the efficiency scales quadratically in the unsaturated regime. Figure 1.20 shows the simulated second harmonic spectrum of a $5 \mathrm{fs}$ pulse with Gaussian distribution in time and space. The beam size is set for $800 \mathrm{GW} / \mathrm{cm}^{2}$ peak intensity on the crystal. The phase-matching angle of a type I BBO crystal is set to $25.6^{\circ}$ and for a type I LBO it is $15.5^{\circ}$. The second-harmonic generation (SHG) efficiency in LBO is much lower than $\mathrm{BBO}$ for the same crystal thickness, but LBO supports a broader second harmonic spectrum. The same spectrum can be achieved for $50 \mu \mathrm{m}$-thick BBO with an efficiency of $9.5 \%$ and $100 \mu \mathrm{m}$-thick LBO with an efficiency of $5 \%$. To overcome this poor conversion efficiency, achromatic SHG technique can be used. Here, the fundamental beam can be angularly dispersed to facilitate the phase-matching of a wide range of frequencies, which leads to efficiencies as high as $20 \%$ (Wnuk et al., 2010). However, the technique requires a complicated setup to minimize the angular dispersion of broadband pulses. Therefore, OPCPA is a more promising route to achieve broadband, high energy pulses in the VIS range. 

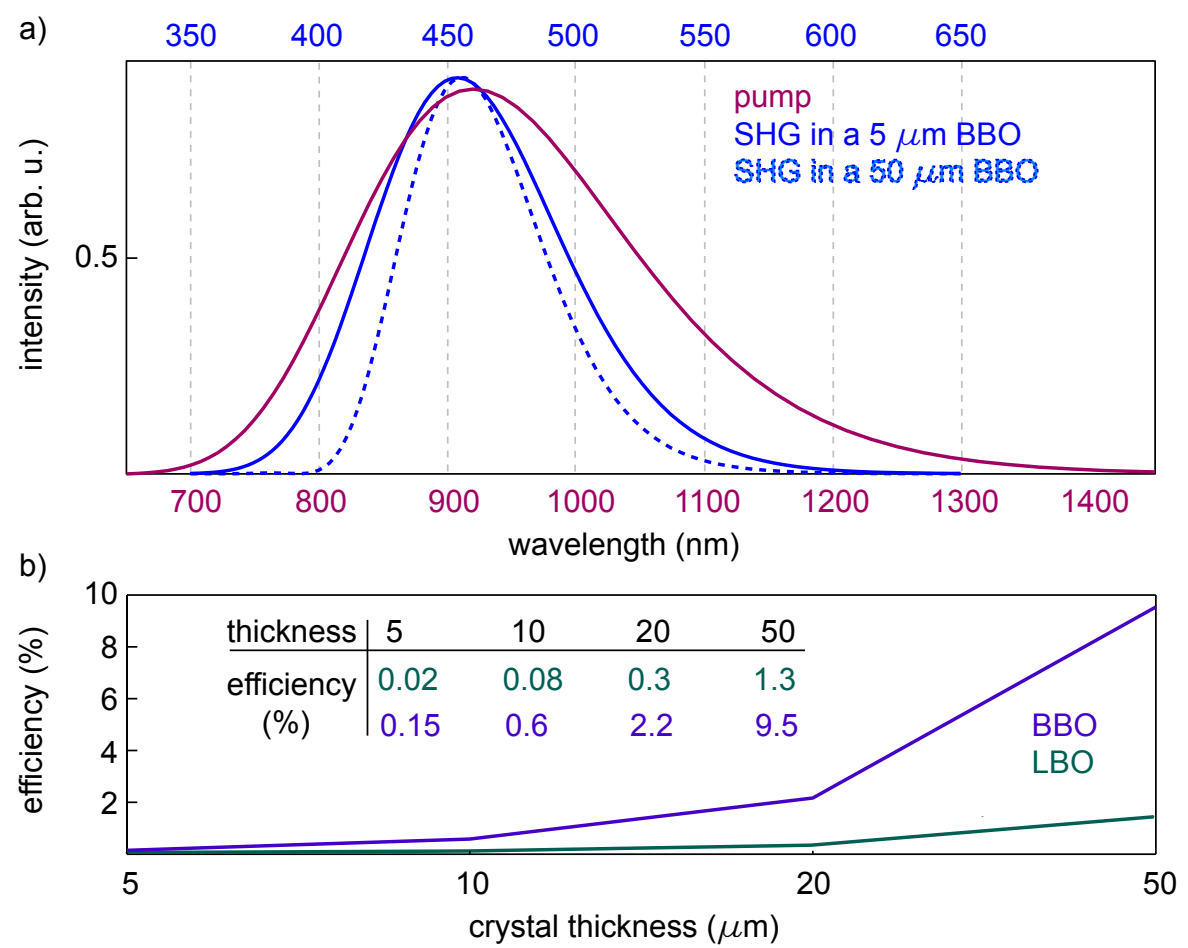

Figure 1.20: a) Simulated SHG in a $5 \mu \mathrm{m}$ and $50 \mu \mathrm{m}$ BBO crystal. b) SHG efficiency for BBO and LBO crystals of different thicknesses.

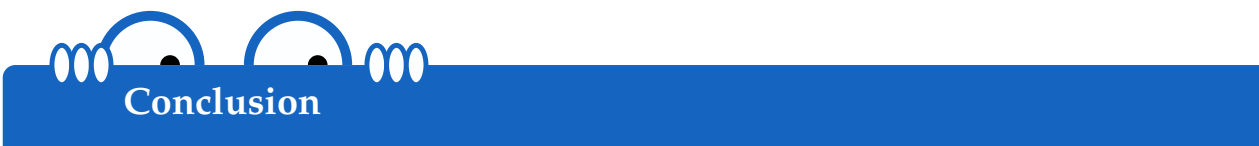

By employing different harmonics of an Yb:YAG thin-disk laser to pump an OPCPA system, few-cycle, high-energy, pulses at different carrier wavelength from visible to mid-infrared can be generated. Among the variety of crystals and phase-matching techniques, degenerate OPCPA in $\mathrm{LiNbO}_{3}$, noncollinear OPCPA in BBO and LBO, and noncollinear OPCPA in BBO pumped by fundamental, second- and third harmonics of an Yb:YAG thin-disk laser, respectively, are the methods of choice in terms of the amplification bandwidth and nonlinearity. These studies are exploited for experimental realization of a mJ-level, sub-two-cycle OPCPA system, centered at $1 \mu \mathrm{m}$ (Chapter 4) and a design study of a multi-channel synthesizer (Chapter 5 ). 


\subsection{Tunable OPCPA}

${ }^{1}$ Time resolved spectroscopy often requires tunable multi-cycle femtosecond pulses. OPCPA systems based on Yb:YAG lasers provide mJ-level, sub-50fs pulses, tunable over a broad spectral range spanning from UV to MIR. In this section, the design and feasibility of such a system is simulated and discussed. The following simulation is performed for a Yb:YAG thin-disk regenerative amplifier yielding 30-mJ, 1.6-ps pulses at $10-\mathrm{kHz}$ repetition rate, i.e. an average power level of $300 \mathrm{~W}$ (Teisset et al., 2013), as high average powers are often desirable in nonlinear spectroscopy to increase the signalto-noise ratio.

The fundamental pulse along with its second and third harmonics, are used to pump and amplify a supercontinuum spanning from $450 \mathrm{~nm}$ to $3 \mu \mathrm{m}$. The seed is divided into three bands centered at carrier wavelengths of $550 \mathrm{~nm}, 1 \mu \mathrm{m}$ and $2 \mu \mathrm{m}$, which are pumped with the third-harmonic, secondharmonic and fundamental pulses of the Yb:YAG laser, respectively. Each OPCPA arm consists of two amplifier stages, where the residual pump energy after the first stage is used to pump the second stage (see Section 4.1), using thin $\mathrm{BBO}, \mathrm{LBO}$ and $\mathrm{LiNbO}_{3}$ crystals, pumped at $343 \mathrm{~nm}, 515 \mathrm{~nm}$ and $1030 \mathrm{~nm}$, respectively.

Pulse-duration control and wavelength tuning of the amplified pulses is accomplished by temporally stretching the seed continua and controlling their delay with respect to the pump pulses. In fact, a pump temporal window of $\tau_{\text {pump }} \simeq 1$ ps slices out a fraction of the bandwidth, $\Delta v_{\text {signal }}$, of the stretched seed continuum, $\Delta v_{\text {seed }}$, which is inversely proportional to the duration $\tau_{\text {seed }}$ of the stretched seed: $\Delta v_{\text {signal }} \simeq \Delta v_{\text {seed }}\left(\tau_{\text {pump }} / \tau_{\text {seed }}\right)$ (Fig. 1.21). Thanks to the near-linear chirp carried by the stretched seed, the carrier frequency of the amplified signal can be tuned by varying the delay of the seed with respect to the pump pulse and by adjusting the phase-matching angle of the amplifier crystal. Fig. 1.21 shows a series of amplified signal spectra from simulations in the three channels pumped with pulses of a duration $\tau_{\text {pump }} \simeq 1.7 \mathrm{ps}$. The continuum is stretched to $\tau_{\text {seed }} \simeq 30 \mathrm{ps}$.

For the numerical study of the tunable OPCPA system, a Gaussian beam profile for both pump and seed and a Gaussian temporal profile of the pump pulses are assumed. By using different harmonics of the regenerative amplifier and different crystals, mJ-energy, sub-40-fs pulses, tunable from $445 \mathrm{~nm}$ to $2750 \mathrm{~nm}$ could be generated. The length of the OPCPA crystals is kept constant for each chain. As the amplification gain is different for different frequencies, the gain saturation of the frequencies in one set of crystal lengths is ensured by shaping and scaling the amplitude of the input signal

${ }^{1}$ This part is adapted from (Fattahi et al., 2014a). The electronic print of this paper is attached in Appendix E with the permission of OSA. 
a)

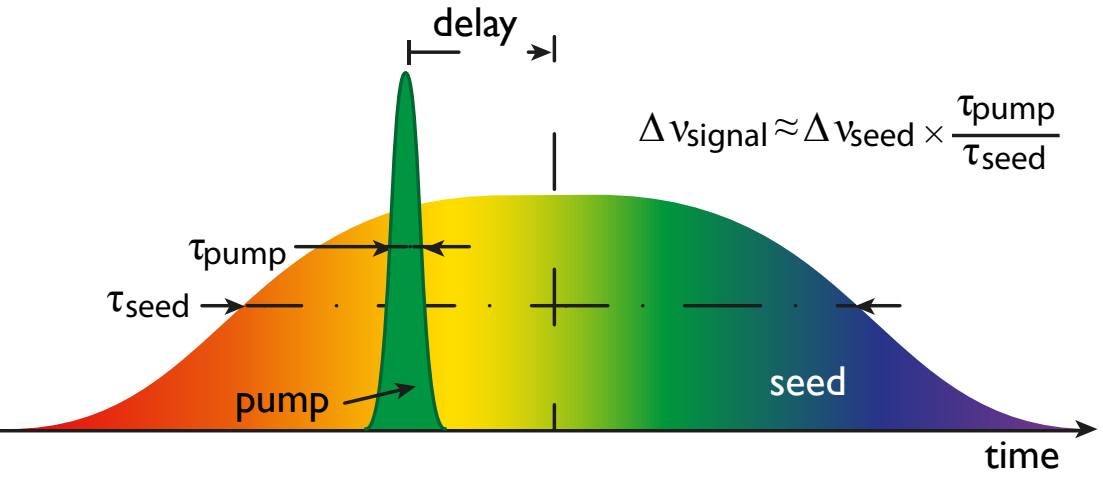

b)

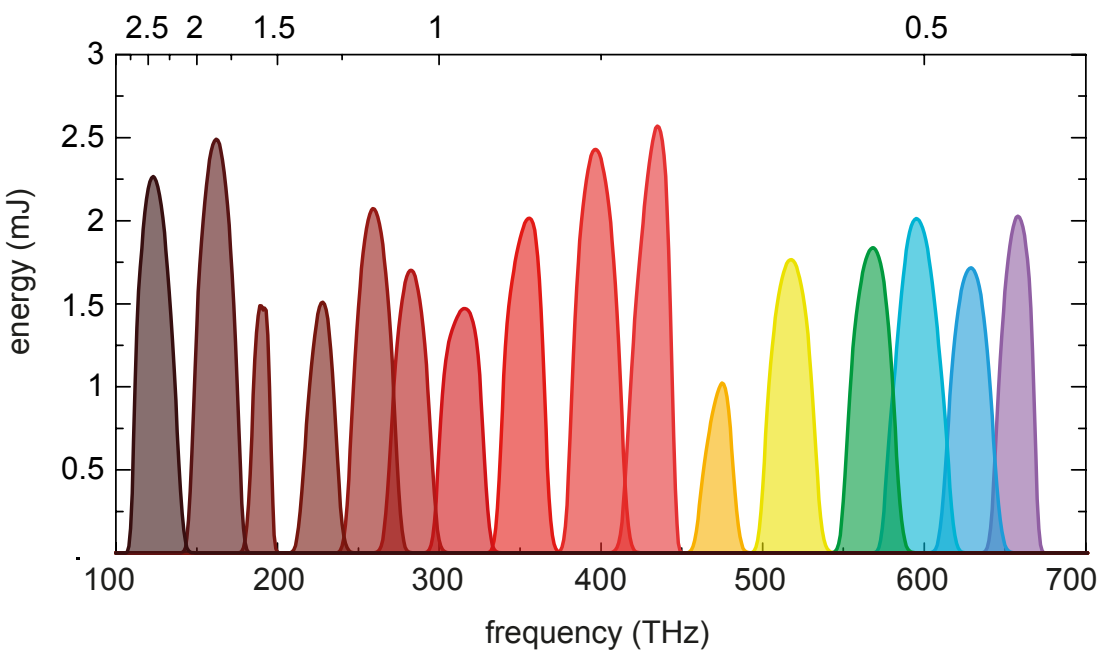

Figure 1.21: Generation of widely tunable femtosecond pulses. a) Scheme for generating spectrally and temporally tunable pulses at any wavelength. The seed pulses are stretched to a significantly longer duration than that of the pump pulse. Amplification in a simple OPCPA setup yields pulses that can spectrally be tuned by changing the temporal delay between pump and seed pulse. b) Amplified spectra of a widely tunable two-stage OPCPA system obtained from simulations. Millijoule-level, sub-40-fs pulses, tunable from 445 to $2750 \mathrm{~nm}$, can be generated by using different harmonics of a $10 \mathrm{kHz}$ Yb:YAG regenerative amplifier. The shown spectra are normalized to their energy. 
to the cascaded OPCPA stages. By utilizing a prism compressor, these pulses can be compressed close to their Fourier transform limits. The detailed parameters of the simulation are summarized in Table 1.1 and Table 1.2.

\begin{tabular}{l|ccc} 
pump harmonics & pump energy & crystal & tunable bandwidth \\
\hline fundamental & $12 \mathrm{~mJ}$ & $\mathrm{LiNbO}_{3}$ & $1500-2750 \mathrm{~nm}$ \\
second harmonic & $10 \mathrm{~mJ}$ & LBO & $670-1420 \mathrm{~nm}$ \\
third harmonic & $8 \mathrm{~mJ}$ & BBO & $445-660 \mathrm{~nm}$
\end{tabular}

Table 1.1: General parameters of the tunable OPCPA system.

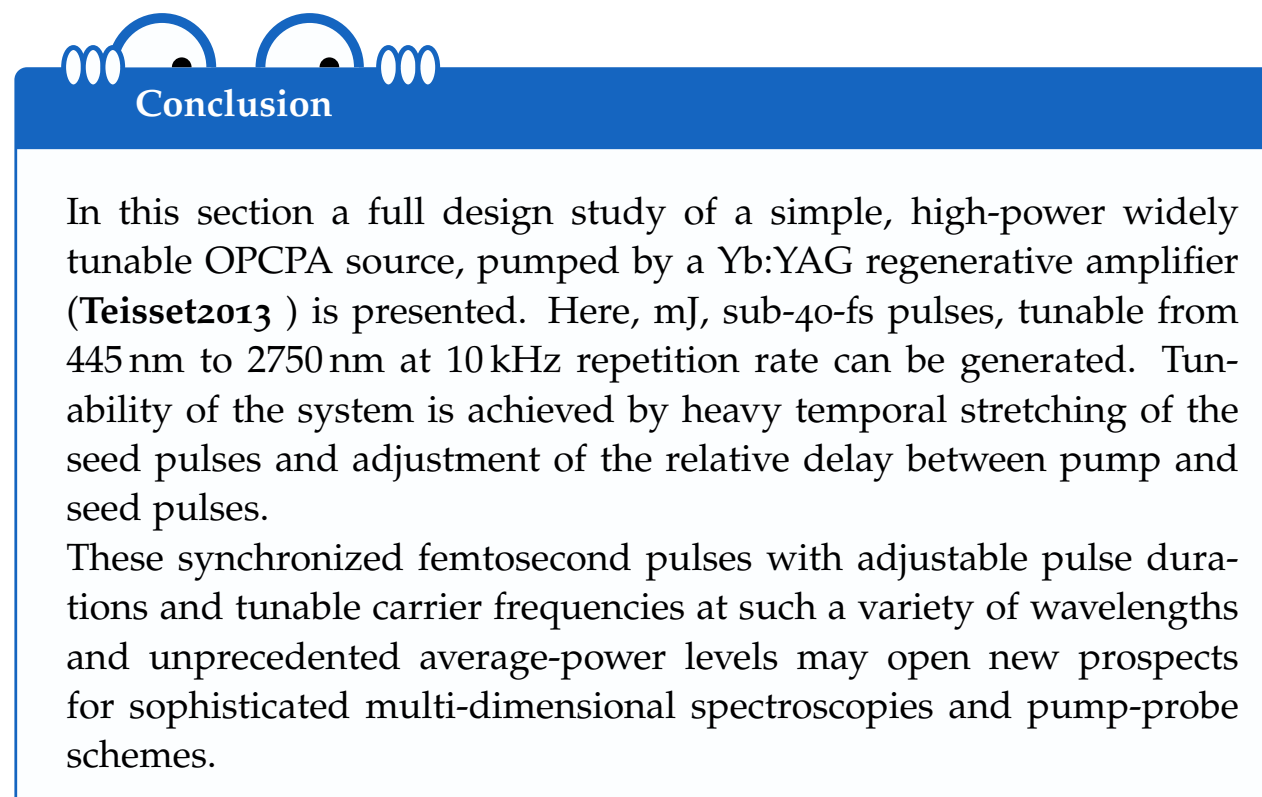

$000 \quad 000$ 


\begin{tabular}{|c|c|c|}
\hline \multicolumn{3}{|c|}{ fundamental-pumped } \\
\hline 2450 & $300 \mathrm{~nJ}$ & 0.7886 \\
\hline 1750 & $300 \mathrm{~nJ}$ & 0.7893 \\
\hline 1550 & $300 \mathrm{~nJ}$ & 0.8 \\
\hline \multicolumn{3}{|c|}{ second harmonic-pumped } \\
\hline wavelength $(\mathrm{nm})$ & input seed energy & phase-matching angle (rad) \\
\hline 1350 & I50 nJ & 0.26 \\
\hline 1150 & $105 \mathrm{~nJ}$ & 0.2714 \\
\hline 1050 & $60 \mathrm{~nJ}$ & 0.2714 \\
\hline 950 & $50 \mathrm{~nJ}$ & 0.2714 \\
\hline 850 & $20 \mathrm{~nJ}$ & 0.2714 \\
\hline 750 & $300 \mathrm{~nJ}$ & 0.2714 \\
\hline 700 & $300 \mathrm{~nJ}$ & 0.2714 \\
\hline
\end{tabular}

\begin{tabular}{|c|c|c|}
\hline \multicolumn{3}{|c|}{ third harmonic-pumped } \\
\hline wavelength (nm) & input seed energy & phase-matching angle (rad) \\
\hline 640 & $10 \mathrm{~nJ}$ & 0.569 \\
\hline 575 & $50 \mathrm{~nJ}$ & 0.5712 \\
\hline 525 & $50 \mathrm{~nJ}$ & 0.5712 \\
\hline 500 & $300 \mathrm{~nJ}$ & 0.5712 \\
\hline 475 & $100 \mathrm{~nJ}$ & 0.5712 \\
\hline 450 & $300 \mathrm{~nJ}$ & 0.5716 \\
\hline
\end{tabular}

Table 1.2: Detailed parameters of the tunable OPCPA system. 



\section{Chapter 2}

\section{Broadband seed generation}

A major requirement for developing the OPCPA systems discussed in Chap$\operatorname{ter} 1$, is the availability of seed pulses with a broadband spectrum, a wellbehaved spectral phase, and preferably a stable carrier envelope phase (CEP).

Traditionally, OPCPA seeds were provided by using low-energy broadband spectrum of a Ti:sapphire oscillator (Ishii et al., 2005). The seed pulses in this case have a good temporal contrast, well-behaved spectral phase and their carrier envelope phase offset can be actively stabilized (Telle et al., 2014). By using the oscillator to seed the OPCPA chain and the OPCPA pump laser, pump and seed pulses are intrinsically synchronized (Teisset et al., 2005). However due to the long optical beam path difference between seed and pump pulses, mechanical vibrations of optical components and temperature drifts additional timing jitter happens, which needs to be compensated by an active temporal synchronization system (Schwarz et al., 2012; Teisset, 2009; Fattahi et al., 2012b). Despite all the above mentioned advantages the pulse energy is limited to several tens of nJ. This can be resolved by using an additional OPCPA stage or generating the seed via supercontinuum after boosting the output of the oscillator in a regenerative amplifier. However, this results in more superfluorescence (Tavella et al., 2006) in the first approach and degraded temporal contrast in the second approach.

Recently demonstrated kerr-lens mode-locked Yb:YAG thin-disk oscillators (Pronin et al., 2011) are a promising alternative to Ti:sapphire as the front-end of OPCPA systems. Their relatively narrowband spectrum can be externally broadened in a cascaded nonlinear stage. Compression of the broadened spectrum results in $10 \mathrm{fs}$ pulses with $260 \mathrm{~nJ}$ of energy at $40 \mathrm{MHz}$ repetition rate (Seidel et al., 2014). The average power of these reproducible waveform-controlled few-cycle pulses exceeds the average power of Ti:sapphire oscillators by more than one order of magnitude. These externally CEP stabilized pulses (Pronin et al., 2013) combined with optical synchronization provides a suitable OPCPA seed at $\mu \mathrm{J}$-levels of energy. 
The need for an active pump-signal temporal synchronization system can be eliminated by generating the broadband seed from a near-1-ps OPCPA pump pulses. In spite of several attempts to generate such a broadband spectrum via supercontinuum generation with sub-10o-fs pump pulses in solids and waveguides (Bradler et al., 2009; Silva et al., 2012; Seidel et al., 2014), the following question remains open: is there a straightforward approach to generate a broadband signal from a near-1-ps pulse? Another appealing question is: What is the best method for generation of a multioctave, CEP-stable spectrum suitable for seeding a multi-channel OPCPA system?

In this chapter different methods for spectral broadening of sub-10o-fs and near-1-ps pulses are discussed. In the beginning spectral broadening of near-1-ps pulses in large mode area (LMA) fibers is shown. Then sub-10ofs-pump spectral broadening in solids and photonic crystal fibers (PCF) are investigated. Cross-polarized wave generation (XPW) as a simple tool for pulse-shortening, which enhances the temporal contrast of the pulse simultaneously, are studied theoretically and experimentally. This chapter culminates with a full study of the difference frequency generation (DFG) process as the most promising route to the generation of a CEP-stable, multi-octave seed for OPCPA systems. 

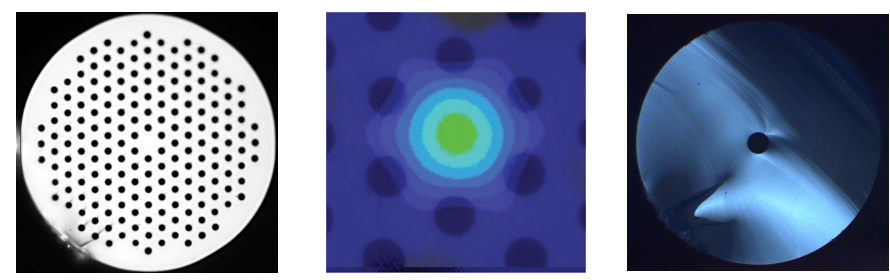

Figure 2.1: Cross-section of an LMA fiber (left) and its beam profile in the far field (middle) (Newport Corporation 2014). Cross section of a HCF (right).

\subsection{Spectral broadening in large mode area Fibers}

Spectral broadening in a waveguide is preferred to spectral broadening in bulk solids due to the longer interaction length resulting in a higher total nonlinearity at a fixed intensity. In PCFs, pioneered by (P. St. J. Russell) in 1996 (Knight et al., 1997; Birks et al., 1997) dispersion and confinement of PCFs are determined by the periodicity of very tiny air holes, contrary to a normal step-index fiber, where the properties are designed by a spatially varying glass composition (Fig. 2.1). PCFs surpass the performance of step-index single-mode fibers, where their single-mode operation is limited to $\sim 630-850 \mathrm{~nm}$. Spectral broadening in the normal dispersion regime is dominated by SPM and Raman scattering, whereas in the anomalous regime, soliton fission (Dudley and Coen, 2006) is responsible for very broad continua. In the anomalous regime the spectrally broadened pulses have a complicated temporal structure which limits their compressibility. Therefore this regime is not an appealing approach to the generation of OPCPA seed pulses.

In the normal regime, LMA fibers have a higher nonlinearity compared to gas-filled HCFs and their higher damage threshold compared to singlemode fibers makes them suitable for the spectral broadening of high power, $\mu J$-energy sources (Südmeyer et al., 2003).

Experiment Fig. 2.2 shows an experimental setup used for spectral broadening of $1 \mathrm{ps}, 11 \mathrm{MHz}, 5 \mu \mathrm{J}$ pulses of a Yb:YAG thin-disk oscillator (Fig. 2.3) (Fattahi et al., 2012b). First the output of the laser was focused into a $4 \mathrm{~mm}$ LBO crystal for SHG. The frequency-converted component of the beam was separated by a dichroic mirror and used later to pump an OPCPA stage, similar to (Fattahi et al., 2012b; Teisset, 2009)).

The residual energy of the fundamental after the SHG stage, which still had a good beam quality (Fig. 2.3), coupled into a quartz LMA fiber with a zero dispersion wavelength at $1.3 \mu \mathrm{m}$. The fiber was placed in an aluminium V-groove on a 3-axis translation stage. The input energy to the fiber was 
adjusted by a tunable attenuator (half-wave plate and a thin film polarizer). A Faraday isolator was placed before the fiber to avoid any back-reflection from the entrance of the fiber to the laser.

Spectral broadening in fibers with different core diameters of $12 \mu \mathrm{m}$, $15 \mu \mathrm{m}$, and $20 \mu \mathrm{m}$ were studied. Fig. 2.2-a shows the broadened spectra in a $65 \mathrm{~mm}$-long, $15 \mu \mathrm{m}$-core diameter LMA fiber for different input average powers. For average powers higher than $7 \mathrm{~W}$ and a fiber diameter smaller than $12 \mu \mathrm{m}$ damage occurred. The spectrum broadened in a $12 \mu \mathrm{m}-$ core-diameter LMA fiber supports $30 \mathrm{fs}$ pulses. The pulses were collimated by using a $3 \mathrm{~mm}$ plano-concave lens and compression was performed in a chirped-mirror compressor with a GDD of $-500 \mathrm{fs}^{2}$ per reflection (Fig. 2.4b), producing a total GDD of $-6000 \mathrm{fs}^{2}$ over 12 reflections.

The total throughput of $57 \%$ for the LMA fiber and the compressor left $4 \mathrm{~W}$ of average power for supercontinuum generation in bulk. The beam is focused into a $4 \mathrm{~mm}$ YAG crystal to generate a single filament with a spectrum reaching $500 \mathrm{~nm}$.

\begin{tabular}{c|cccc} 
crystal & thickness $(\mathrm{mm})$ & input power $(\mathrm{W})$ & output power $(\mathrm{mW})$ & efficiency $(\%)$ \\
\hline YAG & 4 & 4 & 50 & 1.2 \\
\hline $\mathrm{YVO}_{4}$ & 8 & 2.2 & 25 & 1.1
\end{tabular}

Table 2.1: Parameters of the supercontinuum generation in $\mathrm{YAG}$ and $\mathrm{YVO}_{4}$ crystals

Fig. 2.2-c and - $d$ show the generated white light and its spectrum, in two different crystals: $4 \mathrm{~mm}$-long YAG and $10 \mathrm{~mm}$-long $\mathrm{YVO}_{4}$. The average power of the white light was measured behind a high-reflectivity mirror at $1030 \mathrm{~nm}$. The mirror was used to filter out the input spectrum and the scattered laser light. A white paper was used after the filter to diffuse the beam before coupling into the spectrometer. In both cases the efficiency of the supercontinuum generation did not exceed $2 \%$. The generated supercontinuum in $\mathrm{YVO}_{4}$ has a narrower spectrum (Fig. 2.2-d) and lower threshold due to the higher nonlinearity of $\mathrm{YVO}_{4}$ crystal compared to the YAG crystal (Fig. 2.4-a). Accordingly, multi-filamentation and damage occurred at lower pulse energies. The blue color, observable in the $\mathrm{YVO}_{4}$ crystal in Fig. 2.2 is due to the simultaneous THG of the input pulse in the crystal.

The supercontinuum generation also was tried in sapphire. The spectrum and energy in this case was not stable as the peak power of the pulses was just above the critical peak power. Lower nonlinearity of sapphire compared to YAG and $\mathrm{YVO}_{4}$ and hence its higher threshold demands a higher peak power to reach a stable regime of operation. 
$\infty \rightarrow \infty$

Conclusion

Yb:YAG oscillator pulses with $7 \mathrm{~W}$ of average power and a spectral bandwidth of $1.4 \mathrm{~nm}$, centered at $1030 \mathrm{~nm}$ are spectrally broadened in a cascaded broadening stage to a bandwidth supporting few-cycle pulses. Following broadening via SPM in a $12 \mathrm{~mm}$-long LMA fiber and 12 reflections on a chirped-mirror compressor, $30 \mathrm{fs}$ pulses with $4 \mathrm{~W}$ of average power are obtained. Focusing these pulses into a $4^{-}$ mm-thick YAG crystal results in a supercontinuum with $1 \%$ of efficiency in the anti-Stokes wing. In a proof-of-principle experiment, the generated supercontinuum is amplified in a single OPCPA stage similar to (Fattahi et al., 2012b). As the signal and pump pulses are generated from the same laser, they are intrinsically synchronized. Therefore no additional temporal synchronization is required for temporal jitter compensation and amplification in the OPCPA stage. While this approach makes the setup simpler, it still suffers from the lack of carrier envelope phase stability of the generated supercontinuum and is not scalable in energy due to limitations of LMA fibers.

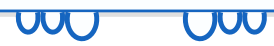



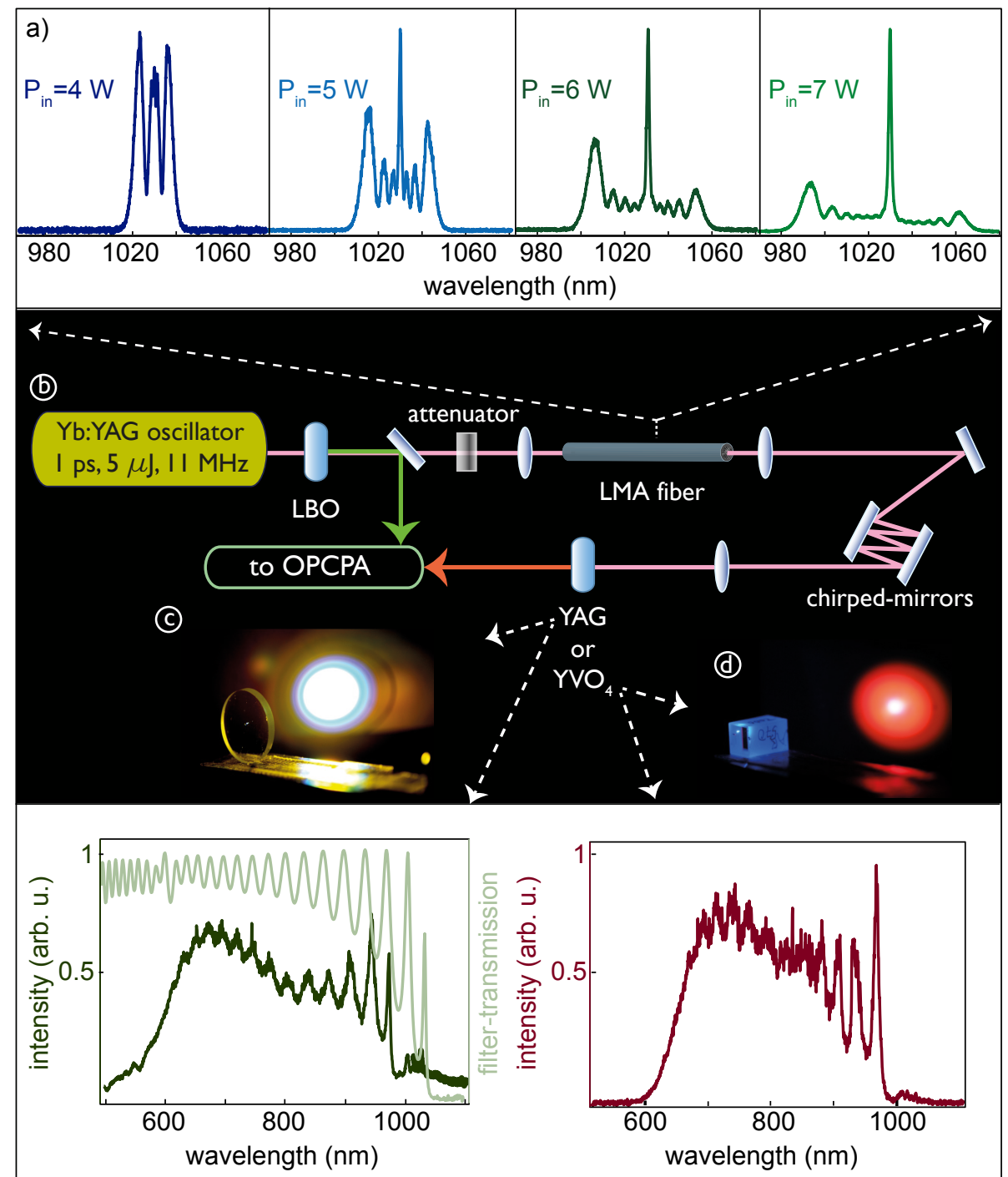

Figure 2.2: a) Spectral broadening in a $15 \mu \mathrm{m}$ core-diameter-LMA fiber for different input average power normalized to one and plotted on a linear scale ( $P_{\text {in }}$ : input average power). b) Schematic layout of the experimental setup. The residual energy after frequency doubling of Yb:YAG oscillator pulses was spectrally broadened in an LMA fiber and later temporally compressed utilizing a chirped-mirror compressor. The compressed pulses are focused into a bulk material for supercontinuum generation which provides a coherent seed for optical parametric amplification. c) Supercontinuum generation in a $4 \mathrm{~mm}-Y A G$ crystal. The transmission curve of a filter used for spectrum and average power measurement is shown in light green. d) Supercontinuum generation in a $\mathrm{YVO}_{4}$ crystal. The generated blue light, visible in the picture of the crystal, is due to the simultaneous third harmonic generation of the input spectrum. 
a)

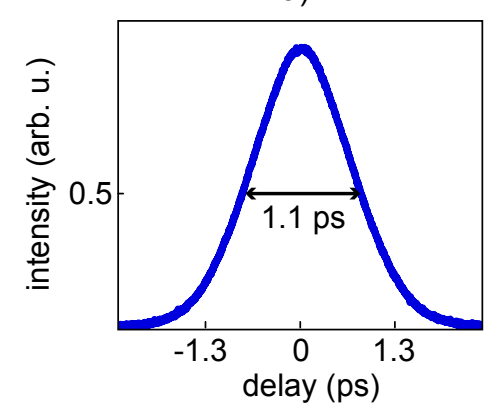

b)

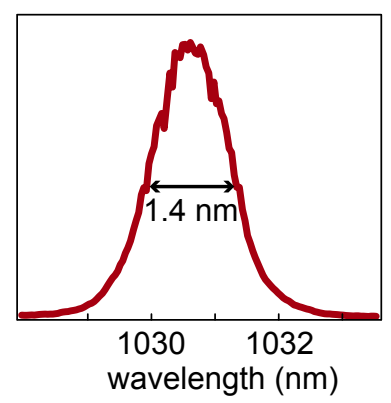

c)

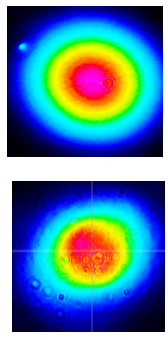

Figure 2.3: Auto-correlation trace (a), spectrum (b) and the beam profile (c) of a SESAM mode-locked Yb:YAG thin-disk oscillator (Fattahi et al., 2012b). The beam profile of the residual of the fundamental after the SHG (d).

a)

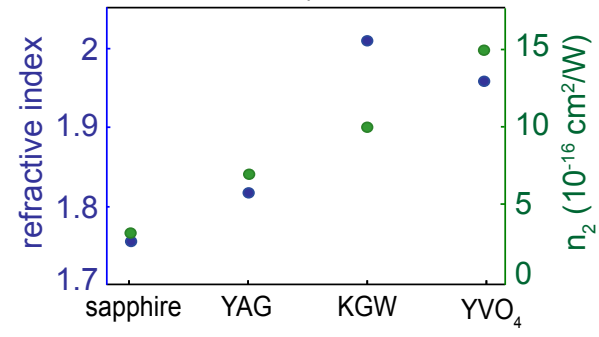

b)

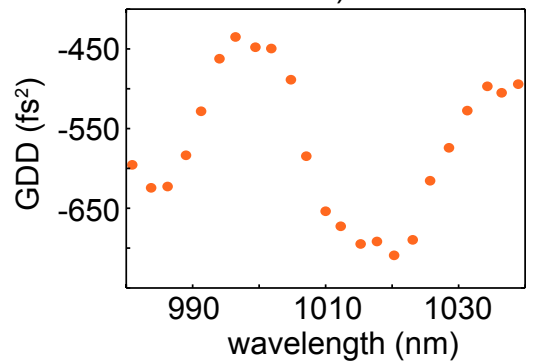

Figure 2.4: a) Refractive index and nonlinear refractive index of different bulk materials. b) Measured GDD curve of the chirped mirrors used for the compression of the broadened spectrum generated in an LMA fiber. 


\subsection{Supercontinuum generation in hollow core fiber}

For large pulse energies, HCFs surpass the performance of PCFs since the optical power is confined to a large-diameter, gas-filled hole and therefore has a higher damage threshold intensity. In a gas-filled HCF, the process of broadening is initiated in the gas and the HCF acts as a dielectric waveguide which provides significantly longer nonlinear interaction length for the light in comparison to the case of filamentation in gas. The energy throughput is limited by damage and plasma formation at the entrance of the fiber as well as self-focusing in the waveguide. The latter limitation is overcome by differentially pumped fibers (Robinson et al., 2006).

Broadening in a HCF takes place in two different regimes. For long pump pulses, purely SPM is observed, whereas for short pulses a more uniformly broadened spectrum indicates the influence of self-steepening (Nisoli et al., 1997). Noble gases have high thresholds for ionization (Nisoli et al., 1996) and are typically preferred. At very high gas pressures, energy loss due to ionization and defocusing at the fiber entrance reduces transmission and produces significant mode splitting, decreasing the bandwidth of the broadened pulses (Wei et al., 2010).

Experiment $5 \%$ of the negatively chirped output of a Ti:sapphire multipass amplifier (Cavalieri et al., 2007) was passed through a pair of SF57 wedges for temporal compression down to $27 \mathrm{fs}$ (Fig. 2.5). The compressed pulses with $60 \mathrm{~nm}$ spectral bandwidth at FWHM, centered at $790 \mathrm{~nm}$, contained $30 \mu \mathrm{J}$ of energy. In an experimental setup shown in Fig. 2.6-a, the beam was focused down to $84 \mu \mathrm{m}$ and coupled into a $120 \mu \mathrm{m}$-core-diameter, $15 \mathrm{~cm}$ long HCF placed on a V-groove in a gas cell, statically filled with 5 bar of krypton.

The gas cell was made of stainless steel with $1 \mathrm{~mm}$ Brewster-cut $\mathrm{CaF}_{2}$ windows. The GDD of the input pulse, adjustable with $\mathrm{SF}_{57}$ wedges, and the pressure of krypton were optimised to obtain the maximum spectral broadening (Fig. 2.6-b) and 50\% throughput in the HCF. The broadened spectrum at 4.3 bar was subsequently focused in a $4 \mathrm{~mm}$ YAG crystal to extend the spectrum to $1400 \mathrm{~nm}$.

\section{Conclusion}

The generated coherent broadband spectrum in a $\mathrm{Kr}$-filled-HCF at 4.5 bar spans from $400 \mathrm{~nm}$ to $1000 \mathrm{~nm}$ and contains $15 \mu \mathrm{J}$ of energy. This spectrum is extended to $1400 \mathrm{~nm}$ by focusing the output of the 
HCF into a 4-mm-thick YAG crystal. These $\mu \mathrm{J}$-level broadband spectra are used to seed a 3-stage OPCPA system, discussed in Section 4.1 and Section $4 \cdot 3$.

uU UOU 

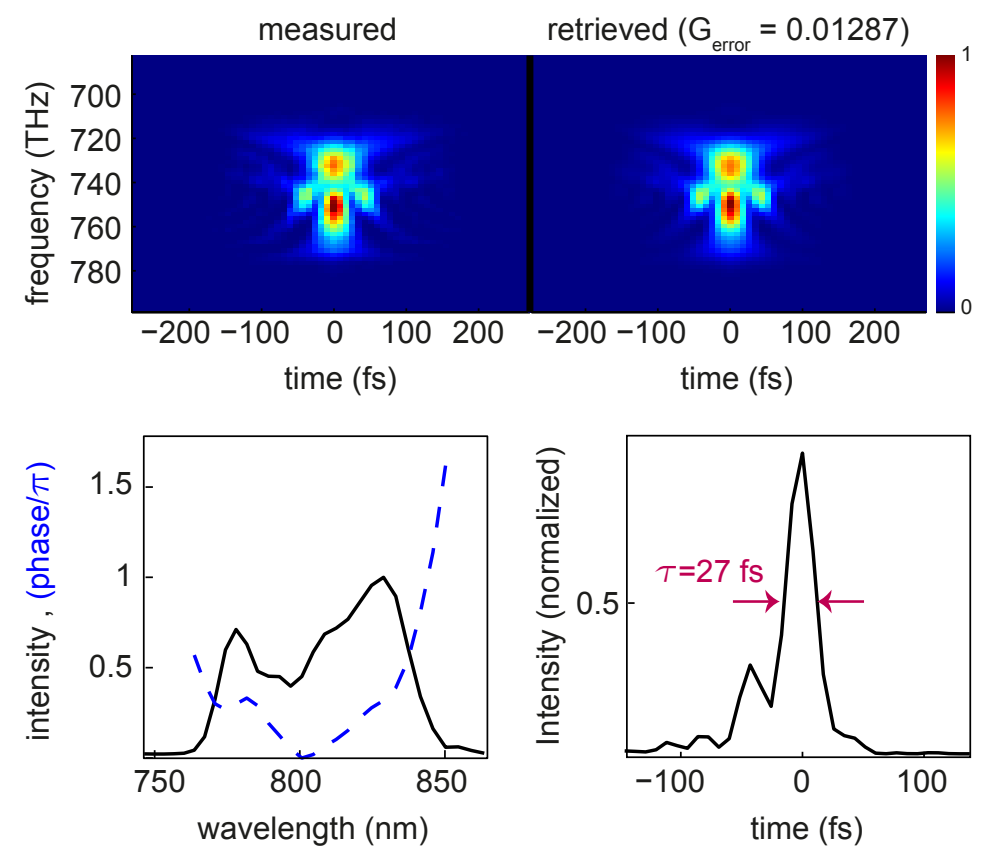

Figure 2.5: Measured and retrieved SH-FROG (Second Harmonic- Frequency Resolved Optical Gating) traces (top) and the retrieved spectrum and temporal profile (bottom) of the pulses at the input of the HCF. The compressed pulse has the pulse duration of $27 \mathrm{fs}$ at FWHM. The uncompressed pre-pulse pedestal, caused by the residual third order chirp, can be compensated by additional specially designed chirped mirrors. 
a)

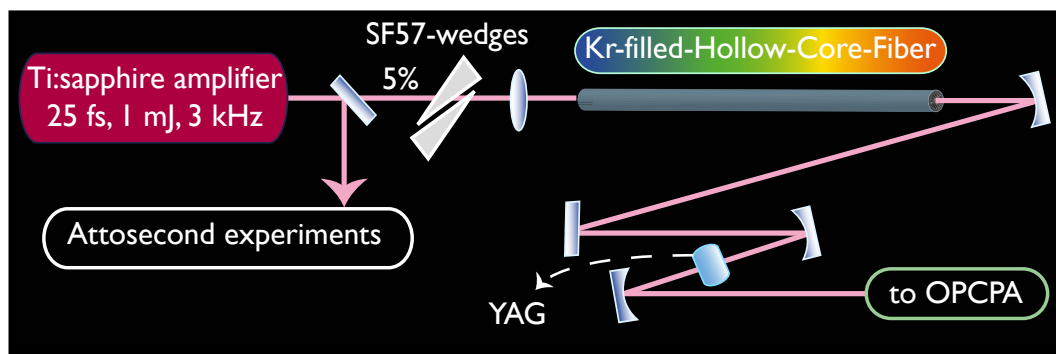

b)
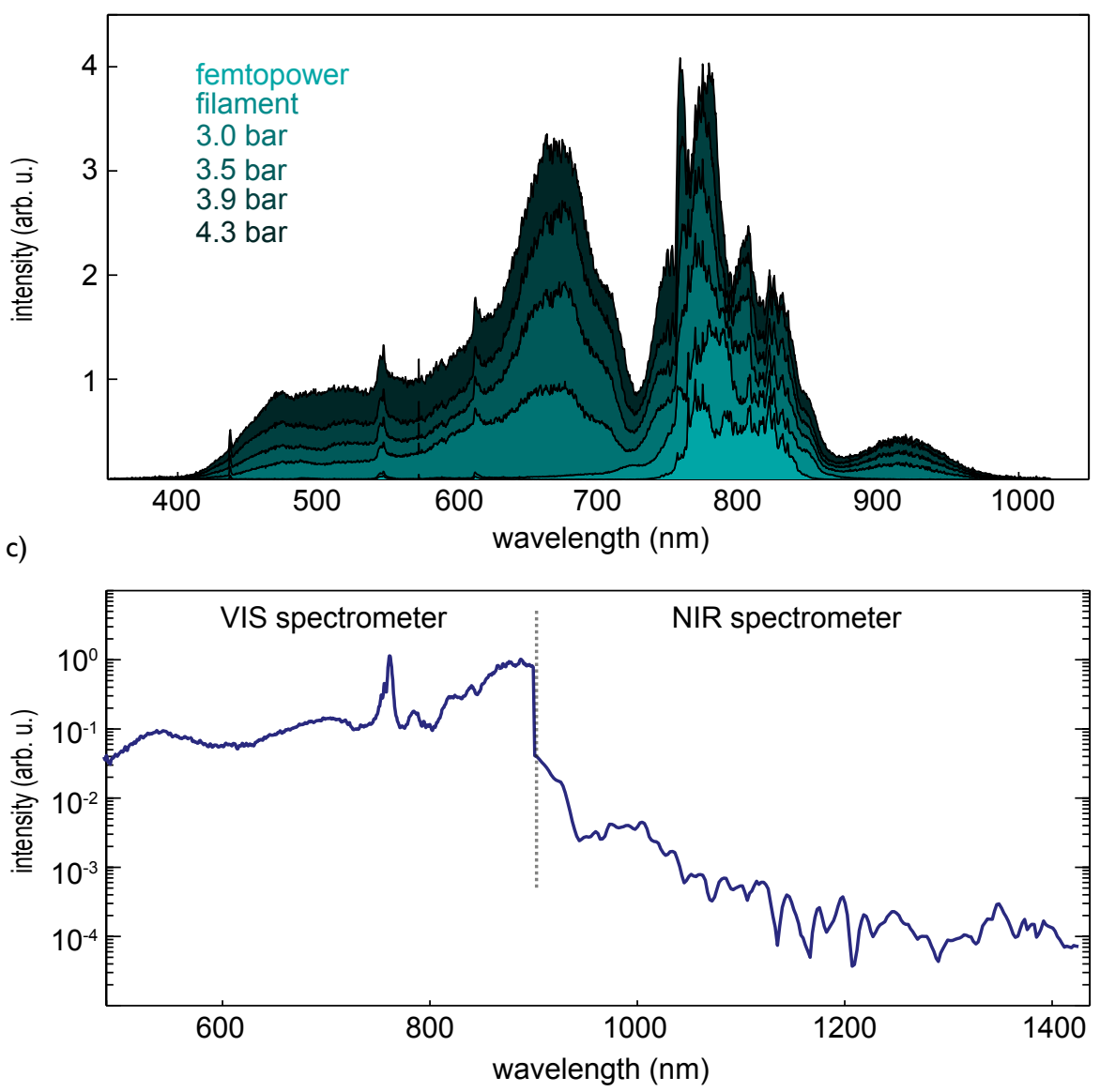

Figure 2.6: a) Layout of the seed generation scheme. A small portion of the energy of a Ti:sapphire multipass amplifier is focused into a $120 \mu \mathrm{m}$-corediameter, $\mathrm{Kr}$-filled HCF. The fine tuning of the group-delay-dispersion of the pulses was performed with a pair of $\mathrm{SF}_{57}$ wedges. b) The spectrum of the Ti:sapphire multipass amplifier (Femtopower), the generated supercontinuum spectrum in $\mathrm{Kr}$ without $\mathrm{HCF}$, and different spectra of the $\mathrm{Kr}$-filled$\mathrm{HCF}$ at different pressures. c) In the second step, the broadband spectrum from the HCF filled with 4.3 bar of $\mathrm{Kr}$, was focused into a $4 \mathrm{~mm}$ YAG crystal to extend the spectrum to $1400 \mathrm{~nm}$. 


\subsection{Cross-polarized wave generation}

XPW (Minkovski et al., 2004) is a degenerate four-wave mixing process which is the result of the interaction of intense, linearly polarized light with a nonlinear medium with an anisotropic third-order nonlinearity and (typically) an isotropic linear index.

The XPW signal has an orthogonal polarization with respect to the pump and it is an automatically phase-matched process. XPW generation preserves the good spatial quality of the input beam and can improve the spatial profile of beams with distorted beam profiles. It also leads to significant shortening of the filtered pulse. The intensity of the generated XPW signal varies as the cubic power of the pump intensity. Suitable crystals for XPW generation are cubic crystals with Fm3m cubic symmetry $\left(\mathrm{BaF}_{2}, \mathrm{CaF}_{2}, \mathrm{LiF}\right)$. Among these crystals $\mathrm{BaF}_{2}$ has a high $\chi^{(3)}$ anisotropy value which allows good conversion efficiency and its linear optical properties allow perfect group velocity matching along the propagation axis and a perfect spatial overlap of the cross-polarized waves. The crystal also has a wide transmission range from $0.2 \mu \mathrm{m}$ to $11 \mu \mathrm{m}$ (Cotel et al., 2006; Ricci et al., 2013).

Modulation in the frequency domain corresponds to satellite pulses in the time domain. In the XPW process these satellite pulses tend to be suppressed, as they have a lower intensity relative to the main pulse. Consequently, this pulse cleaning in the time domain translates to spectral smoothing since only the main peak of the pulse is converted. Therefore the temporal contrast of the generated pulse is greatly enhanced (Jullien et al., 2005).

$\mathrm{XPW}$ also acts as a pulse-shortening process. Here, unlike the other nonlinear phenomena, the generated XPW signal is always shorter than the pump pulse, as the signal is the result of the gating of the pump pulse in the time domain. For long pump pulses, the effect of dispersion of the crystal is negligible and the generated signal stays close to its Fourier transform limit.

In a linear crystal without birefringence, as long as changes in the nonlinear index of refraction can be neglected, the phase-matching condition is always fulfilled: $k_{\perp}=k_{\|}+k_{\|}-k_{\|}$.

Starting from Maxwell's equations and using the slowly varying envelope approximation, the following coupled wave equations for XPW are derived:

$$
\begin{aligned}
-i \frac{d A}{d \xi} & =\gamma_{1} A A^{*} A+\gamma_{2} A A^{*} B+2 \gamma_{2} A A^{*} B \\
& +2 \gamma_{3} A B^{*} B+\gamma_{3} B B A^{*}+\gamma_{4} B B^{*} B
\end{aligned}
$$


a)

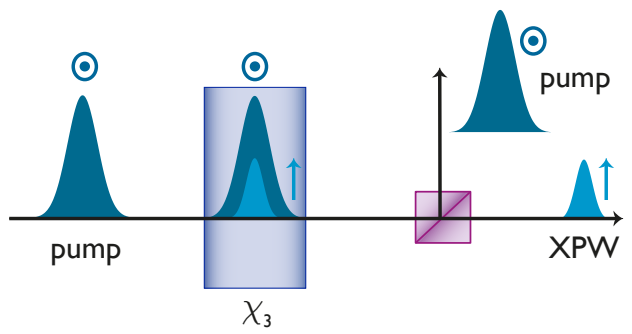

b)

phase matching condition: $\omega^{(\mathbf{I})}=\omega^{(\mathrm{II})}+\omega^{(\mathrm{II})}-\omega^{(\mathrm{II})}$

\begin{tabular}{c|ccc} 
coefficient & $\gamma_{1}, \gamma_{5}$ & $\gamma_{3}$ & $\gamma_{2}, \gamma_{4}$ \\
\hline phenomena & self-phase modultion & cross-phase modulation & $\mathrm{XPW}$
\end{tabular}

Figure 2.7: a) Schematic of crossed-polarized wave generation. Based on a degenerate four-wave mixing process in a nonlinear medium with an anisotropic third-order nonlinearity, a new wave with crossed-polarization with respect to the pump wave and significantly better temporal contrast is generated. b) Role of each coefficient in the coupled wave equations describing the XPW process.

$$
\begin{aligned}
-i \frac{d B}{d \tau} & =\gamma_{5} B B^{*} B+\gamma_{4} B B A^{*}+2 \gamma_{4} A B B^{*} \\
& +2 \gamma_{3} A B A^{*}+\gamma_{3} A A B^{*}+\gamma_{2} A A^{*} A,
\end{aligned}
$$

where $A$ is the complex amplitude of the pump, $B$ is the complex amplitude of the generated signal and $\xi$ is the propagation direction. The coefficients in Eq. (2.1) and Eq. (2.2) describe different degenerate third-order processes that happen in the nonlinear medium simultaneously (Fig. 2.7) (Canova et al., 2008).

To study the XPW process theoretically, the coupled wave equations are solved in a split-step method (Taha and Ablowitz, 1984). The pulse is propagated taking into account dispersion, diffraction and the third order nonlinearity, in the slowly evolving envelope approximation, in two dimensions (cylindrical coordinates with the assumption of radial symmetry). This is efficient enough to calculate not only the effects inside of one crystal, but also the spatio-temporal dynamics of the optimized two crystal systems discussed in (Jullien et al., 2006). The pump pulse in the simulation is assumed to be Gaussian in space and time. 1 ps pulses centered at $1030 \mathrm{~nm}$ are propagated in a $2 \mathrm{~mm}$, [o11]-cut, $\mathrm{BaF}_{2}$ at an intensity of $2 \mathrm{TW}$. It is assumed that $\chi^{(3)}=1.59 \times 10^{-22} \mathrm{~m}^{2} / \mathrm{V}^{2}$ (Jullien et al., 2006) (Appendix B).

As can be seen in Fig. 2.8, in the first $0.5 \mathrm{~mm}$ length of the crystal, the pump pulse does not change its shape during propagation. Here the XPW signal varies as the cube of the input field. In this regime the pulse duration 
is reduced by a factor of $\sqrt{3}$. For a Fourier transform limited pulse, this translates to a spectral bandwidth increased by the same factor.

In a $1 \mathrm{~mm}$-thick crystal, the efficiency of XPW reaches $12 \%$, with a smooth spectral broadening and pulse duration of $900 \mathrm{fs}$ at FWHM. For longer crystal lengths, other third-order processes like SPM and cross phase modulation (XPM) take over XPW and the spectrum becomes modulated. At the same time, with the increase in efficiency of XPW, the pump pulse changes its shape in the time domain, and therefore the generated XPW spectrum becomes modulated. The introduced GDD from the SPM process and the simultaneous change of the shape of the pulse in the time domain makes the XPW pulse longer, while the Fourier transform limit pulse duration of XPW decreases with a sharper slope. This simulation shows that achieving high efficiencies in XPW comes at the expense of a modulated spectrum and therefore a decrease in the temporal contrast of the generated pulse (Jullien et al., 2006).

Experiment In an experimental setup shown in Fig. 2.9, 95\% of the output of the Ti:sapphire multi-pass amplifier (Cavalieri et al., 2007) was focused into a $1.1 \mathrm{~m}$-long, $240 \mu \mathrm{m}$-core-diameter $\mathrm{HCF}$, filled with neon gas at a pressure of 2 bar. The generated white light from the fiber had a total power of $1.5 \mathrm{~W}$ and a spectrum spanning from $400 \mathrm{~nm}$ to $1000 \mathrm{~nm}$ (Cavalieri et al., 2007). Using a chirped-mirror compressor consisting of six mirrors and a pair of thin fused silica wedges, the pulses were compressed down to $4 \mathrm{fs}$ with a total power of $1 \mathrm{~W}$ in the spectral range of $480-1000 \mathrm{~nm}$.

The beam after the fiber was collimated using a $f=1 \mathrm{~m}$ spherical mirror and later by using a set of curved mirrors, the beam size is reduced to $400 \mu \mathrm{m}$. The telescope was misaligned to have a slightly divergent beam. The smallest beam diameter was limited by filamentation in air. To perform an intensity scan of the process, a $1 \mathrm{~mm} \mathrm{BaF}_{2}$ crystal is placed at different positions $\left(P_{0}, P_{1}, P_{2}, P_{3}, P_{4}\right)$ along the divergent beam (Fig. 2.9). The pulses after the HCF are linearly p-polarized with no measurable energy in the s-polarization. Therefore no polarizer is used before the $\mathrm{BaF}_{2}$ crystal. The generated XPW signal was separated from the pump by using a nanoparticle-linear-film polarizer (Thorlabs $\mathrm{GmbH}$ ).

Generated XPW spectra and the corresponding efficiencies are shown in Fig. 2.9-b and Fig. 2.9-e. By increasing the intensity, the XPW spectrum became smoother and the modulations decreased. At position $P_{4}$, the red tail of the spectrum was enhanced which might be caused by other nonlinear processes.

In a second study, the crystal was fixed at position $P_{4}$ and $\mathrm{BaF}_{2}$ with different thicknesses were used. Increasing the crystal thickness from $1 \mathrm{~mm}$ 
to $2 \mathrm{~mm}$ increased the efficiency of the process to $8 \%$ (Fig. 2.9-d).

Later a $1 \mathrm{~mm}$ crystal was placed after the $2 \mathrm{~mm} \mathrm{BaF}_{2}$ to increase the efficiency of XPW by taking advantage of the self focusing after the first crystal (Cotel et al., 2006). The efficiency is slightly increased compared to the single crystal measurement. After switching the order of these two crystals, a yet higher efficiency was achieved as the pulse after propagation in the $1 \mathrm{~mm}$ crystal is less dispersed and the pump peak intensity in the second crystal remained higher. The spectrum of the latter combination is labeled with cascaded-2 in Fig. 2.9-c. The achieved extra efficiency in the cascaded crystal combination, is mostly dominated by extra photons in the red tail of the spectrum, while the blue tail of the spectrum was saturated.

In the third experiment, which was performed for low-energy pumped XPW (for the layout see Fig. 2.11), the spectrally broadened output of the HCF explained in Section 2.2 was compressed to 9 fs (Fig. 2.10) using 10 reflections in a chirped-mirror compressor. The temporally compressed pulses were characterized using a home-built transient grating frequency-resolved optical gating device (TG-FROG) utilizing a $500 \mu \mathrm{m}$ fused silica plate as the nonlinear medium. The spectral components below $650 \mathrm{~nm}$ were not compressed as the design of the chirped-mirror compressor did not support this spectral range. A pair of thin fused silica wedges was used for fine tuning of the dispersion.

Unlike the previous study, in the high pressure gas-filled HCF, $6 \%$ of the energy after the HCF was s-polarized, although the input pulse to the HCF was p-polarized. The generated s-polarized photons resulted from a crossed-phase Kerr effect. In a normal Kerr effect process, three photons with the same polarization are mixed and a fourth photon with the same polarization is generated: $P_{N L} \propto \chi_{p p p p}^{(3)} E_{p} E_{p} E_{p}$. In the presence of the small number of s-polarized photons, crossed-phase Kerr effect, with the nonlinear polarization of $P_{N L}=\chi_{p p s s}^{(3)} E_{p} E_{p} E_{s}$ results in mixing of two p-polarized photons with one s-polarized photon and generation of a new s-polarized photon. At the same time, two photons with s-polarization mix with a ppolarized photon and a new p-polarized photon is generated. In an infinitely long medium the process reaches equilibrium with equal number of s-polarized and p-polarized photons.

By adjusting the polarization of the input pulse to the HCF using a quarter-wave plate, the generated s-polarized photons were minimized to $0.3 \%$ of the total average power. In order to enhance the polarization of the input beam before the XPW crystal, a nanoparticle-linear-film polarizer (Thorlabs $\mathrm{GmbH}$ ) was placed after the chirped-mirror compressor. Then the beam was focused down to $200 \mu \mathrm{m}$ at FWHM using a gold off-axis parabola. $\mathrm{A} \mathrm{BaF}_{2}$ crystal was placed on a translation stage directly after 
the focus to balance the self focusing in the crystal and the divergence of the beam in order to reach a uniform intensity over the crystal length. After the crystal, the beam is collimated using an identical off-axis gold parabola and sent through an identical nanoparticle-linear-film polarizer (Thorlabs $\mathrm{GmbH}$ ) aligned for maximum transmission of the s-polarized light.

Close to the focus of the beam, the beam profile of the XPW signal was distorted (Fig. 2.11-b1 and -b2). Using an uncoated, [011]-cut, $1 \mathrm{~mm}$-thick $\mathrm{BaF}_{2}$ crystal, $3.3 \mathrm{~mW}$ of average power (measured after the second polarizer) in an XPW signal was generated, corresponding to $12.5 \%$ efficiency (Fig. 2.11-b).

In a cascaded-crystal configuration similar to the first study, the generated XPW spectrum was enhanced in the blue tail of the spectrum and the efficiency was increased to $14 \%$. Besides the XPW procces, other third order phenomena like SPM and cross-phase modulation extended the red tail of the XPW spectrum to $1400 \mathrm{~nm}$ (Fig. 2.11-b).

\begin{tabular}{c|c} 
technique & \multicolumn{1}{c}{ cons } \\
\hline plasma mirror & - not suitable for high repetion rate lasers \\
\hline sagnac interferometer & $\begin{array}{l}\text { - hard to align } \\
\text { - complicated set up }\end{array}$ \\
\hline crossed-polarized & $\begin{array}{l}\text { - its contrast enhancement is limited to } \\
\text { polarizer extinction ratio }\end{array}$ \\
\hline self diffraction & - the generated pulse suffers from angular dispersion
\end{tabular}

Table 2.2: Comparison between different techniques to enhance the temporal contrast of ultrashort pulses.

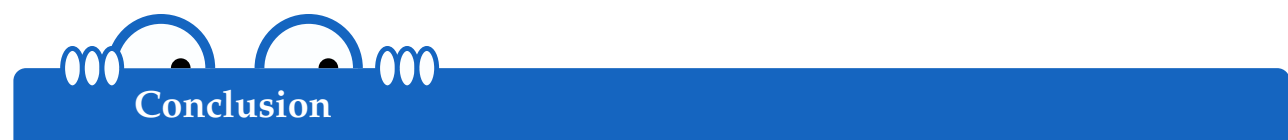

In this section, few-cycle near-infrared pulses are used to generate a cross-polarized wave (XPW). The efficiency of the process is increased to $9 \%$ by employing a cascaded-setup with 1 and $2 \mathrm{~mm} \mathrm{BaF}_{2}$ crystals. To reach a high peak intensity on the second crystal, two parameters are optimized: 1 - temporal dispersion of the pump pulse entering the second stage is minimized by adjusting the length of the crystal in the first stage. 2- The distance between the two cascaded crystals is optimized in order to balance the resulting self-focusing of the pump pulse after the first stage and its divergence. It is observed that the 
generated XPW spectrum contains new frequencies in addition to the pump spectrum.

Beside XPW, other techniques like plasma mirrors (Doumy et al., 2004), Sagnac interferometers (Renault et al., 2005), and self diffraction (Liu and Kobayashi, 2010) can be used to enhance the temporal contrast of laser pulses (Table 2.2). Among these methods, XPW is chosen and implemented in an OPCPA setup described in Section 4.4 due to the possibility of generating coherent photons with new frequencies and enhancing the temporal contrast of the laser pulses simultaneously. Although XPW preserves CEP-phase stability of the pump pulses, a seed generation technique which produces intrinsically CEP-stable pulses combined with a good temporal contrast and a well-behaved spectral phase is desired. The next section presents a study of the DFG process, which meets both of these criteria, making it the ideal OPCPA seed. 
a)
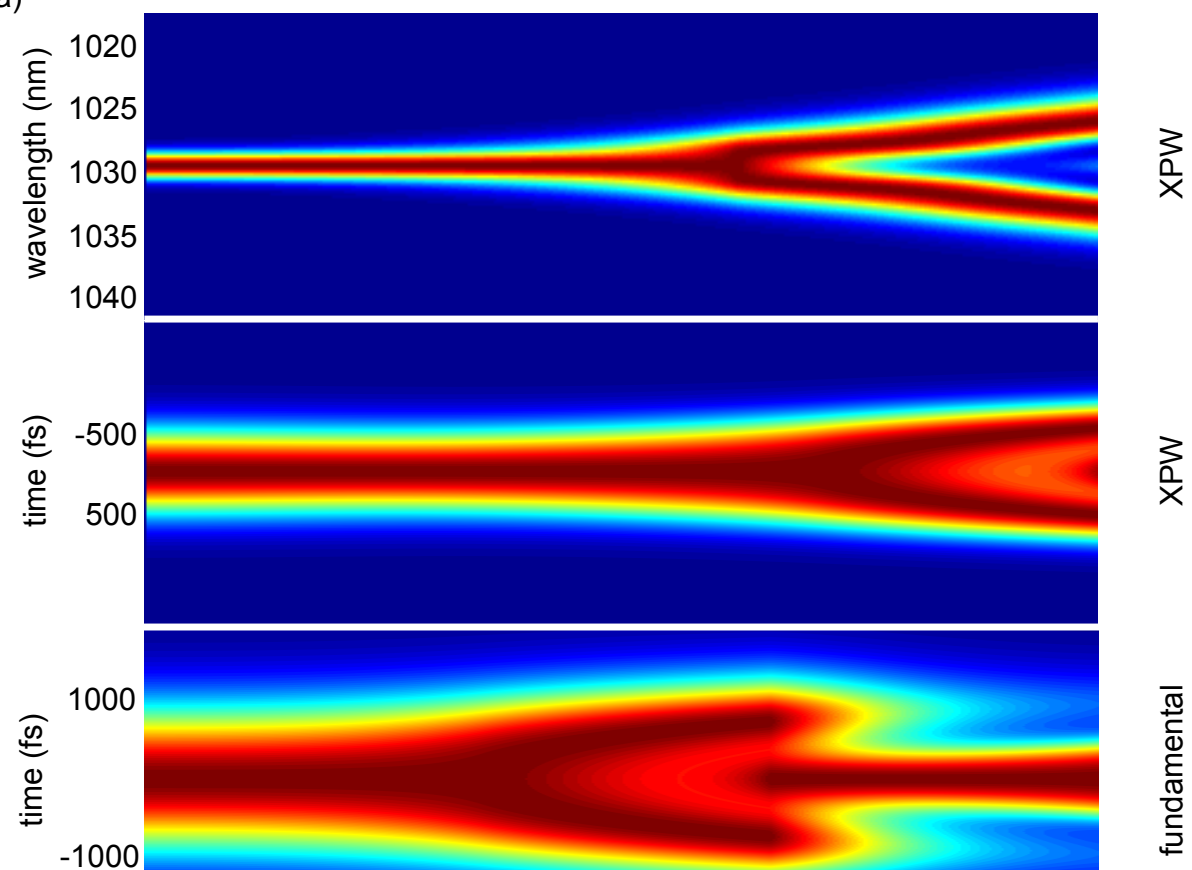

b)

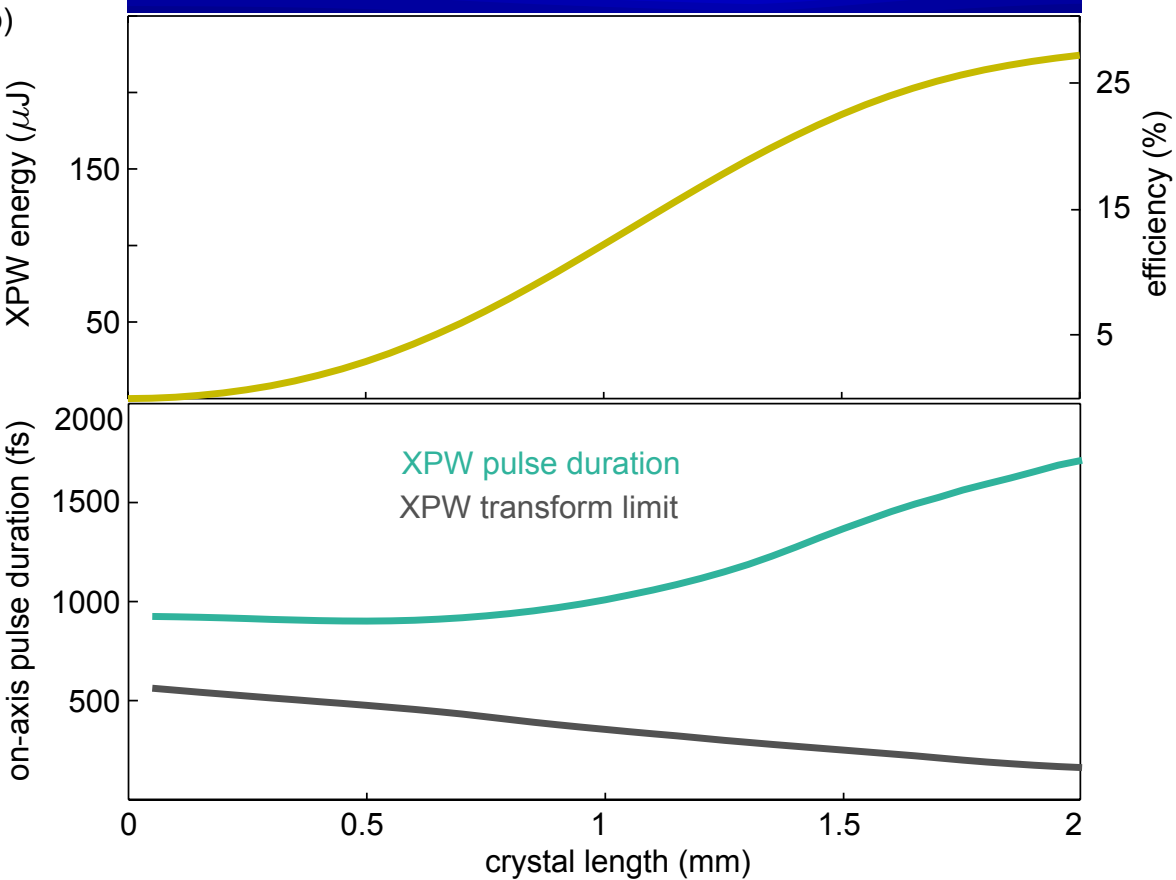

Figure 2.8: a) The generated XPW spectrum, its time domain profile and the temporal profile of the $1.6 \mathrm{ps}, 1030 \mathrm{~nm}$ pump pulses propagating in a $2 \mathrm{~mm}$ $\mathrm{BaF}_{2}$. b) The signal reaches saturation at the end of the crystal, but after $1 \mathrm{~mm}$ of propagation, the spectrum becomes modulated due to SPM, XPM and backconversion of the energy to the pump pulse. Therefore the pulse becomes longer but the Fourier transform of the spectrum supports an even shorter pulse. 
a)

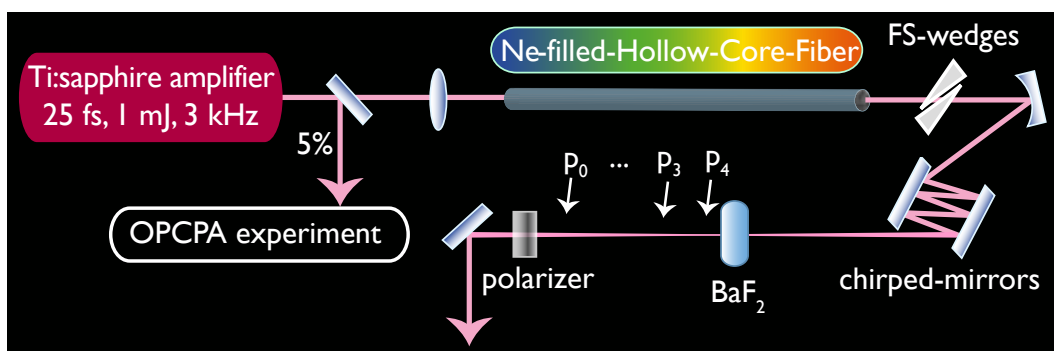

b)

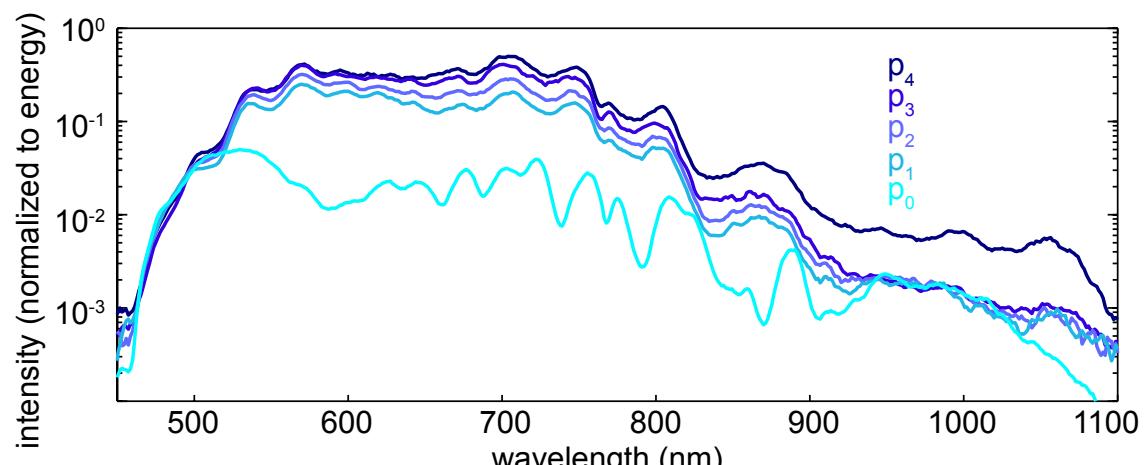

c)

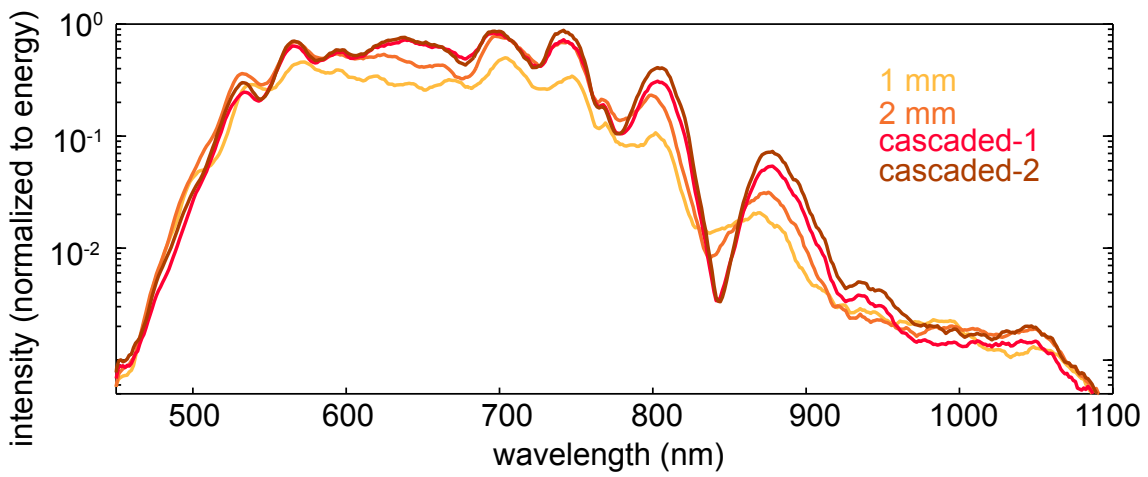

d)

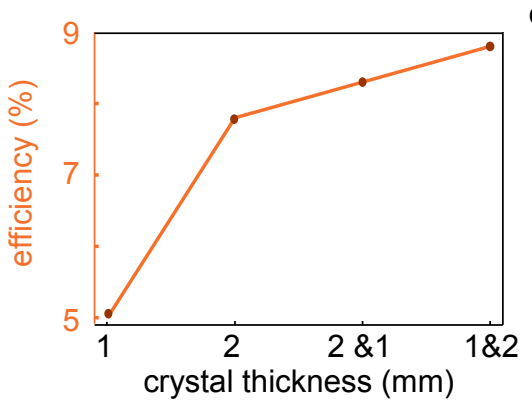

e)

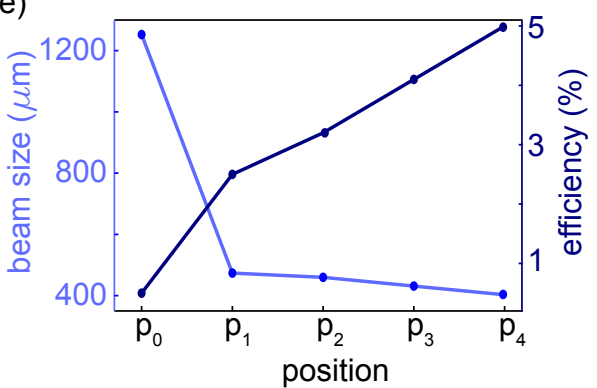

Figure 2.9: a) The experimental setup of XPW generation. b) XPW spectra for different intensities. For the intensity scan the crystal was moved along a slightly divergent beam. $\mathrm{P}_{i}$, where $\mathrm{i}: 0, \ldots, 4$ indicates the position of the crystal on the pump beam (see the layout). c) XPW spectra for different $\mathrm{BaF}_{2}$ thicknesses and the cascaded case. "cascaded- 1 " shows the spectra of the crystal combination when the $2 \mathrm{~mm}$ crystal is placed before the $1 \mathrm{~mm}$ crystal and "cascaded-2" is the spectrum corresponding to the case where two crystals are switched. d) and e) The efficiency of the XPW signal for different intensities and crystal thicknesses. 

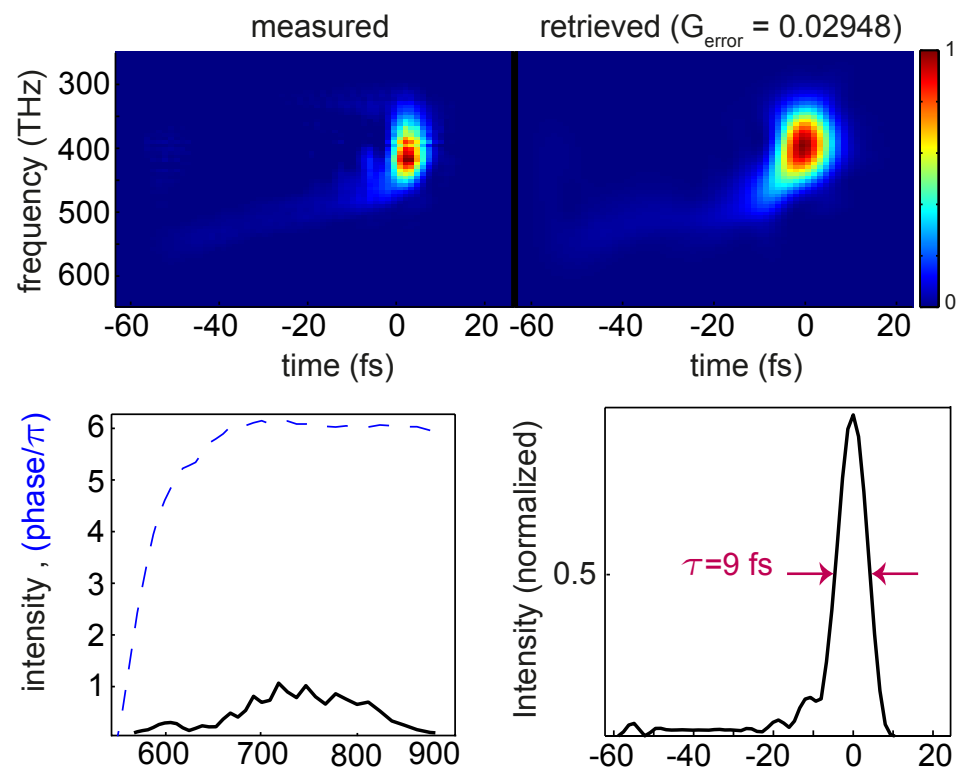

Figure 2.10: Measured and retrieved TG-FROG traces (top) and the retrieved spectrum and temporal profile (bottom) of the measured pulses at the input of the XPW crystal. The compressed pulse has a duration of $9 \mathrm{fs}$ at FWHM. The spectral component below $600 \mathrm{~nm}$ are not compressed which caused a long pedestal in the time domain. 
a)

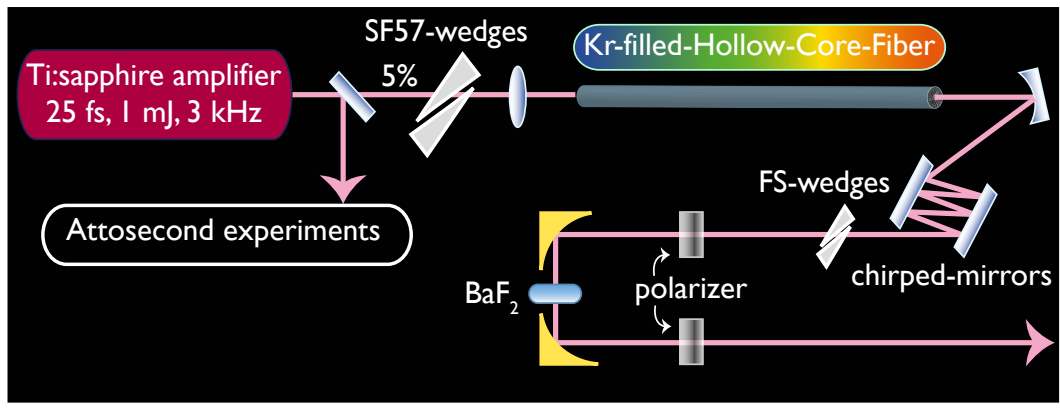

b)

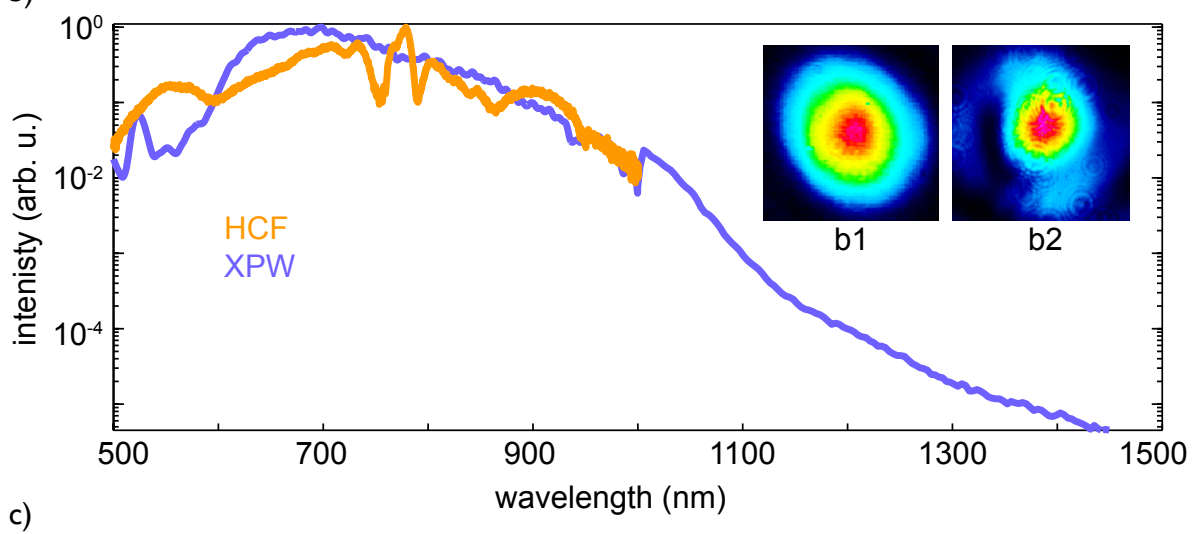

c)

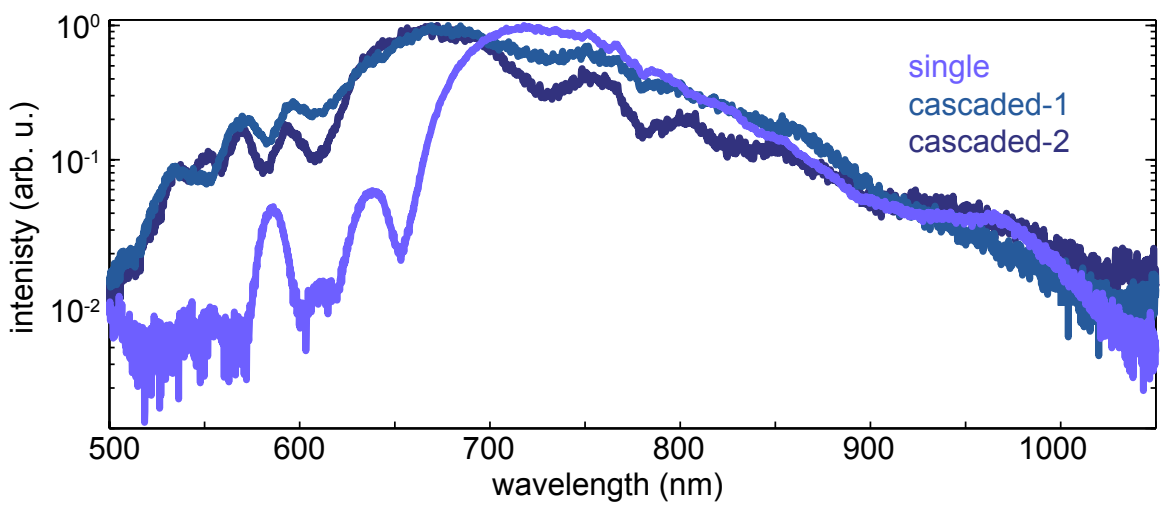

Figure 2.11: a) The experimental setup of XPW generation. b) HCF spectrum (orange) and XPW spectrum (purple) generated in a $1 \mathrm{~mm}$-thick $\mathrm{BaF}_{2}$ crystal (both spectra are normalized to one). bi demonstrates the spatial beam profile of the generated XPW signal and b2 shows the distortion of the XPW beam profile for very high peak intensity in the crystal. c) Generated XPW spectra in the single crystal and cascaded crystals scheme. In the single crystal scheme, the blue tail of the spectrum has lower spectral intensity, as the input pulse is not compressed in this spectral range. The blue tail was enhanced for the case of the cascaded configuration (All spectra are normalized to one). 


\subsection{Difference frequency generation}

${ }^{1}$ The combination of two properties in DFG makes it a popular source for ultrashort pulse generation: the ability to simultaneously phase-match a large fractional bandwidth and the passive CEP stability that can be obtained (Manzoni et al., 2004; Moses et al., 2012; Homann et al., 2012). However, the conversion efficiency into the long wavelengths are usually low, especially in broadband DFG, where the crystal thickness has to be kept short.

In this section different types of phase-matching for generating a broadband signal via DFG are experimentally and theoretically discussed. It is shown that a few-cycle VIS-NIR pulse obtained from spectral broadening of a commercial Ti:sapphire laser can be used to efficiently obtain broadband pulses in the MIR by implementing a simple method. Extending the CEP-stable broadband MIR pulses to VIS is the topic of Section 5.1.

Theory There are three ways of phase-matching for single-pulse DFG in BBO crystals. type I $(e-o \rightarrow o)$ and type II $(e-e \rightarrow o),(e-o \rightarrow e)$. These three cases and their corresponding phase-matching angles are depicted in Fig. 2.12.

A one-dimensional propagation code based on SVEA (Brabec and Krausz, 1997) which includes only second-order nonlinearities, is used for theoretical study of the DFG process. Fig. 2.12-e compares the generated DFG signal from a $3.5 \mathrm{fs}$ Gaussian input pulse in a $500 \mu \mathrm{m}$-thick BBO. In $(e-e \rightarrow o)$ type II and $(e-o \rightarrow o)$ type I phase-matching, the generated spectrum has more than four times the bandwidth of $(e-o \rightarrow e)$ type II phase-matching. Therefore the following study is limited to the two broadband phase-matching configurations.

In type I DFG, the underlying process is very similar to optical parametric amplification. The blue spectral components in the pulse on the fast axis transfer energy to the red components on the slow axis. During this process a phase-stable idler wave is generated and the phase-matching condition is given by:

$$
k_{p}-k_{s}=k_{i}
$$

Where $k_{p}, k_{s}$ and $k_{i}$ are the wave vectors of the blue, red, and the idler wave respectively. As the process is similar to OPA, increasing the number of photons on the fast axis, or pump wave, results in a higher efficiency. Fig. 2.13 and Fig. 2.14-b show the efficiency curves of the DFG process and its corresponding spectrum in a type I BBO crystal for three different polar-

\footnotetext{
${ }^{1}$ This section is adapted from (Fattahi et al., 2013). The electronic print of this paper is attached in Appendix E with the permission of OSA.
} 


\begin{tabular}{cccc}
\multirow{2}{*}{$\Theta$} & type I $(\mathrm{e}-\mathrm{o} \longrightarrow \mathrm{o})$ & type II $(\mathrm{e}-\mathrm{e} \longrightarrow \mathrm{o})$ & type II $(\mathrm{e}-\mathrm{o} \longrightarrow \mathrm{e})$ \\
\cline { 2 - 4 } & $21.4^{\circ}$ & $40^{\circ}$ & $25^{\circ}$ \\
\hline$\Phi$ & $90^{\circ}$ & $0^{\circ}$ & $0^{\circ}$ \\
\hline
\end{tabular}
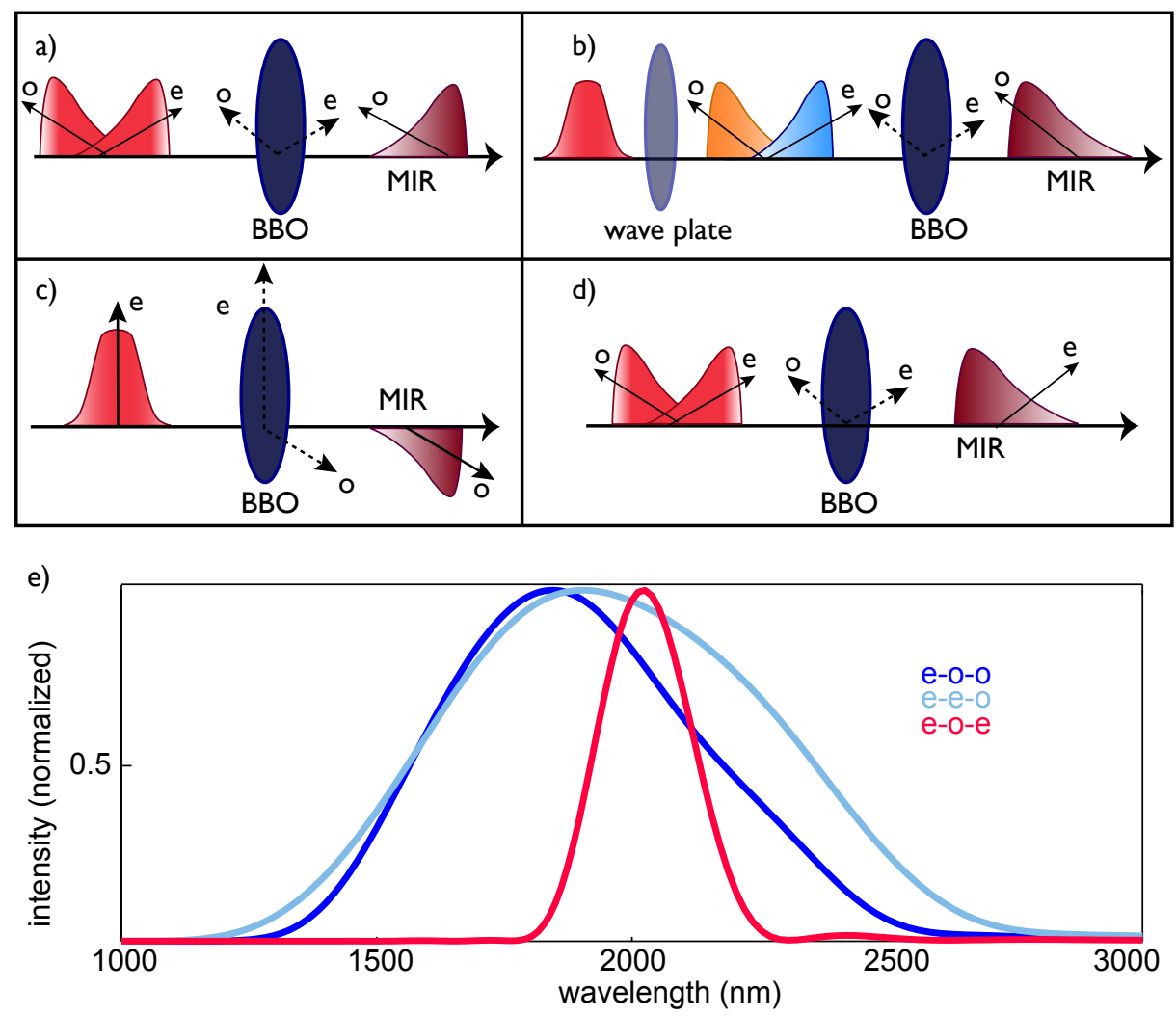

Figure 2.12: Diagram of: a) type I phase-matching with identical pulses sent to the fast and slow axis of a BBO crystal, b) type I phase-matching using a wave plate to optimize the short-wavelength spectral components on the fast axis, c) type II phase-matching with a pulse propagating along the fast axis of the crystal, and d) type II phase-matching with a pulse propagating along the slow and fast axis of the crystal. e) Simulated DFG spectra for the three phase-matching possibilities. The phase-matching angle for different types of phase-matching in a BBO crystal is summarized in the table. 
ization orientations of the pump beam relative to the extra-ordinary axis of the crystal.
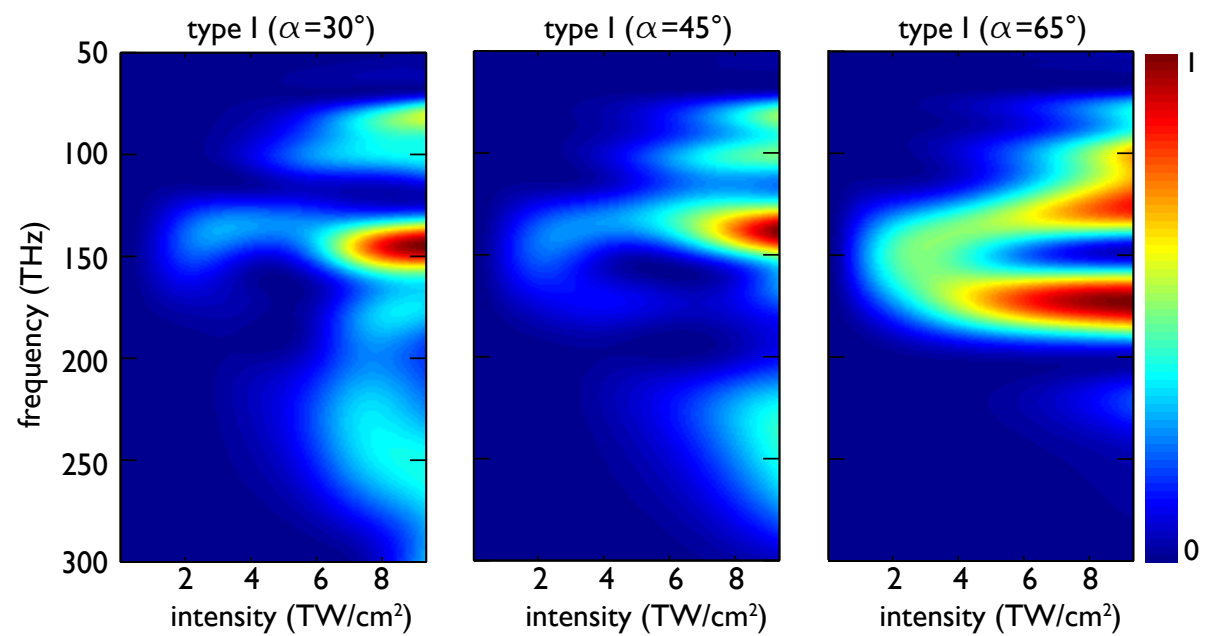

Figure 2.13: Simulated spectra for three different orientations of the crystal relative to the input beam polarization. $\alpha$ shows the angle between the polarization of the incoming beam and the optical axis of the crystal.

The highest achieved efficiency is at $\alpha=30^{\circ}$ where a larger number of photons of the incoming beam are projected onto the extraordinary axis of the crystal. This suggests that the process should become even more efficient by changing the spectral distribution of the input pulse on different axes of the crystal. That is, by sending the blue photons to the extraordinary axis of the crystal and red photons to the ordinary axis. In the presence of a quartz waveplate, the blue spectral components of the pulse obtain more phase shift than its red spectral components and they become separated. Consequently, by implementing a thin waveplate before the BBO crystal the fraction of photons with shorter wavelength on the fast axis can be increased which leads to the rise in the efficiency of the DFG process.

Type II DFG is very similar to optical rectification in the terahertz spectral domain (Bakker et al., 1998): the nonlinear polarization takes the form of the intensity profile of the pulse. By multiplying the phase-matching condition, given in Eq. (2.3) by c:

$$
\omega_{p} n_{p}-\omega_{s} n_{s}=\omega_{i} n_{i}
$$

for broadband pump and signal with the angular frequency of $\omega_{0}$ and the 
spectral width of $\Delta$ :

$$
\begin{aligned}
& \omega_{p}=\omega_{o}+\Delta, \\
& \omega_{s}=\omega_{o}-\Delta, \\
& \omega_{i}=\omega_{p}-\omega_{s}=2 \Delta .
\end{aligned}
$$

The refractive indices of the signal and pump can be expressed to first order in their series expansions

$$
\begin{aligned}
& n_{p}=n_{o}+\left.\Delta \frac{d n_{o}}{d \omega}\right|_{\omega_{0}} . \\
& n_{s}=n_{0}-\left.\Delta \frac{d n_{o}}{d \omega}\right|_{\omega_{0}} .
\end{aligned}
$$

By substituting Eq. (2.5) and Eq. (2.8) in Eq. (2.4):

$$
\omega_{i} n_{o}+\left.\omega_{i} \omega_{o} \frac{d n_{o}}{d \omega}\right|_{\omega_{0}}=\omega_{i} n_{i}
$$

and by dividing the Eq. (2.10) by $\omega_{i}$ :

$$
n_{o}+\left.\omega_{o} \frac{d n_{o}}{d \omega}\right|_{\omega_{o}}=n_{i}
$$

which is the expression for group index. By neglecting higher order terms, we obtain:

$$
n_{g}^{\text {pump }}=n_{0}^{D F G} .
$$

This phase-matching expression shows that the group velocity of the pump pulse should be equal to the phase velocity of the emitted wave. Here the dipole responsible for nonresonant DFG is simply the intensity envelope of the input laser pulse $\left(P^{N L}(t) \propto E^{*}(t) E(t) \propto I(t)\right)$, so matching its velocity to the wavefronts of the DFG pulse results in phase-matching.

The expression also shows intuitively why the $(e-e \rightarrow o)$ type II process is more broadband than the $(e-o \rightarrow e)$ one, namely that the dispersion along the fast axis is greater (more negative) in the infrared than on the slow axis, reducing the range of frequencies where phase-matching is approximately satisfied.

The simulated conversion efficiencies in $500 \mu \mathrm{m}$-thick BBO crystals cut for type I $\left(\theta=21.4^{\circ}, \phi=90^{\circ}\right.$, light polarization $30^{\circ}$ from fast axis) and type II $\left(\theta=40^{\circ}, \phi=0^{\circ}\right)$ are shown in Fig. 2.14. Here, at low pump intensities for unsaturated DFG, the efficiency is linearly proportional to the pump intensity. Increasing the pump intensity leads to higher conversion efficiency. In type I DFG, at high pump intensity, the linearity is broken and the DFG 
process saturates as the energy transfer from the generated MIR pulse to the pump pulse through sum frequency generation is faster than energy transfer from the pump to the DFG signal. Due to extremely short pulses in a single-pulse DFG studied here, the effect of group velocity matching results in rather different behavior of the two phase-matching types at high conversion efficiencies.

a)

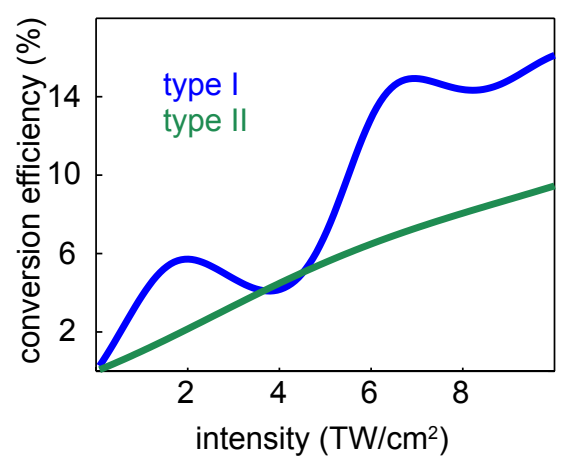

c)

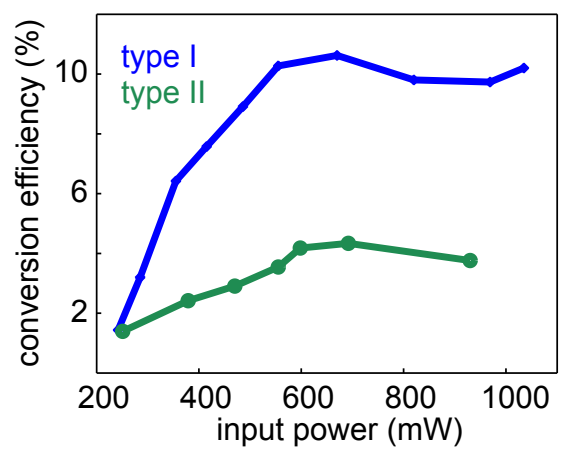

b)

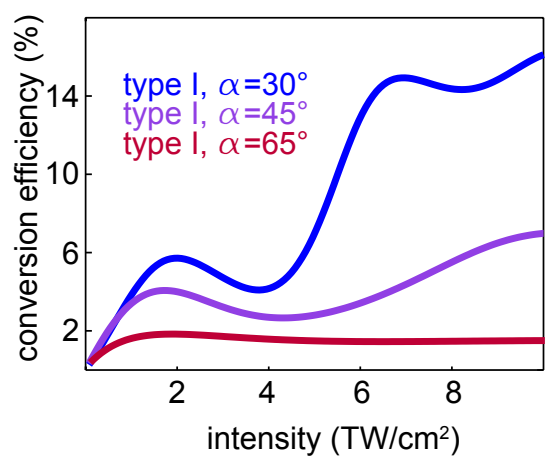

d)

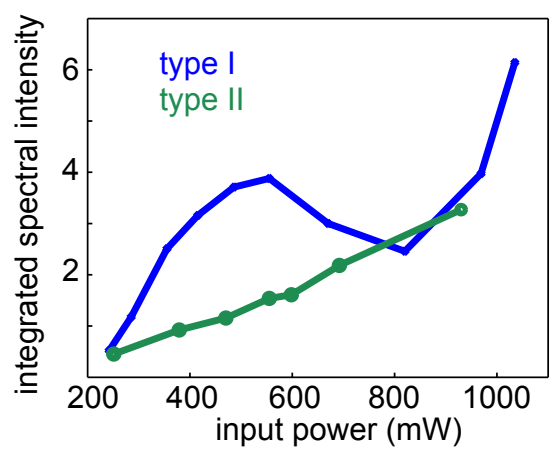

Figure 2.14: Simulated conversion efficiency of light for a) type I and II phase-matching, b) type I phase-matching with different ratios of the beam on each optical axis of the $500 \mu \mathrm{m}$-thick BBO crystal. $\alpha$ shows the angle between the polarization of the incoming beam and the extra-ordinary axis of the crystal. c) Measured scaling of DFG conversion efficiency versus pump power. d) Measured integrated spectral intensity as a function of pump power. The pump power was adjusted with an iris and the spectrum was measured for a beam much larger than the spectrometer entrance slit, indicating the on-axis scaling of the MIR pulse.

In a type I process the group velocity walk-off between the pulses is much less than in type II, which leads to a clear oscillatory behavior in the efficiency curve. Let us consider the case where the MIR wavelength is $2000 \mathrm{~nm}$, with effective signal and pump wavelength of 800 and $580 \mathrm{~nm}$. 
The group indices (Kato, 1986) of DFG, signal and pump are 1.6750, 1.6845, and 1.6932, respectively. Therefore the propagation distance in the crystal for a group delay shift of $10 \mathrm{fs}$ between DFG and signal is $315.5 \mu \mathrm{m}$, and for the same shift between signal and pump is $344.6 \mu \mathrm{m}$. In the case of type II phase-matching, the DFG, signal and pump group indices are 1.6750, 1.6313, 1.6549 , respectively, leading to much shorter distances to separate the DFG from the signal $(68.6 \mu \mathrm{m})$ and the signal from the pump $(127.0 \mu \mathrm{m})$. These relatively short length scales reduce the efficiency of the type II process, but at the same time ensure that backconversion through sum-frequency generation is unlikely at realistic intensities.

The effect of saturation on the DFG spectrum is illustrated in Fig. 2.15. As the intensity is increased, the peak wavelength of the DFG spectra obtained from type I phase-matching exhibits a shift first to longer and then to shorter wavelengths once the local minimum in conversion is reached. The spectral changes in type II phase-matching are more stable and the longwavelength components are gradually added to the spectrum at higher intensities. This is caused by the overall redshift of the input pulse as energy is transferred from the short-wavelength side of the input pulse spectrum to the long-wavelength side, shifting the phase-matching to suit lower frequencies, as shown in Fig. 2.16.

Experiment $4 \mathrm{fs}$ pulses with $1 \mathrm{~W}$ of average power (Cavalieri et al., 2007) were loosely focused down to $0.4 \mathrm{~mm}$ at FWHM by using a spherical mirror with $1 \mathrm{~m}$ focal length. Tighter focusing of the beam resulted in a filament in air and was therefore avoided in the experiment. The $500 \mu \mathrm{m}$-thick, antireflection-coated BBO crystals were placed in the laser focus. A spherical mirror was used for collimating the beam after the $\mathrm{BBO}$ and several gold coated mirrors were used to either send the beam to a spectrometer or to a power-meter. In order to block the residual short-wavelength light, a $2 \mathrm{~mm}$ thick antireflection-coated silicon wafer was placed in the beam to separate the generated DFG from the fundamental beam (Fig. 2.17).

The measurements were performed for both types of phase-matching and the energy of the generated DFG and its corresponding spectrum were measured separately for different input pump energies, scaled by adjusting a pinhole placed before the BBO crystals. The on-axis energy of the DFG signal was calculated by integrating over each DFG spectra, measured for different pump energies (Fig. 2.14-d). The maximum measured DFG efficiency for type I BBO crystal was $11 \%$ and for type II, it was $4.4 \%$. The obtained spectra for each phase-matching scheme and for different pump intensities are shown in Fig. 2.15.

To optimize the efficiency in type I phase-matching, a $42 \mu$ m-thick quartz 
plate was placed far from the focus and at Brewster's angle, such that the short-wavelength components were predominantly on the fast axis of the BBO. By adjusting the quartz plate, the DFG efficiency was increased to $12.4 \%$. The measured spectrum corresponding to the highest DFG pulse energy $(41 \mu \mathrm{J})$, is shown in Fig. 2.17-b, alongside the spectrum of the pump pulses.

In the generation of broadband single-pulse DFG signals, a short interaction length in the material is required to avoid separation between different frequencies in the time domain, caused by dispersion in the crystal. The short pulses used in this experiment are important for reaching high conversion efficiencies as crystals with short thicknesses, could be exposed to higher intensities for reaching higher conversion efficiency. This would not be possible with longer pulses (Lenzner et al., 1998).

In the experiment, the generated signal in type I phase-matching was less dependent on the chirp of the input pulse relative to type II phasematching. In type II phase-matching, the DFG signal is generated from a short pulse which travels on the extraordinary axis of the crystal, but in the type I process the input pulse splits into two beams traveling on different crystal axes. Consequently, the type II phase-matching is more sensitive to dispersion of the input beam as it is very important to have all the frequencies at the same time. But in type I phase-matching the interaction happens between two relatively long pulses traveling on different axis of the crystal and it is less sensitive to the input pulse chirp.

The output power of the type II process had higher stability than the type I process. This behavior can be explained by looking at the conversion efficiency of both cases. In type II phase-matching, the behavior of the efficiency is linear and therefore the energy stability of the generated signal is proportional to the energy stability of the pump pulse. In contrast, for type I phase-matching several local saturation points appear for different intensities. In this case the most stable regime for operation is slightly above saturation, where for slightly different input pulse energies, the same output energy is obtained. However, the shape of the generated spectrum is not robust against intensity fluctuations. The backconversion of the DFG signal to the input signal changes the shape of the input in the time domain and its corresponding spectrum. This can cause spectral instabilities in the generated signal as shown in Fig. 2.15.

The energy stability of type II DFG would make it more attractive, despite its lower conversion efficiency. Also it is an advantage that the emerging white light and MIR pulses are orthogonally polarized: the residual pump light can be non-dispersively suppressed, via a Brewster's angle reflection from a silicon wafer, which we found to reduce the residual white 
light contamination level to a few percent, dominated mostly by residual higher-order modes from hollow-core fiber.

The damage threshold of the $\mathrm{BBO}$ was not reached in the measurement so performing the experiments in vacuum with a smaller focus could lead to yet higher conversion efficiencies.

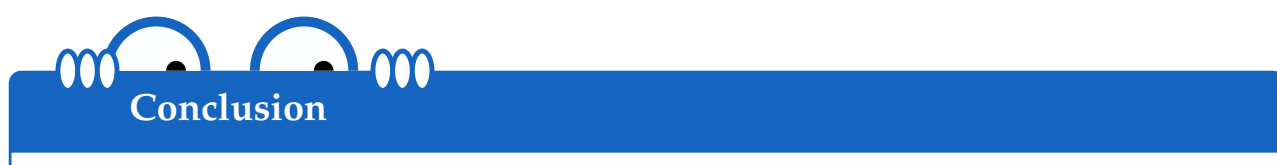

As shown in this chapter, difference frequency generation in a type I BBO crystal using $4.5 \mathrm{fs}$ pulses centered at $800 \mathrm{~nm}$ yields conversion efficiencies above $10 \%$. The presented experimental observations and corresponding numerical simulations show qualitatively different intensity-scaling behavior for type I and type II phase-matching. It is observed that type I DFG has higher overall efficiency than type II DFG. By modifying the polarization state of the pulse using a $42 \mu \mathrm{m}$ zero-order wave plate, conversion efficiency of type I DFG was improved to $12 \%$.

Due to its sensitivity to dispersion, type II DFG has a lower conversion efficiency but enables better stability of the spectrum versus input intensity. The DFG signal was separated from the pump pulses entirely by using a polarizer, as it had a different polarization. These two characteristics of type II DFG, stability and its simple separation, makes it a suitable choice for producing broadband, CEP stable, MIR pulses with enhanced temporal contrast. By focusing the generated pulses in a gas-filled hollow-core-fiber (Schwarz, 2014), the spectrum extends to the VIS range. The remarkable role of such a multi-octave, CEP-stable spectrum to seed an OPCPA system (discussed in Chapter 5) leaves DFG an insuperable technique for generation of broadband, coherent spectrum with the carrier phase locked to the intensity envelope.

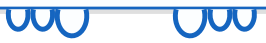


a)

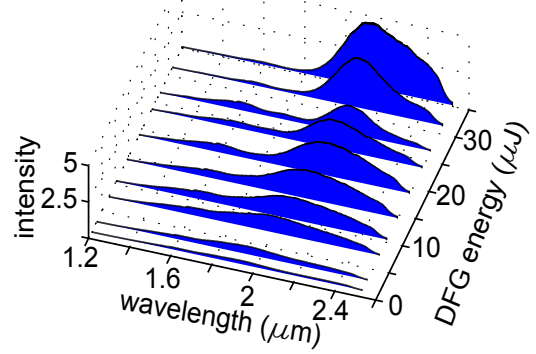

c)

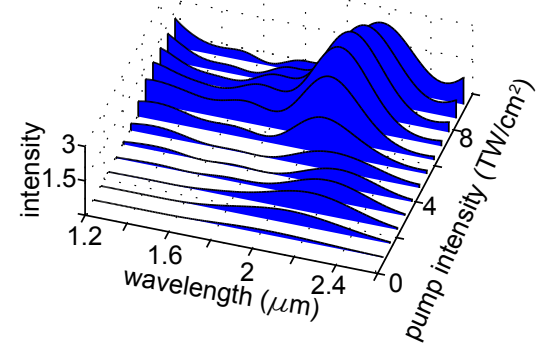

b)

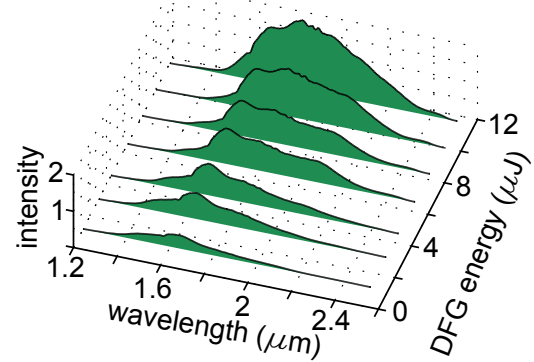

d)

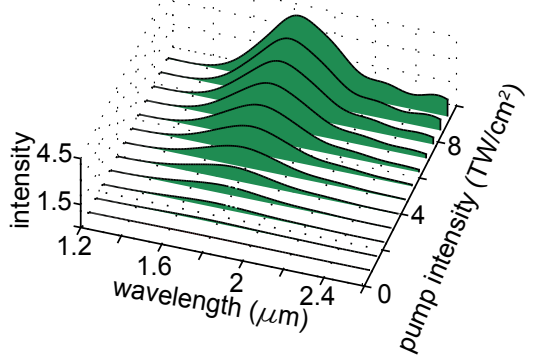

Figure 2.15: Measured and simulated DFG spectra in a $500 \mu$ m-thick BBO crystal for type I $(a, c)$ and type II $(b, d)$ phase-matching at different intensities.

a)

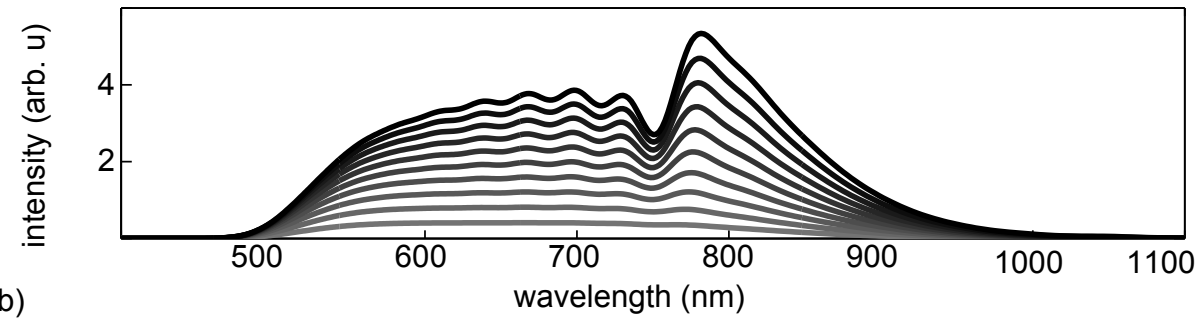

b)

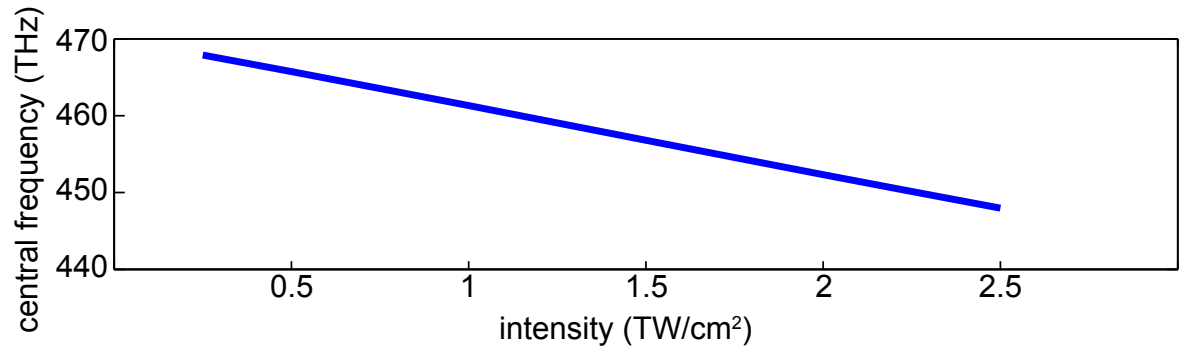

Figure 2.16: Simulated redshift of the pump pulse with increasing intensity. a) Output spectra of the pump pulse emerging from the e axis of the crystal with increasing intensity (from 0.3 to $2.5 \mathrm{TW} / \mathrm{cm}^{2}$ ). b) The first moment frequency of the spectra shown in panel (a) versus intensity. 
a)

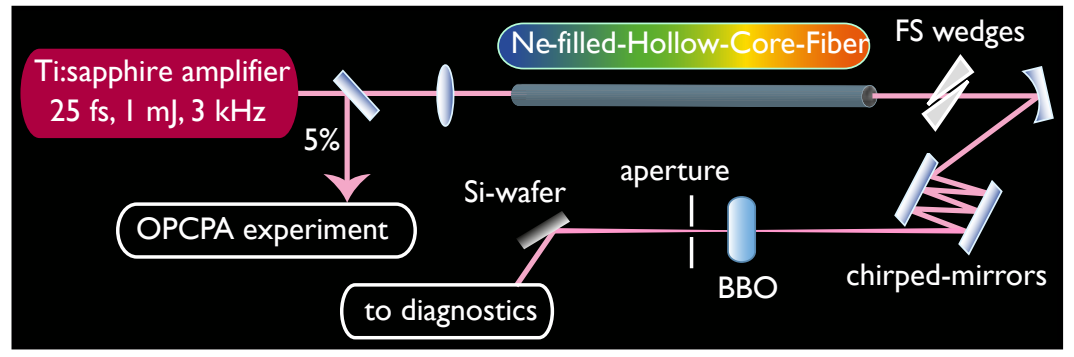

b)

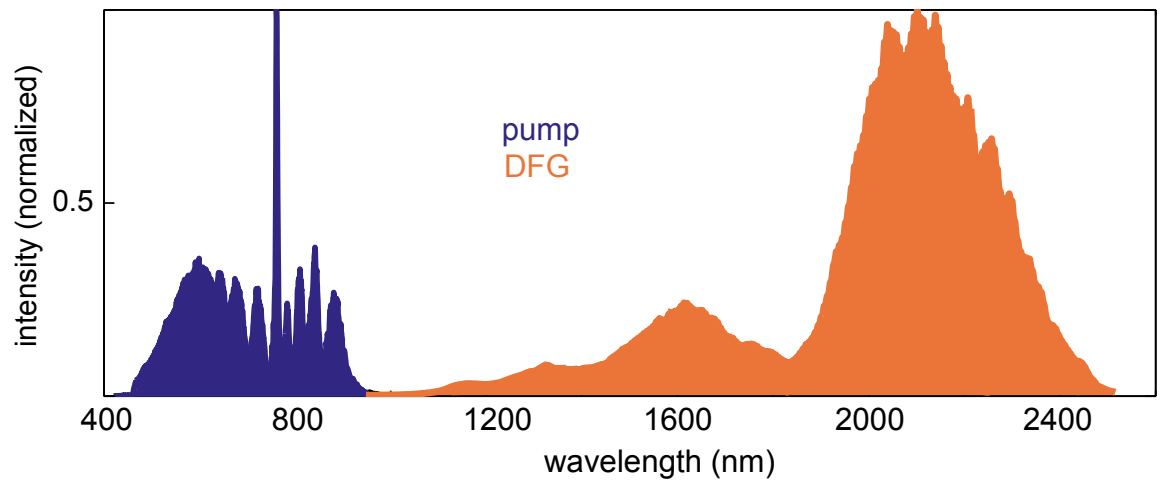

Figure 2.17: a) Layout of measurement set-up. b) Pump spectrum after chirped-mirror compressor and the DFG spectrum with highest efficiency. 



\section{Chapter 3}

\section{Regenerative amplifiers}

\subsection{Yb:YAG thin-disk regenerative amplifier}

${ }^{1}$ Although the construction of high energy laser systems is beyond the scope of this thesis, OPCPA requires a pump laser, whose characteristics form the ultimate limits on the possible specifications. In order to design and create an effective OPCPA, a thorough understanding of the underlying laser technology and its limits is required. The work in this thesis focuses on the use of Yb:YAG-based thin-disk regenerative amplifiers, e.g. (Metzger et al., 2009), as a useful source of high-energy, high power picosecond pulse trains to pump OPCPA systems, and the relevant considerations that must be made in order to fully exploit their capabilities.

In a typical laser gain medium, pump photons at the resonance frequency of the host material excite electrons to an upper atomic level, which subsequently decay to a lower excited state. Interaction of the seed photons with the excited electrons stimulates their decay to a lower energy level (in the ground state manifold) and consequently, photons with the same direction, phase, polarization and frequency of the seed photons are emitted.

In an ideal laser, where the medium consists of infinite numbers of electrons and in the absence of vacuum fluctuations, the amplified energy versus the input pump energy scales linearly. In the presence of vacuum fluctuations, amplification of the spontaneous emission depletes the medium and leaves no opportunity for the amplification of an injected pulse. In a finite medium, due to the restricted amount of atoms in the gain medium and therefore geometry-suppressed spontaneous emission, amplification can take place but still the depletion of the ground state can cause saturation and limit the amplification. Gain media with long upper state lifetimes have the

${ }^{1}$ This chapter is adopted from (Fattahi et al., 2014b). The electronic print of this paper is attached in Appendix E with the permission of OSA. 
potential for substantial population inversion under continuous pumping and are desirable for the construction of high-energy laser sources.

Ytterbium-doped materials have a long upper state lifetime, broad emission, small density of quantum defects (Hönninger et al., 1999) and their broad absorption spectrum in the region accessible by efficient laser diodes makes them the most promising gain medium for amplifiers in disk (Metzger et al., 2009; Teisset et al., 2013), slab (Schulz et al., 2011) or fiber (Jauregui et al., 2013) geometries. However, in continuously pumped regenerative amplifiers, chaotic pulse train dynamics at repetition rates comparable to the inverse of the gain relaxation time restrict the operating parameters of the laser system (Dörring et al., 2004; Grishin et al., 2009). In this chapter, different approaches to overcome these instabilities in order to achieve reproducible, stable pulses with excellent temporal shape, are discussed.

Regenerative amplifiers operating at repetition rates close to the inverse of the inversion build-up time in the gain medium show special amplification dynamics. Here the amplification of a pulse in the cavity is strongly coupled to the previous pulse and can result in stable regimes of operation such as period doubling (bifurcation), tripling, or amplification of pulses with extremely random variations in pulse-to-pulse energy (chaotic regime). To visualize the process, let us consider a pulse entering the cavity and depleting the gain medium. If a second pulse enters the cavity when the population inversion of the gain medium is not at its maximum, then the amplification gain is less.

In the bifurcation regime, the third pulse enters the cavity when the population inversion has fully recovered and therefore carries away a large amount of energy and leaves the gain medium depleted. This cycle repeats and every second pulse gets amplified to the maximum energy. In the chaotic regime, the entrance of the third pulse and subsequent pulses into the cavity are not synchronized with the full recovery of the depleted gain medium and results in random pulse-to-pulse energy. Normal operation of the laser, where every pulse exiting the cavity has the same pulse energy, can be ensured by increasing the number of round trips (passes through the gain medium), such that at a given seed and pump power level, each pulse depletes the gain medium entirely. Increasing the number of round trips increases the number of passes through the Pockels cell (PC) and air. This increases the accumulated nonlinear phase on the amplified pulses and initiates nonlinear effects like SPM which consequently influence the temporal and spectral shape of the pulse.

The induced nonlinear phase is described as:

$$
\frac{\mathrm{d} \Phi_{n l}(z, t)}{\mathrm{d} z}=-\frac{\omega}{c} n_{2} I(t, z) .
$$


This instantaneous phase adds a nonlinear phase to the pulse. After propagating distance $L$ inside the PC and air, the accumulated nonlinear phase in terms of the B-integral is:

$$
B(t, r)=\frac{2 \pi}{\lambda_{\text {vac }}} \int_{0}^{L} n_{2} I(t, r, z) d z,
$$

where $r$ is the transverse coordinate of the laser beam. The spatial variation of the B-integral implies that perfect compensation of the induced nonlinear phase is not possible. The radial variation also forms a nonlinear lens inside the cavity which can cause coupling to higher order modes. These modes are filtered out by the cavity but cause reduction in the efficiency as they increase cavity losses.

B-integral values of $\sim 2$ could double the pulse duration (Chuang et al., 1993). Even for the modest value of $B \leq 1$ significant energy can be removed form the central peak of the pulse and appear as a pedestal, decreasing the contrast and lowering the peak power (Perry et al., 1994). In the SPM process the interference between the newly generated frequencies and the spectrum of the pulse causes a change in the spectral bandwidth of the pulse. In Fig. 3.1 the spectral and temporal effects for different chirp values applied to the pulse are shown. For negatively chirped pulses with a GDD of $-0.27 \times 10^{7} \mathrm{fs}^{2}$, destructive interference between the newly generated photons in SPM and the spectrum of the pulse causes spectral narrowing. For heavily chirped pulses, however, SPM primarily influences the spectral phase of the pulse, leaving the shape and the bandwidth of the spectrum unchanged.

In regenerative amplifiers with low input seed energy, a large number of round trips are required to reach energy saturation. To study the effect of B-integral in low-energy-seeded regenerative amplifiers, a simple 1-D model (Appendix B) based on the rate equations for continuously-pumped Yb:YAG (Antognini et al., 2009) is used. The model includes the wavelengthdependent pulse energy buildup for the full pulse train and the nonlinearity acting on the amplified pulses caused by the PC and air is calculated in each round trip. The gain in each pass is obtained via the temperature-dependent emission cross-section according to (B. Chen et al., 2003).

In the simulation a seed pulse with $10 \mathrm{~nm}$ spectral bandwidth is stretched using GDD of $1.19 \times 10^{8} \mathrm{fs}^{2}$, to approximately $2 \mathrm{~ns}$. The pulse travels for $40 \mathrm{~ns}$ in the cavity in each round trip. A 20-mm-long PC (traversed twice per round trip) with a nonlinearity of $2.99 \times 10^{-20} \mathrm{~cm}^{2} / \mathrm{W}$ (Desalvo et al., 1996) and a beam waist of $3.5 \mathrm{~mm}\left(1 / e^{2}\right)$ on the PC crystal is assumed, similar to (Metzger et al., 2009). $225 \mathrm{~W}$ of pump power with a $2.8 \mathrm{~mm}$ beam waist is used to pump a 10o- $\mu$ m-thick Yb:YAG gain medium. The simulation is performed for two different input seed energies, $80 \mathrm{pJ}$ and $40 \mathrm{~nJ}$. 


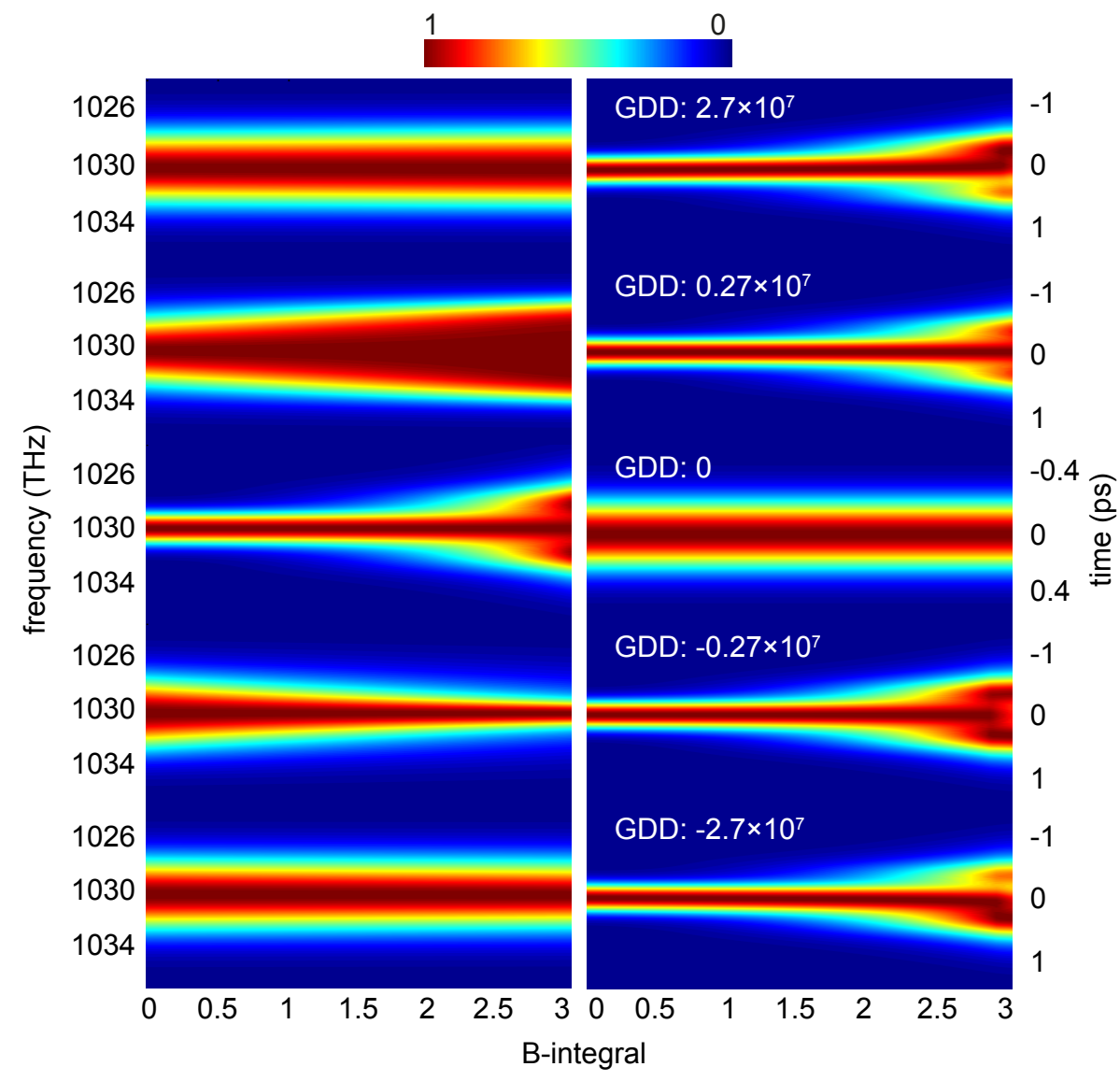

Figure 3.1: Spectral (right panels) and temporal (left panels) evolution of a pulse, stretched with different amounts of dispersion versus B-integral.

Figure 3.2 compares the pulse build-up and spectral and temporal behavior of the amplified pulses for these two cases. In the low-energy-seeded regenerative amplifier, 126 round trips are required to reach saturation. At this number of round trips, the accumulated B-integral causes undesired higherorder nonlinear phase on the amplified pulses, which makes compression of the pulse to its Fourier transform limit challenging. Any additional increase in the round trip numbers causes pulse break-up in the time domain. By increasing the input seed energy level to $40 \mathrm{~nJ}$, the required number of round trips to reach saturation reduces to 90. Here, the introduced nonlinear phase on the amplified pulse is negligible, leaving the spectral phase of the amplified pulse well-behaved as in this case the nonlinear path through the regenerative amplifier is reduced.

In Fig. 3.2, for the case of low-energy-seed, spectral narrowing versus the round trip number is visible, which is caused by the limited bandwidth of 
a)

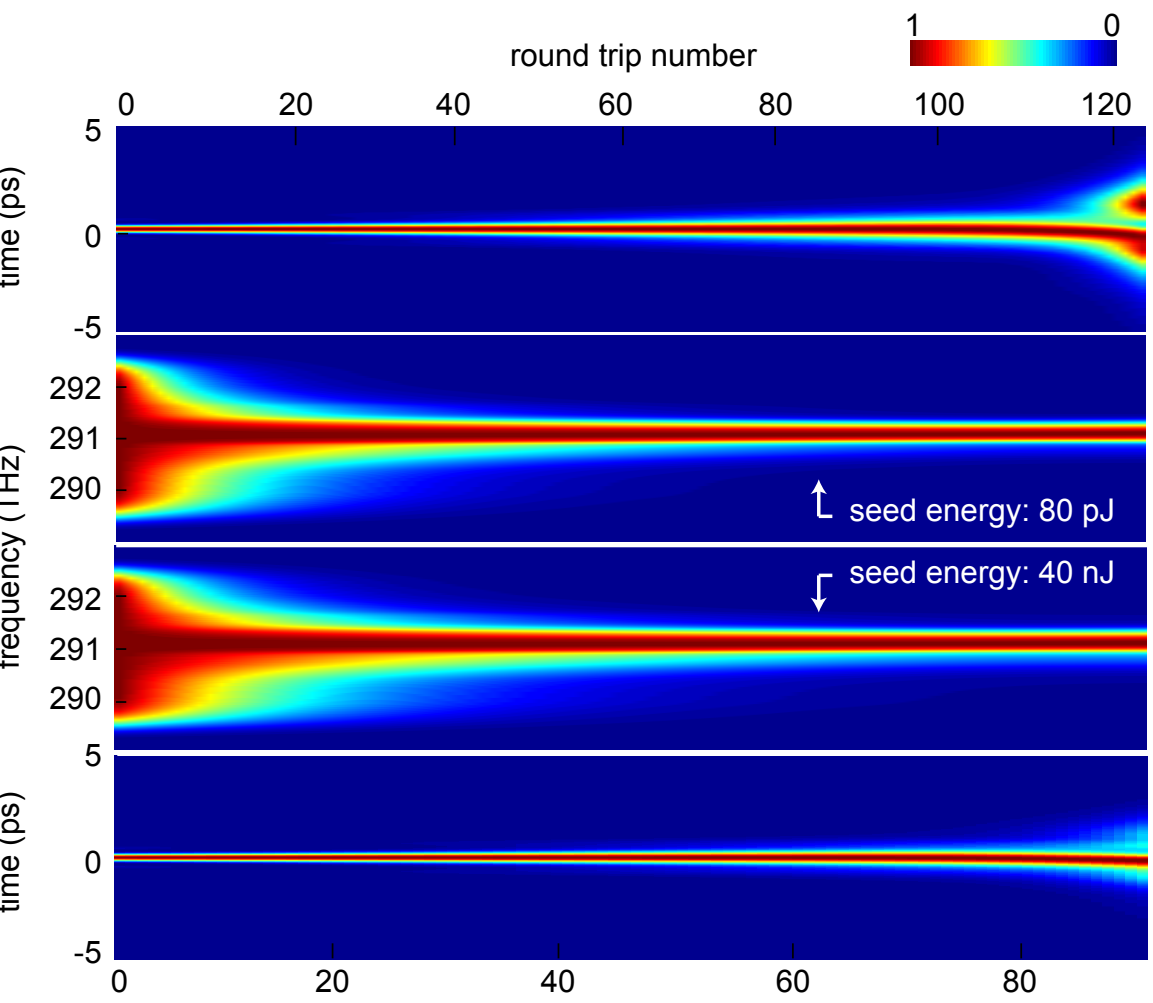

b)

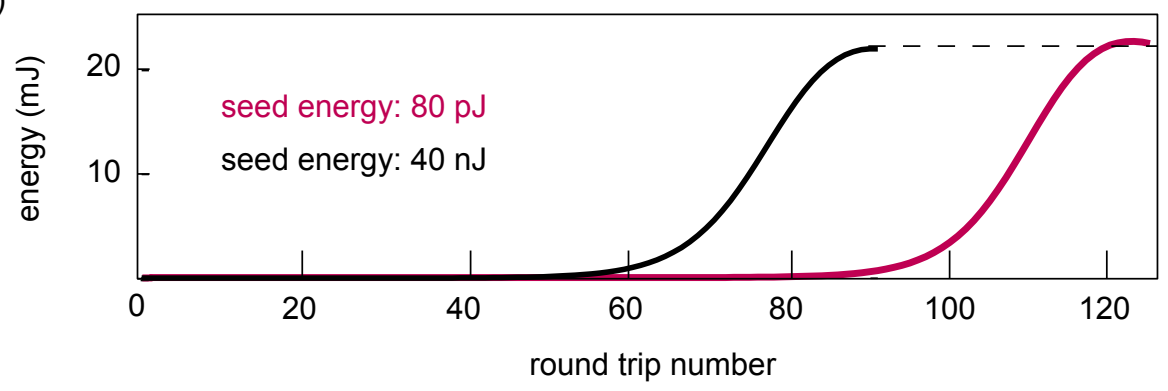

Figure 3.2: a) Simulated temporal and spectral profile of the amplified pulses versus round trip number in a regenerative amplifier for two different input seed energies: $80 \mathrm{pJ}$ and $40 \mathrm{~nJ}$. For the case of low-energy-seed, the accumulated nonlinear phase, causes undesired temporal features on the amplified pulses (The temporal and spectral amplitudes in all cases are normalized to unity). b) Pulse build-up in the regenerative amplifier for the two different input seed energies. For the case of the higher seed energy, saturation happens after a lower number of round trips. 


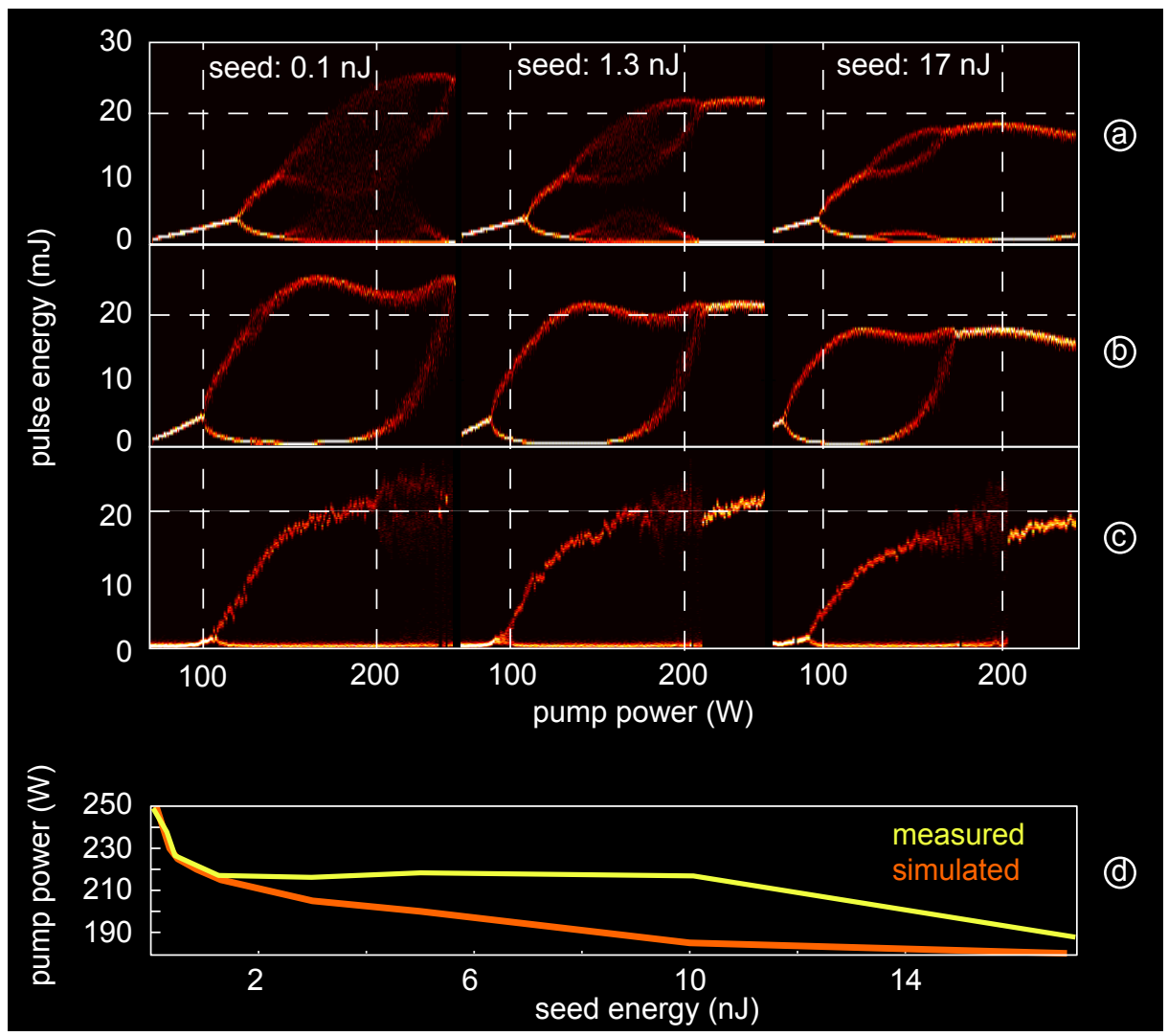

Figure 3.3: Simulated and measured amplification dynamics for CWpumped $\mathrm{Yb}: \mathrm{YAG}$ thin-disk regenerative amplifier operating at different repetition rates and seed energies. a) The amplifier with a PC driven at $6 \mathrm{kHz}$ repetition rate enters a chaotic regime for low seed energy and reaches stable bifurcation at higher pump power. Increasing the input seed energy reduces the chaos in the cavity. b) The same system with a PC driven at $3 \mathrm{kHz}$ repetition rate. Here no chaotic regime is observed and the bifurcation is removed at a higher pump power. The pump power difference for reaching the bifurcation point and the normal operation point is reduced by increasing the input seed energy. c) Measured dynamics at $3 \mathrm{kHz}$. d) Required pump power for stable operation as a function of pulse energy at $3 \mathrm{kHz}$.

the gain medium. In regenerative amplifiers with higher input seed energies, the effect of gain narrowing is reduced, which results in a longer pulse inside of the cavity after amplification and correspondingly lower pulse intensity and less accumulated nonlinear phase.

A Yb:YAG thin-disk regenerative amplifier described in (Metzger et al., 2009), is used to study these effects experimentally. In this system, the $1 \mathrm{pJ}$ 
pulses centered at $1030 \mathrm{~nm}$, driven from a Ti:sapphire oscillator were amplified to an energy of $80 \mathrm{pJ}$ with a diode-pumped fiber pre-amplifier. A second fiber pre-amplifier was placed after the pulse picker for further amplification of the seed pulses up to $40 \mathrm{~nJ}$. In the first measurement, the regenerative amplifier was seeded with $80 \mathrm{pJ}$ of energy. Using 107 cavity round trips, after intermediate chaos, period doubling and a stable regime of operation was achieved. The amplified pulses were passed through a grating pair for temporal compression and characterized by SH-FROG exploiting a $10 \mu \mathrm{m}$ $\mathrm{BBO}$ crystal as the nonlinear medium.

A stable regime of operation with no chaotic behavior or bifurcation was observed after increasing the input seed energy to the cavity (Grishin et al., 2007). Figure 3.3 shows the energy distribution in a bifurcation diagram acquired by incrementing the pump power over the range of 50-250 W with the number of cavity round trips kept constant at 70. Here the PC was driven at $3 \mathrm{kHz}$ repetition rate. For the input seed energy of $0.1 \mathrm{~nJ}$, the bifurcation happened at $100 \mathrm{~W}$ of pump power. By increasing the pump power to $250 \mathrm{~W}$, the amplifier went out of the doubling regime and the amplification happened at $3 \mathrm{kHz}$ repetition rate. By increasing the input seed energy the bifurcation and normal amplification points were shifted to lower pump powers.

The simulated and measured data are in good agreement. In both cases, there is no sign of chaotic behavior for high energy seeding. Figure 3.3-a shows the simulated data in the case of $6 \mathrm{kHz}$ operation similar to (Metzger et al., 2009). Here, the bifurcation point is shifted to a higher pump power compared to $3 \mathrm{kHz}$ operation. After period doubling, the amplifier goes to the chaotic regime and at high pump power reaches a stable doubling regime. The separation of this point and the chaotic zone is determined by the input seed energy level. Running the amplifier with a PC driven at $3 \mathrm{kHz}$ is advantageous as it simplifies synchronization with OPCPA seed sources and reduces the thermal load on the PC crystal. Due to hysteresis, the amplifier has the tendency to stay in the normal operation regime. After emerging from bifurcation, even for slightly reduced pump power, the laser stays in the normal operation mode as the cavity design is optimized for high-power operation. Therefore the cavity efficiency increases slightly as the system enters the normal amplification mode and the output power doubles. The hysteresis and thermal effects which are not included in the simulation are the primary source of discrepancy between the measured and simulated data.

Figure $3 \cdot 4$-b shows the effect of the cavity dynamics on the temporal profile of the compressed pulses. The characterization of the unsaturated amplified pulses at $14 \mathrm{~W}$ seeded with $80 \mathrm{pJ}$ does not show any phase distor- 
a)

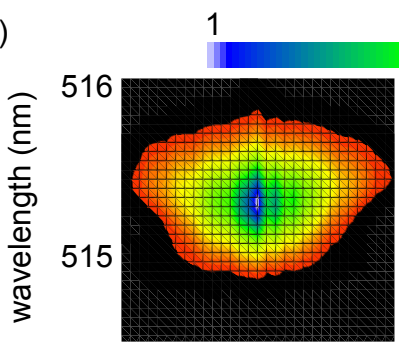

b)
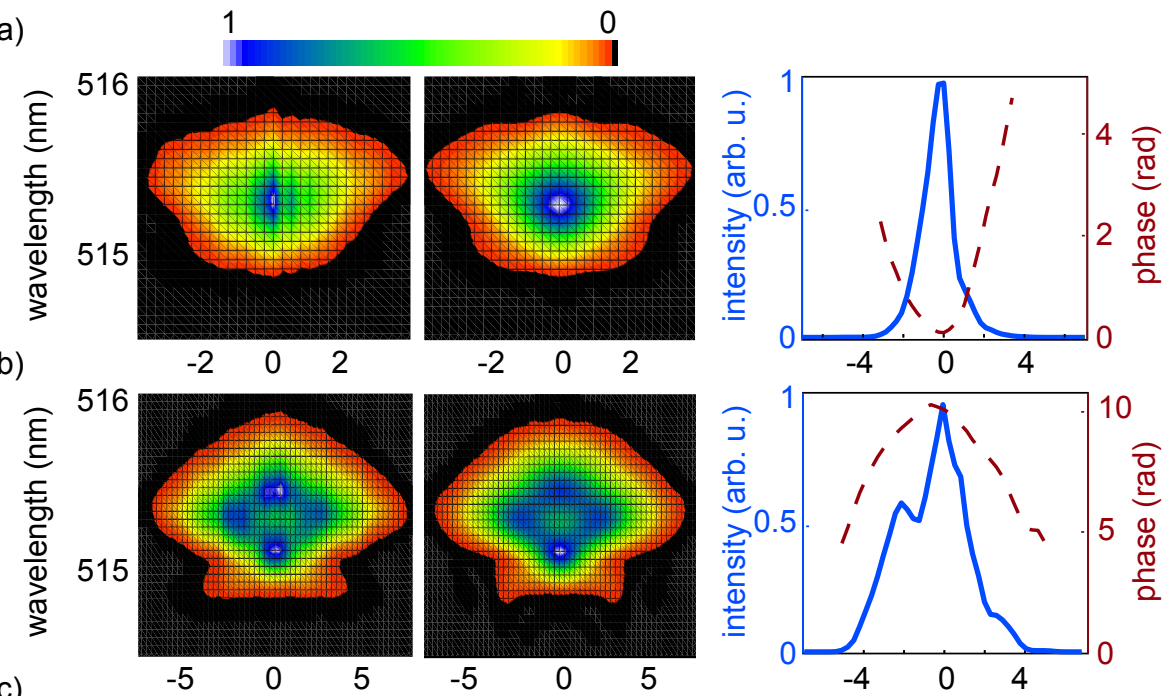

c)
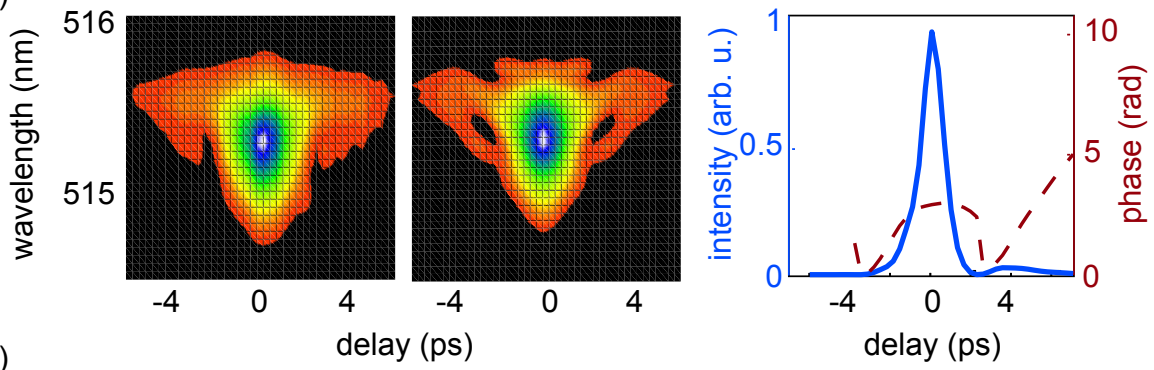

d)

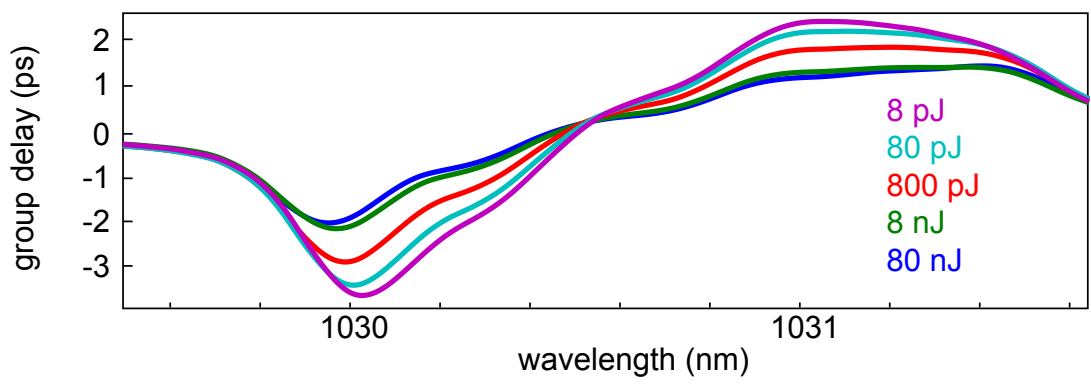

Figure 3.4: a) Measured SH-FROG traces of the $14 \mathrm{~W}$ amplified pulses in a regenerative amplifier seeded with $80 \mathrm{pJ}$ of energy and making 107 cavity round trips (the laser operated in the unsaturated regime with unstable laser output). b) Measured SH-FROG trace for the same pulses amplified to 58 $\mathrm{W}$, showing strong SPM in the time domain. c) Temporal profile of $300 \mathrm{pJ}$ amplified pulses to $61 \mathrm{~W}$ with increased pump power and reduced round trip number. The effect of SPM in this case is negligible. d) Calculated group delay resulting from the induced nonlinear phase on the amplified pulses with different input seed energies. The pump power for all cases was kept constant and saturation was ensured by changing the number of cavity round trips. 
tion and the pulses are nearly compressed to their Fourier-transform limit (Fig. 3.4-a). However, after running the laser in the saturated regime with an output power of $58 \mathrm{~W}$, SPM begins to distort the temporal phase of the amplified pulses, although the shape of the spectrum is not affected. By increasing the input seed energy to $300 \mathrm{pJ}$, increasing the pump power and reducing the cavity round trip number by 10 , the characterized $61 \mathrm{~W}$, compressed pulses show a good temporal profile indicating that the effect of SPM in this case in negligible (Fig. 3.4-c). Figure 3.4-d shows calculated nonlinear group delay distortion of the amplified pulses for varying input seed energies to the amplifier, explaining the temporal behavior of the measured pulse in Fig. 3.4-b. The FROG G-error of the reconstructed trace in Fig. 3.4-b is 0.022 which is more than the the G-error of Fig. 3.4-c, 0.0087 . This could be an indication of pulse-to-pulse variations in temporal profile (Ratner et al., 2012).

Different approaches can be used to eliminate the undesired SPM effects in amplifiers while maintaining stable operation. Table 3.1 summarises different approaches with their pros and cons.

Effect of suboptimal amplifier design in subsequent nonlinear processes In the presence of significant SPM in the system, small changes in the seed energy level and cavity alignment result in large variations of the amplified output pulses. For an OPCPA pumped by such pulses these variations affect the efficiency of the amplified pulses in the subsequent broadband OPCPA stages. For a regenerative amplifier operating at the border of nonlinearity, variations of the spectral phase to the evolution of the pulse inside of the cavity can result in large fluctuations in the OPCPA output, although the pulse-to-pulse energy fluctuations of the pulses of the saturated amplifier are at their minimum. To study these effects, the spectrum and gain of an OPCPA system are simulated.

The pulses from Fig. 3.4-b and Fig. 3.4-c with an identical pulse energy of $3 \mathrm{~mJ}$ and a beam diameter of $1.2 \mathrm{~mm}$ at full width half maximum are used to amplify a broad spectral range in a single OPCPA stage consisting of $1 \mathrm{~mm}$ BBO crystal with a $d_{e f f}$ value of $2.3 \mathrm{pm} / \mathrm{V}$, a phase-matching angle of $24.5^{\circ}$, and noncollinear angle of $2.7^{\circ}$, seeded with $1 \mu \mathrm{J}$ of energy. As shown in Fig. 3.5 this variation in pump pulses results in a factor of 3.5 difference in pump-signal efficiency and modifications in the amplified spectrum. Suboptimal pump pulses also affect the spectrum and efficiency of the generated second harmonic (Fig. 3.5-c). 
a)

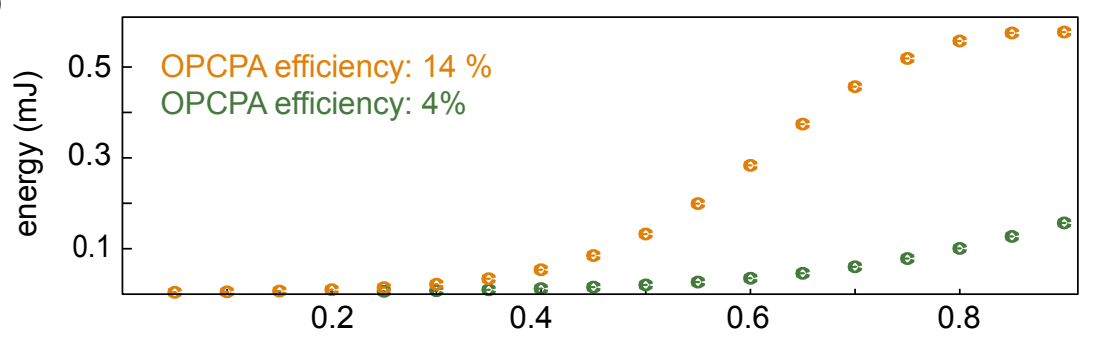

b)

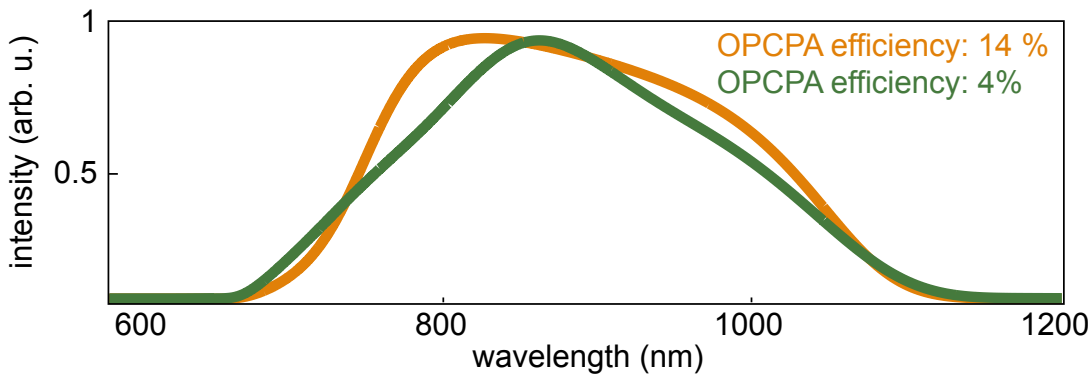

c)

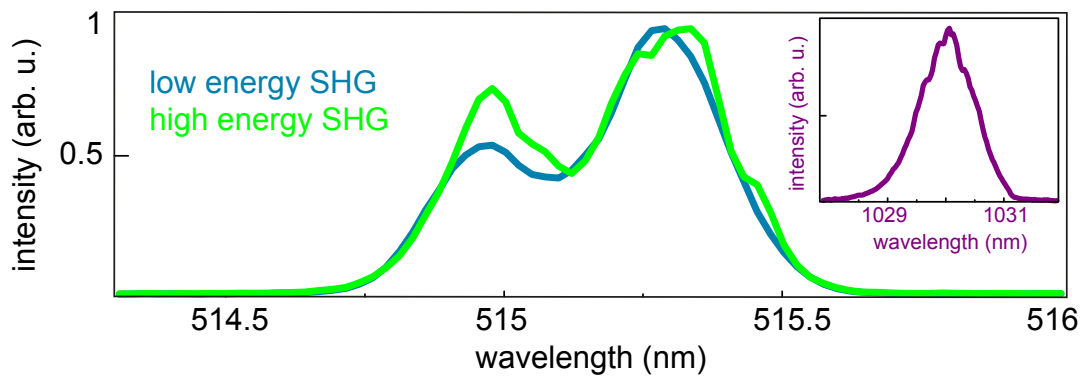

Figure 3.5: Simulation of the effect of amplified SPM on the output of a broadband OPCPA. The orange curves are simulated with the pulse measured in Fig. 3.4-C and the green curves correspond to the pulse in Fig. 3.4-b. a) Gain vs. crystal length in the OPCPA, showing saturation with the SPMfree pulse, and a factor of 3.5 reduction in pulse energy with the distorted pump pulse. b) Modification of the spectrum resulting from the pump temporal profile (normalized). c) The measured second-harmonic spectra of the suboptimal pump pulses (Fig. 3.4-b) in the saturated (high energy) and unsaturated (low energy) regime. The hole in the second harmonic spectra is caused by the distorted spectral phase of the input pump pulses. The pump spectrum is shown in the inset. 


\begin{tabular}{|c|c|c|}
\hline Approach & Mechanisms & Negative consequences \\
\hline $\begin{array}{l}\text { Reduced round trips } \\
\text { with higher pump power }\end{array}$ & $\begin{array}{l}\text { - Reduced nonlinear path length } \\
\text { - Reduced cavity losses leading to } \\
\text { less gain narrowing at same energy }\end{array}$ & $\begin{array}{l}\text { - Increased thermal load } \\
\text { - Smaller range of stable } \\
\text { operating power }\end{array}$ \\
\hline $\begin{array}{l}\text { Increase seed level via } \\
\text { further broadband pre- } \\
\text { amplification }\end{array}$ & $\begin{array}{l}\text { - Reduced gain narrowing } \\
\text { - Reduced nonlinear propagation } \\
\text { distance via reduced round trip number }\end{array}$ & $\begin{array}{l}\text { - Increased system } \\
\text { complexity/cost } \\
\text { - Longer seed beam path }\end{array}$ \\
\hline $\begin{array}{l}\text { Decrease pump spot } \\
\text { size }\end{array}$ & $\begin{array}{l}\text { - Faster inversion buildup for given pump } \\
\text { power, allowing stable operation at lower } \\
\text { power }\end{array}$ & $\begin{array}{l}\text { - Increased thermal gradient in } \\
\text { gain medium } \\
\text { - Reduced saturation energy }\end{array}$ \\
\hline $\begin{array}{l}\text { Increase stretcher and } \\
\text { compressor dispersion }\end{array}$ & - Reduced intensity at given pulse energy & $\begin{array}{l}\text { - Increased cost and alignment } \\
\text { sensitivity of compressor and } \\
\text { stretcher } \\
\text { - Additional timing jitter when } \\
\text { synchronized with external } \\
\text { seed }\end{array}$ \\
\hline $\begin{array}{c}\text { Increased beam size in } \\
\text { nonlinear cavity } \\
\text { elements via reflective } \\
\text { telescope }\end{array}$ & $\begin{array}{l}\text { - If a single element (i.e. Pockels cell) is } \\
\text { responsible, reduced intensity }\end{array}$ & $\begin{array}{l}\text { - Increased complexity of the } \\
\text { laser cavity } \\
\text { - Requires large cavity optics }\end{array}$ \\
\hline Operation in vacuum & - Reduced nonlinearity of free beam path & $\begin{array}{l}\text { - Cost and complexity } \\
\text { - More difficult thermal } \\
\text { management }\end{array}$ \\
\hline
\end{tabular}

Table 3.1: Possible approaches for the reduction of SPM in a thin-disk regenerative amplifier

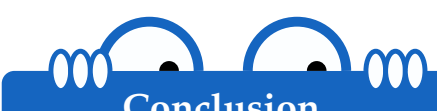

\section{Conclusion}

Stable OPCPA demands not only excellent pump energy stability, but also a high degree of reproducibility of the temporal shape of the pump pulses, which can only be ensured by careful matching of the amplifier design to the seed specifications. The presented simple one-dimensional simulation and the measurements show the chaotic regime in the ytterbium-doped regenerative amplifiers is avoidable by increasing the input seed energy. Here the accumulated nonlinear phase in the cavity is minimized and a reproducible spectral phase is achievable. The effect of a suboptimial amplifier on a subsequent nonlinear stage (here OPCPA) is numerically studied, which emphases the high demands on well-behaved phase of pump pulses in an OPCPA system. By ensuring that these effects are well controlled, thin-disk 
regenerative amplifiers are an ideal route to stable and efficient pumping of high-power, high-energy, broadband OPCPAs.

ou vov 


\begin{tabular}{|c|c|c|c|c|c|c|}
\hline ) & $\mathrm{n}_{2}\left(\mathrm{~cm}^{2} / \mathrm{W}\right)$ & walk-off & available size & $\alpha\left(\mathrm{cm}^{-1}\right)$ & Type I & Type II \\
\hline $\mathrm{BBO}$ & $7.4 \times 10^{-20}$ & $\begin{array}{c}55.3 \mathrm{mrad} \text { (I) } \\
68.47 \mathrm{mrad} \text { (II) }\end{array}$ & $25 \times 25 \mathrm{~mm}^{2}$ & $0.01 @ 532$ & $\Theta=23.4$ & $\Theta=33.7$ \\
\hline LBO & $1.9 \times 10^{-20}$ & $8.16 \mathrm{mrad}$ & $70 \times 70 \mathrm{~mm}^{2}$ & $\begin{array}{l}0.00035 \\
@ 1064 \text { nm }\end{array}$ & $\Phi=13.77^{\circ}$ & $\Theta=25.4^{\circ}$ \\
\hline
\end{tabular}

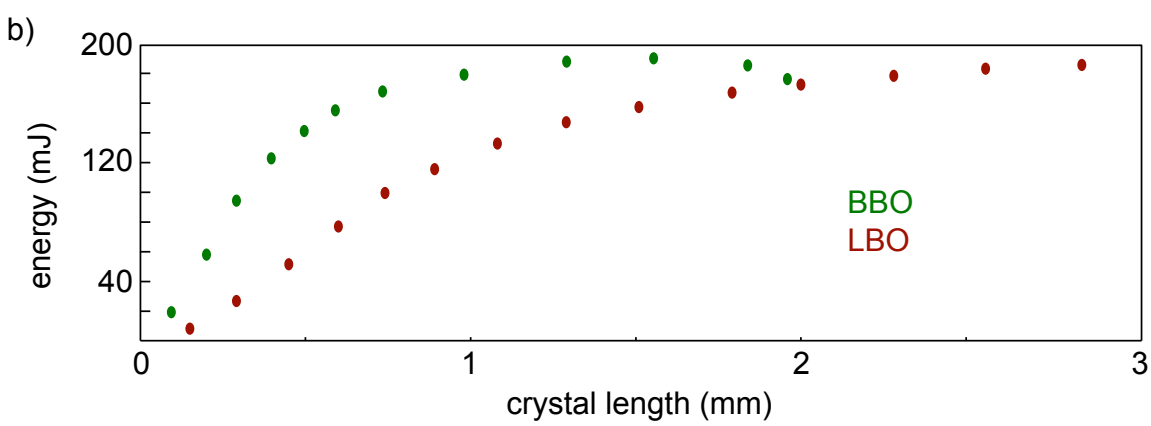

Figure 3.6: a) Parameters of BBO and LBO crystal for SHG. b) SHG gain vs. crystal length of a $200 \mathrm{~mJ}, 1.6$ ps pulses in a type I, BBO and LBO crystal from simulation. $\alpha$ values in the table indicate the absorption coefficient of each crystal.

\subsection{Second harmonic generation}

Pumping OPCPAs in the near infrared and visible requires the generation of low-order harmonics of the near-infrared emission of the Yb:YAG medium (Section 1.2). Although there are numerous options for second harmonic generation, critically phase-matched LBO and BBO are among the best candidates for low-order harmonic generation of near-1-ps pulses at $1030 \mathrm{~nm}$. They have fairly high nonlinear coefficients and damage thresholds. BBO has a higher nonlinearity than LBO but a larger spatial walk-off and is limited in the available aperture, as summarized in Fig. 3.6-a.

Fig. 3.6-b shows the simulated SHG gain versus crystal length for $1.6 \mathrm{ps}$ pulses centered at $1030 \mathrm{~nm}$ with a pulse energy of $200 \mathrm{~mJ}$ and a beam diameter of $10 \mathrm{~mm}$ in type I BBO and LBO crystals. In the LBO crystal with a crystal thickness twice that of the BBO crystal, the generated second harmonic reaches saturation. Due to reduced spatial walk-off between the second harmonic beam and the fundamental beam in LBO, a conversion efficiency similar to BBO can be achieved, despite having a smaller $d_{e f f}$ value than BBO. The calculated conversion efficiency does not reach $100 \%$ becuase of phase-mismatch between the second harmonic and the pump beam, and the temporal and spatial walk-off in the nonlinear crystals. 
Experiment The discussed Yb:YAG thin-disk regenerative amplifier was used to study SHG in LBO and BBO crystals. The output beam profile of the laser after compression became elliptical as the grating pair used for the pulse compression was not entirely flat. The ellipticity was compensated by implementing a home-made adaptive mirror before the grating compressor. By tuning the curvature of the thin, flat mirror the beam ellipticity was improved to 0.78 and the residual ellipticity was further improved to 0.83 by using a set of cylindrical mirrors with radii of curvature of $-1574 \mathrm{~mm}$ and $500 \mathrm{~mm}$ (Fig. 3.7).

A refelective telescope consisting of mirrors with radii of curvature of $-2000 \mathrm{~mm}$ and $800 \mathrm{~mm}$ was used to adjust the beam size on the crystal in order to reach $120 \mathrm{GW} / \mathrm{cm}^{2}$ intensity, assuming a Gaussian beam in time and space. The SHG in a $2 \mathrm{~mm}$ BBO crystal reached $70 \%$ conversion efficiency and saturated at $5 \mathrm{~mJ}$ of input pump energy. By increasing the input pump energy, the energy backconversion from the SHG signal to the pump resulted in oscillatory behavior in the efficiency curve and degradation of the spatial profile of the generated second harmonic (Fig. 3.7-b.3). The generated second harmonic in a $1 \mathrm{~mm}$ BBO crystal with the same pump parameters reached saturation while the spatial profile of the pulses preserved its quality. A pump intensity of $70 \mathrm{GW} / \mathrm{cm}^{2}$ on a $4 \mathrm{~mm}$ LBO crystal resulted in an efficiency of $70 \%$ with an excellent beam quality. SHG could also be used as a measure for fine tuning the grating pair compressor. Fig. 3.7-e) shows the sensitivity of the SHG conversion efficiency to the temporal profile of the pump pulses.

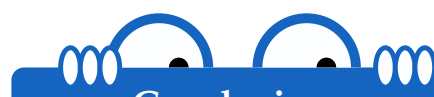

Conclusion

As it is shown in this section, employing BBO or LBO crystals for frequency doubling of a $20 \mathrm{~mJ}, 1.6$ ps pulses of an Yb:YAG amplifier, leads to pump-to-second harmonic conversion efficiency above $65 \%$. This high efficiency indicates the optimum temporal and spatial properties of the amplifier pulses. High conversion efficiencies in both crystals is achieved, even though the nonlinearity of a LBO crystal is about half of the BBO crystal, due to the lower temporal walk-off in a LBO.

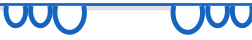



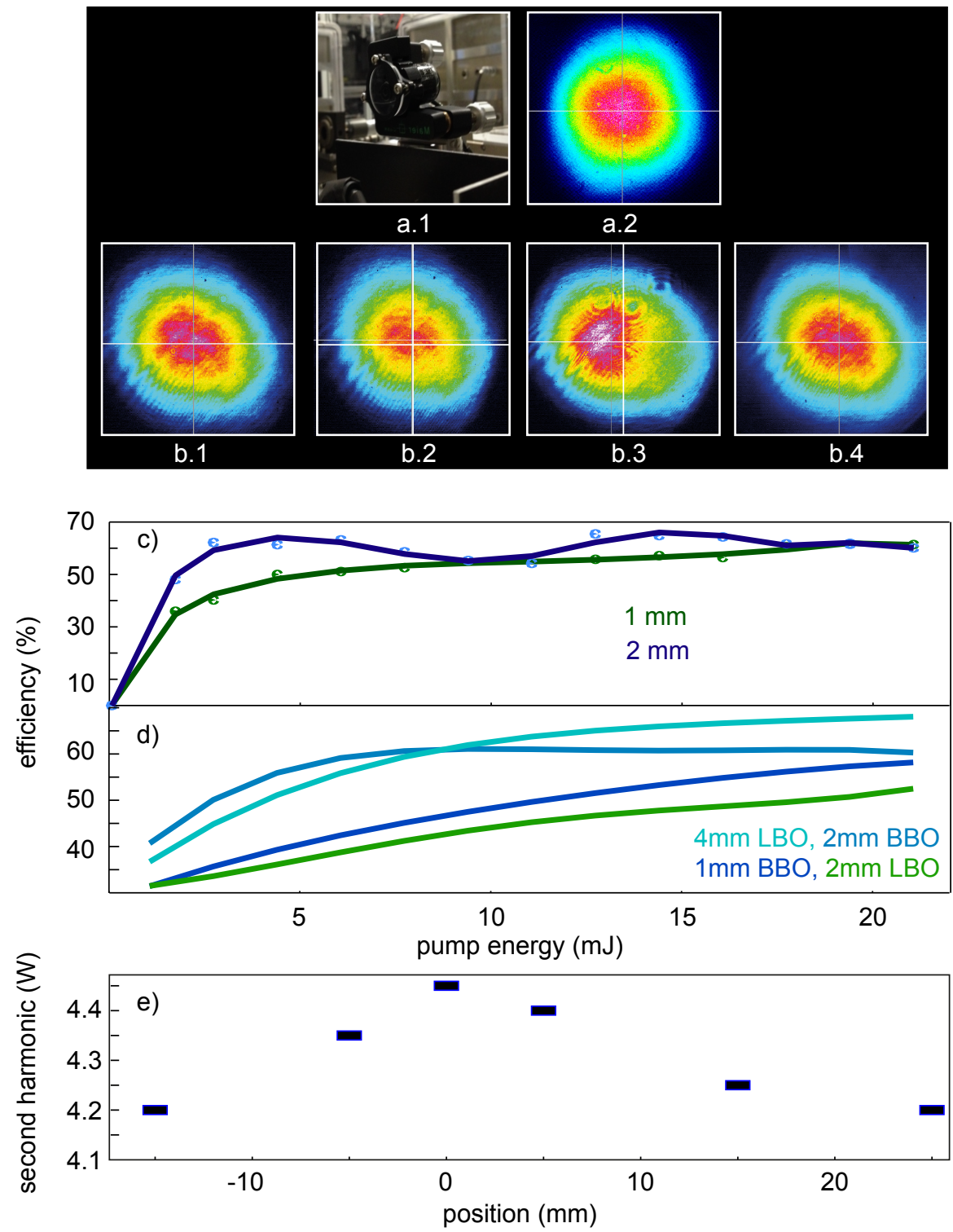

Figure 3.7: $a, b$ ) Home-made passive adaptive mirror used before the grating compressor of the disk laser (a.1). The beam profile of the amplifier after the adaptive mirror and the cylindrical telescope (a.2). The beam profile of the generated second harmonic in a $1 \mathrm{~mm} \mathrm{BBO} \mathrm{(b.1),} 2 \mathrm{~mm}$ LBO (b.2), $2 \mathrm{~mm}$ BBO (b.3) and $4 \mathrm{~mm}$ LBO (b.4) crystals. c) SHG in a $1 \mathrm{~mm}$ and $2 \mathrm{~mm}$ BBO crystal with the intensity of $120 \mathrm{GW} / \mathrm{cm}^{2}$. d) SHG in a $1 \mathrm{~mm}$ and $2 \mathrm{~mm} \mathrm{BBO}$ and $2 \mathrm{~mm}$ and $4 \mathrm{~mm}$ LBO crystals for a pump intensity of $70 \mathrm{GW} / \mathrm{cm}^{2}$. e) Second harmonic signal versus grating separation in a $1 \mathrm{~mm} \mathrm{BBO}$ crystal. 



\section{Chapter 4}

\section{Experimental OPCPA}

In this chapter, broadband seed pulses demonstrated in Chapter 2 and the turn key Yb:YAG pump laser discussed in Chapter 3 are employed to develop OPCPA systems with particularly high conversion efficiency.

Conversion efficiency in an OPCPA is limited by the group velocity mismatch between pump, signal and idler during the amplification process, deficient spatial overlap between the interacting beams caused by spatial walk-off in the nonlinear crystal, the temporal and spatial profiles of the seed and pump beams, and finally, the amplification of superfluorescence.

Overcoming these limitations to increase the efficiency has been the subject of several previous studies. By using a spatiotemporally shaped pump pulse, a pump-to-signal conversion efficiency as high as $40 \%$ (theoretically) (Guardalben et al., 2003) and 25\% (Chekhlov et al., 2006), 29\% (Waxer et al., 2003) and 34\% (Bagnoud et al., 2005) (experimentally) have been reported. However, there is no straightforward possibility for spatiotemporal shape of the ps-pump laser pulses. Also, the amplified spectrum of the mentioned OPCPAs is narrow compared to the bandwidth of few-cycle pulses. For 10o-ps-pumped few-cycle OPCPAs, a 27\% conversion efficiency was demonstrated by applying a complex, double-pass OPCPA configuration (Ishii et al., 2005) and in a near-1ps-pumped OPCPA system, few-cycle pulses with $25 \%$ pump-to-signal efficiency were shown (Fattahi et al., 2012b).

In this chapter, the theoretical analysis and experimental implementation of three different techniques for the simultaneous optimization of conversion efficiency and amplified spectral bandwidth in near-1ps-pumped OPCPA systems are explored. 


\section{I Reusing the pump}

${ }^{1}$ In order to increase the conversion efficiency of the near-1-ps-pumped OPCPAs, the residual of the pump energy from one stage can be reused in a subsequent OPCPA stage (Schultze et al., 2010a). Figure 4.1 compares three different simulated OPCPA designs.

In this calculation, a Gaussian structure for the pump in both space and time and a Gaussian structure for the seed in space is assumed. The fourthorder super-Gaussian seed spectrum is linearly chirped to a $1.6 \mathrm{ps}$ pulse duration with the spectrum spanning from $600-1100 \mathrm{~nm}$. The noncollinear type I geometry in a BBO crystal with an internal pump-signal angle of $2.7^{\circ}$ and a phase-matching angle of $24.5^{\circ}$ is used. The first design consists of a single OPCPA stage using a BBO crystal, pumped with an intensity of approximately $100 \mathrm{GW} / \mathrm{cm}^{2}$. In the second design, firstly, a $0.68 \mathrm{~mm}$-thick $\mathrm{BBO}$ crystal is pumped with $7 \mathrm{~mJ}$ and the residual pump energy is reused in a subsequent stage consisting of another $0.45 \mathrm{~mm}$-thick BBO crystal. In this design a higher pump-to-signal conversion efficiency is expected due to the possibility that the noncollinear pump-signal angle and the phase-matching angle can be readjusted and optimized between the two stages.

The third configuration is a two-stage OPCPA system similar to the second design except that the pump-beam size between the two stages is readjusted to provide about $100 \mathrm{GW} / \mathrm{cm}^{2}$ pump intensity on the second crystal in order to compensate for the reduced pump intensity after its depletion in the first stage. The amplified signal energy as a function of total crystal length for three different designs is shown in Fig. 4.1, where the third design results in a factor of 2.5 increase in the conversion efficiency of the OPCPA system.

The observed increase in efficiency for this scheme arises from the freedom to readjust and optimize the phase-matching angle, noncollinear pumpsignal angle, and spatial and temporal overlap for the second crystal. Importantly, the idler in the second amplification stage is built up from zero energy, and therefore the amplification saturation happens in a longer total crystal length. Additionally, in the third design, the higher pump intensity on the last crystal results in a more efficient energy extraction compared to the second design. In Fig. 4.1-a, the corresponding pump-beam profiles at two different points $\mathrm{A}$ and $\mathrm{B}$ on the amplification gain curve are shown. Although the pump-beam profile changes dramatically from point $A$ to $B$, the amplified signal beams preserve their quality. Therefore, the third design was chosen for experimental realization.

${ }^{1}$ This section is adapted from (Fattahi et al., 2012a). The electronic print of this paper is attached in Appendix E with the permission of OSA. 


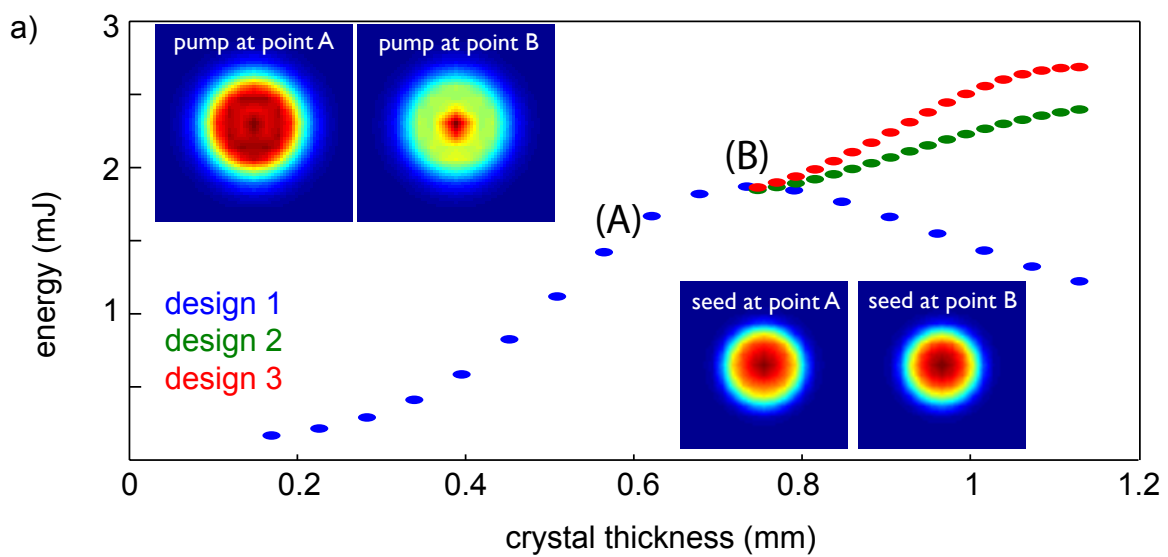

b)

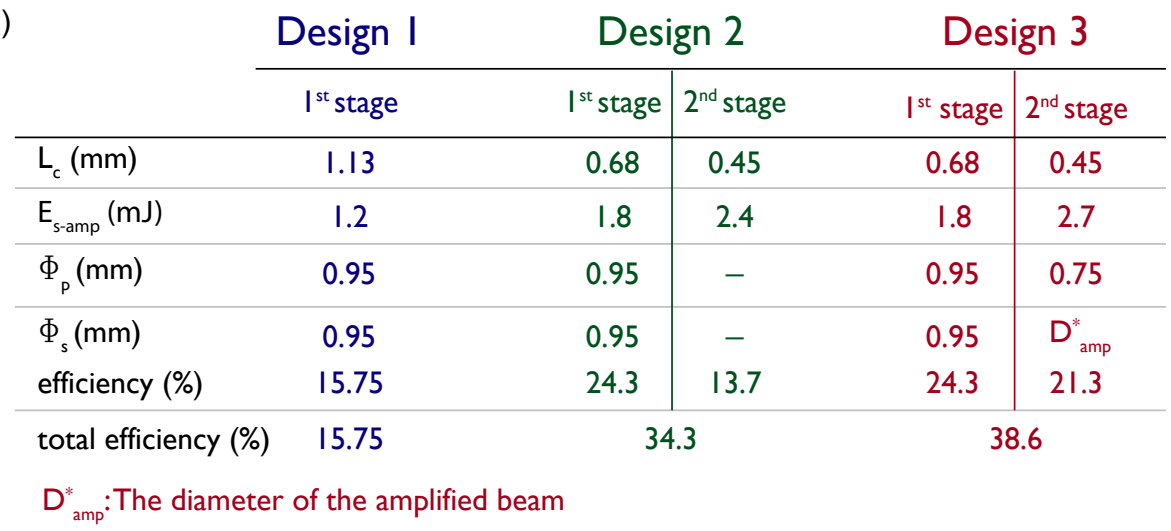

Figure 4.1: a) Simulated amplified signal energy over the crystal length for three different designs. Design 1 consists of one amplification stage. Designs 2 and 3 consist of two amplification stages, where the residual pulse energy after the first amplification stage is used to pump the second stage. In Design 3, the pump after the first amplification stage is resized to keep the pump intensity on the crystal similar to the first stage. The beam profiles of the pump after the first OPCPA stage at points (A) and (B) and their corresponding amplified-signal-beam profiles after the second OPCPA stage are shown in the inset. b) The simulated parameters of the three different designs. 
Experiment The experimental OPCPA setup consisted of five main ingredients: the pump laser (see: Chapter 3), the Ti:sapphire oscillator and amplifier, followed by the broadband nonlinear seed generation (see: Section 2.2), the timing jitter compensation system (Schwarz et al., 2012), and three OPCPA stages followed by a chirped-mirror compressor.

$52 \mathrm{~W}$ of the Yb:YAG regenerative amplifier output was frequency doubled in a $1 \mathrm{~mm}$ type I, BBO crystal with a conversion efficiency as high as $57 \%$ (Fig. 4.2). The choice of the material and the length of the SHG crystal was based on minimizing the total B-integral in the whole OPCPA chain while maintaining a good pump-to-second-harmonic conversion efficiency.

A $4 \mathrm{~mm}$-thick $\mathrm{SF}_{57}$ plate was used to temporally stretch the $10 \mu \mathrm{J}$ broadband OPCPA seed in order to provide good temporal overlap between the pump and signal pulses. The broadband seed was sent through the entire beam path in the absence of amplification consisting in total of $5.5 \mathrm{~mm} \mathrm{BBO}$ from the OPCPA stages, $4 \mathrm{~mm}$ of $\mathrm{SF}_{57}$ glass and air so that the phase could be characterized by a multi-shot, cross-correlation frequency-resolved optical gating (XFROG) device incorporating a $20 \mu \mathrm{m}$ BBO crystal cut at $29^{\circ}$ as the nonlinear medium. A fraction of the Ti:sapphire amplifier output provided the reference pulse (see: Section 2.2). The pulse duration of the seed (assuming a Gaussian fit for the retrieved time structure) is $1.1 \mathrm{ps}$ which ensures sufficiently good temporal matching between the seed and pump pulses in the OPCPA stages (Fig. 4.2). The XFROG measurement indicated that the spectral phase of the OPCPA seed was well-behaved and compressible.

The first OPCPA stage serves as a preamplifier for the low-energy broadband seed pulses. $1 \mathrm{~mJ}$ of the frequency doubled output of the thin-disk amplifier was used for pumping a $1.5 \mathrm{~mm}$ type I, BBO crystal and an output pulse energy of $120 \mu \mathrm{J}$ was achieved. Using a $3 \mathrm{~mm}$ BBO crystal in the first stage resulted in $800 \mathrm{~mW}$ of signal output, but this configuration resulted in a total of $400 \mathrm{~mW}$ of superfluorescence, measured after the third stage when the seed was blocked before the first amplification stage, while no further increase in the efficiency of the system was observed. Therefore the $1.5 \mathrm{~mm}$ $\mathrm{BBO}$ crystal was chosen for the first stage to minimize the superfluorescence after the third stage.

Up to $7.3 \mathrm{~mJ}$ of energy at $515 \mathrm{~nm}$ was used to pump the $2 \mathrm{~mm}$ BBO crystal at the second OPCPA stage. The remaining $5.6 \mathrm{~mJ}$ pump energy after the second stage preserved its good beam quality (Fig. 4.3), (except for the large ellipticity caused by the grating pair compressor of the amplifier, see Chapter 3). Subsequently, the size of the remaining pump beam was reduced to provide $80 \mathrm{GW} / \mathrm{cm}^{2}$ pump intensity on a $2 \mathrm{~mm} \mathrm{BBO}$ crystal in the third amplification stage. In the last two OPCPA stages an optical-to-optical 
a)

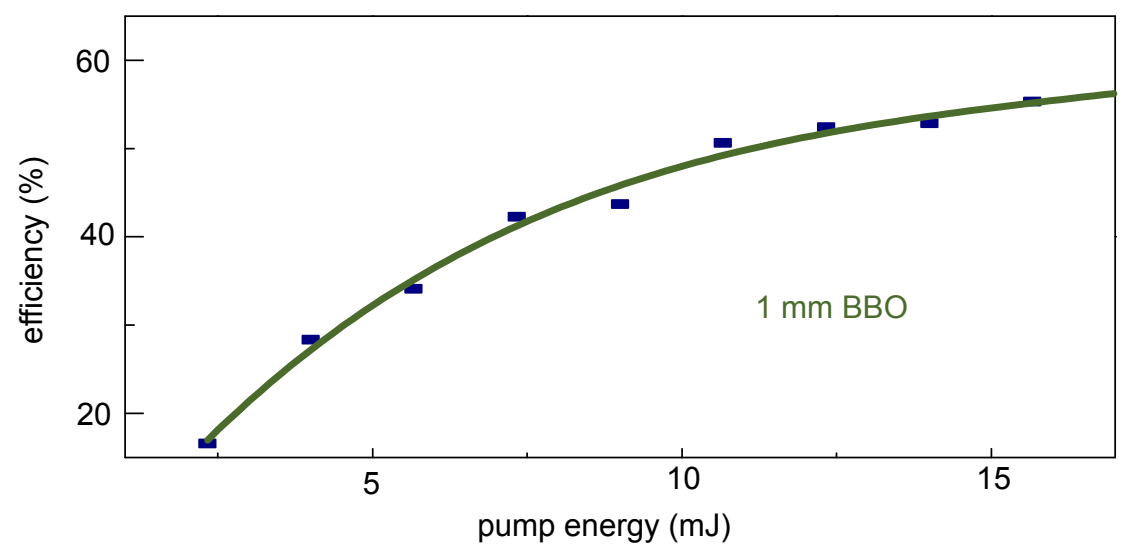

b)
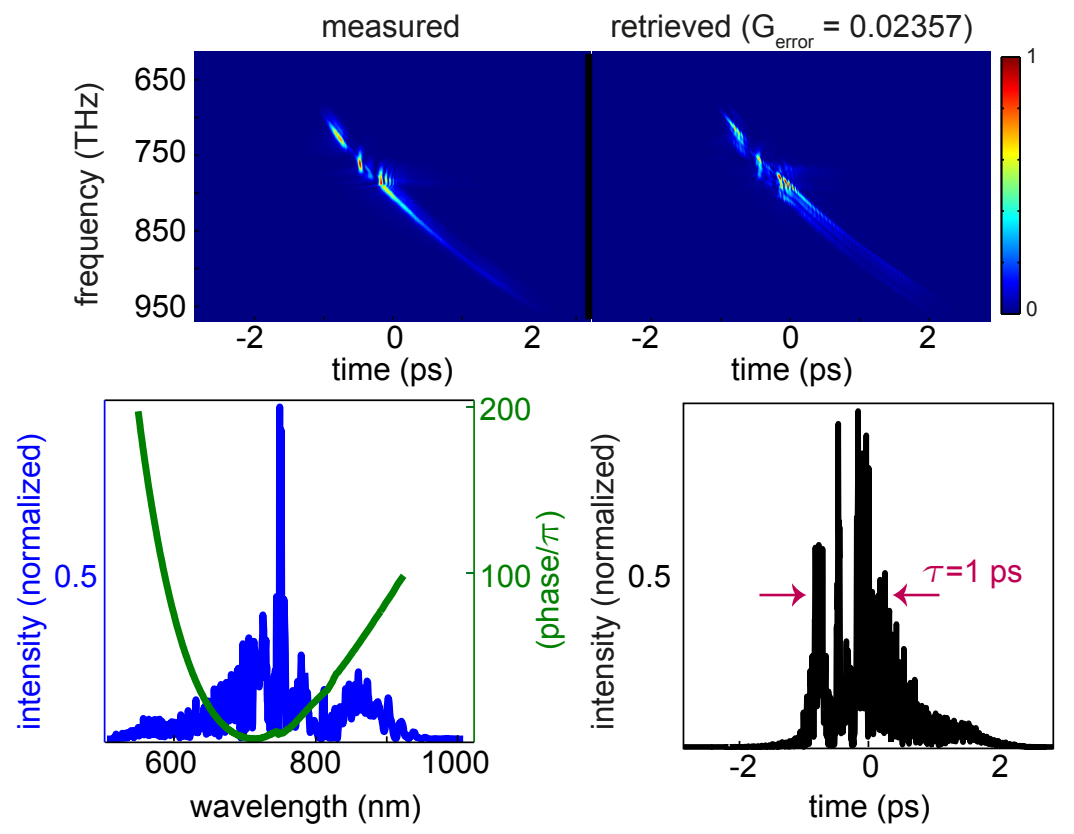

Figure 4.2: a) The efficiency curve of SHG in a $1 \mathrm{~mm}$ BBO crystal. b) The XFROG measurement of the OPCPA signal passing through the entire beam path, consisting altogether $5.5 \mathrm{~mm} \mathrm{BBO}$ and a $4 \mathrm{~mm} \mathrm{SF}_{57} . \mathrm{SF}_{57}$ is used to temporally stretch the signal to ensure a proper temporal overlap between signal and pump pulses. 


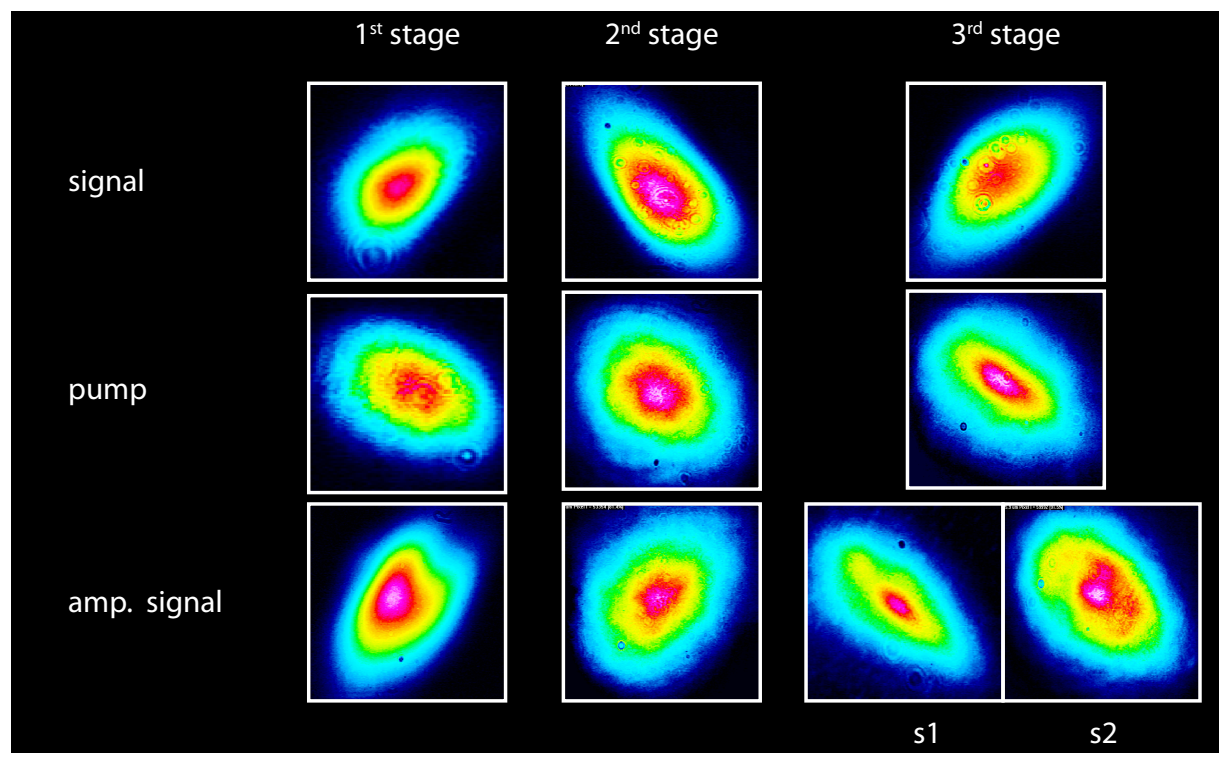

Figure 4.3: Beam profiles of the signal and pump in the OPCPA stages. The ellipticity of the signal beam is caused by the telescope. S1 shows the beam profile of the amplified signal when second stage of the OPCPA runs slightly below saturation. s2 shows the beam profile of the amplified signal when the second stage of the OPCPA runs in saturation.

conversion efficiency of $32 \%$ was achieved (Fig. 4.4). No measurable superfluorescence background was observed when blocking the signal beam in front of the first stage.

The $350 \mathrm{~nm}$-broad-amplified signal supports a Fourier transform limit pulse duration of $5.7 \mathrm{fs}$ as shown in Fig. 4.5. Preliminary compression using a double-angle chirped-mirror compressor similar to (Fattahi et al., 2012b) resulted in a pulse duration of $10 \mathrm{fs}$. The FROG trace and its retrieved counterpart are shown in Fig. 4.5. It can be clearly seen from the retrieved phase and the presence of satellite pulses that the compression was not optimal. Higher-order dispersion and satellite pulses can be compensated by using specially designed chirped mirrors for this system. Also the retrieved spectrum shows amplification of spectral components above $1 \mu \mathrm{m}$, which were not measurable with the available Si-based spectrometer. 

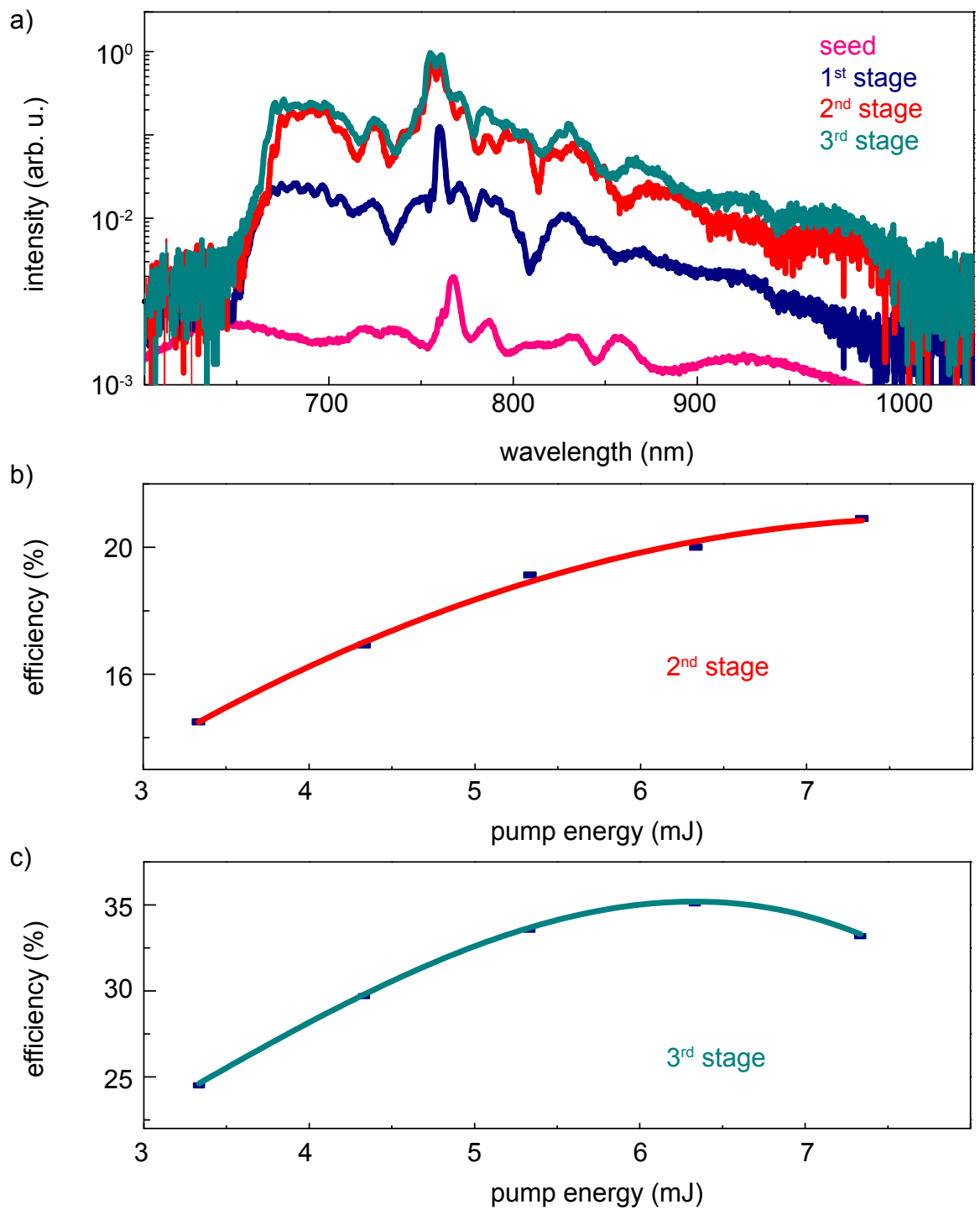

d)

\begin{tabular}{ccccc} 
& $\mathrm{L}_{\mathrm{c}}(\mathrm{mm})$ & $\mathrm{E}_{\text {pump }}(\mathrm{mJ})$ & $\mathrm{E}_{\text {signal }}(\mathrm{mJ})$ & efficiency \\
\hline $1^{\text {st }}$ stage & 1.5 & 1 & 0.12 & $12 \%$ \\
\hline $2^{\text {nd }}$ stage & 2 & 7.3 & 1.7 & $20.5 \%$ \\
\hline $3^{\text {rd }}$ stage & 2 & - & 2.5 & $30.1 \%$
\end{tabular}

Figure 4.4: a) The seed spectrum and the amplified spectra for three OPCPA stages, normalized to the output energy of each stage. b) OPCPA efficiency curve at the second stage. c) OPCPA efficiency curve at the third stage. To calculate the efficiency in (b) and (c) the net amplified signal energy is divided to the pump energy at the corresponding OPCPA stage. d) The detailed parameters of each OPCPA stage. To calculate the efficiency of each stage, the net amplified signal energy is divided by the total pump energy of the OPCPA chain. 
a)

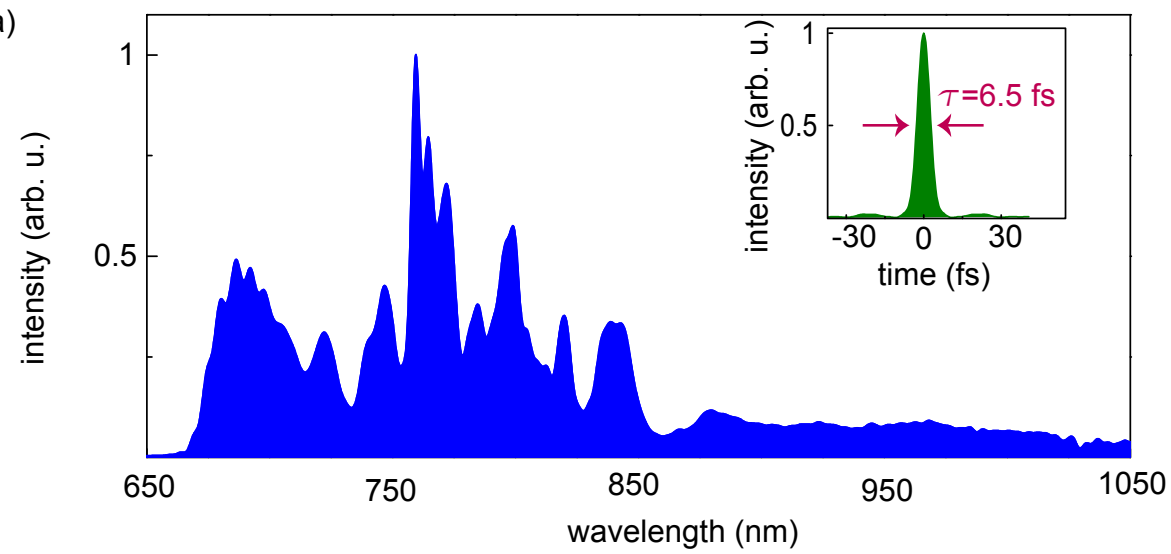

b)
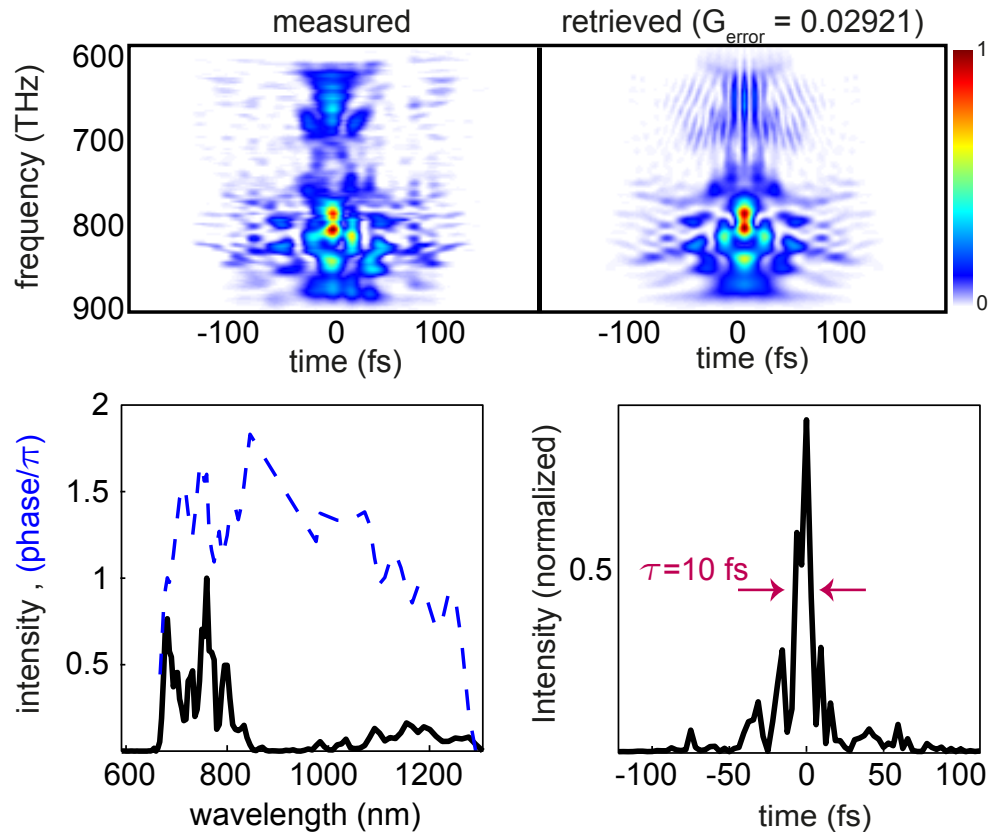

Figure 4.5: a) Amplified spectrum after 3 OPCPA stages on a linear scale, measured with a Si-based spectrometer. Inset: The Fourier transform limit waveform corresponding to the measured spectrum. c) SH-FROG measurement of the compressed pulse utilizing 12 reflections in a double angle chirped-mirror compressor. The retrieved spectrum shows the amplification of spectral components above $1 \mu \mathrm{m}$, not detectable with the available spectrometer. The pulse is compressed to $10 \mathrm{fs}$. 


\section{0}

Conclusion

By careful optimization of the pump intensity on the crystal, temporal stretching ratio between pump and seed, and spatial overlap between the pump and seed via large beam diameters, and by reusing the residual pump energy a high pump to signal conversion efficiency is achieved.

The calculated increase in efficiency by reusing the pump is in good agreement with the experimental results. Quantitative comparison shows, however, that the simulated conversion efficiency is higher than that observed in the experiment. The reason for this overestimation could be explained by the ellipticity of the pump and signal beam profile, which limited the interaction area during the amplification process.

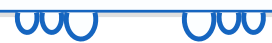




\subsection{Temporal allocation of pump intensity remainder (TAPIR)}

2 In contrast to conventional OPCPA systems, where the seed-pulse duration is a fraction of pump-pulse duration, OPCPA seed pulses can be linearly stretched to be several times longer than the pump-pulse. Once this is done, a different temporal delay between pump and signal pulses in each stage results in temporal and therefore spectral stacking of the signal in the amplification process. In this context, "stacking" refers to the controlled deposition of pump energy in subsequent temporal/spectral locations along the chirped seed pulse (Deng and Krausz, 2013).

This technique enables shaping of the amplified spectrum to overcome the phase-matching deficiencies in broadband OPCPAs, as the phase-matching angle of the crystal can be tuned to the central wavelength of the selected portion of the signal. By reusing the pump after each amplification stage, the total conversion efficiency can be increased. Furthermore, by controlling the amplification gain in each stage, the spatio-temporal profile of the pump pulses can be shaped to a flat-top pulse. On the other hand, this approach demands a complex stretcher and compressor, the B-integral of the reused pump in subsequent OPCPA stages is higher, and most importantly, for the case of unsaturated OPCPA gain, different spectral parts of the amplified signal have varying energy stability which results in spectral beating.

Figure 4.6 shows simulation results for three different OPCPA designs. In the first design, $7 \mathrm{~mJ}$ of pulse energy, centered at $515 \mathrm{~nm}$, with a duration of $1.6 \mathrm{ps}$ at FWHM and beam diameter of $1 \mathrm{~mm}$ at FWHM is used to amplify a fourth-order super-Gaussian broadband spectrum centered at $850 \mathrm{~nm}$, with a pulse duration of $1.6 \mathrm{ps}$ and input energy of $120 \mu \mathrm{J}$. Here, for both the pump and signal, the same pulse duration is considered and a $0.68 \mathrm{~mm} \mathrm{BBO}$ crystal with a phase-matching angle of $24.7^{\circ}$, noncollinear angle of $2.7^{\circ}$ and $d_{e f f}$ value of $2.3168 \mathrm{pm} / \mathrm{V}$ is used as the nonlinear medium. After the first amplification stage, the residual pump energy is used to pump the second stage consisting a $0.45 \mathrm{~mm}$ BBO crystal, while the pump beam diameter is resized and reduced to $78 \%$ of its original diameter to keep the pump intensity at $100 \mathrm{GW} / \mathrm{cm}^{2}$. Finally, the pump after the second stage is used to pump the $0.6 \mathrm{~mm}$ BBO crystal of the third stage while the beam diameter of both the pump and the signal are resized by a factor of 0.78 .

Figure 4.7-a shows the spatio-temporal profile of the pump before each amplification stage. Due to the Gaussian shape of the pump in time and space, the energy extraction took place primarily in the middle of the pump, leaving the wings mostly unaffected with a signature of energy backconver-

\footnotetext{
${ }^{2}$ This technique is based on (Deng and Krausz, 2013)
} 

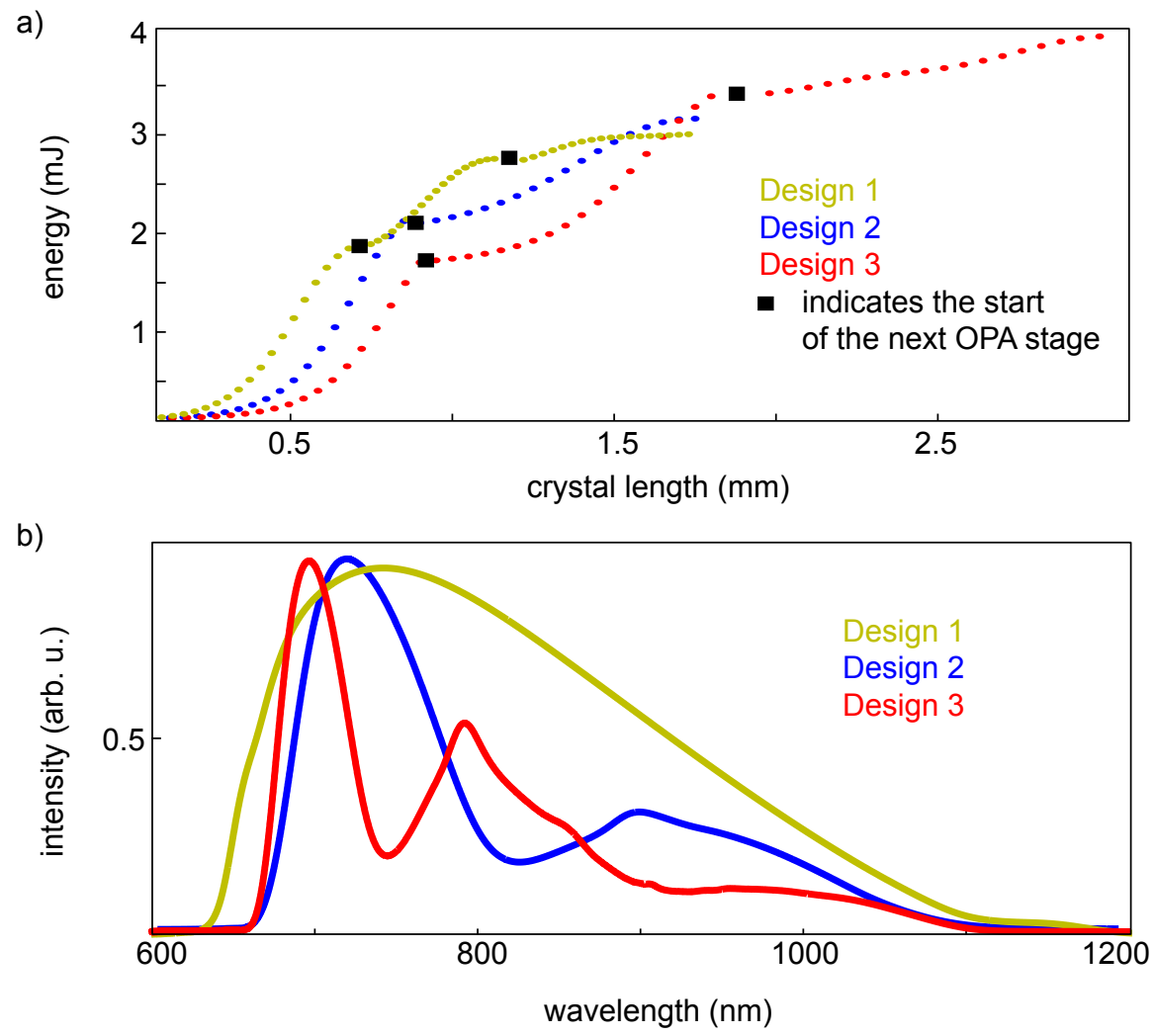

c)

\begin{tabular}{lc|c|cc|cc|c|c} 
& \multicolumn{3}{c}{ Design | } & \multicolumn{2}{c}{ Design 2 } & \multicolumn{2}{c}{ Design 3 } \\
\cline { 2 - 9 } & $\left.\right|^{\text {st }}$ & $2^{\text {nd }}$ & $3^{\text {rd }}$ & $1^{\text {st }}$ & $2^{\text {nd }}$ & $1^{\text {st }}$ & $2^{\text {nd }}$ & $3^{\text {rd }}$ \\
\hline efficiency (\%) & 24.6 & 22.4 & 11.8 & 28.5 & 26.8 & 22.6 & 35.2 & 30 \\
\hline total efficiency (\%) & 43 & \multicolumn{3}{c}{45} & \multicolumn{3}{c}{57}
\end{tabular}

Figure 4.6: a) The amplified pulse energy versus crystal length, b) the amplified spectrum, c) the conversion efficiency for three different OPCPA designs. In Design 1 the signal pulses are stretched to the same pulse duration as the pump pulses and amplified in 3 cascaded OPCPA stages, while the residual pump energy after each stage is used to pump the subsequent stage. In Design 2 the seed pulses are stretched to nearly twice the pumppulse duration and amplified in two cascaded OPCPA stages and in Design 3 , the signal-pulse duration is increased to more than three times the pumppulse duration. In Designs 2 and 3 a different part of the signal spectrum is amplified in each OPCPA stage. 


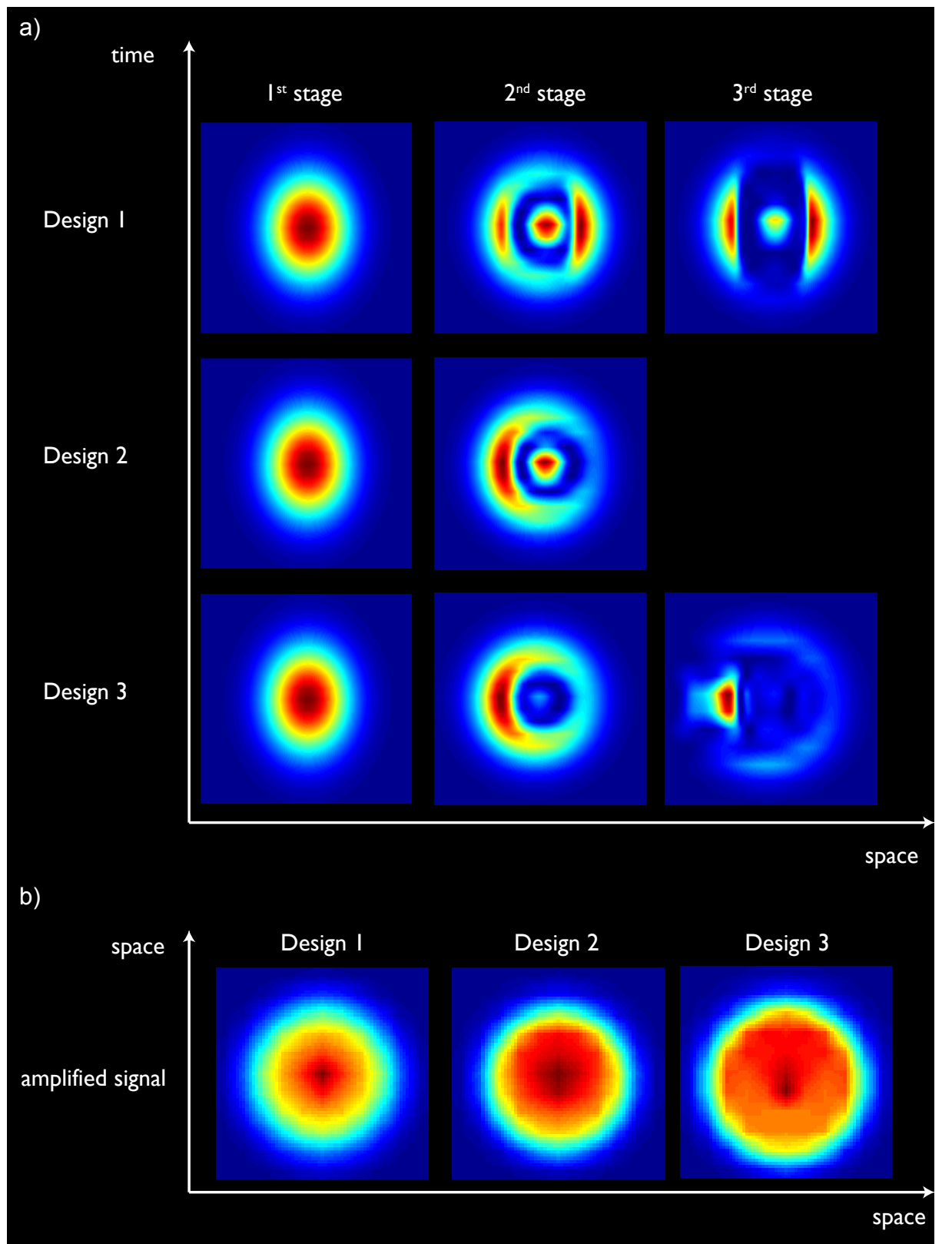

Figure 4.7: a) Spatio-temporal profile of the pump pulses for different OPCPA designs before each OPCPA amplification stage. b) Beam profile of the amplified signal in each OPCPA design. 
sion in the center, owing to the fact that this part of the pump possesses the highest peak intensity. In this design, the OPCPA pump-to-signal conversion efficiency is increased to $43 \%$ with a good spatio-temporal profile for the amplified signal (Fig. 4-7-b). In the second design, the signal pulses were stretched linearly to 4 ps at FWHM and the blue part of the spectrum is amplified in a $0.85 \mathrm{~mm}$ BBO crystal by adjusting the temporal overlap between pump and signal pulses. Subsequently, the pump pulses after the first stage are reused to amplify the red part of the spectrum in a $1 \mathrm{~mm} \mathrm{BBO}$ crystal at the second stage, while the pump beam size is reduced by a factor of 0.78 . In this design the pump-to-signal conversion efficiency reaches $45 \%$ and maintains the beam quality of the amplified signal (Fig. 4.7-b).

The gain and the shape of the amplified spectrum can be further optimized by adjusting the phase-matching angles of the crystal in each stage to tune the amplification for the selected part of the spectrum (this option is not investigated in this study). The spatio-temporal profile of the pump before each OPCPA stage, shown in Fig. 4.7-a, indicates better pump-energyextraction but becomes asymmetric. The asymmetry is caused by the temporal walk-off between signal and pump pulses and can be suppressed by careful adjustment of the initial temporal overlap of the interacting beams. The $45 \%$ conversion efficiency achievable from Design 2, is not drastically different from the achievable $38.6 \%$ efficiency for the case where the signal and pump pulses have the same pulse duration (studied in Section 4.1) as the pump pulses after the first amplification stage in both cases maintain good spatio-temporal profiles.

As shown in Design 1, reusing the pump pulses for a third time results in a comparable efficiency. However, this changes for heavily stretched signal pulses. In design 3, the bluest part of the spectrum of the input signal pulses, which are linearly stretched to 9 ps pulse duration at FWHM, is amplified in a $0.9 \mathrm{~mm}$ BBO crystal. The residual pump diameter is reduced by a factor of 0.78 and used to amplify the middle part of the input spectrum in a $1 \mathrm{~mm}$ BBO crystal and finally, the red wing of the spectrum is amplified in a $1.2 \mathrm{~mm}$ BBO crystal while the input pump beam is shrunk by a factor of 0.68 before the last OPCPA stage. Here the pump-to-signal conversion efficiency reaches $57 \%$, which is a noticably higher value compared to the other designs. The spectral narrowing for Designs 2 and 3 visible in Fig. 4.6$\mathrm{b}$, is caused by a suboptimal stretching factor of the input signal and phasematching angle of the crystal. Optimizing these parameters are cumbersome in simulation but straightforward in an experimental setup.

Experiment An $8 \mathrm{~mm}$-thick $\mathrm{SF}_{57}$ plate was used for stretching the amplified signal pulses after the first OPCPA stage, as described in Section 4.1. 
The glass was used at the Brewster's angle to minimize reflections from the surface of the glass. The delay between pump and signal, and the phasematching angles of the BBO crystal at the second stage were adjusted to shift the amplification to the blue wing of the input spectrum and $4 \mathrm{~W}$ of power was obtained (blue curve in Fig. 4.8-a). In the third OPCPA stage, the phase-matching and temporal overlap between pump and signal pulses were adjusted, to shift the gain to the red wing of the input spectrum with a total amplification power of $6 \mathrm{~W}$ (Fig. 4.8-a, pink curve).

The same setup, after removing the bulk-stretcher, resulted in $6.2 \mathrm{~W}$ amplification after the third stage. Figure 4.8 -b compares the amplified spectra for these two cases. The amplified spectrum of the stacked-OPCPA is smoother and has a square shape as the stretching of the signal spectrum provides comparable amplification gain for different frequencies. The red wing of the spectrum gains more energy in this case, as the phasemismatching could be minimized (see Fig. 1.5). Also the spike around $780 \mathrm{~nm}$ is heavily suppressed, as further stretching of the signal reduces the temporal overlap between different frequencies. Further stretching of the signal in a $12 \mathrm{~mm}$-thick $\mathrm{SF}_{57}$ resulted in a reduced amplified power of $2.75 \mathrm{~W}$ in the second stage, $5 \mathrm{~W}$ in the third stage, and produced a hole in the amplified spectrum at $790 \mathrm{~nm}$ (Fig. 4.8-c), leaving the temporal stretching of the seed using 8-mm-thick SF57, the optimum.

\section{Conclusion}

Temporal stretching of the signal pulses to twice the pump-pulse duration, demonstrated in this section experimentally, did not show any increase in the OPCPA conversion efficiency compared to the scheme realized in Section 4.1. The simulated conversion efficiency for these two schemes is in the same range due to the fact that the residual pump energy after only one amplification stage (without any extra temporal stretching of the signal pulses) preserves good spatiotemporal quality (Fig. 4.7).

As the difference between simulated amplified energy in these two cases is only on the order of $1 \mathrm{~W}$ of average power, their experimental evaluation demands precise control over the signal-pulse duration. However, stretching the duration of the signal pulse to three times (or more) the pump-pulse duration results in a significant increase in conversion efficiency (Fig. 4.6). Here, the advantage of this technique becomes distinguishable, as the residual pump pulses after the second 
amplification stage possess a better temporal and spatial quality for the heavily chirped signal pulses in comparison to the case discussed in Section 4.1. The experimental demonstration of the latter case could be an interesting study for the next generation of PhD students!

ou vov 

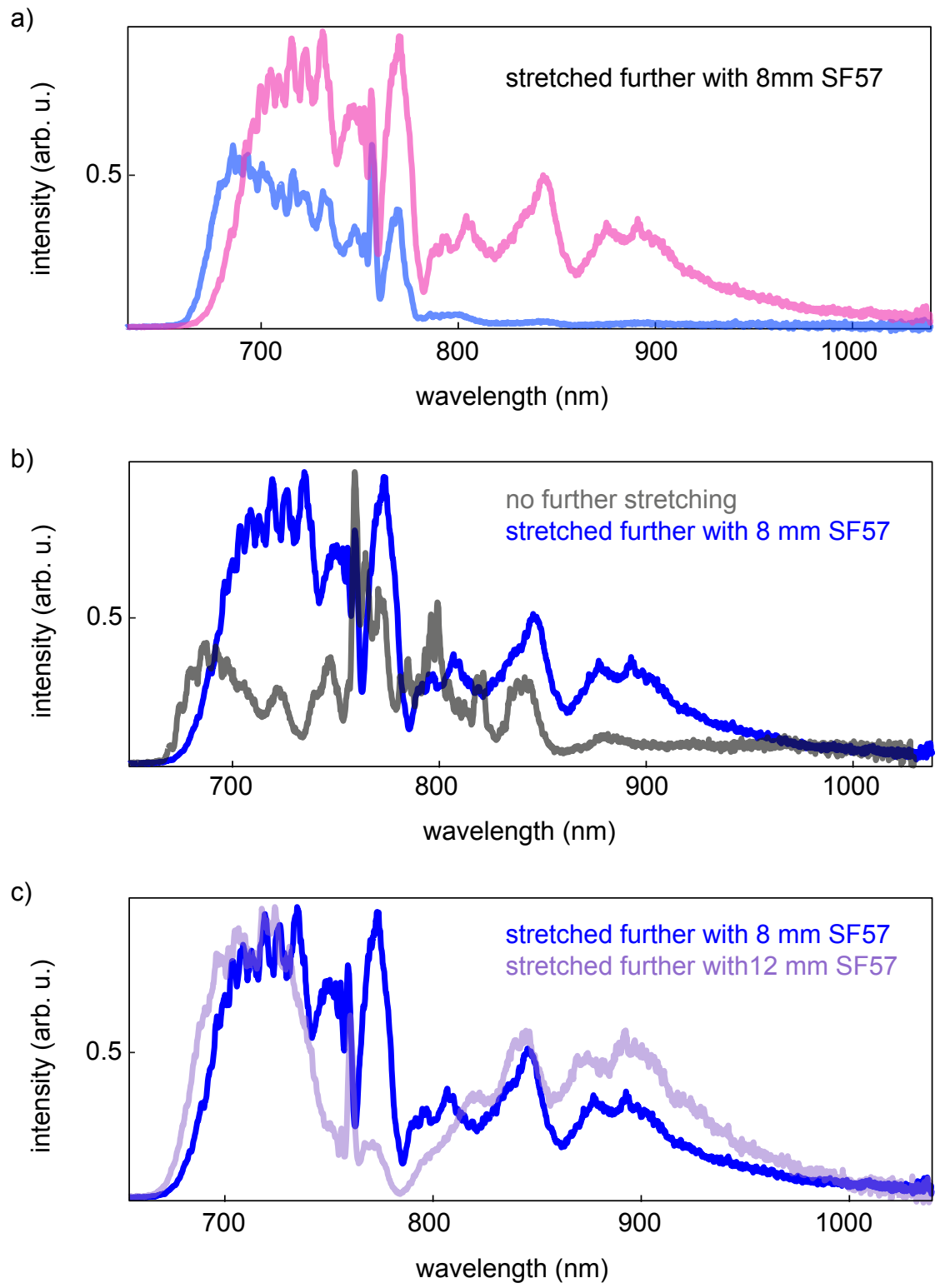

Figure 4.8: a) The stacked spectrum in a two-stage OPCPA system, temporally stretched by $8 \mathrm{~mm}$ of $\mathrm{SF}_{57}$. The high frequencies were amplified in the second stage (blue curve) and the low frequencies were amplified in the third OPCPA stage (pink curve), recycling the pump energy after the second stage. The amplified signal energy in this case is comparable to the amplified energy obtained from the OPCPA system without additional stretching. $b$ ) Comparison between the amplified spectra in a stacked OPCPA (blue curve) and with no additional stretching and reusing the pump (gray curve). c) The amplified spectrum of a pulse stretched using $8 \mathrm{~mm}$ (blue curve) and $12 \mathrm{~mm}$ $\mathrm{SF}_{57}$ (purple curve) and stacked in two-cascaded OPCPA stages. 


\subsection{Bandwidth engineering}

To optimize the amplification bandwidth in an OPCPA system, different crystals or a crystal with different phase-matching angles can be utilized. Figure 4.9-a compares the simulated amplified spectrum from a $2.75 \mathrm{~mm}$ thick LBO crystal at a phase-matching angle of $14.4^{\circ}$ and the noncollinear angle of $1.1^{\circ}$ with an amplified spectrum from a $1 \mathrm{~mm}$-thick BBO crystal with a phase-matching angle of $23.3^{\circ}$ and a noncollinear angle of $0^{\circ}$, and a $1 \mathrm{~mm}$-thick BBO crystal with a phase-matching angle of $24.5^{\circ}$ and a noncollinear angle of $2.7^{\circ}$.

In this study, the pulse duration of the signal and pump pulses are both $1.6 \mathrm{ps}$ at FWHM. The $3 \mathrm{~mJ}$ pulses with a beam diameter of $1.2 \mathrm{~mm}$ at FWHM are used as a pump and the input seed energy is $3 \mu \mathrm{J}$. The same temporal stretching is used for the three cases. Combining the two phase-matching angles in a BBO crystal results in broader amplification compared to LBO. Figure 4.9-b studies the amplification gain in a cascaded OPCPA stage using these two different phase-matching angles. Firstly, the red wing of the spectrum is amplified in the first stage at a noncollinear angle of $0^{\circ}$, and the blue wing of the spectrum in the second stage where the noncollinear angle is set to $2.7^{\circ}$ and pumped with $3 \mathrm{~mJ}$ of energy.

The amplification behavior in the second stage is very interesting. Initially, the spectral components that were amplified in the first stage and are phase-matched in the second stage, namely $800 \mathrm{~nm}$ to $1100 \mathrm{~nm}$, are amplified until they reach saturation. Further amplification of these spectral components is due to the reset of the idler in the second stage. In broadband OPCPA, energy saturation of each frequency component rarely happens at the same crystal position. Later, energy backconversion takes place and at a crystal thickness of $0.6 \mathrm{~mm}$, the amplification is shifted to the blue wing of the input spectrum (Fig. 4.9-b and c). Fig. 4.10 compares the amplified spectra in the two-stage OPCPA using LBO crystal or using BBO crystals at different phase-matching angles. The amplified energy in both cases is similar. $1.1 \mathrm{~mJ}$ in the LBO crystals and $1 \mathrm{~mJ}$ in the BBO crystals for a total pump energy of $6 \mathrm{~mJ}$. The amplified spectrum for the latter case is broader, which reduces the Fourier transform limit of the amplified signal from $5.3 \mathrm{fs}$ to $4.4 \mathrm{fs}$.

Experiment The broadband, octave-spanning spectrum described in Section 2.2 is amplified in an OPCPA system similar to the system described in Section 4.1. A $2 \mathrm{~mm} \mathrm{LBO}, 2 \mathrm{~mm} \mathrm{BBO}$, and $3 \mathrm{~mm}$ LBO crystal was used in each amplification stage alternatively. The first stage was optimized to amplify a broad spectral range spanning from $750 \mathrm{~nm}$ to $1400 \mathrm{~nm}$ up to $50 \mu \mathrm{J}$ of energy. In the second stage, the measured amplified spectrum using a Si- 
a)
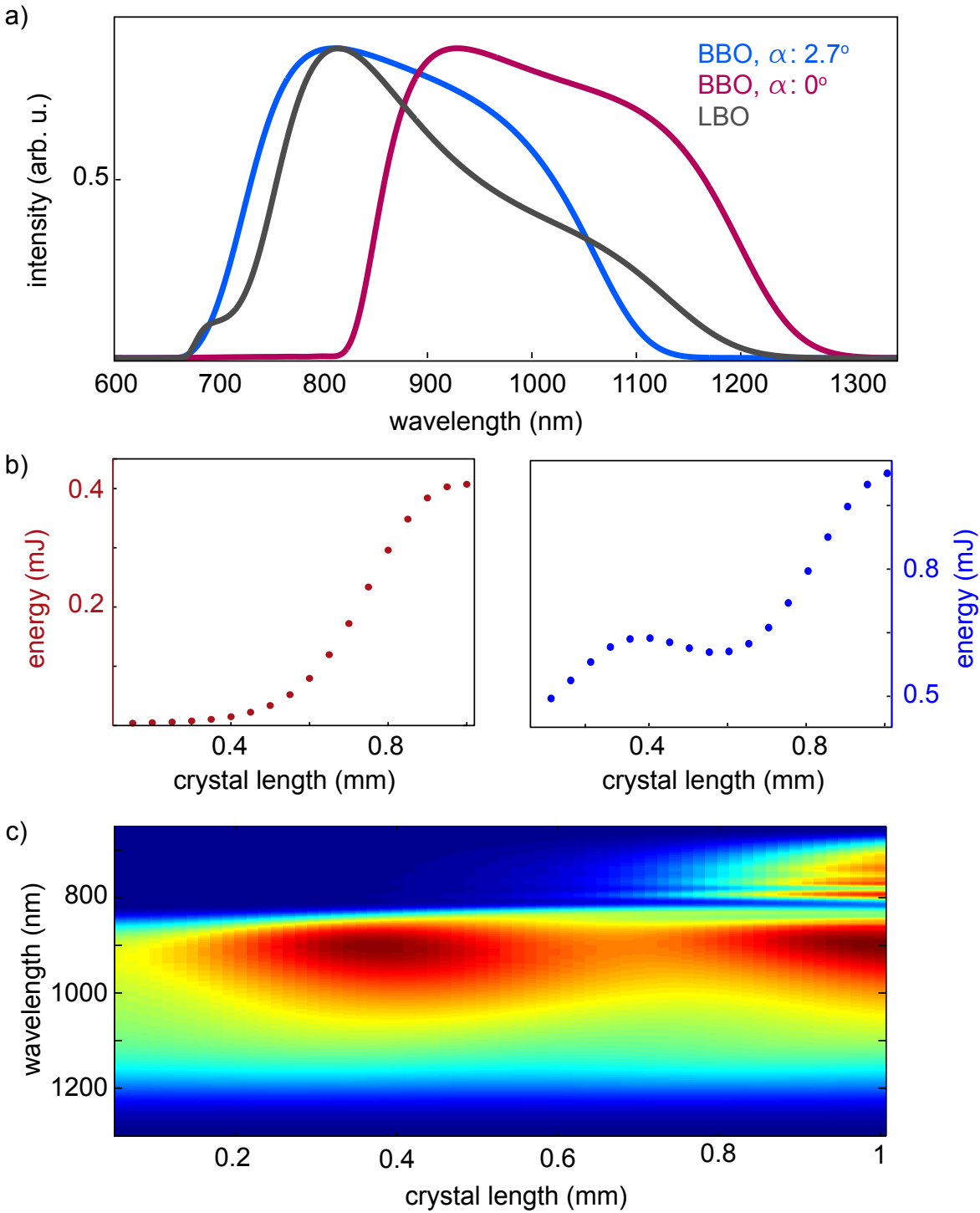

Figure 4.9: a) Simulated amplified spectra in different crystals and different phase-matching and noncollinear angles. b) The OPCPA gain in a 2-stage OPCPA, using two BBO crystals with two different sets of phase-matching angles. Left: the amplified energy vs. crystal length in a BBO crystal with a noncollinear angle of $\alpha=0^{\circ}$ in the first stage. Right: The amplified energy vs. crystal length in a BBO crystal with a noncollinear angle of $\alpha=2.5^{\circ}$ in the second stage. c) The amplified spectrum vs. crystal length in the second stage. 


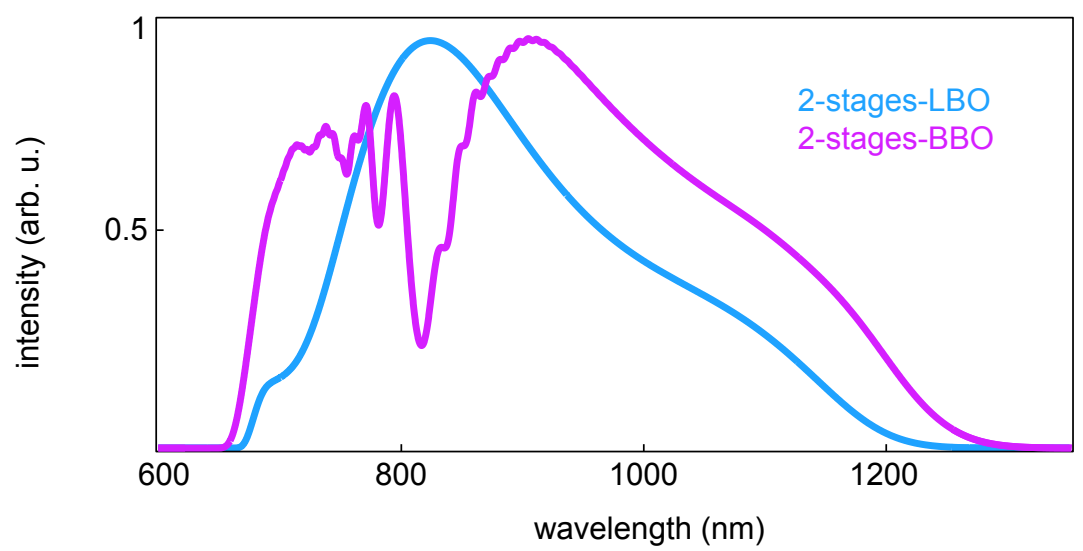

Figure 4.10: Amplified spectra in a 2-stage OPCPA using LBO crystals (blue curve) and BBO crystals with different phase-matching angles (purple curve).

based spectrometer, spans from $670 \mathrm{~nm}$ to $1100 \mathrm{~nm}$ and contained $1.1 \mathrm{~mJ}$ of energy. Finally at the third stage, the amplified energy was scaled to $1.8 \mathrm{~mJ}$ using the LBO crystal. The spectra shown in Fig. 4.11 are normalized to the energy in each amplification stage. Comparing the spectra after the second and third stages indicates energy backconversion for spectral components that reached saturation in the previous stage. The system has a lower efficiency compared to the system described in Section 4.1, as the main goal in this study was the engineering of the spectral bandwidth and the parameters of the system are not optimized in terms of the conversion efficiency.

The amplified spectrum supports a FWHM transform limited pulse duration of $4.3 \mathrm{fs}$. A set of double-angle chirped mirrors designed for spectral wavelengths of $700-1300 \mathrm{~nm}$ (Fig. 4.12-a) was used for the preliminary compression of the amplified pulses. Fig. 4.12-b shows the SH-FROG measurement of the compressed OPCPA seed pulses without any amplification, using 12 reflections on the chirped mirrors. The seed pulses were compressed down to $8 \mathrm{fs}$. The red tail of the spectrum generated via filamentation in the YAG crystal could not be characterized as it contained very little energy compared to the blue part of the spectrum generated via SPM in a HCF. The pre-pulse visible in the SH-FROG trace and also the retrieved pulse in the time domain, indicates uncompensated third-order chirp. After amplification, the signal pulses were characterized after the second and third OPCPA stages. Figure 4.13-a shows the pulse compression after the second OPCPA stage. The higher-order phase developed during the amplification can be seen in the measured FROG trace. The retrieved spectrum includes spec- 
tral components from $680-1100 \mathrm{~nm}$ as the energy in the red wing of the spectrum is much lower than the blue wing. Figure 4.13-b shows the pulse compression down to $9 \mathrm{fs}$ after the third amplification stage. The retrieved spectrum is in good agreement with the measured OPCPA spectrum shown in Fig. 4.14 (red curve). Pulse compression to the Fourier transform limit would require an additional chirped-mirror compressor for compensating the higher-order OPCPA phase and a longer beam time!

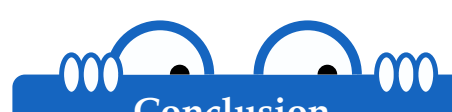

Conclusion

As shown in this section, utilization of well-selected, different nonlinear crystals extends the OPCPA-gain bandwidth substantially. The realized 3-stage OPCPA system, using one BBO and two LBO crystals, delivers $1.8 \mathrm{~mJ}$ pulses with a Fourier transform limit of $4.5 \mathrm{fs}$ and thus supports even shorter pulse-duration than a 3-stage OPCPA system based on LBO alone, which supports only $5.3 \mathrm{fs}$. The reported extension of the supported amplified spectral bandwidth is crucial for experiments which rely on high-energy, single-cycle pulses.

The presented simulation results indicate that rather than using different crystal types, an equally impressive result can be achieved using $\mathrm{BBO}$ crystals at different phase-matching angles.

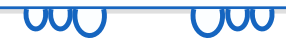




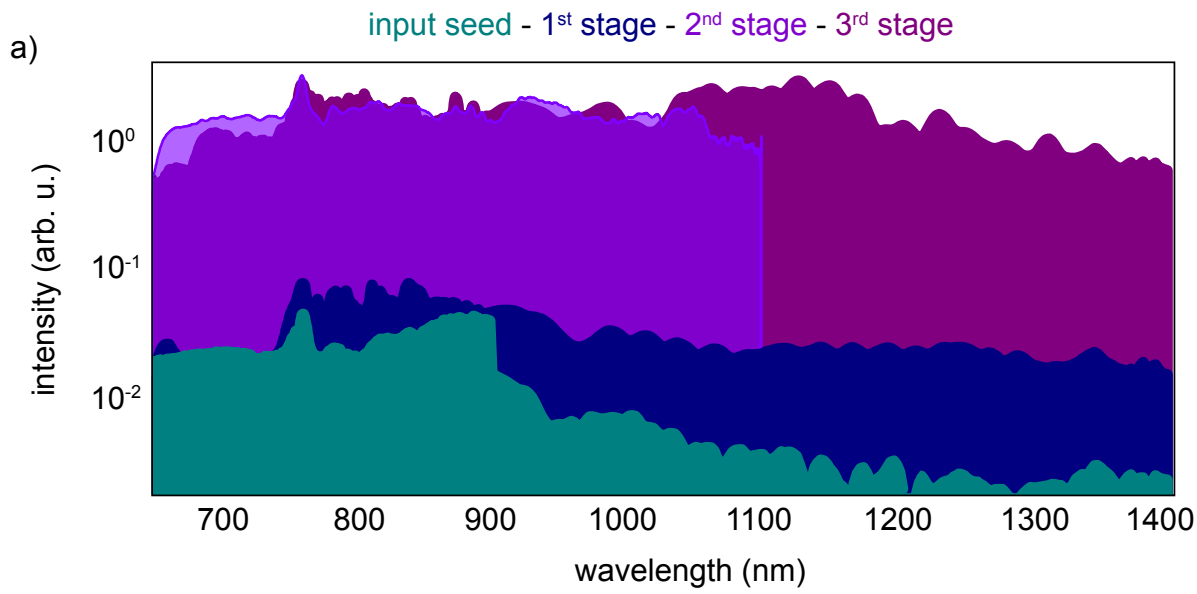

b)

\begin{tabular}{lcccc} 
& $\mathrm{L}_{\mathrm{c}}(\mathrm{mm})$ & $\mathrm{E}_{\text {pump }}(\mathrm{mJ})$ & $\mathrm{E}_{\text {signal }}(\mathrm{mJ})$ & efficiency \\
\hline $1^{\text {st }}$ stage & $2 / \mathrm{LBO}$ & 1 & 0.05 & $5 \%$ \\
\hline $2^{\text {nd }}$ stage & $2 / \mathrm{BBO}$ & 7 & 1.1 & $16 \%$ \\
\hline $3^{\text {rd }}$ stage & $3 / \mathrm{LBO}$ & - & 1.8 & $22 \%$
\end{tabular}

Figure 4.11: a) Amplified spectra in a 3-stage OPCPA. The seed pulses in the first stage were amplified to $50 \mu \mathrm{J}$ using a $2 \mathrm{~mm}$ LBO crystal. In the second stage, the spectral components below $1100 \mathrm{~nm}$ were amplified in a $2 \mathrm{~mm}$ BBO crystal up to $1.1 \mathrm{~mJ}$ of energy, and finally in the last stage, the amplified energy was boosted to $1.8 \mathrm{~mJ}$ using a $3 \mathrm{~mm}$ LBO crystal. The OPCPA seed was generated via cascaded spectral broadening in a HCF and a $4 \mathrm{~mm}$ YAG crystal. b) The parameters of the OPCPA system. 
a)

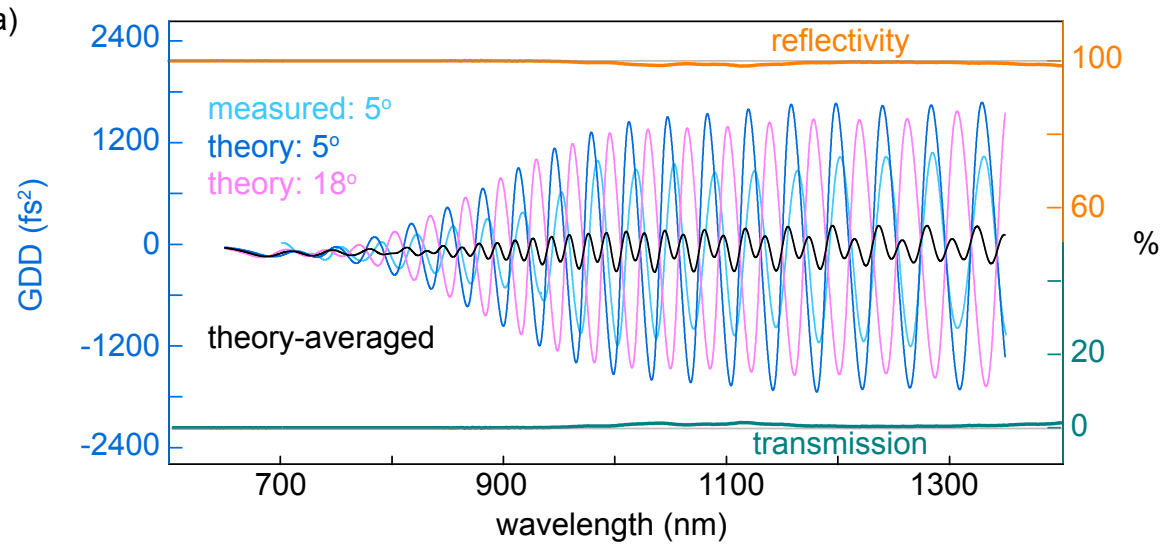

b)
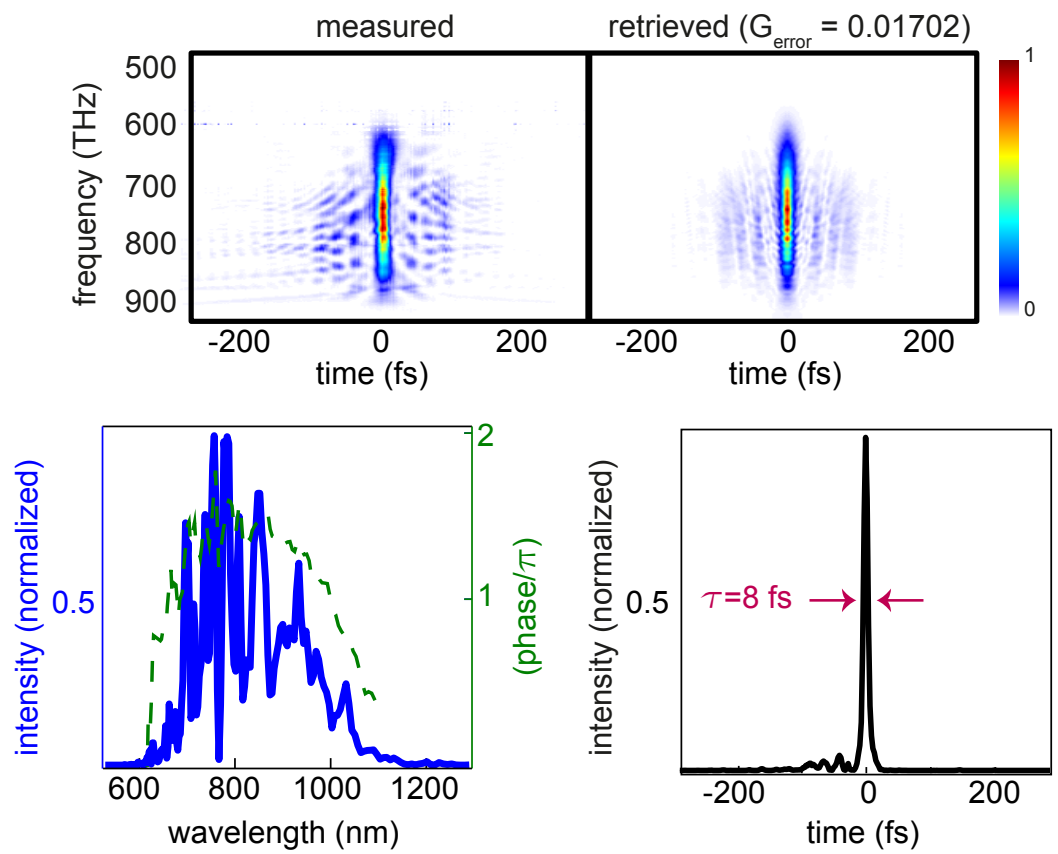

Figure 4.12: a) The GDD, reflectivity and transmission of the double angle chirped mirrors used for compression of the OPCPA system. The black curve shows the average GDD of the two complementary mirrors at two different angles of incidence. b) The SH-FROG measurement of an OPCPA seed without any amplification, using 12 reflections on the chirped-mirror compressor. 
a)
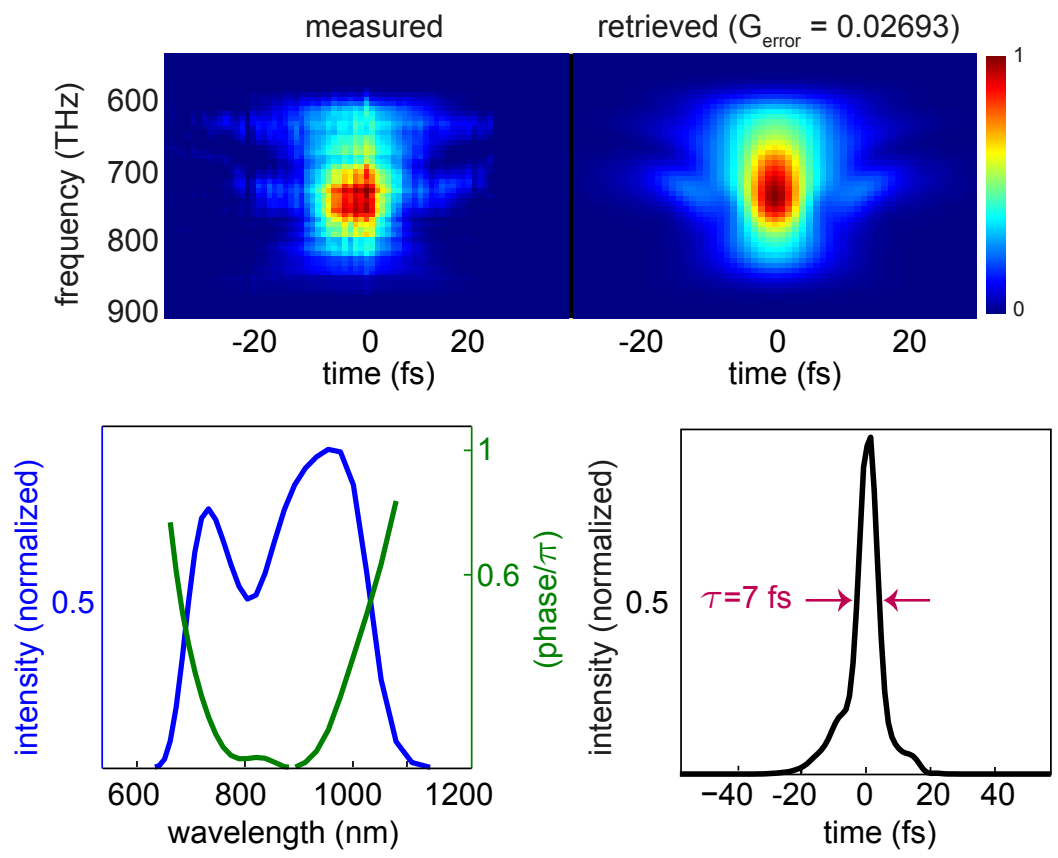

b)
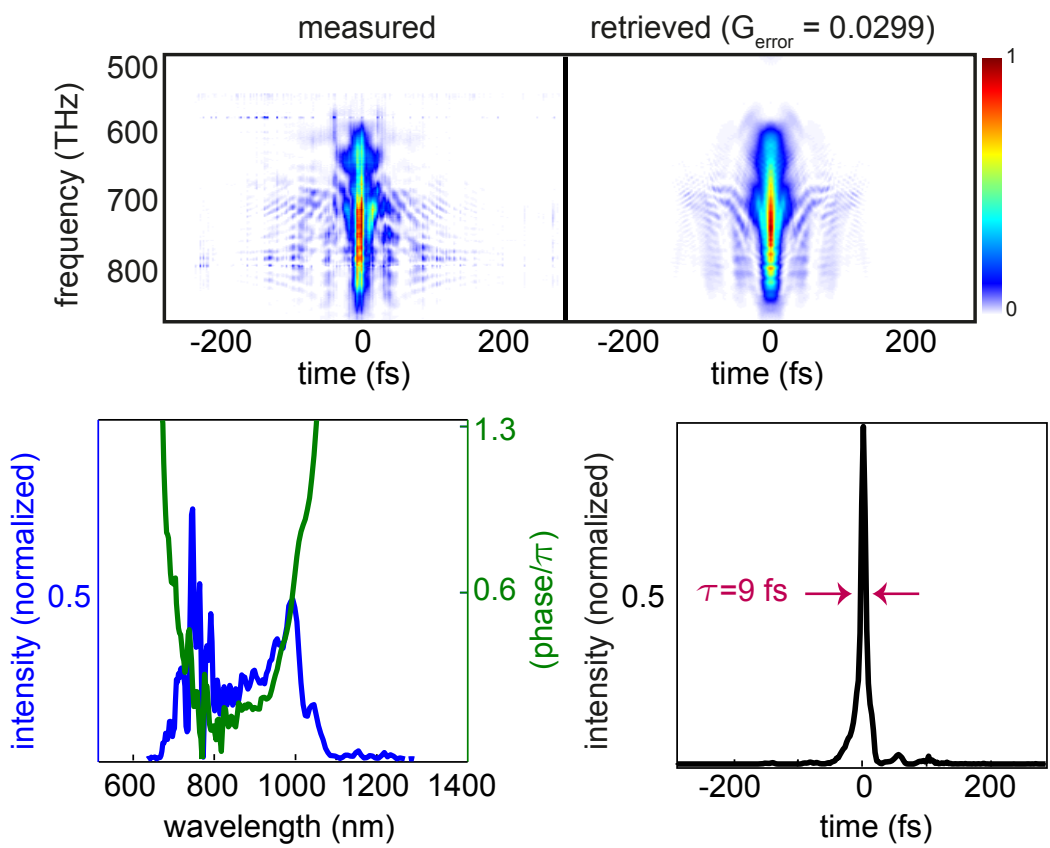

Figure 4.13: Measured and retrieved SH-FROG traces and the retrieved spectrum and temporal profile of the pulses at the second (a) and third (b) stage of the OPCPA measured at an amplified energy of $1.1 \mathrm{~mJ}$. The retrieved spectrum after the third stage is in a good agreement with the measured spectrum shown in Fig. 4.14. 
a)

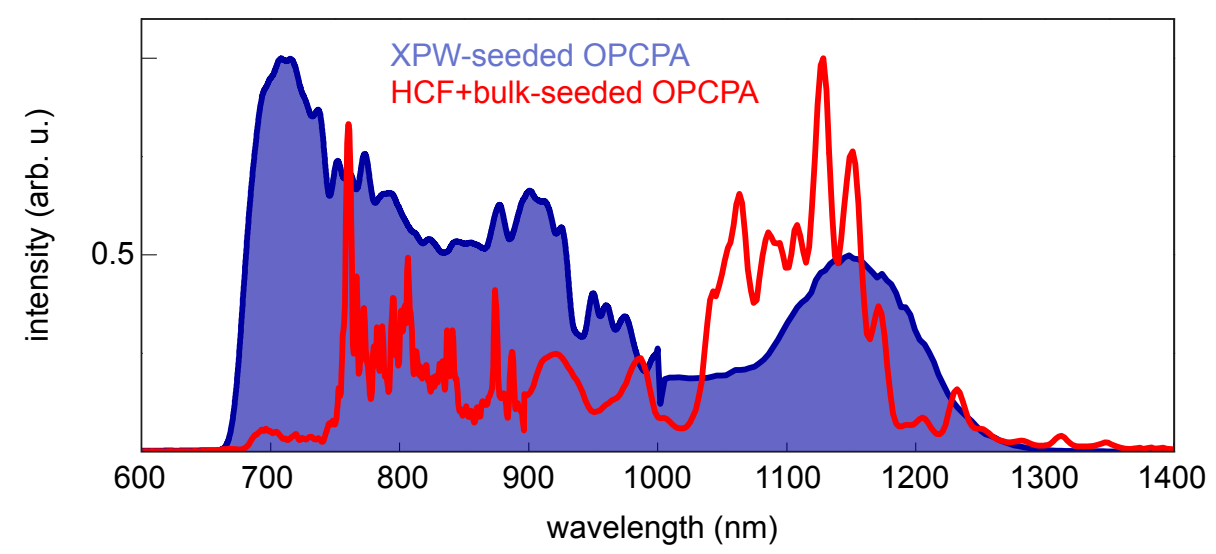

b)

\begin{tabular}{lcccc} 
& $\mathrm{L}_{\mathrm{c}}(\mathrm{mm})$ & $\mathrm{E}_{\text {pump }}(\mathrm{mJ})$ & $\mathrm{E}_{\text {signal }}(\mathrm{mJ})$ & efficiency \\
\cline { 2 - 5 } $1^{\text {st }}$ stage & 2 & 1.5 & 0.03 & $2 \%$ \\
\hline $2^{\text {nd }}$ Stage & 3 & 7 & 0.9 & $13 \%$ \\
\hline $3^{\text {rd }}$ stage & 3 & - & 1.2 & $15 \%$ \\
\hline
\end{tabular}

Figure 4.14: a) Amplified broadband signal generated in XPW process (blue area) and the amplified broadband signal generated in cascaded HCF-bulk stages (red line). b) The parameters of the 3-stage OPCPA system seeded with a broadband signal generated via XPW.

\subsection{XPW seeded OPCPA system}

The generated broadband seed in Section 2.3 is amplified in the 3-stage OPCPA system described in Section 4.1 utilizing LBO crystals in all amplification stages. Fig. 4.14 shows the amplified spectrum compared to the one described in Section 4.3. The smooth amplified spectrum does not contain sharp features, as the temporal contrast of the generated pulses in the XPW process is enhanced. Due to the lower input seed energy in this case compared to the previous OPCPA systems, the pump distribution and crystal thickness in each OPCPA stage should be optimized to achieve the highest conversion efficiency. This proof of principle study shows the potential of $\mathrm{XPW}$ as a temporal filter and a source for generating coherent photons at new frequencies, desired for seeding OPCPA systems. 


\section{Chapter 5}

\section{Design of a multi-terawatt field synthesizer (LWS-pro)}

${ }^{1}$ Near 1ps-pulse-pumped OPCPA offers several advantages over both longpulse-driven OPCPA and conventional CPA implemented in solid-state laser amplifiers. As discussed in Section 1.1, in short-pulse-pumped OPCPA crystals can be pumped at much higher intensities, allowing high gains to be realized with very thin crystals, i.e. in combination with broad amplification bandwidths. Also, the short pump window greatly simplifies the implementation of CPA and dispersion control and, finally and most importantly, improves the temporal contrast of the amplified signal dramatically on the nanosecond to few-picosecond time scale.

The energy scalability of near-1-ps pulses of Yb:YAG thin-disk regenerative amplifiers, their near-diffraction-limited beam and excellent pulse energy stability, makes them promising pump sources for future OPCPA systems.

The gain bandwidth of an Yb:YAG-pumped OPCPA system can be extended up to several octaves, by using different crystals or crystals with different orientations yielding shifted gain bands (as it was experimentally and theoretically demonstrated in Chapter 4) or utilizing multiple pump beams at different wavelengths, i.e. at $1030 \mathrm{~nm}$ and its low-order harmonics at $515 \mathrm{~nm}$, and $343 \mathrm{~nm}$ (see: Section 1.2).

In what follows, the feasibility of super-octave, high-energy, high-power OPCPA sources and their expected performance when being pumped with several-kHz, kW-class thin-disk lasers is discussed.

Driving different OPCPA channels by 1030-nm picosecond pulses from $\mathrm{Yb}$ :YAG thin-disk amplifiers and their second and third harmonics, yield

${ }^{1}$ This chapter is adapted from (Fattahi et al., 2014a). The electronic print of this paper is attached in Appendix E with the permission of OSA. 
few-cycle pulses in the MIR, NIR and VIS spectral ranges, respectively (see: 1.2). These few-cycle pulses may also be superimposed on each other for the synthesis of multi-octave light transients. These options call for a seed coming in the form of a coherent, phase-stable, multi-octave supercontinuum covering the entire wavelength range of interest.

Prototypical OPCPA architecture The common backbone for the proposed and numerically analyzed prototypical architecture in this chapter consists of (i) a high-power femtosecond laser, in our case a 10o-W-scale Kerr lens mode-locked (KLM) Yb:YAG thin-disk oscillator, followed by (ii) the multioctave seed generation, and (iii) a multi-10o-W-to-kW-scale source of multimJ, near-1-ps-pulses, in our case based on Yb:YAG thin-disk amplifiers (Fig. 5.1).

Using chirped dichroic beam splitters, the supercontinuum seed is split into three spectral channels:VIS centered at $550 \mathrm{~nm}$, NIR centered at $1 \mu \mathrm{m}$, and MIR centered at $2 \mu \mathrm{m}$. Each of the three OPCPA channels can be used to obtain few-cycle pulses. Their pump pulses are generated by a simple frequency-converter module comprising two LBO crystals. SHG in the first one yields twin pulses of comparable energy at $515 \mathrm{~nm}$ and $1030 \mathrm{~nm}$, which are mixed in the second crystal to produce a third pump pulse at $343 \mathrm{~nm}$ by sum frequency generation (SFG). Moderate SHG and SFG conversion efficiencies ensure that all beams exiting the frequency converter unit have a good beam quality, which is important for OPCPA pumping (for more details, see: Section 5.2). The three beams are subsequently separated by dichroic beam splitters and directed into three OPCPA channels. 


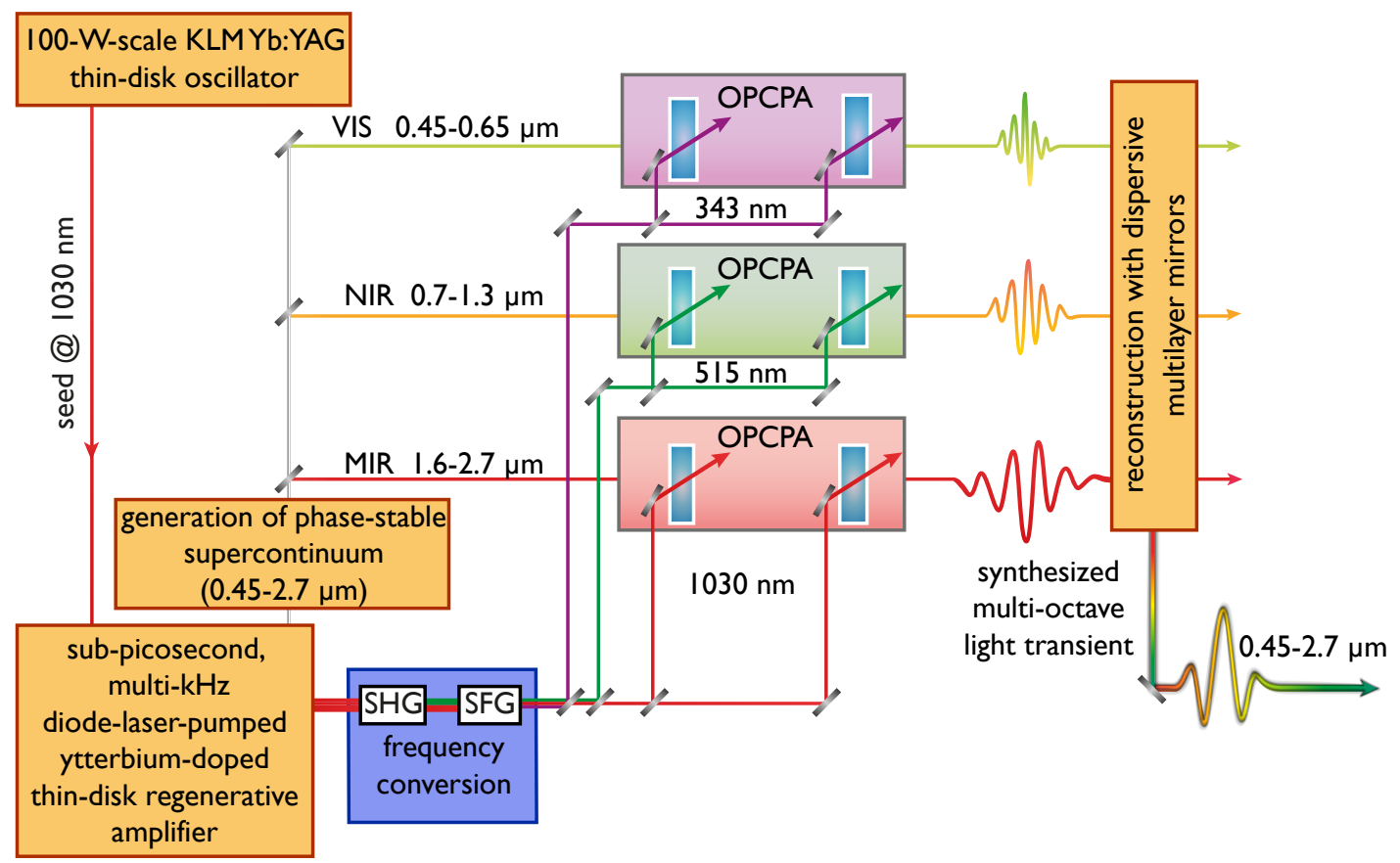

Figure 5.1: Schematic architecture of a three-channel OPCPA system seeded and pumped by near-1-ps ytterbium lasers. A part of its output is used for generating the multi-octave supercontinuum signal, which is split into three channels, centered at $550 \mathrm{~nm}, 1 \mu \mathrm{m}$, and $2 \mu \mathrm{m}$, respectively. The different channels are pumped by different low-order harmonics of the multi-mJ level, $\mathrm{kHz}, \mathrm{Yb}$ :YAG regenerative amplifier output. Each channel supports few-cycle pulses. By coherently combining the three few-cycle channels, amplified, non-sinusoidal, multi-octave light transients can be generated. 


\subsection{Proposed multi-octave seed generation}

DFG is the most powerful technique for the generation of MIR continua among the spectral broadening schemes discussed in detail in Section 2.4. This process creates a CEP-stable output from a non-CEP-stabilized pulse and has been successfully applied to seeding few-cycle MIR-OPCPA systems (Erny et al., 2007; Chalus et al., 2009; Deng et al., 2012; Hong et al., 2011). The DFG signal has good temporal contrast, is scalable in energy, and is passively CEP-stable which provides a reproducible shot-to-shot electric field, as required for HHG. The spectrum of the DFG output at mJ-level or $\mu J$-level can be efficiently extended to cover the multi-octave range of $400-5000 \mathrm{~nm}$ in filament (Kartashov et al., 2012; Silva et al., 2012). Moreover, the spectrum of sub-20-fs pulses from a MIR OPCPA (Deng et al., 2012), could be efficiently broadened in a 1 bar-Ar-filled HCF, to cover the entire VIS and NIR spectral range from $400-2500 \mathrm{~nm}$, as shown in Fig. 5.2. Such continua, at energy levels of hundreds of microjoules, could also be achieved with substantially longer, multi-cycle MIR fs pulses, in agreement with theoretical predictions (Kartashov et al., 2012). The temporal characterization of the generated continuum by SH-FROG shows the smooth spectral phase and its compressibility (Schwarz, 2014).

In what follows, different designs for generation of broadband MIR, > $\mu \mathrm{J}$-level energy, passively CEP stabilized pulses, required for seeding the OPCPA are proposed.

1-ps-pumped multi-octave seed generation The $10 \mathrm{fs}$ pulses required to seed the continuum generator shown in Fig. 5.2, may possibly be derived directly from the output of the near-1-ps Yb:YAG pump source by cascaded temporal compression. This approach would eliminate the need for the active pump-seed synchronization system for the OPCPA, because both the pump and the seed would travel comparable optical paths. In a cascaded gas-filled-HCF, the 1 ps input pulses centered at $1030 \mathrm{~nm}$ could be broadened to $700-1500 \mathrm{~nm}$ (Voronin et al., 2013). Although in this scheme the spectral phase in the vicinity of $1030 \mathrm{~nm}$ is not well-behaved and could cause a long pedestal in the time domain, the spectral phase in the blue and red wings of the spectrum are well-behaved and suitable for a type II DFG process.

In an alternative approach, shown in Fig. 5.3-a, 1-ps-pump pulses could be temporally shortened in an XPW stage (Cotel et al., 2006). As XPW acts as a gate in the time domain, no additional step would be required to compress the generated signal.

Recent experiments by Karpowicz et al. show that the red shift of the laser radiation necessitated by energy conservation in optical rectification 
a)

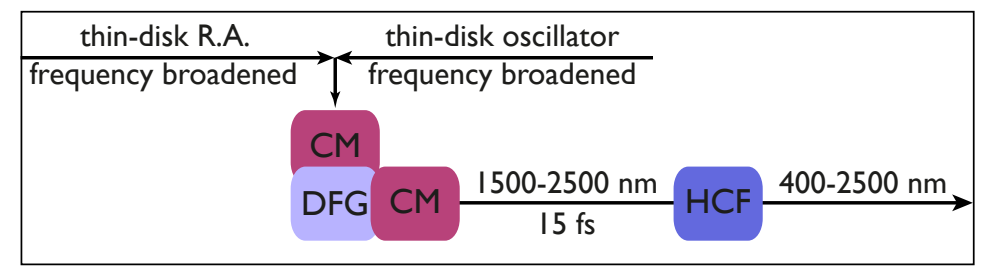

b)

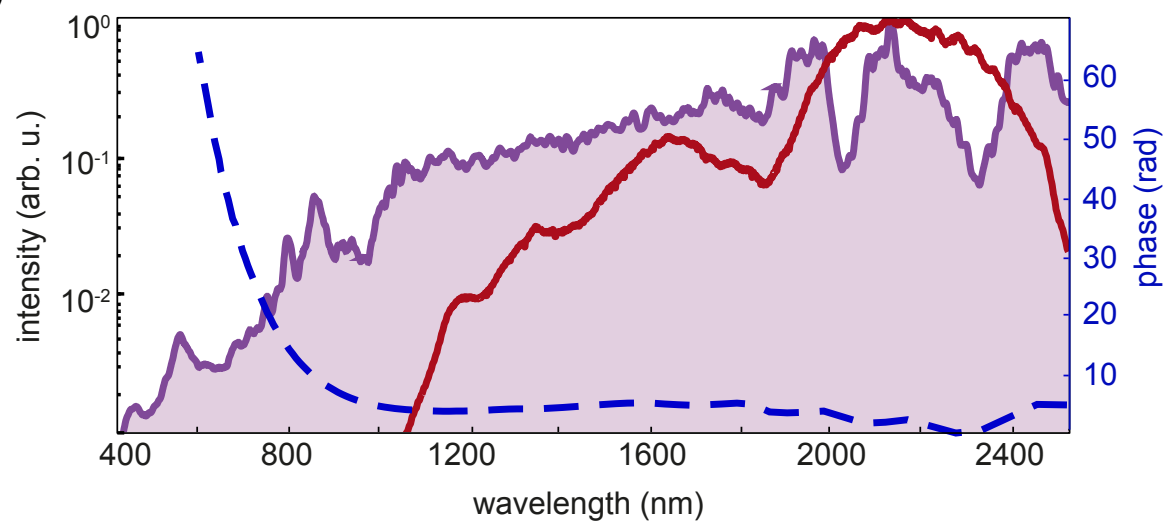

Figure 5.2: Multi-octave seed generation. a) Near-three-octave seed generation schemes based on the output from a KLM oscillator or driven by a ps thin-disk regenerative amplifier. The spectrally broadened and compressed output of both lasers drive the DFG stage. The resultant broadband DFG signal, centered at $2 \mu \mathrm{m}$ is compressed and focused into a gas-filled HCF to extend the spectrum into the visible range. b) Broadband phase-stable continua generated in preliminary experiments. Red curve: Differencefrequency radiation in a $500 \mu \mathrm{m}$-thick BBO crystal. Purple shaded area: Spectral broadening of a parametrically amplified three-cycle DFG signal in a gas-filled HCF to a continuum containing $33 \mu \mathrm{J}$ of energy (Schwarz, 2014). The detected bandwidth of the spectrum in both cases is limited by the sensitivity of the spectrometer in the infrared tail. The dashed blue curve shows the measured spectral phase of the generated supercontinuum using the SH-FROG technique (Schwarz, 2014). 

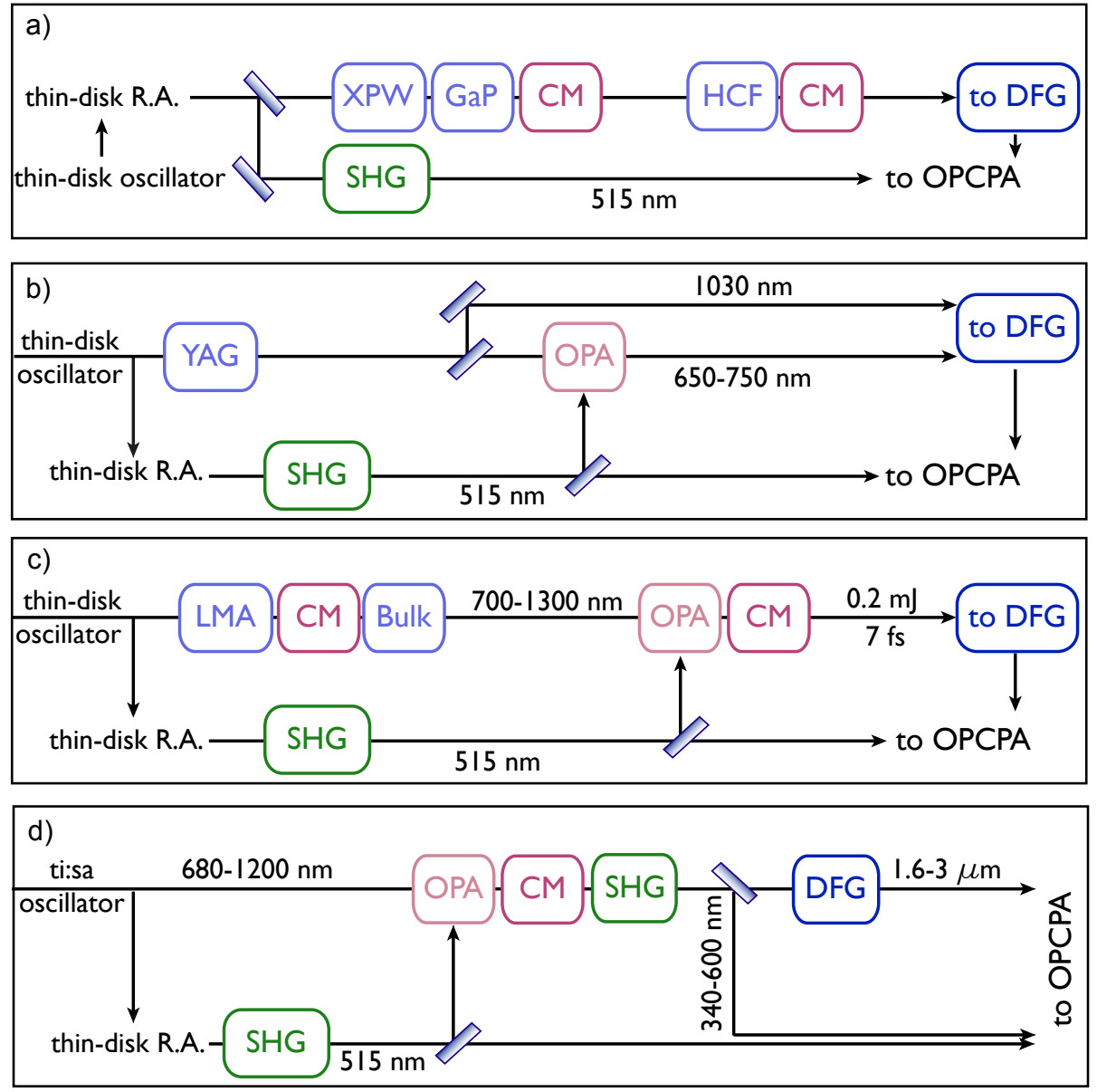

Figure 5.3: a) 1-ps-pumped broadband CEP-stable seed generation scheme based on Yb:YAG regenerative amplifiers and cascaded spectral broadening in an XPW stage, a GaP crystal and a HCF. b) 200-fs pumped broadband CEP-stable seed generation scheme based on Yb:YAG oscillator and supercontinuum generation in YAG crystal. c) Sub-20o-fs pumped broadband CEP-stable seed generation scheme based on Yb:YAG oscillator and cascaded broadening in LMA fibers. d) Ti:sapphire oscillator-based, multioctave seed generation.

for generating terahertz transients leads to pulse shortening. In their experiment, 1.6 ps pulses at $1030 \mathrm{~nm}$ were compressed to $300 \mathrm{fs}$, after propagation in a $5 \mathrm{~mm}[110] \mathrm{GaP}$ and a grating compressor. This scheme can be exploited as the second broadening stage after XPW to shorten pulses down to $100 \mathrm{fs}$, a suitable pulse duration for spectral broadening in a gas-filled HCF. After compression, the $10 \mathrm{fs}$ pulses are a suitable driver for the DFG process and multi-octave seed generation. 
Sub-2oofs-pumped multi-octave seed generation In this scheme, the $200 \mathrm{fs}$, $\mu \mathrm{J}$-level energy pulses directly from a KLM thin-disk oscillator, which serves to seed a thin-disk regenerative amplifier, are focused in a bulk (here YAG) for supercontinuum generation (Fig. 5.3-b). The spectral components at $1030 \mathrm{~nm}$ and $650-750 \mathrm{~nm}$ are separated from the rest of the continuum. The latter one is amplified in an OPCPA stage pumped by the second harmonic of the thin-disk regenerative amplifier and then combined with the $1030 \mathrm{~nm}$ pulses in a DFG stage (Homann et al., 2012). As the efficiency of the generated supercontinuum in bulk does not exceed $2 \%$, the $1030 \mathrm{~nm}$ pulses have $\mu \mathrm{J}$-level energies and the energy of the DFG signal does not exceed several tens of nJ. Although the scheme is simple, it is not scalable to higher pulse energies.

In an alternative approach, the output of the oscillator is broadened in a cascaded SPM stages and compressed down to $10 \mathrm{fs}$ pulses with $\mu \mathrm{J}$-scale energy (Fig. 5.3-c) (Seidel et al., 2014). The spectrum of these pulses with a well-behaved spectral phase and excellent spatial beam quality is matched to the gain band of $\mathrm{BBO}$ and LBO-based optical parametric amplifiers pumped by a fraction of the second harmonic of the Yb:YAG think-disk regenerative amplifier. Amplification in such an OPCPA to the several hundred $\mu \mathrm{J}$ energy level and recompression of the amplified NIR pulse is followed by a DFG stage. Saturated amplification in such an OPCPA provides not only the pulse energy and intensity required for subsequent spectral broadening but also stabilizes the amplified NIR against energy fluctuations accumulating in the preceding nonlinear stages. Here the signal and pump pulses must be synchronized actively.

In the last proposed scheme (Fig. 5.3-d), a broadband spectrum of a Ti:sapphire oscillator, used to seed the thin-disk regenerative amplifier, is amplified in an OPCPA stage. A broadband cascaded SHG and DFG of the amplified pulses after compression, extends the spectrum to VIS and MIR. In this scheme, active synchronization is required and the carrier-envelope phase of the VIS and MIR spectra need to be actively stabilized.

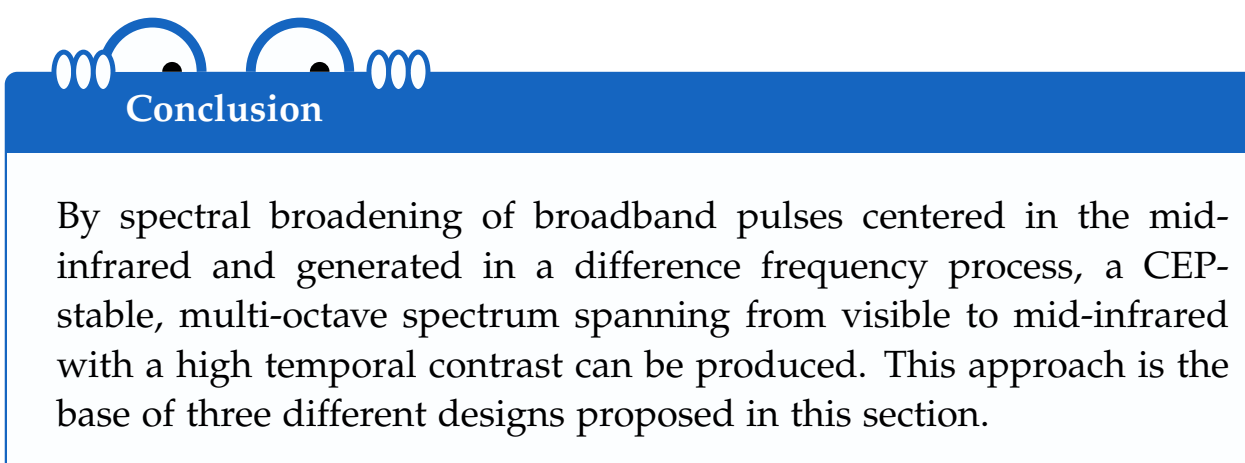


Deriving the required spectrum to pump a DFG stage from a 1-ps thin-disk regenerative amplifier, eliminates the need for a pump-tosignal active temporal synchronisation in the OPCPA chain. This spectrum can be achieved in a setup consisting of three nonlinear stages. However, the proposed scheme consists of three unsaturated nonlinear steps which makes achieving the required energy and spectralbandwidth stability of the generated signal challenging.

The two other proposed approaches in this section, based on sub$300-f s$ pulses of a thin-disk oscillator require active temporal synchronisation of the signal and pump pulses. Here, the setup contains a saturated OPCPA stage which may provide necessitated energy and spectral bandwidth stability for the subsequent DFG stage.

For generality a multi-octave seed generation scheme based on a Ti:sapphire oscillator is discussed, as well. Here the generated spectrum in the mid-infrared is passively CEP-stable while the nearinfrared and visible part of the spectrum has to be actively stabilized. In this scheme, an active temporal synchronization between pump and seed pulses is also required.

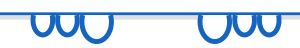




\subsection{Synthesizer channels}

Each of the three prototypical, multi-terawatt OPCPA channels can be designed to generate few-cycle pulses at a single carrier wavelength, by amplifying and recompressing the continua seeded into the amplifier chains. The following model calculations for a prototypical multi-color, multi-terawatt, few-cycle system is performed by assuming the availability of a $200 \mathrm{~mJ} \mathrm{Yb}$ :YAG thin-disk regenerative amplifier and designed for operating at a $5-\mathrm{kHz}$ repetition rate (Fattahi et al., 2014a).

In this design study, the pump energy is distributed preferentially to the NIR and MIR channels. This strategy is motivated by numerous applications benefiting from longer wavelengths, like generating $\mathrm{keV}$ photon energies in HHG due to the $\lambda^{2}$-scaling of the high-harmonic cutoff energy, as illustrated in Section $5 \cdot 3$ (Tate et al., 2007).

Generation of different harmonics of the pump laser In a first step, SHG of the $1030 \mathrm{~nm} \mathrm{Yb}: Y A G$ laser pulses in a type I LBO crystal cut at $40.2^{\circ}$ and the $d_{e f f}$ of $0.7 \mathrm{pm} / \mathrm{V}$, is implemented with an optical-to-optical efficiency of approximately $50 \%$ to share the pump-pulse energy equally between the fundamental laser wavelength and its second harmonic, i.e. $100 \mathrm{~mJ}$ of energy at $515 \mathrm{~nm}$ and $1030 \mathrm{~nm}$ each. LBO is chosen instead of BBO, owing to its availability in large sizes and its small spatial and temporal walk-off, in spite of its smaller effective nonlinear coefficient.

The direct THG in solids has low conversion efficiency reaching $\approx 6 \%$ in BBO crystal (Banks et al., 2002), caused by the small magnitude of the $\chi^{(3)}$ nonlinear polarization compared to the one arising from $\chi^{(2)}$. Hence, for generating the third harmonic of the laser, cascaded third harmonic generation in a simple collinear geometry without the need of an additional delay element is used (Herrmann et al., 2010a). The SHG stage is followed by SFG in another LBO crystal with a conversion efficiency of approximately $20 \%$. This approach yields approximately $40 \mathrm{~mJ}$ at $343 \mathrm{~nm}, 74 \mathrm{~mJ}$ at $515 \mathrm{~nm}$ and $86 \mathrm{~mJ}$ at $1030 \mathrm{~nm}$ for pumping the VIS, NIR, and MIR channels of the OPCPA system, respectively (Fig. 5.4).

The OPCPA is designed for maximum spectral bandwidth and pump-tosignal efficiency. The latter has been optimized, among others, by recycling the residual of the pump energy after the penultimate amplifier stage, a scheme which was successfully implemented in an experiment discussed in detail in Section 4.1. In the last crystal of the chain, the phase-matching angle, noncollinear pump-signal angle, spatial walk-off and spatial overlap of the interacting beams is slightly readjusted for maximum efficiency. In the following simulations, a Gaussian spatial and temporal profile of the pump pulses is assumed. 
a)

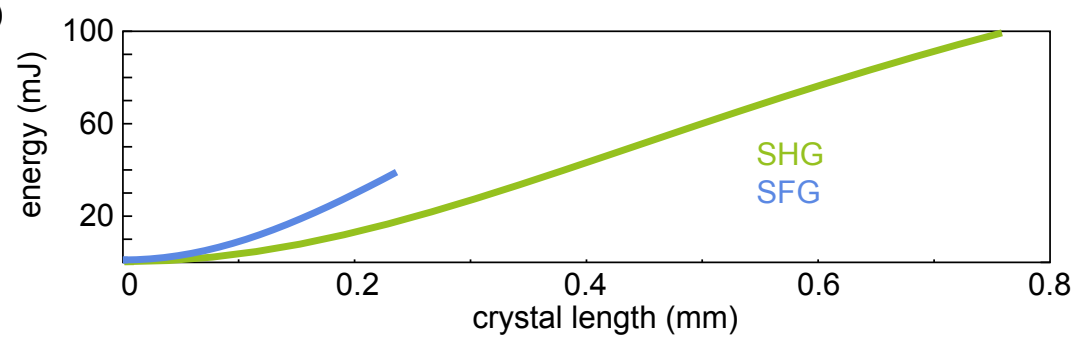

b)

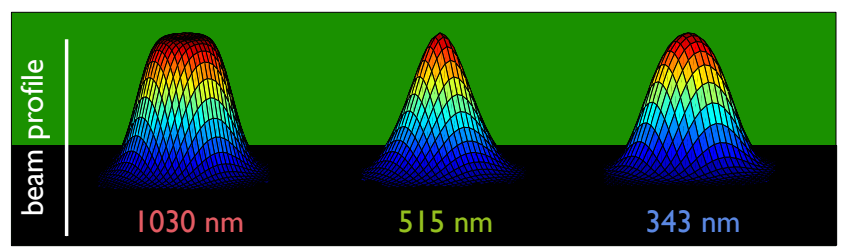

c)

\begin{tabular}{|c|c|c|c|c|c|c|}
\hline & $\mathrm{d}_{\mathrm{eff}}(\mathrm{pm} / \mathrm{V})$ & $\mathrm{L}_{c}(\mathrm{~mm})$ & $\Phi(\mathrm{mm})$ & $\mathrm{E}_{\text {input }}(\mathrm{mJ})$ & $\mathrm{E}_{\text {output }}(\mathrm{mJ})$ & efficiency \\
\hline \multirow{2}{*}{ SHG } & \multirow{2}{*}{0.82} & \multirow{2}{*}{0.75} & \multirow{2}{*}{9.8} & \multirow{2}{*}{200} & 102 & \multirow{2}{*}{$50 \%$} \\
\hline & & & & & 98 & \\
\hline \multirow[b]{2}{*}{ SFG } & \multirow[b]{2}{*}{0.7} & \multirow[b]{2}{*}{0.23} & $\mathrm{D}_{\mathrm{amp}} \times 0.7$ & 100 & 90 & \multirow{2}{*}{$35 \%$} \\
\hline & & & $\mathrm{D}_{\text {amp }} \times 0.85$ & 98 & 73 & \\
\hline
\end{tabular}

Figure 5.4: a) The SHG energy over crystal length for a near-1-ps, $200 \mathrm{~mJ}$, $1030 \mathrm{~nm}$ Yb:YAG regenerative amplifier (green curve) and the SFG between the second harmonic and the fundamental (blue curve). b) The simulated beam profile of the cascaded SHG-SFG frequency converter. c)The detailed parameters of the simulation. $L_{c}$ : length of the crystal, $\phi:$ beam diameter at FWHM, $E_{\text {input }}$ : input energy, $E_{\text {output }}$ : output energy. In the table, red areas indicate the parameters related to fundamental, while green and blue areas are related to the parameters of second and third harmonics of the laser, respectively.

A linearly-chirped fourth-order super-Gaussian spectrum is used for the seed pulses. The pump and seed duration in each channel is set to $1.6 \mathrm{ps}$ at FWHM. After setting the pump-beam diameter such that the desired onaxis peak intensity is realized, the size of the seed beam is optimized for best efficiency and amplified beam profile.

In the spectrum shown in Fig. 5.2, the different spectral regions, $1600 \mathrm{~nm}$ to $2800 \mathrm{~nm}$ (MIR channel), $700 \mathrm{~nm}$ to $1400 \mathrm{~nm}$ (NIR channel) and $450 \mathrm{~nm}$ to $600 \mathrm{~nm}$ (VIS channel) contain $40 \%, 40 \%$, and $3.5 \%$ of the total energy of the super-continuum, respectively. In this study, the same spectral energy distribution for the input seed containing a total energy of $6.5 \mu \mathrm{J}$ is assumed. 
MIR channel $\mathrm{LiNbO}_{3}$ is chosen as the gain medium for a 4-stage MIR OPCPA chain (Section 1.2), with the first three stages pumped with pulses of energy of $1 \mathrm{~mJ}, 7 \mathrm{~mJ}, 78 \mathrm{~mJ}$, respectively, and the residual pump energy surviving the third stage recycled to pump the fourth stage. The pump intensity in all stages is kept at $70 \mathrm{GW} / \mathrm{cm}^{2}$ to avoid photorefraction. The optimized chain yields $19.2 \mathrm{~mJ}$ pulses with a bandwidth-limited pulse duration of $12.3 \mathrm{fs}$ at FWHM, corresponding to less than two cycles of the carrier wave (Table 5.1). A $d_{\text {eff }}$ value of $3.3 \mathrm{pm} / \mathrm{V}$ is assumed in the simulation. The pump-to-signal energy conversion efficiency is $18 \%$, limited by back conversion of the signal into the pump via phase-matched SHG (Fig. 5.5).

NIR channel In the second-harmonic-pumped NIR channel, a broad phasematched bandwidth can be obtained by using a BBO crystal with an internal pump-signal angle of $2.2^{\circ}$ and a phase-matching angle of $22^{\circ}$, or an LBO crystal with an internal pump-signal angle of $1.1^{\circ}$ and a phase-matching angle of $\phi=15.5^{\circ}$ in the noncollinear type I geometry. LBO is chosen as the gain medium because it supports somewhat shorter amplified pulses. The NIR OPCPA chain also consists of 4 crystals being pumped with a total energy of $74 \mathrm{~mJ}$. The $d_{\text {eff }}$ value of $0.82 \mathrm{pm} / \mathrm{V}$ is assumed in the simulation. Adjusting the peak pump intensity to $110 \mathrm{GW} / \mathrm{cm}^{2}, 22.7-\mathrm{mJ}$ pulses with a bandwidth-limited pulse duration of $4.8 \mathrm{fs}$ have been predicted by our simulation (Table 5.1), corresponding to a conversion efficiency of $30 \%$. Thanks to the noncollinear geometry, parasitic backconversion is strongly reduced, giving rise to an excellent efficiency (Fig. 5.5).

VIS channel Finally, the VIS channel pumped with $40 \mathrm{~mJ}, 343 \mathrm{~nm}$ pulses, has been modelled by using type-I BBO crystals phase matched at $32.7^{\circ}$ and an internal noncollinear pump-signal angle of $4.5^{\circ}$ in three amplifier stages. The pump intensity in all stages is kept to $70 \mathrm{GW} / \mathrm{cm}^{2}$ to avoid BBO degradation. With pump energies of $3 \mathrm{~mJ}$ and $37 \mathrm{~mJ}$ for the first two OPCPA stages and reusing the residual pump energy of the second stage in the third stage, $7.1 \mathrm{~mJ}$ pulses with a bandwidth-limited duration of $5.1 \mathrm{fs}$ could be reached (Fig. 5.5). A $d_{\text {eff }}$ value of $1.56 \mathrm{pm} / \mathrm{V}$ is assumed for the calculation (Table 5.1).

Challenges Although parametric amplification, due to the (ideally) instantaneous, non-resonant nature of the light-matter interaction involved should be scalable in average power, limitations and challenges might arise in real crystals due to the absorption of certain frequencies, even with a very small absorption coefficient, in high power systems. This could cause a temperature gradient in the crystal and eventually lead to cracking of the crystal or 


\begin{tabular}{|c|c|c|c|c|}
\hline \multicolumn{5}{|c|}{ MIR channel } \\
\hline & Ist stage & $2^{\text {nd }}$ stage & $3^{\text {rd }}$ stage & $4^{\text {th }}$ stage \\
\hline$E_{p}(m J)$ & I & 7 & 78 & 54 \\
\hline $\mathrm{L}_{c}(\mathrm{~mm})$ & 2.5 & 1.7 & 1.4 & 1.1 \\
\hline $\mathrm{E}_{\text {s-amp }}(\mathrm{mJ})$ & 0.146 & 1.3 & 12 & 19.2 \\
\hline$\Phi_{p}(m m)$ & 0.9 & 2.4 & 7.6 & 6.1 \\
\hline$\Phi_{s}(\mathrm{~mm})$ & 0.9 & $* \mathrm{D}_{\text {amp }} \times 3$ & $* \mathrm{D}_{\mathrm{amp}} \times 3.5$ & $* \mathrm{D}_{\mathrm{amp}} \times 1.2$ \\
\hline \multicolumn{5}{|c|}{ NIR channel } \\
\hline & | st stage & $2^{\text {nd }}$ stage & $3^{\text {rd }}$ stage & $4^{\text {th }}$ stage \\
\hline$E_{p}(m J)$ & I & 7 & 66 & 45 \\
\hline $\mathrm{L}_{\mathrm{c}}(\mathrm{mm})$ & 2.5 & 1.5 & 1.5 & 1.2 \\
\hline $\mathrm{E}_{\text {s-amp }}(\mathrm{mJ})$ & 0.189 & 1.6 & 14 & 22.5 \\
\hline$\Phi_{p}(\mathrm{~mm})$ & 0.7 & 1.8 & 5.6 & 4.5 \\
\hline$\Phi_{s}(\mathrm{~mm})$ & 0.7 & $* \mathrm{D}_{\mathrm{amp}} \times 2.5$ & $* \mathrm{D}_{\mathrm{amp}} \times 3.2$ & $* \mathrm{D}_{\mathrm{amp}} \times 1.2$ \\
\hline \multicolumn{5}{|c|}{ VIS channel } \\
\hline & It stage & $2^{\text {nd }}$ stage & $3^{\text {rd }}$ stage & \\
\hline$E_{p}(m J)$ & 3 & 37 & 29.4 & \\
\hline $\mathrm{L}_{c}(\mathrm{~mm})$ & 1.2 & 0.75 & 0.5 & \\
\hline $\mathrm{E}_{\text {s-amp }}(\mathrm{mJ})$ & 0.474 & 4.8 & 7.1 & \\
\hline$\Phi_{p}(m m)$ & 1.2 & 5.3 & 4.66 & \\
\hline$\Phi_{s}(\mathrm{~mm})$ & 1.2 & $* D_{\text {amp }} \times 4.4$ & $* D_{\text {amp }} \times 1.1$ & \\
\hline
\end{tabular}

Table 5.1: Detailed parameters of the simulated channels. $E_{p}$ : input pump energy. $L_{c}$ : crystal length. $E_{s-a m p}$ : amplified energy of the signal. $\Phi_{p}$ : beam diameter of the pump at FWHM. $\Phi_{s}$ : beam diameter of the signal at FWHM. $D_{a m p}$ : Diameter of the amplified beam at FWHM. 
a) wavelength $(\mathrm{nm})$

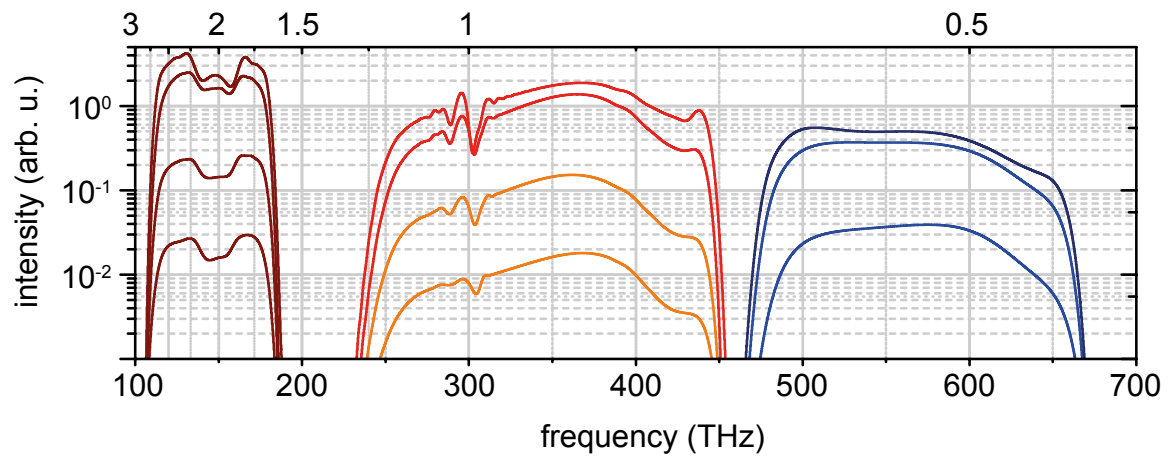

b)

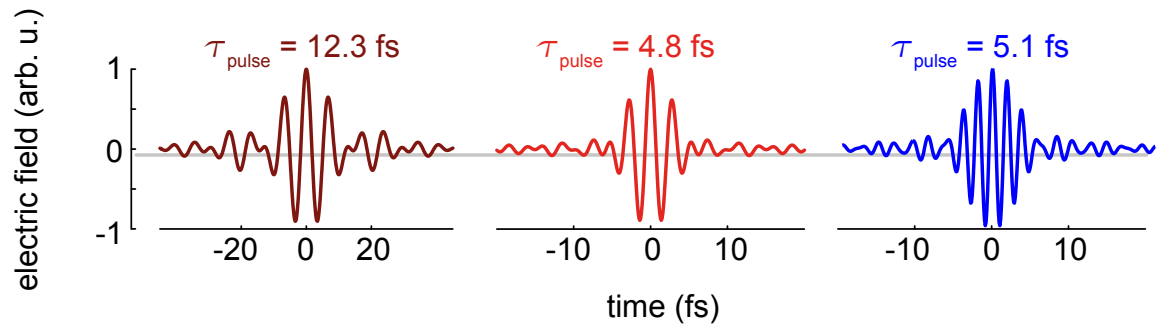

Figure 5.5: a) Amplified spectra of the three channels of the synthesizer obtained from simulation. The spectra of the different stages in the different channels are normalized to their energy and shown on a logarithmic scale. VIS channel is plotted in blue, NIR in red-orange, and MIR in brown. The dip in the amplified spectra of the MIR channel is caused by the phasematched SHG of the signal in the degenerate OPCPA. The strong modulations around $1 \mu \mathrm{m}$ in the NIR channel are also caused by SHG. Here, because of the noncollinear geometry, the dip is narrower. b) Fourier-transformlimited electric field corresponding to the final output spectrum of each channel and their pulse duration at FWHM. All channels support few-cycle pulses. 


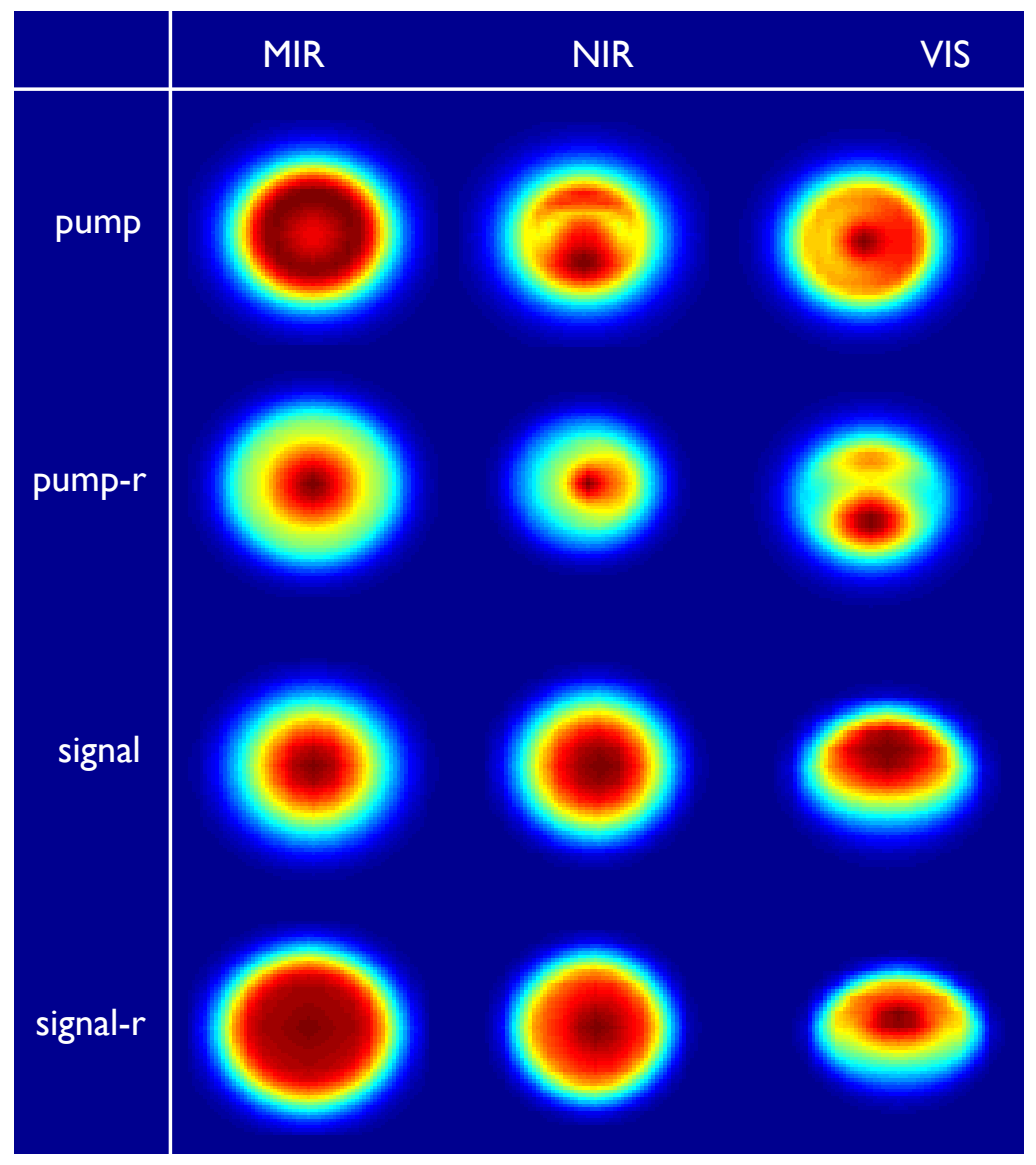

Figure 5.6: Beam profile of the signal and pump pulses at the last (signal$r$ and pump-r) and the penultimate (signal, pump) OPCPA stages of three channels of the synthesizer. The amplified signal in the MIR and NIR channels preserve the Gaussian shape. The asymmetric beam profile of the signal in the VIS channel comes as a consequence of the large noncollinear angle between signal and pump. This effect can be avoided via pulse-front-tilt compensation. 
cause phase-matching changes via the thermo-optic refractive index of the crystal.

In $\mathrm{LBO}$, similar to $\mathrm{BBO}$, a problematic absorption may be expected from the second harmonic of the pump centered at $515 \mathrm{~nm}$ due to the high power density. However, this value is estimated to be negligible for the pump lasers with the average power as high as $1 \mathrm{~kW}$ and should not be problematic in this case (Rothhardt et al., 2013; Prandolini et al., 2014). In the MIR channel the linear absorption in $\mathrm{LiNbO}_{3}$ at $2.6 \mu \mathrm{m}$ could cause a temperature gradient and eventually mechanically damage the crystal. This problem could be avoided by cutting the amplified spectrum to below $2.6 \mu \mathrm{m}$ or heating up the crystal. The main challenge of the visible channel is caused by nonlinear absorption of the pump laser as its second harmonic approaches the UV cut-off edge of the BBO at $189 \mathrm{~nm}$. However, the calculated two-photon absorption in the VIS channel of the synthesizer is negligible due to the low intensity employed. BBO crystal exhibits decreasing transmittance with increasing UV intensity and over time, e.g. when irradiated with ns-laser pulses centered at $262 \mathrm{~nm}$, which could be improved by heating up the crystal (Takachiho et al., 2014).

For frequency-doubling of pulses with an average power of $1 \mathrm{~kW}$, the temperature of the SHG-crystal should be stabilized. Another option for SHG is utilizing crystals with noncritical phase-matching (NCPM) which is characterized by the absence of walk-off, maximized effective nonlinearity and very wide acceptance angle. The temperature bandwidth and the acceptance angle of LBO for NCPM is $4{ }^{\circ} \mathrm{C}$ and $52 \mathrm{mrad} / \mathrm{cm}^{1 / 2}$, respectively. As for the current system, critical phase-matching is temperature and angle dependent, and using NCPM could be preferable. Table 5.2 summarizes some of the challenges with potential solutions. 


\begin{tabular}{|c|c|c|}
\hline challenge & Negative consequences & Proposed solution \\
\hline $\begin{array}{l}\text { Linear absorption at } \\
515 \mathrm{~nm} \text { in } B B O \text { and } \angle B O\end{array}$ & $\begin{array}{l}\text { - temperature gradient } \\
\text { - mechanical damage } \\
\text { - varied phase matching over the beam } \\
\text { cross section } \\
\text { - temperature dependent phase matching }\end{array}$ & $\begin{array}{l}\text { - thermal control of the crystal by } \\
\text { heating it up, utilizing special } \\
\text { holders }\end{array}$ \\
\hline $\begin{array}{l}\text { Linear absorption at } \\
2600 \mathrm{~nm} \text { in } \mathrm{LiNbO}_{3}\end{array}$ & $\begin{array}{l}\text { - temperature gradient } \\
\text { - mechanical damage } \\
\text { - varies phase matching over the beam } \\
\text { cross section } \\
\text { - temperature dependent phase-matching }\end{array}$ & $\begin{array}{l}\text { - thermal control of the crystal by } \\
\text { heating it up, utilizing special } \\
\text { holders } \\
\text { - cutting the amplification } \\
\text { bandwidth in OPCPA }\end{array}$ \\
\hline Nonlinearity in air & $\begin{array}{l}\text { - accumulation of the nonlinear phase, } \\
\text { causing undesired temporal and spatial } \\
\text { changes }\end{array}$ & - operation in vacuum \\
\hline $\begin{array}{l}\text { two-photon absorption in } \\
\text { BBO pumped at } 343 \mathrm{~nm}\end{array}$ & $\begin{array}{l}\text { - mechanical damage } \\
\text { - crystal degradation }\end{array}$ & - reduction of pump intensity \\
\hline $\begin{array}{l}\text { BBO degradation } \\
\text { in the presence of UV }\end{array}$ & $\begin{array}{l}\text { - decreasing transmittance with increasing } \\
\text { intensity }\end{array}$ & $\begin{array}{l}\text { - Heating up the crystal } \\
\text { - ejection of } \mathrm{O}_{2}\end{array}$ \\
\hline $\begin{array}{l}\text { Linear absorption at } \\
515 \mathrm{~nm} \text { in LBO used for } \\
\text { SHG }\end{array}$ & $\begin{array}{l}\text { - temperature gradient } \\
\text { - mechanical damage } \\
\text { - varied phase-matching over the beam } \\
\text { cross section } \\
\text { - temperature dependent phase matching }\end{array}$ & $\begin{array}{l}\text { - thermal control of the crystal } \\
\text { by heating it up, utilizing special } \\
\text { holders } \\
\text { - using noncritical phase- } \\
\text { matching }\end{array}$ \\
\hline
\end{tabular}

Table 5.2: Summary of possible challenges for the described three-channel synthesizer and different approaches to resolve them. 


\subsection{Field synthesizer}

Waveform-controlled light transients with a bandwidth approaching two octaves have been demonstrated at $\mu \mathrm{J}$ energy and GW peak power levels (Hassan et al., 2012; Wirth et al., 2011; Harth et al., 2012; Manzoni et al., 2012). They allow the control of electric fields of femtosecond pulses on a sub-cycle scale (Luu et al., 2013). The described prototypical three-color few-cycle OPCPA system offers a conceptually simple route to scaling multi-octave optical waveform synthesis to the multi-terawatt regime. To this end, the three channels delivering few-cycle pulses in the VIS, NIR, and MIR spectral ranges are recombined into one beam using a set of dichroic chirped mirrors (Huang et al., 2012; Wirth et al., 2011). Due to the difficulties of dispersion management, the high demands on the chirped-mirror compressor, and the required coating for the OPCPA crystals in serial pulse synthesis (Harth et al., 2012; Herrmann et al., 2010b), a parallel-synthesis approach is chosen (Kärtner et al., 2013).

In coherent synthesis of different pulses, fine control over the spectral phase, relative phase of each pulse, and their relative timing jitter are crucial. Fluctuations and drifts in the relative timing of the recombined pulses need to be suppressed to a tiny fraction of the half-field cycle for a stable waveform resulting from the coherent superposition (Bagayev et al., 2013). To control the spectral phase, a Dazzler acousto-optic pulse shaper (Verluise et al., 2000) will be implemented in the MIR OPCPA chain, and later the negatively-chirped, amplified pulses will be compressed to their Fourier transform limit using a spatially tailored chirped-mirror compressor. For the compression of the NIR and VIS channels, a dual spatial light modulator (DSLM) (Binhammer et al., 2005) and two sets of specially designed chirped-mirrors could be utilized. This combination allows fine control over the spectral phase of each channel to minimize the residual spectral phase in each arm separately.

A passively stabilized OPCPA seed ensures a stable carrier-envelope phase, and the relative timing stability between the three arms will be ensured by a balanced optical cross-correlator (BOC) to avoid a phase shift of the waveform at the synthesis point. By utilizing a BOC, a temporal overlap with sub-10o-as precision could be achieved, as in this approach the contribution of the amplitude noise of each OPCPA output on the detected error signal is suppressed (Huang et al., 2012; Manzoni et al., 2012). Besides the precise temporal overlap of pulses, precise spatial synthesis is required for wave-front matching of the pulses. As a majority of applications will employ focusing of light transients in order to attain high intensities, the OPCPA pulses will be combined in a constant-waist-width fashion that allows the temporal pulse form to remain unchanged upon propagation (Zou 
and Lü, 2007).

The feasibility of super-octave optical waveform synthesis was recently demonstrated in the NIR-VIS-UV spectral range by seeding a three-channel (Wirth et al., 2011) and, more recently, four-channel (Luu et al., 2013) synthesizer consisting of broadband chirped mirrors with a continuum originating from a Ti:sapphire-laser-driven HCF. Implementation with an OPCPA system is also being prepared (Kärtner et al., 2013; Rossi et al., 2014; Fang et al., 2013).

Merely the adjustment of the relative timing of the three pulses emerging from the three OPCPA channels can result in a great variety of electric field wave forms on the time scale of the optical cycle. Fig. 5.7 depicts a few representative waveforms that may be synthesized by first compressing the output of the individual channels to their bandwidth limit and then adding the three pulses with various time delays and relative amplitudes. Further degrees of freedom for waveform modification can be introduced by shaping the amplitude and phase of the spectra of the individual channels.

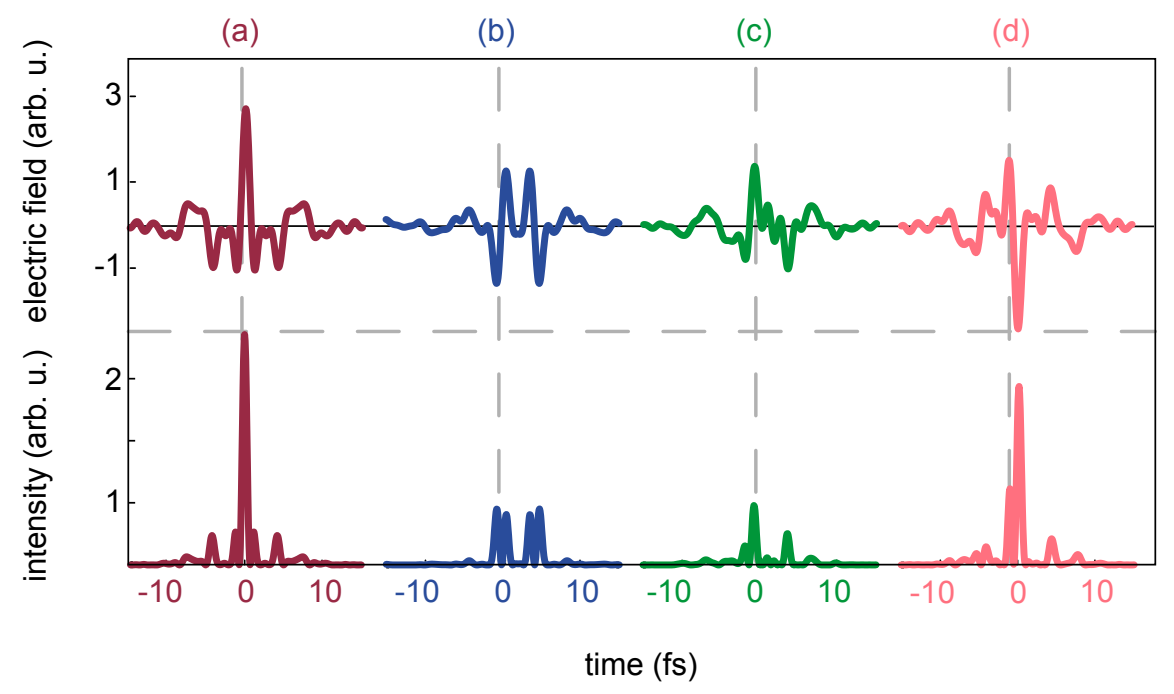

Figure 5.7: Calculated synthesized waveforms from the three-channel OPCPA system. a) Fourier-transform limit waveform of the three OPCPA channels. b) Synthesized waveform generated by changing the amplitude ratio between the three channels to $(1:-4 / 3: 1), c)$ and by changing the amplitude ratio of three different spectra to (1:0.5:1) and the delay between them to (o:-0.6 fs:1 fs). d) Optimized waveform for HHG in terms of the highest cutoff energy of the synthesizer. 


\section{0}

Conclusion

In this section a conceptual design of an OPCPA-based synthesizer is presented and discussed. 1.6-ps pulses from a 200-mJ Yb:YAG thindisk regenerative amplifier operating at $5 \mathrm{kHz}$ are sent to a simple frequency converter module for generation of second and third harmonics. A multi-octave seed spectrum is divided to three parts, centered at $550 \mathrm{~nm}, 1 \mu \mathrm{m}$, and $2 \mu \mathrm{m}$. Consequently, these spectra are amplified to the total energy of $49 \mathrm{~mJ}$ in three OPCPA channels pumped by fundamental, second- and third-harmonic pulses of the regenerative amplifier. Each OPCPA channel supports few-cycle pulses.

Coherent combination of the three channels after their temporal compression results in a sub-cycle waveform. By adjusting the relative timing and amplitude between three arms of the synthesizer, variety of confined, sub-cycle electric fields with different shapes are achievable. 


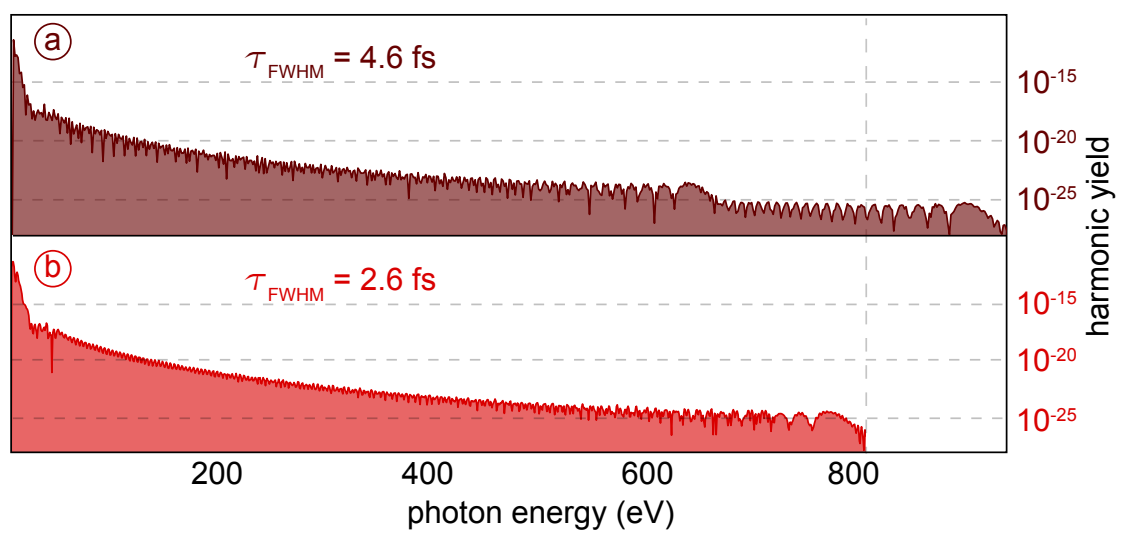

Figure 5.8: a) Simulated HHG spectrum of a $4.6 \mathrm{fs}$ and b) $2.6 \mathrm{fs}$ pulse centered at $800 \mathrm{~nm}$ at the pump intensity of $50 \times 10^{14} \mathrm{~W} / \mathrm{cm}^{2}$ in He. The cutoff decreases for the single-cycle pulse as the envelope of the pulse becomes shorter compared to the cycle.

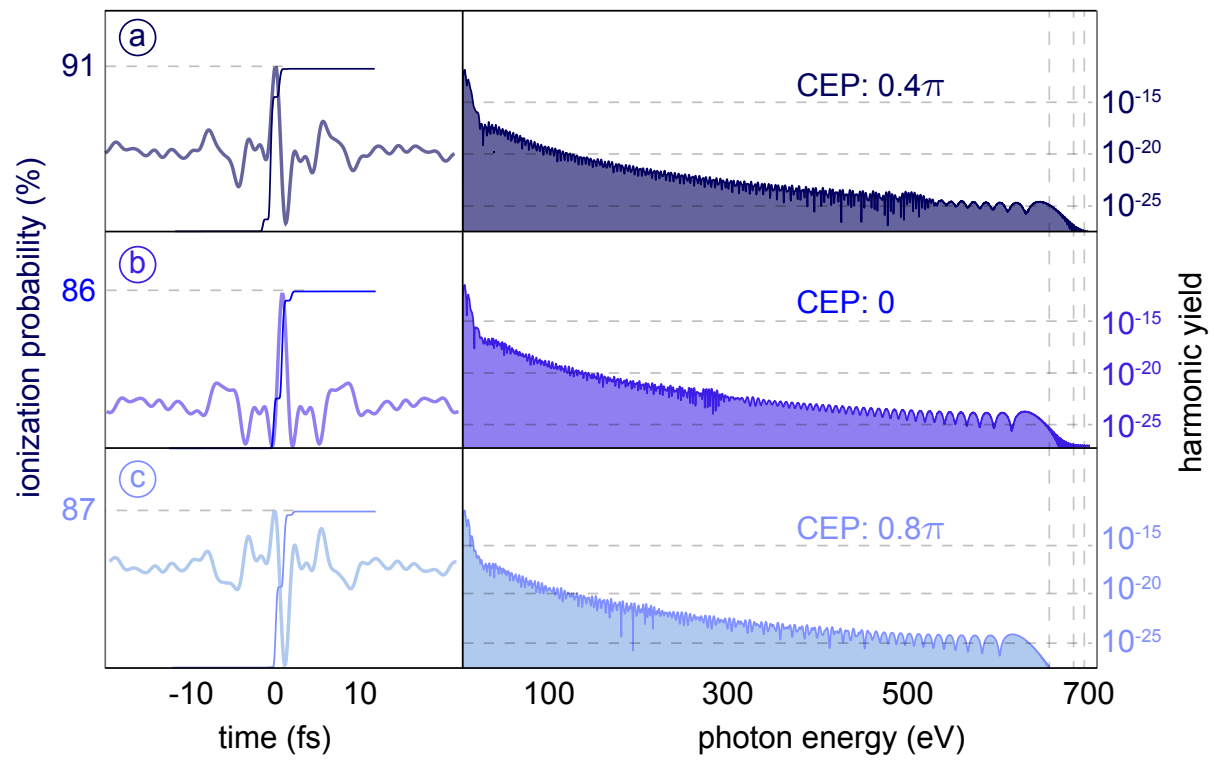

Figure 5.9: The synthesized waveform with the CEP offset of $0.4 \pi$ shows the highest HHG cutoff (a), compared to a waveform with carrier envelope phase offset of $0(\mathrm{~b})$ and $0.8 \pi(\mathrm{c})$.

\subsection{Prospects for experiments}

Multi-terawatt, multi-octave light transients are promising tools to extend the photon energy frontier of attosecond pulses to kilo-electronvolts and the boundary of the hard x-rays regime (Moulet et al., 2014). To explore the potential of keV HHG, the cutoff energy of different waveforms is evaluated by 
calculating the dipole response of one atom using the strong-field approximation (Lewenstein et al., 1994; Antoine et al., 1996) (see: Appendix B). Fig. 5.8 compares the harmonic spectra of a $4.6 \mathrm{fs}$ pulse centered at $800 \mathrm{~nm}$ and a $2.6 \mathrm{fs}$ single-cycle pulse at $800 \mathrm{~nm}$. The dipole response is calculated for a pump intensity of $50 \times 10^{14} \mathrm{~W} / \mathrm{cm}^{2}$ interacting with He atoms.

Without controlling the waveform, the cutoff tends to reduce for singlecycle pulses, as the HHG process requires two half-cycles to push electrons away from their parent core and accelerate them home. Diminishing either of the half-cycles decreases the cutoff. This problem is unavoidable for a single-cycle Gaussian pulse, as the envelope of the pulse becomes shorter compared to the cycle. Controlled-transient waveforms hold promise to overcome this shortcoming, as the half-cycles of the waveform can be fully optimized by adjusting different parameters like spectral phase and amplitude of each channel, the relative temporal delay between different arms of the synthesizer, carrier envelope phase and the spectral chirp of the final waveform.

Fig. 5.9 shows the influence of the carrier envelope phase on the HHG spectrum of the waveform shown in Fig. 5.7-a at a peak intensity of $50 \times$ $10^{14} \mathrm{~W} / \mathrm{cm}^{2}$ in He. The highest cutoff energy is achieved for a global phase of $0.4 \pi$, as in this case the waveform contains two strong half-cycles. The first half-cycle strongly ionizes the atoms and the second one provides the force for recollision of the electrons with their parent ions. The optimized waveform in terms of the CEP offset, has the highest ionization rate compared to the other waveforms.

In the multi-octave transient waveform, the energy distribution between three channels and therefore, the shape of the spectrum plays an important role in defining the cutoff energy. Low-frequency photons extend the cutoff and higher frequency photons are helpful in the ionization process although they reduce the cutoff to lower photon energies. In Fig. 5.10 the cutoff energies for different energy ratios of the three channels of the synthesizer at $50 \times 10^{14} \mathrm{~W} / \mathrm{cm}^{2}$ peak intensity and in a He atom are compared. Reducing the energy of the VIS channel extends the cutoff dramatically while increasing the energy of the MIR channel improves the cutoff energy. For the case of an energy ratio of 3:1:o between the MIR:NIR:VIS channel, the cutoff decreases, emphasizing the role of the VIS channel in the ionization process.

This suggests that the cutoff can be optimized further by changing the relative delay of the VIS channel compare to the other channels in order to shift the high frequency photons in the VIS range to the leading edge of the waveform where the first half-cycle ionizes the atom. As shown in Fig. 5.11, this selectively introduced chirp leads to a substantial increase in the generated photon energies. Comparing the ionization probabilities in 


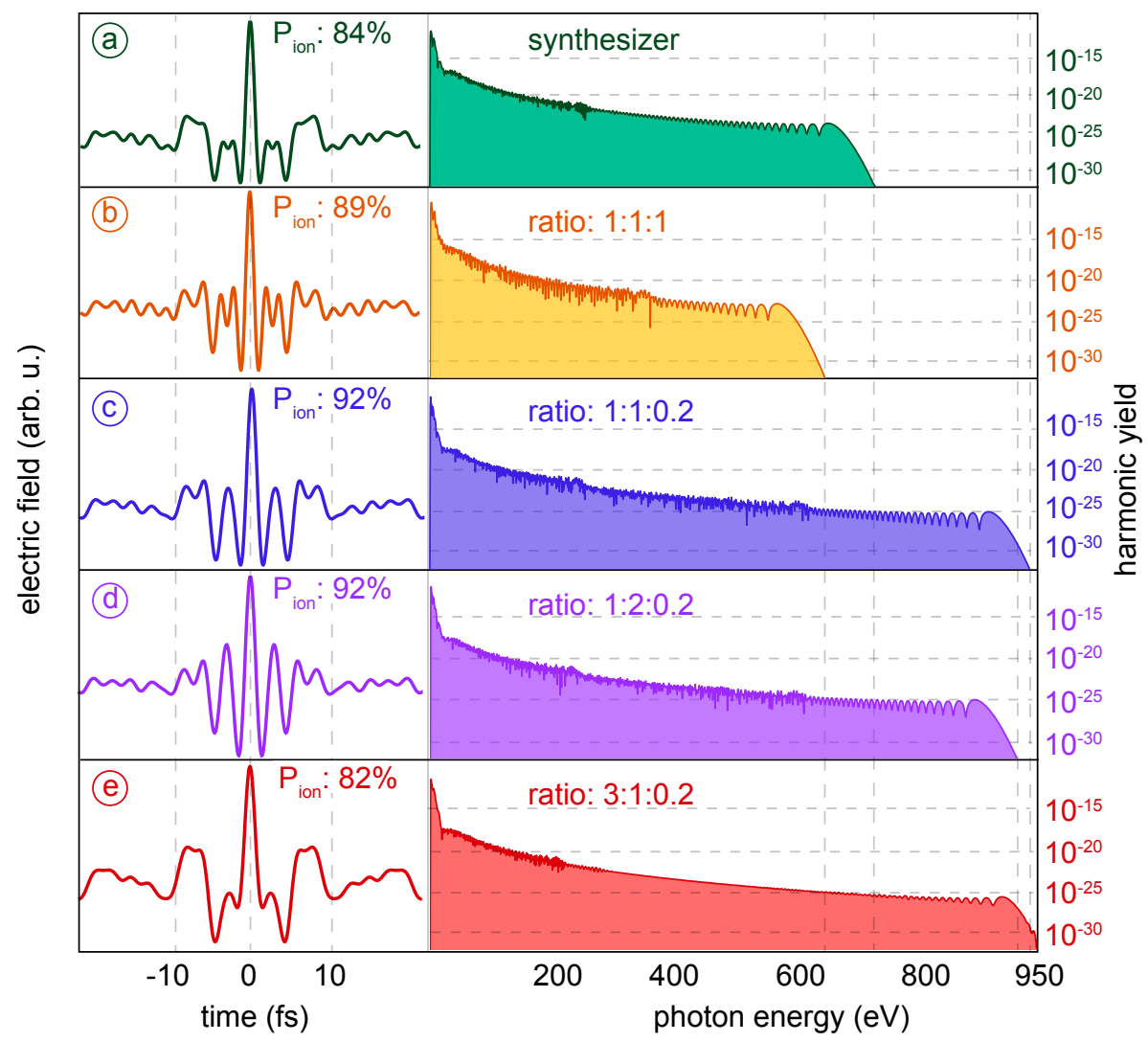

Figure 5.10: Right panels: the simulated HHG spectra for $50 \times 10^{14} \mathrm{~W} / \mathrm{cm}^{2}$ of peak intensity in He gas, for the energy distribution of a) the simulated synthesizer in the previous section b) 1:1:1 between the MIR:NIR:VIS channels c) 1:1:0.2 between the MIR:NIR:VIS channels d) 1:2:0.2 between the MIR:NIR:VIS channels d) 1:2:0.2 between the MIR:NIR:VIS channels e) 3:1:0.2 between the MIR:NIR:VIS channels. Left panels: the corresponding transient waveform responsible for the shown spectra and their corresponding ionization probabilities. The simulation is performed for a peak intensity of $50 \times 10^{14} \mathrm{~W} / \mathrm{cm}^{2}$ and in a He atom. $\mathrm{P}_{\text {ion }}$ : ionisation probability.

Fig. 5.10-a and Fig. 5.11-d suggests that synthesized waveforms are also superior to Gaussian pulses in terms of reaching the same cutoff energy at a lower ionization rate, as one of the fundamental limits in phase-matching high-energy photons is determined by the plasma density. We have neglected the magnetic drift as the electron in this parameter regime is not yet relativistic (Palaniyappan et al., 2006; Reiss, 2014). 


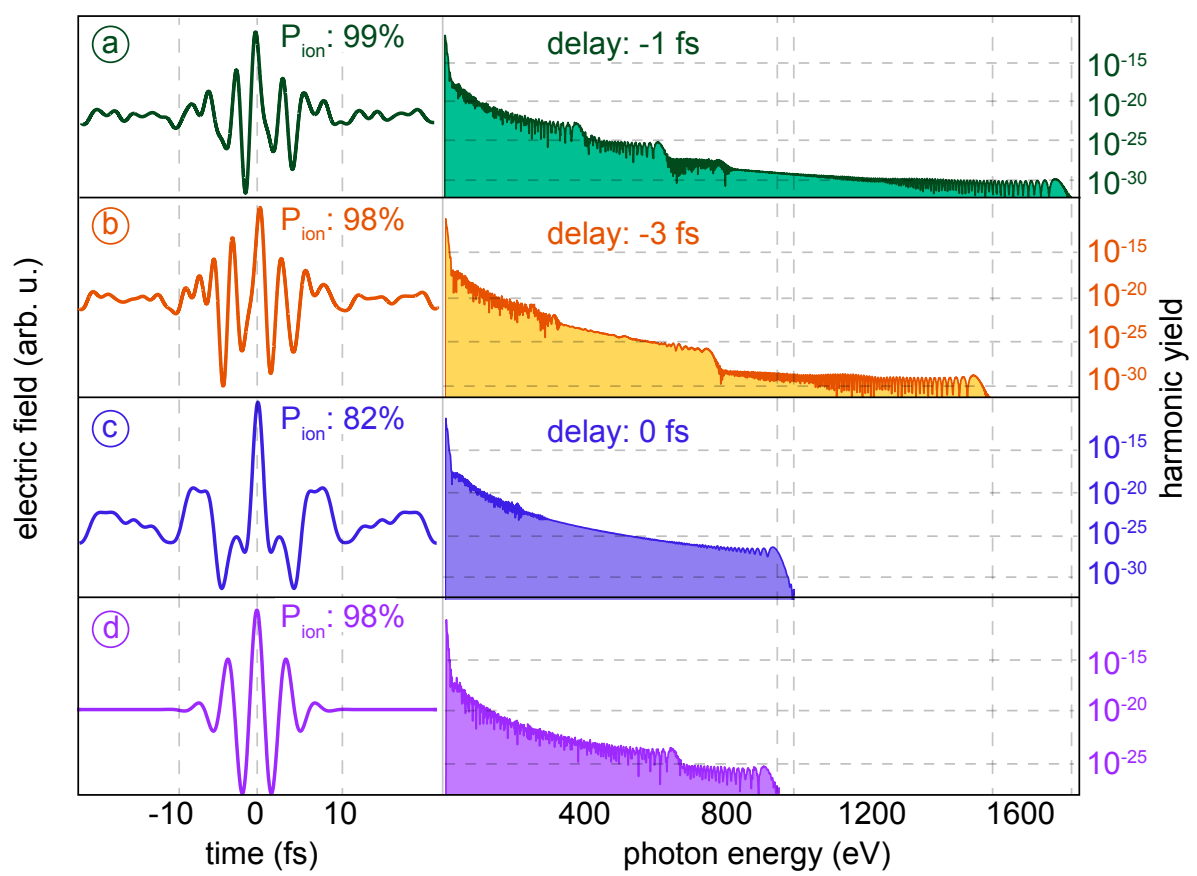

Figure 5.11: Right panels: the HHG spectra generated with a transient waveform of the synthesizer with energy distribution of 3:1:0.2 for VIS:NIR:MIR channels, while the VIS channel is delayed by a) $-1 \mathrm{fs} b)-3 \mathrm{fs}$ and c) $0 \mathrm{fs}$ relative to the other channels. d) The HHG spectrum of a $4.6 \mathrm{fs}$ Gaussian pulse shown for comparison. Left panels: the corresponding synthesized waveform that produced the HHG spectra. $\mathrm{P}_{\text {ion }}$ : ionisation rate.

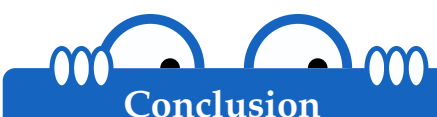

Sub-1-cycle waveforms hold promise to improve the cut-off energy in the HHG process. Due to their confined sub-cycle electric field, they provide the same cut off energy in lower ionization probability compared to Gaussian pulses. This improves the phase-matching over propagation.

It is analytically shown that the cut off of the waveform driven HHG can be extended by pushing the high energy photons to the leading edge of the waveform and leaving the low-energy photons at the trailing edge of the waveform. 



\section{Chapter 6}

\section{Conclusion and outlook}

The first-generation of femtosecond technology relied on dye lasers with $\mathrm{MW}$-scale peak power and average powers up to $100 \mathrm{~mW}$. The spectral bandwidth of these sources was confined to a narrow range between 600 and $900 \mathrm{~nm}$.

The second-generation femtosecond lasers, driven by chirped pulse amplification (CPA) in broadband solid-state laser media, boosted the peak power of lasers to the multi-terawatt level. At this peak power lasers provide enough intensity to efficiently harvest $\chi^{(2)}$ and $\chi^{(3)}$ nonlinearities and reach extreme nonlinearity at low repetition rates but are still limited in terms of reaching high peak power at high repetition rates.

Based on short-pulse-pumped optical parametric chirped pulse amplification (OPCPA), third-generation femtosecond technology allows boosting the peak and average power of coherent femtosecond light sources to the multi-terawatt level with hundreds of Watts average power, at a $\mathrm{MHz}$ repetition rate.

The OPCPA systems designed and realized in this thesis are pumped with near-1-ps Yb:YAG thin-disk lasers and have a modular and robust architecture offering different modes of operation. From a high-power, widely tunable source to few-cycle sources at different carrier wavelengths spanning from visible to mid-infrared or super-octave, subcycle light transients.

Crucial for the performance of this concept is the availability of broadband, high-contrast, CEP-stable seed pulses and powerful and reliable pump sources:

In Chapter 2 of this work, different techniques for the generation of coherent, broadband spectra suitable for seeding OPCPA systems were theoretically and experimentally investigated. Comparison of several techniques revealed that the smooth spectrum and good temporal contrast made cross-polarized wave generation (XPW) and difference frequency generation (DFG) the most favorable seed generation schemes. Additionally, the DFG 
concept offers intrinsic phase-lock between the carrier wave and the intensity envelope (CEP stability). However, the efficiency of these processes is low, for XPW due to the weak third-order nonlinearity and in broadband-DFG because of the limit phase-matching requirements impose on the nonlinear crystal thickness. Here, it is experimentally shown that the efficiency can be doubled by implementing a cascaded XPW setup. Optimization of the DFG process up to $10 \%$ efficiency is achieved by careful projection of the input signal on both the ordinary and extraordinary axis of the nonlinear crystal, making DFG the method of choice for OPCPA seed generation.

Chapter 3 investigates $\mathrm{Yb}$ :YAG thin-disk regenerative amplifiers as the most promising pump sources for high-energy, high-power OPCPA systems. To fully exploit the capabilities of these sources, the underlying dynamics of chirped pulse amplification including the chaotic amplification scheme in an ytterbium-based thin-disk amplifier is examined. It was demonstrated that with adequate input seed energy and optimized cavity design, the nonlinear amplification dynamics and the chaotic regime in regenerative amplifiers are in balance and a safe operation mode is achieved. Yielding $<1 \%$ amplitude stability renders these systems ideal pump sources for nonlinear amplification schemes like OPCPA.

With broadband seed pulses and the reliable, $20 \mathrm{~mJ}$ Yb:YAG thin-disk regenerative amplifier in place, different techniques for further optimization of the conversion efficiency and spectral bandwidth engineering in an OPCPA system were experimentally and theoretically studied (Chapter 4). It was shown that by reusing the residual pump energy in a 3-stage OPCPA system and employing BBO crystals as the nonlinear medium, a pump-tosignal conversion efficiency of $30 \%$ is feasible while the amplified spectrum supports sub-6-fs pulses.

In a conventional OPCPA system, a balance between amplification bandwidth and conversion efficiency has to be found. Necessarily, the seed pulse duration has to be shorter than the pump pulses. Consequently only a fraction of the pump pulse energy can be used for amplification, limiting the overall efficiency. This can be resolved by controlling the deposition of pump energy along the temporal profile of the heavily chirped seed pulse. Chapter 4 presents the theoretical and experimental evidence of this new scheme, promising unprecedented efficiency.

The discussion is concluded by a theoretical outline and experimental verification of a technique to engineer the OPCPA amplification gain bandwidth. Based on this, the bandwidth of a 3 -stage OPCPA system was extended beyond the phase-matching limitations by utilizing different gain media (LBO and BBO in this case). Amplification of near-1-cycle pulses, at $3 \mathrm{kHz}$ repetition rate and centered at $1 \mu \mathrm{m}$ to $1.8 \mathrm{~mJ}$ was demonstrated, 
parameters that have been unachievable with $2^{\text {nd }}$ generation femtosecond technology.

All of the above presented findings are utilized in Chapter 5, for the full design study of the multi-TW, multi-octave, 200-Watt-scale, OPCPA-based field synthesizer currently under development at the Laboratory of Extreme Photonics (LEX) in Garching.

The fundamental, second and third harmonics of a $200 \mathrm{~mJ}, 1 \mathrm{~kW}$ Yb:YAG thin-disk laser will be used to pump three OPCPA channels covering the visible to mid-infrared wavelength range. The superposition of the few-cycle pulses from the individual channels will allow synthesis of sub-cycle waveforms. Analytical methods were employed to identify the ideal waveform that maximises the cutoff energy of the generated photons in the high harmonic generation (HHG) process. With the optimized waveform seeding HHG, the laser system is predicted to become a new source of attosecond $\mathrm{keV}$ x-ray pulses.

Such a laser will equip scientists with a unique tool to explore and scrutinize a new era of physics so far deemed beyond reach. The attempts to better understand and control the underlying principles of lasers in the single cycle limit, discussed in this thesis, are small contributions to the next chapter of the fascinating story of photons. 



\section{Part III}

Appendix 



\section{Appendix A}

\section{In situ spectral interferometry as a pulse compression tool}

Compressing the output of a laser or an OPA requires that both the pulse and the compressor are free of complicated phase features, unless they are equal and opposite. However, once a complicated structure is detected in the final pulse, the question still remains if it is coming from the laser or the compressor. For example, a split pulse could arise either through a strongly amplified reflection, or through an unwanted ripple in the group delay curve of a set of chirped mirrors. Finding the culprit requires a way of measuring at least one of them independently.

FROG and other pulse measurement techniques are based on nonlinear effects, and cannot be easily used for an uncompressed pulse. A simple and effective way to characterize the chirped-mirror compressor is measuring the total group delay response of the compressor using interferometry, which is a linear effect. This method does not require the pulse to be compressed to perform a measurement, can be done in a single shot and requires only a spectrometer capable of detecting the spectrum of the laser or OPA itself.

In order to characterize the chirped-mirror compressor used in Section 4.3, the seed pulse of the OPCPA system was sent to the chirped-mirror compressor. A 4-mm-thick $\mathrm{CaF}_{2}$ plate beam splitter was placed before the compressor, reflecting a reference pulse which bypassed the compressor. After the rest of the light was transmitted through the compressor, it was recombined with the reference light (which passed through a variable time delay), using an identical glass plate beam splitter (Fig. A.1). The combined beams were sent to a spectrometer so that their spectral interference pattern could be observed.

These patterns, accompanied by reference spectra from the two arms independently, are sufficient to completely reconstruct the group delay curve 
of the compressor.

The spectral intensity of the two pulses after interference is described as:

$$
\begin{aligned}
I(\omega) & \propto\left|\tilde{E}_{A}(\omega)+\tilde{E}_{B}(\omega)\right|^{2} \\
& \propto\left|\tilde{E}_{A}(\omega)\right|^{2}+\left|\tilde{E}_{B}(\omega)\right|^{2}+2\left|\tilde{E}_{A}(\omega)\right|\left|\tilde{E}_{B}(\omega)\right| \cos \phi_{r e l}(\omega) \\
& =I_{A}(\omega)+I_{B}(\omega)+2 \sqrt{I_{A} I_{B}} \cos \phi_{r e l}(\omega) .
\end{aligned}
$$

The cosine of the spectral phase can be calculated from the measurement:

$$
\cos \phi_{\text {rel }}(\omega)=\frac{I(\omega)-I_{A}(\omega)-I_{B}(\omega)}{2 \sqrt{I_{A} I_{B}}},
$$

and by using the Hilbert transform:

$$
\sin \phi_{r e l}(\omega)=\frac{1}{\pi} \text { p.v. } \int_{-\infty}^{\infty} \frac{\cos \phi_{r e l}(\omega)}{\omega-\omega^{\prime}} d \omega^{\prime} .
$$

Therefore the spectral phase is:

$$
\phi_{\text {rel }}=\operatorname{atan} 2\left(\sin \phi_{\text {rel }}(\omega), \cos \phi_{\text {rel }}(\omega)\right) .
$$

Measured interference patterns and their corresponding group delay curves for two different time delays are shown in Fig. A.1.

Conclusion Spectral interference is an extremely useful technique for characterizing a compressor without having to perform a pulse retrieval. Because it relies on linear optics, it can be performed with any beam light source without having to approximately compress the pulse beforehand. The simple setup and the single shot measurement makes it possible to optimize the reflection angles of a set of mirrors. 

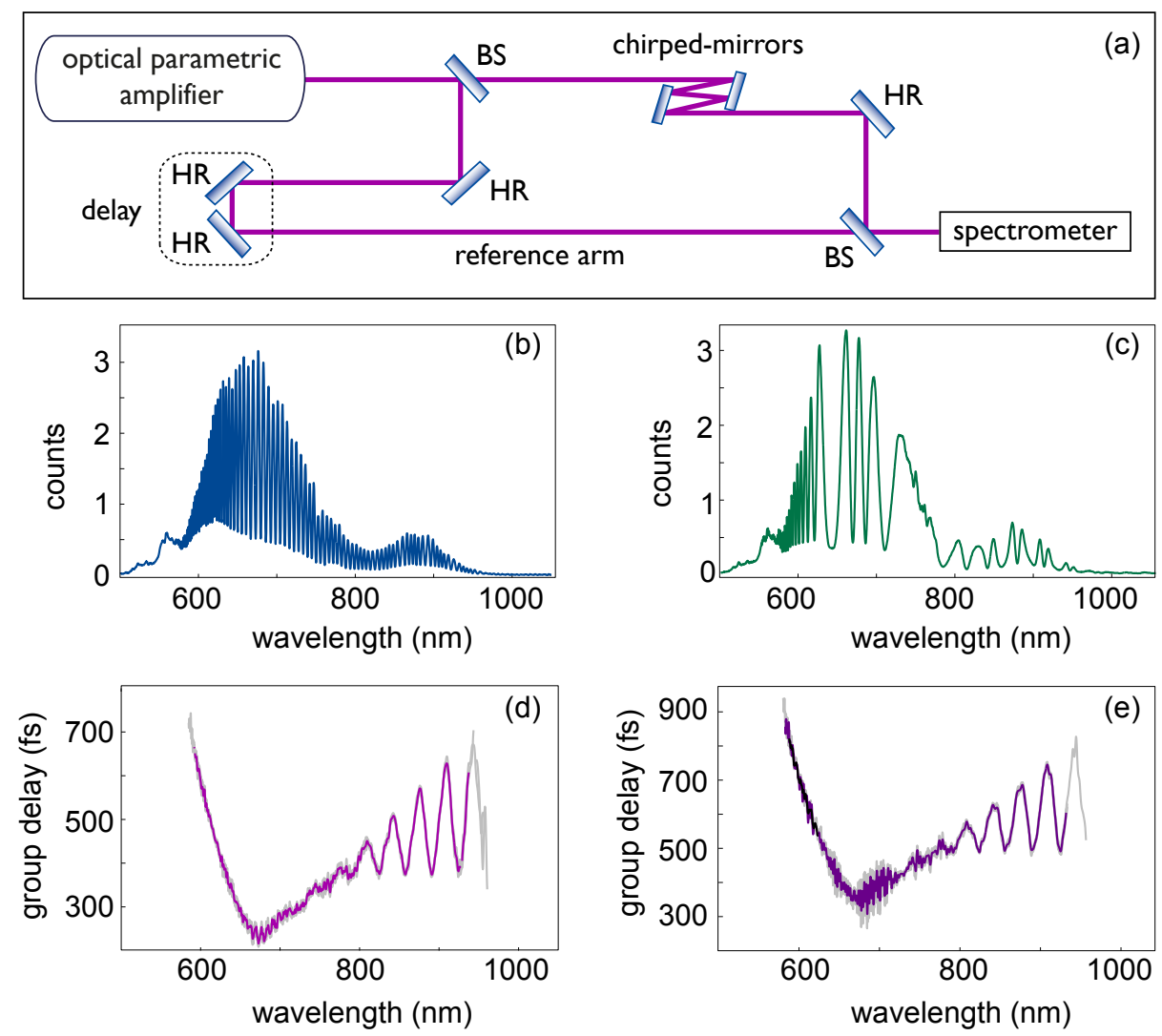

Figure A.1: a) Layout of the spectral interferometer setup; BS, beam splitter; HR, high reflector. b) Recorded spectrum with reference significantly temporally separated from signal. c) Spectrum with a smaller path length difference. d) Reconstructed group delay curve from b). e) Reconstructed group delay curve from c). $12 \mathrm{~mm}$ of glass was inserted in the compressor to remove some of the negative GDD so that the high-order terms were more visible. 



\section{Appendix B}

\section{Toolbox and ideas}

OPCPA simulations The numerical simulation of the tunable OPCPA system, the multi-channel synthesizer and case studies presented in Chapter 1 were performed using the SISYFOS code, developed by Dr. Gunnar Arisholm.

SISYFOS uses a Fourier-space method to simulate second-order nonlinear interactions. In this method, each beam is decomposed into plane-wave eigenmodes and the coupled differential equations are solved for the slowly varying eigenmode amplitudes (Arisholm, 1997).The code is capable of handling wide bandwidth, linear and two-photon absorption, thermal effects, nonlinear refraction, parasitic processes and noncollinear beams with possibly tilted pulse fronts. In the numerical studies in this thesis, thermal effects are not included. and the Sellmeier coefficients are taken from (Kato, 1994; Zelmon et al., 1997; Zhang et al., 2000).

Nick's photonics library The numerical studies of difference frequency generation, cross-polarized wave generation, spectral interferometry, and chaotic behavior in Yb-doped lasers were done using Nick's photonics library. This marvelous library was developed by genius Dr. Nicholas Karpowicz and in the case of cross-polarized wave generation calculation, it was modified in the least clever way by the author of this thesis!

HHG simulation For numerical studies of the single-dipole HHG response in Chapter 5, a code based on the Lewenstein model (Lewenstein et al., 1994) and developed by Dr. Valer Tosa was used (Moulet et al., 2014).

TAPIR idea The idea explained in Section 4.2, was conceived by Dr. Yunpei Deng (Deng and Krausz, 2013).

Patent Number: EP2742384, US14/0293405. 
Title: "Method and device for optical parametric chirped pulse amplification."

Inventors: Yunpei Deng, Ferenc Krausz. 


\section{Appendix C}

\section{Simulation details of Fig. 0.2}

Table C.1 summarizes the parameters of the simulations performed for OPCPA systems depicted with red triangles in Fig. o.2. In all calculations, a pumpto-seed energy ratio of $10^{3}$ is considered. The pump pulses have a Gaussian profile in time and space. For the temporal profile of the signal, a Gaussian distribution of the fourth order is assumed. LBO crystal with a noncollinear pump to signal angle of $1.1^{\circ}$ and a phase-matching angle of $14.4^{\circ}$ is used as a nonlinear medium. The $d_{\text {eff }}$ value is $0.819 \times 10^{-12} \mathrm{pm} / \mathrm{V}$. In all simulations, three-stages of OPCPA is considered while in the last stage the residual pump energy of the second stage is recycled. The amplified spectrum in all cases is centered at $1 \mu \mathrm{m}$. 


\begin{tabular}{|c|l|}
\hline \multicolumn{2}{|c|}{$300 \mathrm{~W}$, ps thin-disk regenerative amplifier at $10 \mathrm{kHz}$} \\
\hline pump laser & $30 \mathrm{~mJ}, 10 \mathrm{kHz}, 1.6 \mathrm{ps}$ \\
second harmonic & $21 \mathrm{~mJ}, 70 \%$ efficiency \\
pump peak intensity & $110 \mathrm{GW} / \mathrm{cm}^{2}$ \\
amplified energy/pulse duration & $6.1 \mathrm{~mJ} / 5 \mathrm{fs}$ \\
\hline Compact diode-pumped 1.1 kW Yb:YAG Innoslab femtosecond amplifier \\
\hline pump laser & $1.38 \mathrm{~mJ}, 800 \mathrm{kHz}, 7.3 \mathrm{ps}$ \\
second harmonic & $700 \mu \mathrm{J}, 50 \%$ efficiency \\
pump peak intensity & $40.7 \mathrm{GW} / \mathrm{cm}^{2}$ \\
amplified energy/pulse duration & $128 \mu \mathrm{J} / 5.24 \mathrm{fs}$ \\
\hline 200 mJ, ps X-CPA thin-disk regenrative amplifier at $5 \mathrm{kHz}$ \\
\hline pump laser & $200 \mathrm{~mJ}, 5 \mathrm{kHz}, 1.6 \mathrm{ps}$ \\
second harmonic & $140 \mathrm{~mJ}, 70 \%$ efficiency \\
pump peak intensity & $110 \mathrm{GW} / \mathrm{cm}^{2}$ \\
amplified energy/pulse duration & $40 \mathrm{~mJ} / 5 \mathrm{fs}$ \\
\hline Envisioned $2 \mathrm{~J}$, thin-disk booster at $10 \mathrm{kHz}$ \\
\hline pump laser & $2 \mathrm{~J}, 10 \mathrm{kHz}, 1.6 \mathrm{ps}$ \\
second harmonic & $1.4 \mathrm{~J}, 70 \%$ efficiency \\
pump peak intensity & $110 \mathrm{GW} / \mathrm{cm}^{2}$ \\
amplified energy/pulse duration & $345 \mathrm{~mJ} / 5 \mathrm{fs}$ \\
\hline
\end{tabular}

Table C.1: Summary of the simulated OPCPA systems depicted in Fig. 0.2 


\section{Appendix D}

\section{1-ps-pumped supercontinuum generation}

In a preliminary experiment, broadband supercontinuum was generated by focusing a small fraction of a $800 \mathrm{fs}$ pulses, centered at $1030 \mathrm{~nm}$ (Klingebiel et al., 2011) to a $20 \mathrm{~mm}$-long TGG crystal.

Single filament, stable regime of operation for the input pulse energies of $70 \mu \mathrm{J}$ to $150 \mu \mathrm{J}$ was achieved. In a $4 \mathrm{~mm}$-long KGW crystal the generated spectrum shows a different behavior which is originated from Raman effect (Fig. D.1-a,-b). Focusing the second harmonic of the laser in $4 \mathrm{~mm}$ YAG and $20 \mathrm{~mm}$ TGG, stable supercontinuum, with the cut-off below $500 \mathrm{~nm}$ was generated. As the photon energies are higher in this case, the damage of the crystal happened easier (Fig. D.1-c). Simultaneous focusing of the pulses at $1030 \mathrm{~nm}$ and $515 \mathrm{~nm}$ resulted in a broadband spectrum covering spectral component from $470 \mathrm{~nm}$ to $1400 \mathrm{~nm}$. These supercontinuum are not scalable in energy, as the generated filament in bulk can carry a certain amount of energy and for peak power above critical power Eq. (D.1), multi-filamentation happens.

$$
P_{c r}=\frac{\pi(0.61)^{2} \lambda_{0}^{2}}{8 n_{0} n_{2}}
$$

Their carrier envelope phase is not stable but they preserve the CEPstability of the pump pulse. Anti-stokes and stokes wings of such supercontinuum have a well-behaved spectral phase although it is distorted at the vicinity of the pump wavelength (Cerullo et al., 1997; Siddiqui et al., 2009), makes it difficult to compress a full supercontinuum spectrum(Antipenkov et al., 2011; Riedle et al., 2000). It is shown recently that the magnitude of this phase jump scales with the pulse duration of the the pump pulses (Harth et al., 2012). 
a)

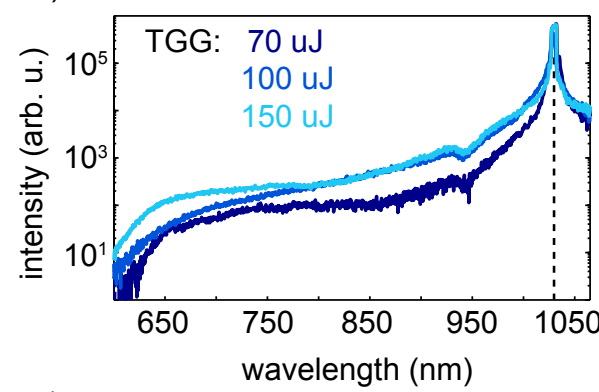

c)

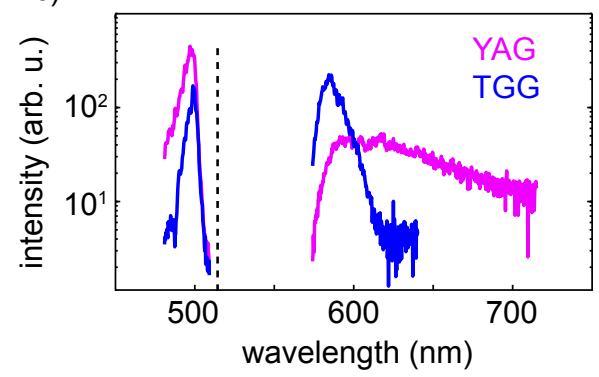

b)

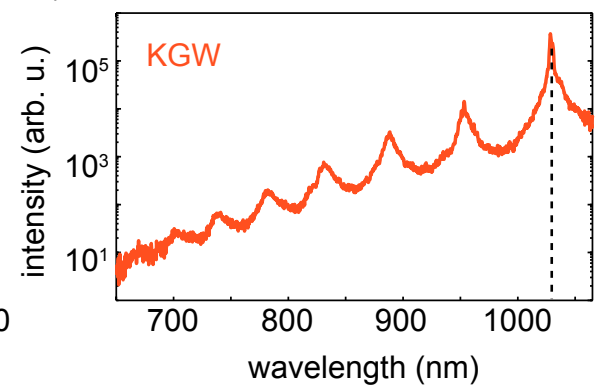

d)

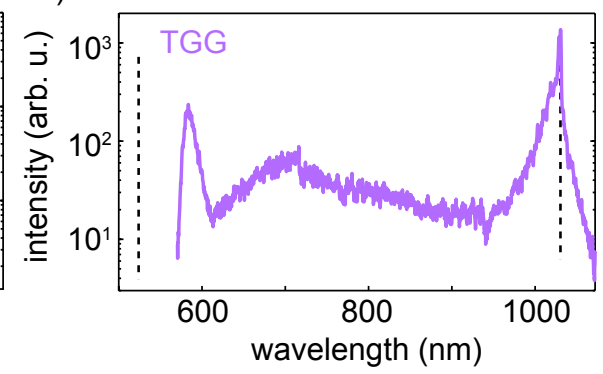

Figure D.1: a) Anti-stoke wing of the generated supercontinuum pumped by 1030-nm, 80o-fs pulses in 20-mm TGG crystal and b) the observed Raman effect in a 4-mm KGW crystal. c) Supercontinuum generation in 4-mm YAG and 20-mm TGG crystal pumped by $800-f s$ pulses and measured after a notch filter at $515-\mathrm{nm}$. $\omega-2 \omega$ pumped supercontinuum in 20-mm TGG. a long-pass filter at 515-nm and a short-pass filter at 1030-nm is used to filter out the pump beam. 


\section{Appendix E}

\section{Publications}

\section{E.1 Decoupling chaotic amplification and nonlinear phase in high-energy thin-disk amplifiers for stable OPCPA pumping}

This paper was published in Optics Express and is made available as an electronic reprint with the permission of OSA. The paper can be found at the following URL on the OSA website: http://www.opticsinfobase.org/oe/ abstract. cfm? uri=0e-22-25-31440. Systematic or multiple reproduction or distribution to multiple locations via electronic or other means is prohibited and is subject to penalties under law. 


\title{
Decoupling chaotic amplification and nonlinear phase in high-energy thin-disk amplifiers for stable OPCPA pumping
}

\author{
Hanieh Fattahi, ${ }^{1,2}$ Alexander Schwarz, ${ }^{1,2}$ Xiao Tao Geng, ${ }^{3,4}$ Sabine Keiber, ${ }^{1,2}$ Dong Eon \\ Kim, ${ }^{3,4}$ Ferenc Krausz, ${ }^{1,2}$ and Nicholas Karpowicz ${ }^{*}$ \\ ${ }^{1}$ Max-Planck-Institut für Quantenoptik, Hans-Kopfermann-Straße 1, 85748 Garching, Germany \\ ${ }^{2}$ Department of Physics, Ludwig-Maximilians-Universität München, Am Coulombwall 1, 85748 Garching, Germany \\ ${ }^{3}$ Department of Physics, Center for Attosecond Science and Technology, Pohang University of Science and \\ Technology, Pohang, 790-784, South Korea \\ ${ }^{4}$ Max Planck Center for Attosecond Science, Pohang, 790-784, South Korea \\ nicholas.karpowicz@mpq.mpg.de
}

\begin{abstract}
The dynamics of chirped pulse amplification in thin-disk regenerative amplifiers relevant to the pumping of optical parametric chirp pulse amplification systems are described. It is shown that the suitability for reproducible pumping of subsequent nonlinear processes requires a balance between the demands of avoiding chaotic pulse train dynamics and providing a reproducible spectral phase. We describe measures that may be taken to ensure that a laser system operates in the desired stable regime.
\end{abstract}

(C)2014 Optical Society of America

OCIS codes: (140.0140) Lasers and laser optics; (140.1540) Chaos; (140.3480) Lasers, diodepumped; (140.3615) Lasers, ytterbium.

\section{References and links}

1. F. Krausz and M. I. Stockman, "Attosecond metrology: from electron capture to future signal processing," Nat. Photonics 8(3), 205-213 (2014).

2. H. Fattahi, H. G. Barros, M. Gorjan, T. Nubbemeyer, B. Alsaif, C. Y. Teisset, M. Schultze, S. Prinz, M. Haefner, M. Ueffing, A. Alismail, L. Vámos, A. Schwarz, O. Pronin, J. Brons, X. T. Geng, G. Arisholm, M. Ciappina, V. S. Yakovlev, D.-E. Kim, A. M. Azzeer, N. Karpowicz, D. Sutter, Z. Major, T. Metzger, and F. Krausz, "Thirdgeneration femtosecond technology," Optica 1(1), 45 (2014).

3. A. Buck, M. Nicolai, K. Schmid, C. M. S. Sears, A. Sävert, J. M. Mikhailova, F. Krausz, M. C. Kaluza, and L. Veisz, "Real-time observation of laser-driven electron acceleration," Nat. Phys. 7(7), 543-548 (2011).

4. A. Dubietis, G. Jonušauskas, and A. Piskarskas, "Powerful femtosecond pulse generation by chirped and stretched pulse parametric amplification in BBO crystal," Opt. Commun. 88(4-6), 437-440 (1992).

5. H. Fattahi, C. Skrobol, M. Ueffing, Y. Deng, A. Schwarz, Y. Kida, V. Pervak, T. Metzger, Z. Major, and F. Krausz, "High efficiency, multi-mJ, sub $10 \mathrm{fs}$, optical parametric amplifier at $3 \mathrm{kHz}$," in Conference on Lasers and Electro-Optics 2012 (OSA, 2012), p. CTh1N.6.

6. T. Metzger, A. Schwarz, C. Y. Teisset, D. Sutter, A. Killi, R. Kienberger, and F. Krausz, "High-repetition-rate picosecond pump laser based on a Yb:YAG disk amplifier for optical parametric amplification,” Opt. Lett. 34(14), 2123-2125 (2009).

7. C. Teisset, M. Schultze, R. Bessing, M. Haefner, S. Prinz, D. Sutter, and T. Metzger, " $300 \mathrm{~W}$ Picosecond ThinDisk Regenerative Amplifier at $10 \mathrm{kHz}$ Repetition Rate," in Advanced Solid-State Lasers Congress Postdeadline, G. and M. P. Huber, ed. (OSA, 2013), p. JTh5A.1.

8. M. Schulz, R. Riedel, A. Willner, T. Mans, C. Schnitzler, P. Russbueldt, J. Dolkemeyer, E. Seise, T. Gottschall, S. Hädrich, S. Duesterer, H. Schlarb, J. Feldhaus, J. Limpert, B. Faatz, A. Tünnermann, J. Rossbach, M. Drescher, and F. Tavella, "Yb:YAG Innoslab amplifier: efficient high repetition rate subpicosecond pumping system for optical parametric chirped pulse amplification," Opt. Lett. 36(13), 2456-2458 (2011).

9. C. Jauregui, J. Limpert, and A. Tünnermann, "High-power fibre lasers," Nat. Photonics 7(11), 861-867 (2013).

10. C. Hönninger, R. Paschotta, M. Graf, F. Morier-Genoud, G. Zhang, M. Moser, S. Biswal, J. Nees, A. Braun, G. A. Mourou, I. Johannsen, A. Giesen, W. Seeber, and U. Keller, "Ultrafast ytterbium-doped bulk lasers and laser amplifiers," Appl. Phys. B Lasers Opt. 69(1), 3-17 (1999).

11. J. Dörring, A. Killi, U. Morgner, A. Lang, M. Lederer, and D. Kopf, "Period doubling and deterministic chaos in continuously pumped regenerative amplifiers," Opt. Express 12(8), 1759-1768 (2004).

12. M. Grishin, V. Gulbinas, and A. Michailovas, "Bifurcation suppression for stability improvement in Nd:YVO4 regenerative amplifier,” Opt. Express 17(18), 15700-15708 (2009). 
13. M. Grishin, V. Gulbinas, and A. Michailovas, "Dynamics of high repetition rate regenerative," Opt. Express 15, 9434-9443 (2007).

14. M. D. Perry, T. Ditmire, and B. C. Stuart, "Self-phase modulation in chirped-pulse amplification," Opt. Lett. 19(24), 2149-2151 (1994).

15. Y.-H. Chuang, L. Zheng, and D. D. Meyerhofer, "Propagation of light pulses in a chirped-pulse-amplification laser," IEEE J. Quantum Electron. 29(1), 270-280 (1993).

16. A. Antognini, K. Schuhmann, F. D. Amaro, F. Biraben, A. Dax, A. Giesen, T. Graf, T. W. Hänsch, P. Indelicato, L. Julien, C. Kao, P. E. Knowles, F. Kottmann, E. Le Bigot, Y. Liu, L. Ludhova, N. Moschüring, F. Mulhauser, T. Nebel, F. Nez, P. Rabinowitz, C. Schwob, D. Taqqu, and R. Pohl, "Thin-Disk Yb : YAG Oscillator-Amplifier Laser, ASE, and Effective Yb : YAG Lifetime," IEEE J. Quantum Electron. 45(8), 993-1005 (2009).

17. B. Chen, J. Dong, M. Patel, Y. Chen, A. Kar, and M. A. Bass, "Modeling of high-power solid-state slab lasers," in SPIE Proceeding, R. Scheps, ed. (2003), pp. 1-10.

18. U. Brauch, A. Giesen, M. Karszewski, C. Stewen, and A. Voss, "Multiwatt diode-pumped Yb:YAG thin disk laser continuously tunable between 1018 and 1053 nm," Opt. Lett. 20(7), 713-715 (1995).

19. M. Baumgartl, F. Jansen, F. Stutzki, C. Jauregui, B. Ortaç, J. Limpert, and A. Tünnermann, "High average and peak power femtosecond large-pitch photonic-crystal-fiber laser," Opt. Lett. 36(2), 244-246 (2011).

20. T. Eidam, J. Rothhardt, F. Stutzki, F. Jansen, S. Hädrich, H. Carstens, C. Jauregui, J. Limpert, and A. Tünnermann, "Fiber chirped-pulse amplification system emitting $3.8 \mathrm{GW}$ peak power," Opt. Express 19(1), 255$260(2011)$.

21. A. Schwarz, M. Ueffing, Y. Deng, X. Gu, H. Fattahi, T. Metzger, M. Ossiander, F. Krausz, and R. Kienberger, "Active stabilization for optically synchronized optical parametric chirped pulse amplification," Opt. Express 20(5), 5557-5565 (2012).

22. J. Ratner, G. Steinmeyer, T. C. Wong, R. Bartels, and R. Trebino, "Coherent artifact in modern pulse measurements," Opt. Lett. 37(14), 2874-2876 (2012).

23. G. Arisholm, "General numerical methods for simulating second-order nonlinear interactions in birefringent media,” J. Opt. Soc. Am. B 14(10), 2543 (1997).

\section{Introduction}

The generation of high energy, few-cycle laser pulses at a high repetition rate is of great interest, as progress in fields such as attosecond science [1,2] and high field physics [3] benefits directly from the availability of such sources. Optical parametric chirped pulse amplification (OPCPA) represents the most promising route to simultaneously achieving high pulse energies and large amplification bandwidths supporting intense, few-cycle pulses [4]. To push the frontiers of ultrafast pulse generation, pump lasers delivering sub-10 ps, highenergy pulses with near diffraction limited beam quality, ideally at repetition rates in the $\mathrm{kHz}$ to $\mathrm{MHz}$ range, are required $[2,5]$.

Amplifiers based on Yb-doped gain media in disk [6,7], slab [8] or fiber [9] geometries are a most promising route as ytterbium has a broad absorption in a spectral region accessible by efficient laser diodes [6,10]. The long fluorescence lifetime of ytterbium makes it an ideal candidate for regenerative amplifiers as it supports substantial population inversion under continuous pumping. However, in continuously pumped regenerative amplifiers when the pulse repetition rate becomes comparable to the inverse of the gain relaxation time, bifurcation and chaotic pulse train dynamics can restrict the operating parameters of the laser system [11,12]. These instabilities can be suppressed by increasing the input seed energy to the regenerative amplifier [13], which may require additional stages of pre-amplification, or by increasing the pump intensity or number of passes of the pulse through the cavity, each of which may have negative consequences in terms of accumulation of nonlinear phase or intracavity power levels approaching the damage threshold of the optical elements. As a consequence, a regenerative amplifier may have excellent energy stability but exhibit variations in the temporal pulse shape that spoil the reproducibility of subsequent nonlinear processes $[14,15]$. It is therefore essential to both understand and manage these effects before a reliable OPCPA can be realized.

\section{Theoretical description and experiment}

In regenerative amplifiers operating at repetition rates close to the inverse of the inversion build-up time in the gain medium, the gain experienced by a pulse in the cavity is strongly coupled to the depletion caused by the previous pulse. This can result in nonlinear 
amplification dynamics, including both stable regimes such as period doubling (bifurcation) and chaotic regimes, where subsequent pulses exhibit extreme, seemingly random variations

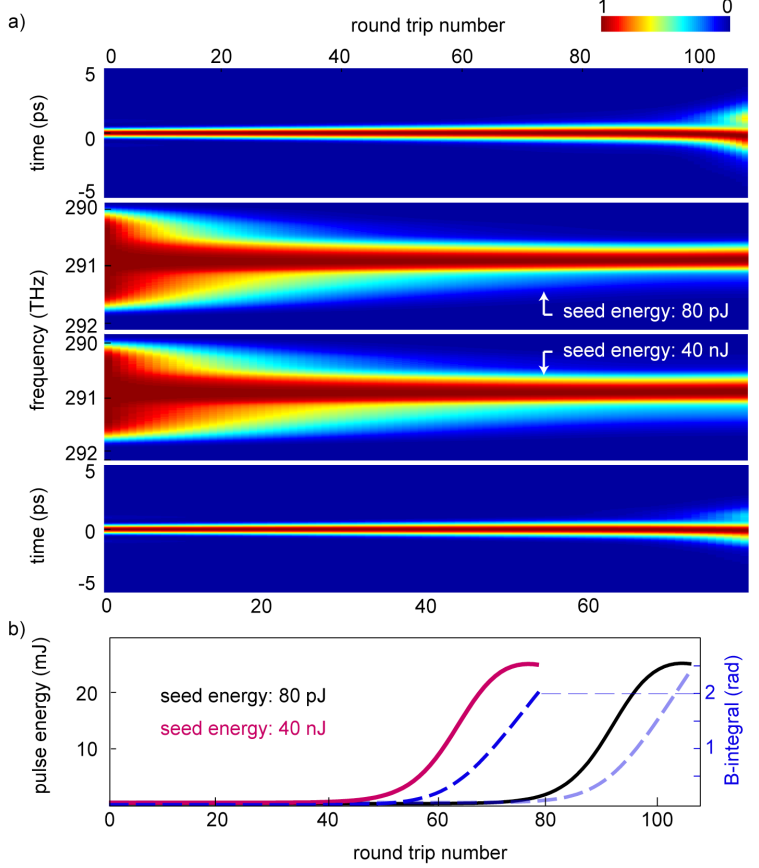

Fig. 1. (a) Simulated temporal and spectral profiles of the amplified pulses versus round trip number in a regenerative amplifier for two different input seed energy: $80 \mathrm{pJ}$ and $40 \mathrm{~nJ}$ (amplitudes normalized). (b) Pulse build up in the regenerative amplifier for two different input seed energies, along with the build-up of B-integral in the cavity. The pulse distorts rapidly as the B-integral exceeds 2 for this output spectrum.

in energy. For example, consider the case of a pulse in a continuous-wave-pumped regenerative amplifier whose gain medium had been entirely depleted by the previous pulse. In the time between the exit of the previous pulse from the cavity and the entrance of the new pulse, the inversion in the medium was not sufficiently recovered to produce a high gain for the new pulse. As a result, its energy remains low for its time in the cavity, and it is unable to extract a significant amount of energy. By the time the next pulse comes, the inversion has recovered to a level where there is significant gain, the new pulse amplifies quickly and carries away a large amount of energy, leaving the medium depleted. This cycle then repeats, creating a stable energy bifurcation, with only every other pulse being amplified. If the inversion can recover to the same level between all pulses, the laser operates "normally", with each pulse entering the cavity exiting with the same energy. This can be ensured by increasing the number of round trips through the cavity, such that at a given seed and pump power level, each pulse will entirely deplete the medium.

In regenerative amplifiers with low input seed energy, a large number of round trips (passes through the gain medium) are required to reach energy saturation. This means a larger number of passes through the Pockels cell (PC) and air, increasing the accumulation of nonlinear phase on the amplified pulses. This self-phase modulation (SPM) consequently influences the temporal and spectral shape of the pulse. In the SPM process the interference between the newly generated frequencies and the spectrum of the pulse causes a decrease or increase in the spectral bandwidth of the pulse. For heavily chirped pulses, however, SPM primarily influences the spectral phase of the pulse, leaving the shape of the spectrum unchanged. To study the effect of B-integral in low-energy-seeded regenerative amplifiers, a 
simple 1-D model propagating the gain medium inversion and wavelength-dependent pulse energy buildup for the full pulse train is developed, where the nonlinearity on the amplified pulse caused by the PC and air is calculated in each round trip. The populations in the ground and excited state manifolds are calculated based on the rate equations for continuouslypumped Yb:YAG [16] and the gain in each pass is obtained via the temperature-dependent emission cross-section according to [17].

The detailed simulation parameters are as follows: A seed pulse with $10 \mathrm{~nm}$ bandwidth is stretched using $1.19 \times 10^{8} \mathrm{fs}^{2}$ dispersion, to approximately $2 \mathrm{~ns}$. After entering the cavity, the pulse travels $40 \mathrm{~ns}$ in each round trip, with a beam waist of $3.4 \mathrm{~mm}\left(1 / \mathrm{e}^{2}\right)$ on the $20-\mathrm{mm}$-long PC (twice per round trip), similar to [6] and with an assumed nonlinear refractive index of $2.99 \times 10^{-20} \mathrm{~cm}^{2} / \mathrm{W}$. The nonlinearity of the gain medium is neglected as it is small compared to that of the PC. $225 \mathrm{~W}$ of pump power with a $2.8 \mathrm{~mm}$ beam waist is used to pump the 100 $\mu \mathrm{m}$-thick gain medium with $12.5 \%$ doping and $1.2 \mathrm{~ms}$ upper state lifetime, with the assumption of $90 \%$ absorption of the incident pump light and $95.5 \%$ overlap of the pumped energy with the cavity mode. The Yb:YAG temperature is $300 \mathrm{~K}$, and the emission spectrum as determined by [18] is used to determine the spectral profile of the gain. The mode of the seed is assumed match that of the cavity. The cavity round trip transmission efficiency is $98 \%$ and each trip takes $40 \mathrm{~ns}$. The repetition rate is set to $3 \mathrm{kHz}$, such that the inversion rebuilds under constant pumping conditions for $333 \mu$ s between pulses. The pulse passes twice through the disk during each round trip. The SPM is calculated in each round trip so that SPM-induced spectral changes will influence the gain and efficiency of the amplifier. The simulation is run over 120 pulses in the pulse train to reach a steady state. The same parameters are used in all calculations.

The calculation is shown in Fig. 1 for two input seed energies, $80 \mathrm{pJ}$ and $40 \mathrm{~nJ}$. Figure 1 compares the pulse build up and spectral and temporal behavior of the amplified pulses for these two cases. For $80 \mathrm{pJ}$ seed energy, 107 round trips are required to reach saturation. At this number of the round trips, the accumulated B-integral causes undesired higher-order nonlinear phase on the amplified pulses, which makes the compression of the pulse to its Fourier transform limit challenging. The spectral narrowing versus the round trip number visible in Fig. 1 is caused by the limited gain bandwidth of the medium. Increasing the input seed energy to $40 \mathrm{~nJ}$ will reduce the required number of round trips to reach saturation to 90 . In this case the introduced nonlinear phase on the pulse is negligible and leaves the temporal phase of the pulse well-behaved. The difference is due to two factors: the reduced nonlinear path through the regenerative amplifier, and more significantly, the reduction of gain narrowing, which results in a longer pulse inside of the cavity after amplification to high energy, and a correspondingly lower intensity. When the B-integral exceeds approximately 2 , the pulse rapidly distorts. This, combined with the fact that the rate of increase of the nonlinear phase is maximized when the amplifier reaches energy saturation, has the end result that there is a sharp threshold where nonlinear phase fluctuations may easily exceed energy fluctuations as a source of changes in the peak power of the laser output. The exact pulse deformation depends on the spectral profile of the amplified light - with linear chirping, the profile of the spectral intensity is imprinted onto the spectral phase.

A Yb:YAG thin-disk regenerative amplifier, as described in [6], is used for experimental verification of these effects in this study. In this system, the $1 \mathrm{pJ}$ pulses centered at $1030 \mathrm{~nm}$, derived from a Ti:sapphire oscillator, were amplified to $80 \mathrm{pJ}$ exploiting a diode-pumped, fiber pre-amplifier $[9,19,20]$. A second fiber pre-amplifier was placed after the pulse picker for further amplification of seed pulses up to $40 \mathrm{~nJ}$. At the seed energy level of $80 \mathrm{pJ}$ and 107 cavity round trips, after intermediate chaos, period doubling and a stable regime of operation was achieved resulting in $20 \mathrm{~mJ}$ pulse energy at $3 \mathrm{kHz}$ repetition rate after passing through a grating pair for temporal compression. The SHG-FROG measurement indicates that the accumulated nonlinear phase, due to the large number of round trips, distorted the temporal phase of the amplified pulse, without affecting the spectrum. 


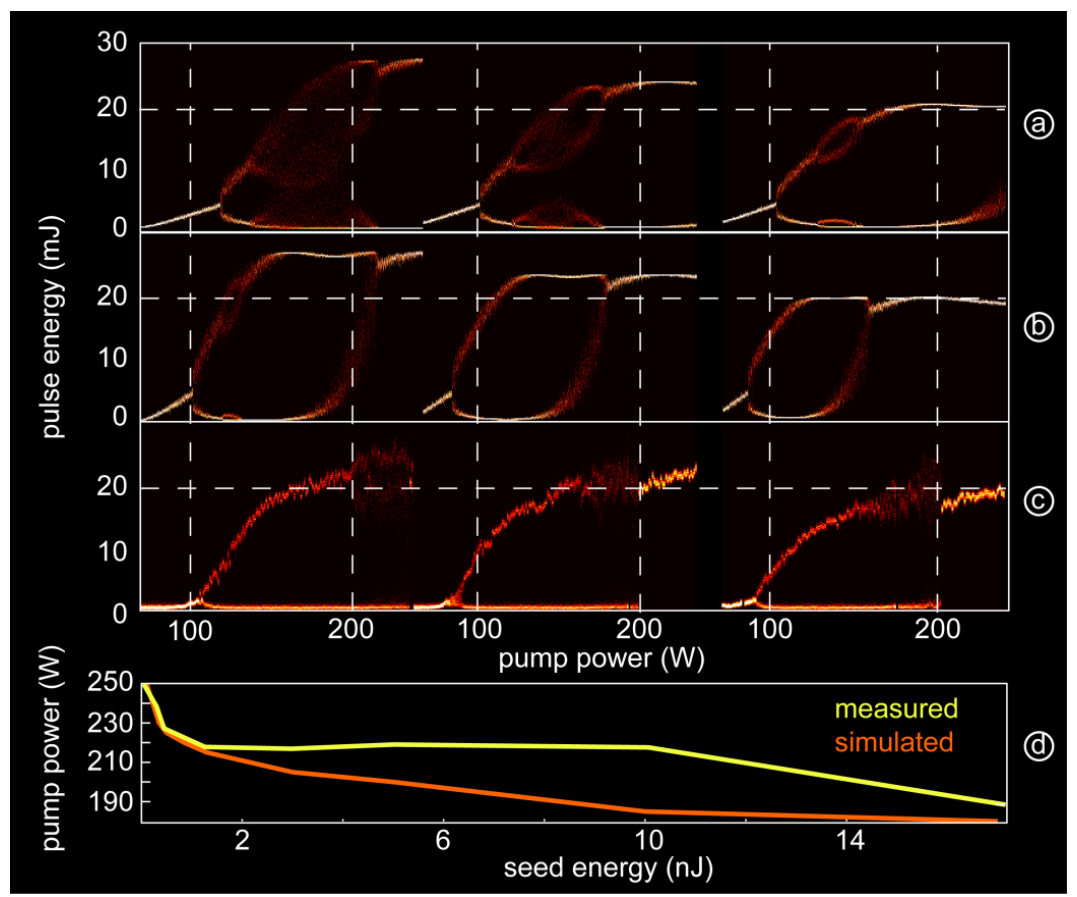

Fig. 2. Simulated and measured pulse train dynamics for CW-pump Yb:YAG thin-disk amplifiers for varying seed levels and repetition rates. (a) Simulated pulse train dynamics at 6 $\mathrm{kHz}$ Pockels cell rate, exhibiting chaotic behavior at low seed energies and stable bifurcation at higher pump powers. (b) The same system simulated at $3 \mathrm{kHz}$, showing the elimination of chaos at all powers and the removal of bifurcation at higher pump powers. (c) Measured dynamics at $3 \mathrm{kHz}$. (d) Required pump power for stable operation as a function of pulse energy at $3 \mathrm{kHz}$.

By increasing the input seed energy, a stable regime of operation with no chaotic behavior and no period doubling was observed. Figure 2 shows the energy distribution in a bifurcation diagram acquired by incrementing the pump power over the range of 50-250 W with the number of round trips in the ampli $\square$ er being kept constant at 100 . For a cavity seeded with 0.1 $\mathrm{nJ}$ of energy, at $100 \mathrm{~W}$ of pump power, bifurcation occurred while the $\mathrm{PC}$ was driven at $3 \mathrm{kHz}$ repetition rate. Increasing the pump power to $250 \mathrm{~W}$ lead to repetition rate preserving amplification. Increasing the input seed energy shifted both the bifurcated and normal amplification points to lower pump powers. The measured data are consistent with the simulation, indicating no chaotic behavior for the case of high energy seeding. In the case of 6 $\mathrm{kHz}$ operation, chaotic behavior appears, as measured in [6], resulting from the stronger pulse-to-pulse coupling. Period doubling persists to higher pump powers, and reaches a stable doubling regime, whose location and separation from chaotic zones is determined by the seed energy. Under the same conditions, when the PC is operated at $6 \mathrm{kHz}$ in period doubling, normal operation may be obtained with the $\mathrm{PC}$ run at $3 \mathrm{kHz}$, reducing the thermal load on the PC crystal and simplifying synchronization with OPCPA seed sources [21]. It is noteworthy that the severity of the nonlinear cavity dynamics decreases as the seed level increases; this is due to the reduced sensitivity of the amplification to the process to the degree of inversion of the medium for an amplifier with lower total gain. This has the end result that wider range of stable operating points is available with higher seed levels, making it more likely that a mode with low B-integral and the desired repetition rate can be found.

The primary difference between the simulation and the experiment is in the tendency of the laser to emerge from bifurcation and stay in the normal operation regime even if the pump power is slightly reduced (hysteresis), due to the fact that the cavity is optimized for high- 
power operation, including thermal effects not present in the simulation: the cavity efficiency increases slightly as the system enters the normal amplification mode and the output power doubles.
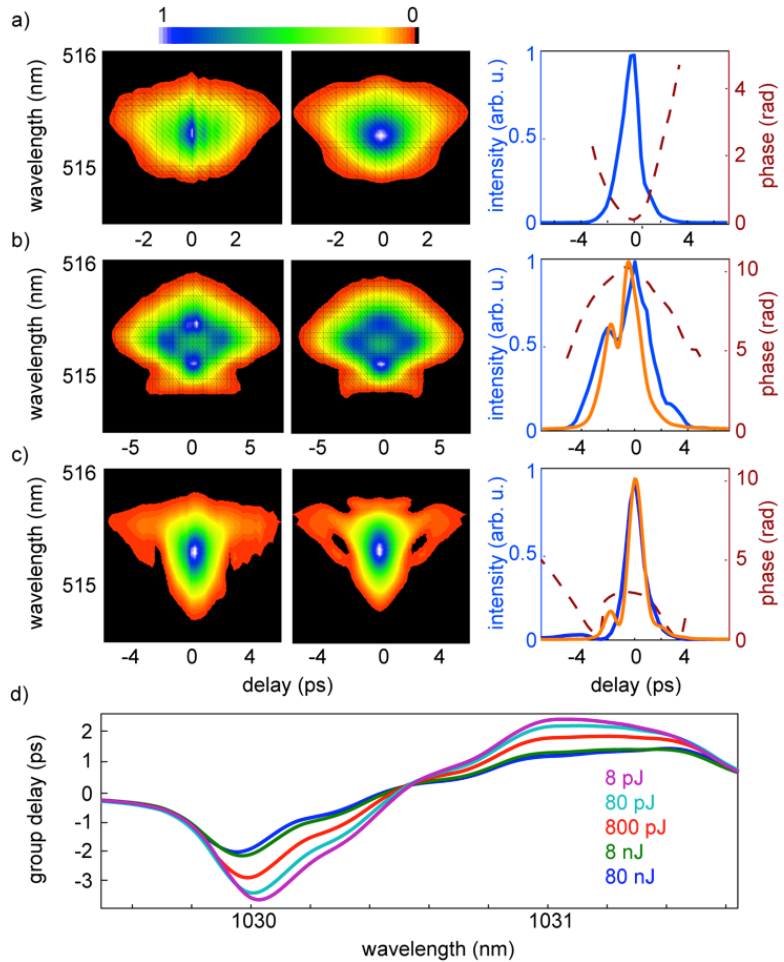

Fig. 3. Pulse dynamics in regenerative amplifier exhibiting SPM and its removal. (a) unsaturated SHG-FROG trace using $80 \mathrm{pJ}$ seed amplified to $14 \mathrm{~W}$ (unstable laser output in the unsaturated regime). (b) $80 \mathrm{pJ}$ seed amplified to $58 \mathrm{~W}$, exhibiting strong SPM. (c) $300 \mathrm{pJ}$ seed amplified to $61 \mathrm{~W}$ with increased pump power and reduced round trips, exhibiting minimal SPM. (d) Additional group delay resulting from nonlinear phase as calculated for varying seed levels, with sufficient number of round trips to reach saturation.

Figure 3 shows how the cavity dynamics affect the temporal profile of the compressed pulse. In Fig. 3(a) the unsaturated output of a laser system seeded with $80 \mathrm{pJ}$, amplified to 14 $\mathrm{W}$ shows a nearly transform-limited profile, but after increasing the energy to $58 \mathrm{~W}$ in Fig. 3(b), the pulse is obviously distorted by SPM. After increasing the seed energy to just $300 \mathrm{pJ}$ and reducing the round trip number by 10 (combined with additional pump power) the compressed pulse characterized with a SHG-FROG indicates a good temporal profile for the pulse (i.e. SPM effects are negligible), at an average power of $61 \mathrm{~W}$ (Fig. 3(c)). A sufficient amount of pre-amplification of pJ-level seed pulses is therefore essential for the reproducibility of the peak power produced by the amplifier. In Fig. 3(d), the numerical results showing the effect of seed energy on the nonlinear group delay distortion of the output pulses are shown, explaining the difference in the three FROG traces. Additionally, the trace in Fig. 3(b) reconstructs with significantly more G-error than the corrected trace in Fig. 3(c), 0.022 compared to 0.0087 , respectively. This may indicate pulse-to-pulse variations in shape [22].

The elimination of SPM effects can be attempted via several approaches, some entailing compromises in other areas of laser performance or system complexity. A number of possible approaches, along with their mechanisms and drawbacks are shown in Table 1, in order of approximate overall difficulty. As opposed to lasers exhibiting no chaotic behavior, the 
simple solution of reducing the pump intensity does not work for these systems: the laser must operate with both a stable pulse train (at the correct repetition rate) and have a clean spectral phase. This can only be accomplished by decoupling the pulse distortion of the pulse from the nonlinear pulse train dynamics by the use of methods that do not affect both characteristics to the same degree.

Table 1. Possible approaches to the reduction of SPM in a thin disk regenerative amplifier

\begin{tabular}{|c|c|c|}
\hline Approach & Mechanisms & Negative consequences \\
\hline $\begin{array}{l}\text { Reduce round } \\
\text { trips with higher pump } \\
\text { power }\end{array}$ & $\begin{array}{l}\text { - Reduced nonlinear path length } \\
\text { - Reduced cavity losses leading to less } \\
\text { gain narrowing at same energy }\end{array}$ & $\begin{array}{l}\text { - Increased thermal load } \\
\text { - Smaller range of stable } \\
\text { operating power }\end{array}$ \\
\hline $\begin{array}{l}\quad \text { Increase seed } \\
\text { level via further } \\
\text { broadband pre- } \\
\text { amplification }\end{array}$ & $\begin{array}{l}\text { - Reduced gain narrowing } \\
\text { - Reduced nonlinear propagation } \\
\text { distance via reduced round trip number }\end{array}$ & $\begin{array}{l}\text { - Increased system } \\
\text { complexity/cost } \\
\text { - Longer seed beam path }\end{array}$ \\
\hline $\begin{array}{l}\text { Decrease pump } \\
\text { spot size }\end{array}$ & $\begin{array}{l}\text { - Faster inversion buildup for given } \\
\text { pump power, allowing stable operation at } \\
\text { lower power }\end{array}$ & $\begin{array}{l}\text { - Increased thermal } \\
\text { gradient in gain medium } \\
\text { - Reduced saturation } \\
\text { energy }\end{array}$ \\
\hline $\begin{array}{l}\text { Increase } \\
\text { stretcher and } \\
\text { compressor dispersion }\end{array}$ & $\begin{array}{l}\text { - Reduced intensity at given pulse } \\
\text { energy }\end{array}$ & $\begin{array}{l}\text { - Increased cost and } \\
\text { alignment sensitivity of } \\
\text { compressor and stretcher } \\
\quad \text { - Additional timing jitter } \\
\text { when synchronized with } \\
\text { external seed }\end{array}$ \\
\hline $\begin{array}{l}\text { Increased beam } \\
\text { size in nonlinear cavity } \\
\text { elements via reflective } \\
\text { telescope }\end{array}$ & $\begin{array}{l}\text { - If a single element (i.e. Pockels cell) } \\
\text { is responsible, reduced intensity }\end{array}$ & $\begin{array}{l}\text { - Increased complexity of } \\
\text { the laser cavity } \\
\text { - Requires larger cavity } \\
\text { optics }\end{array}$ \\
\hline $\begin{array}{l}\text { Operation in } \\
\text { vacuum }\end{array}$ & path - Reduced nonlinearity of free beam & $\begin{array}{l}\text { - Cost and complexity } \\
\text { - More difficult thermal } \\
\text { management }\end{array}$ \\
\hline
\end{tabular}

\section{Effect of suboptimal amplifier design on OPCPA}

In the event that significant SPM can be observed in the pulse emitted from such an amplifier, it is likely that it cannot be used reproducibly until it is eliminated, e.g. by the design changes listed above. In the case of significant SPM, small changes in the seed and cavity alignment result in large variations of the output of an OPCPA pumped by such a pulse. These effects are shown in Fig. 4, where pulses with the same energy and spectrum, but with varying levels of SPM result in significant variations in the efficiency and spectrum of the pulses amplified in a broadband OPCPA. As a result, even in the total absence of energy fluctuations in the output of a saturated amplifier, variations of the spectral phase induced by the sensitivity of the SPM process to the evolution of the pulse inside of the cavity can nevertheless result in large fluctuations in the OPCPA.

This is shown in the simulations presented in Fig. 4, calculated using the SISYFOS 3D code [23]. In the simulation, 20-mJ, 1030-nm pump pulses with the beam diameter of $3.3 \mathrm{~mm}$ at full width half maximum are used to amplify a broad spectral range in a single OPCPA stage. The OPCPA consists of a $2.7 \mathrm{~mm}$-thick $\mathrm{LiNbO}_{3}$ crystal with $\mathrm{d}_{\text {eff }}$ value of $3.3 \mathrm{pm} / \mathrm{V}$, and a phase-matching angle of $45.25^{\circ}$. Here the broadband phase-matching is fulfilled as the OPA works in degeneracy. Seed pulses with energy of $3 \mu \mathrm{J}$ and beam diameter of $3.3 \mathrm{~mm}$ are considered. The pulses from Figs. 3(b)-3(c) are used as the temporal profile of pump pulses in the simulation whereas a super-Gaussian of the order of four is assumed for the seed in the time domain. The 1.6-ps seed pulses are linearly chirped in both cases. The thickness of the $\mathrm{LiNbO}_{3}$ crystal is optimized to reach gain saturation in the OPCPA when the temporal profile of pump is taken from the measurement shown in Fig. 3(c). Using identical parameters for OPCPA and pump pulses with accumulated nonlinear phase (Fig. 3(b)), results in a factor of 
3.5 difference in amplified energy and decreases the conversion efficiency from $12 \%$ to $2.2 \%$. Here the OPA does not reach gain saturation anymore and the amplified spectrum becomes modulated in consistent with the temporal shape of the pump pulses.

a)

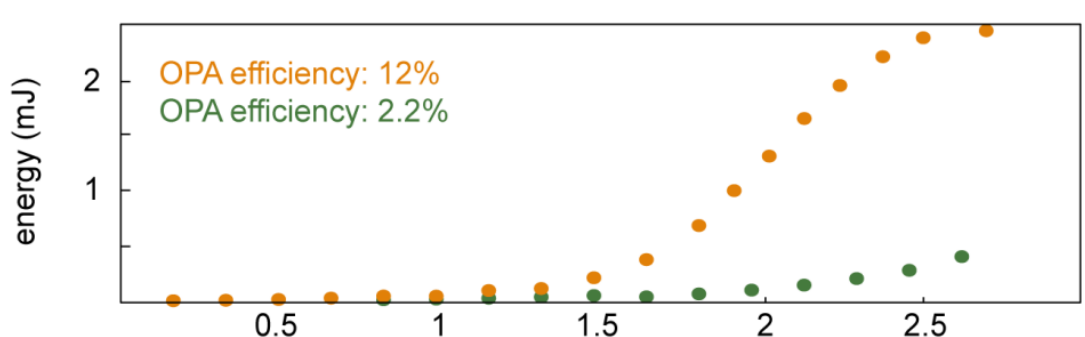

b)

crystal length $(\mathrm{mm})$

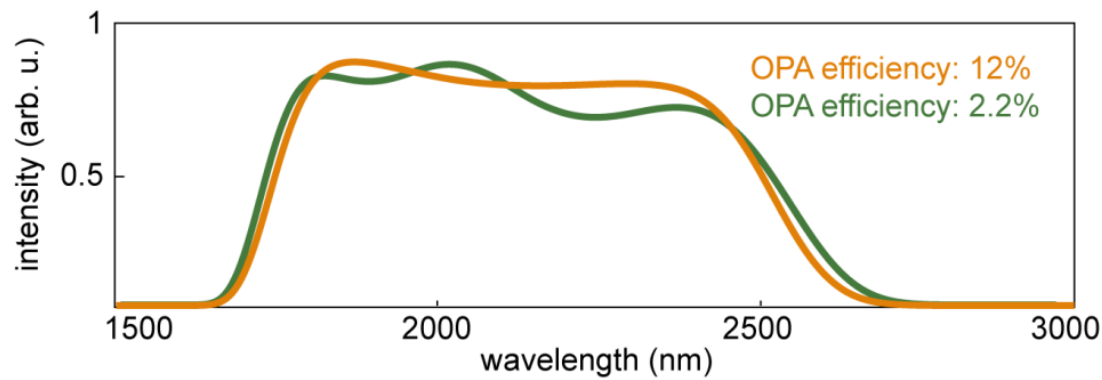

Fig. 4. Simulation of the effects of amplifier SPM on the output of a broadband OPCPA. Orange curves are simulated with the pulse measured in Fig. 3 (c) and green curves with the pulse in Fig. 3 (b), all other parameters are the same: pump energy $20 \mathrm{~mJ}$, seed energy $3 \mu \mathrm{J}$ amplified in $2.7 \mathrm{~mm} \mathrm{LiNbO}_{3}$ with the phasematching angle $45.25^{\circ}$. (a) Gain vs. crystal length in the OPCPA, showing saturation with the SPM-free pulse, and a factor of 3.5 reduction in pulse energy with the distorted pump pulse. (b) Modification of the spectrum resulting from the pump temporal profile (normalized).

\section{Conclusion}

Stable OPCPA demands not only excellent pump energy stability, but also a high degree of reproducibility of the temporal shape of the pump pulses, which can only be ensured by careful matching of the amplifier design to the seed specifications. The relevant effects can be modeled and understood using a simple numerical approach, which when combined with an awareness of the various trade-offs involved, allows an optimal design for a given set of inputs and target parameters. By ensuring that these effects are well controlled, thin-disk regenerative amplifiers are an ideal route to stable and efficient pumping of high-power, highenergy broadband OPCPAs, enabling the exploration of new frontiers in ultrafast science.

\section{Acknowledgments}

We gratefully acknowledge helpful discussions with Gunnar Arisholm and valuable technical support from the group of Jens Limpert and also support from LASERLAB-EUROPE (grant agreement no. 284464, EC's Seventh Framework Programme), the Munich-Centre for Advanced Photonics, and the German Federal Ministry of Education and Science (BMBP) via the NEXUS project. Dong Eon Kim and Xiao Tao Geng thank the support from Global Research Laboratory Program [Grant No 2009-00439] and Max Planck POSTECH/KOREA Research Initiative Program [Grant No 2011-0031558] through the National Research Foundation of Korea (NRF) funded by Ministry of Science, ICT \& Future Planning. 


\section{E.2 Third-generation femtosecond technology}

This paper was published in Optica and is made available as an electronic reprint with the permission of OSA. The paper can be found at the following URL on the OSA website: http://www . opticsinfobase. org/optica/ abstract. cfm?uri=optica-1-1-45. Systematic or multiple reproduction or distribution to multiple locations via electronic or other means is prohibited and is subject to penalties under law. 


\section{Third-generation femtosecond technology}

Hanieh Fattahi, ${ }^{1,2}$ Helena G. Barros, ${ }^{2}$ Martin Gorjan, ${ }^{1,2}$ Thomas Nubbemeyer, ${ }^{2}$ Bidoor Alsaif, ${ }^{1,3,4}$ Catherine Y. Teisset, ${ }^{5}$ Marcel Schultze, ${ }^{5}$ Stephan Prinz, ${ }^{5}$ Matthias Haefner, ${ }^{5}$ Moritz Ueffing, ${ }^{2}$ Ayman Alismall, ${ }^{2,3}$ Lénárd Vámos, ${ }^{2,6}$ Alexander Schwarz, ${ }^{1,2}$ Oleg Pronin, ${ }^{2}$ Jonathan Brons, ${ }^{2}$ Xiao Tao Geng,,${ }^{1,7}$ Gunnar Arisholm, ${ }^{8}$ Marcelo Ciappina, ${ }^{1}$ Vladislav S. Yakovlev, ${ }^{1,2,9}$ Dong-Eon Kim, ${ }^{7}$ Abdallah M. Azzeer, ${ }^{3}$ Nicholas Karpowicz, ${ }^{1}$ Dirk Sutter, ${ }^{10}$ Zsuzsanna Major, ${ }^{1,2}$ Thomas Metzger, ${ }^{5}$ and Ferenc Krausz ${ }^{1,2, *}$

${ }^{1}$ Max-Planck Institut für Quantenoptik, Hans-Kopfermann-Str. 1, D-85748 Garching, Germany

${ }^{2}$ Department für Physik, Ludwig-Maximilians-Universität München, Am Coulombwall 1, D-85748 Garching, Germany

${ }^{3}$ Physics and Astronomy Department, King Saud University, Riyadh 11451, Saudi Arabia

${ }^{4}$ Current address: Electrical Engineering, Computer, Electrical and Mathematical Sciences and Engineering Division, Building 1, 4700 King Abdullah University of Science and Technology, Thuwal 23955-6900, Saudi Arabia

${ }^{5}$ TRUMPF Scientific Lasers GmbH + Co. KG, Feringastr. 10, 85774 München-Unterföhring, Germany

${ }^{6}$ Wigner Research Center for Physics, Konkoly-Thege Miklós út 29-33, H-1121 Budapest, Hungary

${ }^{7}$ Physics Department, POSTECH, San 31 Hyoja-Dong, Namku, Pohang, Kyungbuk 790-784, South Korea

${ }^{8}$ FFI (Norwegian Defence Research Establishment), Postboks 25, NO-2027 Kjeller, Norway

${ }^{9}$ Current address: Center for Nano-Optics (CeNO), Georgia State University, Atlanta, Georgia 30303, USA

${ }^{10}$ TRUMPF Laser GmbH + Co. KG, Aichhalder Str. 39, 78713 Schramberg, Germany

*Corresponding author: krausz@lmu.de

Received 23 May 2014; revised 25 June 2014; accepted 26 June 2014 (Doc. ID 212665); published 22 July 2014

Femtosecond pulse generation was pioneered four decades ago using mode-locked dye lasers, which dominated the field for the following 20 years. Dye lasers were then replaced with titanium-doped sapphire (Ti:Sa) lasers, which have had their own two-decade reign. Broadband optical parametric amplifiers (OPAs) appeared on the horizon more than 20 years ago but have been lacking powerful, cost-effective picosecond pump sources for a long time. Diode-pumped ytterbium-doped solid-state lasers are about to change this state of affairs profoundly. They are able to deliver 1 ps scale pulses at kilowatt-scale average power levels, which, in thin-disk lasers, may come in combination with terawatt-scale peak powers. Broadband OPAs pumped by these sources hold promise for surpassing the performance of current femtosecond systems so dramatically as to justify referring to them as the next generation. Third-generation femtosecond technology (3FST) offers the potential for femtosecond light tunable over several octaves, multi-terawatt few-cycle pulses, and synthesized multi-octave light transients. Unique tunability, temporal confinement, and waveform variety in combination with unprecedented average powers will extend nonlinear optics and laser spectroscopy to previously inaccessible wavelength domains, ranging from the far IR to the $x$-ray regime. Here we review the underlying concepts, technologies, and proof-of-principle experiments. A conceptual design study of a prototypical tunable and wideband source demonstrates the potential of 3FST for pushing the frontiers of femtosecond and attosecond science. () 2014 Optical Society of America

OCIS codes: (140.0140) Lasers and laser optics; (140.3480) Lasers, diode-pumped; (190.4970) Parametric oscillators and amplifiers; (320.7160) Ultrafast technology; (140.3615) Lasers, ytterbium; (230.4480) Optical amplifiers.

http://dx.doi.org/10.1364/OPTICA.1.000045 


\section{INTRODUCTION}

Femtosecond technology was born in the 1970s, when passively mode-locked dye lasers produced the first pulses shorter than 1 ps [1-3] . Subsequent advances led to pulse durations of a few tens of femtoseconds directly from laser oscillators [4-7]. The poor energy storage capability of laser dyes limited amplification to microjoule energies and megawatt peak powers $[\underline{8}, \underline{2}]$. This first-generation femtosecond technology (1FST) opened the door for direct time-domain investigations of hitherto immeasurably fast processes such as molecular dynamics, chemical reactions, and phase transitions in condensed matter $[10,11]$.

Broadband solid-state lasers with large energy storage capabilities appeared by the end of the 1980s [12-14]. They offered the potential for further pulse shortening as well as boosting the pulse energy and peak power by many orders of magnitude. Second-generation femtosecond technology (2FST), based on chirped-pulse amplification (CPA) [15] in solid-state lasers, in particular, in Ti:sapphire-based systems [16-18], and dispersion control by chirped multilayer mirrors (henceforth, for brevity, chirped mirrors) [19-21] paved the way for the emergence of entirely new research fields and technologies such as attosecond science [22] and laser-driven particle acceleration [23].

2FST is now capable of providing pulses with ultrahigh (petawatt) peak power at moderate average power [24] and moderate-peak-power (gigawatt) pulses at ultrahigh (approaching the kilowatt scale) average power levels [25]; see Fig. 1 . Based on optical parametric chirped-pulse amplification (OPCPA) [26] driven by terawatt-scale pulses from ytterbium lasers at kilowatt-scale average power, third-generation femtosecond technology (3FST) will, as a defining characteristic, combine high (terawatt-scale) peak powers with high (kilowattscale) average powers in ultrashort optical pulse generation for the first time. This unprecedented parameter combination will allow us to explore extreme nonlinearities of matter and extend ultrashort pulse generation to short (nanometer to subnanometer) as well as long (multimicrometer) wavelengths at unprecedented flux levels, holding promise for yet another revolution in ultrafast science. Figure $\underline{1}$ shows a summary of the performance of $1 \mathrm{FST}, 2 \mathrm{FST}$, and $3 \mathrm{FST}$ systems.

OPCPA requires intense optical pulses for pumping the nonlinear medium used for the parametric conversion. The optimum duration of these pulses is of the order of $1 \mathrm{ps}$, constituting a trade-off between a high resistance to optical damage (decreasing for longer pulses [27-29]) and a small temporal walk-off $[30,31]$ between pump and signal pulses relative to their duration (increasing for shorter pulses). OPCPA pumped by 1-ps-scale pulses offers octave-spanning light amplification with unprecedented efficiency, not accessible by any other technique known to date. Moreover, for very similar physical reasons, these pulse durations appear to be ideal for efficient frequency conversion of the pump light via low-order harmonic generation and/or frequency mixing $[\underline{32}, \underline{33}]$. Hence, a reliable, cost-effective, and power-scalable source of highenergy 1-ps-scale laser pulses would constitute the ideal basis

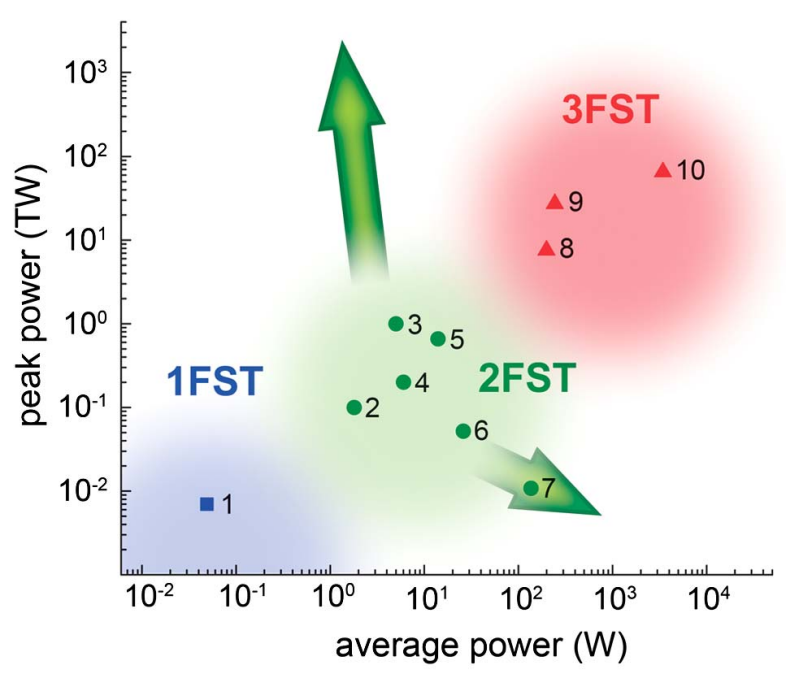

\begin{tabular}{c|c|c|c|c} 
& $\tau_{\text {pulse }}$ & $E_{\text {pulse }}$ & $P_{\text {peak }}$ & $P_{\text {average }}$ \\
\hline system 8 & $5 \mathrm{fs}$ & $40 \mathrm{~mJ}$ & $7.5 \mathrm{TW}$ & $200 \mathrm{~W}$ \\
\hline system 9 & $1.7 \mathrm{fs}$ & $49 \mathrm{~mJ}$ & $27 \mathrm{TW}$ & $245 \mathrm{~W}$ \\
\hline system 10 & $5 \mathrm{fs}$ & $345 \mathrm{~mJ}$ & $65 \mathrm{TW}$ & $3450 \mathrm{~W}$
\end{tabular}

Fig. 1. Summary of recorded performances of $1 F S T$ and $2 F S T$ and the expected performance of 3FST, in terms of average and peak powers. These systems are reviewed in detail in Supplement 1. The blue square represents the best performance achieved by dye-laser technology (1, corresponding to Ref. [9]), the green dots show femtosecond CPA solid-state technology $(2-7$, corresponding to Refs. $[\underline{60}, \underline{71}, \underline{66}, \underline{18}, \underline{67}]$, and [25], respectively), and the red triangles represent the simulated results for OPCPA based on pump sources under development ( 8 and 9 , pumped by a $1 \mathrm{ps}, 5 \mathrm{kHz}, 200 \mathrm{~mJ} \mathrm{Yb:YAG} \mathrm{thin-disk} \mathrm{laser)} \mathrm{and} \mathrm{envisioned} \mathrm{(10,}$ based on a future $1 \mathrm{ps}, 10 \mathrm{kHz}, 2 \mathrm{~J}$ Yb:YAG thin-disk laser system). Systems 8 and 10 use one OPCPA channel in the NIR, whereas in system 9 the output of three OPCPA channels in the VIS-NIR-IR are added for coherent synthesis of a subcycle waveform. The table summarizes the predicted output parameters of these systems. The relevant pump source architectures and multichannel OPCPA system are discussed in Section 2 and Section 3 , respectively. OPCPA systems pumped by Yb:YAG sla $\bar{b}$ and fiber amplifier systems $[108,110]$ have been demonstrated in the performance range of 2FST and are not displayed in the figure.

for exploiting the full potential of OPCPA for ultrashort pulse amplification at a variety of wavelengths.

In this work we show that diode-pumped Yb-doped thindisk lasers based on a technology well established in industrial environments fulfill all these requirements and offer a promising route to implementing 3FST in the conceptual architecture outlined in Fig. 2. In addition to simultaneously reaching peak and average power levels that will outperform 1FST and 2FST by several orders of magnitude (Fig. 1), 3FST systems allow a variety of operational modes, offering multicycle pulses tunable over several octaves, few-cycle pulses at different carrier wavelengths, and multi-octave synthesis of light waveforms.

Our discussion in this paper focuses on powerful ultrashortpulse generation at high $(\geq 1 \mathrm{kHz})$ repetition rates; ultrahighintensity lasers emitting a few pulses per second or less as well as sources delivering moderate-power pulses at high average power are out of the scope of this work (for a review, the 


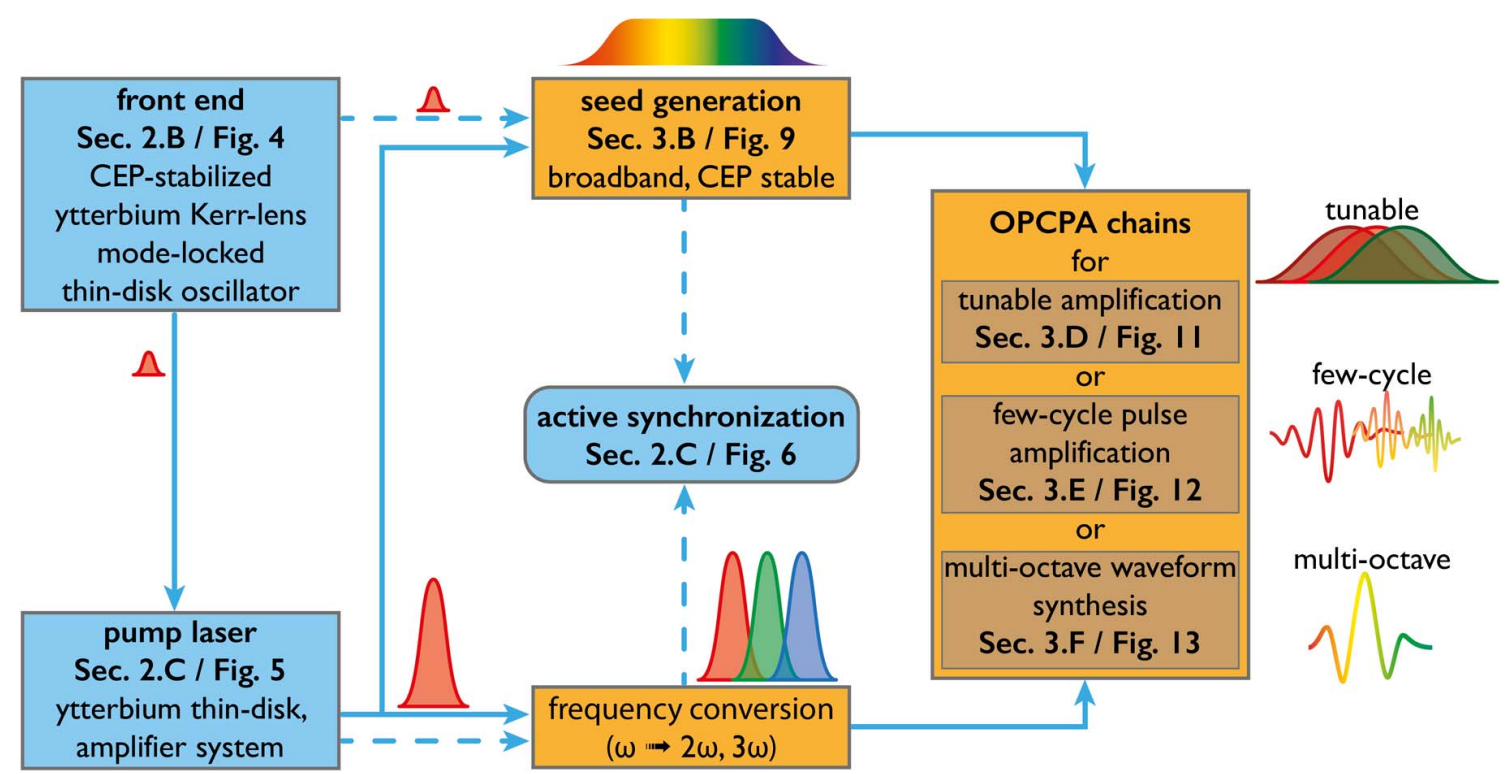

Fig. 2. Basic conceptual architecture of a 3 FST system. A subpicosecond ytterbium laser oscillator seeds the pump source. The broadband seed can be generated either from the output of the picosecond pump laser (solid arrows) or directly from the oscillator (dashed arrows). In the latter case an active temporal synchronization is needed between the pump and seed pulses of the OPCPA chain. The 3FST source can be operated to generate (i) widely tunable pulses of a few tens of femtoseconds duration, (ii) few-cycle pulses in different spectral ranges, or (iii) multi-octave controlled waveforms with a sub-optical-cycle structure. The building blocks of the 3FST system are discussed in detail in the respective sections as indicated in the figure.

interested reader is kindly referred to relevant reviews in [23] and [34], respectively). A brief historical overview of 1 FST $[\underline{1}-\underline{9}, \underline{35}-\underline{45}]$ and 2FST $[\underline{12}-21, \underline{45}-73]$ is presented in Supplement 1. The remaining part of the introduction addresses some of the major milestones of OPCPA history.

\section{A. Conceptual Basis for 3FST}

Optical parametric amplification (OPA) was discovered in the 1960s [74,75], but only nonlinear crystals with a high secondorder nonlinear susceptibility and high resistance to optical damage, such as $\beta$-barium borate (BBO) [76] along with the invention of OPCPA by Piskarskas and co-workers [26] opened the prospect for efficient amplification of femtosecond laser pulses via this mechanism. A prerequisite for OPA being able to provide a competitive alternative to femtosecond laser amplifiers is the availability of power-scalable pump sources with a good wall-plug efficiency. So far, only lasers with pulse durations much longer than 1 ps have been able to meet this requirement. The instantaneous nature of the OPA pump-tosignal energy conversion calls for a signal pulse that is temporally stretched to match the duration of the pump pulse for efficient OPA and then recompressed after amplification.

The bandwidth of OPCPA can be enhanced by a noncollinear pump-signal beam propagation geometry, utilizing the slightly different propagation directions of the interacting beams to compensate the effect of material dispersion in the nonlinear medium $[77,78]$. Drawing on these basic concepts and a variety of pump and seed sources, a large number of OPA experiments aiming at efficient amplification of ultrashort pulses have been performed over the past 20 years. Their review is beyond the scope of this paper; we refer the interested reader to a number of excellent review articles on this subject [79-83].
OPCPA has been demonstrated to be capable of amplifying pulses as short as $4 \mathrm{fs}$ [84], achieving peak-power levels of 16 TW from a tabletop system [85], approaching the petawatt frontier when pumped by large-scale lasers [86-92], and reaching average power levels as high as $22 \mathrm{~W}$ at a $1 \mathrm{MHz}$ repetition rate [93]. However, none of these systems have been capable of achieving high peak and average powers simultaneously. The most powerful OPCPA system of this kind reported to date delivers $0.49 \mathrm{TW}$ pulses at an average power of $2.7 \mathrm{~W}$ [94], which is still inferior to state-of-the-art Ti:Sa systems. The pump laser technology described in Section 2 holds promise for changing this state of affairs dramatically.

In what follows, Section $\underline{2}$ reviews near-1-ps pulse amplification and its implementation with thin-disk lasers, scalable to high peak as well as average powers. Section $\underline{3}$ is devoted to conceptual design studies demonstrating the potential of 3FST for creating a source of femtosecond light with unprecedented characteristics, and Section $\underline{4}$ addresses some of the expected implications.

\section{NEAR-1-PS YTTERBIUM LASERS}

Near-1-ps laser pulses with high peak power have long been available from flashlamp-pumped passively mode-locked neodymium-doped glass lasers-however, only at a very low repetition rate and hence low average power level [95-97]. High average powers ranging from tens to hundreds of watts recently became available from diode-pumped fiber, slab, and cryogenically cooled thick-disk lasers [요 $\underline{109}$ ]. Because of excessive accumulation of nonlinearly induced phase shifts in their long gain media, their scaling to much higher energies requires large-aperture (meter-scale) and hence extremely 
Table 1. Performance of Yb-Doped Few-Picosecond High Average and High Peak Power Systems ${ }^{a}$

\begin{tabular}{|c|c|c|c|c|}
\hline $\boldsymbol{P}_{\text {peak }}$ & $\boldsymbol{P}_{\mathrm{avg}}$ & $\boldsymbol{E}_{\text {pulse }} / \boldsymbol{\tau}_{\text {pulse }}$ & $f_{\text {rep }}$ & Reference \\
\hline \multicolumn{5}{|c|}{ Fiber laser systems } \\
\hline $12 \mathrm{MW}$ & $830 \mathrm{~W}$ & $10.6 \mu \mathrm{J} / 640 \mathrm{fs}$ & $78 \mathrm{MHz}$ & {$[100]$} \\
\hline $0.75 \mathrm{GW}$ & $93 \mathrm{~W}$ & $93 \mu \mathrm{J} / 81 \mathrm{fs}$ & $1 \mathrm{MHz}$ & {$[\overline{102}]$} \\
\hline $1.8 \mathrm{GW}$ & $530 \mathrm{~W}$ & $\begin{array}{l}1.3 \mathrm{~mJ} / 670 \mathrm{fs} \\
\text { Slab laser systems }\end{array}$ & $400 \mathrm{kHz}$ & {$[\overline{106}$} \\
\hline $37 \mathrm{MW}^{b}$ & $140 \mathrm{~W}$ & $43 \mu \mathrm{J} / 1.1 \mathrm{ps}$ & $3.25 \mathrm{MHz}$ & [108] \\
\hline $80 \mathrm{MW}$ & $1.1 \mathrm{~kW}$ & $55 \mu \mathrm{J} / 615 \mathrm{fs}$ & $20 \mathrm{MHz}$ & $\overline{[99]}$ \\
\hline $23 \mathrm{GW}^{b}$ & $250 \mathrm{~W}$ & $20 \mathrm{~mJ} / 830 \mathrm{fs}$ & $12.5 \mathrm{kHz}$ & {$[\overline{110}]$} \\
\hline \multicolumn{5}{|c|}{ Cryogenically cooled laser systems } \\
\hline $0.65 \mathrm{MW}^{b}$ & $430 \mathrm{~W}$ & $8.6 \mu \mathrm{J} / 12.4 \mathrm{ps}$ & $50 \mathrm{MHz}$ & {$[104]$} \\
\hline $8.7 \mathrm{MW}^{b}$ & $93 \mathrm{~W}$ & $93 \mu \mathrm{J} / 10 \mathrm{ps}$ & $1 \mathrm{MHz}$ & {$[\overline{105}$} \\
\hline $73 \mathrm{MW}$ & $19.4 \mathrm{~W}$ & $1 \mathrm{~mJ} / 11.7 \mathrm{ps}$ & $20 \mathrm{kHz}$ & $\overline{[98]}$ \\
\hline $7 \mathrm{GW}^{b}$ & $60 \mathrm{~W}$ & $12 \mathrm{~mJ} / 1.6 \mathrm{ps}$ & $5 \mathrm{kHz}$ & {$[\overline{103}]$} \\
\hline $2 \mathrm{GW}^{b}$ & $64 \mathrm{~W}$ & $32 \mathrm{~mJ} / 15 \mathrm{ps}$ & $2 \mathrm{kHz}$ & {$[\overline{101}]$} \\
\hline $170 \mathrm{GW}$ & $100 \mathrm{~W}$ & $1 \mathrm{~J} / 5.1 \mathrm{ps}$ & $100 \mathrm{~Hz}$ & {$[109]$} \\
\hline \multicolumn{5}{|c|}{ Thin-disk laser systems } \\
\hline $1.4 \mathrm{GW}^{b}$ & $200 \mathrm{~W}$ & $2 \mathrm{~mJ} / 1.3 \mathrm{ps}$ & $100 \mathrm{kHz}$ & {$[206]$} \\
\hline $17.6 \mathrm{GW}^{b}$ & $300 \mathrm{~W}$ & $30 \mathrm{~mJ} / 1.6 \mathrm{ps}$ & $10 \mathrm{kHz}$ & [147] \\
\hline $38.8 \mathrm{GW}^{b}$ & $200 \mathrm{~W}$ & $40 \mathrm{~mJ} / 0.97 \mathrm{ps}$ & $5 \mathrm{kHz}$ & Fig. 5 (b) \\
\hline $27.6 \mathrm{GW}^{b}$ & $100 \mathrm{~W}$ & $50 \mathrm{~mJ} / 1.7 \mathrm{ps}$ & $2 \mathrm{kHz}$ & {$[13 \overline{44}$} \\
\hline 一 & - & $200 \mathrm{~mJ}$ & $5 \mathrm{kHz}$ & $\begin{array}{c}\text { under } \\
\text { development }\end{array}$ \\
\hline - & - & $2 \mathrm{~J}$ & $10 \mathrm{kHz}$ & envisioned \\
\hline
\end{tabular}

${ }^{a}$ Peak power $\left(P_{\text {peak }}\right)$, average power $\left(P_{\text {avg }}\right)$, pulse energy $\left(E_{\text {pulse }}\right)$, pulse duration $\left(\tau_{\text {pulse }}\right)$, and repetition rate $\left(f_{\text {rep }}\right)$ of selected Yb:YAG fiber, slab, cryogenically cooled, and thin-disk amplifier systems delivering pulses with a duration of a few picoseconds or shorter. The data are taken from the corresponding references.

${ }^{b}$ Peak powers have been calculated assuming a Gaussian pulse shape $\left(P_{\text {peak }} \approx 0.94 * E_{\text {pulse }} / \tau_{\text {pulse }}\right)$.

expensive diffraction gratings for implementing CPA $[102,106,110]$ or complex architectures, such as the coherent combination of a large number of parallel beams [111], or possibly coherent pulse stacking [112]. Diode-pumped ytterbiumdoped thin-disk lasers offer energy and peak-power scalability from simple, cost-effective assemblies. Therefore, in what follows we focus on this technology as a promising candidate for driving broadband OPA systems scalable to high average and peak powers. Nevertheless, we stress that diode-pumped fiber, cryogenically cooled thick-disk, and slab lasers constitute a highly competitive alternative at high repetition rates and moderate peak power levels, and we kindly refer the reader to recent reviews of these approaches [33,107,113-116]. Table 1 summarizes the parameters of some of the bestperforming systems based on $\mathrm{Yb}$-doped fiber, slab, cryogenically cooled thick-disk, and thin-disk technology.

\section{A. Toward High Peak and Average Powers}

Ever since its first demonstration in 1994 [117], the thin-disk laser has been one of the most promising concepts for scaling sub-picosecond pulses to the highest peak and average powers. In this section we briefly summarize the basic features of thin-disk technology and refer the reader to Supplement 1 and recent reviews [118-120] for a more detailed discussion of performance, limitations and ways of overcoming them.

In a thin-disk laser the active medium is a thin and relatively large-diameter disk, typically tens to hundreds of micrometers in thickness and few (tens) millimeters in diameter. Crystals are used due to their favorable thermal and mechanical properties compared to glasses, with ytterbium-doped yttrium aluminium garnet (Yb:YAG) being the paradigm material of choice to date, although thin-disk lasers using different disk materials such as $\mathrm{Yb}: \mathrm{Lu}_{2} \mathrm{O}_{3}$ [121,122], Yb:CALGO [123], and ceramic Yb:YAG disks $[\underline{124,125]}$ have also been demonstrated.

A Yb:YAG laser disk is coated on the back side to act as a high-reflective (HR) mirror for both the pump and the laser wavelengths. The other (front) side is antireflection (AR) coated for both wavelengths [see Fig. 3(a)]. The HR-coated side of the disk is firmly fixed onto a supporting substrate, which, in turn, is mounted on a water-cooled assembly. To achieve good ( $90 \%$ or more) absorption of the pump light, the pump beam is delivered at an angle from the front side and reflected in a number of passes using a special imaging multipass assembly [Fig. 3(b)]. Heat removal from the crystal is realized along the optical axis of the resonator. This minimizes thermally induced changes in the optical properties of the laser medium across the laser beam and allows for extremely high pump power densities reaching and exceeding $10 \mathrm{~kW} / \mathrm{cm}^{2}[118,126,127]$. Energy and power scaling can be accomplished by scaling the diameter of the disk along with the pump and laser beams, which is eventually limited by amplified spontaneous emission (ASE) $[\underline{128}, \underline{129}]$.

The small length of the gain medium greatly suppresses nonlinear focusing during the amplification of ultrashort pulses as compared to other laser geometries. As a result, CPA can be implemented with substantially smaller temporal stretching, requiring smaller, less expensive diffraction gratings as compared to other solid-state ultrashort pulse amplifiers $[\underline{99}, 107]$. These superior features of the thin-disk laser geometry come at the expense of a low single-pass gain of typically $10 \%-15 \%$ (small signal). This shortcoming can be mitigated by multiple passages and/or the serial combination of several disks $[\underline{118}, 130,131]$.

Thin-disk gain modules have been used for ultrashort pulse generation in mode-locked oscillators $[\underline{132,133]}$ and regenerative [134] and multipass [135] amplifiers. Thousands of them have been tried and tested in $24 / 7$ service for industry. This mature technology constitutes an ideal basis for scaling subpicosecond pulses to unprecedented combinations of peak (a)

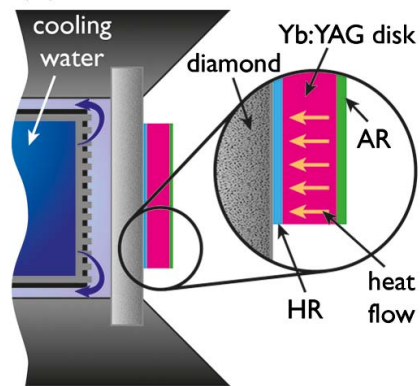

(b)

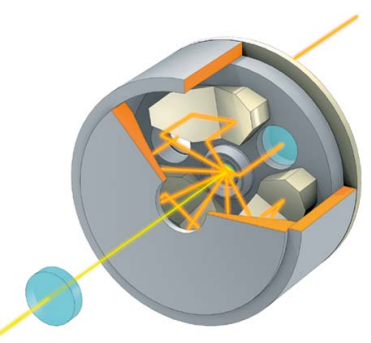

Fig. 3. Schematics of thin-disk laser technology. (a) Cold finger with substrate showing the cooling mechanism in a disk laser head, (b) disk laser head showing the principle of the pump light reimaging technique onto the thin-disk active medium (courtesy of TRUMPF Laser GmbH). 


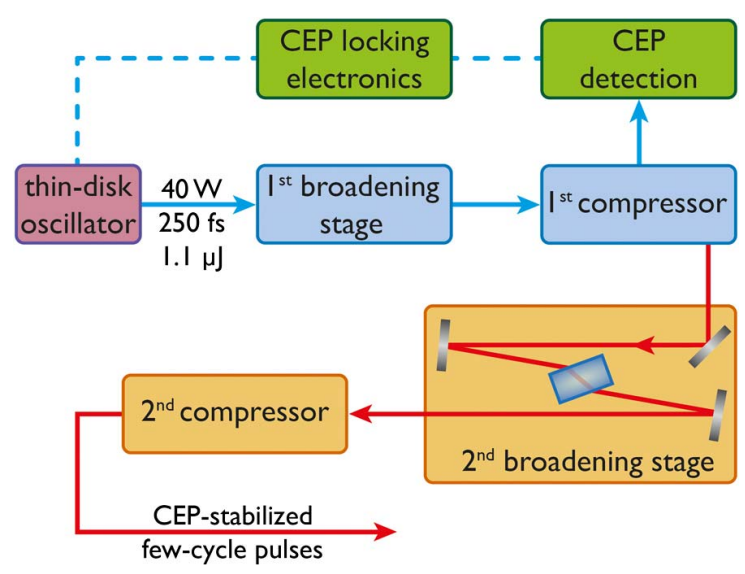

Fig. 4. Schematic of a KLM Yb:YAG laser system. Pulses from the oscillator centered at $1030 \mathrm{~nm}$ with an energy of $1.1 \mu \mathrm{J}$ and a duration of $250 \mathrm{fs}$ at a repetition rate of $38 \mathrm{MHz}$ are passed through a two-stage compressor made up of a solid-core large-mode-area fiber (first broadening stage), a bulk crystal as the nonlinear media (second broadening stage), and chirped mirrors forming the dispersive delay lines (first and second compressors), resulting in carrier-envelope-phase (CEP)stabilized few-cycle output pulses [144].

and average power levels for driving OPAs in 3FST [136]. The remaining part of this section reveals how this technology can provide both the broadband seed and the high-power pump pulses in a perfectly synchronized fashion to this end.

\section{B. Mode-Locked Ytterbium-Doped Thin-Disk Oscillators}

Ultrashort pulse generation from a diode-pumped $\mathrm{Yb}$-doped thin-disk laser oscillator was first demonstrated by Keller and co-workers at the turn of the millennium [137]. The technology was subsequently advanced to average power levels of hundreds of watts [138], pulse energies of several microjoules $[130,139,140]$, and pulse durations shorter than $100 \mathrm{fs}$ $[131,141]$ directly from the oscillator. Kerr-lens mode locking (KLM) [49] and semiconductor saturable absorber mirrors [142] have been the methods of choice for mode locking $[137,143]$.

Figure 4 shows the schematic of a KLM Yb:YAG thindisk-oscillator-based few-cycle source [144]. The system delivers reproducible waveform-controlled few-cycle pulses at an average power exceeding that of few-cycle Ti:Sa oscillators by more than an order of magnitude.

The average power of KLM Yb:YAG thin-disk oscillators was recently increased by nearly an order of magnitude to deliver $14 \mu \mathrm{J}, 330 \mathrm{fs}$ pulses at a $19 \mathrm{MHz}$ repetition rate [140]. These advances open up the prospect of a megahertz source of near-infrared (NIR) femtosecond continua with a peak power of several hundred megawatts at average power levels of the order of $100 \mathrm{~W}$ and, if needed, with a controlled waveform. Such a source holds promise for greatly expanding the range of applications of ultrashort pulsed laser oscillators (as a stand-alone system) and for serving as a front end for gigawatt-to-terawatt 3FST architectures (see Fig. $\underline{2}$ and discussion in Section $\underline{3}$.
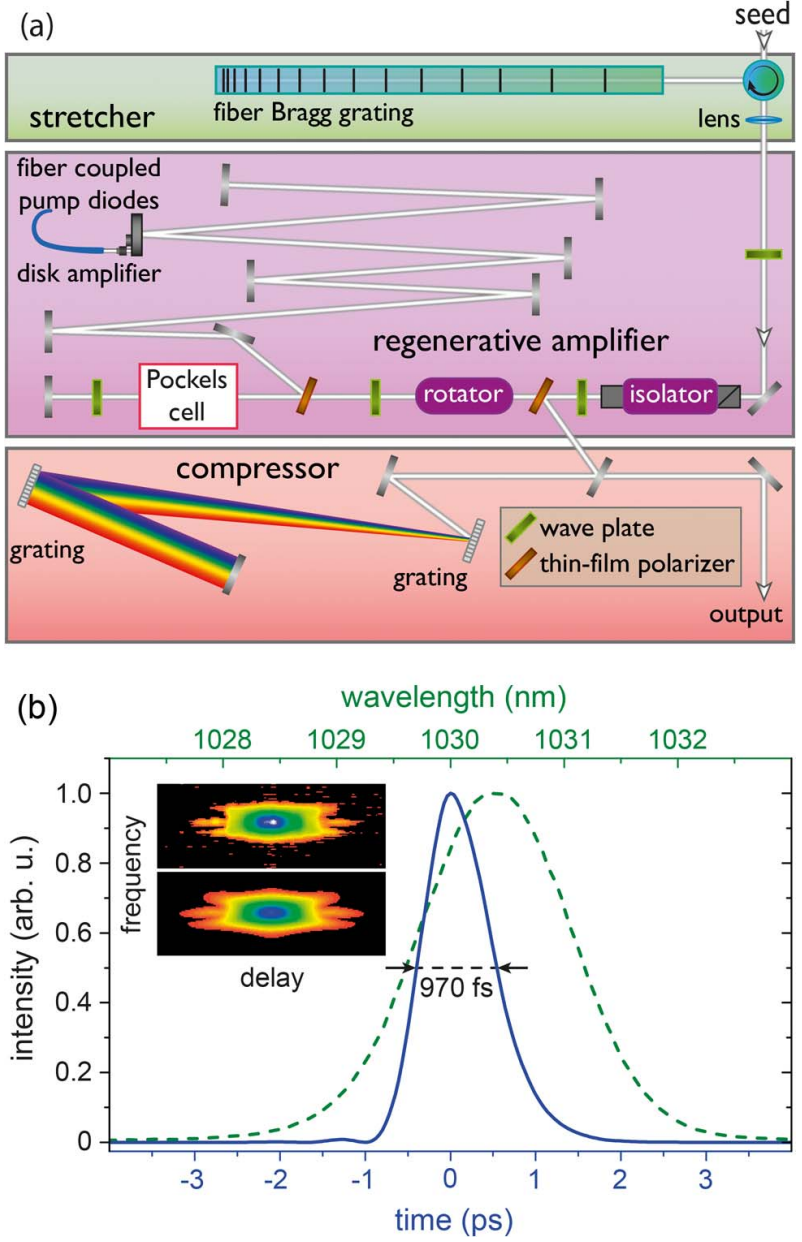

Fig. 5. Thin-disk regenerative amplifier. (a) Typical schematic layout. The subpicosecond seed pulse is temporally stretched before entering the thin-disk Yb:YAG regenerative amplifier. The amplified pulses are recompressed in a grating compressor. (b) Measured spectrum (green dashed line) and temporal intensity profile (blue line) from a system equipped with two disk modules (disk parameters: doping concentration $>7 \%$, thickness $\sim 100 \mu \mathrm{m}$, beveled and roughened edge, TRUMPF Laser $\mathrm{GmbH}$ ). These 0.97 ps pulses carry an energy of $40 \mathrm{~mJ}$ at a repetition rate of $5 \mathrm{kHz}$, corresponding to an average power of $200 \mathrm{~W}$. The corresponding measured (top) and reconstructed (bottom) FROG traces are shown as insets ( $\mathrm{G}$ error: 0.0024).

\section{Thin-Disk Regenerative Amplifiers for Pumping OPCPA}

The first thin-disk-based regenerative amplifier was demonstrated in 1997 and generated 2.3 ps pulses with energies up to $0.18 \mathrm{~mJ}$ and an average power of the order of $1 \mathrm{~W}$ [145]. A decade of development work advanced the technology into the multimillijoule, tens of watts regime [146]. By drawing on commercial Yb:YAG thin-disk modules originally designed for multikilowatt-class $\mathrm{cw}$ products, continued efforts led to near-1-ps pulses with energies as high as $30 \mathrm{~mJ}$ and average powers reaching $300 \mathrm{~W}$ at repetition rate of $10 \mathrm{kHz}$ [147]. Milestones of this evolution are listed in Table $\underline{1}$, and a schematic of the architecture of state-of-the-art systems is shown in Fig. 5(a). All results on picosecond CPA with thin-disk lasers 
referred to or reported directly in this work have so far been achieved with standard Yb:YAG thin-disk laser modules designed and fabricated for industrial lasers. This suggests that there may be some room left for further optimization of thin-disk Yb:YAG chirped-pulse amplifiers.

Thanks to their superior thermal management and low B integral, Yb:YAG thin-disk regenerative amplifiers deliver their near-bandwidth-limited pulses in a near-diffraction-limited beam $\left(M^{2}<1.1\right)$ with excellent pulse-energy stability characterized by a drift smaller than $1 \%$ over $12 \mathrm{~h}$. Figure $5(\mathrm{~b})$ presents the measured optical spectrum and the retrieved temporal profile showing a full width at half-maximum (FWHM) pulse duration of $0.97 \mathrm{ps}$ of an Yb:YAG thin-disk regenerative amplifier composed of two thin-disk amplifying modules within one resonator, delivering $40 \mathrm{~mJ}$ pulses at a repetition rate of $5 \mathrm{kHz}$.

While $40 \mathrm{~mJ}$ at $200 \mathrm{~W}$ and $30 \mathrm{~mJ}$ at $300 \mathrm{~W}$ [147] represent current records in high-energy 1-ps-scale pulse generation with high average power, none of these values individually appear to even come close to the ultimate limits of picosecond thin-disk laser technology. In fact, a regenerative amplifier followed by a multipass amplifier recently boosted the energy of sub-2-ps pulses to more than $500 \mathrm{~mJ}$ at a repetition rate of $100 \mathrm{~Hz}$ [148]. At a much higher repetition rate $(800 \mathrm{kHz}), 7$ ps pulses from a commercial thin-disk laser were amplified to an average power of $1.1 \mathrm{~kW}$ [135]. An amplifier chain containing two thin-disk-based multipass amplifiers as final stages delivers $14 \mathrm{~kW}, 140 \mathrm{~mJ}$ in a $10 \mathrm{~Hz}$ burst mode [149].

Thanks to their optimum pulse duration of the order of $1 \mathrm{ps}$ and excellent beam quality, thin-disk Yb:YAG regenerative amplifiers allow for efficient generation of second-harmonic and third-harmonic light by $\chi^{(2)}$ processes [second-harmonic generation (SHG) and sum-frequency generation (SFG), respectively]. As an example, results achieved with $1.3 \mathrm{ps,}$ $1030 \mathrm{~nm}$ pulses from a multikilohertz Yb:YAG thin-disk laser demonstrate a second-harmonic conversion efficiency as high as $74 \%$ in a $1.5 \mathrm{~mm}$ thick $\mathrm{LiB}_{3} \mathrm{O}_{5}$ (LBO) crystal $\left(\theta=90^{\circ}, \varphi=12.9^{\circ}\right)$.

OPA relies on a spatial as well as temporal overlap of the pump and seed pulses for efficient amplification. Hence, pump and seed pulses are derived from the same femtosecond laser $[136,150]$ serving as the common front end. However, the pump pulse suffers a delay of several microseconds upon passage through the regenerative and/or multipass amplifier(s). This delay is compensated for by selecting a correspondingly delayed seed pulse from the train delivered by the common front end. Already fractional changes as small as $10^{-7} 10^{-8}$ (by air turbulences, mechanical vibrations, and expansion due to temperature drifts) in the microsecond delay of the pump and seed pulses suffered upon passage through different optical systems may cause an excessive timing jitter [151] and require active stabilization.

Spectrally resolved cross correlation of the seed and the pump pulses offers a powerful means of active synchronization [152]. A possible implementation of this concept is based on stretching a small fraction of the broadband seed pulse to a duration of several picoseconds and mixing this pulse with the narrowband pump pulse in a nonlinear crystal. Changes in the carrier wavelength of the resultant sum-frequency output are unambiguously related to the relative timing between pump and seed pulses. In its first demonstration, this stretched-pulse cross-correlation technique was capable of reducing the RMS timing jitter to $\sigma=24$ fs over the frequency band of $20 \mathrm{mHz}$ to $1.5 \mathrm{kHz}$ [153].

Recently, this method was improved by replacing the sumfrequency generator by an OPA stage and deriving the timing information (optical error signal) from the spectrally resolved amplified signal output [154]. For this approach it is sufficient to split off only $\sim 2 \mathrm{pJ}$ of the seed pulse energy, since with the amplification of the seed pulse the OPA inherently delivers an amplified error signal. The concept is schematically depicted in Fig. 6(a). Its first implementation yielded pump-seed timing stabilization with a record residual RMS jitter of less than $2 \mathrm{fs}$ over the frequency band ranging from $0.1 \mathrm{~Hz}$ to $1 \mathrm{kHz}$ as well as long-term timing stability [Fig. 6(b)], ensuring ideal conditions for stable OPCPA operation.

\section{Scaling Thin-Disk Amplifiers-Future Prospects}

Present-day industrial thin-disk laser technology is capable of transforming diode-laser light of poor beam quality into kilowatts of power delivered in a diffraction-limited laser beam. The overriding question is to what extent this tremendous potential can be exploited for simultaneously boosting the energy and average power of near-1-ps laser pulses. Scaling of power and energy have already been demonstrated separately

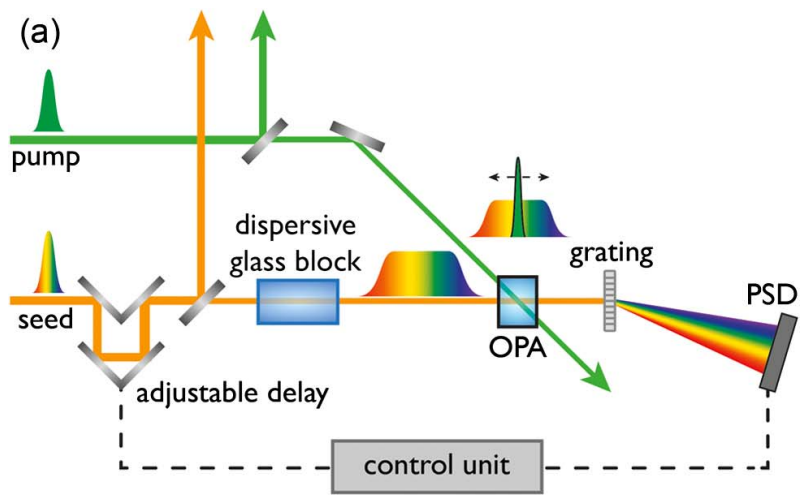

(b)

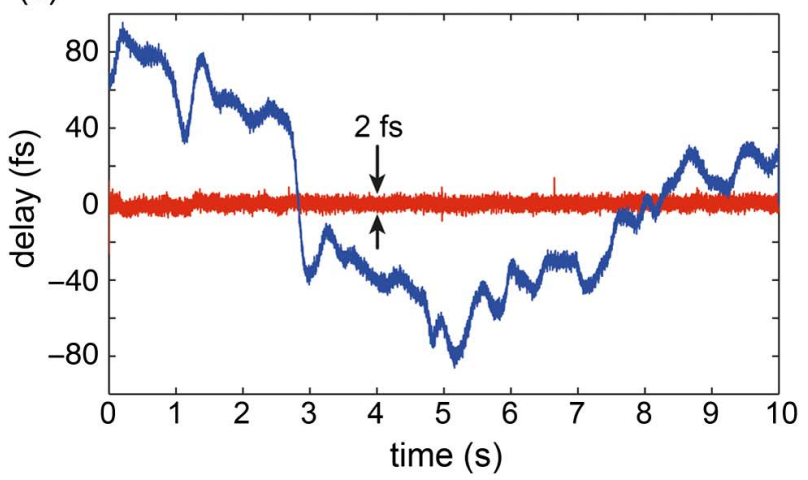

Fig. 6. Active pump-seed temporal synchronization system. (a) Schematic layout. PSD, position sensitive detector. (b) Timing fluctuations on the order of \pm 100 fs are reduced to a residual RMS jitter of less than $1.9 \mathrm{fs}$; for details, see [154]. 


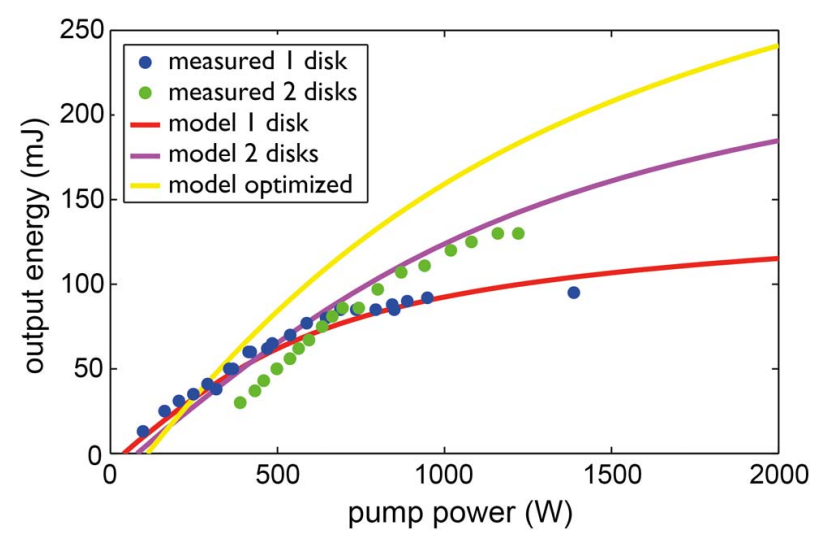

Fig. 7. Performance of $\mathrm{cw}$ diode-pumped regenerative amplifiers utilizing commercial thin-disk modules (disk parameters: doping concentration $>9 \%$, thickness $\sim 120 \mu \mathrm{m}$, beveled and roughened edge, TRUMPF Laser $\mathrm{GmbH}$ ). In a linear cavity setup, $95 \mathrm{~mJ}$ output energy was demonstrated at a $1 \mathrm{kHz}$ repetition rate with one standard disk pumped at $940 \mathrm{~nm}$ (blue dots). While there were indications of energy saturation, the energy was boosted to $130 \mathrm{~mJ}$ by adding a second disk (green dots). By further optimization of both disk parameters and the pump design, more than $200 \mathrm{~mJ}$ energy at $5 \mathrm{kHz}$ can be achieved (yellow line).

beyond $1 \mathrm{~kW}$ [135] and up to $1 \mathrm{~J}$ [155-157], respectively. Achieving these values of both energy and average power in the same thin-disk laser system will require optimal scaling of the disk diameter (increasing the available energy but also the depopulation losses) and disk thickness (increasing the available energy and the deleterious thermal effects) [118].

A regenerative amplifier equipped with commercial $\mathrm{Yb}$ : YAG thin-disk modules is being developed in our laboratory for generating $200 \mathrm{~mJ}$ pulses at a $5 \mathrm{kHz}$ repetition rate. In preliminary experiments with a linear cavity amplifier, 95 and $130 \mathrm{~mJ}$ pulses at a repetition rate of $1 \mathrm{kHz}$ have been demonstrated, with one and two disk modules, respectively (Fig. 7), with the latter being limited by thermal effects in the Faraday isolator preventing feedback into the front end [158]. The use of a ring cavity will remove this limitation and - based on these preliminary results_-holds promise for achieving the above target parameters. A rate-equation model of thin-disk laser energy that takes into account the decrease in the upper level lifetime, caused by ASE [120] and the disk temperature, shows good agreement with the measurements and indicates that the same energy can be extracted up to $5 \mathrm{kHz}$ with the current disks. An optimized design with increased disk thickness pumped at $969 \mathrm{~nm}$ can further increase the extracted energy, using the same beam size of $5 \mathrm{~mm}$.

Much higher energies and powers can be expected from larger apertures [159]. The feasibility of scaling near-1-ps thin-disk amplifiers to the $1 \mathrm{~J}$ frontier was recently demonstrated [155-157]. Further discussion on how careful engineering of large-aperture Yb:YAG disk amplifier modules for minimizing ASE and temperature control could permit scaling of the amplified energy to the joule-kilowatt level is given in Supplement 1.

Such large-aperture Yb:YAG disks have been shown to be capable of handling more than $10 \mathrm{~kW}$ of diode laser power
[160]. Merely a couple of thin-disk amplifier modules equipped with 20-mm-diameter Yb:YAG disks and pumped by approximately $30 \mathrm{~kW}$ of $\mathrm{cw}$ diode laser light each will be sufficient to boost the energy of the $0.2 \mathrm{~J}$ seed pulsesdelivered by two $5 \mathrm{kHz}$ regenerative amplifiers in a parallel architecture-to the level of $2 \mathrm{~J}$ at a $10 \mathrm{kHz}$ repetition rate. A possible approach to this goal is schematically illustrated in Fig. 8. Should scaling to this energy level encounter unexpected difficulties, coherent combination of several amplifiers $[25,161-163]$ or the concept of pulse stacking [112] might provide a remedy. These developments may open the door for a kilowatt-class 3FST source of few-cycle or tunable multiterawatt femtosecond pulses.

\section{BROADBAND OPCPA PUMPED BY NEAR-1-PS PULSES}

With robust nonlinear crystals and a reliable, cost-effective, and power-scalable short-pulsed pump-laser technology along with methods for accurate pump-seed timing synchronization in place (see Section 2.C), near-1-ps-pulse-pumped OPCPA offers several advantages over both long-pulse-driven OPCPA and conventional CPA implemented in solid-state laser amplifiers. First, the amplifier crystals can be pumped at much higher intensities [27-29], allowing high gains to be realized with very thin crystals, i.e., in combination with broad amplification bandwidths. Second, the short pump window also greatly simplifies the implementation of CPA and dispersion control and, finally and most importantly, improves the temporal contrast of the amplified signal dramatically on the nanosecond to few-picosecond time scale.

The gain bandwidth can be even further extended, up to several octaves, by using different crystals or crystals with different orientations yielding shifted gain bands and utilizing multiple pump beams [164] at all wavelengths where they can be made available with good wall-plug efficiency, i.e., at $1030 \mathrm{~nm}$ and its low-order harmonics at 515 and $343 \mathrm{~nm}$. This constitutes the basis for developing the prototypical broadband or broadly tunable sources of 3FST. The very same front end and multicolor pump source may be utilized for both purposes. In what follows, we shall discuss the feasibility of these 3FST sources and their expected performance when being pumped with several-kilohertz, kilowatt-class thin-disk lasers recently demonstrated [147] and systems that are currently under development [158].

\section{A. Basic Theory}

In the OPA process, energy is transferred from a highfrequency, high-intensity (pump) beam to a low-frequency, low-intensity (seed or signal) beam in a birefringent [165] nonlinear crystal, while a third beam, the idler, is generated. By polarizing the pump along the fast axis and the signal or idler or both along the slow axis, conservation of energy and momentum of the participating (pump, signal, idler, labeled with $p$, s, and $i$, respectively) photons can be simultaneously fulfilled:

$$
\hbar \omega_{p}-\hbar \omega_{s}-\hbar \omega_{i}=0
$$




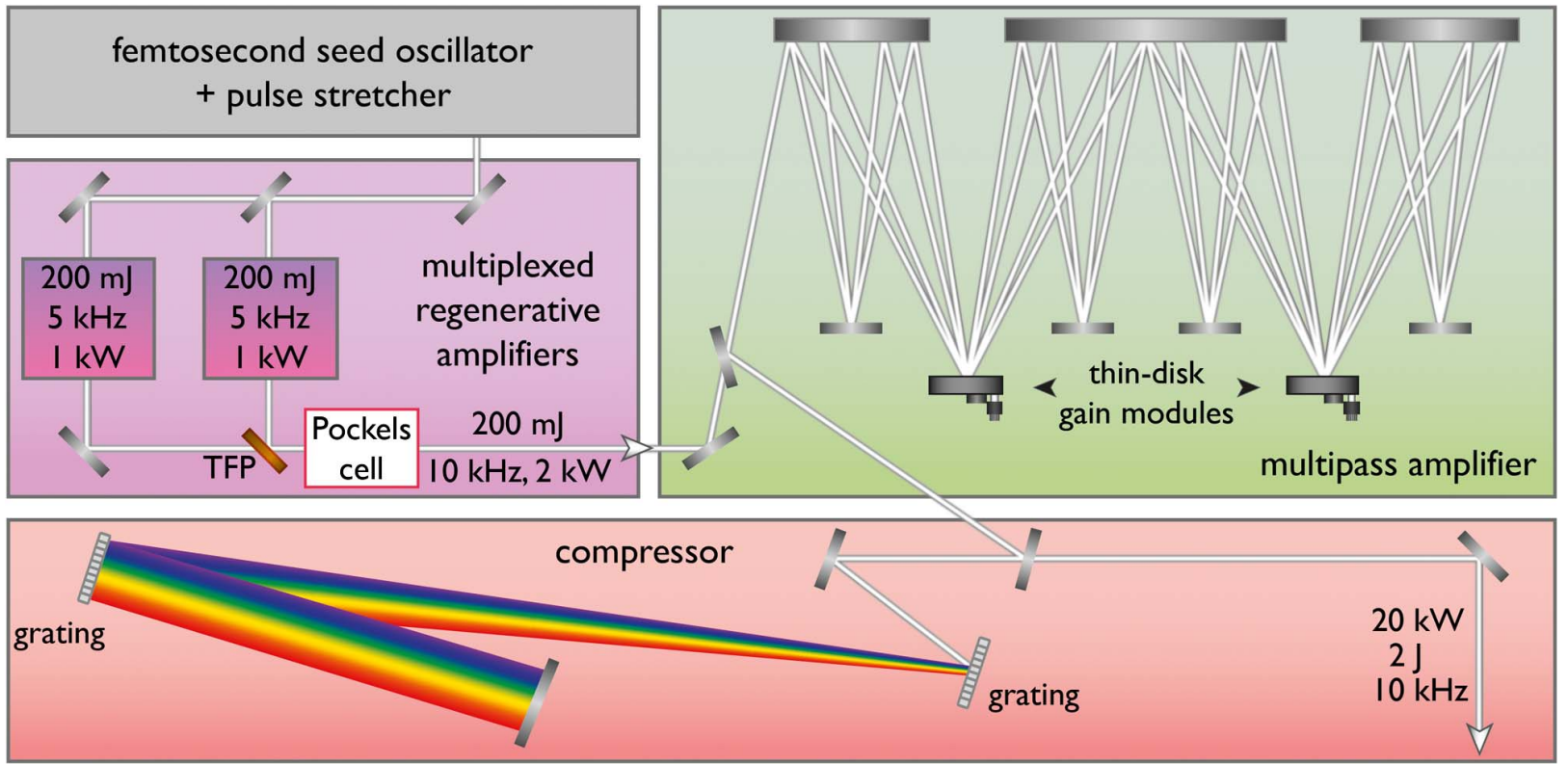

Fig. 8. Schematic layout of the multikilowatt, joule-class picosecond laser setup. A regenerative amplifier and a subsequent multipass amplification stage are used for generating multikilowatt, joule-class picosecond pulses (TFP, thin-film polarizer). Pulses from a front end comprising an oscillator and a pulse stretcher (cf. Fig. 5) are used to seed two regenerative amplifiers working at a $5 \mathrm{kHz}$ repetition rate. The $200 \mathrm{~mJ}$ amplified pulses from both amplifiers are interleaved in time to produce a $10 \mathrm{kHz}, 200 \mathrm{~mJ}$ pulse train. These pulses are then guided to a multipass amplifier using two thin-disk laser heads. Finally, the amplified pulses are compressed in a grating compressor.

$$
\vec{k}_{p}-\vec{k}_{s}-\vec{k}_{i}=0
$$

In the classical description of the process, Eqs. ( 1$)$ and (2) account for the parametric frequency downconversion and phase (velocity) matching of the participating waves, respectively. Due to dispersion, these conditions can be fulfilled over a limited range of signal frequencies only, which manifests itself in a finite parametric gain bandwidth $\Delta \nu$ $[166,167]:$

$$
\Delta \nu=\frac{2(\ln 2)^{1 / 2}}{\pi}\left(\frac{\Gamma}{L}\right)^{1 / 2}\left|\frac{1}{v_{g i}}-\frac{1}{v_{g s}}\right|^{-1}
$$

where $L$ is the length of the nonlinear medium, $\Gamma$ is the parametric gain coefficient proportional to the pump-field amplitude and the effective nonlinear optical coefficient, and $\nu_{g i, s}$ stand for the group velocity of the idler and the signal, respectively [166]. Equation (3) suggests that temporal walk-off between the amplified pulses limits the achievable gain bandwidth.

In sharp contrast with lasers, the central frequency and the width of the OPA gain band can be manipulated by changing the orientation or temperature of the crystal, and/or by the pump-signal propagation geometry. These degrees of freedom can be used to produce tunable femtosecond pulses. Alternatively, degenerate OPA near the wavelength where the groupvelocity dispersion for the signal and idler beams becomes zero $\quad\left(\omega_{s} \simeq \omega_{i} \simeq \omega_{p} / 2\right) \quad[167]$ or the noncollinear $\mathrm{OPA}$ $[\underline{77,78,168-170]}$ permits the amplification of few-cycle pulses
$[171,172]$. Both modes of operation can be simultaneously implemented in several OPA channels driven by the third or second harmonic or the fundamental of the $1030 \mathrm{~nm}$ picosecond pulses from Yb:YAG thin-disk amplifiers to yield synchronized tunable or few-cycle pulses in the visible (VIS), NIR, and mid-infrared (MIR) spectral ranges, respectively. These pulses may also be superimposed on each other for the synthesis of multi-octave light transients [173-175]. These options call for a seed coming in the form of a coherent, phase-stable, multi-octave supercontinuum covering the entire wavelength range of interest.

\section{B. Generation of Waveform-Controlled Continua for OPA Seeding}

The NIR continuum produced by the prototypical carrierenvelope-phase (CEP)-stabilized femtosecond KLM thindisk-laser-based source described in Section 2.B [144], constitutes - after proper active synchronization to the pump pulses (see Section 2.C) —an ideal seed for broadband OPA. In fact, these CEP-stabilized continua exhibit a well-behaved spectral phase and excellent spatial beam quality and are delivered with microjoule-scale energy, allowing efficient OPA with low fluorescence background. The spectrum is perfectly matched to the gain band of $\mathrm{BBO}$ and $\mathrm{LBO}$ parametric amplifiers [136] pumped by the second harmonic of the Yb:YAG laser $(515 \mathrm{~nm})$.

Amplification in such an OPA to the millijoule energy level and recompression of the amplified NIR pulse may be followed by further spectral broadening in a gas-filled hollowcore fiber (HCF). Self-phase modulation and self-steepening broadens the input spectrum predominantly toward shorter 
wavelengths. This approach can provide the broadband seed required by a VIS-OPCPA pumped by the third harmonic of the Yb:YAG laser $(343 \mathrm{~nm})$ but fails to do so for a MIR-OPCPA driven directly at $1030 \mathrm{~nm}$. In the remaining part of this section we discuss the generation of a phase-stable continuum in the MIR and its subsequent extension to shorter wavelengths.

A powerful technique for the generation of CEP-stabilized MIR continua has been difference frequency generation (DFG) $[176,177]$. This process creates a CEP-stable output from a non-CEP-stabilized femtosecond pulse and has been successfully applied to seeding few-cycle MIR-OPA systems $[166,178,179]$. The output of the sub-10-fs NIR source described in Section 2.B can-after preamplification in a single broadband OPA stage pumped at $1030 \mathrm{~nm}$-efficiently drive DFG to yield a continuum in the $1.5-2.5 \mu \mathrm{m}$ range [180]. The oscillator does not need to be CEP stabilized, since the CEP of the fundamental cancels out in the DFG process.

The spectrum of the DFG output can be efficiently extended in a gas-filled HCF [181] to cover the multi-octave range of 400-2500 nm. The main building blocks of such a supercontinuum generator are sketched in Fig. 9(a). The 10 -fs-scale seed pulses may possibly also be derived directly from the output of the near-1-ps Yb:YAG pump source by cascaded temporal compression [182], as indicated by a dashed line in Fig. 9(a). This approach would greatly relax the need for the active pump-seed synchronization system for the OPCPA described in Section 2.C, because both the pump and the seed would travel comparable optical paths.

All essential processes underlying the above concept have already been successfully demonstrated. In fact, we have recently generated IR continua [shown in Fig. 9(b)] from few-cycle NIR pulses with an efficiency exceeding 10\% [180]. Moreover, the spectrum from a MIR-OPA seeded by a similar CEP-stable continuum could be efficiently broadened in a gasfilled HCF to cover the entire VIS-NIR-MIR spectral range of 400-2500 nm, which is also shown in Fig. 9(b). The temporal characterization of the continuum by second-harmonic frequency-resolved optical gating (FROG) shows the high degree of coherence and compressibility of the generated multi-octave spectrum in Fig. 9(b) [183]. This indicates such continua, at energy levels of hundreds of microjoules, are achievable with few-cycle MIR pulses, in agreement with theoretical predictions [181]. The seed signals for the simulated OPCPA systems discussed below are derived from the multi-octave continuum shown in Fig. 9(b).

\section{Prototypical OPCPA Architectures in 3FST}

The common backbone for all prototypical 3FST architectures we propose and numerically analyze in the following sections consists of (i) a high-power femtosecond laser, in our case a 100-W-scale KLM Yb:YAG thin-disk oscillator, followed by (ii) the multi-octave seed generation described in the previous section and (iii) a multi-100-W-to-kW-scale source of multi$\mathrm{mJ}$, near-1-ps-pulses, in our case based on Yb:YAG thin-disk amplifiers, see Fig. 10(a). The supercontinuum seed [see Fig. 10(a)] is split into three spectral channels: VIS centered
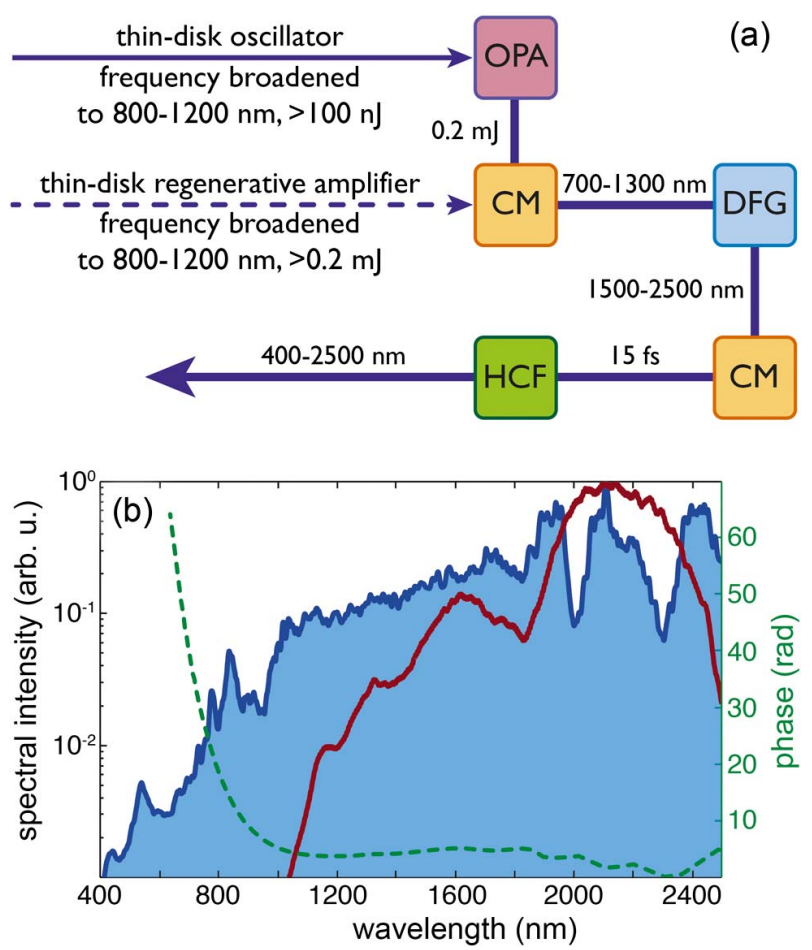

Fig. 9. Multi-octave seed generation. (a) Near-three-octave seed generation schemes based on the output from the oscillator described in Section $2 . \mathrm{B}$ or driven by the amplified subpicosecond pulse characterized in Fig. $\overline{5(\mathrm{~b})}$ (dashed arrow). The spectrally broadened and compressed output of the Yb:YAG oscillator is amplified in an OPA stage to $50 \mu \mathrm{J}$ of energy and subsequently compressed in a chirped-mirror compressor $(\mathrm{CM})$ to about $6 \mathrm{fs}$ for efficient difference frequency generation (DFG). The resultant broadband DFG signal, centered at about $2 \mu \mathrm{m}$, is compressed and focused into a gas-filled hollow-core fiber to extend the spectrum into the visible range. This approach provides a near-three-octave, phase-stable continuum at the energy level of the order of $1 \mu \mathrm{J}$. Alternatively, spectrally broadened subpicosecond millijoule-scale pulses directly from the amplifier circumvent the need for an additional OPA stage and may result in a phase-stable supercontinuum at the level of several hundred microjoules. (b) Broadband phase-stable continua generated in preliminary experiments. Red curve: Difference-frequency radiation in a $500-\mu$ m-thick type-I BBO crystal optimized for $12 \%$ conversion efficiency. Blue shaded area: Spectral broadening of a parametrically amplified three-cycle DFG signal in a gas-filled hollowcore fiber to a supercontinuum containing $330 \mu \mathrm{J}$ of energy. The detected bandwidth of the spectrum in both cases is limited by the sensitivity of the spectrometer in the IR tail. The dashed green curve shows the measured spectral phase of the generated supercontinuum using the FROG technique. The red curve in (b) is reproduced from [180].

at $550 \mathrm{~nm}$, NIR centered at $1 \mu \mathrm{m}$, and MIR centered at $2 \mu \mathrm{m}$, by using chirped dichroic beam splitters [173,184,185].

Each of the three OPA channels can be used to generate tunable multi-cycle pulses which will be described in Section 3.D or to yield few-cycle pulses as will be shown in Section 3.E. Their pump pulses are generated by a simple frequency-converter module comprising two LBO crystals. Our crystal of choice is $\mathrm{LBO}$ instead of $\mathrm{BBO}$, owing to its availability in large sizes and its small spatial and temporal 


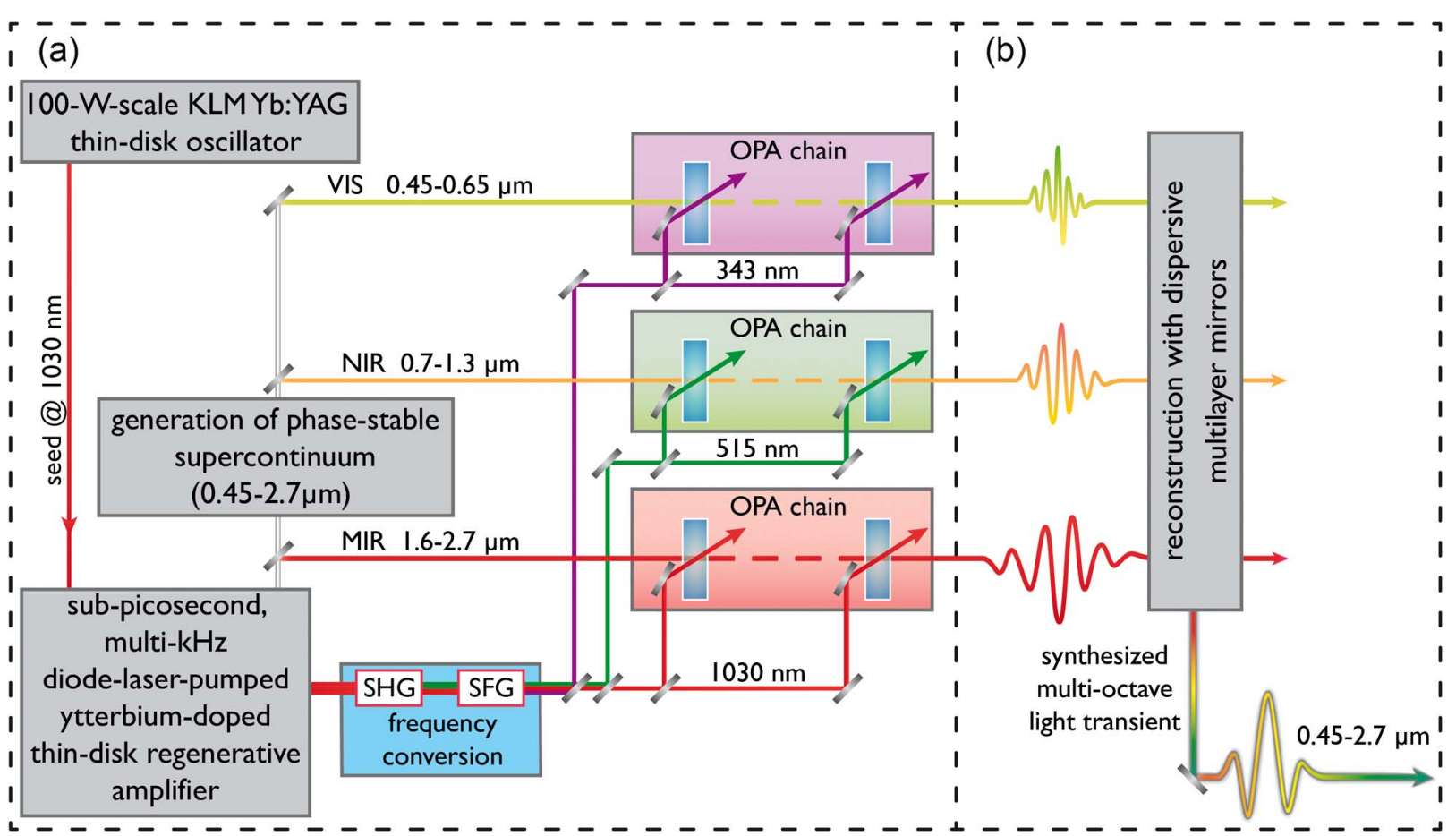

Fig. 10. Prototypical multi-octave 3FST field synthesizer (Section $\underline{3 . E}$ and Section $\underline{3 . F}$ ). (a) Schematic architecture of a three-channel OPCPA system seeded and pumped by subpicosecond ytterbium lasers. A part of its output is used for generating the multi-octave supercontinuum signal, which is split into three channels, centered at $550 \mathrm{~nm}$ and 1 and $2 \mu \mathrm{m}$, respectively. The different channels are pumped by different (low-order) harmonics of the multimillijoule-level, kilohertz Yb:YAG regenerative amplifier output. Each channel supports few-cycle pulses after compression. Alternatively, using a similar concept, several-tens-of-femtoseconds multicycle pulses widely tunable from the UV to the IR spectral range can be produced. (b) By coherently combining the three few-cycle channels amplified in (a), nonsinusoidal multi-octave light transients can be generated.

walk-off, in spite of its smaller effective nonlinear coefficient. Second-harmonic generation (SHG) in the first one yields twin pulses of comparable energy at $515 \mathrm{~nm}$ and $1030 \mathrm{~nm}$, which are mixed in the second crystal to produce a third pump pulse at $343 \mathrm{~nm}$ by sum-frequency generation (SFG). Moderate SHG and SFG conversion efficiencies (of about $50 \%$ and 20\%-30\%, respectively) ensure that all beams exiting the frequency converter unit have a good beam quality, which is important for OPCPA pumping. The three beams are subsequently separated by dichroic beam splitters and directed into the three OPA channels described in the following sections.

\section{Power of 3FST: Tunability over Several Octaves}

Time-resolved spectroscopy often requires tunable multicycle femtosecond pulses. The three OPCPA channels depicted in Fig. 10(a) can be designed to deliver wavelength-tunable femtosecond pulses. Their seed can be generated as described in the preceding section. We propose to produce the primary pump pulses at $1030 \mathrm{~nm}$ with the Yb:YAG thin-disk regenerative amplifier recently demonstrated, yielding $30 \mathrm{~mJ}$, $1.6 \mathrm{ps}$ pulses at a $10 \mathrm{kHz}$ repetition rate, i.e., an average power level of $300 \mathrm{~W}$ [147]. The frequency converter described in the previous section distributes this pump energy among the three OPCPA channels. The super-continuum is divided into three bands centered at carrier wavelengths of $550 \mathrm{~nm}$ and 1 and $2 \mu \mathrm{m}$. They are seeded into the VIS, NIR, and MIR arms of the OPCPA system, each of which consists of two amplifier stages, using thin $\mathrm{BBO}, \mathrm{LBO}$, and $\mathrm{LiNbO}_{3}$ crystals, pumped at 343,515 , and $1030 \mathrm{~nm}$, respectively. For more details, see Supplement 1.

Pulse duration control and wavelength tuning of the amplified pulses is accomplished by temporally stretching the seed continua and controlling their delay with respect to the pump pulses. In fact, the pump temporal window of $\tau_{\text {pump }} \simeq 1 \mathrm{ps}$ slices out a fraction, $\Delta \nu_{\text {signal }}$, of the bandwidth of the (stretched) seed continuum, $\Delta \nu_{\text {seed }}$, which is inversely proportional to the duration $\tau_{\text {seed }}$ of the stretched seed: $\Delta \nu_{\text {signal }} \approx$ $\Delta \nu_{\text {seed }}\left(\tau_{\text {pump }} / \tau_{\text {seed }}\right)$ [see Fig. 11(a)]. Thanks to a near-linear chirp carried by the stretched seed, the carrier frequency of the amplified signal can be tuned by varying the delay of the seed with respect to the pump pulse and by setting the phase-matching angle of the amplifier crystal. Figure 11(b) shows a series of amplified signal spectra from simulations in the three channels pumped with pulses of a duration of $\tau_{\text {pump }} \simeq 1.7$ ps. The continua stretched to $\tau_{\text {seed }} \simeq 30$ ps yield-after recompression—sub-40-fs pulses tunable over several octaves from the VIS to the MIR spectral range. Synchronized femtosecond pulses with adjustable pulse duration and tunable carrier frequency at such a variety of wavelengths and unprecedented average power levels may open new prospects for sophisticated multidimensional spectroscopies and pump-control-probe schemes. 


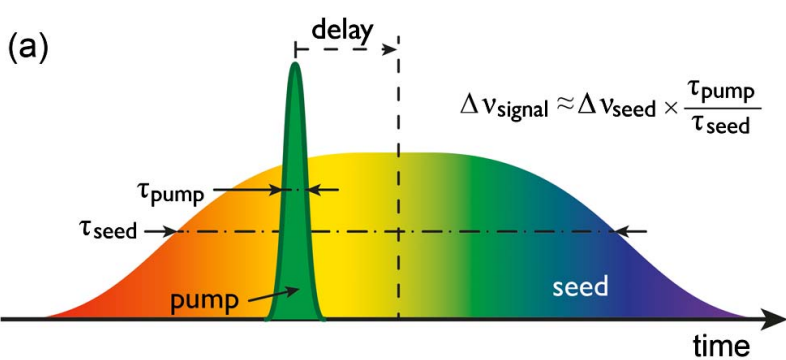

(b)

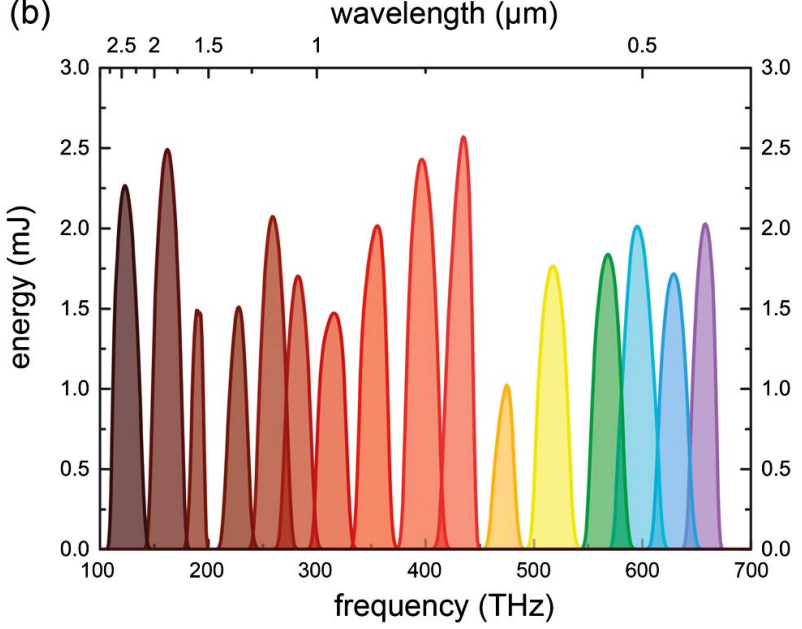

Fig. 11. Generation of widely tunable femtosecond pulses (Section 3.D). (a) Scheme for generating spectrally and temporally tunable pulses at any wavelength. The seed pulses are stretched to a significantly longer duration than that of the pump pulse. Amplification in a simple OPCPA setup yields pulses that can be spectrally tuned by changing the temporal delay between pump and seed pulses. (b) Amplified spectra of a widely tunable two-stage OPCPA system obtained from simulations. Millijoule-level, sub- 40 -fs pulses, tunable from 445 to $2750 \mathrm{~nm}$, can be generated by using different harmonics of a $10 \mathrm{kHz}$ Yb:YAG regenerative amplifier (see Supplement 1).

\section{E. Power of 3FST: Multiterawatt VIS, NIR, and MIR Few-Cycle Waveforms}

As an alternative to delivering tunable multicycle pulses, the three OPCPA channels of our prototypical 3FST system can be designed to generate few-cycle pulses at a single carrier wavelength from each channel, by broadband amplification and subsequent recompression of the continua seeded into the amplifier chains. Typical application fields of few-cycle pulses are attosecond science and extreme nonlinear optics, benefiting from peak powers as high as possible. Few-cycle pulses with multiterawatt peak powers are expected to allow scaling of the flux and/or the photon energy of attosecond pulses by increasing the beam size in high-harmonic generation (HHG) from ionizing atoms [22] or exploiting relativistic interactions with high-density plasmas at the surfaces of solids [186-191]. Therefore, we perform the following model calculations for a prototypical multicolor, multiterawatt few-cycle 3FST system by assuming the availability of the most powerful 3FST driver currently under development: a near-1-ps, $200 \mathrm{~mJ}, 5 \mathrm{kHz}$ Yb:YAG thin-disk regenerative amplifier [158].
In our numerical study, we distribute the pump energy among the three OPA chains in favor of the NIR and MIR channels. This strategy is motivated by numerous applications benefiting from longer wavelengths [192-194]. Frequency conversion to the low-order harmonics as described in Section 3.C yields approximately $40 \mathrm{~mJ}$ at $343 \mathrm{~nm}, 74 \mathrm{~mJ}$ at $515 \mathrm{~nm}$, and $86 \mathrm{~mJ}$ at $1030 \mathrm{~nm}$ for pumping the VIS, NIR, and MIR channels of the OPCPA system, respectively.

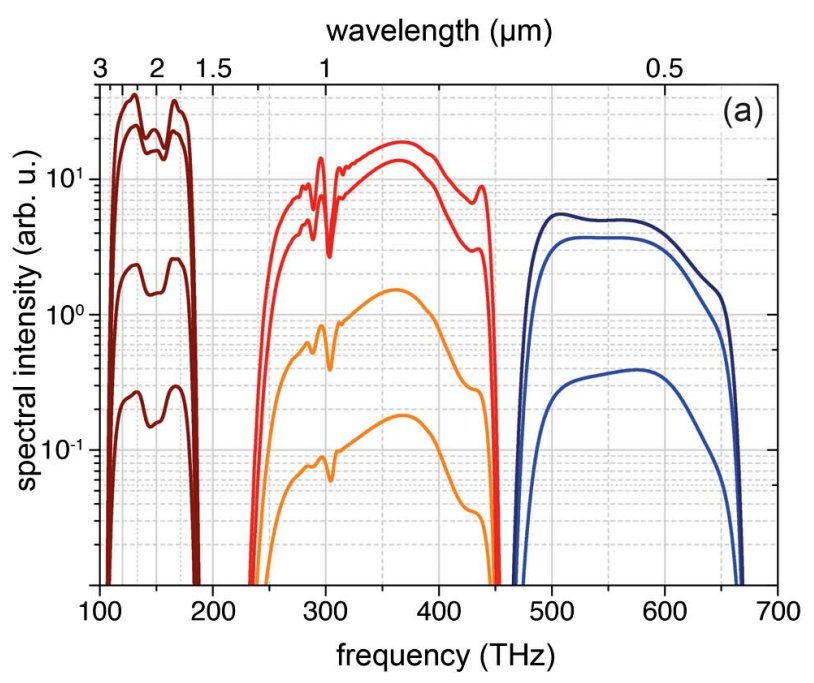

(b)
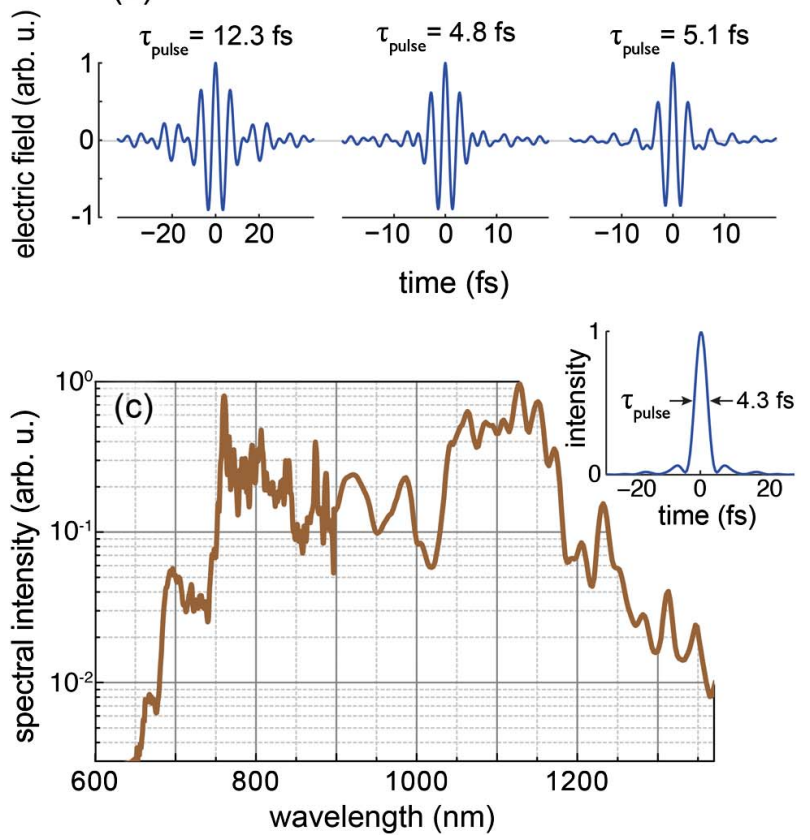

Fig. 12. Multi-octave amplified spectra of the three-channel synthesizer and corresponding waveforms (Section 3.E). (a) Spectra of the three-channel OPCPA synthesizer obtained from simulations. The spectra of the different stages in the different channels are normalized to their energy and shown on a logarithmic scale: VIS, blue; NIR, red-orange; IR, brown. For details of the simulations, see Supplement 1. (b) Fouriertransform-limited electric field associated with the output spectrum of each channel in (a); (c) amplified spectrum in a three-stage OPCPA chain. The spectrum contains $1.8 \mathrm{~mJ}$ and supports sub-5-fs pulses at a $3 \mathrm{kHz}$ repetition rate. 
The high intensity threshold for damage offered by the near1 -ps pump pulses allows a high single-pass gain in very thin (few-millimeter) OPA crystals, yielding a broad amplification bandwidth. The short length of the crystals is optimized for best gain saturation in each amplification stage. The energy is then boosted by using several amplification stages without compromising the bandwidth.

For the MIR channel using four $\mathrm{LiNbO}_{3}$ crystals, our simulations predict an amplified pulse energy of $19.2 \mathrm{~mJ}$, carried at a center wavelength of $2 \mu \mathrm{m}$, with a bandwidth-limited pulse duration of $12.3 \mathrm{fs}$ (FWHM), corresponding to less than two cycles of the carrier wave. The pump-to-signal energy conversion efficiency in this channel is $22 \%$, limited by backconversion of the signal and idler into the pump via phasematched SFG. In the NIR channel, $22.7 \mathrm{~mJ}$ pulses with a bandwidth-limited pulse duration of $4.8 \mathrm{fs}$ can be expected (see Fig. 12), corresponding to a conversion efficiency of $30 \%$. Thanks to the noncollinear geometry, parasitic backconversion is strongly reduced, giving rise to an excellent efficiency. Last but not least, the VIS channel may yield $7.1 \mathrm{~mJ}$ pulses with a bandwidth-limited duration of $5.1 \mathrm{fs}$ (Fig. 12). For details of the simulations, see Supplement 1.

In order to verify the credibility of this design study, we constructed a three-stage OPCPA test, seeded by a continuum derived from a Ti:sapphire front end (Femtopower Compact Pro Ti:sapphire multipass amplifier, Femtolasers $\mathrm{GmbH}$ ) and pumped by an optically synchronized Yb:YAG thin-disk regenerative amplifier [136]. The $3 \mu \mathrm{J}$ seed pulse covering the spectral range of 500-1400 nm was generated in two stages of spectral broadening in a $120-\mu \mathrm{m}$-inner-diameter, $15-\mathrm{cm}$ long HCF filled with $\mathrm{Kr}$ atoms at a pressure of 5 bars and subsequently in a 2 -mm-thick plate of YAG crystal using $30 \mu \mathrm{J}$ of the $1 \mathrm{~mJ}, 25 \mathrm{fs}$ output pulses of the Ti:sapphire amplifier. The three subsequent stages of OPCPA used $2 \mathrm{~mm}$ LBO, $2 \mathrm{~mm}$ $\mathrm{BBO}$, and $4 \mathrm{~mm} \mathrm{LBO}$ as the nonlinear crystal, amplifying the spectral ranges of 800-1350, 670-1000, and 800-1350 nm, respectively. $1 \mathrm{~mJ}$ of a total energy of approximately $8 \mathrm{~mJ}$ of the $1.7 \mathrm{ps}, 515 \mathrm{~nm}$ pump pulse drove the first stage, with the remaining energy and its fraction transmitted by the second stage pumping the second and third stages, respectively.

Figure $12(\mathrm{c})$ shows the spectrum of the amplified pulses supporting a transform-limited pulse duration of $4.3 \mathrm{fs}$ (FWHM). The preliminary compression of the amplified spectrum to sub-10-fs pulses, utilizing a (not-yet-optimized) set of chirped mirrors, reveals a well-behaved spectral phase of the amplified signal, indicating its compressibility to the Fourier limit. The energy of the amplified pulses was $1.8 \mathrm{~mJ}$, with negligible ASE content. The amplified bandwidth supporting sub5 -fs pulses and the conversion efficiency in excess of $20 \%$ achieved already in preliminary experiments create confidence in the predictions of our modeling.

\section{F. Power of 3FST: Synthesis of Multi-Octave, Multiterawatt Light Transients}

Waveform-controlled light transients with a bandwidth approaching two octaves have been demonstrated at microjoule energy and gigawatt peak power levels $[\underline{173}, \underline{184}, \underline{195}, \underline{196}]$. They allow temporal confinement of optical radiation to less than 1 femtosecond in subcycle waveforms $[\underline{197,198]}$. With their power substantially enhanced, these extreme waveforms may open up a new chapter in nonlinear optics and attosecond science (thanks to, among other things, the feasibility of suppressing ionization up to unprecedented peak intensities and instantaneous ionization rates approaching optical frequencies, respectively). The prototypical three-color few-cycle OPCPA system described in the previous section offers a conceptually simple route to scaling multi-octave optical waveform synthesis to the multiterawatt regime.

To this end, the three channels delivering few-cycle pulses in the VIS, NIR, and MIR spectral ranges are recombined using a set of dichroic chirped mirrors to yield one beam in a scheme similar to that reported in $[175,184]$. Due to the difficulties of dispersion management, the high demands on a chirped-mirror compressor, and the required coating for the (a)

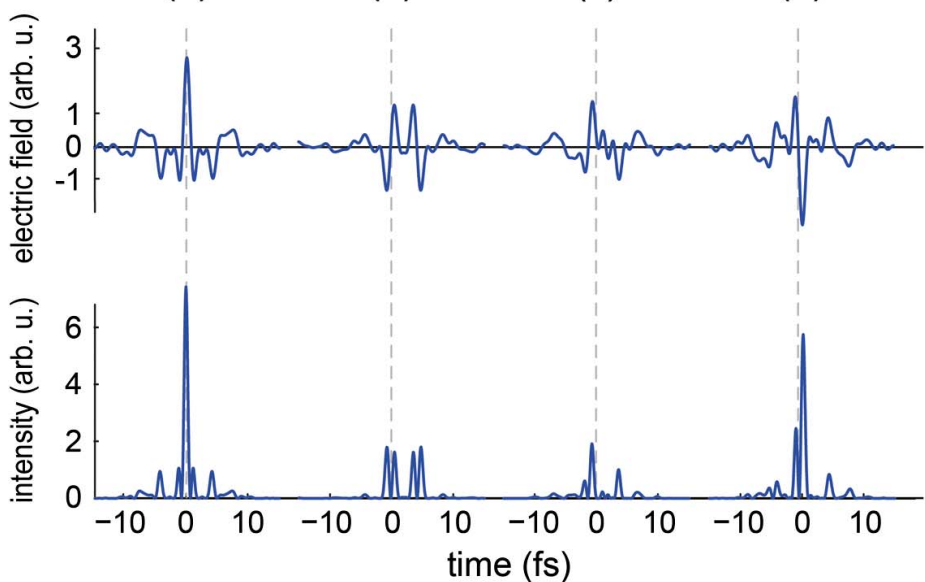

(e)

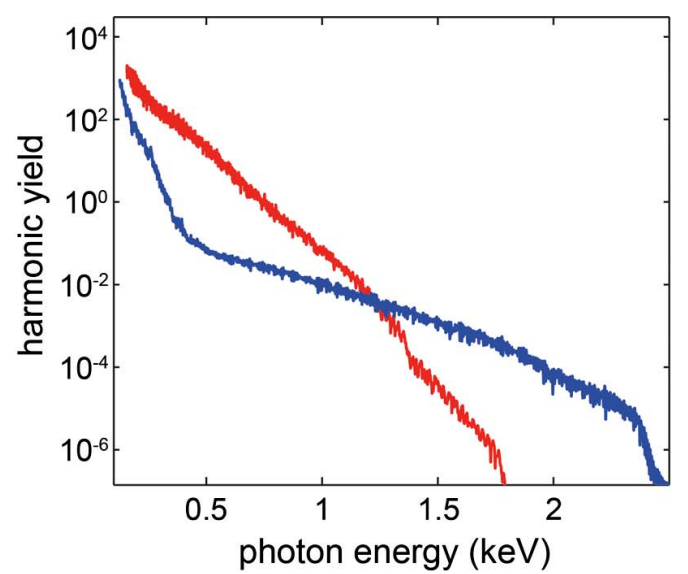

Fig. 13. Calculated synthesized waveforms from the three-channel OPCPA system and high-harmonic generation using synthesized waveforms (Section 3.E). (a) Fourier-transform-limited waveform; (b), (c) synthesized waveforms generated by changing the relative energy and time delay between different arms; (d) optimized waveform for HHG in terms of the highest cutoff energy of the synthesizer; (e) simulated HHG spectrum in helium. The red line represents the Gaussian-shaped pulse with 5 fs FWHM, whereas the blue line corresponds to the preoptimized waveform shown in (d). 
OPCPA crystals in serial pulse synthesis [195], a parallelsynthesis [199] approach is chosen. Furthermore, fluctuations and drifts in the relative timing of the recombined pulses need to be suppressed to a tiny fraction of the half-field cycle for a stable waveform resulting from the coherent superposition [200]. This optical timing synchronization can be accomplished with the required sub-100-as precision with a balanced optical cross correlator demonstrated recently $[\underline{175}, \underline{196}]$. The feasibility of super-octave optical waveform synthesis was recently demonstrated in the NIR-VIS-UV spectral range by seeding a three-channel [184] and, more recently, four-channel [197] synthesizer consisting of broadband chirped mirrors with a continuum originating from a Ti:sapphire-laser-driven hollow-fiber/chirped-mirror compressor. Implementation with an OPA system is also being prepared [199,201,202].

Merely the adjustment of the relative timing of the three pulses emerging from the three OPA channels can result in a great variety of electric field forms on the time scale of the optical cycle. In fact, Figs. 13(a)-13(d) depict a few representative waveforms that may be synthesized from bandwidthlimited pulses emerging from the three channels described in the preceding section by varying their relative timing upon recombination. Further degrees of freedom for waveform sculpting can be introduced by shaping the amplitude and phase of the spectra of the individual channels, e.g., via an acousto-optic pulse shaper [203] and/or a spatial light modulator [204].

\section{CONCLUSIONS AND OUTLOOK}

Femtosecond technology emerged from nonlinear optical techniques allowing both the production and the characterization of femtosecond laser pulses. Its first generation (1FST) relied on dye lasers and delivered femtosecond pulses with peak and average powers up to the $100 \mathrm{MW}$ and $100 \mathrm{~mW}$ ranges, respectively, over a narrow spectral range largely confined to 600-900 nm. Broadband solid-state laser media with high saturation fluence and CPA heralded the second generation of femtosecond technology (2FST), allowing for a boost of the peak and average powers of sub-100-fs pulses to the multiterawatt or $10 \mathrm{~W}$ regime, respectively, but not both of them simultaneously. Powerful 2FST systems are able to produce femtosecond pulses over an extended range of frequencies from the far-IR to the extreme UV via coherent frequency conversion based on $\chi^{(2)}$ and $\chi^{(3)}$ nonlinearities. However, these secondary sources are limited to power levels that are several orders of magnitude lower. The range of carrier wavelengths of powerful primary 2FST sources is - similar to 1FST — rather limited, currently spanning about $0.7-1.1 \mu \mathrm{m}$.

Based on OPCPA driven by terawatt-scale pulses from ytterbium lasers at kilowatt-scale average power (so far demonstrated with water-cooled thin-disk and slab and cryogenically cooled thick-disk technologies), third-generation technology (3FST) allows boosting the peak and average powers of coherent femtosecond light simultaneously to the multiterawatt and hundreds of watts range, respectively. It is capable of doing so over a wavelength range extended to more than two octaves, spanning $0.45-2.5 \mu \mathrm{m}$ with either (i) tunable, synchronized, multicycle, VIS, NIR, MIR tens of femtoseconds duration pulses,

(ii) synchronized, few-cycle VIS, NIR, MIR few-femtosecondduration pulses, or

(iii) subcycle to few-cycle light transients synthesized from all spectral components,

available within the above multi-octave region, in all cases with full control over the generated light waves. These operation modes are being offered by a single basic system architecture and basic instrumentation, providing an unprecedented versatility and variety of methodologies for ultrafast spectroscopy and nonlinear optics.

Driven by the primary 3FST sources outlined in Section $\underline{3}$, secondary sources of femtosecond light are likely to outperform their predecessors based on 2FST in several respects. Not only are 3FST-based secondary sources likely to exceed the power of their 2FST-based predecessors by orders of magnitude, but they may also dramatically extend their spectral coverage. As an example, we have scrutinized the capability of multiterawatt, multi-octave light transients to extend the photon energy frontier of attosecond pulses to several kiloelectron volts, to the boundary of the regime of hard $\mathrm{x}$ rays. With the preoptimized waveform presented in Fig. 13(d), our numerical simulations of $\mathrm{HHG}$, based on the strong field approximation [205] in helium [see caption of Fig. 13(e) for details] show that synthesized multi-octave transients are superior to few-cycle pulses in pushing the frontiers of HHG into the regime of hard $x$ rays. As a matter of fact, our preoptimized subcycle transients substantially increase the photon energy of the cutoff harmonics as compared to those generated by a 5 fs Gaussian pulse of identical peak power. Our preliminary study indicates that 3FST will be beneficial for extending the frontiers of attosecond science into the $\mathrm{x}$-ray regime.

1FST provided real-time access to a wealth of microscopic phenomena for the first time and created the technological basis for the birth of femtochemistry, allowing direct insight into the making and breaking of chemical bonds. 2FST has also created entirely new research fields and technologies, such as laser-driven accelerators and attosecond science. 3FST holds promise for consequences of comparable impact. One of them may be the recording of movies of any microscopic motion outside the atomic core via attosecond $\mathrm{x}$-ray diffraction.

\section{FUNDING INFORMATION}

Center for Advanced Laser Applications (CALA); German Federal Ministry of Education and Research (BMBF); Max-Planck-Institute of Quantum Optics (MPQ); Munich Center for Advanced Photonics (MAP); Deutsche Forschungsgemeinschaft (DFG) (EXC-158).

\section{DEDICATION}

This paper is dedicated to Gerard Mourou on the occasion of his 70th birthday. His invention, chirped-pulse amplification, laid the groundwork for both second-generation and thirdgeneration femtosecond technology, and thereby for all fields of science relying on intense laser light. Thank you, Gerard! 


\section{ACKNOWLEDGMENTS}

The authors acknowledge both direct and indirect contributions from a number of colleagues at MPQ and LMU. Most importantly, the pioneering work of Stefan Karsch, László Veisz, and co-workers in the field of high-power OPCPA has led to relevant progress and insight on which the current work draws. We thank Christian Hackenberger for preparing the graphical illustrations for this paper.

See Supplement 1 for supporting content.

\section{REFERENCES AND NOTES}

1. E. P. Ippen, C. V. Shank, and A. Dienes, "Passive mode locking of the cw dye laser," Appl. Phys. Lett. 21, 348-350 (1972).

2. J. P. Letouzey and S. O. Sari, "Continuous pulse train dye laser using an open flowing passive absorber," Appl. Phys. Lett. 23, 311-313 (1973).

3. C. V. Shank and E. P. Ippen, "Subpicosecond kilowatt pulses from a mode-locked cw dye laser," Appl. Phys. Lett. 24, 373-375 (1974).

4. R. L. Fork, B. I. Greene, and C. V. Shank, "Generation of optical pulses shorter than 0.1 psec by colliding pulse mode-locking," Appl. Phys. Lett. 38, 671-672 (1981).

5. W. Dietel, E. Dopel, D. Kuhlke, and B. Wilhelmi, "Pulses in the femtosecond range from a cw dye ring laser in the colliding pulse mode-locking (CPM) regime with down-chirp," Opt. Commun. 43, 433-436 (1982).

6. W. Dietel, J. J. Fontaine, and J. C. Diels, "Intracavity pulsecompression with glass: a new method of generating pulses shorter than 60 fsec," Opt. Lett. 8, 4-6 (1983).

7. J. A. Valdmanis, R. L. Fork, and J. P. Gordon, "Generation of optical pulses as short as 27 femtoseconds directly from a laser balancing self-phase modulation, group-velocity dispersion, saturable absorption, and saturable gain," Opt. Lett. 10, 131-133 (1985).

8. W. H. Knox, M. C. Downer, R. L. Fork, and C. V. Shank, "Amplified femtosecond optical pulses and continuum generation at $5-\mathrm{kHz}$ repetition rate," Opt. Lett. 9, 552-554 (1984).

9. C. Rolland and P. B. Corkum, "Amplification of 70-fs-pulses in a high repetition rate $\mathrm{XeCl}$ pumped dye-laser amplifier," Opt. Commun. 59, 64-68 (1986).

10. W. Kaiser, D. H. Auston, K. B. Eisenthal, R. M. Hochstrasser, C. K. Johnson, A. Laubereau, D. Linde, A. von der Seilmeier, C. V. Shank, and W. Zinth, Ultrashort Laser Pulses: Generation and Applications (Springer, 1993).

11. A. H. Zewail, "Femtochemistry: atomic-scale dynamics of the chemical bond," J. Phys. Chem. A 104, 5660-5694 (2000).

12. P. F. Moulton, "Spectroscopic and laser characteristics of $\mathrm{Ti}_{-}-\mathrm{Al}_{2} \mathrm{O}_{3}$," J. Opt. Soc. Am. B 3, 125-133 (1986).

13. V. Petričević, S. K. Gayen, R. R. Alfano, K. Yamagishi, H. Anzai, and Y. Yamaguchi, "Laser action in chromium-doped forsterite," Appl. Phys. Lett. 52, 1040-1042 (1988).

14. E. Sorokin, S. Naumov, and I. T. Sorokina, "Ultrabroadband infrared solid-state lasers," IEEE J. Sel. Top. Quantum Electron. 11, 690-712 (2005).

15. D. Strickland and G. Mourou, "Compression of amplified chirped optical pulses," Opt. Commun. 56, 219-221 (1985).

16. D. E. Spence, P. N. Kean, and W. Sibbett, "60-fsec pulse generation from a self-mode-locked Ti:sapphire laser," Opt. Lett. 16, 42-44 (1991).

17. S. Backus, C. G. Durfee, G. Mourou, H. C. Kapteyn, and M. M. Murnane, "0.2-TW laser system at $1 \mathrm{kHz}$," Opt. Lett. 22, 1256-1258 (1997).

18. Y. Nabekawa, Y. Kuramoto, T. Togashi, T. Sekikawa, and S. Watanabe, "Generation of $0.66-\mathrm{TW}$ pulses at $1 \mathrm{kHz}$ by a Ti:sapphire laser," Opt. Lett. 23, 1384-1386 (1998).
19. R. Szipöcs, K. Ferencz, C. Spielmann, and F. Krausz, "Chirped multilayer coatings for broad-band dispersion control in femtosecond lasers," Opt. Lett. 19, 201-203 (1994).

20. M. Nisoli, S. DeSilvestri, O. Svelto, R. Szipöcs, K. Ferencz, C. Spielmann, S. Sartania, and F. Krausz, "Compression of highenergy laser pulses below 5 fs," Opt. Lett. 22, 522-524 (1997).

21. S. Sartania, Z. Cheng, M. Lenzner, G. Tempea, C. Spielmann, F. Krausz, and K. Ferencz, "Generation of O.1-TW 5-fs optical pulses at a 1-kHz repetition rate," Opt. Lett. 22, 1562-1564 (1997).

22. F. Krausz and M. Ivanov, "Attosecond physics," Rev. Mod. Phys. 81, 163-234 (2009).

23. G. A. Mourou, T. Tajima, and S. V. Bulanov, "Optics in the relativistic regime," Rev. Mod. Phys. 78, 309-371 (2006).

24. Y. Chu, X. Liang, L. Yu, Y. Xu, L. Xu, L. Ma, X. Lu, Y. Liu, Y. Leng, R. $\mathrm{Li}$, and Z. Xu, "High-contrast 2.0 petawatt Ti:sapphire laser system," Opt. Express 21, 29231-29239 (2013).

25. S. Hädrich, A. Klenke, A. Hoffmann, T. Eidam, T. Gottschall, J. Rothhardt, J. Limpert, and A. Tünnermann, "Nonlinear compression to sub-30-fs, $0.5 \mathrm{~mJ}$ pulses at $135 \mathrm{~W}$ of average power," Opt. Lett. 38, 3866-3869 (2013).

26. A. Dubietis, G. Jonusauskas, and A. Piskarskas, "Powerful femtosecond pulse generation by chirped and stretched pulse parametric amplification in BBO crystal," Opt. Commun. 88, 437-440 (1992).

27. D. Du, X. Liu, G. Korn, J. Squier, and G. Mourou, "Laser-induced breakdown by impact ionization in $\mathrm{SiO}_{2}$ with pulse widths from 7 ns to 150 fs," Appl. Phys. Lett. 64, 3071-3073 (1994).

28. B. C. Stuart, M. D. Feit, A. M. Rubenchik, B. W. Shore, and M. D. Perry, "Laser-induced damage in dielectrics with nanosecond to subpicosecond pulses," Phys. Rev. Lett. 74, 2248-2251 (1995).

29. M. Lenzner, J. Krüger, S. Sartania, Z. Cheng, C. Spielmann, G. Mourou, W. Kautek, and F. Krausz, "Femtosecond optical breakdown in dielectrics," Phys. Rev. Lett. 80, 4076-4079 (1998).

30. C. Radzewicz, Y. B. Band, G. W. Pearson, and J. S. Krasinski, "Short pulse nonlinear frequency conversion without groupvelocity-mismatch broadening," Opt. Commun. 117, 295-302 (1995).

31. S. M. Saltiel, K. Koynov, B. Agate, and W. Sibbett, "Secondharmonic generation with focused beams under conditions of large group-velocity mismatch," J. Opt. Soc. Am. B 21, 591-598 (2004).

32. C. Y. Chien, G. Korn, J. S. Coe, J. Squier, and G. Mourou, "Highly efficient second-harmonic generation of ultraintense $\mathrm{Nd}$-glass laser pulses," Opt. Lett. 20, 353-355 (1995).

33. P. Rußbüldt, J. Weitenberg, T. Sartorius, G. Rotarius, H. D. Hoffmann, and R. Poprawe, "Ytterbium Innoslab amplifiers-the high average power approach of ultrafast lasers," AIP Conf. Proc. 1462, 120-123 (2012).

34. P. Russbueldt, T. Mans, G. Rotarius, J. Weitenberg, H. D. Hoffmann, and R. Poprawe, "400 W Yb:YAG Innoslab fs-amplifier," Opt. Express 17, 12230-12245 (2009).

35. O. G. Peterson, S. A. Tuccio, and B. B. Snavely, "CW operation of an organic dye solution laser," Appl. Phys. Lett. 17, 245-247 (1970).

36. M. DiDomenico, "Small-signal analysis of internal (coupling-type) modulation of lasers," J. Appl. Phys. 35, 2870-2876 (1964).

37. L. E. Hargrove, R. L. Fork, and M. A. Pollack, "Locking of He- $\mathrm{Ne}$ laser modes induced by synchronous intracavity modulation," Appl. Phys. Lett. 5, 4-5 (1964).

38. A. Yariv, "Internal modulation in multimode laser oscillators," J. Appl. Phys. 36, 388-391 (1965).

39. E. P. Ippen and C. V. Shank, "Dynamic spectroscopy and subpicosecond pulse compression," Appl. Phys. Lett. 27, 488-490 (1975).

40. J. C. Diels, E. Vanstryland, and G. Benedict, "Generation and measurement of 200 femtosecond optical pulses," Opt. Commun. 25, 93-96 (1978).

41. E. B. Treacy, "Measurement of picosecond pulse substructure using compression techniques," Appl. Phys. Lett. 14, 112-114 (1969).

42. R. L. Fork, C. H. B. Cruz, P. C. Becker, and C. V. Shank, "Compression of optical pulses to six femtoseconds by using cubic phase compensation," Opt. Lett. 12, 483-485 (1987). 
43. C. V. Shank, "Generation of ultrashort optical pulses," Top. Appl. Phys. 60, 5-34 (1988).

44. J. C. Diels, "Femtosecond dye lasers," in Dye Laser Principles, F. J. Duarte and L. W. Hillman, eds. (Academic, 1990), Chap. 3, pp. 41-132.

45. P. M. W. French, "The generation of ultrashort laser pulses," Rep. Prog. Phys. 58, 169-262 (1995).

46. U. Keller, G. W. Thooft, W. H. Knox, and J. E. Cunningham, "Femtosecond pulses from a continuously self-starting passively modelocked Ti:sapphire laser," Opt. Lett. 16, 1022-1024 (1991).

47. L. Spinelli, B. Couillaud, N. Goldblatt, and D. K. Negus, "Starting and generation of sub-100 fs pulses in $\mathrm{Ti}^{\mathrm{A}} \mathrm{Al}_{2} \mathrm{O}_{3}$ by self-focusing," in Conference on Lasers and Electro-Optics, J. Bufton, A. Glass, T. Hsu, and W. Krupke, eds., Vol. 10 of OSA Technical Digest (Optical Society of America, 1991), paper CPD7.

48. M. Piche, "Beam reshaping and self-mode-locking in nonlinear laser resonators," Opt. Commun. 86, 156-160 (1991).

49. T. Brabec, C. Spielmann, P. F. Curley, and F. Krausz, "Kerr lens mode-locking," Opt. Lett. 17, 1292-1294 (1992).

50. H. A. Haus, J. G. Fujimoto, and E. P. Ippen, "Analytic theory of additive pulse and Kerr lens mode locking," IEEE J. Quantum Electron. 28, 2086-2096 (1992).

51. V. P. Kalosha, M. Muller, J. Herrmann, and S. Gatz, "Spatiotemporal model of femtosecond pulse generation in Kerr-lens mode-locked solid-state lasers," J. Opt. Soc. Am. B 15, 535-550 (1998).

52. R. Szipöcs, A. Stingl, C. Spielmann, and F. Krausz, "Chirped dielectric mirrors for dispersion control in femtosecond laser systems," Proc. SPIE 2377, 11-22 (1995).

53. A. Stingl, M. Lenzner, C. Spielmann, F. Krausz, and R. Szipöcs, "Sub-10-fs mirror-dispersion-controlled Ti:sapphire laser," Opt. Lett. 20, 602-604 (1995)

54. A. Kasper and K. J. Witte, "10-fs pulse generation from a unidirectional Kerr-lens mode-locked Ti:sapphire ring laser," Opt. Lett. 21 360-362 (1996).

55. T. Brabec and F. Krausz, "Intense few-cycle laser fields: frontiers of nonlinear optics," Rev. Mod. Phys. 72, 545-591 (2000).

56. P. Maine, D. Strickland, P. Bado, M. Pessot, and G. Mourou, "Generation of ultrahigh peak power pulses by chirped pulse amplification," IEEE J. Quantum Electron. 24, 398-403 (1988).

57. J. V. Rudd, G. Korn, S. Kane, J. Squier, G. Mourou, and P. Bado, "Chirped-pulse amplification of 55 -fs pulses at a $1-\mathrm{kHz}$ repetition rate in a $\mathrm{Ti}_{-} \mathrm{Al}_{2} \mathrm{O}_{3}$ regenerative amplifier," Opt. Lett. 18, 2044-2046 (1993).

58. O. E. Martinez, "Design of high-power ultrashort pulse amplifiers by expansion and recompression," IEEE J. Quantum Electron. 23, 1385-1387 (1987).

59. C. P. J. Barty, G. Korn, F. Raksi, A. C. Tien, K. R. Wilson, V. V. Yakovlev, C. Rose-Petruck, J. Squier, and K. Yamakawa, "Regenerative pulse shaping and amplification of ultrabroadband optical pulses," Opt. Lett. 21, 219-221 (1996).

60. M. Hentschel, Z. Cheng, F. Krausz, and C. Spielmann, "Generation of 0.1-TW optical pulses with a single-stage Ti:sapphire amplifier at a 1-kHz repetition rate," Appl. Phys. B 70, S161-S164 (2000).

61. J. Z. H. Yang and B. C. Walker, "0.09-terawatt pulses with a $31 \%$ efficient, kilohertz repetition-rate Ti:sapphire regenerative amplifier," Opt. Lett. 26, 453-455 (2001).

62. S. Backus, R. Bartels, S. Thompson, R. Dollinger, M. Murnane, and $\mathrm{H}$. Kapteyn, "High efficiency, single-stage, $7 \mathrm{kHz}$, high average power ultrafast laser system," in Conference on Lasers and Electro-Optics, Baltimore, Maryland, 2001.

63. D. M. Gaudiosi, A. L. Lytle, P. Kohl, M. M. Murnane, H. C. Kapteyn, and S. Backus, "11-W average power Ti:sapphire amplifier system using downchirped pulse amplification," Opt. Lett. 29, 2665-2667 (2004).

64. J. Huve, T. Haarlammert, T. Steinbruck, J. Kutzner, G. Tsilimis, and $\mathrm{H}$. Zacharias, "High-flux high harmonic soft x-ray generation up to $10 \mathrm{kHz}$ repetition rate," Opt. Commun. 266, 261-265 (2006).

65. T. Imahoko, N. Inoue, K. Takasago, T. Sumiyoshi, H. Sekita, and M. Obara, "Development of a $50 \mathrm{kHz}, 13 \mathrm{~W}$ Ti:sapphire femtosecond regenerative amplifier," in Pacific Rim Conference on Lasers and Electro-Optics (IEEE, 2007), pp. 774-775.

66. S. Chen, M. Chini, H. Wang, C. Yun, H. Mashiko, Y. Wu, and Z. Chang, "Carrier-envelope phase stabilization and control of $1 \mathrm{kHz}, 6 \mathrm{~mJ}, 30 \mathrm{fs}$ laser pulses from a Ti:sapphire regenerative amplifier," Appl. Opt. 48, 5692-5695 (2009).

67. I. Matsushima, H. Yashiro, and T. Tomie, "10 kHz 40 W Ti:sapphire regenerative ring amplifier," Opt. Lett. 31, 2066-2068 (2006).

68. M. Nisoli, S. DeSilvestri, and O. Svelto, "Generation of high energy 10 fs pulses by a new pulse compression technique," Appl. Phys. Lett. 68, 2793-2795 (1996).

69. E. Goulielmakis, M. Schultze, M. Hofstetter, V. S. Yakovlev, J. Gagnon, M. Uiberacker, A. L. Aquila, E. M. Gullikson, D. T. Attwood, R. Kienberger, F. Krausz, and U. Kleineberg, "Single-cycle nonlinear optics," Science 320, 1614-1617 (2008).

70. A. L. Cavalieri, E. Goulielmakis, B. Horvath, W. Helml, M. Schultze, M. Fieß, V. Pervak, L. Veisz, V. S. Yakovlev, M. Uiberacker, A. Apolonski, F. Krausz, and R. Kienberger, "Intense 1.5-cycle near infrared laser waveforms and their use for the generation of ultrabroadband soft-x-ray harmonic continua," New J. Phys. 9, 242 (2007).

71. S. Bohman, A. Suda, T. Kanai, S. Yamaguchi, and K. Midorikawa, "Generation of $5.0 \mathrm{fs}, 5.0 \mathrm{~mJ}$ pulses at $1 \mathrm{kHz}$ using hollow-fiber pulse compression," Opt. Lett. 35, 1887-1889 (2010).

72. W. Schweinberger, A. Sommer, E. Bothschafter, J. Li, F. Krausz, R. Kienberger, and M. Schultze, "Waveform-controlled near-singlecycle milli-joule laser pulses generate sub-10 nm extreme ultraviolet continua," Opt. Lett. 37, 3573-3575 (2012).

73. U. Morgner, F. X. Kartner, S. H. Cho, Y. Chen, H. A. Haus, J. G. Fujimoto, E. P. Ippen, V. Scheuer, G. Angelow, and T. Tschudi, "Sub-two-cycle pulses from a Kerr-lens mode-locked Ti:sapphire laser," Opt. Lett. 24, 411-413 (1999).

74. C. C. Wang and G. W. Racette, "Measurement of parametric gain accompanying optical difference frequency generation," Appl. Phys. Lett. 6, 169-171 (1965).

75. S. E. Harris, M. K. Oshman, and R. L. Byer, "Observation of tunable optical parametric fluorescence," Phys. Rev. Lett. 18, 732-734 (1967).

76. C. Chen, B. Wu, A. Jiang, and G. You, "A new-type ultraviolet SHG crystal: $\beta-\mathrm{BaB}_{2} \mathrm{O}_{4}$, , Sci. Sin. Ser. B 28, 235-243 (1985).

77. T. Wilhelm, J. Piel, and E. Riedle, "Sub-20-fs pulses tunable across the visible from a blue-pumped single-pass noncollinear parametric converter," Opt. Lett. 22, 1494-1496 (1997).

78. E. Riedle, M. Beutter, S. Lochbrunner, J. Piel, S. Schenkl, S. Sporlein, and W. Zinth, "Generation of 10 to 50 fs pulses tunable through all of the visible and the NIR," Appl. Phys. B 71, 457-465 (2000).

79. R. Butkus, R. Danielius, A. Dubietis, A. Piskarskas, and A. Stabinis "Progress in chirped pulse optical parametric amplifiers," Appl. Phys. B 79, 693-700 (2004)

80. A. Dubietis, R. Butkus, and A. P. Piskarskas, "Trends in chirped pulse optical parametric amplification," IEEE J. Sel. Top. Quantum Electron. 12, 163-172 (2006).

81. S. Witte and K. S. E. Eikema, "Ultrafast optical parametric chirpedpulse amplification," IEEE J. Sel. Top. Quantum Electron. 18, 296-307 (2012).

82. A. Vaupel, N. Bodnar, B. Webb, L. Shah, and M. Richardson, "Concepts, performance review, and prospects of table-top, few-cycle optical parametric chirped-pulse amplification," Opt. Eng. 53, 051507 (2014)

83. G. Cerullo and S. De Silvestri, "Ultrafast optical parametric amplifiers," Rev. Sci. Instrum. 74, 1-18 (2003).

84. A. Baltuska, T. Fuji, and T. Kobayashi, "Visible pulse compression to $4 \mathrm{fs}$ by optical parametric amplification and programmable dispersion control," Opt. Lett. 27, 306-308 (2002).

85. D. Herrmann, L. Veisz, R. Tautz, F. Tavella, K. Schmid, V. Pervak, and F. Krausz, "Generation of sub-three-cycle, 16 TW light pulses by using noncollinear optical parametric chirped-pulse amplification," Opt. Lett. 34, 2459-2461 (2009). 
86. I. N. Ross, P. Matousek, M. Towrie, A. J. Langley, and J. L. Collier, "The prospects for ultrashort pulse duration and ultrahigh intensity using optical parametric chirped pulse amplifiers," Opt. Commun. 144, 125-133 (1997).

87. I. N. Ross, P. Matousek, G. H. C. New, and K. Osvay, "Analysis and optimization of optical parametric chirped pulse amplification," J. Opt. Soc. Am. B 19, 2945-2956 (2002).

88. C. N. Danson, P. A. Brummitt, R. J. Clarke, J. L. Collier, B. Fell, A. J. Frackiewicz, S. Hawkes, C. Hernandez-Gomez, P. Holligan, M. H. R. Hutchinson, A. Kidd, W. J. Lester, I. O. Musgrave, D. Neely, D. R. Neville, P. A. Norreys, D. A. Pepler, C. Reason, W. Shaikh, T. B. Winstone, R. W. W. Wyatt, and B. E. Wyborn, "Vulcan petawatt: design, operation and interactions at $5 \times 10^{20} \mathrm{Wcm}^{-2}$," Laser Part. Beams 23, 87-93 (2005).

89. O. V. Chekhlov, J. L. Collier, I. N. Ross, P. K. Bates, M. Notley, C. Hernandez-Gomez, W. Shaikh, C. N. Danson, D. Neely, P. Matousek, S. Hancock, and L. Cardoso, "35 J broadband femtosecond optical parametric chirped pulse amplification system," Opt. Lett. 31, 3665-3667 (2006)

90. V. V. Lozhkarev, G. I. Freidman, V. N. Ginzburg, E. V. Katin, E. A. Khazanov, A. V. Kirsanov, G. A. Luchinin, A. N. Mal'shakov, M. A. Martyanov, O. V. Palashov, A. K. Poteomkin, A. M. Sergeev, A. A. Shaykin, and I. V. Yakovlev, "Compact 0.56 petawatt laser system based on optical parametric chirped pulse amplification in KD*P crystals," Laser Phys. Lett. 4, 421-427 (2007).

91. Z. Major, S. Trushin, I. Ahmad, M. Siebold, C. Wandt, S. Klingebiel, T.-J. Wang, J. A. Fülöp, A. Henig, S. Kruber, R. Weingartner, A. Popp, J. Osterhoff, R. Hörlein, J. Hein, V. Pervak, A. Apolonski, F. Krausz, and S. Karsch, "Basic concepts and current status of the petawatt field synthesizer-a new approach to ultrahigh field generation," Rev. Laser Eng. 37, 431-436 (2009).

92. O. Novak, M. Divoky, H. Turcicova, and P. Straka, "Design of a petawatt optical parametric chirped pulse amplification upgrade of the kilojoule iodine laser PALS," Laser Part. Beams 31, 211-218 (2013).

93. J. Rothhardt, S. Demmler, S. Hadrich, J. Limpert, and A. Tunnermann, "Octave-spanning OPCPA system delivering CEPstable few-cycle pulses and $22 \mathrm{~W}$ of average power at $1 \mathrm{MHz}$ repetition rate," Opt. Express 20, 10870-10878 (2012).

94. S. Adachi, N. Ishii, T. Kanai, A. Kosuge, J. Itatani, Y. Kobayashi, D. Yoshitomi, K. Torizuka, and S. Watanabe, "5-fs, multi-mJ, CEP-locked parametric chirped-pulse amplifier pumped by a 450-nm source at $1 \mathrm{kHz}$," Opt. Express 16, 14341-14352 (2008).

95. D. J. Bradley and W. Sibbett, "Streak-camera studies of picosecond pulses from a mode-locked Nd: glass laser," Opt. Commun. 9, 17-20 (1973)

96. T. R. Royt, "Passive mode-locking of the Nd-glass oscillator at high repetition rate with thermally compensated phosphate glasses," Opt. Commun. 35, 271-276 (1980).

97. L. S. Goldberg, P. E. Schoen, and M. J. Marrone, "Repetitively pulsed mode-locked Nd:phosphate glass laser oscillator-amplifier system," Appl. Opt. 21, 1474-1477 (1982)

98. S. Tokita, J. Kawanaka, Y. Izawa, M. Fujita, and T. Kawashima, "23.7-W picosecond cryogenic-Yb:YAG multipass amplifier," Opt. Express 15, 3955-3961 (2007).

99. P. Russbueldt, T. Mans, J. Weitenberg, H. D. Hoffmann, and R. Poprawe, "Compact diode-pumped 1.1 kW Yb:YAG Innoslab femtosecond amplifier," Opt. Lett. 35, 4169-4171 (2010).

100. T. Eidam, S. Hanf, E. Seise, T. V. Andersen, T. Gabler, C. Wirth, T. Schreiber, J. Limpert, and A. Tünnermann, "Femtosecond fiber CPA system emitting $830 \mathrm{~W}$ average output power," Opt. Lett. 35, 94-96 (2010).

101. K.-H. Hong, J. T. Gopinath, D. Rand, A. M. Siddiqui, S.-W. Huang, E. Li, B. J. Eggleton, J. D. Hybl, T. Y. Fan, and F. X. Kärtner, "Highenergy, kHz-repetition-rate, ps cryogenic Yb:YAG chirped-pulse amplifier," Opt. Lett. 35, 1752-1754 (2010).

102. J. Rothhardt, S. Hädrich, H. Carstens, N. Herrick, S. Demmler, J. Limpert, and A. Tünnermann, "1 $\mathrm{MHz}$ repetition rate hollow fiber pulse compression to sub-100-fs duration at $100 \mathrm{~W}$ average power," Opt. Lett. 36, 4605-4607 (2011).
103. D. A. Rand, S. E. J. Shaw, J. R. Ochoa, D. J. Ripin, A. Taylor, T. Y. Fan, H. Martin, S. Hawes, J. Zhang, S. Sarkisyan, E. Wilson, and P. Lundquist, "Picosecond pulses from a cryogenically cooled, composite amplifier using Yb:YAG and Yb:GSAG," Opt. Lett. 36, 340-342 (2011)

104. K. Kowalewski, J. Zembek, V. Envid, and D. C. Brown, "201 W picosecond green laser using a mode-locked fiber laser driven cryogenic Yb:YAG amplifier system," Opt. Lett. 37, 4633-4635 (2012).

105. K. F. Wall, D. E. Miller, and T. Y. Fan, "Cryo-Yb:YAG lasers for next-generation photoinjector applications," Proc. SPIE $\mathbf{8 2 3 5}$, 823512 (2012).

106. A. Klenke, S. Breitkopf, M. Kienel, T. Gottschall, T. Eidam, S. Hädrich, J. Rothhardt, J. Limpert, and A. Tünnermann, "530 W, $1.3 \mathrm{~mJ}$, four-channel coherently combined femtosecond fiber chirped-pulse amplification system,” Opt. Lett. 38, 2283-2285 (2013).

107. C. Jauregui, J. Limpert, and A. Tünnermann, "High-power fibre lasers," Nat. Photonics 7, 861-867 (2013).

108. R. Riedel, A. Stephanides, M. J. Prandolini, B. Gronloh, B Jungbluth, T. Mans, and F. Tavella, "Power scaling of supercontinuum seeded megahertz-repetition rate optical parametric chirped pulse amplifiers," Opt. Lett. 39, 1422-1424 (2014).

109. B. A. Reagan, C. Baumgarten, K. Wernsing, H. Bravo, M. Woolston, A. Curtis, F. J. Furch, B. Luther, D. Patel, C. Menoni, and J. J. Rocca, "1 Joule, $100 \mathrm{~Hz}$ repetition rate, picosecond CPA laser for driving high average power soft x-ray lasers," in CLEO, OSA Technical Digest (online) (Optical Society of America, 2014), paper SM1F.4.

110. M. Schulz, R. Riedel, A. Willner, T. Mans, C. Schnitzler, P. Russbueldt, J. Dolkemeyer, E. Seise, T. Gottschall, S. Hädrich, S. Duesterer, H. Schlarb, J. Feldhaus, J. Limpert, B. Faatz, A. Tünnermann, J. Rossbach, M. Drescher, and F. Tavella, "Yb: YAG Innoslab amplifier: efficient high repetition rate subpicosecond pumping system for optical parametric chirped pulse amplification," Opt. Lett. 36, 2456-2458 (2011).

111. G. Mourou, B. Brocklesby, T. Tajima, and J. Limpert, "The future is fibre accelerators," Nat. Photonics 7, 258-261 (2013).

112. S. Breitkopf, "A path to terawatt peak-power fibre laser systems" (submitted).

113. G. Huber, C. Kränkel, and K. Petermann, "Solid-state lasers: status and future," J. Opt. Soc. Am. B 27, B93-B105 (2010).

114. D. J. Richardson, J. Nilsson, and W. A. Clarkson, "High power fiber lasers: current status and future perspectives," J. Opt. Soc. Am. B 27, B63-B92 (2010).

115. J. W. Dawson, J. K. Crane, M. J. Messerly, M. A. Prantil, P. H. Pax, A. K. Sridharan, G. S. Allen, D. R. Drachenberg, H. H. Phan, J. E. Heebner, C. A. Ebbers, R. J. Beach, E. P. Hartouni, C. W. Siders, T. M. Spinka, C. P. J. Barty, A. J. Bayramian, L. C. Haefner, F. Albert, W. H. Lowdermilk, A. M. Rubenchik, and R. E. Bonanno, "High average power lasers for future particle accelerators," AIP Conf. Proc. 1507, 147-153 (2012).

116. M. E. Fermann and I. Hartl, "Ultrafast fibre lasers," Nat. Photonics 7, 868-874 (2013).

117. A. Giesen, H. Hügel, A. Voss, K. Wittig, U. Brauch, and H. Opower, "Scalable concept for diode-pumped high-power solid-state lasers," Appl. Phys. B 58, 365-372 (1994).

118. A. Giesen and J. Speiser, "Fifteen years of work on thin-disk lasers: results and scaling laws," IEEE J. Sel. Top. Quantum Electron. 13, 598-609 (2007).

119. D. Kouznetsov, J.-F. Bisson, and K. Ueda, "Scaling laws of disk lasers," Opt. Mater. 31, 754-759 (2009).

120. J. Speiser, "Scaling of thin-disk lasers-influence of amplified spontaneous emission," J. Opt. Soc. Am. B 26, 26-35 (2009).

121. S. V. Marchese, C. R. E. Baer, R. Peters, C. Kränkel, A. G Engqvist, M. Golling, D. J. H. C. Maas, K. Petermann, T. Südmeyer, G. Huber, and U. Keller, "Efficient femtosecond high power $\mathrm{Yb}: \mathrm{Lu}_{2} \mathrm{O}_{3}$ thin disk laser," Opt. Express 15, 16966-16971 (2007). 
122. C. R. E. Baer, C. Kränkel, C. J. Saraceno, O. H. Heckl, M. Golling, T. Südmeyer, R. Peters, K. Petermann, G. Huber, and U. Keller, "Femtosecond $\mathrm{Yb}: \mathrm{Lu}_{2} \mathrm{O}_{3}$ thin disk laser with $63 \mathrm{~W}$ of average power," Opt. Lett. 34, 2823-2825 (2009).

123. S. Ricaud, A. Jaffres, P. Loiseau, B. Viana, B. Weichelt, M. Abdou-Ahmed, A. Voss, T. Graf, D. Rytz, M. Delaigue, E. Mottay, $\mathrm{P}$. Georges, and F. Druon, "Yb:CaGdAlO ${ }_{4}$ thin-disk laser," Opt. Lett. 36, 4134-4136 (2011).

124. W. P. Latham, A. Lobad, T. C. Newell, and D. Stalnaker, "6.5 kW, Yb:YAG ceramic thin disk laser," AIP Conf. Proc. 1278, 758-764 (2010).

125. M. Suzuki, H. Kiriyama, I. Daito, Y. Ochi, H. Okada, M. Sato, Y. Tamaoki, T. Yoshii, J. Maeda, S. Matsuoka, H. Kan, P. R. Bolton, A. Sugiyama, K. Kondo, and S. Kawanishi, "Hundred mJ, subpicoseconds, high temporal contrast OPCPA/Yb:YAG ceramic thin disk hybrid laser system," Appl. Phys. B 105, 181-184 (2011).

126. J. Mende, G. Spindler, J. Speiser, W. L. Bohn, and A. Giesen, "Mode dynamics and thermal lens effects of thin-disk lasers," Proc. SPIE 6871, 68710M (2008).

127. A. Killi, C. Stolzenburg, I. Zawischa, D. Sutter, J. Kleinbauer, S. Schad, R. Brockmann, S. Weiler, J. Neuhaus, S. Kalfhues, E. Mehner, D. Bauer, H. Schlueter, and C. Schmitz, "The broad applicability of the disk laser principle: from CW to ps," Proc. SPIE 7193, 71931T (2009).

128. A. Antognini, K. Schuhmann, F. D. Amaro, F. Biraben, A. Dax, A. Giesen, T. Graf, T. W. Hansch, P. Indelicato, L. Julien, C.-Y. Kao, P. E. Knowles, F. Kottmann, E. Le Bigot, Y.-W. Liu, L. Ludhova, N. Moschuring, F. Mulhauser, T. Nebel, F. Nez, P. Rabinowitz, C. Schwob, D. Taqqu, and R. Pohl, "Thin-disk Yb:YAG oscillatoramplifier laser, ASE, and effective Yb:YAG lifetime," IEEE J. Quantum Electron. 45, 993-1005 (2009).

129. H. Furuse, H. Chosrowjan, J. Kawanaka, N. Miyanaga, M. Fujita, and Y. Izawa, "ASE and parasitic lasing in thin disk laser with antiASE cap," Opt. Express 21, 13118-13124 (2013).

130. D. Bauer, I. Zawischa, D. H. Sutter, A. Killi, and T. Dekorsy, "Modelocked Yb:YAG thin-disk oscillator with $41 \mu \mathrm{J}$ pulse energy at 145 W average infrared power and high power frequency conversion," Opt Express 20, 9698-9704 (2012).

131. S. Ricaud, A. Jaffres, K. Wentsch, A. Suganuma, B. Viana, P. Loiseau, B. Weichelt, M. Abdou-Ahmed, A. Voss, T. Graf, D. Rytz, C. Hönninger, E. Mottay, P. Georges, and F. Druon, "Femtosecond $\mathrm{Yb}: \mathrm{CaGdAIO}_{4}$ thin-disk oscillator," Opt. Lett. 37, 3984-3986 (2012).

132. O. Pronin, J. Brons, C. Grasse, V. Pervak, G. Boehm, M. C. Amann, A. Apolonski, V. L. Kalashnikov, and F. Krausz, "High-power Kerrlens mode-locked Yb:YAG thin-disk oscillator in the positive dispersion regime," Opt. Lett. 37, 3543-3545 (2012).

133. C. J. Saraceno, F. Emaury, C. Schriber, M. Hoffmann, M. Golling, T. Südmeyer, and U. Keller, "Ultrafast thin-disk laser with $80 \mu \mathrm{J}$ pulse energy and $242 \mathrm{~W}$ of average power," Opt. Lett. 39, 9-12 (2014).

134. C. Teisset, M. Schultze, R. Bessing, M. Häfner, J. Rauschenberger, D. Sutter, and T. Metzger, "Picosecond thin-disk regenerative amplifier with high average power for pumping optical parametric amplifiers," in CLEO, OSA Postdeadline Paper Digest (online) (Optical Society of America, 2013), paper CTh5C.6.

135. J.-P. Negel, A. Voss, M. A. Ahmed, D. Bauer, D. Sutter, A. Killi, and T. Graf, "1.1 kW average output power from a thin-disk multipass amplifier for ultrashort laser pulses," Opt. Lett. 38, 5442-5445 (2013).

136. H. Fattahi, C. Skrobol, M. Ueffing, Y. Deng, A. Schwarz, Y. Kida, V. Pervak, T. Metzger, Z. Major, and F. Krausz, "High efficiency, multi-mJ, sub $10 \mathrm{fs}$, optical parametric amplifier at $3 \mathrm{kHz}$," in Conference on Lasers and Electro-Optics, OSA Technical Digest (online) (Optical Society of America, 2012), paper CTh1N.6.

137. J. Aus der Au, G. J. Spuhler, T. Südmeyer, R. Paschotta, R. Hovel, M. Moser, S. Erhard, M. Karszewski, A. Giesen, and U. Keller, "16.2-W average power from a diode-pumped femtosecond $\mathrm{Yb}$ : YAG thin disk laser," Opt. Lett. 25, 859-861 (2000).
138. C. J. Saraceno, F. Emaury, O. H. Heckl, C. R. E. Baer, M. Hoffmann, C. Schriber, M. Golling, T. Südmeyer, and U. Keller, "275 W average output power from a femtosecond thin disk oscillator operated in a vacuum environment," Opt. Express 20 , 23535-23541 (2012).

139. S. V. Marchese, C. R. Baer, A. G. Engqvist, S. Hashimoto, D. J. Maas, M. Golling, T. Sudmeyer, and U. Keller, "Femtosecond thin disk laser oscillator with pulse energy beyond the 10-microjoule level," Opt Express 16, 6397-6407 (2008).

140. J. Brons, V. Pervak, E. Fedulova, M. Seidel, D. Bauer, D. Sutter, V. L. Kalashnikov, A. Apolonski, O. Pronin, and F. Krausz, "Power-scaling of Kerr-lens mode-locked Yb:YAG thin-disk oscillators," in CLEO, OSA Technical Digest (online) (Optical Society of America, 2014), paper SM4F.7.

141. A. Diebold, F. Emaury, C. Schriber, M. Golling, C. J. Saraceno, T. Südmeyer, and U. Keller, "SESAM mode-locked $\mathrm{Yb}: \mathrm{CaGdAIO}_{4}$ thin disk laser with 62 fs pulse generation," Opt. Lett. 38, 3842-3845 (2013).

142. C. J.Saraceno, C. Schriber, M. Mangold, M. Hoffmann, O. H. Heckl, C. R. Baer, M. Golling, T. Südmeyer, and U. Keller, "SESAMs for high-power oscillators: design guidelines and damage thresholds," IEEE J. Sel. Top. Quantum Electron. 18, 29-41 (2012).

143. O. Pronin, J. Brons, C. Grasse, V. Pervak, G. Boehm, M. C. Amann, V. L. Kalashnikov, A. Apolonski, and F. Krausz, "High-power 200 fs Kerr-lens mode-locked Yb:YAG thin-disk oscillator," Opt. Lett. 36, 4746-4748 (2011).

144. O. Pronin, M. Seidel, F. Lücking, J. Brons, V. Pervak, A. Apolonski, T. Udem, and F. Krausz, "Next-generation source of waveformcontrolled light" (submitted).

145. C. Hönninger, I. Johannsen, M. Moser, G. Zhang, A. Giesen, and U. Keller, "Diode-pumped thin-disk Yb:YAG regenerative amplifier," Appl. Phys. B 65, 423-426 (1997).

146. T. Metzger, C. Y. Teisset, and F. Krausz, "High-repetition-rate picosecond pump laser based on a Yb:YAG disk amplifier for optical parametric amplification," in Advanced Solid-State Photonics, OSA Technical Digest Series (CD) (Optical Society of America, 2008), paper TuA2.

147. C. Teisset, M. Schultze, R. Bessing, M. Haefner, S. Prinz, D. Sutter, and T. Metzger, "300 W picosecond thin-disk regenerative amplifier at $10 \mathrm{kHz}$ repetition rate," in Advanced Solid-State Lasers Congress Postdeadline, OSA Postdeadline Paper Digest (online) (Optical Society of America, 2013), paper JTh5A.1.

148. J. Tuemmler, R. Jung, T. Nubbemeyer, I. Will, and W. Sandner, "Providing thin-disk technology for high laser pulse energy at high average power," in Frontiers in Optics 2011/Laser Science XXVII, OSA Technical Digest (Optical Society of America, 2011), paper FThB3.

149. M. Schulz, H. Hoeppner, M. Temme, R. Riedel, B. Faatz, M. J. Prandolini, M. Drescher, and F. Tavella, "14 kilowatt burst average power from 2-stage cascaded Yb:YAG thin-disk multipass amplifier," in Frontiers in Optics, OSA Technical Digest (online) (Optical Society of America, 2013), paper FTu4A.2.

150. C. Skrobol, I. Ahmad, S. Klingebiel, C. Wandt, S. A. Trushin, Z. Major, F. Krausz, and S. Karsch, "Broadband amplification by picosecond OPCPA in DKDP pumped at 515 nm," Opt. Express 20, 4619-4629 (2012).

151. S. Klingebiel, I. Ahmad, C. Wandt, C. Skrobol, S. A. Trushin, Z. Major, F. Krausz, and S. Karsch, "Experimental and theoretical investigation of timing jitter inside a stretcher-compressor setup," Opt. Express 20, 3443-3455 (2012).

152. T. Miura, K. Takasago, A. Endo, K. Torizuka, and F. Kannari, "Timing stabilization of the 1-kHz femtosecond pulses with active control by means of the spectral-resolved upconversion," in The 4th Pacific Rim Conference on Lasers and Electro-Optics (IEEE, 2001), Vol. 2, pp. 520-521.

153. A. Schwarz, M. Ueffing, Y. Deng, X. Gu, H. Fattahi, T. Metzger, M. Ossiander, F. Krausz, and R. Kienberger, "Active stabilization for optically synchronized optical parametric chirped pulse amplification," Opt. Express 20, 5557-5565 (2012). 
154. S. Prinz, "Sub-2-fs active pump-seed synchronization for OPCPA" (submitted).

155. J. Tümmler, R. Jung, H. Stiel, P. V. Nickles, and W. Sandner, "High-repetition-rate chirped-pulse-amplification thin-disk laser system with joule-level pulse energy," Opt. Lett. 34, 1378-1380 (2009).

156. J. Tümmler, R. Jung, H. Stiel, P. V. Nickles, and W. Sandner, "High repetition rate diode pumped CPA thin disk laser of the joule class," in Conference on Lasers and Electro-Optics/International Quantum Electronics Conference, OSA Technical Digest (CD) (Optical Society of America, 2009), paper CFD4.

157. http://www.mb-berlin.de/de/research/projects/1.2/topics/1_ power_disk_laser/.

158. M. Gorjan and T. Metzger, (unpublished).

159. J. Speiser, "Thin disk laser-energy scaling," Laser Phys. 19, 274-280 (2009).

160. T. Gottwald, C. Stolzenburg, D. Bauer, J. Kleinbauer, V. Kuhn, T. Metzger, S. Schad, D. Sutter, and A. Killi, "Recent disk laser development at Trumpf," Proc. SPIE 8547, 85470C (2012).

161. M. Kienel, M. Müller, S. Demmler, J. Rothhardt, A. Klenke, T. Eidam, J. Limpert, and A. Tünnermann, "Coherent beam combination of Yb:YAG single-crystal rod amplifiers," Opt. Lett. 39, 3278-3281 (2014)

162. J. Limpert, "Performance scaling of ultrafast laser systems by coherent addition of femtosecond pulses," in CLEO, OSA Technical Digest (online) (Optical Society of America, 2014), paper SW3E.3.

163. M. Kienel, A. Klenke, T. Eidam, S. Hädrich, J. Limpert, and A. Tünnermann, "Energy scaling of femtosecond amplifiers using actively controlled divided-pulse amplification," Opt. Lett. 39, 1049-1052 (2014).

164. D. Herrmann, C. Homann, R. Tautz, M. Scharrer, P. S. J. Russell, F. Krausz, L. Veisz, and E. Riedle, "Approaching the full octave: noncollinear optical parametric chirped pulse amplification with twocolor pumping," Opt. Express 18, 18752-18762 (2010).

165. Alternatively, momentum conservation can also be fulfilled by periodic modulation of some optical property of the nonlinear crystal (quasi-phase matching) instead of exploiting birefringence. Periodically poled crystals are being widely used for this purpose.

166. D. Brida, C. Manzoni, G. Cirmi, M. Marangoni, S. Bonora, P. Villoresi, S. D. Silvestri, and G. Cerullo, "Few-optical-cycle pulses tunable from the visible to the mid-infrared by optical parametric amplifiers," J. Opt. 12, 013001 (2010).

167. R. Danielius, A. Piskarskas, A. Stabinis, G. P. Banfi, P. Di Trapani, and R. Righini, "Traveling-wave parametric generation of widely tunable, highly coherent femtosecond light pulses," J. Opt. Soc. Am. B 10, 2222-2232 (1993).

168. T. J. Driscoll, G. M. Gale, and F. Hache, "Ti:sapphire secondharmonic-pumped visible range femtosecond optical parametric oscillator," Opt. Commun. 110, 638-644 (1994).

169. G. M. Gale, M. Cavallari, T. J. Driscoll, and F. Hache, "Sub-20-fs tunable pulses in the visible from an $82-\mathrm{MHz}$ optical parametric oscillator," Opt. Lett. 20, 1562-1564 (1995).

170. G. M. Gale, M. Cavallari, and F. Hache, "Femtosecond visible optical parametric oscillator," J. Opt. Soc. Am. B 15, 702-714 (1998).

171. Optical parametric amplification done in the frequency domain holds promise for relaxing restrictions arising from phase mismatch in OPA

172. B. E. Schmidt, N. Thiré, M. Boivin, A. Laramée, F. Poitras, G. Lebrun, T. Ozaki, H. Ibrahim, and F. Légaré, "Frequency domain optical parametric amplification," Nat. Commun. 5, 3643 (2014).

173. M. T. Hassan, A. Wirth, I. Grguraš, A. Moulet, T. T. Luu, J. Gagnon, V. Pervak, and E. Goulielmakis, "Invited Article: Attosecond photonics: synthesis and control of light transients," Rev. Sci. Instrum. 83, 111301 (2012).

174. S.-W. Huang, G. Cirmi, J. Moses, K.-H. Hong, S. Bhardwaj, J. R. Birge, L.-J. Chen, E. Li, B. J. Eggleton, G. Cerullo, and F. X. Kartner, "High-energy pulse synthesis with sub-cycle waveform control for strong-field physics," Nat. Photonics 5, 475-479 (2011).
175. S.-W. Huang, G. Cirmi, J. Moses, K.-H. Hong, S. Bhardwaj, J. R. Birge, L.-J. Chen, I. V. Kabakova, E. Li, B. J. Eggleton, G. Cerullo, and F. X. Kärtner, "Optical waveform synthesizer and its application to high-harmonic generation," J. Phys. B 45, 074009 (2012).

176. C. Manzoni, G. Cerullo, and S. De Silvestri, "Ultrabroadband selfphase-stabilized pulses by difference-frequency generation," Opt. Lett. 29, 2668-2670 (2004)

177. G. Cerullo, A. Baltuška, O. D. Mücke, and C. Vozzi, "Few-opticalcycle light pulses with passive carrier-envelope phase stabilization," Laser Photon. Rev. 5, 323-351 (2011).

178. K.-H. Hong, S.-W. Huang, J. Moses, X. Fu, C.-J. Lai, G. Cirmi, A Sell, E. Granados, P. Keathley, and F. X. Kärtner, "High-energy, phase-stable, ultrabroadband $\mathrm{kHz}$ OPCPA at $2.1 \mu \mathrm{m}$ pumped by a picosecond cryogenic Yb:YAG laser," Opt. Express 19, 15538-15548 (2011).

179. Y. Deng, A. Schwarz, H. Fattahi, M. Ueffing, X. Gu, M. Ossiander, T. Metzger, V. Pervak, H. Ishizuki, T. Taira, T. Kobayashi, G. Marcus, F. Krausz, R. Kienberger, and N. Karpowicz, "Carrierenvelope-phase-stable, $1.2 \mathrm{~mJ}, 1.5$ cycle laser pulses at 2.1 нm," Opt. Lett. 37, 4973-4975 (2012).

180. H. Fattahi, A. Schwarz, S. Keiber, and N. Karpowicz, "Efficient, octave-spanning difference-frequency generation using few-cycle pulses in simple collinear geometry," Opt. Lett. 38, 4216-4219 (2013)

181. D. Kartashov, S. Ališauskas, A. Pugžlys, A. Voronin, A. Zheltikov, M. Petrarca, P. Béjot, J. Kasparian, J.-P. Wolf, and A. Baltuška, "White light generation over three octaves by femtosecond filament at $3.9 \mu \mathrm{m}$ in argon," Opt. Lett. 37, 3456-3458 (2012).

182. A. A. Voronin, J. M. Mikhailova, M. Gorjan, Z. Major, and A. M. Zheltikov, "Pulse compression to subcycle field waveforms with split-dispersion cascaded hollow fibers," Opt. Lett. $\mathbf{3 8}$ 4354-4357 (2013).

183. A. Schwarz, "Few-cycle, phase-stable infrared OPCPA," Ph.D. dissertation (LMU, 2014)

184. A. Wirth, M. T. Hassan, I. Grguraš, J. Gagnon, A. Moulet, T. T. Luu, S. Pabst, R. Santra, Z. A. Alahmed, A. M. Azzeer, V. S. Yakovlev, V. Pervak, F. Krausz, and E. Goulielmakis, "Synthesized light transients," Science 334, 195-200 (2011).

185. O. Razskazovskaya, et al., is preparing an article.

186. S. V. Bulanov, N. M. Naumova, and F. Pegoraro, "Interaction of an ultrashort, relativistically strong laser pulse with an overdense plasma," Phys. Plasmas 1, 745-757 (1994).

187. R. Lichters, J. Meyer-ter-Vehn, and A. Pukhov, "Short-pulse laser harmonics from oscillating plasma surfaces driven at relativistic intensity," Phys. Plasmas 3, 3425-3437 (1996).

188. T. Baeva, S. Gordienko, and A. Pukhov, "Theory of high-order harmonic generation in relativistic laser interaction with overdense plasma," Phys. Rev. E 74, 046404 (2006).

189. G. D. Tsakiris, K. Eidmann, J. Meyer-ter-Vehn, and F. Krausz, "Route to intense single attosecond pulses," New J. Phys. 8, 19 (2006).

190. P. Heissler, R. Hörlein, J. M. Mikhailova, L. Waldecker, P. Tzallas, A. Buck, K. Schmid, C. M. S. Sears, F. Krausz, L. Veisz, M. Zepf, and G. D. Tsakiris, "Few-cycle driven relativistically oscillating plasma mirrors: a source of intense isolated attosecond pulses," Phys. Rev. Lett. 108, 235003 (2012).

191. J. M. Mikhailova, M. V. Fedorov, N. Karpowicz, P. Gibbon, V. T. Platonenko, A. M. Zheltikov, and F. Krausz, "Isolated attosecond pulses from laser-driven synchrotron radiation," Phys. Rev. Lett. 109, 245005 (2012).

192. J. Tate, T. Auguste, H. G. Muller, P. Salières, P. Agostini, and L. F. DiMauro, "Scaling of wave-packet dynamics in an intense midinfrared field," Phys. Rev. Lett. 98, 013901 (2007).

193. A. D. Shiner, C. Trallero-Herrero, N. Kajumba, H. C. Bandulet, D. Comtois, F. Légaré, M. Giguère, J. C. Kieffer, P. B. Corkum, and D. M. Villeneuve, "Wavelength scaling of high harmonic generation efficiency," Phys. Rev. Lett. 103, 073902 (2009). 
194. F. Krausz and M. I. Stockman, "Attosecond metrology: from electron capture to future signal processing," Nat. Photonics $\mathbf{8}$, 205-213 (2014).

195. A. Harth, M. Schultze, T. Lang, T. Binhammer, S. Rausch, and U. Morgner, "Two-color pumped OPCPA system emitting spectra spanning 1.5 octaves from VIS to NIR," Opt. Express 20, 3076-3081 (2012).

196. C. Manzoni, S. W. Huang, G. Cirmi, P. Farinello, J. Moses, F. X. Kärtner, and G. Cerullo, "Coherent synthesis of ultra-broadband optical parametric amplifiers," Opt. Lett. 37, 1880-1882 (2012).

197. T. T. Luu, M. T. Hassan, A. Moulet, O. Razskazovskaya, N. Kaprowicz, V. Pervak, F. Krausz, and E. Goulielmakis, "Isolated optical attosecond pulses," in CLEO, OSA Technical Digest (online) (Optical Society of America, 2013), paper QF1C.6.

198. M. T. Hassan, "Attosecond control of bound electrons" (submitted).

199. F. X. Kärtner, O. Mücke, G. Cirmi, S. Fang, S.-H. Chia, C. Manzoni, P. Farinello, and G. Cerullo, "High energy sub-cycle optical waveform synthesizer," in Advanced Solid-State Lasers Congress, OSA Technical Digest (online) (Optical Society of America, 2013), paper AW2A.1.

200. S. N. Bagayev, V. I. Trunov, E. V. Pestryakov, V. E. Leschenko, S. A. Frolov, and V. A. Vasiliev, "High-intensity femtosecond laser systems based on coherent combining of optical fields," Opt. Spectrosc. 115, 311-319 (2013).
201. G. M. Rossi, G. Cirmi, S. Fang, S.-H. Chia, O. D. Muecke, F. Kärtner, C. Manzoni, P. Farinello, and G. Cerullo, "Spectrotemporal characterization of all channels in a sub-optical-cycle parametric waveform synthesizer," in CLEO, OSA Technical Digest (online) (Optical Society of America, 2014), paper SF1E.3.

202. F. Shaobo, G. Cirmi, S. H. Chia, O. D. Mucke, F. X. Kartner, C. Manzoni, P. Farinello, and G. Cerullo, "Multi-mJ parametric synthesizer generating two-octave-wide optical waveforms," in Conference on Lasers and Electro-Optics Pacific Rim (Optical Society of America, 2013), paper WB3_1.

203. F. Verluise, V. Laude, Z. Cheng, C. Spielmann, and P. Tournois, "Amplitude and phase control of ultrashort pulses by use of an acousto-optic programmable dispersive filter: pulse compression and shaping," Opt. Lett. 25, 575-577 (2000).

204. T. Binhammer, E. Rittweger, R. Ell, F. X. Kärtner, and U. Morgner, "Prism-based pulse shaper for octave spanning spectra," IEEE J. Quantum Electron. 41, 1552-1557 (2005).

205. M. Lewenstein, P. Balcou, M. Y. Ivanov, A. L'Huillier, and P. B. Corkum, "Theory of high-harmonic generation by low-frequency laser fields," Phys. Rev. A 49, 2117-2132 (1994).

206. M. Schultze, C. Y. Teisset, S. Prinz, D. H. Sutter, K. Michel, and T. Metzger, "Highly-efficient, optically synchronized thin disk amplifier for pumping OPCPA at high repetition rates between 100-300 kHz," Solid State Lasers XXIII: Technology and Devices, 2014, to be published. 


\section{E.3 Efficient, octave-spanning difference-frequency generation using few-cycle pulses in simple collinear geometry}

This paper was published in Optics Letter and is made available as an electronic reprint with the permission of OSA. The paper can be found at the following URL on the OSA website: http://www.opticsinfobase.org/ol/ abstract. cfm? uri=ol-38-20-4216. Systematic or multiple reproduction or distribution to multiple locations via electronic or other means is prohibited and is subject to penalties under law. 


\title{
Efficient, octave-spanning difference-frequency generation using few-cycle pulses in simple collinear geometry
}

\author{
Hanieh Fattahi, ${ }^{1,2}$ Alexander Schwarz, ${ }^{1,2}$ Sabine Keiber, ${ }^{1,2}$ and Nicholas Karpowicz ${ }^{1, *}$ \\ ${ }^{1}$ Max Planck Institut für Quantenoptik, Hans-Kopfermann-Straße 1, 85748 Garching, Germany \\ ${ }^{2}$ Department of Physics, Ludwig-Maximilians-Universität München, Am Coulombwall 1, 85748 Garching, Germany \\ ${ }^{*}$ Corresponding author: nicholas.karpowicz@mpq.mpg.de
}

Received August 21, 2013; revised September 17, 2013; accepted September 18, 2013; posted September 19, 2013 (Doc. ID 196144); published October 14, 2013

We present experimental observations and corresponding numerical simulations illustrating the differencefrequency generation of mid-infrared radiation using few-cycle near-infrared-to-visible pulses, which yields conversion efficiencies above $12 \%$ in beta-barium borate crystal. Type I and type II phase-matching are shown to yield qualitatively different intensity-scaling behavior, with the former showing higher overall efficiency, especially with the addition of a zero-order wave plate for modifying the polarization state of the pulse, and the latter having a better stability of the spectrum versus input intensity. (c) 2013 Optical Society of America

OCIS codes: (190.7110) Ultrafast nonlinear optics; (190.4410) Nonlinear optics, parametric processes; (190.4223)

Nonlinear wave mixing.

http://dx.doi.org/10.1364/OL.38.004216

Sources of intense, broadband, coherent, and phasestabilized light in the mid-infrared (MIR) are seeing increasing interest in the ultrafast community, especially in the realm of attosecond physics [ $[1,2]$. Both technologically, where the demand for higher photon energies from the high-harmonic-generation process makes longer wavelength-driving pulses advantageous [3-5], and for experiments where either the long wavelength is desirable for avoiding electronic resonances in a solid-state system $[\underline{6}, 7]$ or for matching the oscillations of the applied field to the dynamics being studied []ㅡ, there is a great demand for few-cycle MIR light sources. This is now being achieved with optical parametric amplifiers (OPAs), either seeded via difference-frequency generation (DFG) directly in the MIR [ $\underline{9}-\underline{14}$ ] or by utilizing the long-wavelength idler pulse of a near-infrared (NIR) OPA [15-19].

$\mathrm{D} \overline{\mathrm{FG}}$ is a popular OPA seed source due to the combination of two properties: the ability to simultaneously phase-match a large fractional bandwidth [20-22] and the intrinsic stability of the carrier-envelope phase (CEP) $[\underline{17}, \underline{23}]$ when the signal is derived in a phase-preserving process from the pump. Due to the usually low conversion into the long wavelengths, parametric amplification is used to boost the energy of the MIR pulse. However, there are many experiments where it would be useful to have the MIR pulse precisely synchronized with a NIR or visible pulse, either to use as a pump or a probe. When reaching the microjoule level through a multistage OPA, there is a significant path length, several nonlinear processes and a high-power pump beam all adding complexity and, typically, phase and amplitude noise to any pump-probe experiment. For this reason, simple methods of reaching the MIR with high conversion efficiency would be quite helpful. We show here that a few-cycle visible-NIR pulse obtained from spectral broadening of a commercial Ti:sapphire laser can be used to efficiently obtain broadband pulses in the MIR.
To summarize the experiment, the majority of the output of a nine-pass Ti:sapphire amplifier (FemtoLasers; $\sim 25 \mathrm{fs}, 2.8 \mathrm{~W}$ at $3 \mathrm{kHz}$ repetition rate) is focused into a $1.1 \mathrm{~m}$ hollow-core fiber with $240 \mu \mathrm{m}$ core diameter, filled with neon gas at a pressure of 2 bar. A white-light continuum emerges from the fiber, with a total power of $1.5 \mathrm{~W}$, and a spectrum from 400 to $1000 \mathrm{~nm}$ [24]. This light is compressed using a set of six chirped mirrors [25] and a thin fused silica wedge pair. The compressed pulse duration is below $4 \mathrm{fs}$, with a total power of $1.0 \mathrm{~W}$ in the spectral range $480-1000 \mathrm{~nm}$. The beam was loosely focused by slightly increasing the distance between the end of the fiber and a $f=1 \mathrm{~m}$ collimating spherical mirror to produce a $0.4 \mathrm{~mm}$ FWHM focus $\sim 2 \mathrm{~m}$ away. The $500 \mu \mathrm{m}$ thick, antireflection-coated beta-barium borate crystals (BBOs, Castech) were placed in the laser focus, followed by a collimating mirror, and gold-coated mirrors to either send the beam to a spectrometer (Ocean Optics) or for power measurement. A $2 \mathrm{~mm}$ thick antireflectioncoated silicon wafer was placed in the beam in order to block the residual short-wavelength light.

The highest conversion efficiency obtained was $12.4 \%$, using type I phase-matching and an additional wave plate inserted in the beam path in order to control the spectrum of the light incident on each axis of the crystal. By tuning the wave plate, a $42 \mu \mathrm{m}$ quartz plate placed far from the focus and at Brewster's angle, such that the short-wavelength components are predominantly on the fast axis of the BBO, the efficiency of the DFG process is improved. The spectrum obtained corresponding to the highest DFG pulse energy $(41 \mu \mathrm{J})$, is shown in Fig. 1(a) alongside the spectrum of the compressed white light pulse after the chirped mirror compressor. Without the wave plate, the efficiency reached $11 \%$ for type I and $4.4 \%$ for type II. Tighter focusing would result in a filament in air; performing experiments in vacuum with a smaller focus could lead to yet higher conversion efficiencies, since the damage threshold of the $\mathrm{BBO}$ was not reached in the measurements. 

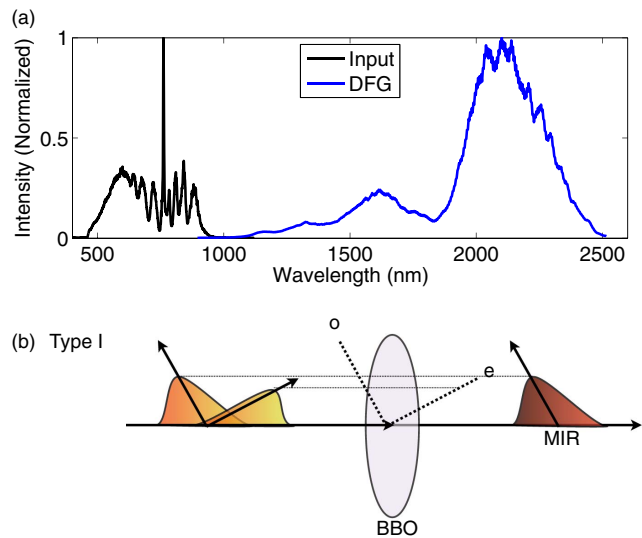

(c) Type I with wave plate for polarization shaping

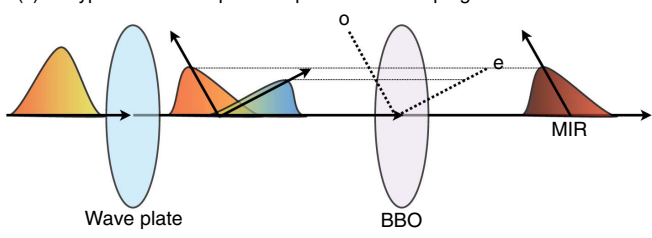

(d) Type II

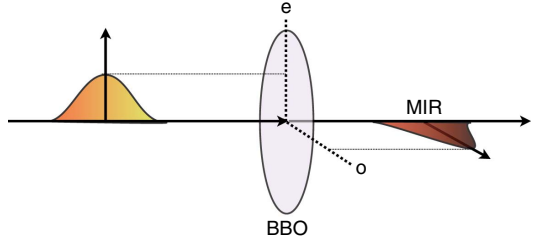

Fig. 1. (a) Input pulse spectrum after chirped mirror compressor and output spectrum of the DFG process showing highest efficiency (see text). (b)-(d) Diagrams of the DFG interactions tested. (b) Type I phase-matching with identical pulses sent to the fast and slow axes of the crystal. (c) Type I phase-matching using a wave plate to send a greater proportion of shortwavelength spectral components to the fast axis. (d) Type II phase-matching with a single, short pulse propagating along the fast axis of the crystal. In all cases, the MIR pulse is created on the slow axis.

There are two ways to phase-match the single-pulse DFG process in BBO crystal for broadband output: type I $(e-o \rightarrow o)$ and type II $(e-e \rightarrow o)$, illustrated in Fig. 1. They exhibit qualitatively different behavior once the energy conversion reaches several percent. In type I DFG, the blue spectral components in the pulse on the fast axis transfer energy to the red components on the slow axis, and, in the process generate a phase-stable idler wave, the DFG output, with the phase-matching condition $k_{p}-k_{s}=k_{i}$, where $k_{p}$ is the wave vector of the blue wave, $k_{s}$ is that of the red wave, and $k_{i}$ is that of the idler. Type II DFG, on the other hand, is similar to optical rectification in the terahertz spectral domain [26]: the nonlinear polarization takes the form of the intensity profile of the pulse, with the alternative phase-matching condition $n_{g}^{(\text {pump) }}=n_{o}^{(\mathrm{DFG})}$, i.e., the group velocity of the pump pulse is equal to the phase velocity of the emitted wave. DFG can also be phase-matched in the type II $e-o \rightarrow e$ configuration, but this is not employed here due to its relatively narrow bandwidth.

At low intensities, the efficiency of DFG tends to be linearly proportional to intensity. However, when the conversion efficiency is high, this linearity is broken as
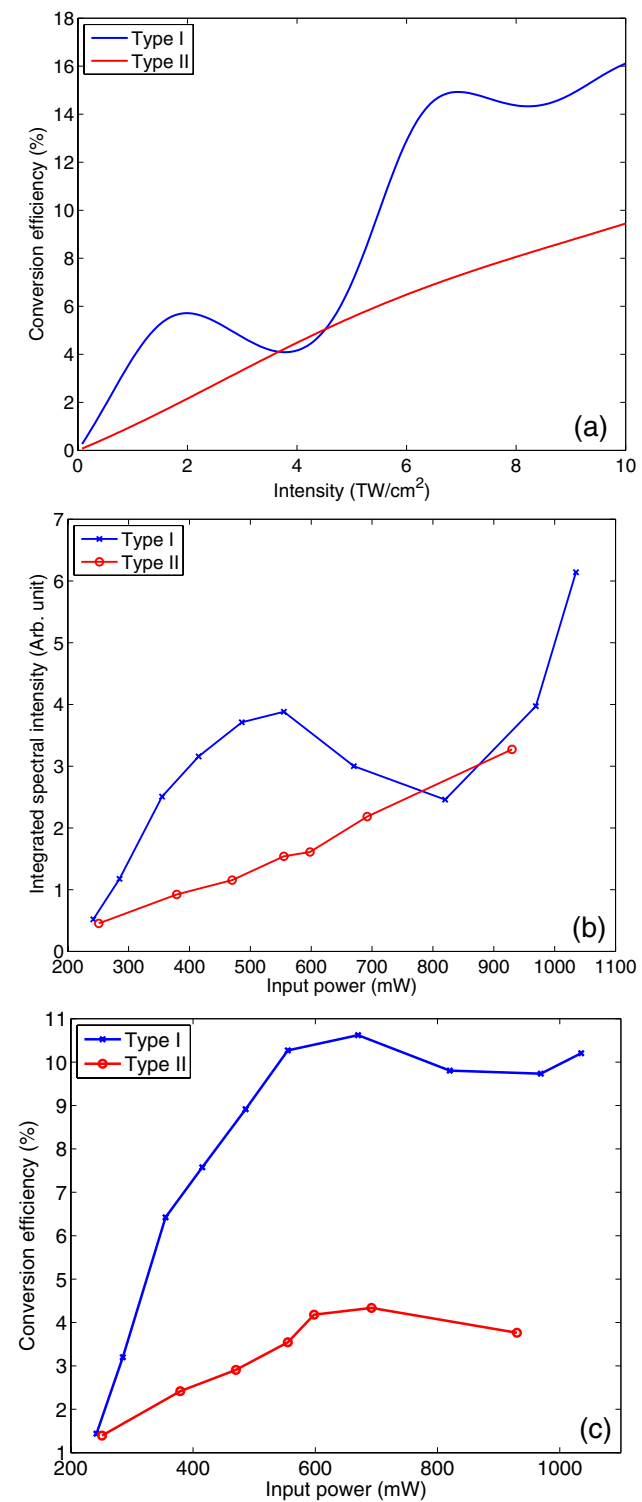

Fig. 2. Power scaling behavior. (a) Simulated conversion efficiency of light into phase-stable, difference-frequency radiation, using $1 \mathrm{D}$ propagation in $500 \mu \mathrm{m}$ thick BBO cut for type I $\left(\theta=21.4^{\circ}, \phi=90^{\circ}\right.$, light polarization $30^{\circ}$ from fast axis) and type II $\left(\theta=40^{\circ}, \phi=0^{\circ}\right)$. (b) Measured integrated spectral intensity as a function of input power as adjusted with an iris, measured with a beam much larger than the spectrometer entrance slit, indicating the on-axis scaling of the IR radiation. (c) Measured scaling of overall conversion efficiency versus input power.

the process saturates. Given a long phase-matched interaction length, saturation is reached when energy returns from the MIR pulse to the input pulse through sumfrequency generation faster than it is transferred to it through DFG. Due to the extremely short pulses used here, the effects of group velocity matching result in rather different behavior of the two phase-matching types at high conversion efficiencies. This can be seen both in simulations and experiments in Fig. 2, where the type I process exhibits a clear oscillatory behavior not seen in the type II interaction. The reason for this is straightforward: in type I, the group velocity walk-off between the pulses is much less than in type II. 
For example, let us consider the case where the MIR wavelength is $2000 \mathrm{~nm}$, with effective signal and pump wavelengths of 800 and $580 \mathrm{~nm}$. The group indices [27] of DFG, signal and pump are $1.6750,1.6845$, and 1.6932, respectively. This means that the propagation distance in the crystal for a group delay shift of 10 fs between DFG and signal is $315.5 \mu \mathrm{m}$, and, for the same shift between signal and pump, the distance is $344.6 \mu \mathrm{m}$. In the case of type II phase-matching, the DFG, signal, and pump group indices are $1.6750,1.6313$, and 1.6549 , respectively, leading to much shorter distances to separate the DFG from the signal $(68.6 \mu \mathrm{m})$ and the signal from the pump $(127.0 \mu \mathrm{m})$. These relatively short length scales reduce the efficiency of the type II process, but at the same time ensure that back-conversion through sum-frequency generation is unlikely at realistic intensities.

Despite the effects of group velocity walk-off in the crystals, the short pulses used in this experiment are important for reaching high conversion efficiencies. Because the damage threshold intensity increases as the pulse duration is decreased, the crystals can be exposed to higher intensities than would be possible with longer pulses [28], compensating for the walk-off effects. When the desired output spectrum is very broadband, short interaction lengths are required in any case.

Higher conversion rates also affect the spectrum obtained through the DFG process. As illustrated in Fig. $\underline{3}$, at the intensity is increased, the type I process exhibits a shift of the peak wavelength of the spectrum, first to longer and then to shorter wavelengths once the local minimum in conversion is reached. The type II process exhibits a more subtle change, gradually adding

(a)
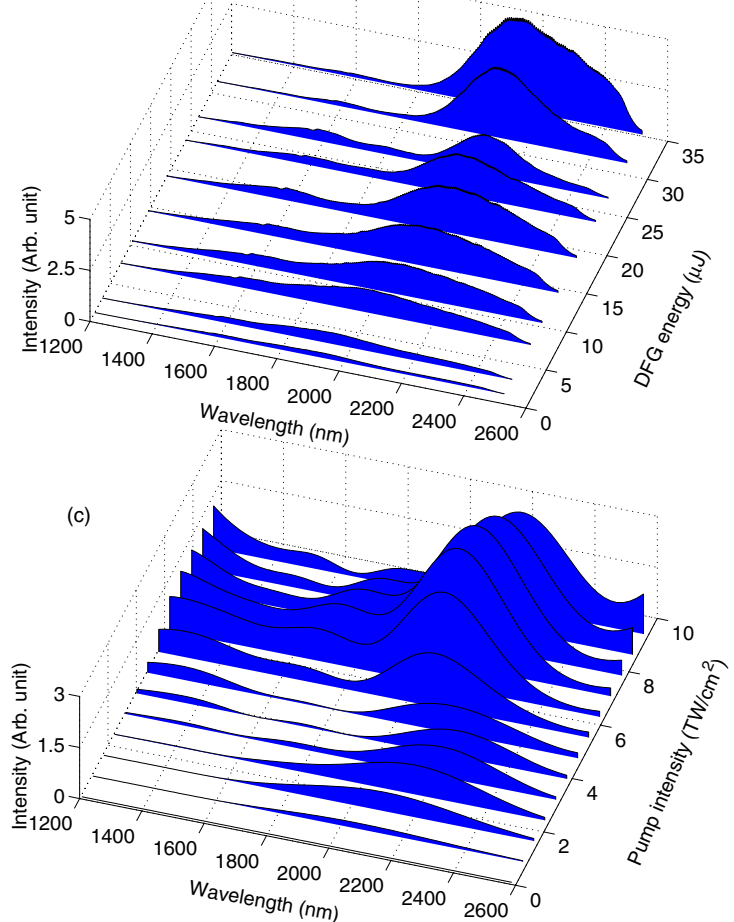

long-wavelength components at higher intensities, due to the overall redshift of the input pulse as energy is transferred from the short-wavelength side of the input pulse spectrum to the long-wavelength side, shifting the phase-matching to suit lower frequencies. These changes are reproduced by numerical simulations (based on the slowly evolving envelope approximation [29]) including only second-order nonlinearities, indicating that thirdorder effects are not yet playing a significant role in the process.

The relative stability of the type II process as the input pulse parameters are changed may make it more attractive in many applications, despite its lower conversion efficiency. The fact that the emerging white light and MIR pulses are orthogonally polarized is an advantage: the residual pump light can be nondispersively suppressed, via a Brewster's angle reflection from a silicon wafer, which we found reduces the residual white light contamination level to a few percent, dominated mostly by residual higher-order modes from the hollow-core fiber.

In conclusion, using few-cycle pulses at high intensities in BBO crystal is a promising method for producing broadband MIR pulses for experiments in attosecond and ultrafast science. Conversion efficiencies above $12 \%$ can be obtained using type I phase-matching and employing a thin wave plate for spectral polarization control. There are significant differences in the saturation behaviors of the type I and type II phase-matched interactions, which may influence their relative suitability given a specific experimental arrangement. Since only one input pulse, of the type typically already being employed in
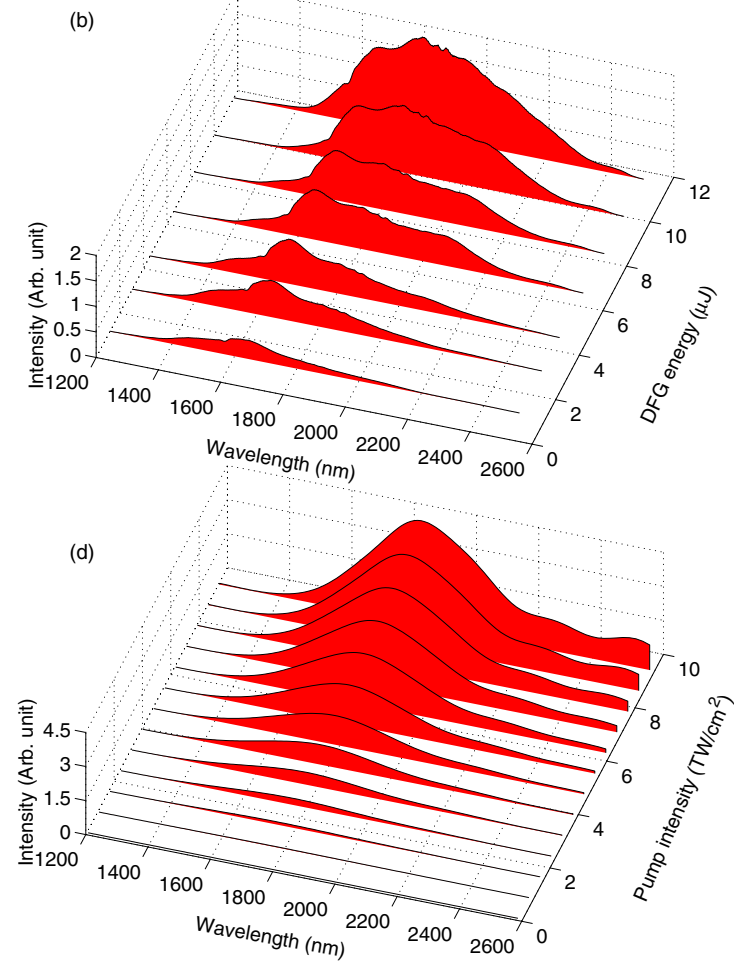

Fig. 3. Difference frequency spectra: (a) and (b) measured spectra for type I (blue) and type II (red) phase-matching, respectively, versus output MIR energy; (c) and (d) corresponding spectra from numerical simulations for type I and type II, respectively. The calculated spectra extend slightly beyond the spectrometer range, but are truncated for comparison. 
attosecond beam lines, is required, this technique can be used to easily add an MIR pump or probe pulse to attosecond experiments without the complexity of an additional parametric amplifier.

We gratefully acknowledge helpful discussions with Ferenc Krausz and support from the Munich-Centre for Advanced Photonics, and the German Federal Ministry of Education and Science (BMBP) via the NEXUS project.

\section{References}

1. C. Vozzi, M. Negro, and S. Stagira, J. Mod. Opt. 59, 1283 (2012).

2. K. Schultz, C. Blaga, R. Chirla, P. Colosimo, J. Cryan, A. March, C. Roedig, E. Sistrunk, J. Tate, J. Wheeler, P. Agostini, and L. F. Dimauro, J. Mod. Opt. 54, 1075 (2007).

3. J. Tate, T. Auguste, H.-G. Muller, P. Salieres, P. Agostini, and L. F. DiMauro, Phys. Rev. Lett. 98, 013901 (2007).

4. V. Yakovlev, M. Ivanov, and F. Krausz, Opt. Express 15, 15351 (2007).

5. T. Popmintchev, M.-C. Chen, D. Popmintchev, P. Arpin, S. Brown, S. Alisauskas, G. Andriukaitis, T. Balciunas, O. D. Mucke, A. Pugzlys, A. Baltuska, B. Shim, S. E. Schrauth, A. Gaeta, C. Hernandez-Garcia, L. Plaja, A. Becker, A. Jaron-Becker, M. M. Murnane, and H. C. Kapteyn, Science 336, 1287 (2012).

6. S. Ghimire, A. D. Dichiara, E. Sistrunk, P. Agostini, L. F. Dimauro, and D. A. Reis, Nat. Phys. 7, 138 (2010).

7. A. Schiffrin, T. Paasch-Colberg, N. Karpowicz, V. Apalkov, D. Gerster, S. Mühlbrandt, M. Korbman, J. Reichert, M. Schultze, S. Holzner, J. V. Barth, R. Kienberger, R. Ernstorfer, V. S. Yakovlev, M. I. Stockman, and F. Krausz, Nature 493, 70 (2013).

8. I. Znakovskaya, P. von den Hoff, G. Marcus, S. Zherebtsov, B. Bergues, X. Gu, Y. Deng, M. J. J. Vrakking, R. Kienberger, F. Krausz, R. de Vivie-Riedle, and M. F. Kling, Phys. Rev. Lett. 108, 063002 (2012).

9. J. Darginavičius, N. Garejev, and A. Dubietis, Opt. Lett. 37, 4805 (2012)

10. C. Manzoni, C. Vozzi, E. Benedetti, G. Sansone, S. Stagira, O. Svelto, S. D. Silvestri, M. Nisoli, and G. Cerullo, Opt. Lett. 31, 963 (2006).

11. Y. Deng, A. Schwarz, H. Fattahi, M. Ueffing, X. Gu, M. Ossiander, T. Metzger, V. Pervak, H. Ishizuki, T. Taira,
T. Kobayashi, G. Marcus, F. Krausz, R. Kienberger, and N. Karpowicz, Opt. Lett. 37, 4973 (2012).

12. C. Vozzi, C. Manzoni, F. Calegari, E. Benedetti, G. Sansone, G. Cerullo, M. Nisoli, S. D. Silvestri, and S. Stagira, J. Opt. Soc. Am. B 25, B112 (2008).

13. K.-H. Hong, S.-W. Huang, J. Moses, X. Fu, C.-J. Lai, G. Cirmi, A. Sell, E. Granados, P. Keathley, and F. X. Kärtner, Opt. Express 19, 15538 (2011).

14. N. Ishii, K. Kaneshima, K. Kitano, T. Kanai, S. Watanabe, and J. Itatani, Opt. Lett. 37, 4182 (2012).

15. C. P. Hauri, R. B. Lopez-Martens, C. I. Blaga, K. D. Schultz, J. Cryan, R. Chirla, P. Colosimo, G. Doumy, A. M. March, C. Roedig, E. Sistrunk, J. Tate, J. Wheeler, L. F. Dimauro, and E. P. Power, Opt. Lett. 32, 868 (2007).

16. F. Silva, P. K. Bates, A. Esteban-Martin, M. Ebrahim-Zadeh, and J. Biegert, Opt. Lett. 37, 933 (2012).

17. A. Baltuška, T. Fuji, and T. Kobayashi, Phys. Rev. Lett. 88, 133901 (2002).

18. C. Li, D. Wang, L. Song, J. Liu, P. Liu, C. Xu, Y. Leng, R. Li, and Z. Xu, Opt. Express 19, 6783 (2011).

19. B. E. Schmidt, A. D. Shiner, P. Lassonde, J.-C. Kieffer, P. B. Corkum, D. M. Villeneuve, and F. Légaré, Opt. Express 19, 6858 (2011).

20. C. Manzoni, G. Cerullo, and S. D. Silvestri, Opt. Lett. 29, 2668 (2004)

21. J. Moses, H. Suchowski, and F. Kärtner, Opt. Lett. 37, 1589 (2012).

22. C. Homann, M. Bradler, M. Förster, P. Hommelhoff, and E. Riedle, Opt. Lett. 37, 1673 (2012).

23. G. Cerullo, A. Baltuška, O. Mücke, and C. Vozzi, Laser Photon. Rev. 5, 323 (2010).

24. A. L. Cavalieri, E. Goulielmakis, B. Horvath, W. Helml, M. Schultze, M. Fieß, V. Pervak, L. Veisz, V. S. Yakovlev, M. Uiberacker, A. Apolonski, F. Krausz, and R. Kienberger, New J. Phys. 9, 242 (2007).

25. V. Pervak, Appl. Opt. 50, C55 (2011).

26. H. Bakker, G. Cho, H. Kurz, Q. Wu, and X.-C. Zhang, J. Opt. Soc. Am. B 15, 1795 (1998).

27. K. Kato, IEEE J. Quantum Electron. 22, 1013 (1986).

28. M. Lenzner, J. Krüger, S. Sartania, Z. Cheng, C. Spielmann, G. Mourou, W. Kautek, and F. Krausz, Phys. Rev. Lett. 80, 4076 (1998).

29. T. Brabec and F. Krausz, Phys. Rev. Lett. 78, 3282 (1997). 


\section{E.4 High efficiency, multi-mJ, sub $10 \mathrm{fs,} \mathrm{optical}$ parametric amplifier at $3 \mathrm{kHz}$}

This paper was published in Optics CLEO2012 and is made available as an electronic reprint with the permission of OSA. The paper can be found at the following URL on the OSA website: http://www.opticsinfobase.org/ abstract.cfm?uri=CLEO_SI-2012-CTh1N.6. Systematic or multiple reproduction or distribution to multiple locations via electronic or other means is prohibited and is subject to penalties under law. 


\title{
High efficiency, multi-mJ, sub 10 fs, optical parametric amplifier at $3 \mathrm{kHz}$
}

\author{
Hanieh Fattahi ${ }^{1,2}$, Christoph Skrobol ${ }^{1,2}$, Moritz Ueffing ${ }^{1}$, Yunpei Deng ${ }^{1}$, Alexander Schwarz ${ }^{1}$, Yuichiro Kida ${ }^{1}$, \\ Vladimir Pervak $^{1,2}$, Thomas Metzger ${ }^{1,2}$, Zsuzsanna Major ${ }^{1,2}$ and Ferenc Krausz ${ }^{1,2}$ \\ ${ }^{1}$ Max-Planck-Institute of Quantum Optics (MPQ), Hans-Kopfermann-Straße 1, 85748 Garching, Germany \\ ${ }^{2}$ Ludwig-Maximilians-University München (LMU), Department of Physics, Am Coulombwall 1, 85748 Garching, Germany \\ Hanieh.Fattahi@mpq.mpg.de
}

Abstract: We present a short-pulse pumped optical parametric amplifier (OPA) with a broadband spectrum from $670 \mathrm{~nm}$ to $1000 \mathrm{~nm}$, supporting $5.7 \mathrm{fs}$ pulses with > $32 \%$ pump-to-signal conversion efficiency resulting in pulse energies of $2.5 \mathrm{~mJ}$.

OCIS codes: $190.4970,140.7090,140.3280$.

\section{Introduction}

Optical parametric chirped pulse amplification (OPCPA) represents the most promising route towards high-energy few-cycle pulses [1]. OPCPA allows ultra-broad-band amplification far above the multimillijoule level [2] overcoming the limitation of conventional chirped pulse amplifiers [3]. However, to reach the few-cycle regime and high pulse energies simultaneously, a modified implementation is required, where the pump pulse duration is reduced to a few ps. Since the unsaturated parametric gain scales as the exponential of the square root of the pump intensity, it is desirable to carry out the parametric amplification with pump intensities as high as permitted by the onset of detrimental nonlinear optical phenomena such as self-focusing and self phase modulation [4]. With ps-scale pumping the high gain allows for the use of very thin crystals that support a large bandwidth. Although the shortpulse pumped OPCPA scheme supports high-power, few-cycle pulse generation, it requires laser sources specially tailored for pumping OPCPA [5]. It has been shown that the residual pump beam after an OPCPA stage still possesses a sufficiently good beam quality for being reused in a subsequent stage and consequently increasing the pump to signal conversion efficiency while maintaining the broad bandwidth [6]. In this paper we present a compact, 3-stage, multi-mJ OPCPA system at $3 \mathrm{kHz}$ repetition rate based on a non-collinear phase matching geometry (NOPA), where the residual pump beam of the $2^{\text {nd }}$ stage is used to pump the further stage. The broadband amplified spectrum supports $5.7 \mathrm{fs}$ pulses. A pump-to-seed conversion efficiency of $>32 \%$ was achieved in the last two stages, which to our knowledge is the highest efficiency reported in a broadband optical parametric amplification (OPA).

\section{Experimental setup and results}

The OPCPA experimental setup is schematically shown in Fig. 1. It consists of a Ti:Sapphire based oscillator and amplifier followed by a broadband nonlinear seed generation scheme, a pump laser, a jitter compensation system, three OPA stages and a chirped mirror compressor [7]. 5\% of the $60 \mathrm{~nm}$ (FWHM) broad output spectrum of the Ti:Sapphire amplifier centered at a wavelength of $790 \mathrm{~nm}$ containing $30 \mu \mathrm{J}$ pulse energy was focused into a $15 \mathrm{~cm}$ long $120 \mu \mathrm{m}$ diameter hollow core fiber filled with 5 bar of Krypton where the pulse broadens its spectrum to cover a spectral range from $\sim 500$ to $\sim 1050 \mathrm{~nm}$. The Yb:YAG regenerative amplifier optically synchronized with the OPCPA seed [8] delivers $20 \mathrm{~mJ}, 1.6 \mathrm{ps}$ (FWHM) pulses at $3 \mathrm{kHz}$ and its frequency doubled output is used for pumping the OPA. However, owing to the long optical beam path difference between seed and pump pulses, additional timing fluctuations occur due to air turbulences, mechanical vibrations of optical components, temperature drifts and the finite stability of the frontend, which is needed to be compensated by an active stabilization system. The timing jitter in our system is compensated to a level of 24 fs (RMS) by an active stabilization system based on a spectrally resolved cross correlation between the stretched seed and the pump pulse [9]. For frequency doubling of the pump laser, a $1 \mathrm{~mm} \mathrm{BBO}$ crystal was used. $50 \%$ SHG efficiency confirms the excellent beam quality and clean output pulses of the regenerative amplifier. After the hollow core fiber the signal pulses were stretched with $4 \mathrm{~mm}$ SF57 to nearly $1.1 \mathrm{ps}$ to provide good temporal overlap between seed and pump pulses in the OPA chain. The first OPA stage serves as a preamplifier for the low-energetic broadband seed pulse. We used $1 \mathrm{~mJ}$ of the frequency doubled output of the disk amplifier for pumping and achieved in a $1.5 \mathrm{~mm}$ BBO crystal $\left(\theta=23.3^{\circ}, \alpha=2.4^{\circ}\right)$ an output pulse energy of $120 \mu \mathrm{J}$ and a $450 \mathrm{~nm}$ broad spectrum shown in Fig. 1 . In the following two stages we were aiming to reach the highest possible pump-to-signal conversion efficiency. Up to 7.3 $\mathrm{mJ}$ of energy at $515 \mathrm{~nm}$ were used to pump the $2^{\text {nd }}$ OPA stage $\left(B B O, 2 \mathrm{~mm}, \theta=23.3^{\circ}, \alpha=2.4^{\circ}\right.$ ), resulting in a pump intensity on the crystal of approximately $100 \mathrm{GW} / \mathrm{cm}^{2}$. We obtained an amplified pulse with $1.77 \mathrm{~mJ}$ energy while keeping the full spectral bandwidth as shown in Fig. 1. Subsequently the size of the remaining pump pulse was 
reduced and used to pump the $3^{\text {rd }}$ amplification stage. The pump intensity of approximately $60 \mathrm{GW} / \mathrm{cm}^{2}$ on a $2 \mathrm{~mm}$ BBO crystal $\left(\theta=23.3^{\circ}\right)$ amplified the pulse to the energy of $2.5 \mathrm{~mJ}$ (cf. Fig. 1 for the spectrum). In the last two OPA stages an optical-to-optical conversion efficiency of $33 \%$ has been achieved, which to our knowledge is the highest efficiency reported. No measurable superfluorescence background was observed, when blocking the signal beam in front of the $2^{\text {nd }}$ and $3^{\text {rd }}$ stages. To achieve stable output and low energy fluctuations the last two OPA stages were run in saturation [10]. The careful optimization of the pump intensity on the crystal, temporal stretching ratio between pump and seed and reusing the residual pump energy allowed reaching the high conversion efficiency. The experimental results are fully consistent with the outcome from our " $2 \mathrm{D}$ " in house-written code [11]. The $350 \mathrm{~nm}$ broad OPA signal supports a time bandwidth limited pulse duration of $5.7 \mathrm{fs}$ (FWHM). The preliminary compression by using double angle chirped mirrors resulted in a pulse duration of $9.5 \mathrm{fs}$ for the main part of the pulse. Higher order dispersion and the satellite pulses can be compensated by using specially designed chirped mirrors.
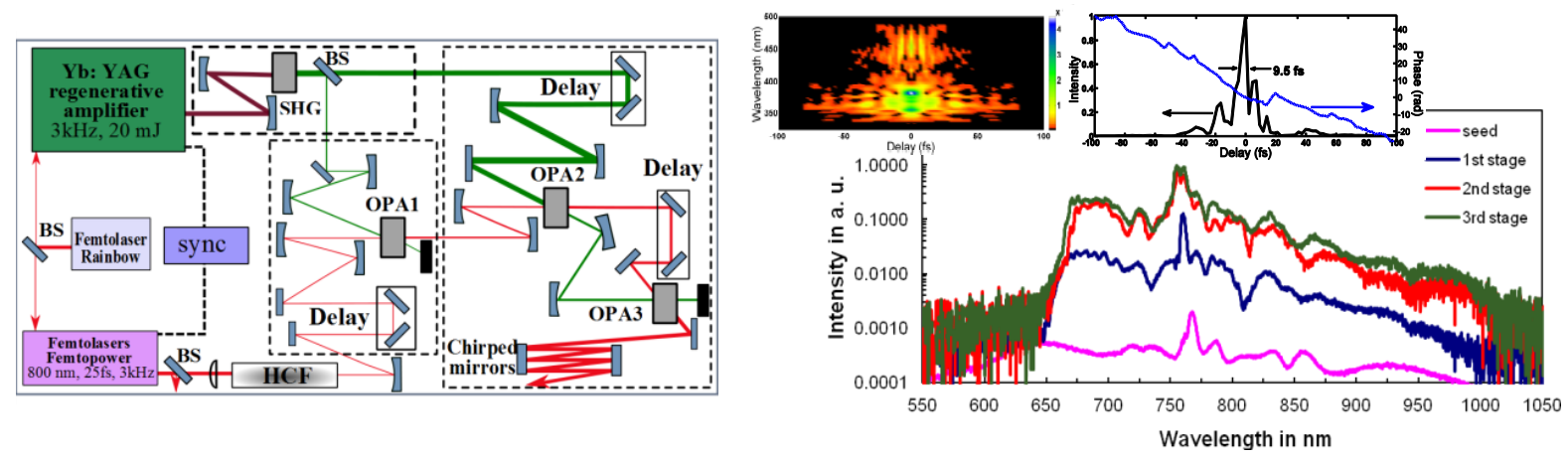

Fig. 1: Schematic OPA layout (left). Input and amplified seed spectrum (right). Retrieved FROG trace of the compressed pulse and retrieved structure of the pulse in the time domain (inset). OPA, optical parametric amplification; BS, beam splitter; HCF, hollow core fiber; SHG, second harmonic generation; sync, synchronization setup.

\section{Conclusion and Outlook}

In the course of the development of new few-cycle laser sources for attosecond experiments [12], we have demonstrated a highly efficient compact OPCPA system delivering broadband pulses with more than $2 \mathrm{~mJ}$ pulse energy supporting two-cycle pulse durations at $3 \mathrm{kHz}$ repetition rate. Good stability and high efficiency of the system indicate that the OPA system runs in the saturated regime. The extraction efficiency is expected to be further increased by using a thicker crystal in the last stage without reducing the amplification bandwidth.

\section{References}

[1] A. Dubietis, G. Jonusauskas, and A. Piskarskas, "Powerful femtosecond pulse generation by chirped and stretched pulse parametric amplification in BBO crystal," Opt. Comm. 88, 437 (1992).

[2] D. Herrmann, L. Veisz, R. Tautz, F. Tavella, K. Schmid, V. Pervak, and F. Krausz, "Generation of sub-three-cycle, 16 TW light pulses by using noncollinear optical parametric chirped-pulse amplification,” Opt. Lett. 34, 2459 (2009).

[3] D. Strickland, and G. Mourou, "Compression of amplified chirped optical pulses," Opt. Commun. 56, 219-221 (1985).

[4] D. Brida, C. Manzoni, G. Cirmi, M. Marangoni, S.Bonora, P. Villoresi, S.De Silvestri, and G. Cerullo, "Few-optical-cycle pulses tunable from the visible to the mid-infrared by optical parametric amplifiers," J. Opt. 12, 013001 (2010).

[5] T. Metzger, A. Schwarz, C. Y. Teisset, D. Sutter, A. Killi, R. Kienberger, and F. Krausz, "High-repetition-rate picosecond pump laser based on a Yb:YAG disk amplifier for optical parametric amplification," Opt. Lett. 34, 2123-2125 (2009).

[6] M. Schultze, T. Binhammer, G. Palmer, M. Emons, T. Lang, and U. Morgner, "Multi- $\mu$ J, CEP-stabilized, two-cycle pulses from an OPCPA system with up to $500 \mathrm{kHz}$ repetition rate," Opt. Express 18, 27291-27297(2010).

[7] R. Szipocs, K. Ferencz, C. Spielmann, and F. Krausz, "Chirped multilayer coatings for broadband dispersion control in femtosecond lasers," Opt. Lett. 19, 201-203 (1994).

[8] C. Y. Teisset, N. Ishii, T. Fuji, T. Metzger, S. Köhler, A. Baltuska, and Ferenc Krausz, “All-Optical Pump-Seed Synchronization for FewCycle OPCPA," in Conference on Lasers and Electro-Optics/Quantum Electronics and Laser Science and Photonic Applications Systems Technologies, Technical Digest (CD) (Optical Society of America, 2005), paper CMB3.

[9] A. Schwarz, M. Ueffing, Y. Deng, X. Gu, H. Fattahi, T. Metzger, M. Ossiander, R. Kienberger, and F. Krausz, "Active stabilization for optically synchronized OPCPA," submitted for publication (2011).

[10] M. J. Guardalben, J. Keegan, L. J. Waxer, V. Bagnoud, I. A. Begishev, J. Puth, and J. D. Zuegel, "Design of a highly stable, highconversion-efficiency, optical parametric chirped-pulse amplification system with good beam quality," Opt. Express 11, 2511-2524 (2003).

[11] C. Skrobol, I. Ahmad, S. Klingebiel, C. Wandt, S. A. Trushin, Zs. Major, F. Krausz, and S. Karsch, “ Broadband Amplification by Picosecond OPCPA in DKDP Pumped at $515 \mathrm{~nm}$," submitted for publication (2011).

[12] F. Krausz and M. Ivanov, “Attosecond Physics,” Rev. Mod. Phys. 81, 163 (2009). 


\section{E.5 Pump-seed synchronization for $\mathrm{MHz}$ repetition rate, high-power optical parametric chirped pulse amplification}

This paper was published in Optics Express and is made available as an electronic reprint with the permission of OSA. The paper can be found at the following URL on the OSA website: http://www.opticsinfobase.org/oe/ abstract. cfm?uri=oe-20-9-9833. Systematic or multiple reproduction or distribution to multiple locations via electronic or other means is prohibited and is subject to penalties under law. 


\title{
Pump-seed synchronization for MHz repetition rate, high-power optical parametric chirped pulse amplification
}

\author{
Hanieh Fattahi, ${ }^{1,2, *}$ Catherine Yuriko Teisset, ${ }^{2}$ Oleg Pronin, ${ }^{1}$ Atsushi Sugita, ${ }^{1}$ \\ Roswitha Graf, ${ }^{1,2}$ Vladimir Pervak, ${ }^{2}$ Xun Gu, ${ }^{1}$ Thomas Metzger, ${ }^{1,2}$ Zsuzsanna Major, ${ }^{1,2}$ \\ Ferenc Krausz, ${ }^{1,2}$ and Alexander Apolonski ${ }^{1,2}$ \\ ${ }^{1}$ Max-Planck-Institut für Quantenoptik, Hans-Kopfermann-Str. 1, 85748 Garching, Germany \\ ${ }^{2}$ Ludwig-Maximilians-Universität München (LMU), Am Coulombwall 1, 85748 Garching, Germany \\ *hanieh.fattahi@mpq.mpg.de
}

\begin{abstract}
We report on an active synchronization between two independent mode-locked lasers using a combined electronic-optical feedback. With this scheme, seed pulses at $\mathrm{MHz}$ repetition rate were amplified in a noncollinear optical parametric chirped pulse amplifier (OPCPA). The amplifier was seeded with stretched $1.5 \mathrm{~nJ}$ pulses from a femtosecond Ti:Sapphire oscillator, while pumped with the $1 \mathrm{ps}, 2.9 \mu \mathrm{J}$ frequencydoubled output of an Yb:YAG thin-disk oscillator. The residual timing jitter between the two oscillators was suppressed to 120 fs (RMS), allowing for an efficient and broadband amplification at $11.5 \mathrm{MHz}$ to a pulse energy of $700 \mathrm{~nJ}$ and an average power of $8 \mathrm{~W}$. First compression experiment with $240 \mathrm{~nJ}$ amplified pulse energy resulted in a pulse duration of $\sim 10 \mathrm{fs}$.

(C)2012 Optical Society of America

OCIS codes: (140.7090) Ultrafast lasers; (190.4410) Parametric processes; (320.7110) Ultrafast nonlinear optics; (190.4970) Parametric oscillators and amplifiers.
\end{abstract}

\section{References and links}

1. J. Lin, N. Weber, A. Wirth, S. H. Chew, M. Escher, M. Merkel, M. F. Kling, M. I. Stockman, F. Krausz, and U. Kleineberg, "Time of flight-photoemission electron microscope for ultrahigh spatiotemporal probing of nanoplasmonic optical fields,” J. Phys. Condens. Matter 21(31), 314005 (2009).

2. J. Ullrich, R. Moshammer, A. Dorn, R. Dörner, L. P. H. Schmidt, and H. Schmidt-Böcking, "Recoil-ion and electron momentum spectroscopy: reaction-microscopes," Rep. Prog. Phys. 66(9), 1463-1545 (2003).

3. S. Naumov, A. Fernandez, R. Graf, P. Dombi, F. Krausz, and A. Apolonski, "Approaching the microjoule frontier with femtosecond laser oscillators," New J. Phys. 7, 216 (2005).

4. A. Dubietis, G. Jonusauskas, and A. Piskarskas, "Powerful femtosecond pulse generation by chirped and stretched pulse parametric amplification in BBO crystal," Opt. Commun. 88(4-6), 437-440 (1992).

5. J. Neuhaus, D. Bauer, J. Zhang, A. Killi, J. Kleinbauer, M. Kumkar, S. Weiler, M. Guina, D. H. Sutter, and T. Dekorsy, "Subpicosecond thin-disk laser oscillator with pulse energies of up to 25.9 microjoules by use of an active multipass geometry," Opt. Express 16(25), 20530-20539 (2008).

6. M. Schultze, T. Binhammer, G. Palmer, M. Emons, T. Lang, and U. Morgner, "Multi- $\mu$ J, CEP-stabilized, twocycle pulses from an OPCPA system with up to $500 \mathrm{kHz}$ repetition rate," Opt. Express 18(26), 27291-27297 (2010).

7. M. Emons, A. Steinmann, T. Binhammer, G. Palmer, M. Schultze, and U. Morgner, "Sub-10-fs pulses from a MHz-NOPA with pulse energies of $0.4 \mu \mathrm{J}$," Opt. Express 18(2), 1191-1196 (2010).

8. C. Skrobol, I. Ahmad, S. Klingebiel, C. Wandt, S. A. Trushin, Z. Major, F. Krausz, and S. Karsch, "Broadband amplification by picosecond OPCPA in DKDP pumped at 515 nm," Opt. Express 20(4), 4619-4629 (2012).

9. C. Y. Teisset, N. Ishii, T. Fuji, T. Metzger, S. Köhler, R. Holzwarth, A. Baltuška, A. M. Zheltikov, and F. Krausz, "Soliton-based pump-seed synchronization for few-cycle OPCPA," Opt. Express 13(17), 6550-6557 (2005).

10. I. Ahmad, S. A. Trushin, Z. Major, C. Wandt, S. Klingebiel, T. J. Wang, V. Pervak, A. Popp, M. Siebold, F. Krausz, and S. Karsch, "Frontend light source for short-pulse pumped OPCPA system," Appl. Phys. B 97(3), 529-536 (2009)

11. N. Ishii, L. Turi, V. S. Yakovlev, T. Fuji, F. Krausz, A. Baltuška, R. Butkus, G. Veitas, V. Smilgevicius, R. Danielius, and A. Piskarskas, "Multimillijoule chirped parametric amplification of few-cycle pulses," Opt. Lett. 30(5), 567-569 (2005). 
12. T. Binhammer, S. Rausch, M. Jackstadt, G. Palmer, and U. Morgner, "Phase-stable Ti:sapphire oscillator quasisynchronously pumped by a thin-disk laser,” Appl. Phys. B 100(1), 219-223 (2010).

13. T. R. Schibli, J. Kim, O. Kuzucu, J. T. Gopinath, S. N. Tandon, G. S. Petrich, L. A. Kolodziejski, J. G. Fujimoto, E. P. Ippen, and F. X. Kaertner, "Attosecond active synchronization of passively mode-locked lasers by balanced cross correlation," Opt. Lett. 28(11), 947-949 (2003).

14. S. V. Marchese, T. Südmeyer, M. Golling, R. Grange, and U. Keller, "Pulse energy scaling to $5 \mu \mathrm{J}$ from a femtosecond thin disk laser,” Opt. Lett. 31(18), 2728-2730 (2006).

15. V. Pervak, C. Y. Teisset, A. Sugita, S. Naumov, F. Krausz, and A. Apolonski, "High-dispersive mirrors for femtosecond lasers," Opt. Express 16(14), 10220-10233 (2008).

16. T. Fuji, A. Unterhuber, V. S. Yakovlev, G. Tempea, A. Stingl, F. Krausz, and W. Drexler, "Generation of smooth, ultra-broadband spectra directly from a prism-less Ti:sapphire laser,” Appl. Phys. B 77(1), 125-128 (2003).

17. R. Paschotta, "Noise of mode-locked lasers (Part II): timing jitter and other fluctuations," Appl. Phys. B 79(2), 163-173 (2004)

18. C. Hönninger, R. Paschotta, F. Morier-Genoud, M. Moser, and U. Keller, "Q-switching stability limits of continuous-wave passive mode locking," J. Opt. Soc. Am. B 16(1), 46-56 (1999).

19. V. Pervak, I. Ahmad, M. K. Trubetskov, A. V. Tikhonravov, and F. Krausz, "Double-angle multilayer mirrors with smooth dispersion characteristics," Opt. Express 17(10), 7943-7951 (2009).

20. I. T. Sorokina, " $\mathrm{Cr}^{2+}$-doped II-VI materials for lasers and nonlinear optics," Opt. Mater. 26(4), $395-412$ (2004).

21. W. Cao and Y. Duan, "Breath analysis: potential for clinical diagnosis and exposure assessment," Clin. Chem. 52(5), 800-811 (2006).

22. O. Pronin, J. Brons, C. Grasse, V. Pervak, G. Boehm, M. C. Amann, V. L. Kalashnikov, A. Apolonski, and F Krausz, "High-power 200 fs Kerr-lens mode-locked Yb:YAG thin-disk oscillator," Opt. Lett. 36(24), 4746-4748 (2011).

\section{Introduction}

Ultrashort laser pulses with high average power at high repetition rates are highly demanded in many ultrafast experiments in order to decrease the measurement time and to increase the signal-to-noise ratio $[1,2]$. Considering the scalability limitations of the Ti:Sapphire (Ti:Sa) technology in terms of pulse energy and repetition rate [3], optical parametric chirped pulse amplification (OPCPA) is a powerful alternative method for creating broadband few-cycle pulses [4]. The latest development of thin-disk oscillators resulted in $30 \mu \mathrm{J}$, sub-ps, MW peak power pulses at $\mathrm{MHz}$ repetition rates [5] making them suitable for pumping OPCPAs. Shortpulse-pumped OPCPA allows the use of thinner amplifier crystals resulting in a large amplification bandwidth, while keeping the same gain level due to the higher possible pump intensity. $3 \mu \mathrm{J}$ pulses at $143 \mathrm{kHz}$ have been demonstrated by using an optically synchronized regenerative amplifier and a Ti:Sa oscillator (two-laser approach) [6]. At a higher repetition rate of $1 \mathrm{MHz}$, sub-10 fs pulses with $420 \mathrm{~nJ}$ pulse energy and $0.4 \mathrm{~W}$ of average power have been shown [7]. In this one-laser approach a supercontinuum generated in bulk material provides the OPCPA seed. However, this scheme has the following drawbacks: i) the continuum is always centered at the wavelength of the pump laser and cannot be shifted to other spectral ranges and ii) it does not provide carrier-envelope-phase (CEP) stabilization. Therefore combining a Ti:Sa seed oscillator and an electronically synchronized pump oscillator would permit the generation of CEP-stabilized, few-cycle pulses at high repetition rates. Moreover, electronic synchronization between two independent mode-locked lasers is a universal approach and can be implemented for lasers operating in substantially different spectral ranges.

To maintain stable operation of the parametric amplifier and to avoid fluctuations of the amplified spectrum, pulse energy and pulse duration, the maximum timing jitter between the pump and seed sources needs to be less than $10 \%$ of the pump-pulse duration [8], imposing strict constraints on the pump-seed synchronization when picoseconds-scale pump pulses are used. In order to achieve this goal, several synchronization schemes have been reported. In passive synchronization [9-11], the OPCPA pump sources such as regenerative amplifiers or fiber lasers are seeded with the OPCPA seed oscillator. Synchronization can be achieved also by quasi-synchronously pumping of the seed oscillator [12]. Although in the above mentioned cases the pump and seed pulses are intrinsically synchronized, further synchronization of the seed and pump pulse trains at the OPCPA stage is required due to additional jitter introduced 
by air turbulences, mechanical vibrations of optical components, temperature drifts and the finite stability of the frontend. In an active synchronization scheme, an electronic feedback loop controls the cavity length of one of the oscillators in order to control the timing jitter at the OPCPA stage. The feedback signal can be measured by electronic or optical detection. For the case of electronic detection the electronic phase measurement is done by comparing the repetition rates of two lasers, while the optical method uses a nonlinear interaction to measure directly the timing offset. Designing a control loop based on both electronic and an optical detection would allow the combination of the large dynamic range of electronic phase-locked loops (PLLs) with the timing precision of the optical detection. Using this principle, a jitter as low as 80 as has already been demonstrated for low-energy Ti:Sa and Cr:Forsterite oscillators [13]. Obviously such timing precision would be unnecessary for parametric amplifiers driven by 1-ps pump pulses. So far, active synchronization of lasers is mostly realized for modelocked oscillators, with a typical output power of a few hundred milliwatt, which are intrinsically very stable. In this work, we developed a synchronization scheme combining an electronic loop based on a PLL and an optical control loop using balanced cross-correlation as the optical nonlinear interaction to synchronize a narrowband thin-disk oscillator with an average output power of tens of watts and a low-power Ti:Sa oscillator with $300 \mathrm{~nm}$ bandwidth. To our best knowledge, this is the first successful attempt of such type.

\section{Mode-locked thin-disk oscillator}

The pump source is based on an Yb:YAG thin-disk and long-cavity oscillator. The resonator, shown in Fig. 1, contains the Yb:YAG thin-disk (7\%-doped, $200 \mu \mathrm{m}$-thick) used as a turning mirror. The thin-disk laser module from Dausinger \& Giesen $\mathrm{GmbH}$ is pumped by fibercoupled diodes at $940 \mathrm{~nm}$ wavelength. The pump spot diameter on the disk is $2.8 \mathrm{~mm}$.

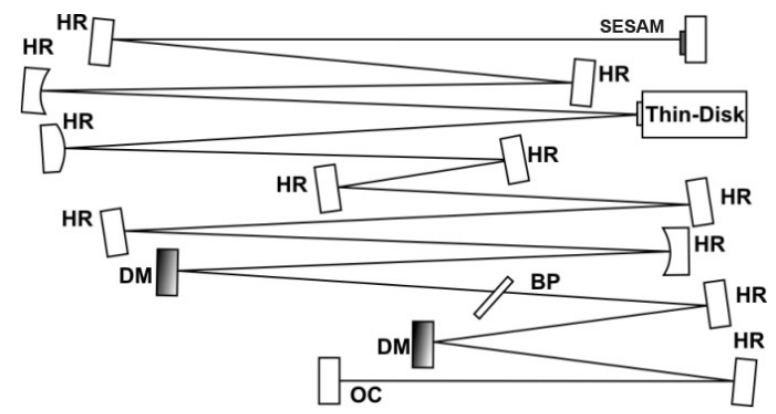

Fig. 1. Schematic of the Yb:YAG oscillator. HR, high reflector; DM, dispersive mirror; OC, output coupler; BP, Brewster plate; SESAM, Semiconductor Saturable Absorber Mirror.

A $1 \mathrm{~mm}$ thick fused silica Brewster plate is inserted to enforce linear polarisation. The oscillator is operating with a $12 \%$ output coupler. Passive mode-locking of the oscillator is achieved by using a commercially available Semiconductor Saturable Absorber Mirror (SESAM, BATOP, SAM-1040-1-500fs) as an end mirror of the cavity. The SESAM with a modulation depth of $0.5 \%$ is impinged by pulses with a peak intensity exceeding $4 \mathrm{GW} / \mathrm{cm}^{2}$. With a pump power of $260 \mathrm{~W}, 70 \mathrm{~W}$ of average power is obtained resulting in an optical-tooptical efficiency of $27 \%$. In contrast to [14], the laser operates in air and delivers up to $6 \mu \mathrm{J}$ pulse energy at $11.5 \mathrm{MHz}$ repetition rate with near-diffraction limited beam quality of $\mathrm{M}^{2}<$ 1.1 (Fig. 2). Stable single-pulse operation was obtained with $-18000 \mathrm{fs}^{2}$ of negative group delay dispersion (GDD) per round-trip. The dispersion was introduced by two highly dispersive mirrors [15] with $-4500 \mathrm{fs}^{2} \mathrm{GDD}$ per bounce. At $60 \mathrm{~W}$ output power, the oscillator exhibits a pulse duration of $1 \mathrm{ps}$ and a bandwidth of $1.4 \mathrm{~nm}$ (FWHM) at a center wavelength of $1030 \mathrm{~nm}$ (Fig. 2). The RF-spectrum indicates strong sideband suppression above $60 \mathrm{~dB}$ (Fig. 2) around the repetition rate frequency. 

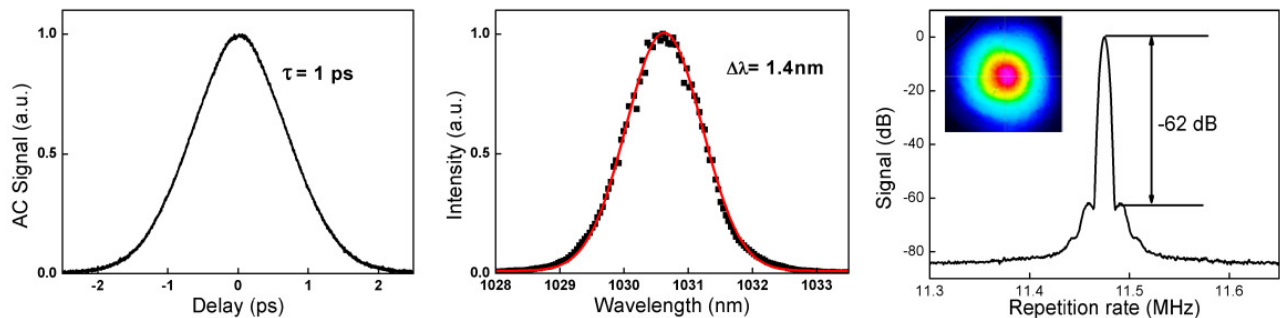

Fig. 2. The autocorrelation trace (left), optical spectrum (middle), the RF spectrum (right) and the beam profile (inset) of the Yb:YAG thin-disk oscillator.

\section{Synchronization scheme}

In order to synchronize the two independent lasers, we use an electronic synchronization with a feedback loop to correct the timing jitter. To increase the precision of the measured error signal, we developed a scheme combining an electronic and an optical control loop (Fig. 3) similar to that presented in [13].

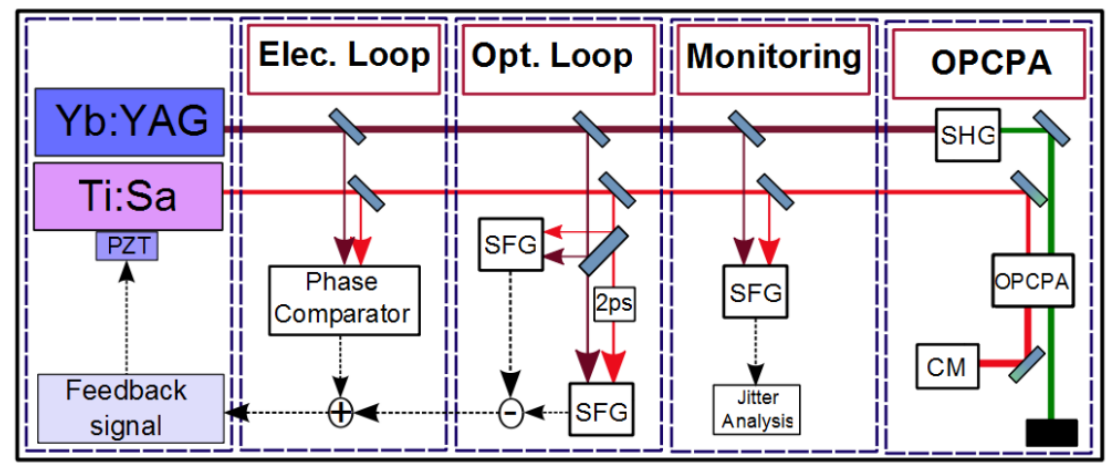

Fig. 3. Block diagram of the system; CM, chirped mirror.

In the system, the $7 \mathrm{fs} \mathrm{Ti}: \mathrm{Sa}$ oscillator with $69 \mathrm{MHz}$ repetition rate [16] is locked to the 11.5 MHz Yb:YAG laser. One of the folding mirrors in the Ti:Sa oscillator is mounted on a piezoelectric transducer (PZT). This configuration was chosen to avoid Q-switching instabilities in the Yb:YAG laser arising from sudden changes in the cavity. The PZT (PiezoJena P 25/10) has a response time of $\mathrm{ms}$ and a travel range of $50 \mu \mathrm{m}$. The locking proceeds in two steps. First, the electronic phase loop locks the pulse trains of the two oscillators. This is done by comparing the phase of the $36^{\text {th }}$ harmonic of the repetition rate of the Yb:YAG and the $6^{\text {th }}$ harmonic of the repetition rate of Ti:Sa oscillator at $414 \mathrm{MHz}$. The loop employs two standard 1-GHz-bandwidth photodiodes to detect the two pulse trains. After electronic locking, the sum frequency signal (SFG) of the optical loop can already be observed and traced (Fig. 4) although unstable at this point due to the high remaining timing jitter. In the second step, a balanced cross-correlator provides the feedback signal for the optical loop. As shown in Fig. 3, a fraction of the two laser pulses is directed onto two identical 2-mm thick BBO crystals for SFG. In one of the SFG setups, a 2 ps delay between the Ti:Sa and Yb:YAG pulses is introduced in order to determine the direction of the temporal offset. To produce the optical feedback signal, the two signals are recorded by two photodiodes and subtracted. When the temporal overlap between the two pulses at the position of the BBO crystals drifts, an asymmetric error signal is generated to readjust the position of the PZT (Fig. 5). This optical error signal adds a further correction to the electronic loop. 
To investigate the locking performance of the synchronization, we set up a monitoring stage and measured the cross-correlation between the pulses of the lasers (Fig. 4). The timing jitter between the two lasers can be estimated from the width of the cross-correlation trace and the pulse durations of the two lasers. The monitoring stage (Fig. 3) consists of a 1-mm-thick BBO crystal and a photomultiplier for detecting the SFG. Assuming that the temporal shape of both laser pulses and the probability distribution of the timing jitter have a Gaussian distribution, the cross-correlation function is given by:

$$
\tau_{c}=\sqrt{\tau_{T: S a}^{2}+\tau_{Y b: Y A G}^{2}+\tau_{j}^{2}}
$$

where $\tau_{j}$ is the full width at half maximum (FWHM) of the timing jitter and $\tau_{c}$ is the FWHM of the cross-correlation. $\tau_{T i: S a}$ and $\tau_{Y b: Y A G}$ are the pulse durations (FWHM) of the Ti:Sa and Yb:YAG oscillators, respectively. Due to the beam path in air and the transmission optics, the duration of the Ti:Sa pulses at the monitoring stage is elongated to 150 fs. From Eq. (1), the FWHM of the timing jitter between the two mode-locked oscillators can be estimated to an RMS value of 120 fs. For further characterization of the timing jitter, the pulse-to-pulse fluctuations of the SFG signal were analyzed at a delay of approximately half of the pump pulse duration. In this configuration, the timing jitter $\Delta \mathrm{t}$ is directly proportional to the measured intensity fluctuations $\Delta \mathrm{I}$ (Fig. 6) and the RMS timing jitter $\sigma_{\mathrm{j}}$ can be retrieved from the RMS intensity fluctuations and the calibrated slope of the correlation curve. The coupling of intensity noise into timing noise has been thoroughly investigated in the case of passively mode-locked lasers [17]. To suppress the discrete nature of the pulse train at 11.5 $\mathrm{MHz}$, a photomultiplier with a bandwidth below $500 \mathrm{kHz}$ is used to measure the SFG. Figure 7 shows the recorded signal over $2 \mathrm{~s}$. By calculating the Allan variance [17] of the recorded intensity noise, the frequency distribution of the timing jitter and thereby the time scale on which the timing jitter mostly occurs can be identified. From these data, the timing jitter is quantified in the range between $2 \mu$ s and $1 \mathrm{~s}$ as shown in Fig. 6. It can be seen that the main contribution of the timing jitter is located near $100 \mu \mathrm{s}$. The relaxation oscillation frequencies of the Ti:Sa and Yb:YAG lasers are estimated to be $140 \mathrm{kHz}$ and $6.5 \mathrm{kHz}$, respectively [18]. The second value is very close to the highest component of the timing jitter located around 5 $\mathrm{kHz}$. Therefore, it is most likely that the relaxation oscillations of the Yb:YAG are the main jitter source in our system.
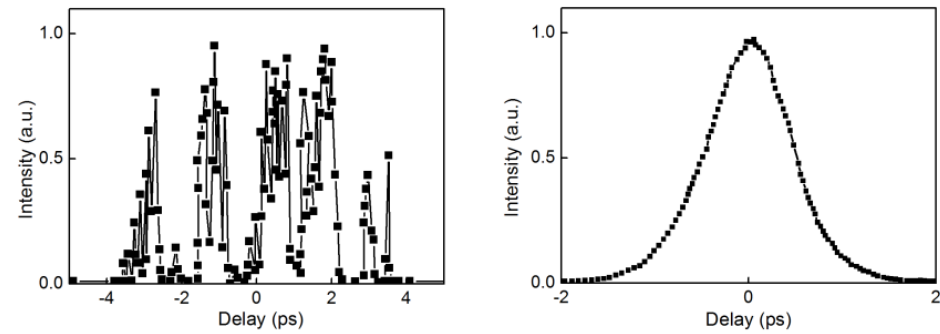

Fig. 4. The cross-correlation trace at the monitoring stage with only the electronic loop (left) and after activating the optical loop (right). 

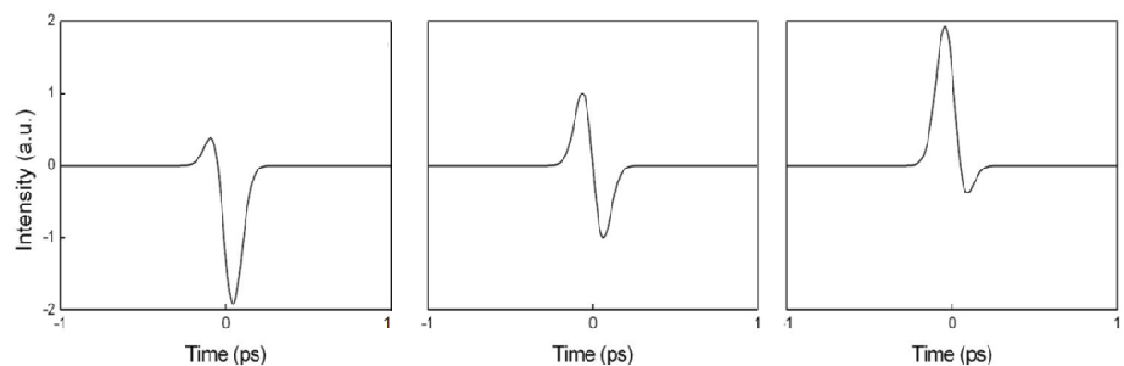

Fig. 5. Calculated error signal of the optical loop for $-50 \mathrm{fs}$ (left), $0 \mathrm{fs}$ (middle) and $+50 \mathrm{fs}$ (right) timing offset between the two pulse trains.
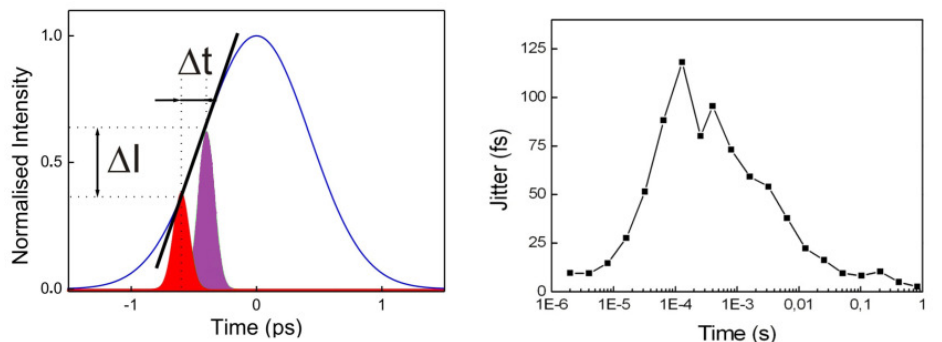

Fig. 6. Schematic representation of the timing jitter. Solid blue line, Yb:YAG pulse. Red and purple filled areas: SFG signal for different relative timing (left). The Allan variance of the measured timing jitter (right).
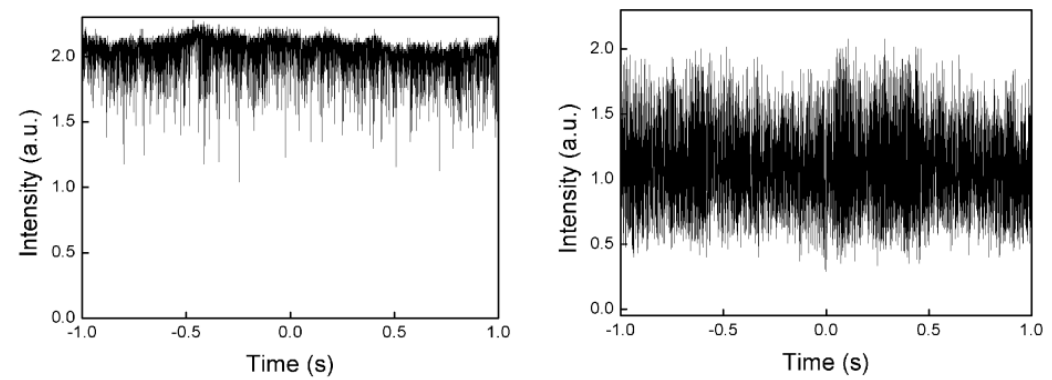

Fig. 7. The measured SFG at the monitoring stage: at the peak of the SFG signal (left), at the half of the SFG peak (right).

The quality of synchronization would certainly benefit from an optimization of the SESAM parameters and the cavity design to improve the stability performances of the oscillator. Another improvement of the timing jitter could be obtained by using a faster PZT thus increasing the feedback bandwidth of the active control loop. The residual jitter after the electronic and optical control loops is still within approximately $10 \%$ of the pump pulse width. These results allow for stable parametric amplification of the Ti:Sa seed pulses by using the frequency-doubled output of the Yb:YAG oscillator as a pump.

\section{MHz OPCPA}

The amplifier system consists of a single-stage non-collinear OPCPA and a chirped mirror compressor. To pump the parametric amplifier, the $\mathrm{Yb}$ :YAG pulses are frequency doubled in a $4 \mathrm{~mm}$ critically-phase-matched type-I LBO crystal, generating $43.5 \mathrm{~W}$ of $515 \mathrm{~nm}$ radiation with excellent beam quality. This corresponds to an optical efficiency of $65 \%$. Due to strong saturation in the frequency conversion process, the pulse-to-pulse energy fluctuations at 515 
$\mathrm{nm}$ are similar to that of the fundamental. The seed energy in front of the OPCPA crystal amounts to $1.4 \mathrm{~nJ}$. The pump is focused to a beam diameter of $200 \mu \mathrm{m}$, slightly larger than the seed. Pump and seed are crossed at an internal angle $\alpha=2.5^{\circ}$ inside a 4-mm BBO crystal cut at $\Theta=24^{\circ}$ for type-I phase-matching. In this geometric configuration, the interaction length is longer than the crystal, ensuring good conversion efficiency and uniform amplification. Pumping the crystal with $34 \mathrm{~W}$ of average power and an intensity of $18 \mathrm{GW} / \mathrm{cm}^{2}$ results in 5.6 $\mathrm{W}$ of amplified output power and a pump-to-signal conversion efficiency of $17 \%$ (Fig. 8). To improve the temporal overlap between pump and seed pulses, $16 \mathrm{~mm}$ uncoated fused silica was used to stretch the seed pulses to 500 fs resulting in $8 \mathrm{~W}$ of the amplified average power. This corresponds to a pulse energy of $700 \mathrm{~nJ}$ and a conversion efficiency of $24 \%$. In this case, the amplified spectral bandwidth narrows, as shown in Fig. 8 but still supports a sub-7-fs Fourier-transform-limited pulse duration.
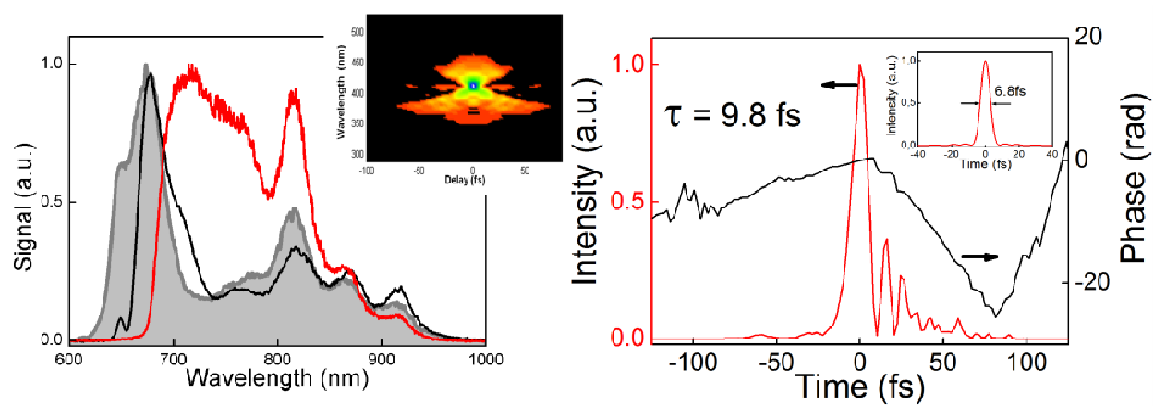

Fig. 8. Left: The seed spectrum (gray) and the amplified spectra at $490 \mathrm{~nJ}$ (black) and $700 \mathrm{~nJ}$ (red). Insert: retrieved FROG trace for $240 \mathrm{~nJ}$. Right: FROG retrieval in the time domain. Insert: the transformed-limited pulse.

The superfluorescence background could not be measured with the power meter available to us. The superfluorescence ring was only visible by using a CCD camera and by removing the neutral density filter in front of it. The superfluorescence-to-signal ratio could be estimated to be on the order of $0.1 \%$. On a time scale of $10 \mathrm{~ms}$, the fluctuations of the amplified pulse train were similar to the fluctuations of the pump pulses. The preliminary compression was carried out by using a double-angle chirped-mirror compressor [19]. Since the only mirrors available to us had a relatively low GDD ( $-30 \mathrm{fs}^{2}$ per bounce), we minimised the introduced dispersion to the seed in order to reduce the amount of necessary bounces in the compressor. For this purpose the seed pulse duration was again decreased, by removing the stretcher and by using a thinner crystal in the OPCPA stage. Amplifying the seed in a 3 mm BBO crystal, we obtained $240 \mathrm{~nJ}$ pulse energy and a compressed pulse duration of $9.8 \mathrm{fs}$. The FROG trace (Fig. 8) shows satellite pulses which are caused by uncompensated higherorder dispersion. The quality of the compression can be improved further by using specially designed chirped mirrors.

\section{Conclusion}

In conclusion, we have realised a synchronization scheme with $<120$ fs (RMS) timing jitter suitable for OPCPA systems pumped by a 1-ps laser source. Using this approach the $300 \mathrm{~nm}$ broadband spectrum of a Ti:Sa oscillator was amplified in a $4 \mathrm{~mm}$ BBO crystal to $700 \mathrm{~nJ}$ and $8 \mathrm{~W}$ of average power at $11.5 \mathrm{MHz}$ repetition rate. $10 \mathrm{fs}, 240 \mathrm{~nJ}$ pulses were realized with a 3 $\mathrm{mm}$ BBO crystal. Among others, this approach promises to reach CEP-stable $\mu \mathrm{J}$-level pulses at $\mathrm{MHz}$ repetition rate in the mid-infrared region [20], representing an attractive source e.g. for gas spectroscopy and gas analysis [21]. On the other hand, recent progress in generating even shorter pulses with high-power Yb-based oscillators [22] requires further improvement of the two-laser synchronization approach when short-pulse-pumped OPCPA is considered. 


\section{Acknowledgments}

We acknowledge the technical support by L. Turi and S. Herbst. The work was supported by the Munich Centre for Advanced Photonics (MAP). Current address for C. Y. Teisset is: Coherent GmbH-Niederlassung Lübeck, Seeland str. 9, 23569 Lübeck, Germany. Current address for A. Sugita is: Department of Materials Science, Shizuoka University, 3-5-1 Johoku, Naka-ku, Hamamatsu 432-8561, Japan. 


\section{Appendix F}

\section{Data archiving}

The original data and the analysis programs developed for data evaluation or theoretical studies in this thesis are stored on the Data Archive Computer (DAC) of the Division for Attosecond Physics at Max Planck Institute of Quantum Optics. The raw data and all Matlab codes used for obtaining the figures are stored in separate folders for each chapter.

In the following, a complete list of the folders containing figures, measurement and/or simulation data is given.

\begin{tabular}{|l|l|l|l|l|}
\hline \multicolumn{5}{|c|}{ data archive } \\
\hline \multicolumn{3}{|l|}{} & & folder \\
\hline Introduction & & - & Fig01 \\
\hline Fig.0.1 & Pdf file & - & Fig02 \\
\hline Fig.0.2 & Pdf file & simulated data & - & \\
\hline Fig.1.1 & Pdf file & simulated data & - & Fig1.1 \\
Fig.1.2 & Pdf file & - & Fig1.2 \\
Fig.1.3 & Pdf file & simulated data & - & Fig1.3 \\
Fig.1.4 & Pdf file & simulated data & - & Fig1.4 \\
Fig.1.5 & Pdf file & simulated data & - & Fig1.5 \\
Fig.1.6 & Pdf file & simulated data & - & Fig1.6 \\
Fig.1.7 & Pdf file & simulated data & - & Fig1.7 \\
Fig.1.8 & Pdf file & simulated data & - & Fig1.8 \\
Fig.1.9 & Pdf file & simulated data & - & Fig1.9 \\
Fig.1.10 & Pdf file & simulated data & - & Fig1.10 \\
Fig.1.11 & Pdf file & simulated data & - & Fig1.11 \\
Fig.1.12 & Pdf file & simulated data & - & Fig1.12 \\
Fig.1.13 & Pdf file & simulated data & - & Fig1.13 \\
Fig.1.14 & Pdf file & simulated data & - & Fig1.14 \\
\hline
\end{tabular}


Fig.1.15

Fig.1.16

Fig.1.17

Fig.1.18

Fig.1.19

Fig.1.20

Fig.1.21

Chapter 2

Fig. 2.1

Fig. 2.2

Fig. 2.3

Fig. 2.4

Fig. 2.5

Fig. 2.6

Fig. 2.7

Fig. 2.8

Fig. 2.9

Fig. 2.10

Fig. 2.11

Fig. 2.12

Fig. 2.13

Fig. 2.14

Fig. 2.15

Fig. 2.16

Fig. 2.17

Chapter 3

Fig. 3.1

Fig. 3.2

Fig. 3.3

Fig. 3.4

Fig. 3.5

Fig. 3.6

Fig. 3.7

Chapter 4

Fig.4.1

Fig. 4.2

Fig. 4.3

Fig. 4.4

Fig. 4.5

Fig. 4.6

\begin{tabular}{l|r|l|} 
Pdf file & simulated data & - \\
Pdf file & simulated data & measured data \\
Pdf file & simulated data & - \\
Pdf file & simulated data & measured data \\
Pdf file & simulated data & - \\
Pdf file & simulated data & - \\
Pdf file & simulated data & -
\end{tabular}

Fig1.15

Fig1.16

Fig1.17

Fig1.18

Fig1.19

Fig1.20

Fig1.21

folder

Fig2.1

Pdf file

Pdf file

- measured data

Fig2.2

\section{Pdf file}

\section{Pdf file}

measured data

measured data

measured data

measured data

Pdf file

Fig2.3

Fig2. 4

Fig2.5

Fig2.6

Fig2.7

Fig2.8

Fig2.9

measured data

measured data

Fig2.10

measured data

Fig2.11

Fig2.12

Fig2.13

Fig2.14

Fig2.15

Fig2.16

Pdf file

simulated data

measured data

measured data

simulated data

-

Fig2.17

measured data

folder

Fig3.1

Fig3.2

Fig3.3

Fig3.4

Fig3.5

Fig3.6

Fig3.7

folder

Fig4.1

Fig4.2

measured data

measured data

Fig4. 3

measured data

Fig4. 4

measured data

Fig4.5

Fig4.6 


\begin{tabular}{|l|l|l|l|l|} 
Fig.4.7 & Pdf file & simulated data & - & Fig4.7 \\
Fig.4.8 & Pdf file & - & measured data & Fig4.8 \\
Fig.4.9 & Pdf file & simulated data & - & Fig4.9 \\
Fig.4.10 & Pdf file & simulated data & - & Fig4.10 \\
Fig.4.11 & Pdf file & - & measured data & Fig4.11 \\
Fig.4.12 & Pdf file & - & measured data & Fig4.12 \\
Fig.4.13 & Pdf file & - & measured data & Fig4.13 \\
Fig.4.14 & Pdf file & - & measured data & Fig4.14 \\
\hline Chapter 5 & & & & folder \\
\hline Fig.5.1 & Pdf file & - & - & Fig5.1 \\
Fig.5.2 & Pdf file & - & measured data & Fig5.2 \\
Fig.5.3 & Pdf file & - & - & Fig5.3 \\
Fig.5.4 & Pdf file & simulated data & - & Fig5.4 \\
Fig.5.5 & Pdf file & simulated data & - & Fig5.5 \\
Fig.5.6 & Pdf file & simulated data & - & Fig5.6 \\
Fig.5.7 & Pdf file & simulated data & - & Fig5.7 \\
Fig.5.8 & Pdf file & simulated data & - & Fig5.8 \\
Fig.5.9 & Pdf file & simulated data & - & Fig5.9 \\
Fig.5.10 & Pdf file & simulated data & - & Fig5.10 \\
Fig.5.11 & Pdf file & simulated data & - & Fig5.11 \\
\hline Appendix & & & & folder \\
\hline Fig.A.1 & Pdf file & simulated data & measured data & FigA.1 \\
Fig.D.1 & Pdf file & - & measured data & FigD.1 \\
\hline
\end{tabular}





\section{References}

Agostini, P., Fabre, F., Mainfray, G., Petite, G., and Rahman, N. (1979). "FreeFree Transitions Following Six-Photon Ionization of Xenon Atoms". In: Physical Review Letters 42.17, pp. 1127-1130.

Antipenkov, R., Varanavičius, A., Zaukevičius, A., and Piskarskas, A. (2011). "Femtosecond $\mathrm{Yb}: \mathrm{KGW}$ MOPA driven broadband NOPA as a frontend for TW few-cycle pulse systems". In: Optics Express 19.4, pp. 3519-24.

Antognini, A., Schuhmann, K., Amaro, F. D., Biraben, F., Dax, A., Giesen, A., Graf, T., Hansch, T. W., Indelicato, P., Julien, L., Kao, C.-Y., Knowles, P. E., Kottmann, F., Le Bigot, E., Liu, Y.-W., Ludhova, L., Moschuring, N., Mulhauser, F., Nebel, T., Nez, F., Rabinowitz, P., Schwob, C., Taqqu, D., and Pohl, R. (2009). "Thin-Disk Yb:YAG Oscillator-Amplifier Laser, ASE, and Effective Yb:YAG Lifetime". In: IEEE Journal of Quantum Electronics 45.8, pp. 993-1005.

Antoine, P., L'Huillier, A., Lewenstein, M., Salières, P., and Carré, B. (1996). "Theory of high-order harmonic generation by an elliptically polarized laser field". In: Physical Review A 53.3, pp. 1725-1745.

Arisholm, G. (1997). "General numerical methods for simulating secondorder nonlinear interactions in birefringent media". In: Journal of the Optical Society of America B 14.10, pp. 2543-2549.

Augstov, P. A. and Shvarts, K. K. (1980). "The temperature and light intensity dependence of photorefraction in $\mathrm{LiNbO}_{3}$ ". In: Applied Physics 21.2, pp. 191194 .

Bagayev, S. N., Trunov, V. I., Pestryakov, E. V., Leschenko, V. E., Frolov, S. A., and Vasiliev, V. A. (2013). "High-intensity femtosecond laser systems based on coherent combining of optical fields". In: Optics and Spectroscopy 115.3, pp. 311-319.

Bagnoud, V., Begishev, I. A., Guardalben, M. J., Puth, J., and Zuegel, J. D. (2005). " $5 \mathrm{~Hz},>250 \mathrm{~mJ}$ optical parametric chirped-pulse amplifier at 1053 nm". In: Optics Letters 30.14, pp. 1843-1845. 
Bakker, H. J., Cho, G. C., Kurz, H., Wu, Q., and Zhang, X.-C. (1998). “Distortion of terahertz pulses in electro-optic sampling". In: Journal of the Optical Society of America B 15.6, pp. 1795-1801.

Banks, P. S., Feit, M. D., and Perry, M. D. (2002). "High-intensity thirdharmonic generation". In: Journal of the Optical Society of America B 19.1, pp. 102-118.

Binhammer, T., Rittweger, E., Ell, R., Kartner, F., and Morgner, U. (2005). "Prism-based pulse shaper for octave spanning spectra". In: IEEE Journal of Quantum Electronics 41.12, pp. 1552-1557.

Birks, T. a., Knight, J. C., and Russell, P. S. (1997). "Endlessly single-mode photonic crystal fiber". In: Optics Letters 22.13, pp. 961-3.

Bohman, S., Suda, A., Kanai, T., Yamaguchi, S., and Midorikawa, K. (2010). "Generation of $5.0 \mathrm{fs}, 5.0 \mathrm{~mJ}$ pulses at $1 \mathrm{kHz}$ using hollow-fiber pulse compression". In: Optics Letters 35.11, pp. 1887-9.

Boyd, R. W. (2003). Nonlinear Optics.

Brabec, T. and Krausz, F. (1997). "Nonlinear Optical Pulse Propagation in the Single-Cycle Regime". In: Physical Review Letters 78.17, pp. 3282-3285.

Bradler, M., Baum, P., and Riedle, E. (2009). "Femtosecond continuum generation in bulk laser host materials with sub- $\mu \mathrm{J}$ pump pulses". In: Applied Physics B 97.3, pp. 561-574.

Bromage, J., Rothhardt, J., Hädrich, S., Dorrer, C., Jocher, C., Demmler, S., Limpert, J., Tünnermann, A., and Zuegel, J. D. (2011). "Analysis and suppression of parasitic processes in noncollinear optical parametric amplifiers". In: Optics Express 19.18, pp. 16797-808.

Buth, C., He, F., Ullrich, J., Keitel, C. H., and Hatsagortsyan, K. Z. (2013). "Attosecond pulses at kiloelectronvolt photon energies from high-orderharmonic generation with core electrons". In: Physical Review A 88.3, pp. 03384857 .

Canova, L., Kourtev, S., Minkovski, N., Jullien, A., Lopez-Martens, R., Albert, O., and Saltiel, S. M. (2008). "Efficient generation of cross-polarized femtosecond pulses in cubic crystals with holographic cut orientation". In: Applied Physics Letters 92.23, p. 231102.

Cavalieri, A. L., Goulielmakis, E., Horvath, B., Helml, W., Schultze, M., Fieß, M., Pervak, V., Veisz, L., Yakovlev, V. S., Uiberacker, M., Apolonski, A., Krausz, F., and Kienberger, R. (2007). "Intense 1.5-cycle near infrared laser 
waveforms and their use for the generation of ultra-broadband soft-x-ray harmonic continua". In: New Journal of Physics 9.7, pp. 2421-29.

Cerullo, G. and De Silvestri, S. (2003). "Ultrafast optical parametric amplifiers". In: Review of Scientific Instruments 74.1, pp. 1-18.

Cerullo, G., Nisoli, M., and De Silvestri, S. (1997). “Generation of 11 fs pulses tunable across the visible by optical parametric amplification". In: Applied Physics Letters 71.25, pp. 3616-3618.

Chalus, O., Bates, P. K., Smolarski, M., and Biegert, J. (2009). “Mid-IR shortpulse OPCPA with micro-Joule energy at 1ookHz". In: Optics Express 17.5, pp. 3587-3594.

Chekhlov, O. V., Collier, J. L., Ross, I. N., Bates, P. K., Notley, M., HernandezGomez, C., Shaikh, W., Danson, C. N., Neely, D., Matousek, P., Hancock, S., and Cardoso, L. (2006). " 35 J broadband femtosecond optical parametric chirped pulse amplification system". In: Optics Letters 31.24, pp. 3665-7.

Chen, B., Dong, J., Patel, M., Chen, Y., Kar, A., and Bass, M. A. (2003). “Modeling of high-power solid-state slab lasers". In: SPIE Proceeding. Ed. by R. Scheps, pp. 1-10.

Chen, S., Chini, M., Wang, H., Yun, C., Mashiko, H., Wu, Y., and Chang, Z. (2009). "Carrier-envelope phase stabilization and control of $1 \mathrm{kHz}, 6 \mathrm{~mJ}, 30$ fs laser pulses from a Ti:sapphire regenerative amplifier". In: Applied Optics 48.30, pp. 5692-5.

Chu, Y., Liang, X., Yu, L., Xu, Y., Xu, L., Ma, L., Lu, X., Liu, Y., Leng, Y., Li, R., and $\mathrm{Xu}, \mathrm{Z}$. (2013). "High-contrast 2.o Petawatt Ti:sapphire laser system". In: Optics Express 21.24, pp. 29231-9.

Chuang, Y.-H., Zheng, L., and Meyerhofer, D. (1993). "Propagation of light pulses in a chirped-pulse-amplification laser". In: IEEE Journal of Quantum Electronics 29.1, pp. 270-280.

Cook, C. (1960). "Pulse Compression-Key to More Efficient Radar Transmission". In: Proceedings of the IRE 48.3, pp. 310-316.

Cotel, A., Jullien, A., Forget, N., Albert, O., Chériaux, G., and Le Blanc, C. (2006). "Nonlinear temporal pulse cleaning of a $1-\mu \mathrm{m}$ optical parametric chirped-pulse amplification system". In: Applied Physics B 83.1, pp. 7-10.

Deng, Y. and Krausz, F. (2013). Method and device for optical parametric chirped pulse amplification. 
Deng, Y., Schwarz, A., Fattahi, H., Ueffing, M., Gu, X., Ossiander, M., Metzger, T., Pervak, V., Ishizuki, H., Taira, T., Kobayashi, T., Marcus, G., Krausz, F., Kienberger, R., and Karpowicz, N. (2012). "Carrier-envelope-phase-stable, $1.2 \mathrm{~mJ}, 1.5$ cycle laser pulses at $2.1 \mu \mathrm{m}$ ". In: Optics Letters 37.23, pp. 4973-5.

Desalvo, R., Said, A. A., Hagan, D. J., Stryland, E. W. V., Member, S., and Sheik-bahae, M. (1996). "Infrared to Ultraviolet Measurements of Two-Photon Absorption and n2 in Wide Bandgap Solids". In: IEEE Journal of Quantum Electronics 32.8, pp. 1324-1333.

Dmitriev, V. G., Gurzadyan, G. G., and Nikogosyan, D. N. (1999). Handbook of Nonlinear Optical Crystals, p. 414.

Dörring, J., Killi, A., Morgner, U., Lang, A., Lederer, M., and Kopf, D. (2004). "Period doubling and deterministic chaos in continuously pumped regenerative amplifiers". In: Optics Express 12.8, pp. 1759-68.

Doumy, G., Quéré, F., Gobert, O., Perdrix, M., and Martin, P. (2004). “Complete characterization of a plasma mirror for the production of high-contrast ultraintense laser pulses". In: Physical Review E 69.2, pp. 026402-14.

Dubietis, A., Jonušauskas, G., and Piskarskas, A. (1992). “Powerful femtosecond pulse generation by chirped and stretched pulse parametric amplification in BBO crystal". In: Optics Communications 88.4-6, pp. 437-440.

Dubietis, A., Tamošauskas, G., Varanavičius, A., and Valiulis, G. (2000). "TwoPhoton Absorbing Properties of Ultraviolet Phase-Matchable Crystals at 264 and 211 nm". In: Applied Optics 39.15, pp. 2437-40.

Dudley, J. M. and Coen, S. (2006). "Supercontinuum generation in photonic crystal fiber". In: Reviews of Modern Physics 78.4, pp. 1135-1184.

Einstein, A. (1917). "Zur Quantentheorie der Strahlung". In: Physikalische Zeitschrift 18, pp. 121-128.

Erny, C., Moutzouris, K., Biegert, J., Kühlke, D., Adler, F., Leitenstorfer, A., and Keller, U. (2007). "Mid-infrared difference-frequency generation of ultrashort pulses tunable between 3.2 and $4.8 \mu \mathrm{m}$ from a compact fiber source". In: Optics Letters 32.9, pp. 1138-1140.

Fang, S., Cirmi, G., Chia, S.-H., Mücke, O. D., Kärtner, F. X., Manzoni, C., Farinello, P., and Cerullo, G. (2013). "Multi-mJ Parametric Synthesizer Generating Two-Octave-Wide Optical Waveforms". In: 2013 Conference on Lasers and Electro-Optics Pacific Rim. Kyoto: Optical Society of America, WB3_1. 
Fattahi, H., Barros, H. G., Gorjan, M., Nubbemeyer, T., Alsaif, B., Teisset, C. Y., Schultze, M., Prinz, S., Haefner, M., Ueffing, M., Alismail, A., Vámos, L., Schwarz, A., Pronin, O., Brons, J., Geng, X. T., Arisholm, G., Ciappina, M., Yakovlev, V. S., Kim, D.-E., Azzeer, A. M., Karpowicz, N., Sutter, D., Major, Z., Metzger, T., and Krausz, F. (2014a). "Third-generation femtosecond technology". In: Optica 1.1, pp. 45-63.

Fattahi, H., Schwarz, A., Geng, X. T., Keiber, S., Kim, D. E., Krausz, F., and Karpowicz, N. (2014b). "Decoupling chaotic amplification and nonlinear phase in high-energy thin-disk amplifiers for stable OPCPA pumping". In: Optics Express 22.25, pp. 31440-7.

Fattahi, H., Schwarz, A., Keiber, S., and Karpowicz, N. (2013). "Efficient, octave-spanning difference-frequency generation using few-cycle pulses in simple collinear geometry". In: Optics Letters 38.20, pp. 4216-9.

Fattahi, H., Skrobol, C., Ueffing, M., Deng, Y., Schwarz, A., Kida, Y., Pervak, V., Metzger, T., Major, Z., and Krausz, F. (2012a). "High efficiency, multi-mJ, sub $10 \mathrm{fs}$, optical parametric amplifier at $3 \mathrm{kHz}$ ". In: Conference on Lasers and Electro-Optics 2012. Washington, D.C.: OSA, CThiN.6.

Fattahi, H., Teisset, C. Y., Pronin, O., Sugita, A., Graf, R., Pervak, V., Gu, X., Metzger, T., Major, Z., Krausz, F., and Apolonski, A. (2012b). "Pumpseed synchronization for $\mathrm{MHz}$ repetition rate, high-power optical parametric chirped pulse amplification". In: Optics Express 20.9, pp. 9833-40.

Fork, R. L., Greene, B. I., and Shank, C. V. (1981). "Generation of optical pulses shorter than 0.1 psec by colliding pulse mode locking". In: Applied Physics Letters 38.9, pp. 671-2.

Franken, P., Hill, A., Peters, C., and Weinreich, G. (1961). "Generation of Optical Harmonics". In: Physical Review Letters 7.4, pp. 118-119.

Furukawa, Y., Kitamura, K., Takekawa, S., Miyamoto, A., Terao, M., and Suda, N. (2000). "Photorefraction in $\mathrm{LiNbO}_{3}$ as a function of $[\mathrm{Li}] /[\mathrm{Nb}]$ and MgO concentrations". In: Applied Physics Letters 77.16, pp. 2494-96.

Gale, G. M., Cavallari, M., Driscoll, T. J., and Hache, F. (1995). "Sub-20-fs tunable pulses in the visible from an $82-\mathrm{MHz}$ optical parametric oscillator". In: Optics Letters 20.14, pp. 1562-1564.

Gale, G. M., Cavallari, M., and Hache, F. (1998). "Femtosecond visible optical parametric oscillator". In: Journal of the Optical Society of America B 15.2, pp. 702-14. 
Gordon, J., Zeiger, H., and Townes, C. (1955). "The Maser-New Type of Microwave Amplifier, Frequency Standard, and Spectrometer". In: Physical Review 99.4, pp. 1264-1274.

Grishin, M., Gulbinas, V., and Michailovas, A. (2007). “Dynamics of high repetition rate regenerative". In: Optics Express 15.15, pp. 9434-9443.

Grishin, M., Gulbinas, V., and Michailovas, A. (2009). "Bifurcation suppression for stability improvement in $\mathrm{Nd}: \mathrm{YVO}_{4}$ regenerative amplifier". In: $\mathrm{Op}$ tics Express 17.18, pp. 15700-8.

Guardalben, M., Keegan, J., Waxer, L., Bagnoud, V., Begishev, I., Puth, J., and Zuegel, J. (2003). "Design of a highly stable, high-conversion-efficiency, optical parametric chirped-pulse amplification system with good beam quality". In: Optics Express 11.20, pp. 2511-24.

Hädrich, S., Klenke, A., Hoffmann, A., Eidam, T., Gottschall, T., Rothhardt, J., Limpert, J., and Tünnermann, A. (2013). "Nonlinear compression to sub30-fs, $0.5 \mathrm{~mJ}$ pulses at $135 \mathrm{~W}$ of average power". In: Optics Letters 38.19, pp. 3866-9.

Harth, A., Schultze, M., Lang, T., Binhammer, T., Rausch, S., and Morgner, U. (2012). "Two-color pumped OPCPA system emitting spectra spanning 1.5 octaves from VIS to NIR". In: Optics Express 20.3, pp. 3076-81.

Hassan, M. T., Wirth, A., Grguraš, I., Moulet, A., Luu, T. T., Gagnon, J., Pervak, V., and Goulielmakis, E. (2012). "Invited article: attosecond photonics: synthesis and control of light transients". In: The Review of Scientific Instruments 83.11, pp. 1113011-19.

Hentschel, M., Cheng, Z., Krausz, F., and Spielmann, C. (2000). “Generation of o.1-TW optical pulses with a single-stage Ti:sapphire amplifier at a $1-\mathrm{kHz}$ repetition rate". In: Applied Physics B 70.S1, S161-S164.

Herrmann, D., Homann, C., Tautz, R., Scharrer, M., Russell, P. S. J., Krausz, F., Veisz, L., and Riedle, E. (2010a). "Approaching the full octave: noncollinear optical parametric chirped pulse amplification with two-color pumping". In: Optics Express 18.18, pp. 18752-62.

- (2010b). "Approaching the full octave: noncollinear optical parametric chirped pulse amplification with two-color pumping". In: Optics Express 18.18, pp. 18752-62.

Herrmann, D., Tautz, R., Tavella, F., Krausz, F., and Veisz, L. (2010c). “Investigation of two-beam-pumped noncollinear optical parametric chirped-pulse 
amplification for the generation of few-cycle light pulses". In: Optics Express 18.5, pp. $4170-83$.

Homann, C., Bradler, M., Förster, M., Hommelhoff, P., and Riedle, E. (2012). "Carrier-envelope phase stable sub-two-cycle pulses tunable around $1.8 \mu \mathrm{m}$ at $100 \mathrm{kHz}$ ". In: Optics Letters 37.10, pp. 1673-5.

Hong, K. han, Huang, S. wei, Moses, J., Fu, X., Lai, C. jen, Cirmi, G., Sell, A., Granados, E., Keathley, P., and Franz, X. (2011). "High-energy, phase-stable, ultrabroadband $\mathrm{kHz}$ OPCPA at $2.1 \mu \mathrm{m}$ pumped by a picosecond cryogenic Yb:YAG laser". In: Optics Express 19.16, pp. 15538-15548.

Hönninger, C., Paschotta, R., Graf, M., Morier-Genoud, F., Zhang, G., Moser, M., Biswal, S., Nees, J., Braun, A., Mourou, G., Johannsen, I., Giesen, A., Seeber, W., and Keller, U. (1999). "Ultrafast ytterbium-doped bulk lasers and laser amplifiers". In: Applied Physics B: Lasers and Optics 69.1, pp. 3-17.

Huang, S.-W., Cirmi, G., Moses, J., Hong, K.-H., Bhardwaj, S., Birge, J. R., Chen, L.-J., Kabakova, I. V., Li, E., Eggleton, B. J., Cerullo, G., and Kärtner, F. X. (2012). "Optical waveform synthesizer and its application to highharmonic generation". In: Journal of Physics B: Atomic, Molecular and Optical Physics 45.7, pp. 0740091-14.

Ippen, E., Shank, C. V., and Dienes, A. (1972). "Passive mode locking of the cw dye laser". In: Applied Physics Letters 21.8, pp. 348-50.

Ishii, N., Turi, L., Yakovlev, V. S., Fuji, T., Krausz, F., Baltuska, A., Butkus, R., Veitas, G., Smilgevicius, V., Danielius, R., and Piskarskas, A. (2005). "Multimillijoule chirped parametric amplification of few-cycle pulses". In: Optics Letters 30.5, pp. 567-69.

Jauregui, C., Limpert, J., and Tünnermann, A. (2013). "High-power fibre lasers". In: Nature Photonics 7.11, pp. 861-867.

Jullien, A., Albert, O., Chériaux, G., Etchepare, J., Kourtev, S., Minkovski, N., and Saltiel, S. M. (2006). "A two crystal arrangement to fight efficiency saturation in cross-polarized wave generation". In: Optics Express 14.7, pp. 27609.

Jullien, A., Albert, O., Burgy, F., Hamoniaux, G., Rousseau, J.-P., Chambaret, J.-P., Augé-Rochereau, F., Chériaux, G., Etchepare, J., Minkovski, N., and Saltiel, S. M. (2005). "10^-10 temporal contrast for femtosecond ultraintense lasers by cross-polarized wave generation". In: Optics Letters 30.8, pp. 920922. 
Kartashov, D., Ališauskas, S., Pugžlys, A., Voronin, A., Zheltikov, A., Petrarca, M., Béjot, P., Kasparian, J., Wolf, J.-P., and Baltuška, A. (2012). "White light generation over three octaves by femtosecond filament at $3.9 \mu \mathrm{m}$ in argon". In: Optics Letters 37.16, pp. 3456-8.

Kärtner, F. X., Mücke, O., Cirmi, G., Fang, S., Chia, S.-H., Manzoni, C., Farinello, P., and Cerullo, G. (2013). "High Energy Sub-cycle Optical Waveform Synthesizer". In: Advanced Solid-State Lasers Congress. Ed. by G. Huber and P. Moulton. Washington, D.C.: OSA, AW2A.1.

Kastler, A. (1950). "Quelques suggestions concernant la production optique et la détection optique d'une inégalité de population des niveaux de quantifigation spatiale des atomes. Application à l'expérience de Stern et Gerlach et à la résonance magnétique". In: J. Phys. Radium 11, pp. 255-265.

Kato, K. (1986). "Second-harmonic generation to 2048 Åin $\beta$-Ba2O4". In: IEEE Journal of Quantum Electronics 22.7, pp. 1013-1014.

- (1994). “Temperature-tuned $90^{\circ}$ phase-matching properties of $\mathrm{LiB}_{-} \mathrm{O}_{-}$ 5". In: IEEE Journal of Quantum Electronics 30.12, pp. 2950-2952.

Klingebiel, S., Wandt, C., Skrobol, C., Ahmad, I., Trushin, S. A., Major, Z., Krausz, F., and Karsch, S. (2011). "High energy picosecond Yb:YAG CPA system at $10 \mathrm{~Hz}$ repetition rate for pumping optical parametric amplifiers". In: Optics Express 19.6, pp. 5357-63.

Knight, J. C., Birks, T. a., Russell, P. S., and Atkin, D. M. (1997). “All-silica single-mode optical fiber with photonic crystal cladding: errata". In: Optics Letters 22.7, pp. 484-5.

Knox, W. H., Downer, M. C., Fork, R. L., and Shank, C. V. (1984). “Amplified femtosecond optical pulses and continuum generation at $5-\mathrm{kHz}$ repetition rate". In: Optics Letters 9.12, pp. 552-4.

Krausz, F. and Ivanov, M. (2009). "Attosecond physics". In: Reviews of Modern Physics 81.1, pp. 163-234.

Krausz, F. and Stockman, M. I. (2014). "Attosecond metrology: from electron capture to future signal processing". In: Nature Photonics 8.3, pp. 205-213.

Lenzner, M., Krüger, J., Sartania, S., Cheng, Z., Spielmann, C., Mourou, G., Kautek, W., and Krausz, F. (1998). "Femtosecond Optical Breakdown in Dielectrics". In: Physical Review Letters 80.18, pp. 4076-4079. 
Lewenstein, M., Balcou, P., Ivanov, M., L'Huillier, A., and Corkum, P. (1994). "Theory of high-harmonic generation by low-frequency laser fields". In: Physical Review A 49.3, pp. 2117-2132.

Liu, J. and Kobayashi, T. (2010). "Generation and amplification of tunable multicolored femtosecond laser pulses by using cascaded four-wave mixing in transparent bulk media". In: Sensors (Basel, Switzerland) 10.5, pp. 4296341.

Luu, T. T., Hassan, M. T., Moulet, A., Razskazovskaya, O., Kaprowicz, N., Pervak, V., Krausz, F., and Goulielmakis, E. (2013). "Isolated optical attosecond pulses - OSA Technical Digest (online)". In: CLEO: 2013. San Jose, California: Optical Society of America, QFiC.6.

Maiman, T. H. (1960). "Stimulated Optical Radiation in Ruby". In: Nature 187.4736, pp. 493-494.

Manzoni, C., Cerullo, G., and De Silvestri, S. (2004). "Ultrabroadband selfphase-stabilized pulses by difference-frequency generation". In: Optics Letters 29.22, pp. 2668-2670.

Manzoni, C., Huang, S.-W., Cirmi, G., Farinello, P., Moses, J., Kärtner, F. X., and Cerullo, G. (2012). "Coherent synthesis of ultra-broadband optical parametric amplifiers". In: Optics Letters 37.11, pp. 1880-2.

Matsushima, I., Yashiro, H., and Tomie, T. (2006). “1o kHz 40 W Ti:sapphire regenerative ring amplifier". In: Optics Letters 31.13, pp. 2066-8.

Metzger, T., Schwarz, A., Teisset, C. Y., Sutter, D., Killi, A., Kienberger, R., and Krausz, F. (2009). "High-repetition-rate picosecond pump laser based on a Yb:YAG disk amplifier for optical parametric amplification". In: Optics Letters 34.14, pp. 2123-25.

Minkovski, N., Petrov, G. I., Saltiel, S. M., Albert, O., and Etchepare, J. (2004). "Nonlinear polarization rotation and orthogonal polarization generation experienced in a single-beam configuration". In: Journal of the Optical Society of America B 21.9, pp. 1659-64.

Moses, J., Suchowski, H., and Kärtner, F. X. (2012). "Fully efficient adiabatic frequency conversion of broadband Ti:sapphire oscillator pulses". In: Optics Letters 37.9, pp. 1589-91.

Moulet, A., Tosa, V., and Goulielmakis, E. (2014). “Coherent kiloelectronvolt x-rays generated by subcycle optical drivers: a feasibility study". In: Optics Letters 39.21, pp. 6189-92. 
Moulton, P. F. (1986). "Spectroscopic and laser characteristics of Ti:Al_2O_3". In: Journal of the Optical Society of America B 3.1, pp. 125-133.

Nabekawa, Y., Kuramoto, Y., Togashi, T., Sekikawa, T., and Watanabe, S. (1998). "Generation of o.66-TW pulses at $1 \mathrm{kHz}$ by a Ti:sapphire laser". In: Optics Letters 23.17, pp. 1384-86.

Nakatani, H., Bosenberg, W. R., Cheng, L. K., and Tang, C. L. (1988). "Laserinduced damage in beta-barium metaborate". In: Applied Physics Letters 53.26, pp. $2587-89$.

Newport Corporation (2014).

Nisoli, M., De Silvestri, S., and Svelto, O. (1996). "Generation of high energy 10 fs pulses by a new pulse compression technique". In: Applied Physics Letters 68.20, pp. 2793-95.

Nisoli, M., Stagira, S., De Silvestri, S., Svelto, O., Sartania, S., Cheng, Z., Lenzner, M., Spielmann, C., and Krausz, F. (1997). "A novel-high energy pulse compression system: generation of multigigawatt sub-5-fs pulses". In: Applied Physics B: Lasers and Optics 65.2, pp. 189-196.

Palaniyappan, S., Ghebregziabher, I., DiChiara, A., MacDonald, J., and Walker, B. (2006). "Emergence from nonrelativistic strong-field rescattering to ultrastrongfield laser-atom physics: A semiclassical analysis". In: Physical Review A 74.3, pp. 033403-11.

Perry, M. D., Ditmire, T., and Stuart, B. C. (1994). “Self-phase modulation in chirped-pulse amplification". In: Optics Letters 19.24, pp. 2149-51.

Petričević, V., Gayen, S. K., Alfano, R. R., Yamagishi, K., Anzai, H., and Yamaguchi, Y. (1988). "Laser action in chromium-doped forsterite". In: Applied Physics Letters 52.13, pp. 1040-42.

Pires, H., Galimberti, M., and Figueira, G. (2014). "Numerical evaluation of ultrabroadband parametric amplification in YCOB". In: Journal of the Optical Society of America B 31.11, pp. 2608-14.

Popmintchev, T., Chen, M.-C., Popmintchev, D., Arpin, P., Brown, S., Alisauskas, S., Andriukaitis, G., Balciunas, T., Mücke, O. D., Pugzlys, A., Baltuska, A., Shim, B., Schrauth, S. E., Gaeta, A., Hernández-García, C., Plaja, L., Becker, A., Jaron-Becker, A., Murnane, M. M., and Kapteyn, H. C. (2012). "Bright coherent ultrahigh harmonics in the $\mathrm{keV} x$-ray regime from midinfrared femtosecond lasers". In: Science (New York, N.Y.) 336.6o86, pp. 128791. 
Prandolini, M. J., Riedel, R., Schulz, M., Hage, A., Tavella, F., and Jena, D.-. (2014). "Design considerations for a high power, ultrabroadband optical parametric chirped-pulse amplifier". In: Optics Express 22.2, pp. 1594-1607.

Pronin, O., Brons, J., Grasse, C., Pervak, V., Boehm, G., Amann, M.-C., Kalashnikov, V. L., Apolonski, A., and Krausz, F. (2011). "High-power 200 fs Kerrlens mode-locked Yb:YAG thin-disk oscillator". In: Optics Letters 36.24, pp. 47468.

Pronin, O., Seidel, M., Brons, J., Lücking, F., Pervak, V., Apolonski, A., Udem, T., and Krausz, F. (2013). "Carrier-envelope phase stabilized thin-disk oscillator". In: Advanced Solid-State Lasers Congress. Ed. by G. Huber and P. Moulton. Washington, D.C.: OSA, AF3A.5.

Ratner, J., Steinmeyer, G., Wong, T. C., Bartels, R., and Trebino, R. (2012). "Coherent artifact in modern pulse measurements". In: Optics Letters 37.14, pp. 2874-6.

Reiss, H. R. (2014). "The tunneling model of laser-induced ionization and its failure at low frequencies". In: arXiv.org arXiv:1403, pp. 1-19. arXiv: arXiv: $1403.0568 v 1$.

Renault, A., Augé-Rochereau, F., Planchon, T., D'Oliveira, P., Auguste, T., Chériaux, G., and Chambaret, J.-P. (2005). "ASE contrast improvement with a non-linear filtering Sagnac interferometer". In: Optics Communications 248.46, pp. 535-541.

Ricci, A., Silva, F., Jullien, A., Cousin, S. L., Austin, D. R., Biegert, J., and Lopez-Martens, R. (2013). "Generation of high-fidelity few-cycle pulses at 2.1 $\mu \mathrm{m}$ via cross-polarized wave generation". In: Optics Express 21.8, pp. 971121.

Riedle, E., Beutter, M., Lochbrunner, S., Piel, J., Schenkl, S., Spörlein, S., and Zinth, W. (2000). "Generation of 10 to 50 fs pulses tunable through all of the visible and the NIR". In: Applied Physics B 71.3, pp. 457-465.

Robinson, J., Haworth, C., Teng, H., Smith, R., Marangos, J., and Tisch, J. (2006). "The generation of intense, transform-limited laser pulses with tunable duration from 6 to 30 fs in a differentially pumped hollow fibre". In: Applied Physics B 85.4, pp. 525-529.

Rolland, C. and Corkum, P. (1986). "Amplification of 70 fs pulses in a high repetition rate $\mathrm{XeCl}$ pumped dye laser amplifier". In: Optics Communications 59.1, pp. 64-68. 
Rossi, G. M., Cirmi, G., Fang, S., Chia, S.-H., Muecke, O. D., Kärtner, F., Manzoni, C., Farinello, P., and Cerullo, G. (2014). "Spectro-Temporal Characterization of All Channels in a Sub-Optical-Cycle Parametric Waveform Synthesizer". In: CLEO: 2014. Washington, D.C.: OSA, SF1E.3.

Rothhardt, J., Demmler, S., Hädrich, S., Limpert, J., and Tünnermann, A. (2012). "Octave-spanning OPCPA system delivering CEP-stable few-cycle pulses and $22 \mathrm{~W}$ of average power at $1 \mathrm{MHz}$ repetition rate". In: Optics Express 20.10, pp. 10870-8.

Rothhardt, J., Demmler, S., Hädrich, S., Peschel, T., Limpert, J., and Tünnermann, A. (2013). "Thermal effects in high average power optical parametric amplifiers". In: Optics Letters 38.5, pp. 763-5.

Rowlinson, J. S. (1969). "Thomas Andrews and the Critical Point". In: Nature 224.5219, pp. 541-543.

Schawlow, A. and Townes, C. (1958). "Infrared and Optical Masers". In: Physical Review 112.6, pp. 1940-1949.

Schmidt, B. E., Thiré, N., Boivin, M., Laramée, A., Poitras, F., Lebrun, G., Ozaki, T., Ibrahim, H., and Légaré, F. (2014). "Frequency domain optical parametric amplification". In: Nature communications 5, pp. 36431-8.

Schultze, M., Binhammer, T., Palmer, G., Emons, M., Lang, T., and Morgner, U. (2010a). "Multi- $\mu$ J, CEP-stabilized, two-cycle pulses from an OPCPA system with up to $500 \mathrm{kHz}$ repetition rate". In: Optics Express 18.26, pp. 2729197.

Schultze, M., Binhammer, T., Steinmann, A., Palmer, G., Emons, M., and Morgner, U. (2010b). "Few-cycle OPCPA system at $143 \mathrm{kHz}$ with more than 1 microJ of pulse energy". In: Optics Express 18.3, pp. 2836-41.

Schulz, M., Riedel, R., Willner, A., Mans, T., Schnitzler, C., Russbueldt, P., Dolkemeyer, J., Seise, E., Gottschall, T., Hädrich, S., Duesterer, S., Schlarb, H., Feldhaus, J., Limpert, J., Faatz, B., Tünnermann, A., Rossbach, J., Drescher, M., and Tavella, F. (2011). "Yb:YAG Innoslab amplifier: efficient high repetition rate subpicosecond pumping system for optical parametric chirped pulse amplification". In: Optics Letters 36.13, pp. 2456-8.

Schwarz, A. (2014). "Few-cycle phase-stable infrared OPCPA". PhD thesis.

Schwarz, A., Ueffing, M., Deng, Y., Gu, X., Fattahi, H., Metzger, T., Ossiander, M., Krausz, F., and Kienberger, R. (2012). "Active stabilization for optically 
synchronized optical parametric chirped pulse amplification". In: Optics Express 20.5 , pp. 5557-5565.

Seidel, M., Brons, J., Fedulova, E., Pervak, V., Apolonski, A., Pronin, O., and Krausz, F. (2014). "High-Power Few-Cycle Pulse Generation by Spectral Broadening in Bulk Material". In: CLEO: 2014 Postdeadline Paper Digest. Washington, D.C.: OSA, STh5C.9.

Seres, J., Seres, E., Verhoef, A. J., Tempea, G., Streli, C., Wobrauschek, P., Yakovlev, V., Scrinzi, A., Spielmann, C., and Krausz, F. (2005). "Laser technology: source of coherent kiloelectronvolt X-rays." In: Nature 433.7026, p. 596.

Siddiqui, a. M., Cirmi, G., Brida, D., Kärtner, F. X., and Cerullo, G. (2009). "Generation of $<7$ fs pulses at $800 \mathrm{~nm}$ from a blue-pumped optical parametric amplifier at degeneracy". In: Optics Letters 34.22, pp. 3592-4.

Silva, F., Austin, D. R., Thai, A., Baudisch, M., Hemmer, M., Faccio, D., Couairon, A., and Biegert, J. (2012). "Multi-octave supercontinuum generation from mid-infrared filamentation in a bulk crystal". In: Nature communications 3.May, pp. 8071-5.

Skrobol, C., Ahmad, I., Klingebiel, S., Wandt, C., Trushin, S. A., Major, Z., Krausz, F., and Karsch, S. (2012). "Broadband amplification by picosecond OPCPA in DKDP pumped at 515 nm". In: Optics Express 20.4, pp. 4619-29.

Strickland, D. and Mourou, G. (1985). "Compression of amplified chirped optical pulses". In: Optics Communications 55.6, pp. 447-449.

Stuart, B., Feit, M., Herman, S., Rubenchik, A., Shore, B., and Perry, M. (1996). "Nanosecond-to-femtosecond laser-induced breakdown in dielectrics". In: Physical Review B 53.4, pp. 1749-1761.

Südmeyer, T., Brunner, F., Innerhofer, E., Paschotta, R., Furusawa, K., Baggett, J. C., Monro, T. M., Richardson, D. J., and Keller, U. (2003). “Nonlinear femtosecond pulse compression at high average power levels by use of a largemode-area holey fiber". In: Optics Letters 28.20, pp. 1951-3.

Sung, J., Lee, S., Yu, T., Jeong, T., and Lee, J. (2010). “o.1 Hz 1.o PW Ti: sapphire laser". In: Optics Letters 35.18, pp. 3021-3023.

Taha, T. R. and Ablowitz, M. I. (1984). "Analytical and numerical aspects of certain nonlinear evolution equations. II. Numerical, nonlinear Schrödinger equation". In: Journal of Computational Physics 55.2, pp. 203-230. 
Takachiho, K., Yoshimura, M., Takahashi, Y., Imade, M., Sasaki, T., and Mori, Y. (2014). "Ultraviolet laser-induced degradation of CsLiB6O1o and $\beta-\mathrm{BaB}_{2} \mathrm{O}_{4}$ ". In: Optical Materials Express 4.3, pp. 559-567.

Tate, J., Auguste, T., Muller, H., Salières, P., Agostini, P., and DiMauro, L. (2007). "Scaling of Wave-Packet Dynamics in an Intense Midinfrared Field". In: Physical Review Letters 98.1, pp. 0139011-0139014.

Tavella, F., Marcinkevičius, A., and Krausz, F. (2006). "Investigation of the superfluorescence and signal amplification in an ultrabroadband multiterawatt optical parametric chirped pulse amplifier system". In: New Journal of Physics 8.10, pp. 2191-21911.

Teisset, C. (2009). "Few-cycle high-repetition-rate optical parametric amplifiers and their synchronisation schemes". PhD thesis.

Teisset, C., Schultze, M., Bessing, R., Haefner, M., Prinz, S., Sutter, D., and Metzger, T. (2013). "300 W Picosecond Thin-Disk Regenerative Amplifier at $10 \mathrm{kHz}$ Repetition Rate". In: Advanced Solid-State Lasers Congress Postdeadline, JTh5A.1.

Teisset, C., Ishii, N., Fuji, T., Metzger, T., Kohler, S., Baltuska, A., Krausz, F., and Zheltikov, A. (2005). "All-optical pump-seed synchronization for fewcycle OPCPA". In: (CLEO). Conference on Lasers and Electro-Optics, 2005. IEEE, 25-27 Vol. 1 .

Telle, H., Steinmeyer, G., Dunlop, A., Stenger, J., Sutter, D., and Keller, U. (2014). "Carrier-envelope offset phase control: A novel concept for absolute optical frequency measurement and ultrashort pulse generation". In: Applied Physics B 69.4, pp. 327-332.

Thai, A., Skrobol, C., Bates, P. K., Arisholm, G., Major, Z., Krausz, F., Karsch, S., and Biegert, J. (2010). "Simulations of petawatt-class few-cycle opticalparametric chirped-pulse amplification, including nonlinear refractive index effects". In: Optics Letters 35.20, pp. 3471-3.

Verluise, F., Laude, V., Cheng, Z., Spielmann, C., and Tournois, P. (2000). "Amplitude and phase control of ultrashort pulses by use of an acoustooptic programmable dispersive filter: pulse compression and shaping". In: Optics Letters 25.8, pp. 575-7.

Voronin, A. A., Mikhailova, J. M., Gorjan, M., Major, Z., and Zheltikov, A. M. (2013). "Pulse compression to subcycle field waveforms with split-dispersion cascaded hollow fibers". In: Optics Letters 38.21, pp. 4354-7. 
Waxer, L. J., Bagnoud, V., Begishev, I. A., Guardalben, M. J., Puth, J., and Zuegel, J. D. (2003). "High-conversion-efficiency optical parametric chirpedpulse amplification system using spatiotemporally shaped pump pulses". In: Optics Letters 28.14, pp. 1245-1247.

Wei, Z., Hao, T., Chen-Xia, Y., Xin, Z., Xun, H., and Zhi-Yi, W. (2010). “Generation of Sub-2 Cycle Optical Pulses with a Differentially Pumped Hollow Fiber". In: Chinese Physics Letters 27.5, pp. 0542111-4.

Wirth, A., Hassan, M. T., Grguras, I., Gagnon, J., Moulet, A., Luu, T. T., Pabst, S., Santra, R., Alahmed, Z. A., Azzeer, A. M., Yakovlev, V. S., Pervak, V., Krausz, F., and Goulielmakis, E. (2011). "Synthesized light transients". In: Science (New York, N.Y.) 334.6053, pp. 195-200.

Wnuk, P., Stepanenko, Y., and Radzewicz, C. (2010). “High gain broadband amplification of ultraviolet pulses in optical parametric chirped pulse amplifier". In: Optics Express 18.8, pp. 7911-6.

Yanovsky, V., Chvykov, V., Kalinchenko, G., Rousseau, P., Planchon, T., Matsuoka, T., Maksimchuk, A., Nees, J., Cheriaux, G., Mourou, G., and Krushelnick, K. (2008). "Ultra-high intensity- 300-TW laser at 0.1 Hz repetition rate". In: Optics Express 16.3, pp. 2109-14.

Zelmon, D. E., Small, D. L., and Jundt, D. (1997). “Infrared corrected Sellmeier coefficients for congruently grown lithium niobate and $5 \mathrm{~mol} \% \mathrm{mag}$ nesium oxide -doped lithium niobate". In: Journal of the Optical Society of America B 14.12, pp. 3319-22.

Zewail, A. H. (2000). "Femtochemistry: Atomic-Scale Dynamics of the Chemical Bond †". In: The Journal of Physical Chemistry A 104.24, pp. 5660-5694.

Zhang, D., Kong, Y., and Zhang, J.-y. (2000). “Optical parametric properties of 532-nm-pumped beta-barium-borate near the infrared absorption edge". In: Optics Communications 184.5-6, pp. 485-491.

Zhao, B., Jiang, Y., Sueda, K., Miyanaga, N., and Kobayashi, T. (2008). “Ultrabroadband noncollinear optical parametric amplification with LBO crystal". In: Optics Express 16.23, pp. 18863-8.

Zou, Q. and Lü, B. (2007). "Propagation properties of ultrashort pulsed beams with constant waist width in free space". In: Optics $\mathcal{E}$ Laser Technology 39.3, pp. 619-625. 



\section{Acknowledgment}

Finally this page, the most important one! In my opinion doing a $\mathrm{PhD}$ is not just about feeding ones curiosity, but also a half-a-decade combat to overcome difficulties and to test one's willpower. It is about us, with all our weaknesses and strengths, dreams and hopes, teaming up to explore an unknown. Sometimes we succeed, sometimes not.

During my $\mathrm{PhD}$, beside the exciting and joyful moments in the lab and enjoyable discussions about Physics, I also learned a lot about us, human beings. In the last six years I had the chance to work on several projects with many different groups of colleagues which was a unique experience and for sure I could not see the light at the end of the tunnel without the help of my colleagues, the facility at Attosecond division and the provided environment at the Max Planck Institute.

In the very first place, I would like to thank Prof. Ferenc Krausz.

Kedves Ferenc! Először is köszönöm, hogy megbíztál bennem. Világosan emlékszem 2008 novemberére, amikor egy időpontot kértem az MPQ igazgatójánál és te elmagyaráztad nekem hogyan is múködik egy OPA és egy "enhancement cavity". Egy egész évembe került mire teljesen meggyőztelek, hogy felvegyél. Remélem ezen döntésedet azóta sem bántad meg!

Köszönet a sok lehetőségért, amiket az utóbbi hat évben biztosítottál számomra, és az élvezetes tudományos megbeszélésekért, különösen az elmúlt évben. Nagyon sokat tanultam tőled, azonban az akaraterőd, lelkesedésed, energiád és határozottságod, olyan leckék voltak, amik egész szakmai életem során kiváló példaként fognak szolgálni. (ne lepődj meg a folyékony magyar tudásomon, ugyanis egy kicsit segített László is a fordításban ;)

My very big and fat and special thanks goes to Dr. Nicholas Karpowicz for being such an excellent scientist, person, and friend. Nick! Thanks for all the help in the last three years. Thanks for being so patient in listening to me the whole day! That's not easy, I know! Thanks for all the physics plus wine evenings at MPQ! Thanks for the joyful discussions in G2.22 (the best office in the world). Thanks for everything! 
Dear Dr. Thomas Metzger! Many thanks! Thanks for the encouragements, for being there whenever we were frustrated with the laser and whenever I was frustrated with my life!

Dear Prof. Reinhard Kienberger! Millions of thanks for everything!

Many thanks to Dr. Alexander Apolonskiy , Prof. Reinhard Kienberger, Dr. Zsuzsanna Major and Dr. Nicholas Karpowicz, the project coordinators that I had the pleasure to work as a member of their team during the last six years.

Thanks to Dr. Nicholas Karpowicz, Dr. Zsuzsanna Major, Dr. Shawn Sederberg, Dr. Wolfgang Schweinberger and Clemens Jakubeit for the proof reading of my thesis and to Dr. Martin Schultze for helping me with writing the Zusammenfassung and for making my Abstract fancy!

Martin! Thanks for the support, for the Duplos, verdicts, and for the fun lunch, dinner, and bakery breaks!

Dear Dr. Elisabeth Bothschafter! My girl! thanks for the rides to Alte Heide, for the midnight-coffee-breaks at MPQ and for all the help and support.

Dear Dr. Alexander Schwarz! SBCE! Thanks for suffering with me in the lab aligning the THG FROG and running the devil IR wincam! Thanks for pizza-at-11-pm and the night-shifts in the lab. No thanks for your continuous complaints and thanks for listening to my continuous complaints!

Very special thank to the "dinner at MPQ" team, for cheese+tomato+bread every night and for their scientific and social support: lovely Annkatrin Sommer, Sabine Keiber, and Elisabeth Bothschafter, Martin Schultze, Michael Jobst, Tobias Latka, Markus Ossiander, Johann Riemensberger, Nicholas Karpowicz, Clemens Jakubeit, Agustin Schiffrin, and Tim Paasch-Colberg.

Very special thanks to Dr. Volodymyr Pervak for the chirped mirrors and for being a cool friend!

Special thanks to Prof. Matthias Kling and Prof. Peter Hommelhoff, Dr. Nicholas Karpowicz, and Dr. Vladislav Yakovlev for all the help and support.

Many many thanks to the best Roswitha Graf ever, the most supportive Wolfgang Schweinberger, lovely Biddor Alsaif, the clever Antoine Moulet, Xia Tau Geng, the kindest Dr. Wolfram Helml, Dr. Catherine Teisset, Dr. Oleg Pronin, Dr. Christoph Skrobol, Olga Razskazovskaya, Dr. Yunpei Deng, Tran Trung Luu, Dr. Xun Gu, Dr. Thomas Ganz, Lauryna Lötscher, Dr. Ioachim Pupeza, Waldemar Schneider, Tibbor Wittmann, Alexander Kessel, Dr. Antonin Borot, Dr. Julian Bertrand, Dr. Matthew Weidman, Dr. Agustin Schiffrin, Dr. Julia Mikhailova, Dr. Patrik Heißler, Dr. Martin Gorjan, Jakub Novak, Michal Chyla, and Shao-Wei Chou.

Thanks to Dr. Laszlo Veisz, Prof. Stephan Karsch, Dr. Eleftherios 
Goulielmakis, Dr. Sergei Trushin, Dr. Hatmut Schröder and Dr. Boris Bergues for the scientific discussions and support.

Thanks to the best master students ever, the very young Dominik Franz, Martin Schmidberger, Arohi Jain, and Amelia Wigianto!

Special thanks to Moritz Ueffing, Simon Holzner, Clemens Jakubeit, Elisabeth Bothschafter and Alexander Schwarz for calling me the "best colleague ever" in their thesis and indeed they are "The best colleagues ever!" ;).

Very special thanks to the members of the "Italian Restaurant" project.

A very special thank to Olga Razskazovskaya for "preparing an article"!

A very very special thank to the kindest Frau Wild in the world, and lovely Tanya Bergues, Franziska Hoss, Simone Mann and Renate Sailer; dear Klaus Franke, Christian Hackenberger, Nils Haag and Thorsten Naeser.

Thanks to MPQ workshop.

My lovely parents! thanks for every single moment of my life.

And the biggest thanks to Asghar, the kindest, most supportive, cleverest husband ever! 



\title{
Curriculum Vitae
}

\section{Personal data}

\author{
Name Hanieh Fattahi \\ Address Max Planck Institute of Quantum Optics (MPQ), \\ Hans-Kopfermann-Str. 1, 85748 Garching \\ Phone $\quad+49-89-32905732$ \\ E-mail hanieh.fattahi@mpq.mpg.de \\ Date of birth $\quad$ 16th September 1981 \\ Place of birth Tehran, Iran
}

\section{Education}

2008-present Doctoral candidate in the group of Prof. Ferenc Krausz at Max Planck Institute of Quantum Optics

Member of the International Max Planck Research School of Advanced Photon Science (IMPRS-APS)

2005-2007 M.Sc. in Applied Physics

Department of Physics

Sharif University of Technology, Tehran, Iran

2000-2004 B.Sc. in Applied Physics

Department of Physics

Zanjan University, Zanjan, Iran

\section{Scholarships}

2008-2012 The International Max Planck Research School of Advanced Photon Science 


\section{Professional affiliation}

2009-present Optical Society of America (OSA) 